FHWA/IN/JTRP-2000/6

Final Report

EVALUATION OF LIGHTWEIGHT NON-CONTACT PROFILERS

\author{
Amit Mondal \\ Adam J. Hand \\ David R. Ward
}

July 2000 
Final Report

FHWA/IN/JTRP-2000/6

\title{
Evaluation of Lightweight Non-Contact Profilers
}

\author{
by \\ Amit Mondal \\ Adam J. Hand \\ and \\ David R. Ward \\ Joint Transportation Research Program \\ Project Number: C-36-630 \\ File Number: 09-07-15 \\ SPR-2302 \\ Prepared as Part of an Investigation Conducted by the \\ Joint Transportation Research Program \\ Purdue University \\ In cooperation with the \\ U.S. Department of Transportation \\ Federal Highway Administration
}

The contents of this report reflect the views of the authors who are responsible for the facts and accuracy of the data presented herein. The contents do not necessarily reflect the official views or policies of the Indiana Department of Transportation or the Federal Highway Administration at the time of publication. This report does not constitute a standard, specification, or regulation.

\author{
School of Civil Engineering \\ Purdue University \\ West Lafayette, Indiana 47907
}

July 2000 


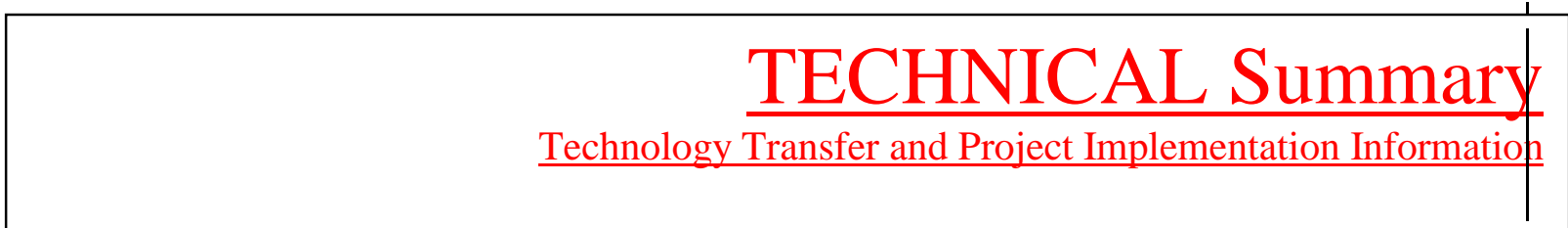

July 2ф00

Final Report

\section{Evaluation of Lightweight Non-Contact Profilers}

\section{Introduction}

In a recent FHWA survey pavement smoothness was identified as the most significant factor the motoring public uses to judge the quality of our Nation's roadways. A new generation of lightweight profilers has been developed which have the potential to provide nearly instantaneous pavement smoothness measurements providing contractors with a tool to identify process control issues more promptly and cost effectively. This could ultimately translate into more rapidly constructed smoother pavements for motorists. A team of INDOT and Purdue University researchers cooperatively evaluated the new generation of profilers through a JTRP research project with partial support from a FHWA initiative.

The objective of the research was to field test and evaluate various stateof-the-art lightweight non-contact profilers, which would provide the data from which end result guide smoothness specifications could be developed for QC/QA purposes. The project was focused directly on one of the primary objectives of the INDOT Strategic Plan, improving pavement smoothness.

Several lightweight profilers along with conventional tools were used to measure the roughness of three each PCC and HMA pavements located near
West Lafayette Indiana during the Summer of 1999. Four lightweight profilers and the INDOT RIP Van performed five replicate measurements at each site. Additionally, California Profilograph tests and a precision rod and level survey were also conducted at one site for comparison with the lightweight profilers. Each test section was 0.1 miles in length and several incorporated curves and grades to test the sensitivity of the profilers to such geometric features. At two of the test sites boards were placed in the test wheel path to simulate bumps and/or bridge abutments, again to assess the response of the profilers to such features.

Consultants and contractors currently performing smoothness measurements on INDOT projects were encouraged to observe/participate in the field evaluation. A field open house was held during one day of the testing and was attended by over forty INDOT, FHWA, consultant, contractor, and producer personnel. This provided excellent exposure of the new technology and demonstrated INDOTs willingness to involve industry in specification development.

The field generated data were analyzed to evaluate the repeatability and reproducibility of the profilers. In other words within and between vendor profiler performance was assessed. The 
smoothness indices considered were IRI and PI with 0.0 and 0.2 inch blanking bands. Poor reproducibility was identified which led to an analysis of the actual profiles, rather than smoothness statistics. The ability of the profilers to identify bumps or must grind locations was also considered in this process.

The data were then used to develop precision statements for the lightweight profilers in accordance with ASTM standards. Data were also obtained from a concurrent lightweight profiler evaluation performed by the Connecticut DOT. Precision statements were developed for the Connecticut data as well as for the Indiana and Connecticut data pooled together.

A review of current trends in the use of smoothness specifications, indices and equipment employed in them, and pay adjustment factors was performed along with a review of critical aspects of smoothness specifications. Other state specifications were also obtained and reviewed. This information was used to develop a draft smoothness specification relying on the use of lightweight profilers for INDOT. The specification was then applied to the field generated data to assess its validity.

\section{Findings}

Analysis of the collected field test data showed that the quality of the data was good. The data clearly showed that PI with a 0.0 inch blanking band is better index for assessing pavement smoothness then PI with a 0.2 inch blanking band. Reported PI values with the 0.2 inch blanking band at several sites were 0in/mi. However when observed values of PI with a 0.0 inch blanking band for the same sites were reviewed measurable differences in smoothness were observed.

Statistical analysis of the field generated data showed that the individual lightweight profilers exhibited good consistency or repeatability. The data did not suggest that boards used at two sites or the geometric features at any of the sites impacted the performance of the profilers. However, the analysis revealed that the profilers exhibited poor reproducibility or between vendor consistency. In fact, paired t-tests of all possible one-to-one vendor combinations showed that the different devices only reported similar average values for individual test sites approximately 25 percent of the time, regardless of the smoothness index considered. The data generated with the INDOT Rip Van did not correlate well with any of the lightweight profilers. Similar findings were observed in a recent Connecticut DOT evaluation of the same lightweight profilers. This finding was very disturbing and led to an analysis of the actual profiles generated by each profiler at each site.

The profiles were compared among themselves and between profilers. The comparisons again showed good individual profiler repeatability, but they did reveal the fact that the performance of some profilers was adversely affected by the boards used at one site and the geometric features at two sites. The between vendor profile comparisons showed very poor reproducibility. This provided an explanation for the poor IRI and PI reproducibility. The INDOT RIP Van, California Profilograph and precision rod and level survey data were included in the analysis. The California 
Profilograph profile was more similar to the rod and level survey data than the lightweight profilers. However, the lightweight profilers identified handtooled transverse joints in the pavement that the other techniques were insensitive to. The INDOT Rip Van Profiles were not similar to any of the other profiles.

An analysis of the ability of the lightweight profilers to provide bump identification or must grind locations given a specified maximum allowable bump was also conducted. All of the devices were capable of identifying the location and magnitude of thin boards placed in the wheel path at one site and three of four also identified the location and magnitude of thick boards used at another other site. The thick boards did induce significant dynamics to several of the profilers and the data suggests that the performance of the accelerometers used by two of the vendors were adversely affected by the dynamics.

With recognition of the poor reproducibility observed, precision statements were developed in accordance with ASTM standards for IRI under five cases and PI under three cases in an effort to establish overall estimates of repeatability and reproducibility for the lightweight profilers. These efforts ultimately revealed good repeatability and poor reproducibility as expected.

The data generated in a recent Connecticut DOT evaluation of the same lightweight profilers was obtained and a precision statement was generated with it for IRI. This data resulted in similar estimates of repeatability and reproducibility to those observed with the Indiana generated data. In order to improve the power of the precision estimates for IRI, the Indiana and Connecticut data were pooled to develop another precision statement. This resulted in precision estimates similar to those observed in other analyses.

Unfortunately, the precision analysis revealed that the lightweight profiler technology needs to be further refined in order to develop smoothness specifications that rely upon their use, at least those evaluated in Indiana and Connecticut. The profilers evaluated are actually the only devices commercially available at this point in time. The reason refinements are needed is that both the repeatability in most cases and reproducibility limits in all cases observed for IRI and PI both exceed the increments in smoothness (5 to $10 \mathrm{in} / \mathrm{mi}$ for pay factor adjustments) used in most specifications today.

The observed reproducibility limit for IRI with the Indiana and Connecticut data pooled was $31.8 \mathrm{in} / \mathrm{mi}$. This means that based on the performance of the profilers evaluated, the difference in IRI reported by two different vendors at a single site could be up to $31.8 \mathrm{in} / \mathrm{mi}$ simply due to the inherent variability of the test. This value $(31.8 \mathrm{in} / \mathrm{mi})$ is obviously much larger than the 5 to $10 \mathrm{in} / \mathrm{mi}$ increments currently in specifications. The observed repeatability limit for the pooled data was $10.7 \mathrm{in} / \mathrm{mi}$. This suggests that if the same device and operator perform replicate measurements at the same site the difference in IRI reported for the two measurements could be up to $10.7 \mathrm{in} / \mathrm{mi}$ simply due to the variability of the test. This difference in itself is greater than the limits used in specifications for 
payment. Similar observations are made when PI was considered.

What the precision statements as well as the statistical analysis of means actually indicate is that the incremental pay adjustment steps used in specifications could very likely lead to situations where one lightweight would indicate that a significant penalty should be imposed while another would indicate that a significant bonus should be paid. The data also suggest that the variability observed for the profilers evaluated could very easily lead to disputes in QC/QA situations when different brands of profilers were used by different parties. The disputes could also occur if the same brand of profiler were used by both parties. This means that disputes between the state and the contractor, as well as the contractor and his/her subcontractor could easily develop which none of the potentially involved parties like to see occur.

Even though the statistical analysis and precision statement tasks revealed unacceptable inconsistency among the lightweight profilers a smoothness specification was drafted.

The developed draft specification relies on IRI and PI with a 0.0 inch blanking band for smoothness measures of HMA and PCC pavements, respectively. It also incorporates pay adjustment factors for both pavement types. The data generated as part of the lightweight profiler evaluation was substituted into several state smoothness specifications and reviewed in light of inspections of the field sites tests. Based on this pay adjustment factors were proposed for the Indiana specification. The proposed specifications (pay adjustment factors) were then applied to the field test data generated in Indiana. The payment based on this process appeared to be reasonable for the sites considered. It is important to note however that the sites evaluated were either very smooth or very rough. Therefore the proposed draft specification should be viewed with caution.

\section{Implementation}

The findings of this research should be implemented as soon as practically possible. The implementation would require that an implementation study be initiated in which personnel be assigned to monitor developments and review forthcoming state and FHWA reports on concurrent evaluations in other states. They should also obtain and review the forthcoming AASHTO Guide Specification in light of the evaluation and findings stated in this report.

The current INDOT smoothness specification should be modified to incorporate PI with a 0.0 inch blanking band, rather than a 0.2 inch blanking band. This should be done based on the pay factor adjustments in the proposed draft specification presented in Section 6 of this report, the other specifications reviewed in this report, and through interaction with industry in the process.

The modified specification should then be evaluated on a trial basis on several projects in the coming construction season for informational purposes rather than payment. The data generated through this process should then be used to validate or further refine the proposed pay factor adjustments.

INDOT should support and cooperate in future efforts to refine the 
lightweight profiler technology. It should also make every effort possible to implement the use of lightweight profilers for smoothness measurements once the technology is refined. This may even dictate the investment in one or more lightweight profilers in the near future.

\section{Contacts}

For more information contact:

Professor Adam J. Hand

Principal Investigator

School of Civil Engineering

Purdue University

West Lafayette, IN 47907-1284

Phone: (765) 496-3996

Facsimile: (765) 496-1364

\section{Mr. David R. Ward}

Co-Principal Investigator

Indiana Department of Transportation

Division of Research

1205 Montgomery Street

P.O. Box 2279

West Lafayette, IN 47906

Phone: (765) 463-1521

Facsimile: (765) 497-1665

\section{Purdue University}

Joint Transportation Research Program School of Civil Engineering

West Lafayette, IN 47907-1284

Phone: (765) 494-9310

Facsimile: (765) 496-1105 


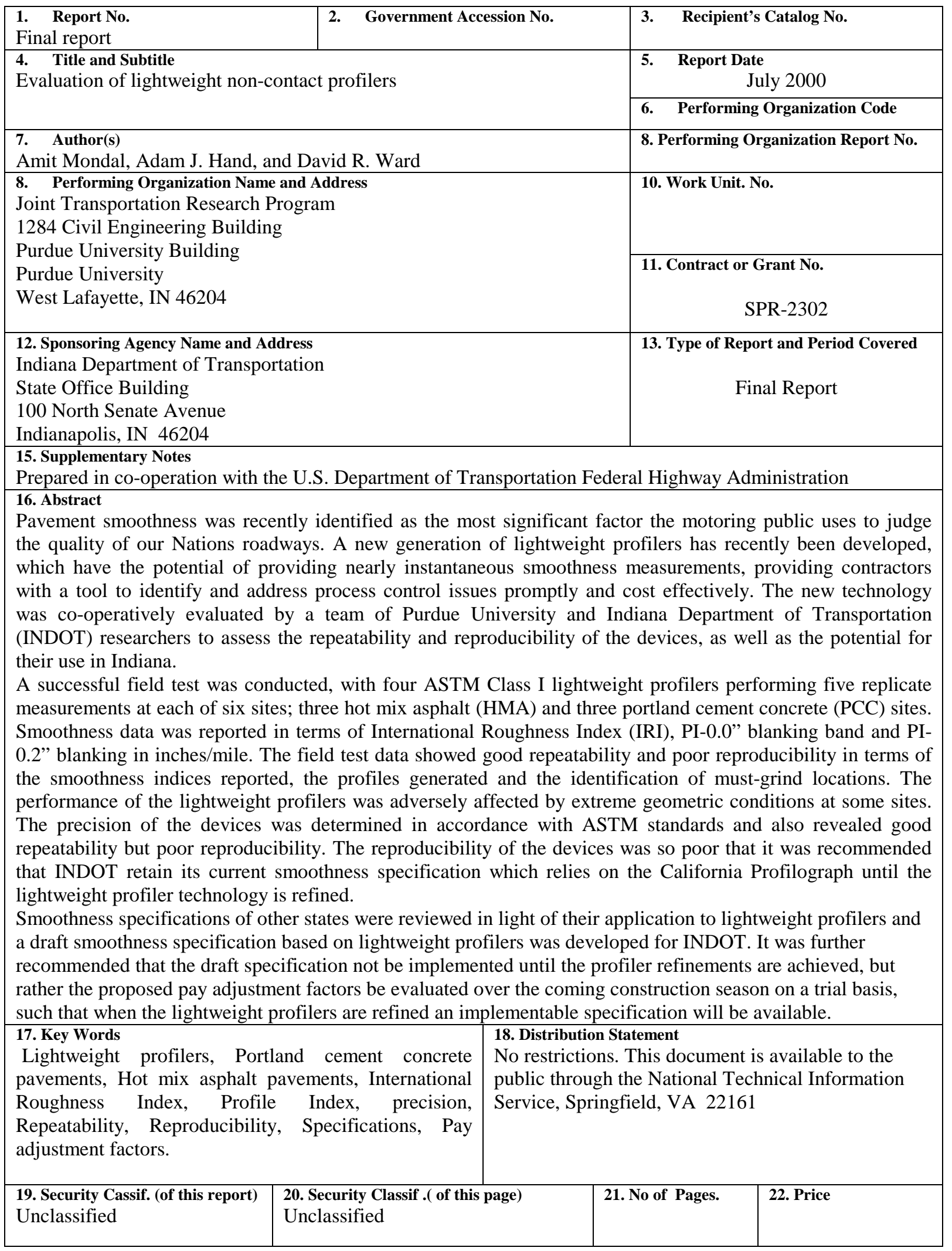




\section{Table of Contents}

LIST OF FIGURES.............................................................

LIST OF TABLES .......................................................

IMPLEMENTATION RECOMMENDATIONS ...............................................

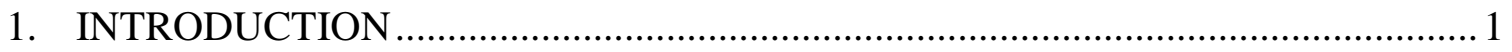

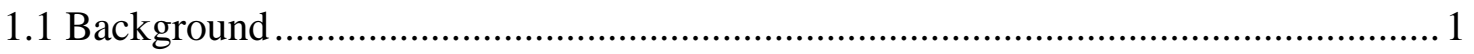

1.2 Project Scope and Objectives......................................................................... 4

2. HISTORY AND PERFORMANCE OF PROFILERS …................................. 7

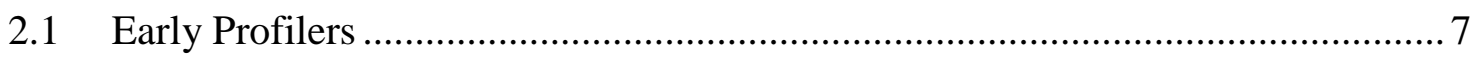

2.1.1 Via-Graph.........................................................

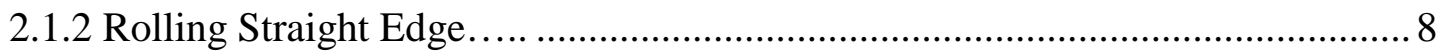

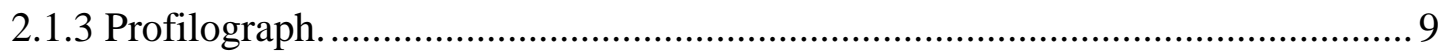

2.1.3.1 California Profilograph........................................ 11

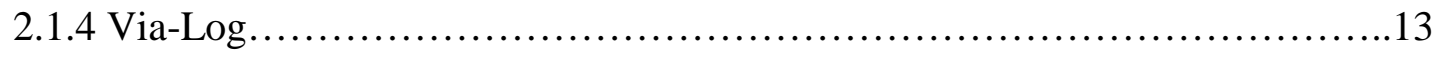

2.1.5 Bureau of Public Roads Roughometer (BPR)...........................13

2.1.6 CHLOE............................................................. 14

2.1.7 Response-Type Road Roughness Measuring Systems (RTRRMS)..........15

2.1.8 Inertial Profilers...................................................... 16

2.1.8.1 South Dakota Profiling System............................... 19

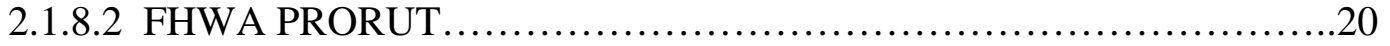




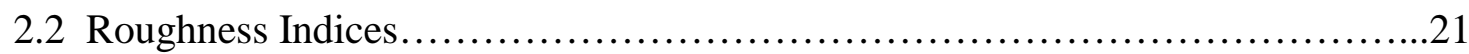

2.2.1 International Roughness Index...................................... 21

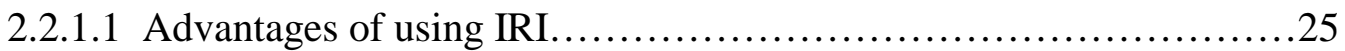

2.2.1.2 Disadvantages of using IRI.................................... 25

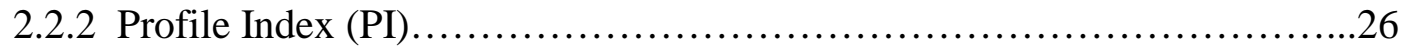

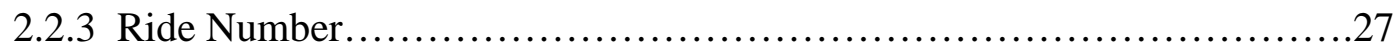

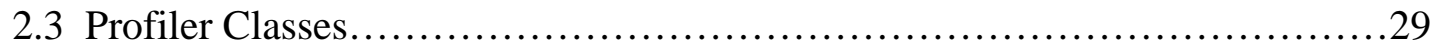

2.3.1 Rod and Level Surveys........................................... 30

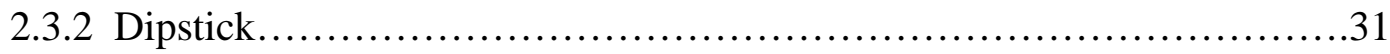

2.3.3 Modern Inertial Profiler...............................................33

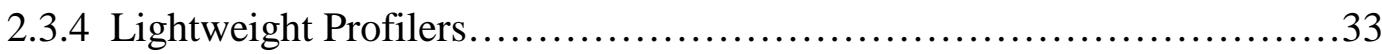

2.4 Factors affecting profile measurement........................................

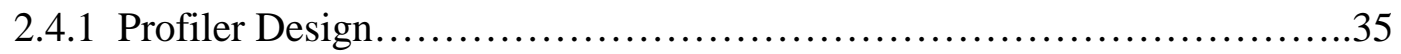

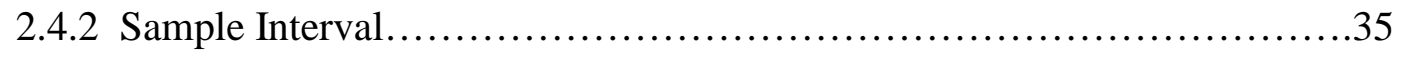

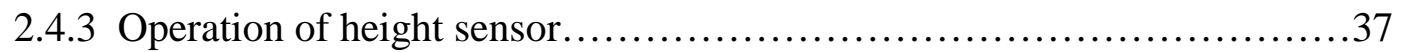

2.4.4 Operation of accelerometer.......................................... 38

2.4.5 Longitudinal Distance Measurement......................................38

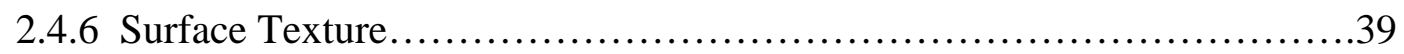

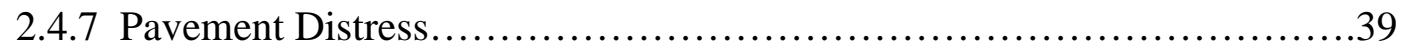

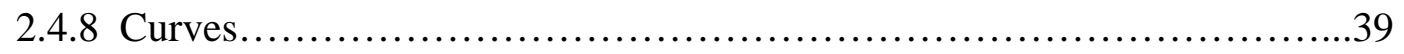

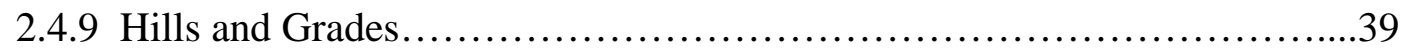

2.4.10 Measuring Environment...........................................40 
2.4.11 Profiler Operation.................................................. 40

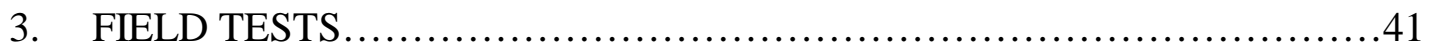

3.1 Identification of Lightweight Profiler Vendors.............................41

3.2 Experimental Design.................................................. 42

3.3 Site Selection Process................................................ .45

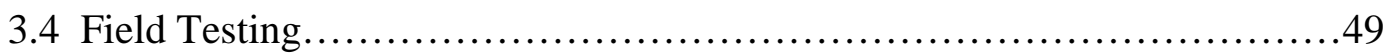

3.5 Description of Profilers................................................ 52

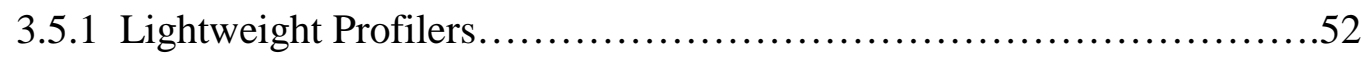

3.5.1.1 Ames Engineering Incorporated.............................53

3.5.1.2 International Cybernetics Corporation............................54

3.5.1.3 K J Law Engineering............................................56

3.5.1.4 Pathway Services Incorporated..............................5

3.5.2 INDOT Rip Van Inertial High Speed Profiler...........................59

3.5.3 California Profilograph.............................................60

3.5.4 Precision Survey .....................................................61

3.6 Distance Calibration.................................................62

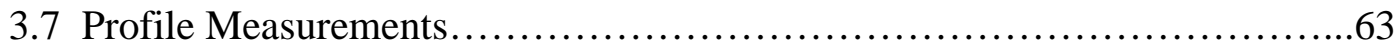

3.7.1 Site 1 (Covington Street) Measurements.................................64

3.7.2 Site 2 (Interstate 65 ) Measurements.................................64

3.7.3 Site 3 (Prairie Street) Measurements................................65

3.7.4 Site 4 (Vinton Street) Measurements............................... 65

3.7.5 Site 5 (US 231 A) Measurements.....................................66 


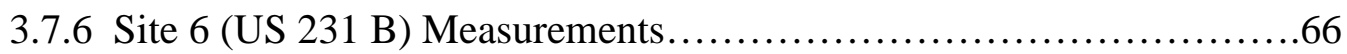

3.8 Other Field Activities....................................................66

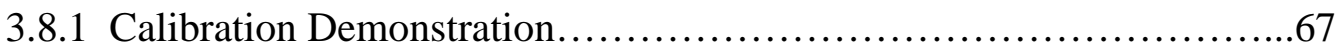

3.8.2 Field Open House.................................................67

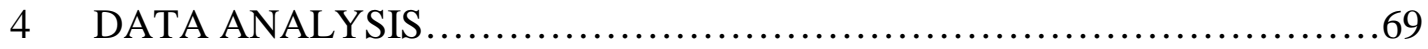

4.1 Smoothness Indices Data by Vendor....................................70

4.1.1 Within Vendor Comparision...................................... . 86

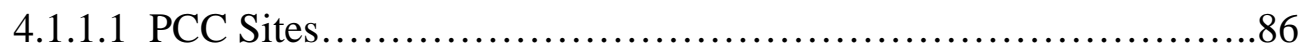

4.1.1.2 HMA Sites...............................................96

4.1.1.3 Within Vendor Standard Deviations.............................111

4.1.2 Between Vendor Comparison......................................113

4.1.2.1 PCC Sites....................................................... 113

4.1.2.2 HMA Sites................................................ 118

4.2 Smoothness Rankings by Vendor......................................125

4.3 Statistical Analysis of Between Vendor Performance.......................128

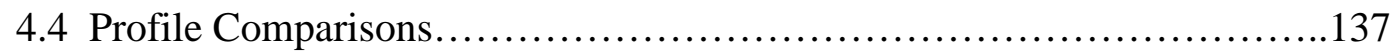

4.4.1 Within Vendor Profile Comparison.................................138

4.4.1.1 Vendor A Profile Comparison.................................. 138

4.4.1.2 Vendor B Profile Comparison.................................142

4.4.1.3 Vendor C Profile Comparison................................. 146

4.4.1.4 Vendor D Profile Comparison................................. 149

4.4.1.5 Overall Within Vendor Profile Performance.......................152 
4.4.2 Between vendor Profile Comparison............................. 153

4.4.2.1 Covington Street (Site-1) Profile Comparison...................154

4.4.2.2 I-65 (Site 2) with Plywood Strips Profile Comparison.............155

4.4.2.3 I-65 (Site 2b) Without Plywood Strips Profile Comparison........156

4.4.2.4 Prairie Street (Site 3) with Plywood Strips Profile Comparison...157

4.4.2.5 Prairie Street (Site 3b) without Plywood Strips Profile

Comparison...........................................158

4.4.2.6 Vinton Street (Site 4) Profile Comparison.........................158

4.4.2.7 US 231A (Site 5) Profile Comparison..........................159

4.4.2.8 US 231B (Site 6) Profile Comparison..........................159

4.4.2.9 Overall Between Vendor Profile Performance.......................160

4.5 Must Grind Locations................................................ 161

PRECISION ANALYSIS ............................................ 165

5.1 Test Method Precision............................................. 166

5.1.1 Application of Analysis of Variance (ANOVA) in Generating Precision

Indices.................................................. 171

5.1.2 Basis of Precision Statements.................................... 173

5.1.2.1 Basis of Acceptable Range of Results.........................175

5.2 Development of Precision Statements................................ 175

5.2.1 Case 1 : Precision Based on Indiana Generated Data with Boards........175

5.2.1.1 Analysis of IRI Data....................................... 176

5.2.1.2 Analysis of PI-0.0" Data.................................... 184 


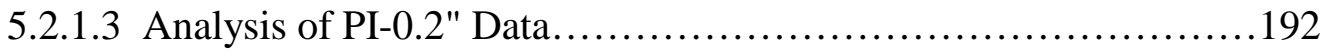

5.2.2 Case 2 : Precision Based on Indiana Generated Data without Boards....200

5.2.2.1 Analysis of IRI Data......................................200

5.2.2.2 Analysis of PI-0.0" Data..................................208

5.2.2.3 Analysis of PI-0.2" Data.................................. 215

5.2.3 Case 3 : Precision Based on Indiana Generated Data with Boards

Excluding Vendor A.........................................223

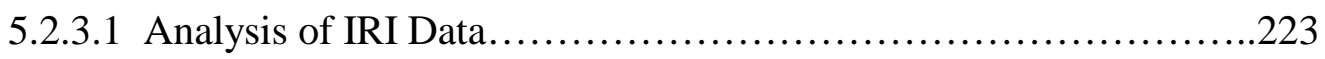

5.2.3.2 Analysis of PI-0.0" Data.................................230

5.2.3.3 Analysis of PI-0.2" Data.....................................237

5.2.4 Case 4 : Precision Based on Connecticut Generated Data with Boards...245

5.2.4.1 Analysis of Data...............................................246

5.2.5 Case 5 : Precision Based on pooled Indiana and Connecticut data........253

5.2 .4 .1 Analysis of Data......................................... 254

5.3 Comparison of Developed Statistics...............................261

6 SMOOTHNESS SPECIFICATIONS ...............................263

6.1 Smoothness Specification Literature...................................264

6.2 Critical Aspects of Smoothness Specifications Based on the Use of Inertial

Profilers.........................................................266

6.2.1 Choice of Smoothness Index...................................266

6.2.2 Choice of Wheel Path.........................................267

6.2.3 Section Length............................................268 
6.2.4 Number of Replicate Measurements

6.3 Review of Smoothness Specifications 268

6.3.1 Rigid Pavement Smoothness Specifications.... .269

6.3.1.1 Connecticut DOT- Rigid Pavement Smoothness Specification....269

6.3.1.2 Georgia DOT- Rigid Pavement Smoothness Specification.........271

6.3.1.1 Kansas DOT- Rigid Pavement Smoothness Specification. .272

6.3.1.1 Pennsylvania DOT- Rigid Pavement Smoothness Specification...275

6.3.2 Smoothness Specification of Flexible Pavements. .278

6.3.2.1 Arizona DOT- Flexible Pavement Smoothness Specification.....279

6.3.2.2 Connecticut DOT- Flexible Pavement Smoothness Specification.282

6.3.2.3 Florida DOT- Flexible Pavement Smoothness Specification......284

6.3.2.4 Kansas DOT- Flexible Pavement Smoothness Specification.......285

6.3.2.5 Virginia DOT- Flexible Pavement Smoothness Specification......288

6.3.2.6 Virginia DOT- New Flexible Pavement Smoothness Specification..290

6.3.2.7 Pennsylvania DOT- Flexible Pavement Smoothness Specification..292

6.3.2.8 Draft of the Lightweight Profiler Equipment Smoothness

Specification prepared by the Texas Department of Transportation..294

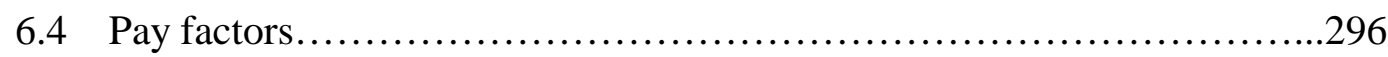

6.5 Draft Guide Pavement Smoothness Specification Based on the Use of Lightweight Non-Contact Profiler. 299

6.5.1 Test method. .299

6.5.2 Calibration and Verification 301 


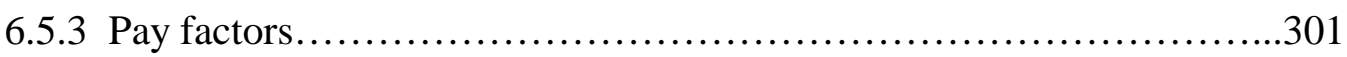

6.6 Assessment of the Proposed Guide Specification........................303

$7 \quad$ SUMMARY AND CONCLUSION.................................. 307

7.1 Recommendations ............................................ 314

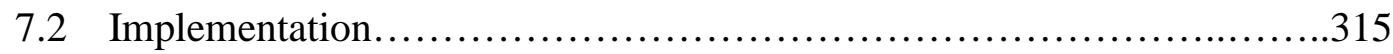

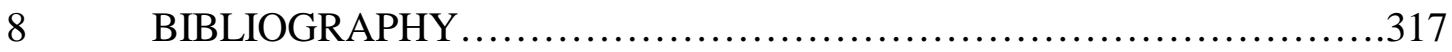

APPENDIX A - IRI Comparison Within Vendors............................. 322

APPENDIX B - PI-0.0" Comparison - Within Vendors.............................339

APPENDIX C - PI-0.2” Comparison - Within Vendors.......................... 356

APPENDIX D - Between Vendors Comparison of Smoothness Indices............371

APPENDIX E - Within Vendor Profile Comparison..........................384

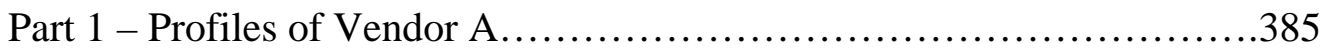

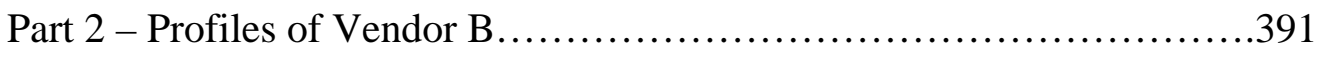

Part 3 - Profiles of Vendor C .....................................397

Part 4 - Profiles of Vendor D.......................................403

APPENDIX F - Between Vendor Profile Comparison..........................409

APPENDIX $\mathrm{G}-$ Summary $\mathrm{h}$ and $\mathrm{k}$ analysis.............................418 


\section{List of Figures}

Figure $\quad$ Page

2-1 Viagraph- Profiler Used in the Early 1900's...............................

2-2 California Profilograph............................................... 9

2-3 Top and Side View of a Rainhart Profilograph............................. 10

2-4 BPR Roughometer.................................................. 14

2-5 CHLOE........................................................... 14

2-6 May's Ride Meter.................................................... 16

2-7 Schematic diagram of an inertial profiler................................. 17

2-8 Quarter-Car Model......................................................23

2-9 Schematic diagram of Rod and Level Survey.............................. 31

2-10 Dipstick device......................................................

2-11 Profiling with DipStick............................................... 32

2-12 Lightweight Non-Contact Profiler........................................ 34

3-1 Ames Engineering Incorporated Lightweight Profiler.........................54

3-2 International Cybernetics Corporation Lightweight Profiler.....................55

3-3 K J Law Engineering Lightweight Profiler................................57

3-4 Pathway Services Incorporated Lightweight Profiler.........................58

3-5 INDOT Rip Van High Speed Profiler.....................................60

3-6 California Profilograph (Manufacturer Ames Engineering Inc.)................61

3-7 Laying out Wheel Paths with Paint Blotches...............................63

4-1 Replicate IRI measurements by Vendor A at Site $1 \ldots \ldots \ldots \ldots \ldots \ldots \ldots \ldots \ldots \ldots . \ldots \ldots$ 
$\begin{array}{lll}\text { Figure } & \text { Page }\end{array}$

4-2 Replicate IRI measurements by Vendor B at Site $1 \ldots \ldots \ldots \ldots \ldots \ldots \ldots \ldots \ldots \ldots . \ldots 8$

4-3 Replicate IRI measurements by Vendor C at Site $1 \ldots \ldots \ldots \ldots \ldots \ldots \ldots \ldots \ldots \ldots . \ldots \ldots$

4-4 Replicate IRI measurements by Vendor D at Site $1 \ldots \ldots \ldots \ldots \ldots \ldots \ldots \ldots \ldots \ldots \ldots . \ldots \ldots$

4-5 Replicate PI-0.0" measurements by Vendor A at Site 1 ........................90

4-6 Replicate PI-0.0" measurements by Vendor B at Site $1 \ldots \ldots \ldots \ldots \ldots \ldots \ldots \ldots \ldots \ldots . \ldots 9$

4-7 Replicate PI-0.0" measurements by Vendor C at Site $1 \ldots \ldots \ldots \ldots \ldots \ldots \ldots \ldots \ldots \ldots . . . . \ldots 1$

4-8 Replicate PI-0.0" measurements by Vendor D at Site $1 \ldots \ldots \ldots \ldots \ldots \ldots \ldots \ldots \ldots \ldots \ldots 1$

4-9 Replicate PI-0.2" measurements by Vendor A at Site $1 \ldots \ldots \ldots \ldots \ldots \ldots \ldots \ldots \ldots \ldots . \ldots 2$

4-10 Replicate PI-0.2” measurements by Vendor B at Site $1 \ldots \ldots \ldots \ldots \ldots \ldots \ldots \ldots \ldots . \ldots 92$

4-11 Replicate PI-0.2" measurements by Vendor C at Site $1 \ldots \ldots \ldots \ldots \ldots \ldots \ldots \ldots \ldots . . \ldots 9$

4-12 Replicate PI-0.2" measurements by Vendor D at Site 1........................93

4-13 Replicate IRI measurements by Vendor A at Site 2

(I-65 with board) ................................................ 99

4-14 Replicate IRI measurements by Vendor B at Site 2

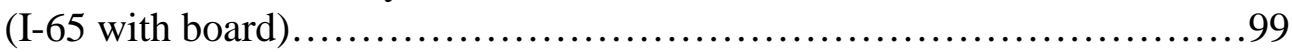

4-15 Replicate IRI measurements by Vendor C at Site 2

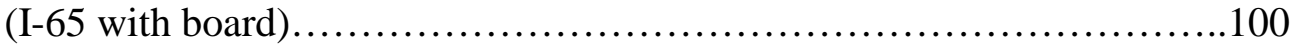

4-16 Replicate IRI measurements by Vendor D at Site 2

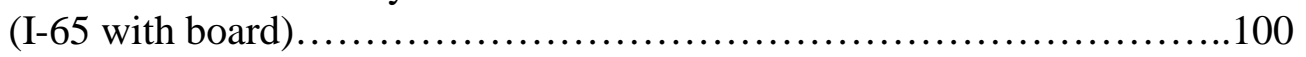

4-17 Replicate PI-0.0" measurements by Vendor A at Site 2

(I-65 with board) .....................................................

4-18 Replicate PI-0.0" measurements by Vendor B at Site 2

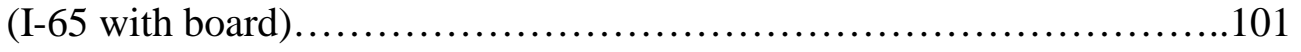

4-19 Replicate PI-0.0" measurements by Vendor C at Site 2

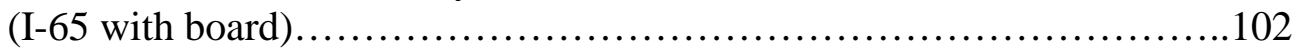


Figure

4-20 Replicate PI-0.0" measurements by Vendor D at Site 2

(I-65 with board)

4-21 Replicate PI-0.2" measurements by Vendor A at Site 2

(I-65 with board)

4-22 Replicate PI-0.2" measurements by Vendor B at Site 2

(I-65 with board)

4-23 Replicate PI-0.2" measurements by Vendor C at Site 2

(I-65 with board)

4-24 Replicate PI-0.2" measurements by Vendor D at Site 2

(I-65 with board)

4-25 Replicate IRI measurements by Vendor A at Site $2 b$

(I-65 without board)

4-26 Replicate IRI measurements by Vendor B at Site 2b

(I-65 without board)

4-27 Replicate IRI measurements by Vendor $\mathrm{C}$ at Site $2 \mathrm{~b}$

(I-65 without board)

4-28 Replicate IRI measurements by Vendor D at Site $2 b$

(I-65 without board)

4-29 Replicate PI-0.0" measurements by Vendor A at Site 2b

(I-65 without board)

4-30 Replicate PI-0.0" measurements by Vendor B at Site $2 b$

(I-65 without board)

4-31 Replicate PI-0.0" measurements by Vendor C at Site $2 \mathrm{~b}$

(I-65 without board) 108

4-32 Replicate PI-0.0" measurements by Vendor D at Site $2 b$

(I-65 without board)

4-33 Between Vendor IRI Comparison for Covington Street (Site 1) 
Figure

4-34 Between Vendor PI-0.0” Comparison for Covington Street (Site 1).............116 4-35 Between Vendor PI-0.2” Comparison for Covington Street (Site 1)..............116

4-36 IRI Between Vendor Comparison for Site 2b (I-65 without board)..............120

4-37 IRI Between Vendor Comparison for Site 2 (I-65 with board).................120

4-38 PI-0.0” Between Vendor Comparison for Site 2b (I-65 without board)............122

4-39 PI-0.0" Between Vendor Comparison for Site 2 (I-65 with board)..............122

4-40 PI-0.2” Between Vendor Comparison for Site 2b (I-65 with board)...............123

4-41 Profiles from Replicate Runs by Vendor A at Covington Street (Site 1)...........139

4-42 Profiles from Replicate Runs by Vendor A at I-65 (Site 2) with (wb)

And Without (nb) plywood strips............................... 140

4-43 Profiles from Replicate Runs by Vendor B at Covington Street (Site 1)...........143

4-44 Profiles from Replicate Runs by Vendor B at I-65 (Site 2) with (wb)

And Without (nb) plywood strips...............................144

4-45 Profiles from Replicate Runs by Vendor C at Covington Street (Site 1)...........146

4-46 Profiles from Replicate Runs by Vendor C at I-65 (Site 2) with (wb)

And Without (nb) plywood strips...............................147

4-47 Profiles from Replicate Runs by Vendor D at Covington Street (Site 1)...........150

4-48 Profiles from Replicate Runs by Vendor D at I-65 (Site 2) with (wb)

And Without $(\mathrm{nb})$ plywood strips................................151

4-49 Between Vendor Profile Comparison for Covington Street (Site-1)..............155

4-50 Between Vendor Profile Comparison for I-65 with Plywood Strips

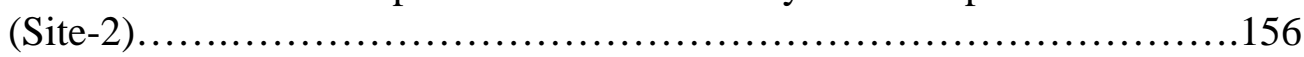

4-51 Between Vendor Profile Comparison for I-65 without Plywood Strips

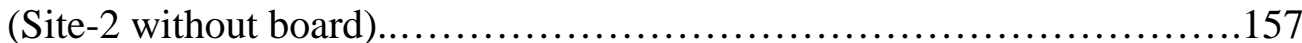


Figure $\quad$ Page

5-1 Case 1 Average Plot by Vendor for IRI................................. 180

5-2 Case $1 \mathrm{~h}$ and $\mathrm{k}$ plots by Vendors for IRI...................................

5-3 Case $1 \mathrm{~h}$ an k plots by Sites for IRI.................................. 182

5-4 Case 1 Repeatability and Reproducibility Statistics by Site for IRI..............183

5-5 Case 1 Average Plot by Vendor for PI-0.0" ..............................188

5-6 Case $1 \mathrm{~h}$ and k plots by Vendors for PI-0.0"............................... 189

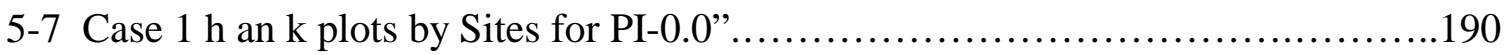

5-8 Case 1 Repeatability and Reproducibility Statistics by Site for PI-0.0”...........191

5-9 Case 1 Average Plot by Vendor for PI-0.2" ............................. 196

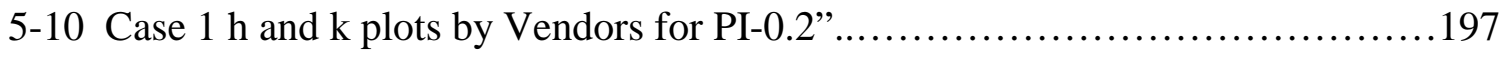

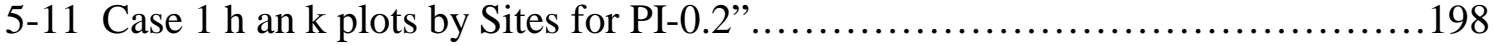

5-12 Case 1 Repeatability and Reproducibility Statistics by Site for PI-0.2”.........199

5-13 Case 2 Average Plot by Vendor for IRI...............................204

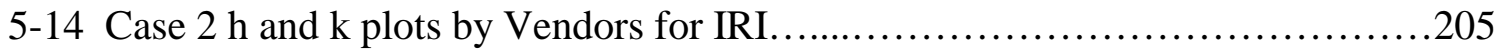

5-15 Case $2 \mathrm{~h}$ an k plots by Sites for IRI...................................... 206

5-16 Case 2 Repeatability and Reproducibility Statistics by Site for IRI.............207

5-17 Case 2 Average Plot by Vendor for PI-0.0"............................. 211

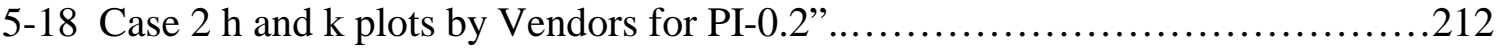

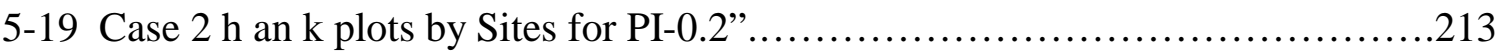

5-20 Case 2 Repeatability and Reproducibility Statistics by Site for PI-0.2”.........214

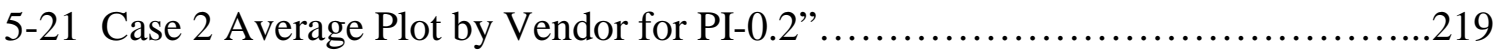


Figure $\quad$ Page

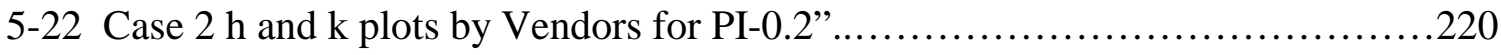

5-23 Case $2 \mathrm{~h}$ an k plots by Sites for PI-0.2" ..................................... 221

5-24 Case 2 Repeatability and Reproducibility Statistics by Site for PI-0.2”........222

5-25 Case 3 Average Plot by Vendor for IRI...................................... 227

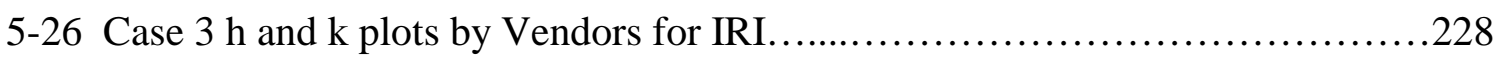

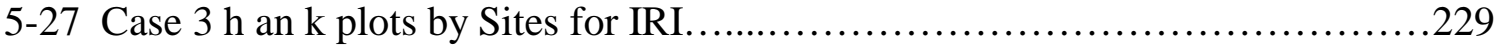

5-28 Case 3 Repeatability and Reproducibility Statistics by Site for IRI.............230

5-29 Case 3 Average Plot by Vendor for PI-0.0" ................................

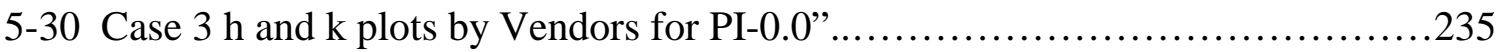

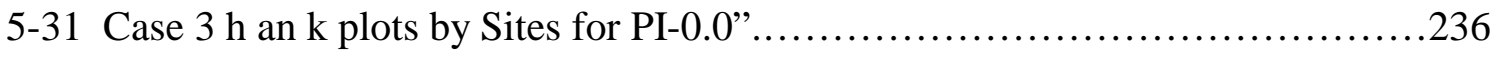

5-32 Case 3 Repeatability and Reproducibility Statistics by Site for PI-0.0".........237

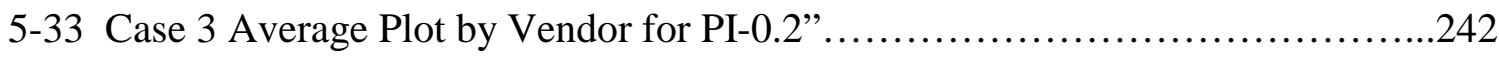

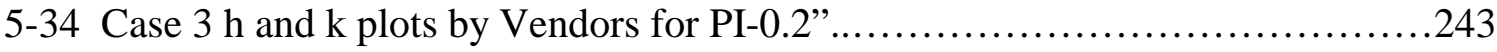

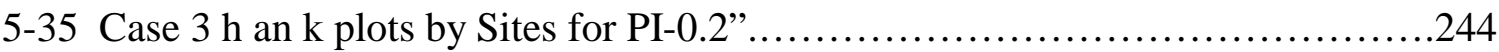

5-36 Case 3 Repeatability and Reproducibility Statistics by Site for PI-0.2”.........245

5-37 Case 4 Average Plot by Vendor for IRI................................. 250

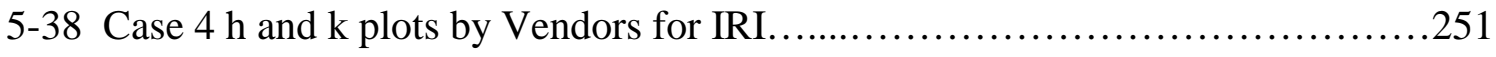

5-39 Case $4 \mathrm{~h}$ an k plots by Sites for IRI.......................................

5-40 Case 4 Repeatability and Reproducibility Statistics by Site for IRI.............253

5-41 Case 5 Average Plot by Vendor for IRI................................ 258

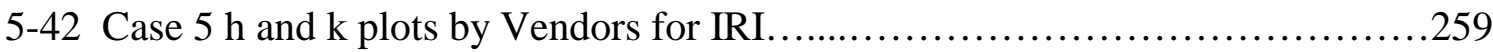


Figure

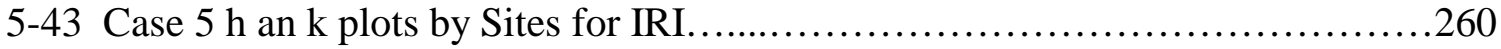

5-44 Case 5 Repeatability and Reproducibility Statistics by Site for IRI.............261 


\section{List of Tables}

Table

Page

2-1 Quarter-Car Model Parameters...........................................24

2-2 Wavelengths used by the Common Indies...............................29

2-3 ASTM Profiler Class Characteristics...................................... 30

3-1 List of Potential Vendors.............................................41

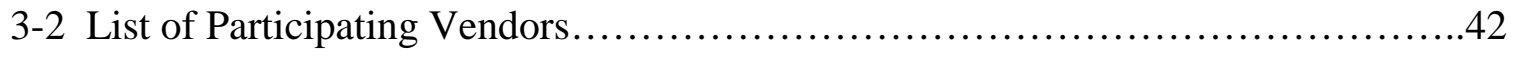

3-3 Experimental Design................................................44

3-4 Potential Sites for Evaluation of Lightweight Non-Contact Profilers..............46

3-5 Shortlist of Potential Sites Provided by Lafayette City Engineers office.............47

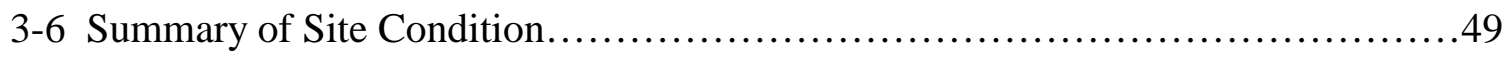

3-7 Individual Site Testing Order Established by Random Drawing..................52

4-1 Vendor A IRI Data and Summary Statistics for All Sites.......................73

4-2 Vendor A PI with a 0.2” Blanking Band and Summary Statistics for

All Sites.................................................. 74

4-3 Vendor A PI with a 0.0" Blanking Band and Summary Statistics for All Sites........................................................ 75

4-4 Vendor B IRI Data and Summary Statistics for All Sites....................... 76

4-5 Vendor B PI with a 0.2” Blanking Band and Summary Statistics for All Sites........................................................ 77

4-6 Vendor B PI with a 0.0” Blanking Band and Summary Statistics for All Sites.................................................. 77

4-7 Vendor C IRI Data and Summary Statistics for All Sites.......................79 
Table

4-8 Vendor C PI with a 0.2” Blanking Band and Summary Statistics for All Sites

4-9 Vendor C PI with a 0.0" Blanking Band and Summary Statistics for All Sites.......................................................... 81

4-10 Vendor D IRI Data and Summary Statistics for All Sites....................82

4-11 Vendor D PI with a 0.2" Blanking Band and Summary Statistics for All Sites

4-12 Vendor D PI with a 0.0" Blanking Band and Summary Statistics for All Sites

4-13 INDOT Rip Van IRI Data and Summary Statistics for All Sites................85

4-14 Summary Smoothness Statistic for PCC Sites..............................87

4-15 Summary Smoothness Statistic for the HMA Sites........................96

4-16 Observed Standard Deviations ............................................112

4-17 Summary of Smoothness Indices for PCC Sites........................... 114

4-18 Summary of Smoothness Indices for PCC Sites......................... 118

4-19 Ranking by Vendors for HMA Sites.................................... 126

4-20 Ranking by Vendors for PCC Sites.................................. 127

4-21 Number of Times Each Received Each Rank.............................127

4-22 Percentage of Times Each Received Each Rank.............................. 127

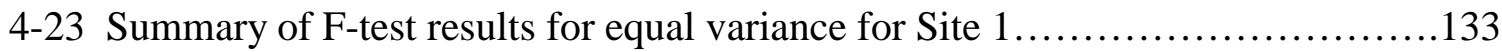

4-24 Summary of F-test results for equal variance for Site 2 with Plywood Strips................................................133

4-25 Summary of F-test results for equal variance for Site 2 without Plywood Strips..................................................133 
4-26 Summary of F-test results for equal variance for Site 3 with

Plywood Strips

4-27 Summary of F-test results for equal variance for Site 3 without

Plywood Strips...............................................134

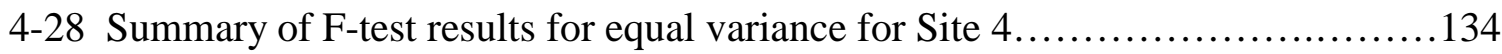

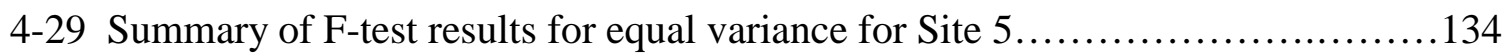

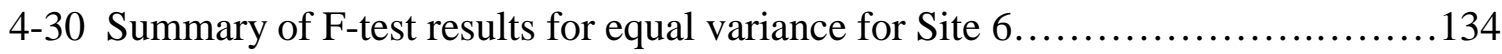

4-31 Summary of paired t-test results for equal means for Site $1 \ldots \ldots \ldots \ldots \ldots \ldots \ldots \ldots 135$

4-32 Summary of paired t-test results for equal means for Site 2 with

Plywood Strips.................................................. 135

4-33 Summary of paired t-test results for equal means for Site 2 without

Plywood Strips................................................ 135

4-34 Summary of paired t-test results for equal means for Site 3 with

Plywood Strips.................................................. 135

4-35 Summary of paired t-test results for equal means for Site 3 without

Plywood Strips.................................................136

4-36 Summary of paired t-test results for equal means for Site $4 \ldots \ldots \ldots \ldots \ldots \ldots \ldots \ldots \ldots$

4-37 Summary of paired t-test results for equal means for Site $5 \ldots \ldots \ldots \ldots \ldots \ldots \ldots \ldots \ldots$

4-38 Summary of paired t-test results for equal means for Site $6 \ldots \ldots \ldots \ldots \ldots \ldots \ldots \ldots \ldots$

4-39 Overall Within Vendor Profile Performance...............................153

4-40 Comparison of Must Grind Locations identified by each Vendor................162

4-41 Repeatability performance in the identification of Must Grind

Locations for Vendor D.........................................164

5-1 Summary of Case 1 Precision Analysis Considerations.......................176

5-2 Case 1 IRI Precision Statement Summary Statistics.........................180 
Table

5-3 Case 1 PI-0.0" Precision Statement Summary Statistics.......................187

5-4 Case 1 PI-0.2" Precision Statement Summary Statistics......................... 195

5-5 Summary of Case 2 Precision Analysis Considerations.......................200

5-6 Case 2 IRI Precision Statement Summary Statistics..........................203

5-7 Case 2 PI-0.0" Precision Statement Summary Statistics.......................211

5-8 Case 2 PI-0.2" Precision Statement Summary Statistics.......................218

5-9 Summary of Case 3 Precision Analysis Considerations..........................223

5-10 Case 3 IRI Precision Statement Summary Statistics.......................226

5-11 Case 3 PI-0.0" Precision Statement Summary Statistics......................233

5-12 Case 3 PI-0.2" Precision Statement Summary Statistics.....................241

5-13 Summary of Case 4 Precision Analysis Considerations.........................246

5-14 Case 4 IRI Precision Statement Summary Statistics........................249

5-15 Comparison of Case 1 through 4 IRI Precision Statistics.....................249

5-16 Summary of Case 5 Precision Analysis Considerations.........................254

5-17 Case 5 IRI Precision Statement Summary Statistics........................257

5-18 Comparison of Case 1 through 5 IRI Precision Statistics.....................261

5-19 Comparison of Case 1 through 3 PI-0.0" Precision Statistics...................262

5-20 Comparison of Case 1 through 3 PI-0.2" Precision Statistics...................262

6-1 Pay Factor Adjustment of Rigid Pavements, Connecticut DOT.................270

6-2 Schedule for Adjusted Payment of Rigid Pavements, Kansas DOT...............274

6-3 Schedule A-Ride Quality Index, Pennsylvania DOT .........................277 
Table

6-4 Schedule B-Ride Quality Index, Pennsylvania DOT. .277

6-5 Schedule C-Ride Quality Index, Pennsylvania DOT.... .277

6-6 Schedule for Payment of Flexible Pavements, Connecticut DOT .................283

6-7 Pay Factor Adjustment for Flexible Pavement Smoothness, Florida DOT..........284

6-8 Schedule for Adjusted Payment of Flexible Pavements, Kansas DOT.............287

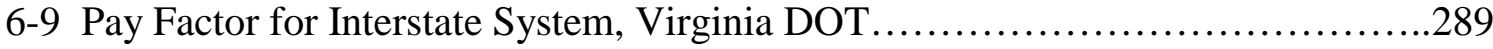

6-10 Pay Factor for Primary System, Virginia DOT ..............................289

6-11 Pay Factor Adjustment for new Flexible Pavement, Virginia DOT..............291

6-12 Pay Factors for Flexible Pavements, Pennsylvania DOT ......................293

6-13 Payment based on Various State Specifications for Rigid Pavements............298

6-14 Payment based on Various State Specifications for Flexible Pavements..........299

6-15 Rigid Pavement Pay Factor Adjustments...................................302

6-16 Flexible Pavement Pay Factor Adjustments.................................302

6-17 Comparison of Repeatability Statistic of Indiana Test Sites.....................304

6-18 Comparison of Reproducibility Statistic for Indiana Test Sites.................305

6-19 Bonus Schedule Based on Proposed Specification...........................305 


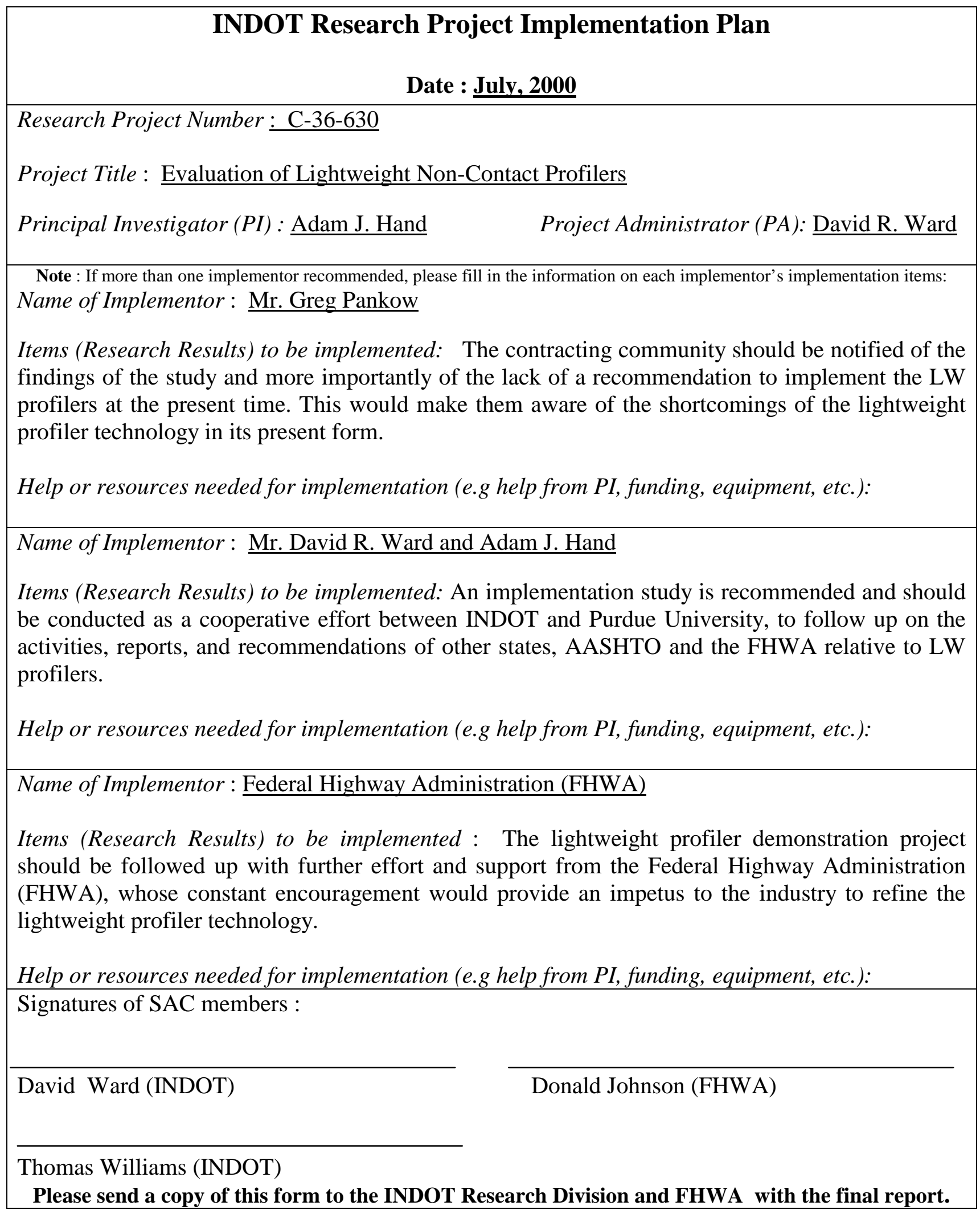




\section{Acknowledgements}

The authors would like to acknowledge the Federal Highway Administration (FHWA), the Indiana Department of Transportation (INDOT), and the Joint Transportation Research Program for providing the funding to support this research. They would also like to thank Pathway Services Incorporated, K J Law Engineering, Ames Engineering Incorporated and International Cybernetics Corporation for their participation, without which this project would not have been possible. Finally, they would also like to acknowledge the project Study Advisory Committee (SAC) members for their critical support and guidance. 


\section{Introduction}

This section provides a brief background for the research project, presents the problem statement, specifies the research objective, and covers the scope of the research.

\subsection{Background}

Pavement smoothness was recently identified as the most significant factor the motoring public uses to judge the quality of our Nation's roadways through a National Quality Initiative survey conducted by the Federal Highway Administration (FHWA, 1998). At the same time, the relationship between pavement smoothness and driver comfort has been well documented over the last fifty years. In recognition of this these facts and in a progressive manner, one of the primary objectives of the Indiana Department of Transportation (INDOT) Strategic Plan is improving pavement smoothness (INDOT, 1999). The contracting community has proven it is possible to construct smooth pavements, but historically there has not been a single uniform industry-wide standard measure of pavement smoothness (smoothness index). Additionally, a plethora of different equipment has been developed and used to measure pavement smoothness over the years. Unfortunately the different equipment has showed sensitivity to different physical pavement features thus providing different indications of pavement smoothness (FHWA, 1998; INDOT, 1999). 
Smoothness can be defined as the condition of the road, which will provide the users with a comfortable and safe ride at optimum travel speeds and thus ensure organized traffic flow. Smooth pavements are important to both the motoring public and the agency who owns the roads. Smooth pavements are not only comfortable for the motoring public, but vehicle operating costs (vehicle depreciation, fuel costs, repair costs etc.) are reduced on smooth pavements. From the roadway-owners point of view it is important because it is a direct reflection of the construction and maintenance policies and proficiency of the owner agency (State Highway Agencies, cities, counties or toll road authorities). Even more importantly pavement service life is directly effected by pavement smoothness, with smoother pavements providing longer service lives and ultimately saving tax-payer's money (Mcghee, K. K., 1999).

A recently completed National Cooperative Highway Research Program (NCHRP) project indicated that smooth pavements last longer and are more costeffective (Smith K.L, et al. 1997). The study suggests that a twenty-five percent increase in initial pavement smoothness yields a nine percent increase in pavement service life. Various models have been developed which predict pavement smoothness as a function of time or total pavement life from initial pavement smoothness. Because pavement smoothness is a well recognized indicator of pavement quality, most State Highway agencies incorporate smoothness into construction specifications and forty five percent use it as a pay item (Russell J.S., Hanna A.S., Bahia H.U., Schmitt R.L., Jung G.S., 1998). Another recently completed NCHRP study focussed on the effect of smoothness specifications on initial smoothness as well as the cost effectiveness of achieving specific 
initial smoothness levels.(Smith K.L, et al. 1997) The project report indicates that initial smoothness is one of the most important aspects that agencies consider while constructing a pavement, which in turn is consistent with the fact that most State Departments of Transportation (DOT's) use it as a pay item.

Many States are using quality control/quality assurance (QC/QA) or end result smoothness specifications in which the contractors pay is commensurate with the quality delivered. These types of specifications drive the contractor to strive for the highest quality possible in an effort to ensure full payment and potentially even bonus. This demands that the contractor have a process control tool(smoothness measuring device) that can quickly and easily generate data that can in turn be used to make process control decisions. Unfortunately, the industry standard California Profilograph is just not such a tool. The manually operated device is quite cumbersome, operates only at walking speed, and generates data which has been manually analyzed historically, although newer devices are capable of generating real time test results.

A new generation of lightweight profilers has recently been developed which have the potential of providing nearly instantaneous smoothness measurements. They employ state-of-the-art measuring equipment (laser and accelerometer technology) mounted to small vehicles at a fraction of the size and cost associated with network level assessment equipment. They incorporate on-board computers automating data collection and analysis, resulting in instantaneous test results. The key advantage of this new generation of profilers is that they are said to be capable of providing more accurate 
profile measurements in less time than the conventional California Profilograph. Profiles may be generated as soon as a pavement is stiff enough to support these lightweight devices on. It is possible that results may be generated within a matter of hours after placement, providing the contractor with an accurate tool to base further paving decisions. It is likely the devices will ultimately be used in this manner of process control (QC). In other words, contractors will be making corrections within hours rather than days. This will ultimately translate into more rapidly constructed smoother pavements and generate direct savings for both the contractor as well as the motoring public. The FHWA initiated a demonstration project in the Spring of 1999 in which support was provided to State DOT's to evaluate the new lightweight profilers. The objective was not only to evaluate the functionality of the devices but also to provide exposure of the new technology to both DOT and industry personnel.

As stated in the Indiana Department of Transportation (INDOT) Strategic Plan, improving pavement smoothness is one of its primary objectives. Therefore INDOT took advantage of the FHWA offer and sponsored a cooperative research effort with Purdue University, through the Joint Transportation Research Program(JTRP), to evaluate the lightweight profilers.

\subsection{Project Scope and Objectives}

The new generation of lightweight profilers offer significant promise. However concerns have been raised regarding the accuracy and other capabilities of the devices. This project was undertaken to address the concerns of the profiling community. The 
objective of the project was to field test and evaluate various state-of-the-art lightweight non-contact profilers, which would provide the data from which end result guide smoothness specifications could be developed. Consultants currently performing smoothness measurements on INDOT projects and contractors were encouraged to observe/participate in the field evaluation. The purpose of this effort was to keep all those that would be affected by a new smoothness specification abreast of the project, fostering a partnering atmosphere.

An attempt was made to include all the lightweight profilers commercially available at the time the project was initiated. These lightweight profilers along with the conventional California Profilograph and the INDOT high speed profiler were used to measure the smoothness of both hot mix asphalt (HMA) and portland cement concrete (PCC) pavements during the summer of 1999. Five replicate smoothness measurements were made with four lightweight profilers and the INDOT high-speed profiler at each of six field sites. The measurements were analyzed to determine the repeatability and reproducibility of these lightweight profilers. This information was used along with a review of current State DOT and AASHTO specifications to develop a guide smoothness specification for INDOT in the Spring of 2000.

The evaluation was to provide INDOT with a proposed guide specification that could be used to assist with the implementation of the use of the lightweight non-contact profiler technology in Indiana. It was felt that the specification would be implementable because it would be based on knowledge of the precision of the lightweight profilers used 
to measure pavement smoothness. The industry was very aware of the potential benefits of the use of the lightweight profilers in construction and indicated it was anxiously awaiting the opportunity to make use of the devices in Indiana. This suggested that a new lightweight smoothness specification would be well received. 


\section{History and Performance of Profilers}

For as long as pavements have been constructed motorists using them have been keenly aware of the relative degree of comfort or discomfort (smoothness versus roughness) experienced in travelling over them.(Carey W.N, Irick P.E, 1960) This has led to extensive research on ride quality over the years which in turn has resulted in the development of many profiling devices and ride quality indices. A review of equipment/techniques developed and the indices they provide are presented in this section along with a description of what roughness is and the factors that effect it.

\subsection{Early Profilers}

The first longitudinal pavement profiler was developed in the early 1900's. Since then significant modification has taken place in profiler design in efforts to obtain more accurate and consistent smoothness measurements. A brief description of early profilers is provided below.

\subsubsection{Via-Graph}

The earliest form of profile measuring instrument was the "Via-graph" developed around the turn of the $20^{\text {th }}$ century. The instrument consisted of a straight edge, and roughness was assessed by recording the deviation at the center point of the sliding straight edge. The instrument was very crude and long wavelengths produced no response. Additionally significant wear and tear was involved, as the straightedge was slid along the pavement surface as depicted in Figure 2-1 (Gillespie, T.D, 1992) . 


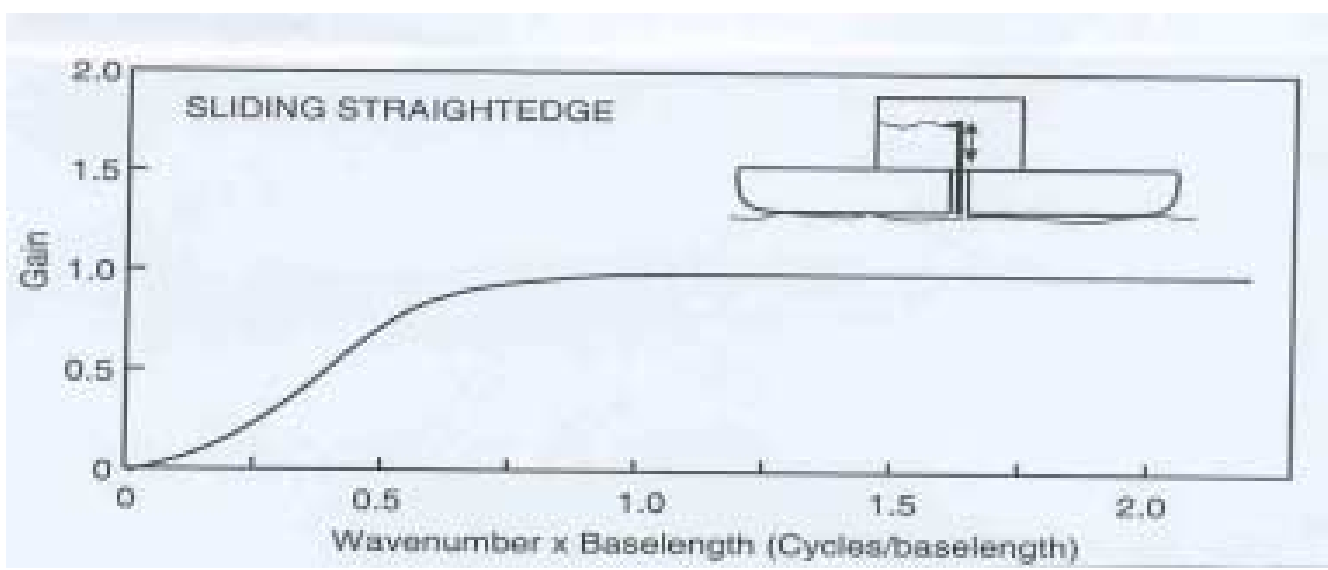

Figure 2-1 Viagraph - Profiler Used in the Early 1900's

\subsubsection{Rolling Straight Edge}

The next instrument that came into use was the rolling straight edge. It was a three wheel device with the measurement wheel in the center. The device had its unique response to roughness, characterized by the fact that it recorded every bump three times, once when the front wheel passed over, a second time when the measuring wheel passed over and a third time when the rear wheel passed over. As the straightedge contacted the road surface at three points, bumps of certain wavelengths were recorded at double their amplitude while others were not recorded at all. Thus the rolling straightedge was tuned to specific wavelengths of roughness in the road, while ignoring others. The rolling straight edge is slow and incapable of measuring true pavement surface profile. However it has been found to be useful for bridge decks and small projects. (Smith K.L et al. 1997). 


\subsubsection{Profilograph}

This problem of measuring each bump three times with the rolling straight edge was overcome with the Profilograph by adding an array of wheels, which established a reference plane with respect to which deviations in elevation could be measured. Bogey attachments for the array of wheels averaged the elevation of all points under the wheels, and roughness was measured as vertical deviation of the center wheel from this reference. This instrument was memorialized as the Profilograph and is depicted in Figure 2-2.

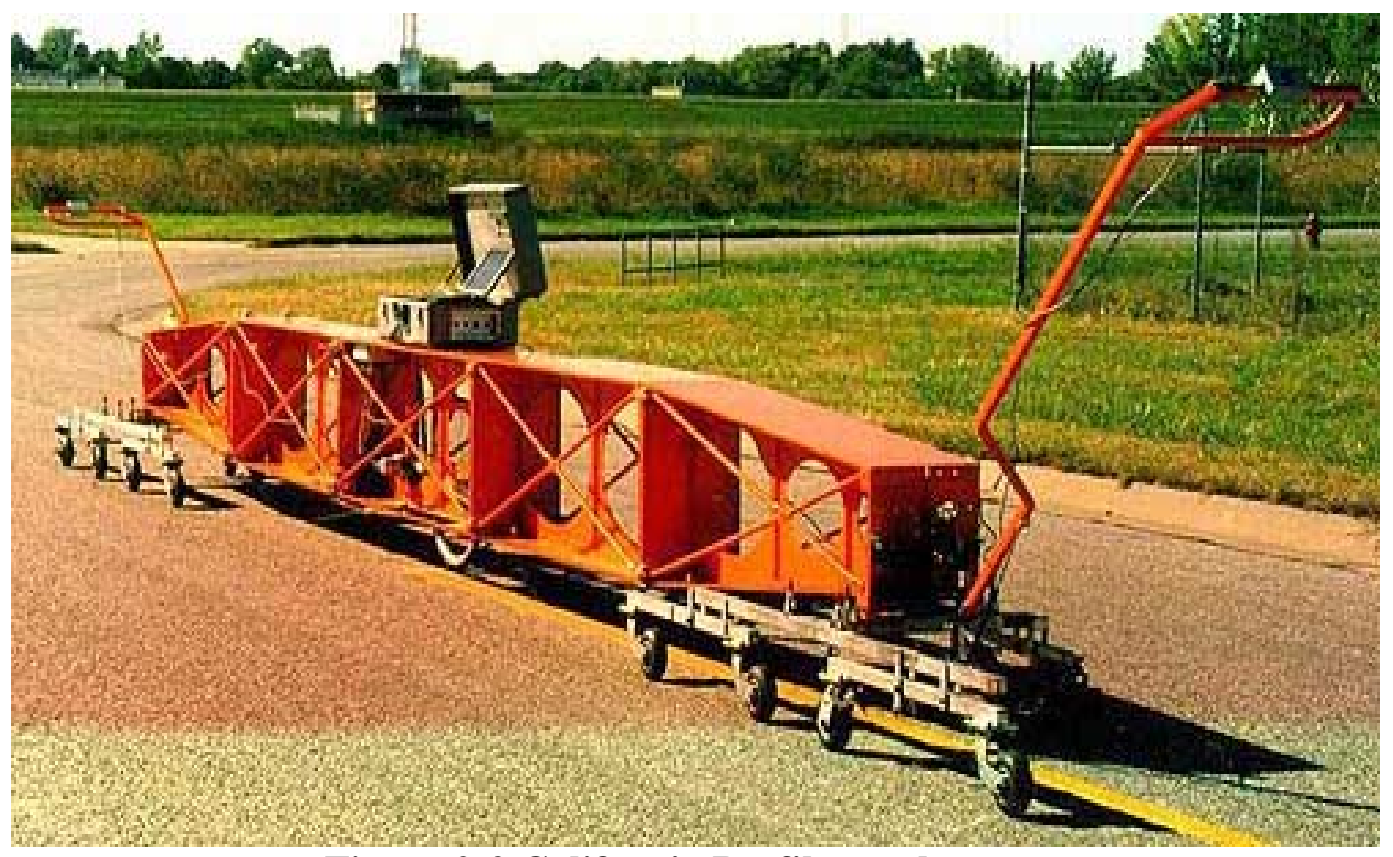

Figure 2-2 California Profilograph

Two basic types of profilographs have evolved, differing in support wheel configuration. Both are manually operated at walking speed. Support wheels on the California type profilograph have varied in number from four to twelve, with systems in many States using twelve wheels. These wheels are attached to the ends of a 25 foot 
(7.6m) long truss and mounted on a multiple axle carriage that includes four wheels spaced 17inches $(0.43 \mathrm{~m})$ from the truss centerline and two wheels spaced 17 inches $(0.43 \mathrm{~m})$ on the opposite side of the truss centerline. The support wheels are commonly spaced at 2.7 feet $(0.82 \mathrm{~m})$ intervals and positioned near the ends of the truss, resulting in an overall profilograph span of 33 foot $(10 \mathrm{~m})$. The twelve support wheels of the Rainharttype profilograph are evenly spaced along its 24.75 foot $(7.5 \mathrm{~m})$ span at offsets up to 22inches $(0.56 \mathrm{~m})$ such that no wheels follow the same path, as depicted in Figure 2-3. On both systems, the front wheel can be steered by the operator.

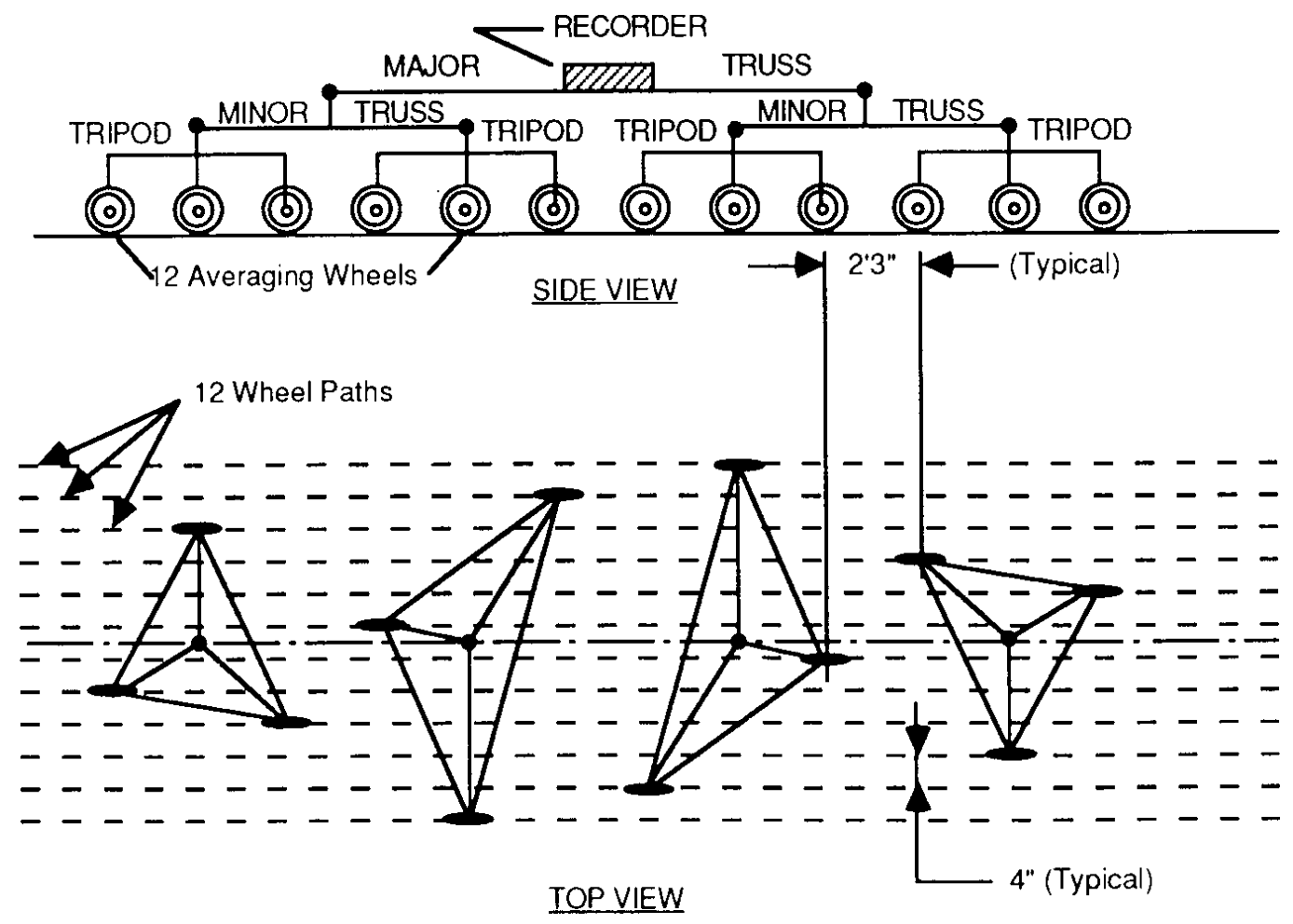

Figure 2-3 Top and Side View of a Rainhart Profilograph

\section{$\underline{\text { 2.1.3.1 California Profilograph }}$}


Most agencies still use the modern version of the profilograph commonly referred to as the California Profilograph. The development of this device can be traced back to the period from 1930-1950. Francis Hveem, former Materials Research and Research Engineer for the California Department of Transportation (CALTRANS) developed the device. The original California Profilograph was typically supported by a carriage of six wheels at each end, arranged in an articulated manner, and provided manual propulsion and steering capability. The current models of the California profilograph consist of a single axle and two wheels at each end of a $25 \mathrm{ft}(7.6 \mathrm{~m})$ long beam. A profile wheel is located at the beam mid-point and is linked to a recorder that provides a paper strip chart showing changes in the distance between the pavement at the point of the profile wheel and the datum established by the carrying wheels. The strip chart produces the deviations on a true vertical scale and on a 1:300 horizontal scale. (Scofield. L.A et. al 1992).

Pavement smoothness is measured in terms of the Profile Index(PI). PI is expressed in units of inches per mile(or millimeters per kilometer) and represents the total accumulated excursion of the strip chart trace beyond a tolerance zone. The tolerance zone typically ranges from 0.2 inch $(0.5 \mathrm{~mm})$ to 0.0 inch $(0.025 \mathrm{~mm})$. For individual bumps, the tolerance limit is expressed as some height, usually 0.3 inch (7.6mm) over a $25 \mathrm{ft}(7.6 \mathrm{~m})$ base-length as read on the strip chart. Some agencies however use $0.4 \mathrm{inch}(10.2 \mathrm{~mm})$ or $0.5 \mathrm{inch}(12.7 \mathrm{~mm})$ tolerance levels (Kulakowski, B.T., 1989).

The strip chart generated is analyzed either manually or by means of a computer. Manual evaluation is carried out with two clear plastic templates. Different blanking 
bands are used by different agencies. Generally it is either 0.2 inch, 0.1 inch or 0.0 inch wide. Individual bumps are manually identified using a second clear plastic template. The computerized analysis varies among different manufacturers as the manufacturers use different data filters to analyze their data. (Kulakowski T.B, et. al. 1989).

As other agencies adopted the California Profilograph, obtaining the device was somewhat difficult. Hence some States constructed devices in house or had them made by local machine shops using plans developed by CALTRANS. INDOT currently uses the California Profilograph as the standard measurement tool in its smoothness specifications.

The device is currently marketed by five firms: Cox and Sons, McCracken Division of International Pipe Machinery Corporation, Ames Engineering, ELE International-Soiltest, and Surface Systems and Instruments-LLC. However these devices have certain limitations. For example, they are manually operated (pushed) at a slow operating speed of 2 to 3 miles/hour $(3.2-4.8 \mathrm{~km} / \mathrm{hr})$. The precision of the devices is poor. Studies conducted by the Arizona DOT have found that the average standard deviation for these devices was $1.9 \mathrm{in} / \mathrm{mi}$ for new pavements with an average PI ranging from 2.6$4.8 \mathrm{in} / \mathrm{mi}$. On rougher sections with an average PI of $8.6 \mathrm{in} / \mathrm{mi}(136 \mathrm{~mm} / \mathrm{km})$, the average standard deviation was $1.6 \mathrm{in} / \mathrm{mi}$. For mechanical and computerized profilographs, the range of test results were between 3.5 and $7.0 \mathrm{in} / \mathrm{mi}$ for a smooth section and between 7.0 and $11.0 \mathrm{in} / \mathrm{mi}$ for a rougher section. This is clearly more variability than can be considered acceptable for implementing incentive/disincentive specifications (Scofield 
L.A, et. al. 1992). Additionally new smoothness indices seem to have some advantages over Profile Index (PI). Finally as the Profilograph amplifies and attenuates the true pavement surface profile, there is some doubt whether the Profilograph output really relates to the wavelengths of a pavement profile that are felt by the highway users. Often poor correlation has been observed between measured PI for a pavement and user response to ride quality (Spangler E.B et. al. 1995).

\subsubsection{Via-Log}

Highway engineers recognized in the 1920's that the roughness properties of greatest importance were those responsible for causing vibrations of motor vehicles. (Gillespie, T.D,1992) The "Via-Log", developed by the State of New York evidenced this thinking by measuring the suspension travel of a passenger car as an indication of roughness level. This device initially recorded the suspension motion, but was soon modified to sum the motion on a mechanical counter and measure an "inches per mile" statistic. A significant shortcoming of the Via-Log was the difficulty in obtaining consistent measurements with it due to the variation in dynamics of individual motor vehicles. Hence there was a drive to standardize the vehicle. (Hveem F.N., 1960).

\subsubsection{Bureau of Public Roads Roughometer (BPR)}

The drive to standardize the Via-Log led to the development of Bureau of Public Roads (BPR) Roughometer depicted in Figure 2-4 in 1925. It was a single wheel trailer in which all dimensions, mass properties, and tire and suspension properties were standardized in an effort to achieve comparable performance from replicate devices. The 
device was later adopted in a similar form, as the Bump Integrator by the Transport and Road Research Laboratory in England. However after some period of use, it was determined that the equipment was highly susceptible to changes in temperature and to the condition of its mechanical components. In addition to that it had a resonant frequency problem. As a result its use gradually declined.

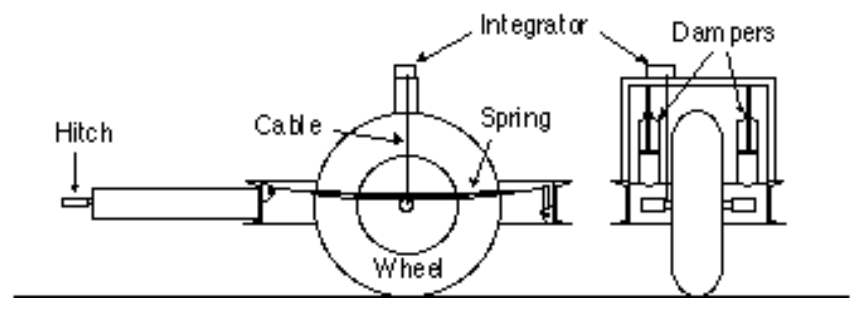

Figure 2-4 BPR Roughometer

\subsubsection{CHLOE}

Another important roughness measurement device was developed at the time of the AASHO Road Test was the CHLOE .

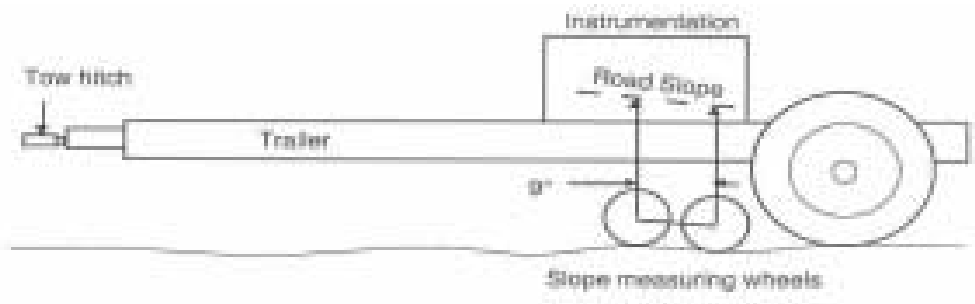

Figure 2-5 CHLOE- Profiler Used at AASHO Road Test

CHLOE is an acronym formed from the first letters of the founder's names Carey, Huckins, Leathers and Other Engineers. The CHLOE consisted of a trailer towed at low 
speed, on which was mounted two small wheels 9 inches $(0.225 \mathrm{~m})$ apart with instrumentation to measure the local road slope as depicted in Figure 2-5. Hence slope deviation or slope variance(SV) in inches per mile was the statistic that was measured. In fact this instrument was used in the development of the AASHO Pavement Serviceability concept. The AASHO Present Serviceability Index (PSI) is a function of SV and actually dominates the equation used to calculate PSI. This is reasonable because the studies of the AASHO Road Test indicated that approximately ninety-five percent of the information about pavement serviceability was contributed by the roughness of the pavement surface profile.

\subsubsection{Response-Type Road Roughness Measuring Systems (RTRRMS)}

The attraction of being able to measure roughness properties with a moving vehicle motivated the development of Response-Type Road Roughness Measurement Systems (RTRRMS). These instruments popularly known as "roadmeters", consist of a transducer that accumulates suspension motions induced by road roughness in typical motor vehicles. However the statistic of inches/mile deviation which they measured are affected by the dynamics of individual vehicles. The more popular models of RTRRMS are the May's Ride Meter, PCA meter, Cox meter and individually made models. A schematic of a May's Ride Meter is presented in Figure 2-6.

The measurements made with these systems were not stable with time nor were they transportable. Simply adding fuel or passengers, adjusting tire pressure, and balancing tires changed the calibration of the device. Another problem was the lack of a 
standard roughness scale. Therefore it was difficult to develop or maintain a database of road roughness conditions using RTRRMS, without excessive effort placed on controlling or compensating for vehicle changes through frequent calibration exercises. (Gillespie T.D., et. al. ,1980 ).

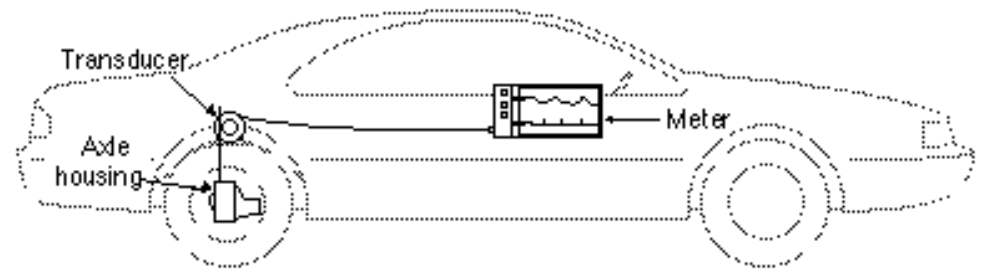

Figure 2-6 May's Ride Meter

This problem was addressed with National Cooperative Highway Research Program (NCHRP) Project 1-18 in 1978. The sources of variability in roughness measurement with RTRRMS were examined and calibration procedures were identified to compensate for each such that consistent roughness measurements could be obtained with different systems. This lead to the development of more sophisticated, precise profilers called inertial profilers.

\subsubsection{Inertial Profilers}

Inertial profilers are commonly used today to measure road profiles particularly for network level pavement management purposes. The use of this high speed profiling technology however dates back to the 1960's when Elson Spangler and William Kelly developed an inertial profilometer at the General Motors Research Laboratory. Since then 
such profilers have undergone dramatic changes which have improved their performance and accuracy. (McQuirt J.E., et. al., 1986).

An inertial profiler generates a series of numbers related, in a well-defined way, to a true surface profile. This set of generated numbers may not be the true elevation of the road. However there lies a relationship between the measured numbers and the true profile.

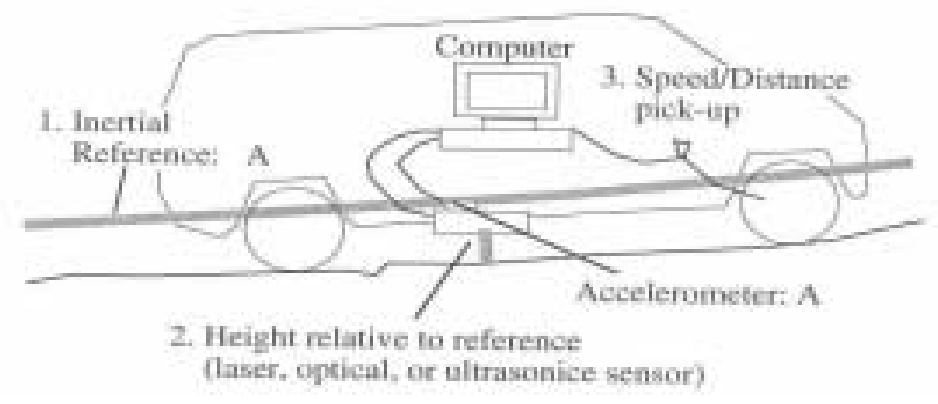

Figure 2-7 Schematic diagram of an inertial profiler

An inertial profiler works by combining three items:

1. A reference elevation;

2. A height relative to the reference elevation; and

3. Longitudinal distance

The inertial profiler computes profile from a combination of the output of three sensors: a height sensor, an accelerometer, and a longitudinal distance pulser as depicted in Figure 2-7. Vertical acceleration measured at a point fixed on the vehicle body is integrated twice to construct a temporary reference height. The height sensor, mounted in the same position as the accelerometer, measures the distance from this temporary reference to the 
road surface. The height sensor signal is subtracted from the height of the temporary reference to compute the profile elevation. The longitudinal distance measurement is needed to associate a position with each profile elevation. The method was invented by Elson Spangler and William Kelly (Spangler E.B. et. al. , 1966).

Mathematically the profile is described as:

$$
Z(x)=H(x)+\iint_{x} A_{t}(s) / V^{2} d s d s
$$

Where $\mathrm{x}=$ Longitudinal distance

$\mathrm{Z}(\mathrm{x})=$ Computed Profile

$H(x)=$ Height sensor measurement (it is the distance from the vehicle to the ground and hence is always negative)

$\mathrm{A}_{\mathrm{t}}(\mathrm{s})=($ Temporal $)$ Vertical acceleration

$\mathrm{V}=$ Forward speed

All inertial profilers use some discrete adaptation of the formula (2.1) to compute profile. Most profilers also apply a high-pass filter as a final step in the computation. Inertial profilers do not measure extremely long wavelengths validly. Hence the highpass filter removes incorrect information and passes the valid part of the profile through. The most common high-pass filter cutoff in use is $300 \mathrm{ft}$ (91 meters) (NCHRP Report10$47,1999)$. However it varies from one software to another used to compute the profile.

Original inertial profilers sensed the height of the vehicle relative to the ground using an instrument called a follower wheel. However because follower wheels were fragile, the testing had to be done at low speeds to avoid vehicle dynamics (bouncing). The 
profile calculations were performed electronically and required that the vehicle operate at constant forward speed which could be difficult to maintain. In modern profilers, the follower wheel has been replaced by a non-contacting sensor. They also correct for minor variations in speed and perform the calculations numerically with on-board computers.

Several inertial profilers have been used in the past. The most popular among them, being the South Dakota Profiling System and the FHWA PRORUT. Several State agencies have also manufactured inertial profilers in-house. The INDOT Rip Van is a typical example.

\subsubsection{South Dakota Profiling System}

The South Dakota Profiling System was developed by the South Dakota DOT in 1981. It is mounted in a small to mid-sized van and measures pavement profile and rutdepth, using the inertial-reference profiling previously explained. An accelerometer and ultrasonic sensor are mounted over the wheel path to measure the profile. Profile elevations are reported at 12 inch $(0.3 \mathrm{~m})$ intervals and the testing speed can range up to $65 \mathrm{mph}$ (105kph).

PSI was the roughness statistic originally generated by the South Dakota Profiling System. However the present software has been upgraded to generate International Roughness Index (IRI) and Mays Output (MO) values from the profile data.

\subsubsection{FHWA PRORUT}


The PRORUT device was developed through an FHWA contract with the University of Michigan Transportation Research Institute (UMTRI) in 1983. The PRORUT can be used to measure and record various roadway characteristics, including longitudinal pavement profiles, rutting, and roughness levels of both wheel tracks. (Ksaibati K., Kercher K., Gulen S., White T.D., 1989).

Laser sensors and accelerometers are used to obtain the profile measurement in each wheel track. Data generated can be analyzed and presented both graphically and numerically. International Roughness Index (IRI) is the roughness statistic generated from the output.

Several other profilers have been developed over the last seventy five years and some were marketed commercially. The discussion above focussed on the most commonly used in the U.S. Each of these devices had some shortcomings and hence the search for new profilers, capable of generating accurate profiles at highway speed has never ended.

\section{$\underline{\text { 2.2 Roughness Indices }}$}

Several roughness indices exist. Most are based on specific profilers and measure different ranges of wavelengths. Hence each of them has a different correlation with highway user response. However, International Roughness Index (IRI), Profile Index (PI) 
and Ride Number $(\mathrm{RN})$ are the most common. The basis of each is subsequently discussed.

\subsubsection{International Roughness Index}

The World Bank provided loans for development of pavement systems to a number of countries (Gillespie T. D., et. al., 1992) It faced problems in obtaining comparable measurements of roughness for data input to highway cost models, so that appropriate funds could be allocated to different projects. This led to the development of a roughness measurement through a correlation experiment. It was independent of the device employed and could be used as a standard throughout the world.

The correlation experiment which was initiated in 1980 lead to the development of a longitudinal pavement profile index that was intended to work with different types of profilers. It was defined as a property of the true pavement profile and was termed the International Roughness Index (IRI). IRI is a scale of roughness based on the response of a generic motor vehicle to the roughness of a pavement surface. IRI is obtained by processing a suitably accurate measurement of longitudinal pavement surface profile through an algorithm which simulates the way a reference vehicle would respond to the profile and accumulates expected suspension travel.

The response properties of an automobile are simulated by a simple dynamic model commonly known as the quarter car model. At each wheel position the vehicle behaves as a sprung mass resting on a suspension system with stiffness and damping, which in turn is attached to the unsprung mass of the wheel, brake, and suspension 
components. The wheel contacts the road through a tire which acts like a spring. Pavement surface characteristics provide inputs to the car which flex the tire, stroke the suspension and cause the sprung and unsprung masses to vibrate in the vertical direction. This simulated suspension motion response is accumulated and divided by the distance traversed to give an index with units of slope (m/km, in/mi etc.) (Sayers, M.W., 1985).

The concept of quarter-car simulation as a method for analyzing pavement profile data was originally an attempt to simulate the output of the BPR Roughometer. The parameters of the quarter-car model are shown in Figure 2-6. They include the sprung mass of the vehicle body; the suspension spring and damper(shock absorber) constants; the unsprung mass of the suspension, tire, and wheel; and the spring constant of the tire. Theoretical correctness would require a damper constant for the tire, however practical application generally ignores the term. Mathematically the behavior of a quarter-car can be described with the two second order equations:

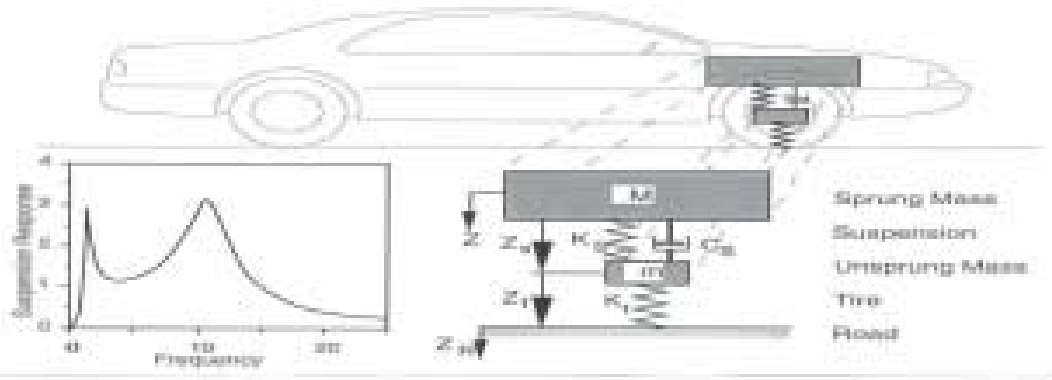

Figure 2-8 Quarter Car Model

and

$$
\begin{aligned}
& M \ddot{Z}_{s}+C_{s}\left(\dot{Z}_{s}-\dot{Z}_{t}\right)+K_{s}\left(Z_{s}-Z_{t}\right)=0 \\
& M \ddot{Z}_{s}+m \ddot{Z}_{t}+K_{t}\left(Z_{t}-Z_{R}\right)=0
\end{aligned}
$$


where

$$
\begin{aligned}
& Z_{R}=\text { road profile elevation points } \\
& Z_{t}=\text { elevation of unsprung mass (axle) } \\
& Z_{\mathrm{s}}=\text { elevation of sprung mass (body) } \\
& \mathrm{K}_{\mathrm{t}}=\text { tire spring constant } \\
& \mathrm{K}_{\mathrm{s}}=\text { suspension spring constant } \\
& \mathrm{C}_{\mathrm{S}}=\text { shock absorber constant } \\
& \mathrm{m}=\text { unsprung mass (axle) } \\
& \mathrm{M}=\text { sprung mass }
\end{aligned}
$$

The double dot notation above the elevation terms represents acceleration while the single dot represents velocity.( Sayers, M.W., 1995).

Since RTRRMS devices generally measure the movement between the vehicle axle and body, simulation requires calculation of the difference in elevation between the body and axle in response to the road profile and forward motion of the vehicle. This is accomplished by integrating the difference in the velocities between the sprung and unsprung mass, producing the quarter car statistic, QCS:

$$
Q C S=\frac{1}{C} \int_{0}^{t}\left|\dot{Z}_{s}-\dot{Z}_{t}\right| d t
$$

The term $\mathrm{C}$ represents either the total time required to traverse the section of pavement being simulated, T, or the length of the section, $\mathrm{L}$. If the time factor is used to normalize the quarter-car statistic, the calculation results in average rectified velocity, 
while a distance base yields the average rectified slope. IRI is the QCS computed using the parameters recommended by the Highway Safety Research Institute (HRSI).

The set of vehicle parameters used to compute IRI have been provided in Table 2-1.

Table 2-1 Quarter-Car Model Parameters [ Gillespie ]

\begin{tabular}{|c|c|c|c|c|}
\hline Vehicle Type & $\mathrm{K}_{1}\left(\mathrm{sec}^{-2}\right)$ & $\mathrm{K}_{2}\left(\mathrm{sec}^{-2}\right)$ & $\mathrm{M}$ & $\mathrm{C}\left(\mathrm{sec}^{-1}\right)$ \\
\hline HRSI Reference & 653 & 62.3 & 0.15 & 6.0 \\
\hline
\end{tabular}

IRI is a general purpose roughness index which is highly correlated to the three vehicle response variables that are of the greatest interests to motorists:

1. Road meter response (for historical continuity);

2. Vertical passenger acceleration (for ride quality); and

3. Tire load (for safety)

IRI ranges from zero (for a perfectly smooth profile) to positive values, proportional to the roughness of the road. The smoothness index has been commonly used in assessing network level and pavement performance in state pavement management systems. (Gillespie, T.D., 1992).

\subsubsection{Advantages of using IRI}

Use of IRI provides several advantages over other roughness statistics. They include :

1. IRI is portable, reproducible and stable with time;

2. IRI is independent of the equipment used to measure it. Hence a number of pavement roughness equipment could be used, thus making the measurement cost-effective; 
3. General use of IRI could lead to more equitable allocation of pavement preservation funds as pavement serviceability would be directly compared across any and all boundaries; and

4. Because IRI is directly related to pavement profile, designing appropriate process control, maintenance and rehabilitation treatments for site-specific locations is easy.

\subsubsection{Disadvantages of using IRI}

There are some disadvantages to the use of IRI which include:

1. IRI is insensitive to shorter wavelengths due to the higher damping used in the quarter-car model and hence does not correlate well with other vehicles (eg. Commercial Trucks); and

2. There is no uniformity in reporting IRI values for specific wheel paths and it should be noted that roughness does vary between wheel paths.

Despite some of these issues associated with IRI, it is currently the standard roughness measurement index employed by most agencies, particularly for pavement management system purposes. Some agencies use it in smoothness specifications also and it is rapidly gaining popularity for this purpose.

\subsubsection{Profile Index (PI)}

Profile Index(PI) is derived from the profilograph output. PI is expressed in units of inches per mile (in/mi) or millimeters per kilometer $(\mathrm{mm} / \mathrm{km})$ and represents the total accumulated excursion of the strip chart trace(which is the profilograph output) beyond a tolerance zone (blanking band). Blanking band width varies for different analysis but 
typically ranges from 0.0 to 0.2 inch. With most States now using the California Profilograph for measuring smoothness, PI has become a standard index for smoothness measurement in construction specifications. (Philips M.B. et. al., 1969).

PI can be evaluated both manually with a plastic template or with the help of software. Manually PI is determined for each 0.1 mile of pavement using a plastic template 21.12inches long. The template is scribed in 0.1inch vertical increments to allow the vertical excursions to be counted. A smoothness tolerance represented by the blanking band runs the length of the template. The blanking band is placed on the profile to cover as much of the trace as possible. The accumulated distances of the trace above and below the blanking band are used to determine PI. For inertial profilers PI can be evaluated by performing a simulation of a profilograph measurement on the generated profile using software, which varies from one manufacturer to another.

Using the inches per mile statistic for PI attenuates longer pavement wavelengths and amplifies shorter wavelengths. Thus use of the inches per mile statistic effectively filters the profile data placing a linearly increasing emphasis on shorter wavelengths.

PI has been used for many years for initial smoothness quality control operations, resulting in improved pavement rideability. However recent studies have indicated that PI does not correlate well with user response. In addition, one study showed that PI correlated only moderately well with PSI, with $\mathrm{R}^{2}$ values of 0.705 using a 0.2 inch blanking band on roadways with report PI values of less than 20 inches/mile (Smith K.L. et al, 1997). However better correlation was observed with a thinner blanking band. 
Reducing or totally eliminating the blanking band should make PI more sensitive to shorter wavelength oscillations that can occur in the paving operation, and hence improve the relationship between PI and user response.

Because PI can be computed using inertial based higher speed profile measurements, data collection, repeatability, and correlation with other smoothness indices could potentially be greatly improved by the new technology. Hence PI will likely continue to serve the pavement community as an effective tool for quality control purposes.

\subsubsection{Ride Number $(\mathbf{R N})$}

Ride Number $(\mathrm{RN})$ indicates rideability of a pavement on a 0 to 5 scale. It is calculated by a non-linear transformation of the PI statistic. Generally a perfectly smooth road has a value of five and zero indicates the maximum possible roughness. RN was developed by Sayers and Karamihas and also uses a quarter-car filter with properties that reportedly allow the index to correlate well with user response (Sayers M.W. and Karamihas S.M.,1996). However Ride Number did exist prior to this development. Janoff developed a RN statistic as part of NCHRP Project 1-23 based on measurements of 282 pavement sections in 5 States spanning a range from 0.4 to 4.5 on a 0 to 5 rideability scale. However it was not used by any highway agency for initial pavement smoothness control. A simultaneous project funded by the Ohio DOT and led by Spangler and Kelly also led to another Ride Number being formulated known as $\mathrm{RN}_{\text {Spangler. }}$ However the algorithm used for calculating this index included an error that caused a significant bias 
as a function of sample interval. Hence Ride Number developed by Sayers called $\mathrm{RN}_{\text {Sayers }}$ is the most popular of the Ride Number indices used by the pavement community.

Ride Number as computed by Sayers was derived by an exponential transformation of a Root Mean Square (RMS) slope statistic called the Sayers PI with units of slope (in/in), using the following relationship:

$$
\left.\mathrm{RN}=5 \mathrm{e}^{-160(\mathrm{PI}} \text { Sayers }\right)
$$

Sayers PI involved the modification of the IRI algorithm by replacing the quarter car coefficients with different values $\left(K_{1}=5120, K_{2}=390, C=17\right.$ and $\left.M=0.036\right)$, changing the initialization length from $36.0 \mathrm{ft}$ to 62.3 feet $(11.0$ to $19.0 \mathrm{~m})$, and changing the accumulation computation from mean absolute to root mean square.

The primary objective in formulating $\mathrm{RN}$ were to provide a roughness statistic that was insensitive to sample interval that provided good agreement when calculated using profiles from different devices, and that used a currently available algorithm to permit easy changeover to a new index. The correlation of RN Sayers with user response using the data from the 1988 NCHRP study was good. (Ksaibati K., Mcnamara R., Miley W. and Armaghani J., 1998).

Each of the indices previously discussed capture different wavelength ranges. The bandwidth of wavelengths each is sensitive to is summarized in Table 2-2. It is a matter 
of research whether the range of wavelengths associated with different smoothness statistics cater to the wavelengths which truly affect the ride quality.

Table 2-2 Wavelengths used by the Common Indices [Smith K.L. et al 1997]

\begin{tabular}{|c|c|c|}
\hline Index & Source & Reported Bandwidth \\
\hline IRI & Paterson $1987 \mathrm{a}$ & $3.0-80$ feet \\
\hline PI & Scofield 1992 & $1.0-75$ feet \\
\hline $\mathrm{RN}_{\text {Sayers }}$ & Sayers 1996 & $1.7-36$ feet \\
\hline $\mathrm{RN}_{\text {Janoff }}$ & Janoff 1986 & $1.6-8$ feet \\
\hline
\end{tabular}

\section{$\underline{\text { 2.3 Profiler Classes }}$}

There are several types of surface profilers in use today. They range in sophistication, from manual rod and level surveys to inertial high speed profilers. The American Society for Testing and Materials (ASTM) defines them by class in ASTM E 950-94, "Standard Test Method for Measuring the Longitudinal Profile of traveled Syrfaces with an Accelerometer Established Inertial Profiling Reference." The classes essentially represent different levels of sampling interval, vertical measurement resolution and most importantly precision.

The specific characteristics associated with each of the four ASTM classes have been defined in Table2-3.

\section{Table 2-3 ASTM Profiler Class Characteristics}

\begin{tabular}{|c|c|c|c|c|}
\hline $\begin{array}{c}\text { Profiler } \\
\text { Class }\end{array}$ & $\begin{array}{c}\text { Longitudinal } \\
\text { Sampling }\end{array}$ & $\begin{array}{c}\text { Vertical Measurement } \\
\text { Resolution }\end{array}$ & $\begin{array}{c}\text { Precision (one } \\
\text { standard deviation)* }\end{array}$ & $\begin{array}{c}\text { Bias (difference from the mean } \\
\text { value of repeated measurements) }\end{array}$ \\
\hline Class 1 & $\leq 1 \mathrm{in}$ & $\leq 0.005 \mathrm{in}$ & $\leq 0.015 \mathrm{in}$ & $\leq 0.05 \mathrm{in}$ \\
\hline
\end{tabular}




\begin{tabular}{|c|c|c|c|c|}
\hline Class 2 & $>$ in to 6in & $>0.005$ in to 0.01in & $\leq 0.03$ in & $\leq 0.1$ in \\
\hline Class 3 & $>$ 6in to 12in & $>0.01$ in to 0.02in & $\leq 0.1$ in & $\leq 0.25$ in \\
\hline Class 4 & $>12$ in & $>0.02$ in & Not defined & Not defined \\
\hline
\end{tabular}

* Precision here implies the repeatability standard deviation measured over a 0.2 mile section at one thousand fifty seven locations spaced 12 inches apart.

The accuracy of a profiler is one of the criteria used for classifying it. A Class 1 instrument must be so accurate that the random error involved would be negligible, for all practical purposes. The commonly used modern devices for profiling such as rod and level, Dipstick and Inertial Profilers all fall into Class 1.

\subsubsection{Rod and Level Surveys}

Rod and level measurement is a static method of measuring a surface profile and is conducted using high-precision surveying tools. A schematic diagram is presented in Figure 2-9. The level provides the height relative to the reference and the tape/laser combination provides individual elevation measurements. ASTM Standard E1364-95 provides guidelines for measuring profiles by this method. It is very precise, but very impractical for most applications other than verifying the performance of another profiler or profiling technique because it is extremely time consuming. 


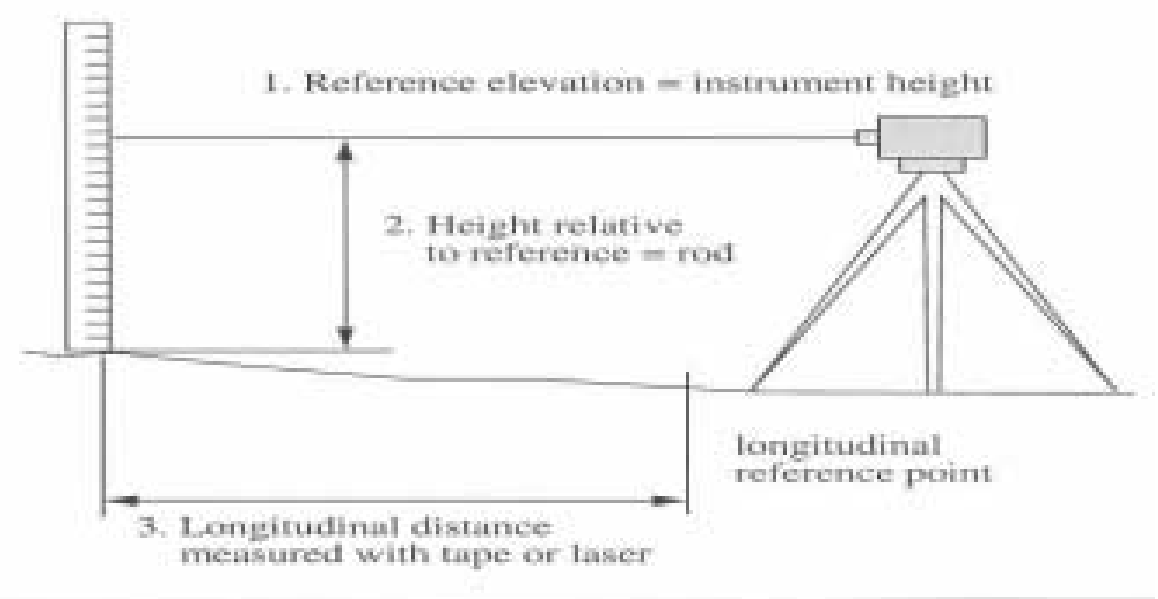

Figure 2-9 Schematic diagram of Rod and Level Survey

\subsubsection{Dipstick}

A device called the Dipstick is faster than the rod and level method of measuring profiles and is suited for roughness analysis. It includes a battery-powered on-board computer to automatically record data and perform the arithmetic needed to produce a profile. The device is very compact, simple to use and is depicted in Figure 2-10.

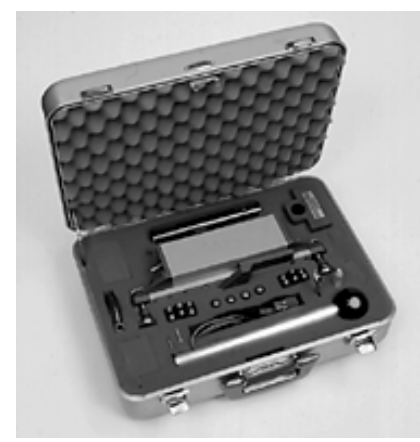

Fig 2-10 DipStick device

It contains a precision inclinometer that measures the difference in height between the two supports, normally spaced 12 inches $(0.3 \mathrm{~m})$ apart. To profile a line along the 
pavement, the device is leaned so that all its weight is on the leading foot, by raising the rear foot slightly off the ground. Then it is pivoted through 180 degrees about the leading foot, locating the other foot (formerly behind) in front, along the line being profiled as depicted in Figure2-11. The computer monitors the sensor continuously. When it senses that the instrument has stabilized, it automatically records the change in elevation and beeps, signaling that the next step may be taken. Thus the reference elevation is the value calculated for the previous point. The height relative to the reference is deduced by the angle of the device relative to gravity, together with the spacing between its supports. The longitudinal distance is determined by multiplying the number of measures made with the known spacing.

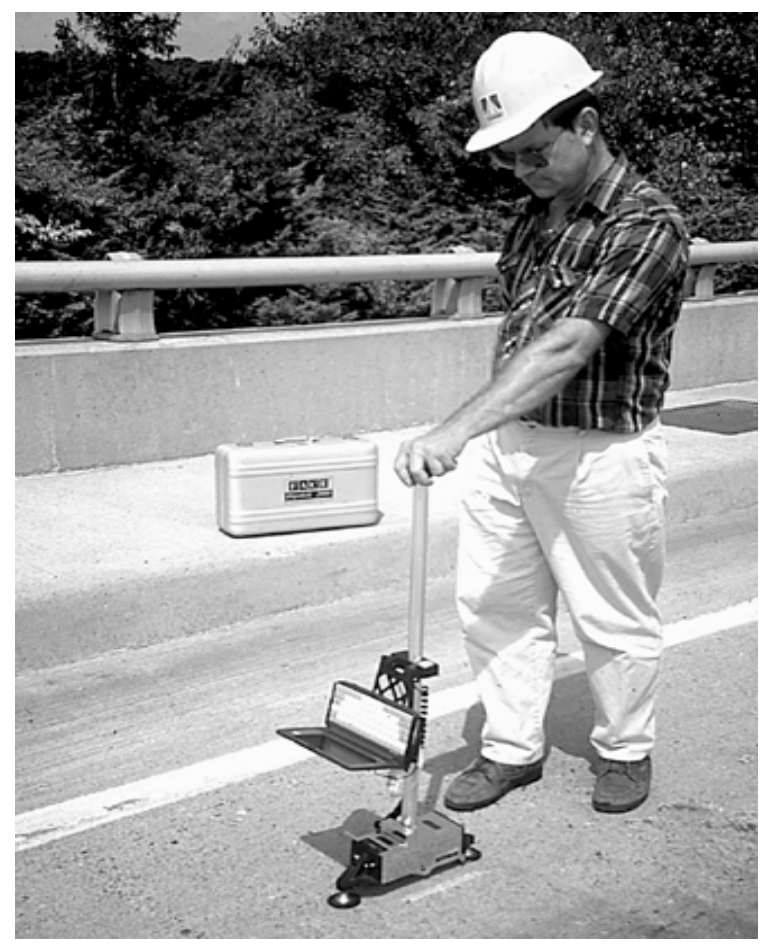

Fig 2-11 Profiling with DipStick

An operational protocol for the Dipstick is presented in Appendix B of SHRP-P338, (SHRP 1993). 


\subsubsection{Modern Inertial Profilers}

Inertial profilers are used in high speed profiling and are very useful for monitoring large road networks. They were discussed in detail in Section 2.1.8. Most modern inertial profilers classify as ASTM Class 1.

\subsubsection{Lightweight Profilers}

A new generation of lightweight inertial profilers has recently been developed specifically for construction quality control and quality acceptance (QC/QA) purposes. They are much smaller and lighter than the network level inertial profilers, providing the benefit of use immediately after hot mix asphalt (HMA) construction and much sooner than would be possible with the network level devices on new portland cement concrete (PCC) pavements. They meet ASTM Class 1 specifications and operate at speeds between 10 and $20 \mathrm{mph}$. On board computers provide instantaneous profile measurement and smoothness summary statistic (eg. IRI, PI, and RN).

The key to the devices is that they provide contractors with a tool to rapidly identify process control issues such that they make corrections promptly and cost

effectively. It is anticipated that lightweight profilers will replace the California Profilograph for construction QC/QA purposes in the near future. Figure 2-12 is an example of a lightweight profiler. Several are currently commercially available and in most cases they incorporate the same hardware used in high speed inertial profilers simply mounted on golf carts or small all terrain vehicles. 


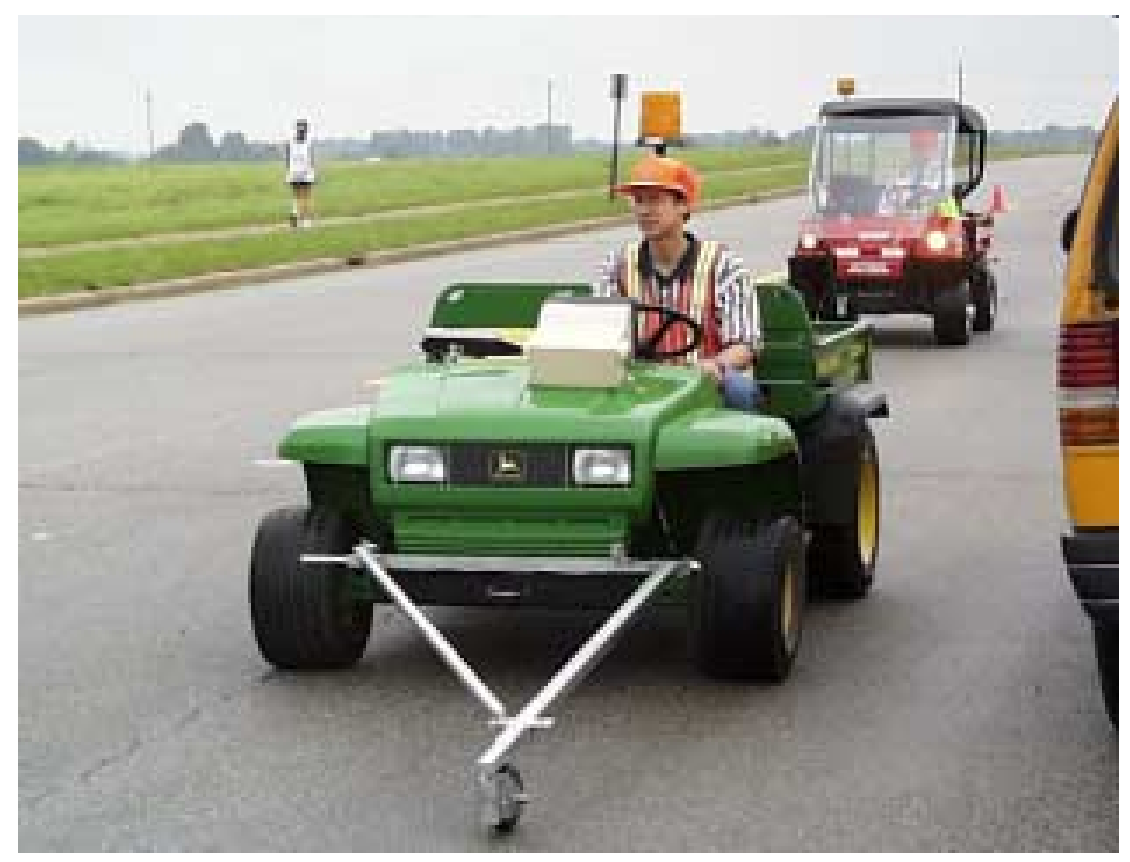

Figure 2-12 Lightweight Non-Contact Profiler

\section{$\underline{\text { 2.4 Factors affecting profile measurement }}$}

Accurate profile measurement is very difficult and it depends on a number of factors. Unless each of the factors are given due attention measurements will not be accurate. In a recent NCHRP study the primary factors affecting profile measurements made with simple inertial profilers were reviewed (NCHRP Report 10-47, 1999). The factors were divided into five broad categories. The following represents a discussion of the factors relative to this project.

\subsubsection{Profiler design}


Several aspects of profiler design affect their accuracy, the most important of which is the sample interval employed. The choice of sample interval should be such that it accurately measures the components within the wavelength range of interest.

\subsubsection{Sample Interval}

A pavement profile represents the elevation of the pavement along a continuous imaginary line on the pavement surface. Since profilers are based on digital equipment, hence only a discrete collection of sampled points are usually measured. The profile is computed from a combination of longitudinal distance, height and acceleration measurements. Accelerometers in a profiler are analog sensors. They output a voltage that is continuous and proportional to the acceleration. Height sensors on the other hand are usually digital transducers, and hence can make a finite number of measurements in a second. Data is digitized and fed into the profile computation algorithm at certain discrete intervals. This interval is called the sample interval. In common practice, sample interval ranges from less than 1 inch to 15 inches ( $25 \mathrm{~mm}$ to $380 \mathrm{~mm}$ ).

A pavement profile consists of significant components of small wavelengths. Sampling a pavement profile at a finite interval can lead to the introduction of a larger wavelength component, which will increase the roughness of a pavement. Hence before the sensor readings are digitized, a set of measurements around a particular reading are averaged, so that the longer wavelength component (the aliased sine wave) is virtually eliminated. In reality, the signals from the height sensors and accelerometers are passed through an analog filter to eliminate the short-wavelength component, before they are 
digitized. This process is called anti-aliasing. Removing the short-wavelength content involves using a low-pass filter, which removes the short wavelengths that will not affect the ride quality of the pavement. It is also important to use the same filter on the height sensor and accelerometer signals in order to avoid aliasing.

The recommended anti-aliasing filter and sampling interval are highly interrelated. In general the sample interval should be selected such that it is half the shortest wavelength of interest in the profile (cutoff wavelength). This is based on Nyquist Sampling Theorem (Smith K.L et al , 1997).

After anti-aliasing, digitizing, and profile computation, the moving average is applied to the digitized sample points. Application of a moving average to the sampled points, generally with a base-length equal to 9.8 inches $(250 \mathrm{~mm})$, also effects the determination of sample interval. The reason is that in order to choose an integer number of data points for analysis inside the moving average base length, the effective baselength differs from the actual one. This shift in the wavelength content of the profile introduces a certain amount of error in the indices calculated based on the profile. Hence sample intervals less than 2.2 inches $(55 \mathrm{~mm})$ and 1 inch $(25 \mathrm{~mm})$ or less are generally chosen for IRI and RN, respectively.

A high pass filter with a cut off of $300 \mathrm{ft}(91 \mathrm{~m})$ is also applied to the profile, in order to improve the quality of the profile. The high pass filter removes long wavelengths that are not of much interest from the viewpoint of comfortable driving on the road. 
Besides long wavelengths are not accurately measured by the profiler. In other words the high-pass filter also helps to remove incorrect information.

\subsubsection{Operation of height sensor}

Four types of height sensors, laser, infrared, optical and ultrasonic are commonly used for profile measurement. However these four types differ in their sampling rates, resolution, size of footprint and sensitivity to the environment. Ultrasonic profilers are unable to sample at fast rates and their resolution is also insufficient for measurement of roughness on smooth roads. In order to use high speed profilers for extremely smooth roads(e.g new construction) the resolution of the sensor signals must be very high. Laser sensors are therefore best suited for this purpose. The size of a laser footprint is very small making them sensitive to coarse pavement macro-texture. However this does not present a problem because of the effective anti-aliasing filtering employed on the height sensor signals.

Lateral sensor spacing along with the lateral vehicle tracking position are also important from the point of view of roughness measurement as they determine where profiles are measured and consequently the roughness measured. The number of sensors on a profiler determines the utility of the device. Generally two sets of sensors, one each on the right and left side, are used for network level profiling applications.

\subsubsection{Operation of Accelerometer}


Accelerometers used in inertial profilers must be valid up to a frequency of $150 \mathrm{~Hz}$ in order to measure roughness accurately within the wavelength range of interest to determine IRI and PI. They must have a total range of approximately $10 \mathrm{~g}$. Errors due to accelerometer performance are generally limited to situations in which it tilts when the host vehicle undergoes pitch or roll as it travels over uneven roads. However the error is small if the lateral and longitudinal acceleration is within 0.1g. In fact accelerations greater than this rarely occur on roads conforming to the maximum allowable grade changes for the US Highway System.

\subsubsection{Longitudinal Distance Measurement}

Distance can be measured using a speedometer or a pulser installed on one of the front wheels of the host vehicle. Generally the only error that occurs with these systems is due to the calibration error or a lack of routine calibration. Several factors such as tread wear, change of tires, change in tire inflation pressure, tire warm up or even operating speed can change the effective rolling radius of a tire from what it was during calibration. This error can cause a small shift in the wavelength content of a profile. The associated values of roughness indices can then also change as they depend on the longitudinal distance over which a profile is obtained. Hence distance calibration conducted at regular intervals will effectively eliminate these errors completely.

\subsubsection{Surface Texture}


Coarse macro texture increases the potential for aliasing errors in roughness measurements. Tined texture, chip sealed asphalt or exposed aggregates do tend to make the texture rougher. However anti-aliasing filters tend to take care of the small spikes caused by these pavement surface characteristics.

\subsubsection{Pavement Distress}

Transverse cracks, opened joints, faulting, spalling and alligator cracking are some of the common distresses observed on pavements. The severity of each of these play an important role in the roughness of a pavement. However none of them should exist on new pavements.

\subsubsection{Curves}

Accelerometers tend to tilt while operating on curves. However if the lateral acceleration is small (e.g an acceleration of $0.15 \mathrm{~g}$ for a curvature with super-elevation of 4 percent, with the vehicle moving at the design speed - AASHTO Green Book, 1994) then the acceleration error is very small. Lateral acceleration normally affects longer wavelengths and hence does not affect roughness indices as these wavelengths are generally outside the range used to generate the indices.

\subsubsection{Hills and Grades}

The AASHTO Policy for Geometric Design of Highways and Streets recommends a maximum grade of 3 percent on a freeway built with a design speed of 70mi/hr (AASHTO Green Book, 1994). It also recommends the sight distance at this 
transition to be 449feet (137m). Once again hills and grades within the AASHTO Green Book Specifications introduce long wavelength curvature in the roughness measurements and hence may cause minor errors in roughness indices.

\subsubsection{Measuring Environment}

Wind, temperature, humidity and precipitation are all factors that can affect profile measurement. Moisture and surface contaminants on a pavement can cause problems with height sensors and accelerometers. It is recommended that pavements be dry and relatively free of surface contaminants prior to profile measurement.

\subsubsection{Profiler Operation}

The efficiency of the operator is one of the most important factors in profile measurement. His or her awareness and knowledge of the equipment as well as all the factors that can affect profile measurements are critical to obtaining reliable profiles.

A key example is that profilers must be operated at relatively constant speeds. Erratic speed changes do affect profile measurements. Another example is that when replicate measurements are made on a given pavement extreme caution must be given to following, as closely as possibly, the exact same wheel path location for each measurement as deviations will result in differences in reported smoothness.

\section{Field Tests}


transition to be 449feet (137m). Once again hills and grades within the AASHTO Green Book Specifications introduce long wavelength curvature in the roughness measurements and hence may cause minor errors in roughness indices.

\subsubsection{Measuring Environment}

Wind, temperature, humidity and precipitation are all factors that can affect profile measurement. Moisture and surface contaminants on a pavement can cause problems with height sensors and accelerometers. It is recommended that pavements be dry and relatively free of surface contaminants prior to profile measurement.

\subsubsection{Profiler Operation}

The efficiency of the operator is one of the most important factors in profile measurement. His or her awareness and knowledge of the equipment as well as all the factors that can affect profile measurements are critical to obtaining reliable profiles.

A key example is that profilers must be operated at relatively constant speeds. Erratic speed changes do affect profile measurements. Another example is that when replicate measurements are made on a given pavement extreme caution must be given to following, as closely as possibly, the exact same wheel path location for each measurement as deviations will result in differences in reported smoothness.

\section{Field Tests}


Significant logistics were associated with preparation for the actual field evaluation of lightweight profilers. The three key components of the field evaluation that had to be addressed prior to making the field measurements included:

1. Identification of lightweight profiler vendors willing to participate in the experiment;

2. Development of an experimental design complying with minimum FHWA requirements; and

3. Identification of test sites.

Each of these items is briefly described in the subsequent sections.

\subsection{Identification of Lightweight Profiler Vendors}

The FHWA provided the research team with a list of potential lightweight profiler vendors. At the time the study was initiated seven potential vendors were identified. The individual vendors and the names/types of equipment they had available are listed in Table 3-1.

Table 3-1 List of Potential Vendors

\begin{tabular}{|c|c|}
\hline Vendor & Equipment \\
\hline Ames Engineering Incorporated & LISA mounted on a John Deere Gator \\
\hline K.J. Law Engineering & T6400 mounted on a Kawasaki Mule \\
\hline Surface Systems and Instruments (SSI) & Laser mounted on Club Car \\
\hline International Cybernetics Corporation (ICC) & Laser mounted on an ATV \\
\hline Surfan Engineering & ROSAN mounted on your vehicle \\
\hline Trigg Industries International Incorporated & 1, 2, and 3 laser profiler mounted to your vehicle \\
\hline Pathway Services Incorporated & PathRunner LITE mounted on golf cart \\
\hline
\end{tabular}


A solicitation for participation was extended to all of the vendors listed in Table 3-1. At the recommendation of FHWA, the offer was made to only provide funding to cover transportation, lodging and per diem. All of the vendors with the exception of Surfan Engineering were receptive to the offer and willing to participate. Surface Systems and Instruments indicated that their device was in the prototype phase of development and that they would attempt to have it ready by early June 1999. Shortly there after it became clear that this was not a reality and the research team was notified. This left five potential vendors. At that point potential field test dates were selected to accommodate as many of the remaining vendors as possible. Unfortunately, it was only possible to identify dates that four of the five remaining vendors could actually be in Indiana for field testing. Thus four lightweight profilers, as identified in Table 3-2, were evaluated in the study.

Table 3-2 List of Participating Vendors

\begin{tabular}{|c|c|}
\hline Vendor & Equipment \\
\hline Ames Engineering Incorporated & LISA mounted on a John Deere Gator \\
\hline International Cybernetics Corporation (ICC) & Laser mounted on an ATV \\
\hline K.J. Law Engineering & T6400 mounted on a Kawasaki Mule \\
\hline Pathway Services Incorporated & PathRunner LITE mounted on golf cart \\
\hline
\end{tabular}

\subsection{Experimental Design}

The experimental design was driven by two factors. They were the need to obtain adequate data to evaluate the repeatability and reproducibility of the lightweight profilers and at the same time satisfy minimum requirements place on the project by FHWA in order to obtain the financial support it offered. The FHWA requirements included: 
1. A minimum of three lightweight profilers had to be evaluated in the study;

2. Test sections had to be a minimum of one mile in length and it was recommended that one or more sites incorporate a curve(s); and

3. Five replicate measurements had to be made on a 0.1 mile portion of each test section using each lightweight profiler.

In order to develop an estimate of the precision of a test method ASTM C802, "Standard Practice for Conducting an Interlaboratory Test Program to Determine the Precision of Test Methods for Construction Materials" specifies that an experiment which incorporates a minimum of thirty degrees of freedom be conducted. It was important to INDOT that both hot mix asphalt (HMA) and portland cement concrete (PCC) pavements be equally represented in the experiment. The participating vendors suggested that it would be possible to perform the testing at a maximum of three sites per day. Therefore it was decided that three each HMA and PCC test sites would be included in the experiment in order to limit the field testing to a two day period. This resulted in an experiment with a total of sixty $[(6$ sites -1$) \times(4$ vendors -1$) \times(5$ measurements/site/vendor -1$)=60]$ degrees of freedom. The experimental design is summarized in Table 3-3.

As designed, the experiment provided adequate data to assess the repeatability and reproducibility of the lightweight profilers in accordance with ASTM standards and at the same time satisfied the FHWA requirements of including a minimum of the three lightweight profilers and performing five replicate measurements per test site and profiler. 
Table 3-3 Experimental Design

\begin{tabular}{|c|c|c|c|c|c|c|c|c|}
\hline \multirow{4}{*}{$\begin{array}{c}\text { Pavement } \\
\text { Type }\end{array}$} & \multirow{4}{*}{ Sites } & \multicolumn{7}{|c|}{ Profiling Technique } \\
\hline & & \multicolumn{4}{|c|}{ Lightweight Profilers } & \multirow{2}{*}{$\begin{array}{l}\text { High Speed } \\
\text { Inertial } \\
\text { Profiler } \\
\text { INDOT } \\
\text { RIP Van }\end{array}$} & \multirow{2}{*}{$\begin{array}{c}\begin{array}{c}\text { California } \\
\text { Profilograph }\end{array} \\
\text { Ames }\end{array}$} & \multirow{2}{*}{$\begin{array}{c}\begin{array}{c}\text { Rod and } \\
\text { Level } \\
\text { Survey }\end{array} \\
\text { N/A }\end{array}$} \\
\hline & & Ames & ICC & $\begin{array}{c}\text { K J } \\
\text { Law }\end{array}$ & Pathway & & & \\
\hline & & \multicolumn{7}{|c|}{ Replicate Measurements per Site } \\
\hline PCC & 1 & 5 & 5 & 5 & 5 & 5 & 4 & 1 \\
\hline HMA & $2 *$ & 8 & 8 & 8 & 8 & 5 & & \\
\hline HMA & $3 * *$ & 10 & 10 & 10 & 10 & 5 & & \\
\hline HMA & 4 & 5 & 5 & 5 & 5 & 5 & & \\
\hline PCC & 5 & 5 & 5 & 5 & 5 & 5 & & \\
\hline $\mathrm{PCC}$ & 6 & 5 & 5 & 5 & 5 & 5 & & \\
\hline
\end{tabular}

* Site 2 had 8 replicate runs, 5 with board and 3 without board

** Site 3 had 10 replicate runs, 5 with board and 5 without board

In addition to the lightweight profilers, the INDOT high speed inertial profiler, referred to as the "RIP Van" by INDOT personnel, was also used to make five profile measurements per site. The obvious reason for including the INDOT RIP Van was to evaluate performance of the lightweight profilers relative to the van. At one site, four replicate measurements were planned with a California Profilograph and a precision rod and level survey was planned for the same site. The California Profilograph was incorporated into the experiment because INDOT's current smoothness specification is based on the use of a California Profilograph. The rod and level survey was included to provide a "true" profile, or at least as true a profile as possible. It would have been impractical time wise to make five measurements per site with the California Profilograph and the Rod and Level survey, thus their limited use.

It should be noted that the experimental design specified that measurements be made on a given section with all devices on a given day in a well marked left wheel path 
in as short a time period as possible. It also specified that the testing (order in which individual vendors performed measurements) be conducted in a random fashion. The intention of these specifications were to eliminate any ambiguity due to changes in roughness with time, time of day, temperature, and environmental factors, as well as to eliminate any systematic error due to testing order.

\subsection{Site Selection Process}

Test sites were selected by researching various ongoing and recently completed paving projects that would provide relatively new surfaces suitable for evaluating the lightweight non-contact profilers. Potential sites were identified through several sources including the local newspaper, the INDOT web-site, and communication with INDOT, specifically the Research Division Section Manager and the Crawfordsville District Construction Engineer. The potential sites that were identified through these sources are listed in Table 3-4 . 
Table 3-4: Potential Sites for Evaluation of Lightweight Non-Contact Profilers.

\begin{tabular}{|c|c|c|l|l|}
\hline Surface & Route & District & County \\
\hline $\begin{array}{c}\text { Full Depth } \\
\text { HMA(Over } \\
\text { Rubblized CRC } \\
\text { Pavement) }\end{array}$ & I-65 & $\begin{array}{c}\text { On I-65 from 2.75km N of SR 43 to } \\
1.61 \mathrm{~km} \text { N of Rest Area ( White County) } \\
\text { RP 180+074 to 197+067 }\end{array}$ & Crawfordsville & Tippecanoe \\
\hline $\begin{array}{c}\text { Full Depth } \\
\text { HMA(0.9 miles } \\
\text { Net Length) }\end{array}$ & SR 267 & $\begin{array}{c}\text { CRC RR Over SR 267 (RP 22+74 to RP } \\
\text { 23+68) and SR 267 Over Wiley } \\
\text { Thompson Ditch (RP23+70), 4.6 \& 5.8 } \\
\text { km North of I-74 }\end{array}$ & Crawfordsville & Hendricks \\
\hline HMA & I-70 & $\begin{array}{c}\text { On I-70 From The Illinois/Indiana State } \\
\text { Line to the West End of the Wabash River } \\
\text { Bridge (RP 0+00 to 5+42) }\end{array}$ & Crawfordsville & Vigo \\
\hline HMA & US 231 & $\begin{array}{c}\text { On US 231 From the North Junction of } \\
\text { SR 25 to 1km South of Wabash River } \\
\text { ( Harrison Bridge) in Lafayette }\end{array}$ & Crawfordsville & Tippecanoe \\
\hline PCC Pavement & US 231 & $\begin{array}{c}\text { US 231 From 0.03 Mile South of SR 25 } \\
\text { to 0.02 mile North of SR 25 }\end{array}$ & Crawfordsville & Tippecanoe \\
\hline PCC Pavement & N 231 & $\begin{array}{c}\text { From 0.82 of Mile South of County Road } \\
500 S \text { (RP 900+00) to 0.03 of Mile South } \\
\text { of SR 25 (RP 903+85) }\end{array}$ & Crawfordsville & Tippecanoe \\
\hline PCC Pavement & SR 231 & $\begin{array}{c}\text { On SR231 between Country Roads 300 } \\
\text { and 500 South }\end{array}$ & Crawfordsville & Tippecanoe \\
\hline HMA Overlay & SR 25 & $\begin{array}{c}\text { On SR25 from US52 to 0.5 miles east of } \\
\text { US 52 }\end{array}$ & Crawfordsville & Tippecanoe \\
\hline HMA & I-74 & $\begin{array}{c}\text { From 0.5miles East of SR 32 to Walnut } \\
\text { Fork Creek }\end{array}$ & Crawfordsville & \\
\hline bound) & $\begin{array}{c}\text { From 0.9miles East of US 41 to 6.6miles } \\
\text { East of the East junction of SR 18 }\end{array}$ & Crawfordsville & \\
\hline
\end{tabular}

The Lafayette City Engineer's office was also contacted and was very receptive to inclusion of site within the city for the project. All potential sites were located and highlighted on city and state maps. Much of the city resurfacing activities had been performed in the downtown area. These sites were dropped from consideration and a short-list of potential sites within the city was generated. The list is summarized in Table 3-5. 
Table 3-5 Shortlist of Potential Sites Provided by Lafayette City Engineers office.

\begin{tabular}{|c|c|c|}
\hline Road Name & From & To \\
\hline $27^{\mathrm{TH}}$ Street & Elmwood Avenue & Vinton Street \\
\hline Elmwood Court & Dead End & Elmwood Avenue \\
\hline Elmwood Court & Elmwood Avenue & Elmwood Avenue \\
\hline Longlois Drive & $29^{\mathrm{TH}}$ Street & Elmwood Avenue \\
\hline Prairie Lane & Vinton Street & Elmwood Avenue \\
\hline Vinton Street & Elmwood Avenue & Longlois Drive \\
\hline Mulberry Drive & Cedar Lane & Pine Lane \\
\hline Cedar Lane & Catalpa Court & Dead End \\
\hline Sequoya Drive & Beck Lane & Teal road \\
\hline
\end{tabular}

The suitability of each remaining potential site was determined by assessing its length, whether a curve were present, and safety. Based on a review of the sites pinpointed on the maps the sites were then surveyed by the research team. The twelve sites that were surveyed included:

1. $27^{\text {th }}$ Street (from Elmwood Avenue to Vinton Street);

2. Elmwood Court (from a dead end to Elmwood Avenue);

3. Elmwood Court (from a point on Elmwood Avenue to another point on Elmwood avenue);

4. Longlois Drive (from $29^{\text {th }}$ Street to Elmwood Avenue);

5. Prairie Lane (from Vinton Street to Elmwood Avenue);

6. Vinton Street (from Elmwood Avenue to Longlois Drive);

7. Mulberry Drive (from Cedar Lane to Pine Lane);

8. Cedar Lane (from Catalpa Court to a dead end);

9. Sequoya Drive (from Beck Lane to Teal Road);

10. US 231 (North of CO RD 500S); 
11. I-65 (On I-65 from $2.75 \mathrm{~km}$ North of SR 43 to $1.61 \mathrm{~km}$ North of Rest Area); and

12. Convinton Street between Dubois and Pike Streets.

The sites were then narrowed down to a few potential sites through consideration of the following factors :

1. Safety of personnel involved in testing;

2. Location relative to West Lafayette;

3. Necessity for closure;

4. Required traffic control;

5. Geometric considerations (length, curvature and grade);

6. Suitability of pavement surface (type, age and condition);

7. FHWA requirements; and

8. Adequacy of staging area;

Based on these considerations, six sites were identified as appropriate for actual profiler evaluation. The selected sites are listed in Table 3-6. Pavement types, geometric considerations and general pavement conditions at each site are provided in the table. 


\section{Table 3-6 Summary of Site Condition}

\begin{tabular}{|c|c|c|}
\hline $\begin{array}{l}\text { Site number and } \\
\text { description }\end{array}$ & Geometric Considerations & Pavement Condition \\
\hline $\begin{array}{c}1 \\
\text { (Covington St.) }\end{array}$ & $\begin{array}{l}\text { No grade; } \\
\text { Slight curve at approximately } \\
180 \text { feet from start; } \\
\text { Standard cross slope; }\end{array}$ & $\begin{array}{l}\text { PCC; Approximately } 6 \text { months old (as new); } \\
\text { Broom finish; well sealed hand cut joints, } \\
\text { No visual distress; } \\
\text { Wet surface (no ponding); }\end{array}$ \\
\hline $\begin{array}{c}2 \\
\text { (Interstate 65) }\end{array}$ & $\begin{array}{l}\text { No grade; } \\
\text { Continuous curve throughout } \\
\text { section; } \\
\text { Standard cross slope; }\end{array}$ & $\begin{array}{l}\text { Full Depth HMA; New (untrafficked); Tight } \\
\text { surface texture; No visual distress; Wet surface } \\
\text { (no ponding); }\end{array}$ \\
\hline $\begin{array}{c}3 \\
\text { (Prairie St.) }\end{array}$ & $\begin{array}{l}\text { Significant uphill grade } \\
\text { followed by slight downhill } \\
\text { grade and slight uphill grade; } \\
\text { Curve through first } \\
\text { approximately } 200 \text { feet; } \\
\text { Cross slope in excess of } 2 \% \text {; }\end{array}$ & $\begin{array}{l}\text { HMA Overlay; Approximately } 10 \text { months old; } \\
\text { Two manholes in right wheel path; } \\
\text { No visual distress; }\end{array}$ \\
\hline $\begin{array}{c}4 \\
\text { (Vinton St.) }\end{array}$ & $\begin{array}{l}\text { Downhill grade throughout } \\
\text { section; } \\
\text { Continuous curve throughout } \\
\text { section; } \\
\text { Standard cross slope in } \\
\text { excess of } 2 \% \text {; }\end{array}$ & $\begin{array}{l}\text { HMA Overlay; Approximately } 10 \text { months old; } \\
\text { Full width utility patch at approximately } 150 \\
\text { feet; Possible transverse reflective cracking }(\approx 5) \\
\text { noticeable due to moist surface; }\end{array}$ \\
\hline $\begin{array}{c}5 \\
\text { (US 231 A) }\end{array}$ & $\begin{array}{l}\text { No grade; } \\
\text { Continuous curve throughout } \\
\text { section; } \\
\text { Standard cross slope; }\end{array}$ & $\begin{array}{l}\text { PCC; Approximately } 24 \text { months old (as new); } \\
\text { Random tining; well sealed saw cut joints; } \\
\text { No visual distress; }\end{array}$ \\
\hline $\begin{array}{c}6 \\
\text { (US 231 B) }\end{array}$ & $\begin{array}{l}\text { Slight downhill grade; } \\
\text { Continuous curve throughout } \\
\text { section; } \\
\text { Standard cross slope; }\end{array}$ & $\begin{array}{l}\text { PCC; Approximately } 24 \text { months old (as new); } \\
\text { Random tining; well sealed saw cut joints; } \\
\text { No visual distress; }\end{array}$ \\
\hline
\end{tabular}

Permission was formally sought and obtained from the City Engineer prior to conducting tests on the city owned sites.

\subsection{Field Testing}

The field evaluation was conducted June $24^{\text {th }}$ and $25^{\text {th }} 1999$ at sites surrounding the West Lafayette, Indiana area. Testing was conducted at the six sites listed in Table 35. Three of the sites were HMA and three were PCC pavement. All four lightweight profilers, as well as the INDOT RIP Van were used at each site. A California 
Profilograph, manufactured by Ames Engineering, as well as a precision rod and level survey were employed at the PCC Covington Street site.

A meeting of all participants and the research team was held at the INDOT Research Division on the morning of June $24^{\text {th }}$. The meeting served several purposes. Most importantly it was a forum for the vendors, researchers, INDOT and FHWA personnel to meet with each other and discuss the field testing prior to actually conducting it. Following introductions the researchers provided a description of the planned field testing and asked for input from all present to facilitate a partnering atmosphere, ensure that the plan was reasonable, and describe detailed test protocol. Items discussed included the need to insure that each vendor:

1. Had calibrated their respective devices (lasers and accelerometers) prior to initiating the testing;

2. Employed the same high and low pass filtering;

3. Understood the data that would be requested from them (eg. profiles and smoothness indices) including electronic file format and naming structure as well as reporting units;

4. Became familiar with local maps in case someone were inadvertently separated from the planned caravan;

5. Understood how each site were physically laid out (beginning and ending points and test wheel path); and

6. Most importantly were conscious of safety in the field (INDOT provided each participant with blaze orange hats and vests). 
Other items that were discussed were a distance calibration that would be conducted immediately following the meeting and the order in which the testing would be conducted at each site. A random drawing was performed to establish testing order at each site. Five pieces of paper with the numbers one through five were placed in a hat and each participant drew one piece of paper from the hat. The number drawn by each vendor defined their place in the testing order at a given site. Therefore the drawing was repeated six times to establish the order for all sites. The order established through the random drawing is summarized in Table 3-7. Five replicate measurements were to be performed with each device at each site. Each vendor was to perform one measurement in the order established through the random drawing. The measurements were then to be repeated four more time in the same order within a site. In other words, five measurements were to be performed with each device at each site in the order presented in Table 3-7. The reason for this was to prevent any bias that could possibly be introduced into the results by changes in the actual profile due to environmental conditions and to eliminate systematic errors. 
Table 3-7 Individual Site Testing Order Established by Random Drawing

\begin{tabular}{|c|c|c|}
\hline Site & Vendor & Testing Order by Random Drawing \\
\hline \multirow{5}{*}{$\begin{array}{c}1 \\
\text { Covington Street }\end{array}$} & Ames & 2 \\
\hline & ICC & 3 \\
\hline & K.J. Law & 1 \\
\hline & Pathway & 4 \\
\hline & InDOT & 5 \\
\hline \multirow{5}{*}{$\begin{array}{c}2 \\
\text { Interstate } 65\end{array}$} & Ames & 4 \\
\hline & ICC & 1 \\
\hline & K.J. Law & 3 \\
\hline & Pathway & 2 \\
\hline & InDOT & 5 \\
\hline \multirow{5}{*}{$\begin{array}{l}3 \text { and } 4 \\
\text { Prairie and Vinton Streets }\end{array}$} & Ames & 4 \\
\hline & ICC & 3 \\
\hline & K.J. Law & 1 \\
\hline & Pathway & 5 \\
\hline & InDOT & 2 \\
\hline \multirow{5}{*}{$\begin{array}{c}5 \\
\text { US } 231 \mathrm{~A}\end{array}$} & Ames & 1 \\
\hline & ICC & 4 \\
\hline & K.J. Law & 2 \\
\hline & Pathway & 5 \\
\hline & InDOT & 3 \\
\hline \multirow{5}{*}{$\begin{array}{c}6 \\
\text { US } 231 \mathrm{~B}\end{array}$} & Ames & 2 \\
\hline & ICC & 5 \\
\hline & K.J. Law & 4 \\
\hline & Pathway & 1 \\
\hline & InDOT & 3 \\
\hline
\end{tabular}

\subsection{Description of Profilers}

A brief description of the profiling equipment and techniques employed for the study is presented in this section prior to describing details of the actual measurements made at each site.

\subsubsection{Lightweight Profilers}

A brief description of each of the four lightweight profilers employed is provided in the following subsections. Fourteen critical profiler characteristics are provided with a picture of each device. 


\subsubsection{Ames Engineering Incorporated}

Figure 3-1 is a picture of the LISA device manufactured by Ames Engineering Incorporated.

Profiler Name : Ames Lightweight Profiler (LISA)

Method of data collection : Accelerometer, Laser and Distance Measurement equipment

Classification: Class 1 road profiler devices ( according to ASTM E950-94)

Indices: International Roughness index (IRI), Profile Index (PI), Ride Number (RN) in in $/ \mathrm{mi}$ or $\mathrm{mm} / \mathrm{km}$.

Sampling rate: 1 inch

Storage Interval:

Wavelengths : $1.8-120$ feet

Sensor Resolution: 0.001 inch

Distance Accuracy:

Controls : Pentium, $133 \mathrm{Mhz}$, IBM compatible

Speed $\quad: 8-12$ miles/hour

Vehicle Used : John Deere 4 wheel Gator

Bump identification: Must Grind Locations can be identified

Additional Features : Start-Stop Triggering and Lead-in 


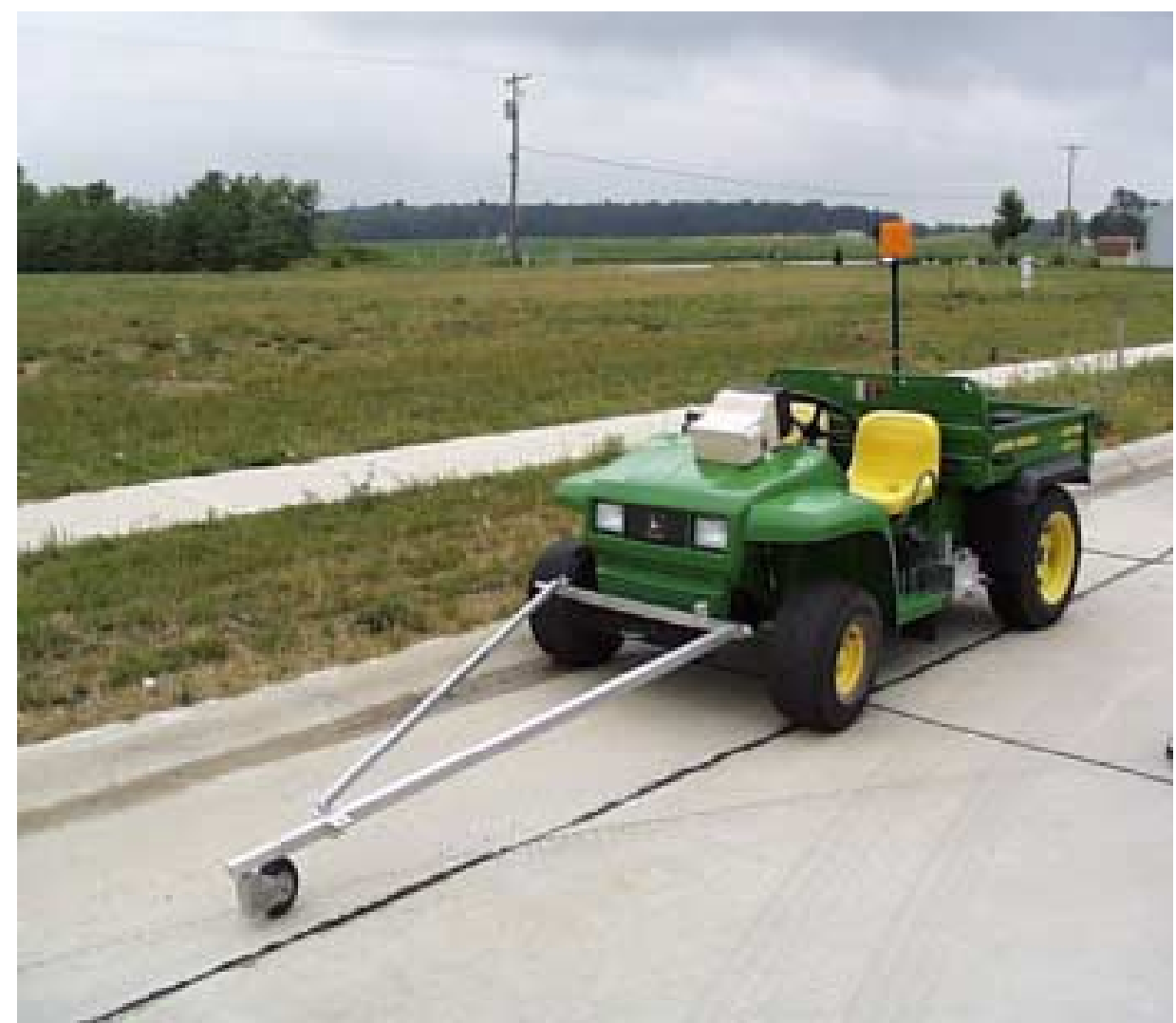

Figure 3-1 Ames Engineering Incorporated Lightweight Profiler

\subsubsection{International Cybernetics Corporation}

Figure 3-2 is a picture of the Mobile Data Recording System manufactured by International Cybernetics Corporation.

Profiler Name : Mobile Data Recording System

Method of data collection : Accelerometer, Laser and Distance Measurement equipment

Classification: Class 1 road profiler devices ( according to ASTM E950-94)

Indices: International Roughness index (IRI), Profile Index (PI), Ride Number (RN) in in $/ \mathrm{mi}$ or $\mathrm{mm} / \mathrm{km}$.

Sampling rate: 1 inch 
Storage interval: 1 inch

Wavelengths : $1.8-1000$ feet

Sensor Resolution: 0.001 inch

Distance accuracy: 0.1\%

Controls : MDR-4080 Road Profiler Family Mobile Data Recorder

Speed : up to 40 miles/hour

Vehicle Used : Laser mounted on an ATV

Bump identification: Must Grind Locations can be identified

Additional Features : Start-Stop Triggering and Lead-in

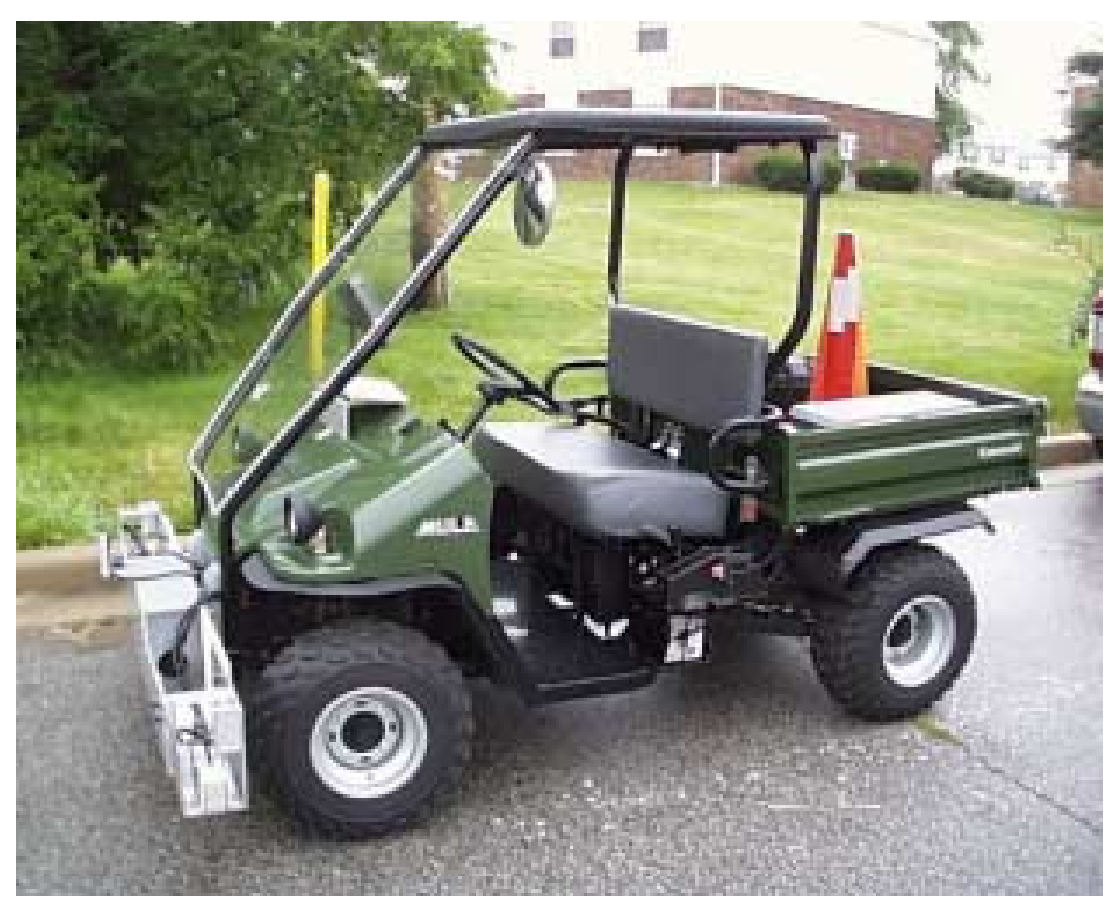

Figure 3-2 International Cybernetics Corporation Lightweight Profiler 


\subsubsection{K J Law Engineering}

Figure 3-3 is a picture of the T6400 Lightweight Profilometer manufactured by K J Law Engineering.

Profiler Name : T6400 Lightweight Profilometer

Method of data collection : Accelerometer, Laser and Distance Measurement equipment

Classification: Class 1 road profiler devices ( according to ASTM E950-94)

Indices: International Roughness index (IRI), Profile Index (PI), Ride Number (RN) in $\mathrm{in} / \mathrm{mi}$ or $\mathrm{mm} / \mathrm{km}$.

Sampling rate: $1 \mathrm{inch}$

Storage interval: 6 inch / 1 inch if required

Wavelengths:

Sensor Resolution: 0.001 inch

Distance accuracy: $0.1 \%$

Controls : IBM compatible PC with state-of-the-art CPU

Speed $\quad: 10-25$ miles/hour

Vehicle Used : T6400 mounted on a Kawasaki Mule

Bump identification: Must Grind Locations can be identified

Additional Features : Start-Stop Triggering and Lead-in 


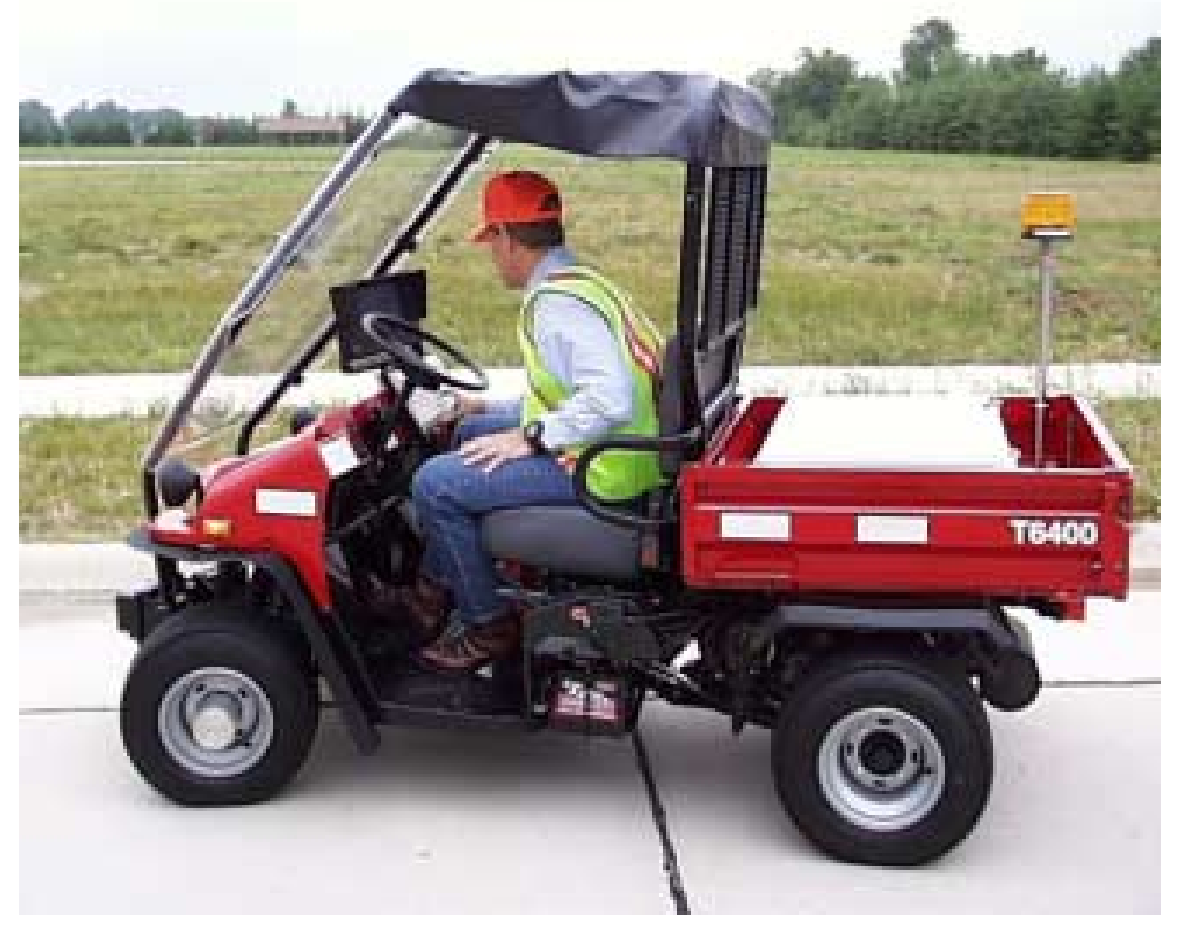

Figure 3-3 K J Law Engineering Lightweight Profilometer

\subsubsection{Pathway Services Incorporated}

Figure 3-4 is a picture of the PathRunner Lite manufactured by Pathway Services Incorporated.

Profiler Name : PathRunner LITE

Method of data collection : Accelerometer, Laser and Distance Measurement equipment Classification: Class 1 road profiler devices ( according to ASTM E950-94)

Indices: International Roughness index (IRI), Profile Index (PI), Ride Number (RN) in in $/ \mathrm{mi}$ or $\mathrm{mm} / \mathrm{km}$.

Sampling rate: 1 inch

Storage interval: 1 inch 
Wavelengths : $1-300$ feet

Sensor Resolution: 0.001 inch

Distance accuracy: $0.1 \%$ per mile

Controls : PCMC1A Based Pentium II PC Computer System

Speed $\quad: 5-20$ miles/hour

Vehicle Used : PathRunner LITE mounted on golf cart

Bump identification: Must Grind Locations can be identified

Additional Features : Start-Stop Triggering and Lead-in

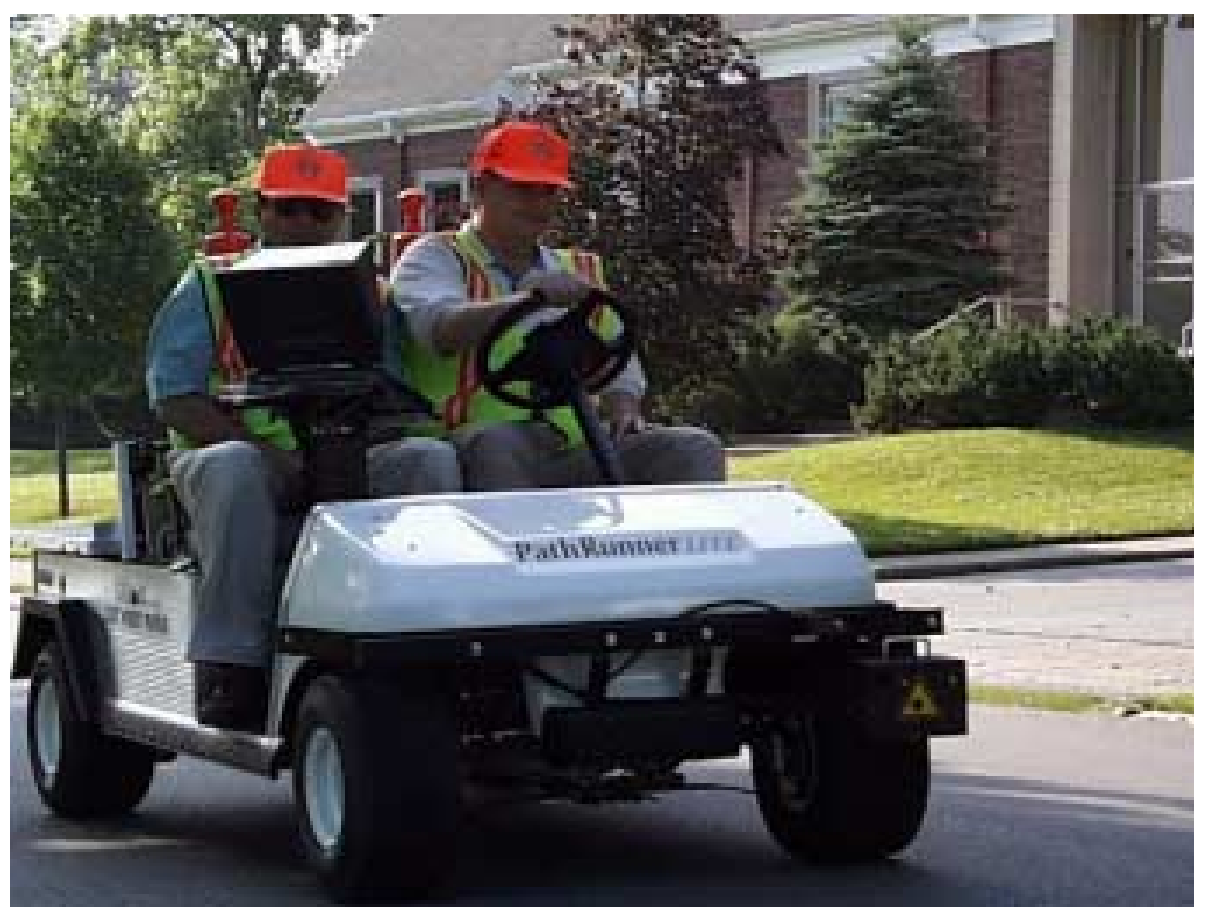

Figure 3-4 Pathway Services Incorporated Lightweight Profiler 


\subsubsection{INDOT RIP VAN Inertial High Speed Profiler}

Figure 3-5 is a picture of the Rip Van manufactured by INDOT.

Profiler Name : RIP Van

Indices : International Roughness Index (IRI) and Rut-Depth.

Method of data collection : Accelerometer, Laser and Distance Measurement equipment

Classification: Class 1 road profiler devices ( according to ASTM E950-94)

Distance measurement : Arthur Allen model \# AA-1422-20 hall-effect transducer

Profile measurement : Both left and right wheel paths possible as one accelerometer and one opticator have been placed in both wheel paths

Sampling Rate :

Storage Interval :

Wavelengths:

Sensor Resolution:

Distance Accuracy:

Controls:

Speed:

Sensitivity of accelerometer $: \pm 2 \mathrm{~g}$

Vehicle Used : 1986 GMC van

Typical Use - High Speed, Network Level PMS

Operational Characteristics : Speed, lead-in, data collection 


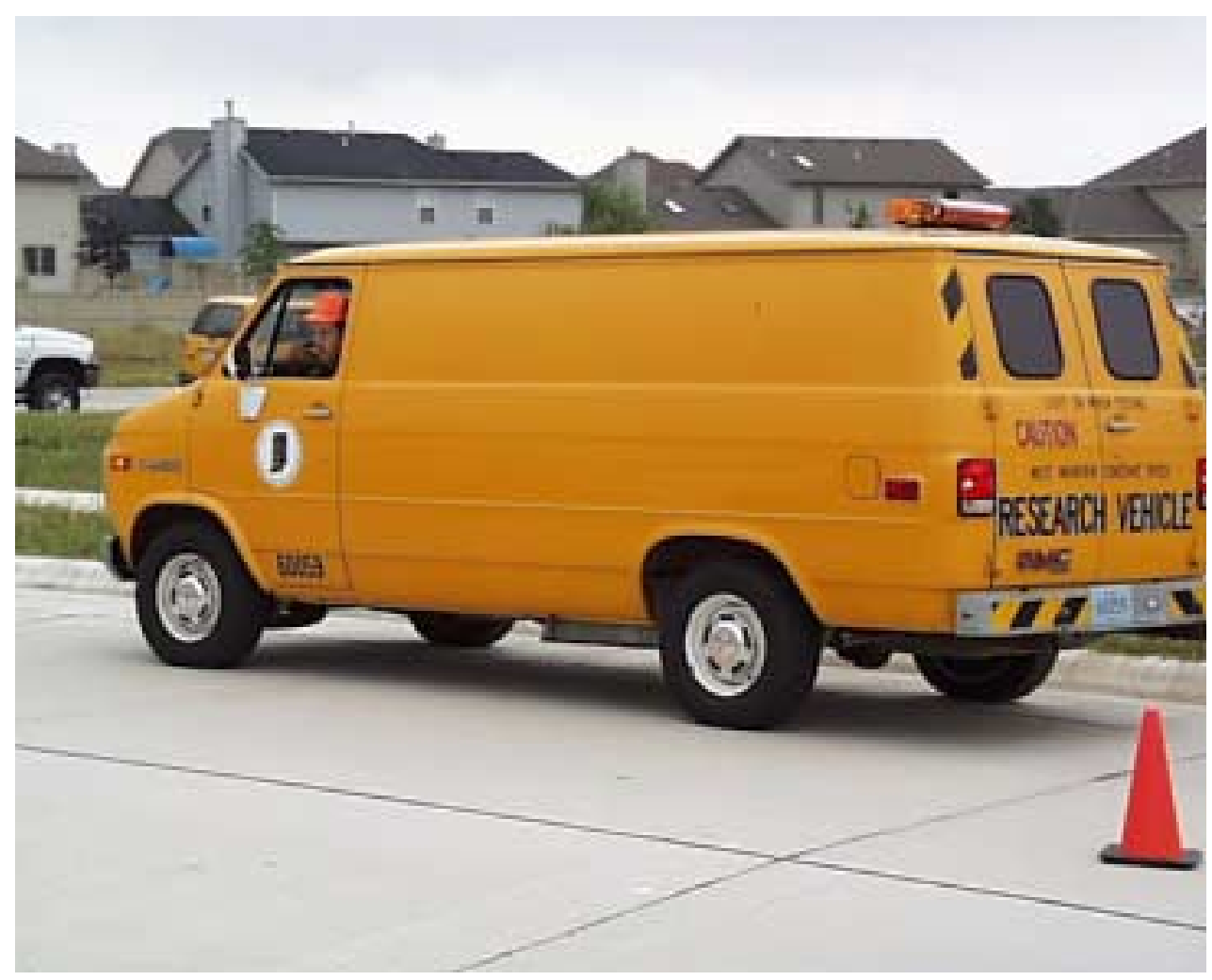

Figure3-5 INDOT Rip Van- High Speed Profiler

\subsubsection{California Profilograph}

A California Profilograph manufactured by Ames Engineering as depicted in

Figure 3-6 was used. It was a manual device owned and operated by INDOT. The profilograph was developed in 1986. The system consists of a total of six wheels used to support each end of the device. However instead of using a truss type framework (which all the other profilographs use), the $25 \mathrm{ft}$ beam portion of the Ames device is a 2 in by 6in aluminium box channel. A profile wheel is located at its midpoint, and a non-contact ultrasonic transducer system transmits movement of the profile wheel to a recorder located at the rear end of the unit. It provides strip chart output at the end of the run showing the profilogram trace. The device was tested and certified by the INDOT 
Division of Materials and Tests prior to its use on this project. Experienced INDOT personnel conducted the tests with this device in order to increase the accuracy of the measurement.

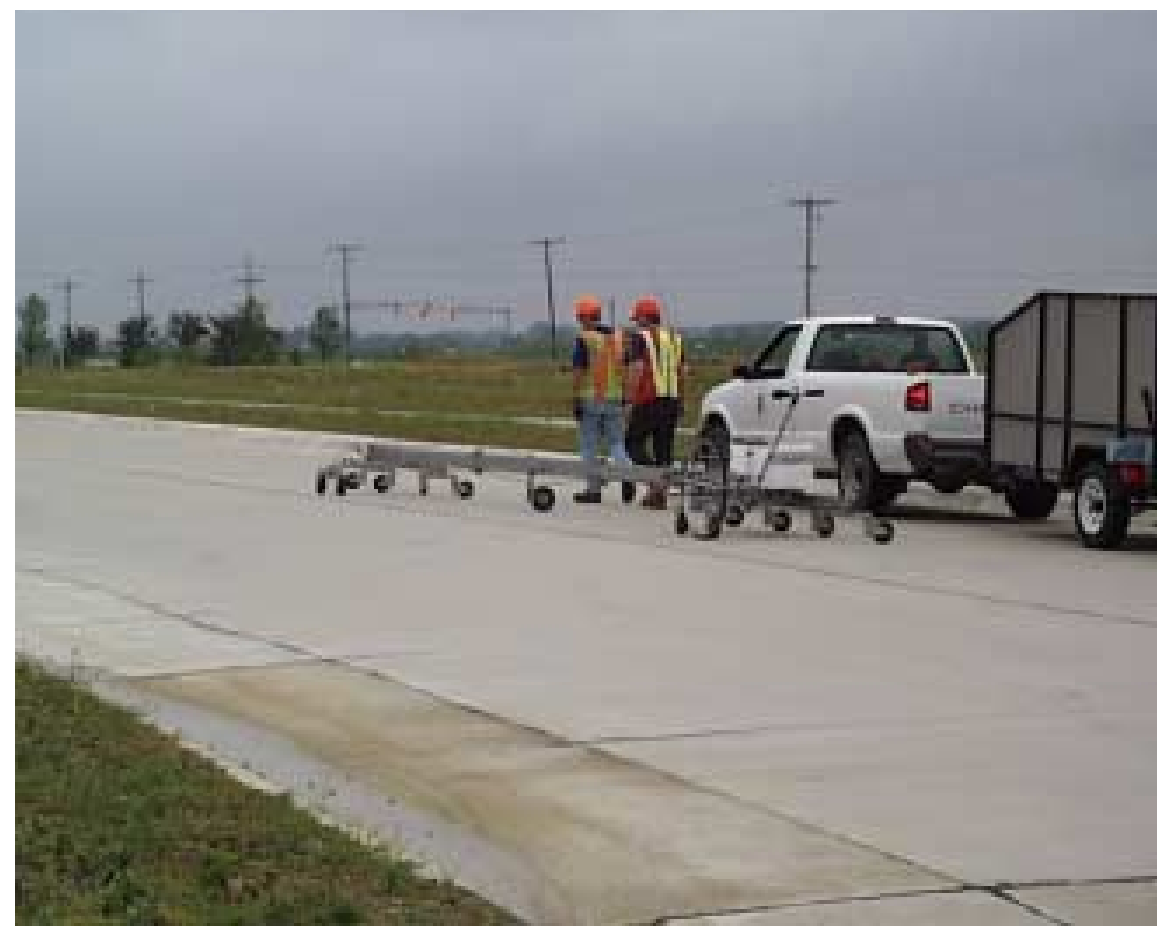

Figure 3-6 California Profilograph (Manufacturer Ames Engineering Inc.)

\subsubsection{Precision Survey}

A three-wire precision rod and level survey was performed at Site 1. A Sokkisha micrometer level was used for the survey along with a precision-scaled rod. The use of this instrument made it possible to achieve the required precision of 0.0005 inch $(0.0125 \mathrm{~mm})$ stated in ASTM E1364-95. The survey was conducted in accordance with the same ASTM standard. Measurements were obtained at 12 inches $(0.3 \mathrm{~m})$ longitudinal increments over a length of approximately five hundred forty-eight feet $(167 \mathrm{~m})$. At each 
station three reading were obtained; top-wire, middle-wire, and bottom-wire. This method along with calculations acted as a check for each reading such that errors in both elevation readings and note taking would be avoided. This was important because this type of survey is very tedious. A temporary benchmark was established to ensure closure of the level loop. The manually recorded data were entered in Microsoft Excel for plotting.

\subsection{Distance Calibration}

Prior to performing any field measurements, all devices with the exception of the California Profilograph, participated in a distance calibration. It should be noted however that a distance calibration was included in the profilograph certification which the device went through the week prior to use.

The calibration site was located on Yeager Road between Cumberland Street and County Road 350 North in West Lafayette. The site layout was performed by INDOT Research Division personnel. A steel tape, corrected for temperature, was used for distance measurement. The left wheel path of the north bound lane was marked with a blotch of white paint every 10 feet to provide a guide for the profile operators to follow. This was in an effort to minimize lateral or transverse wander that could result in inaccurate distance measurement. The beginning and ending points were identified with a nail driven into the pavement through a small piece of surveyors tape. They were also marked with a strip of white paint. Figure 3-7 is an example of marking the wheel path location. 


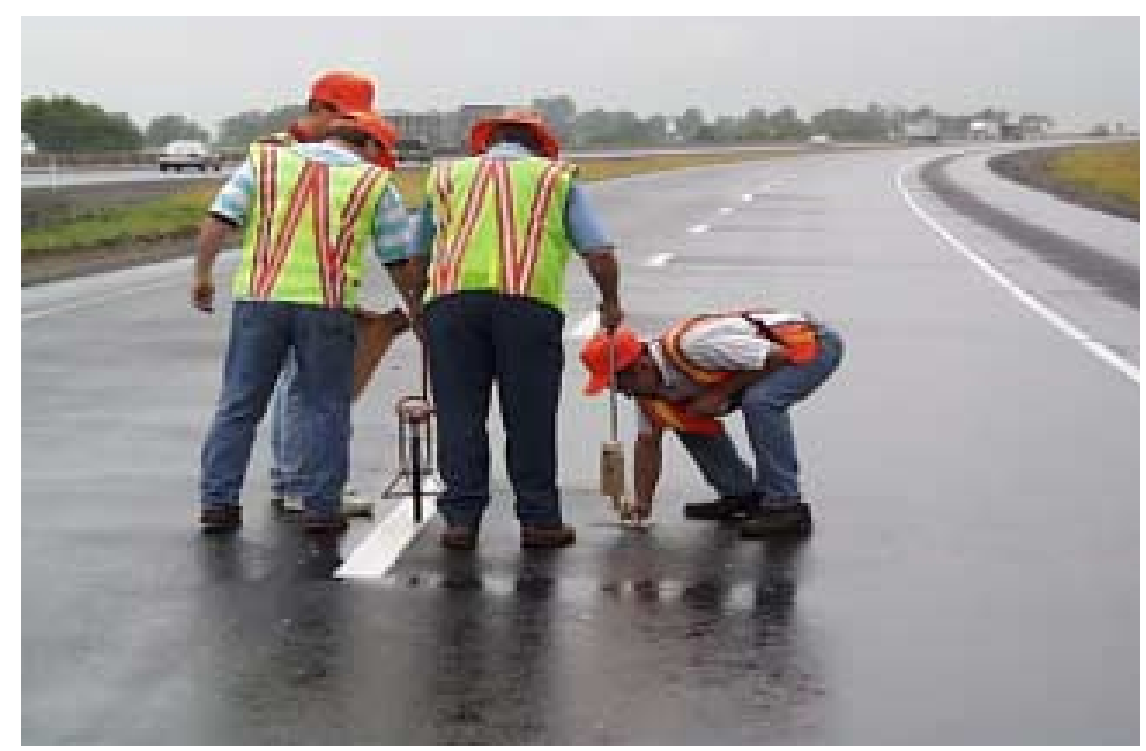

Figure 3-7 Laying out Wheel Path with Paint Blotches

A calibration distance of $1000 \mathrm{ft}(305 \mathrm{~m})$ was employed. Because the actual test sections were $0.1 \mathrm{mi}(177 \mathrm{~m})$ in length, distance errors attributable to calibration would be limited to approximately one half of any error introduced in the calibration process.

\section{$\underline{3.7 \quad \text { Profile Measurements }}$}

A $0.1 \mathrm{mi}(177 \mathrm{~m})$ test section was laid out at each test site by INDOT personnel. The same techniques used to mark the start and finish points, as well as the testing wheel path (left) at the calibration site, were used for each of the test sites. A rolling wheel was used for distance measurement rather than a steel tape. All measurements were made in the left wheel path and only left wheel path data were used for analyses. 
A hat drawing (random number drawing) was used to establish the order in which the profilers would perform measurements at each site. Only five drawings were conducted because measurements at Sites 3 and 4 were taken in a single circuit.

\subsubsection{Site 1 (Covington Street) Measurements}

The rod and level survey was conducted over a period of two days surrounding the day the measurements were made with the lightweight profilers. The survey was performed in accordance with ASTM E1364-95, under the most restrictive precision requirements. In addition to the test section, an addition $10 \mathrm{ft}(3 \mathrm{~m})$ leading into and out of the test section were surveyed. This was done so that moving averages could be calculated if necessary to compare the multiple profile measurements. A single survey was conducted (no replicate measurements). The weather conditions were clear, warm, dry, and there was no wind during the survey. The pavement surface was dry. Five measurements were performed with each of the lightweight profilers and the INDOT Rip Van in the order presented in Table 3-4. Four measurements were made with an Ames California Profilograph by INDOT personnel.

\subsubsection{Site 2 (Interstate 65) Measurements}

Five measurements were initially performed with each of the lightweight profilers and the INDOT RIP Van in the order presented in Table 3-4. At a distance of 10 feet (3m) from the start and finish of the test section $3 / 4$ inch $(20 \mathrm{~mm})$ thick by $2 \mathrm{ft}(0.61 \mathrm{~m})$ wide sheets of plywood extending the full lane with were placed to simulate bumps and/or bridge abutments. After five measurements had been made with each device, the 
plywood strips were removed and an additional three measurements were made with each device in the same testing order. The plywood induced significant dynamics to all of the lightweight profilers. The weather conditions were warm, damp, overcast, and there was no wind during the testing. The pavement surface was damp, but there was no standing water and all of the vendors were comfortable with the surface conditions (dampness) at the time of testing.

\subsubsection{Site 3 (Prairie Street) Measurements}

Five measurements were initially performed with each of the lightweight profilers and the INDOT Rip Van in the order presented in Table 3-4. At a distance of 10 feet (3m) from the start and finish of the test section $3 / 8$ inch $(10 \mathrm{~mm})$ thick by 2 feet $(0.61 \mathrm{~m})$ wide sheets of particle board extending from the centerline through the left wheel path (testing wheel path) were placed to simulate bumps and/or bridge abutments. After five measurements were made with each device, the particle boards were removed and an additional five measurements were made with each device in the same testing order. The thinner particle board strips did not induced the dynamics that the plywood did at Site 2 . The weather conditions were warm, dry, sunny, and there was no wind during the testing. The pavement surface was dry.

\subsubsection{Site 4 (Vinton Street) Measurements}

Five measurements were performed with each of the lightweight profilers and the INDOT Rip Van in the order presented in Table 3-4. No bumps were employed to 
simulate bridge abutments. The weather conditions were warm, dry, sunny, and there was a slight breeze during the testing. The pavement surface was dry.

\subsubsection{Site 5 (US 231 A) Measurements}

Five measurements were performed with each of the lightweight profilers and the INDOT Rip Van in the order presented in Table 3-4. No bumps were employed to simulate bridge abutments. The weather conditions were warm, dry, sunny, and there was no wind during the testing. The pavement surface was dry.

\subsubsection{Site 6 (US 231 B) Measurements}

Five measurements were performed with each of the lightweight profilers and the INDOT Rip Van in the order presented in Table 3-4. No bumps were employed to simulate bridge abutments. The weather conditions were warm, dry, sunny, and there was no wind during the testing. The pavement surface was dry.

\subsection{Other Field Activities}

In addition to performing the measurements described above, two other field activities took place. At the end of the first day of testing, the vendors provided demonstrations of height sensor and accelerometer, calibration verifications. On the afternoon on the second day, a field open house was held. 


\subsubsection{Calibration Demonstration}

All vendors used similar techniques to calibrate height sensors and accelerometers, including simple bump tests. However, they all used somewhat different techniques to verify performance of these sensors, because they all used different software. An example is the Pathway device provides real time displays of accelerometer and height reading. A glance at the computer monitor provides an instant indication that the sensors are working while profiling. A flat line for either sensor while profiling or while simply bouncing of the device would indicate that a sensor was not functioning properly. In the case of the $\mathrm{K} \mathrm{J}$ Law device, a real time profile is generated while profiling. Unfortunately, a trace would continue to be generated even if one of the sensors were not working. However in the calibration mode, the $\mathrm{K} \mathrm{J}$ Law device actually has redundant checks in the form of the bounce test and a signal is fed to the accelerometer while the accelerometer output is monitored for compliance (shunt type test). These were simply examples to demonstrate the types of differences that exist between the profiler calibration methods.

\subsubsection{Field Open House}

Approximately forty people including FHWA, INDOT, contractor, consultant, and user producer agency personnel attended the field demonstration. An INDOT public information officer and local television media were on site also. In fact all persons who would be affected by a new smoothness specification were invited to attend the field test, thus fostering a partnering atmosphere. When the testing was completed, all vendors performed demonstration tests for anyone and everyone who was interested. Interested 
persons also rode with vendors while they profiled the test site. It appeared as though both the visitors and vendors had positive experiences during the field test. The open house was also reported on the local evening news and in the FHWA Focus publication. 


\section{DATA ANALYSIS}

The analysis of the field test data considered the following major characteristics:

1. Smoothness indices (IRI and PI);

2. Profile comparisons; and

3. Identification of bumps and "must grind" locations.

The field tests were performed on both HMA and PCC sites in an effort to ensure that the profilers provided valid results for both pavement types. Tests were conducted with four lightweight profilers and the INDOT Rip Van, with each profiler performing five replicate measurements at each test site. This provided adequate data to evaluate the repeatability and reproducibility of the devices. Additional tests were conducted at two HMA sites with plywood strips being placed in the wheel path(s) of the operating vehicles. These thin plywood strips were used to simulate bridge abutments and provide an indication of how the profilers would respond to such geometric features.

Both within and between vendor comparisons were made for each major data analysis characteristic. Within vendor comparisons provide and indication of the repeatability of the devices, while between vendor comparisons provide an indication of their reproducibility. Good between vendor performance would ensure that all the profilers would give approximately the same results and hence they could be operated interchangeably. Poor between vendor correlation would help in the identification of 
profilers for which some modification(s) were required or extra precautions are required for operating them.

Within and between vendor profile comparisons were made to see how they compared at each site. They were also compared to a rod and level survey as well as California Profilograph data at one site. The precision rod and level survey data represents as true a profile as is obtainable. The California Profilograph comparison was used to compare INDOT's current technique with the lightweight profiler output. Another reason for comparing profiles was that it is possible to obtain similar smoothness indices from very different profiles depending on how profiles are processed. Finally the ability of the devices to identify bumps could only be assessed through a comparison of profiles and vendor indicated must grind locations.

\subsection{Smoothness Indices Data by Vendor}

The smoothness indices data for the replicate measurements made with each device at each site were pooled and analyzed. The data are presented in Tables 4-1 through 4-13. Three indices were analyzed:

1. International Roughness Index (IRI);

2. Profile Index using a 0.0” blanking band (PI-0.0”); and

3. Profile Index using a 0.2 " blanking band (PI-0.2").

The three indices were considered because they are all in vogue and at the time of the analysis INDOT did not indicate a preference toward any specific one. Hence the 
analysis would be useful irrespective of whatever smoothness index ultimately were incorporated into a smoothness specification.

In addition to the five individual measurements per site, the mean, standard deviation, coefficient of variation, minimum, maximum, and range values are presented in Tables 4-1 through 4-13 for each smoothness index by site. The summary tables brought to light the repeatability performance of the individual profilers. All of the devices appear to exhibit reasonable repeatability. A high standard deviation and/or high coefficient of variation (>15\%) would imply that a profiler provided inconsistent results for a particular site. The coefficients of variation were typically much smaller than fifteen percent. However, for some of the PI data with a 0.2 " blanking band very high coefficients of variation were observed. The reason for this is that relative to this data the reported PI values were typically $0 \mathrm{in} / \mathrm{mi}$ with an occasional value of $1 \mathrm{in} / \mathrm{mi}$. Coefficient of variation is very sensitive to such conditions and the high values observed under this condition should be expected. This is discussed in more detail in a subsequent section.

The data were also reasonable in that PI values with a 0.2 " blanking band were all smaller than the values with the 0.0" blanking band for all vendors and as IRI increased or decreased from site to site PI values generally tracked the directional changes. In other words the field data all appeared reasonable, thus further analyses of it were warranted.

One other interesting observation was that inconsistent shifts in PI were observed with the 0.0 " and 0.2 " blanking band for each device. For example, Vendor A reported an 
average PI with the 0.2 " blanking band of $0 \mathrm{in} / \mathrm{mi}$ for site numbers 3 and 7 (Tables 4-2 and 4-3). However, with the 0.0 " blanking band it reported PI values of 5 and $13 \mathrm{in} / \mathrm{mi}$ for the same sites. Similar differences were observed for all the other vendors also. This clearly suggests that the 0.2 " blanking band is inappropriate in that it masks significant differences in the pavement features, which the 0.0 " blanking band identify. 
Table 4-1 Vendor A IRI Data and Summary Statistics for All Sites

\begin{tabular}{|c|c|c|c|c|c|c|c|c|}
\hline \multirow{2}{*}{$\begin{array}{c}\text { Site No } \\
\& \\
\text { Description }\end{array}$} & \multirow[b]{2}{*}{$\begin{array}{l}\text { Replicate } \\
\text { Measure }\end{array}$} & \multicolumn{7}{|c|}{ IRI (in/mi) } \\
\hline & & Values & Mean & $\begin{array}{c}\text { Std } \\
\text { Dev }\end{array}$ & $\begin{array}{l}\mathrm{COV} \\
(\%)\end{array}$ & Min & Max & Range \\
\hline \multirow{5}{*}{$\begin{array}{c}1 \\
\text { Covington } \\
\text { Street }\end{array}$} & 1 & 104 & \multirow{5}{*}{107} & \multirow{5}{*}{5} & \multirow{5}{*}{4} & \multirow{5}{*}{100} & \multirow{5}{*}{111} & \multirow{5}{*}{11} \\
\hline & 2 & 109 & & & & & & \\
\hline & 3 & 111 & & & & & & \\
\hline & 4 & 110 & & & & & & \\
\hline & 5 & 100 & & & & & & \\
\hline \multirow{5}{*}{$\begin{array}{c}2 \\
\text { I-65 } \\
\text { With Board }\end{array}$} & 1 & 183 & \multirow{5}{*}{218} & \multirow{5}{*}{24} & \multirow{5}{*}{11} & \multirow{5}{*}{183} & \multirow{5}{*}{240} & \multirow{5}{*}{57} \\
\hline & 2 & 240 & & & & & & \\
\hline & 3 & 205 & & & & & & \\
\hline & 4 & 238 & & & & & & \\
\hline & 5 & 223 & & & & & & \\
\hline \multirow{3}{*}{$\begin{array}{c}2 \mathrm{~b} \\
\mathrm{I}-65 \\
\text { No Board } \\
\end{array}$} & 1 & 58 & \multirow{3}{*}{59} & \multirow{3}{*}{1} & \multirow{3}{*}{2} & \multirow{3}{*}{58} & \multirow{3}{*}{60} & \multirow{3}{*}{2} \\
\hline & 2 & 60 & & & & & & \\
\hline & 3 & 60 & & & & & & \\
\hline \multirow{5}{*}{$\begin{array}{c}3 \\
\text { Prairie } \\
\text { Street } \\
\text { With Board }\end{array}$} & 1 & 141 & & & & & & \\
\hline & 2 & 150 & & & & & & \\
\hline & 3 & 155 & 148 & 5 & 4 & 141 & 155 & 14 \\
\hline & 4 & 144 & & & & & & \\
\hline & 5 & 148 & & & & & & \\
\hline & 1 & 130 & & & & & & \\
\hline $3 \mathrm{~b}$ & 2 & 126 & & & & & & \\
\hline Street & 3 & 126 & 130 & 6 & 5 & 126 & 141 & 15 \\
\hline street & 4 & 129 & & & & & & \\
\hline & 5 & 141 & & & & & & \\
\hline & 1 & 91 & & & & & & \\
\hline 4 & 2 & 87 & & & & & & \\
\hline Vinton & 3 & 93 & 92 & 4 & 5 & 87 & 98 & 11 \\
\hline Street & 4 & 89 & & & & & & \\
\hline & 5 & 98 & & & & & & \\
\hline & 1 & 65 & & & & & & \\
\hline & 2 & 65 & & & & & & \\
\hline $\begin{array}{c}J \\
\text { UST } 2314\end{array}$ & 3 & 65 & 65 & 0 & 0 & 65 & 65 & 0 \\
\hline & 4 & 65 & & & & & & \\
\hline & 5 & 65 & & & & & & \\
\hline & 1 & 73 & & & & & & \\
\hline & 2 & 78 & & & & & & \\
\hline $\begin{array}{l}0 \\
0 \\
231 \mathrm{P}\end{array}$ & 3 & 70 & 77 & 5 & 7 & 70 & 83 & 13 \\
\hline & 4 & 80 & & & & & & \\
\hline & 5 & 83 & & & & & & \\
\hline
\end{tabular}


Table 4-2 Vendor A, PI with a 0.2" Blanking Band and Summary Statistics for All Sites

\begin{tabular}{|c|c|c|c|c|c|c|c|c|}
\hline \multirow{2}{*}{$\begin{array}{c}\text { Site No } \\
\& \\
\text { Description }\end{array}$} & \multirow{2}{*}{$\begin{array}{c}\text { Replicate } \\
\text { Measure }\end{array}$} & \multicolumn{7}{|c|}{ PI - 0.2"(in/mi) } \\
\hline & & Values & Mean & $\begin{array}{l}\text { Std } \\
\text { Dev }\end{array}$ & $\begin{array}{c}\mathrm{COV} \\
(\%)\end{array}$ & Min & $\operatorname{Max}$ & Range \\
\hline \multirow{5}{*}{$\begin{array}{c}1 \\
\text { Covington } \\
\text { Street }\end{array}$} & 1 & 6 & \multirow{5}{*}{8} & \multirow{5}{*}{1} & \multirow{5}{*}{18} & \multirow{5}{*}{6} & \multirow{5}{*}{9} & \multirow{5}{*}{3} \\
\hline & 2 & 9 & & & & & & \\
\hline & 3 & 9 & & & & & & \\
\hline & 4 & 9 & & & & & & \\
\hline & 5 & 7 & & & & & & \\
\hline \multirow{5}{*}{$\begin{array}{c}2 \\
\text { I-65 } \\
\text { With Board }\end{array}$} & 1 & 46 & \multirow{5}{*}{52} & \multirow{5}{*}{5} & \multirow{5}{*}{9} & \multirow{5}{*}{46} & \multirow{5}{*}{56} & \multirow{5}{*}{10} \\
\hline & 2 & 56 & & & & & & \\
\hline & 3 & 56 & & & & & & \\
\hline & 4 & 55 & & & & & & \\
\hline & 5 & 48 & & & & & & \\
\hline \multirow{3}{*}{$\begin{array}{c}2 \mathrm{~b} \\
\mathrm{I}-65 \\
\text { No Board }\end{array}$} & 1 & 0 & \multirow{3}{*}{0} & \multirow{3}{*}{0} & \multirow{3}{*}{0} & \multirow{3}{*}{0} & \multirow{3}{*}{0} & \multirow{3}{*}{0} \\
\hline & 2 & 0 & & & & & & \\
\hline & 3 & 0 & & & & & & \\
\hline \multirow{5}{*}{$\begin{array}{c}3 \\
\text { Prairie } \\
\text { Street } \\
\text { With Board }\end{array}$} & 1 & 33 & & & & & & \\
\hline & 2 & 32 & & & & & & \\
\hline & 3 & 37 & 34 & 2 & 7 & 32 & 37 & 5 \\
\hline & 4 & 36 & & & & & & \\
\hline & 5 & 32 & & & & & & \\
\hline & 1 & 32 & & & & & & \\
\hline $\begin{array}{c}3 \mathrm{~b} \\
\text { Proirio }\end{array}$ & 2 & 32 & & & & & & \\
\hline $\begin{array}{l}\text { Pralrie } \\
\text { Street }\end{array}$ & 3 & 28 & 32 & 3 & 8 & 28 & 35 & 7 \\
\hline Street & 4 & 33 & & & & & & \\
\hline & 5 & 35 & & & & & & \\
\hline & 1 & 14 & & & & & & \\
\hline 4 & 2 & 19 & & & & & & \\
\hline Vinton & 3 & 16 & 18 & 3 & 15 & 14 & 20 & 6 \\
\hline Street & 4 & 20 & & & & & & \\
\hline & 5 & 20 & & & & & & \\
\hline & 1 & 0 & & & & & & \\
\hline & 2 & 0 & & & & & & \\
\hline IIS $231 \mathrm{~A}$ & 3 & 0 & 0 & 0 & 224 & 0 & 1 & 1 \\
\hline US $231 A$ & 4 & 0 & & & & & & \\
\hline & 5 & 1 & & & & & & \\
\hline & 1 & 2 & & & & & & \\
\hline & 2 & 0 & & & & & & \\
\hline $\begin{array}{c}6 \\
031 \mathrm{R}\end{array}$ & 3 & 1 & 1 & 1 & 70 & 0 & 2 & 2 \\
\hline US $231 B$ & 4 & 2 & & & & & & \\
\hline & 5 & 1 & & & & & & \\
\hline
\end{tabular}


Table 4-3 Vendor A PI with a 0.0" Blanking Band and Summary Statistics for All Sites

\begin{tabular}{|c|c|c|c|c|c|c|c|c|}
\hline \multirow{2}{*}{$\begin{array}{c}\text { Site No } \\
\& \\
\text { Description }\end{array}$} & \multirow{2}{*}{$\begin{array}{l}\text { Replicate } \\
\text { Measure }\end{array}$} & \multicolumn{7}{|c|}{ PI - 0.0"(in/mi) } \\
\hline & & Values & Mean & $\begin{array}{l}\text { Std } \\
\text { Dev }\end{array}$ & $\begin{array}{c}\mathrm{COV} \\
(\%)\end{array}$ & Min & $\operatorname{Max}$ & Range \\
\hline \multirow{5}{*}{$\begin{array}{c}1 \\
\text { Covington } \\
\text { Street }\end{array}$} & 1 & 21 & \multirow{5}{*}{27} & \multirow{5}{*}{3} & \multirow{5}{*}{12} & \multirow{5}{*}{21} & \multirow{5}{*}{29} & \multirow{5}{*}{8} \\
\hline & 2 & 29 & & & & & & \\
\hline & 3 & 28 & & & & & & \\
\hline & 4 & 29 & & & & & & \\
\hline & 5 & 27 & & & & & & \\
\hline \multirow{5}{*}{$\begin{array}{c}2 \\
\text { I-65 } \\
\text { With Board }\end{array}$} & 1 & 69 & \multirow{5}{*}{68} & \multirow{5}{*}{2} & \multirow{5}{*}{3} & \multirow{5}{*}{65} & \multirow{5}{*}{69} & \multirow{5}{*}{4} \\
\hline & 2 & 69 & & & & & & \\
\hline & 3 & 66 & & & & & & \\
\hline & 4 & 69 & & & & & & \\
\hline & 5 & 65 & & & & & & \\
\hline \multirow{3}{*}{$\begin{array}{c}2 \mathrm{~b} \\
\mathrm{I}-65 \\
\text { No Board }\end{array}$} & 1 & 5 & \multirow{3}{*}{5} & \multirow{3}{*}{1} & \multirow{3}{*}{11} & \multirow{3}{*}{5} & \multirow{3}{*}{6} & \multirow{3}{*}{1} \\
\hline & 2 & 6 & & & & & & \\
\hline & 3 & 5 & & & & & & \\
\hline \multirow{5}{*}{$\begin{array}{c}3 \\
\text { Prairie } \\
\text { Street } \\
\text { With Board }\end{array}$} & 1 & 33 & & & & & & \\
\hline & 2 & 35 & & & & & & \\
\hline & 3 & 39 & 36 & 2 & 6 & 33 & 39 & 6 \\
\hline & 4 & 36 & & & & & & \\
\hline & 5 & 36 & & & & & & \\
\hline & 1 & 34 & & & & & & \\
\hline $\begin{array}{c}3 \mathrm{~b} \\
\text { Prairie }\end{array}$ & 2 & 35 & & & & & & \\
\hline $\begin{array}{l}\text { Prairie } \\
\text { Street }\end{array}$ & 3 & 36 & 36 & 1 & 3 & 34 & 37 & 3 \\
\hline Street & 4 & 36 & & & & & & \\
\hline & 5 & 37 & & & & & & \\
\hline & 1 & 23 & & & & & & \\
\hline 4 & 2 & 23 & & & & & & \\
\hline Vinton & 3 & 23 & 23 & 1 & 2 & 23 & 24 & 1 \\
\hline Street & 4 & 24 & & & & & & \\
\hline & 5 & 24 & & & & & & \\
\hline & 1 & 15 & & & & & & \\
\hline & 2 & 13 & & & & & & \\
\hline $\begin{array}{c}5 \\
2314\end{array}$ & 3 & 11 & 13 & 1 & 12 & 11 & 15 & 4 \\
\hline US 231A & 4 & 12 & & & & & & \\
\hline & 5 & 13 & & & & & & \\
\hline & 1 & 17 & & & & & & \\
\hline & 2 & 17 & & & & & & \\
\hline $\begin{array}{c}6 \\
031 \mathrm{R}\end{array}$ & 3 & 15 & 17 & 2 & 10 & 15 & 20 & 5 \\
\hline US $231 B$ & 4 & 18 & & & & & & \\
\hline & 5 & 20 & & & & & & \\
\hline
\end{tabular}


Table 4-4 Vendor B IRI Data and Summary Statistics for All Sites

\begin{tabular}{|c|c|c|c|c|c|c|c|c|}
\hline \multirow{2}{*}{$\begin{array}{c}\text { Site No } \\
\& \\
\text { Description } \\
\end{array}$} & \multirow[b]{2}{*}{$\begin{array}{l}\text { Replicate } \\
\text { Measure }\end{array}$} & \multicolumn{7}{|c|}{ IRI (in/mi) } \\
\hline & & Values & Mean & $\begin{array}{l}\text { Std } \\
\text { Dev }\end{array}$ & $\begin{array}{c}\mathrm{COV} \\
(\%)\end{array}$ & Min & $\operatorname{Max}$ & Range \\
\hline \multirow{5}{*}{$\begin{array}{c}1 \\
\text { Covington } \\
\text { Street }\end{array}$} & 1 & 149 & \multirow{5}{*}{152} & \multirow{5}{*}{3} & \multirow{5}{*}{2} & \multirow{5}{*}{149} & \multirow{5}{*}{155} & \multirow{5}{*}{6} \\
\hline & 2 & 154 & & & & & & \\
\hline & 3 & 154 & & & & & & \\
\hline & 4 & 150 & & & & & & \\
\hline & 5 & 154 & & & & & & \\
\hline \multirow{5}{*}{$\begin{array}{c}2 \\
\text { I-65 } \\
\text { With Board }\end{array}$} & 1 & 86 & \multirow{5}{*}{91} & \multirow{5}{*}{4} & \multirow{5}{*}{5} & \multirow{5}{*}{86} & \multirow{5}{*}{96} & \multirow{5}{*}{10} \\
\hline & 2 & 94 & & & & & & \\
\hline & 3 & 96 & & & & & & \\
\hline & 4 & 93 & & & & & & \\
\hline & 5 & 86 & & & & & & \\
\hline \multirow{3}{*}{$\begin{array}{c}2 \mathrm{~b} \\
\text { I-65 } \\
\text { No Board }\end{array}$} & 1 & 41 & \multirow{3}{*}{39} & \multirow{3}{*}{1} & \multirow{3}{*}{3} & \multirow{3}{*}{38} & \multirow{3}{*}{41} & \multirow{3}{*}{3} \\
\hline & 2 & 40 & & & & & & \\
\hline & 3 & 38 & & & & & & \\
\hline \multirow{5}{*}{$\begin{array}{c}3 \\
\text { Prairie } \\
\text { Street } \\
\text { With Board }\end{array}$} & 1 & 154 & & & & & & \\
\hline & 2 & 151 & & & & & & \\
\hline & 3 & 150 & 150 & 3 & 2 & 146 & 154 & 8 \\
\hline & 4 & 149 & & & & & & \\
\hline & 5 & 146 & & & & & & \\
\hline & 1 & 140 & & & & & & \\
\hline $\begin{array}{c}3 \mathrm{~b} \\
\text { Prairio }\end{array}$ & 2 & 143 & & & & & & \\
\hline Prairle & 3 & 140 & 141 & 1 & 1 & 140 & 143 & 3 \\
\hline No Board & 4 & 141 & & & & & & \\
\hline & 5 & 141 & & & & & & \\
\hline & 1 & 95 & & & & & & \\
\hline 4 & 2 & 102 & & & & & & \\
\hline Vinton & 3 & 101 & 101 & 3 & 3 & 95 & 102 & 7 \\
\hline Street & 4 & 102 & & & & & & \\
\hline & 5 & 102 & & & & & & \\
\hline & 1 & 46 & & & & & & \\
\hline & 2 & 43 & & & & & & \\
\hline US $231 \mathrm{~A}$ & 3 & 44 & 44 & 2 & 3 & 43 & 46 & 4 \\
\hline & 4 & 45 & & & & & & \\
\hline & 5 & 43 & & & & & & \\
\hline & 1 & 57 & & & & & & \\
\hline & 2 & 54 & & & & & & \\
\hline $\begin{array}{c}0 \\
031 \mathrm{~B}\end{array}$ & 3 & 55 & 55 & 1 & 3 & 54 & 55 & 1 \\
\hline US $201 \mathrm{D}$ & 4 & 54 & & & & & & \\
\hline & 5 & 54 & & & & & & \\
\hline
\end{tabular}


Table 4-5 Vendor B PI with a 0.2" Blanking Band and Summary Statistics for All Sites

\begin{tabular}{|c|c|c|c|c|c|c|c|c|}
\hline \multirow{2}{*}{$\begin{array}{c}\text { Site No } \\
\& \\
\text { Description }\end{array}$} & \multirow{2}{*}{$\begin{array}{c}\text { Replicate } \\
\text { Measure }\end{array}$} & \multicolumn{7}{|c|}{ PI - 0.2"(in/mi) } \\
\hline & & Values & Mean & $\begin{array}{c}\text { Std } \\
\text { Dev }\end{array}$ & $\begin{array}{c}\mathrm{COV} \\
(\%)\end{array}$ & Min & $\operatorname{Max}$ & Range \\
\hline \multirow{5}{*}{$\begin{array}{c}1 \\
\text { Covington } \\
\text { Street }\end{array}$} & 1 & 44 & \multirow{5}{*}{44} & \multirow{5}{*}{2} & \multirow{5}{*}{4} & \multirow{5}{*}{41} & \multirow{5}{*}{45} & \multirow{5}{*}{4} \\
\hline & 2 & 44 & & & & & & \\
\hline & 3 & 45 & & & & & & \\
\hline & 4 & 41 & & & & & & \\
\hline & 5 & 44 & & & & & & \\
\hline \multirow{5}{*}{$\begin{array}{c}2 \\
\text { I-65 } \\
\text { With Board }\end{array}$} & 1 & 13 & \multirow{5}{*}{14} & \multirow{5}{*}{1} & \multirow{5}{*}{10} & \multirow{5}{*}{13} & \multirow{5}{*}{17} & \multirow{5}{*}{4} \\
\hline & 2 & 17 & & & & & & \\
\hline & 3 & 14 & & & & & & \\
\hline & 4 & 15 & & & & & & \\
\hline & 5 & 14 & & & & & & \\
\hline \multirow{3}{*}{$\begin{array}{c}2 \mathrm{~b} \\
\mathrm{I}-65 \\
\text { No Board } \\
\end{array}$} & 1 & 0 & \multirow{3}{*}{0} & \multirow{3}{*}{0} & \multirow{3}{*}{0} & \multirow{3}{*}{0} & \multirow{3}{*}{0} & \multirow{3}{*}{0} \\
\hline & 2 & 0 & & & & & & \\
\hline & 3 & 0 & & & & & & \\
\hline \multirow{5}{*}{$\begin{array}{c}3 \\
\text { Prairie } \\
\text { Street } \\
\text { With Board }\end{array}$} & 1 & 45 & & & & & & \\
\hline & 2 & 44 & & & & & & \\
\hline & 3 & 42 & 43 & 1 & 3 & 42 & 45 & 3 \\
\hline & 4 & 42 & & & & & & \\
\hline & 5 & 42 & & & & & & \\
\hline & 1 & 37 & & & & & & \\
\hline $\begin{array}{c}30 \\
\text { Prairie }\end{array}$ & 2 & 39 & & & & & & \\
\hline Street & 3 & 39 & 39 & 1 & 2 & 37 & 39 & 2 \\
\hline No Board & 4 & 39 & & & & & & \\
\hline & 5 & 39 & & & & & & \\
\hline & 1 & 14 & & & & & & \\
\hline 4 & 2 & 17 & & & & & & \\
\hline Vinton & 3 & 17 & 16 & 1 & 7 & 14 & 17 & 3 \\
\hline Street & 4 & 16 & & & & & & \\
\hline & 5 & 16 & & & & & & \\
\hline & 1 & 0 & & & & & & \\
\hline & 2 & 0 & & & & & & \\
\hline US 2314 & 3 & 0 & 0 & 0 & 0 & 0 & 0 & 0 \\
\hline & 4 & 0 & & & & & & \\
\hline & 5 & 0 & & & & & & \\
\hline & 1 & 4 & & & & & & \\
\hline & 2 & 3 & & & & & & \\
\hline US $231 \mathrm{R}$ & 3 & 4 & 3 & 0 & 15 & 3 & 4 & 1 \\
\hline & 4 & 4 & & & & & & \\
\hline & 5 & 3 & & & & & & \\
\hline
\end{tabular}


Table 4-6 Vendor B PI with a 0.0" Blanking Band and Summary Statistics for All Sites

\begin{tabular}{|c|c|c|c|c|c|c|c|c|}
\hline \multirow{2}{*}{$\begin{array}{c}\text { Site No } \\
\& \\
\text { Description }\end{array}$} & \multirow[b]{2}{*}{$\begin{array}{l}\text { Replicate } \\
\text { Measure }\end{array}$} & \multicolumn{7}{|c|}{$\mathrm{PI}-0.0 "(\mathrm{in} / \mathrm{mi})$} \\
\hline & & Values & Mean & $\begin{array}{c}\text { Std } \\
\text { Dev }\end{array}$ & $\begin{array}{c}\mathrm{COV} \\
(\%)\end{array}$ & Min & Max & Range \\
\hline \multirow{5}{*}{$\begin{array}{c}1 \\
\text { Covington } \\
\text { Street }\end{array}$} & 1 & 76 & \multirow{5}{*}{76} & \multirow{5}{*}{1} & \multirow{5}{*}{1} & \multirow{5}{*}{75} & \multirow{5}{*}{77} & \multirow{5}{*}{2} \\
\hline & 2 & 76 & & & & & & \\
\hline & 3 & 77 & & & & & & \\
\hline & 4 & 75 & & & & & & \\
\hline & 5 & 76 & & & & & & \\
\hline \multirow{5}{*}{$\begin{array}{c}2 \\
\text { I-65 } \\
\text { With Board }\end{array}$} & 1 & 33 & \multirow{5}{*}{34} & \multirow{5}{*}{2} & \multirow{5}{*}{7} & \multirow{5}{*}{32} & \multirow{5}{*}{38} & \multirow{5}{*}{6} \\
\hline & 2 & 38 & & & & & & \\
\hline & 3 & 35 & & & & & & \\
\hline & 4 & 35 & & & & & & \\
\hline & 5 & 32 & & & & & & \\
\hline \multirow{3}{*}{$\begin{array}{c}2 b \\
\text { I-65 } \\
\text { No Board }\end{array}$} & 1 & 16 & \multirow{3}{*}{14} & \multirow{3}{*}{2} & \multirow{3}{*}{11} & \multirow{3}{*}{13} & \multirow{3}{*}{16} & \multirow{3}{*}{3} \\
\hline & 2 & 14 & & & & & & \\
\hline & 3 & 13 & & & & & & \\
\hline \multirow{5}{*}{$\begin{array}{c}3 \\
\text { Prairie } \\
\text { Street } \\
\text { With Board }\end{array}$} & 1 & 61 & & & & & & \\
\hline & 2 & 62 & & & & & & \\
\hline & 3 & 61 & 61 & 1 & 1 & 60 & 62 & 2 \\
\hline & 4 & 61 & & & & & & \\
\hline & 5 & 60 & & & & & & \\
\hline & 1 & 55 & & & & & & \\
\hline $\begin{array}{c}3 b \\
\text { Droirio }\end{array}$ & 2 & 57 & & & & & & \\
\hline Prairie & 3 & 56 & 56 & 1 & 2 & 55 & 57 & 2 \\
\hline Street & 4 & 56 & & & & & & \\
\hline & 5 & 55 & & & & & & \\
\hline & 1 & 37 & & & & & & \\
\hline 4 & 2 & 36 & & & & & & \\
\hline Vinton & 3 & 40 & 37 & 2 & 5 & 36 & 40 & 4 \\
\hline Street & 4 & 36 & & & & & & \\
\hline & 5 & 38 & & & & & & \\
\hline & 1 & 21 & & & & & & \\
\hline & 2 & 20 & & & & & & \\
\hline $\begin{array}{l}5 \\
\text { IS } 2314\end{array}$ & 3 & 18 & 19 & 1 & 7 & 18 & 21 & 3 \\
\hline US 231A & 4 & 20 & & & & & & \\
\hline & 5 & 18 & & & & & & \\
\hline & 1 & 24 & & & & & & \\
\hline & 2 & 23 & & & & & & \\
\hline $\begin{array}{c}6 \\
231 \mathrm{R}\end{array}$ & 3 & 24 & 23 & 1 & 3 & 23 & 24 & 1 \\
\hline US $231 B$ & 4 & 23 & & & & & & \\
\hline & 5 & 23 & & & & & & \\
\hline
\end{tabular}


Table 4-7 Vendor C IRI Data and Summary Statistics for All Sites

\begin{tabular}{|c|c|c|c|c|c|c|c|c|}
\hline \multirow{2}{*}{$\begin{array}{c}\text { Site No } \\
\& \\
\text { Description }\end{array}$} & \multirow[b]{2}{*}{$\begin{array}{l}\text { Replicate } \\
\text { Measure }\end{array}$} & \multicolumn{7}{|c|}{ IRI (in/mi) } \\
\hline & & Values & Mean & $\begin{array}{l}\text { Std } \\
\text { Dev }\end{array}$ & $\begin{array}{l}\mathrm{COV} \\
(\%)\end{array}$ & Min & Max & Range \\
\hline \multirow{5}{*}{$\begin{array}{c}1 \\
\text { Covington } \\
\text { Street }\end{array}$} & 1 & 154 & \multirow{5}{*}{153} & \multirow{5}{*}{1} & \multirow{5}{*}{1} & \multirow{5}{*}{153} & \multirow{5}{*}{155} & \multirow{5}{*}{2} \\
\hline & 2 & 153 & & & & & & \\
\hline & 3 & 154 & & & & & & \\
\hline & 4 & 153 & & & & & & \\
\hline & 5 & 153 & & & & & & \\
\hline \multirow{5}{*}{$\begin{array}{c}2 \\
\text { I-65 } \\
\text { With Board }\end{array}$} & 1 & 32 & \multirow{5}{*}{32} & \multirow{5}{*}{0} & \multirow{5}{*}{1} & \multirow{5}{*}{31} & \multirow{5}{*}{32} & \multirow{5}{*}{1} \\
\hline & 2 & 32 & & & & & & \\
\hline & 3 & 32 & & & & & & \\
\hline & 4 & 31 & & & & & & \\
\hline & 5 & 32 & & & & & & \\
\hline \multirow{3}{*}{$\begin{array}{c}2 \mathrm{~b} \\
\text { I-65 } \\
\text { No Board } \\
\end{array}$} & 1 & 32 & \multirow{3}{*}{31} & \multirow{3}{*}{1} & \multirow{3}{*}{4} & \multirow{3}{*}{30} & \multirow{3}{*}{32} & \multirow{3}{*}{2} \\
\hline & 2 & 30 & & & & & & \\
\hline & 3 & 31 & & & & & & \\
\hline \multirow{5}{*}{$\begin{array}{c}3 \\
\text { Prairie } \\
\text { Street } \\
\text { With Board }\end{array}$} & 1 & 187 & & & & & & \\
\hline & 2 & 166 & & & & & & \\
\hline & 3 & 170 & 171 & 9 & 5 & 166 & 187 & 21 \\
\hline & 4 & 168 & & & & & & \\
\hline & 5 & 166 & & & & & & \\
\hline & 1 & 164 & & & & & & \\
\hline $\begin{array}{c}50 \\
\text { Prairis }\end{array}$ & 2 & 165 & & & & & & \\
\hline Street & 3 & 161 & 165 & 4 & 2 & 161 & 172 & 11 \\
\hline No Board & 4 & 172 & & & & & & \\
\hline & 5 & 165 & & & & & & \\
\hline & 1 & 103 & & & & & & \\
\hline 4 & 2 & 103 & & & & & & \\
\hline Vinton & 3 & 100 & 102 & 1 & 1 & 100 & 103 & 3 \\
\hline Street & 4 & 102 & & & & & & \\
\hline & 5 & 101 & & & & & & \\
\hline & 1 & 44 & & & & & & \\
\hline & 2 & 43 & & & & & & \\
\hline $\begin{array}{c}5 \\
2314\end{array}$ & 3 & 44 & 43 & 1 & 1 & 43 & 44 & 1 \\
\hline & 4 & 43 & & & & & & \\
\hline & 5 & 43 & & & & & & \\
\hline & 1 & 58 & & & & & & \\
\hline & 2 & 59 & & & & & & \\
\hline US $231 \mathrm{~B}$ & 3 & 57 & 57 & 1 & 2 & 56 & 59 & 3 \\
\hline & 4 & 57 & & & & & & \\
\hline & 5 & 56 & & & & & & \\
\hline
\end{tabular}


Table 4-8 Vendor C PI with a 0.2" Blanking Band and Summary Statistics for All Sites

\begin{tabular}{|c|c|c|c|c|c|c|c|c|}
\hline \multirow{2}{*}{$\begin{array}{c}\text { Site No } \\
\& \\
\text { Description }\end{array}$} & \multirow{2}{*}{$\begin{array}{c}\text { Replicate } \\
\text { Measure }\end{array}$} & \multicolumn{7}{|c|}{ PI - 0.2"(in/mi) } \\
\hline & & Values & Mean & $\begin{array}{l}\text { Std } \\
\text { Dev }\end{array}$ & $\begin{array}{c}\mathrm{COV} \\
(\%)\end{array}$ & Min & $\operatorname{Max}$ & Range \\
\hline \multirow{5}{*}{$\begin{array}{c}1 \\
\text { Covington } \\
\text { Street }\end{array}$} & 1 & 49 & \multirow{5}{*}{49} & \multirow{5}{*}{0} & \multirow{5}{*}{0} & \multirow{5}{*}{49} & \multirow{5}{*}{49} & \multirow{5}{*}{0} \\
\hline & 2 & 49 & & & & & & \\
\hline & 3 & 49 & & & & & & \\
\hline & 4 & 49 & & & & & & \\
\hline & 5 & 49 & & & & & & \\
\hline \multirow{5}{*}{$\begin{array}{c}2 \\
\text { I-65 } \\
\text { With Board }\end{array}$} & 1 & 1 & \multirow{5}{*}{1} & \multirow{5}{*}{1} & \multirow{5}{*}{39} & \multirow{5}{*}{1} & \multirow{5}{*}{2} & \multirow{5}{*}{1} \\
\hline & 2 & 2 & & & & & & \\
\hline & 3 & 2 & & & & & & \\
\hline & 4 & 1 & & & & & & \\
\hline & 5 & 2 & & & & & & \\
\hline \multirow{3}{*}{$\begin{array}{c}2 \mathrm{~b} \\
\mathrm{I}-65 \\
\text { No Board }\end{array}$} & 1 & 0 & \multirow{3}{*}{0} & \multirow{3}{*}{0} & \multirow{3}{*}{0} & \multirow{3}{*}{0} & \multirow{3}{*}{0} & \multirow{3}{*}{0} \\
\hline & 2 & 0 & & & & & & \\
\hline & 3 & 0 & & & & & & \\
\hline \multirow{5}{*}{$\begin{array}{c}3 \\
\text { Prairie } \\
\text { Street } \\
\text { With Board }\end{array}$} & 1 & 55 & & & & & & \\
\hline & 2 & 50 & & & & & & \\
\hline & 3 & 48 & 49 & 4 & 9 & 45 & 55 & 10 \\
\hline & 4 & 45 & & & & & & \\
\hline & 5 & 45 & & & & & & \\
\hline & 1 & 47 & & & & & & \\
\hline $\begin{array}{c}3 \mathrm{~b} \\
\text { Proirio }\end{array}$ & 2 & 47 & & & & & & \\
\hline Prairie & 3 & 49 & 48 & 1 & 3 & 46 & 49 & 3 \\
\hline No Board & 4 & 49 & & & & & & \\
\hline & 5 & 46 & & & & & & \\
\hline & 1 & 20 & & & & & & \\
\hline 4 & 2 & 18 & & & & & & \\
\hline Vinton & 3 & 19 & 18 & 1 & 7 & 17 & 20 & 3 \\
\hline Street & 4 & 17 & & & & & & \\
\hline & 5 & 19 & & & & & & \\
\hline & 1 & 1 & & & & & & \\
\hline & 2 & 0 & & & & & & \\
\hline $\begin{array}{c}5 \\
2314\end{array}$ & 3 & 0 & 0 & 0 & 135 & 0 & 1 & 1 \\
\hline US $231 A$ & 4 & 1 & & & & & & \\
\hline & 5 & 0 & & & & & & \\
\hline & 1 & 3 & & & & & & \\
\hline & 2 & 2 & & & & & & \\
\hline $\begin{array}{c}6 \\
031 \mathrm{R}\end{array}$ & 3 & 3 & 2 & 0 & 10 & 2 & 3 & 1 \\
\hline US $231 B$ & 4 & 2 & & & & & & \\
\hline & 5 & 3 & & & & & & \\
\hline
\end{tabular}


Table 4-9 Vendor C PI with a 0.0" Blanking Band and Summary Statistics for All Sites

\begin{tabular}{|c|c|c|c|c|c|c|c|c|}
\hline \multirow{2}{*}{$\begin{array}{c}\text { Site No } \\
\& \\
\text { Description }\end{array}$} & \multirow{2}{*}{$\begin{array}{c}\text { Replicate } \\
\text { Measure }\end{array}$} & \multicolumn{7}{|c|}{ PI - 0.0"(in/mi) } \\
\hline & & Values & Mean & $\begin{array}{c}\text { Std } \\
\text { Dev }\end{array}$ & $\begin{array}{l}\mathrm{COV} \\
(\%)\end{array}$ & Min & Max & Range \\
\hline \multirow{5}{*}{$\begin{array}{c}1 \\
\text { Covington } \\
\text { Street }\end{array}$} & 1 & 84 & \multirow{5}{*}{83} & \multirow{5}{*}{1} & \multirow{5}{*}{1} & \multirow{5}{*}{82} & \multirow{5}{*}{84} & \multirow{5}{*}{2} \\
\hline & 2 & 83 & & & & & & \\
\hline & 3 & 84 & & & & & & \\
\hline & 4 & 82 & & & & & & \\
\hline & 5 & 83 & & & & & & \\
\hline \multirow{5}{*}{$\begin{array}{c}2 \\
\text { I-65 } \\
\text { With Board }\end{array}$} & 1 & 11 & \multirow{5}{*}{11} & \multirow{5}{*}{1} & \multirow{5}{*}{8} & \multirow{5}{*}{9} & \multirow{5}{*}{11} & \multirow{5}{*}{2} \\
\hline & 2 & 10 & & & & & & \\
\hline & 3 & 9 & & & & & & \\
\hline & 4 & 11 & & & & & & \\
\hline & 5 & 11 & & & & & & \\
\hline \multirow{3}{*}{$\begin{array}{c}2 b \\
\text { I-65 } \\
\text { No Board } \\
\end{array}$} & 1 & 6 & \multirow{3}{*}{7} & \multirow{3}{*}{0} & \multirow{3}{*}{4} & \multirow{3}{*}{6} & \multirow{3}{*}{7} & \multirow{3}{*}{1} \\
\hline & 2 & 7 & & & & & & \\
\hline & 3 & 7 & & & & & & \\
\hline \multirow{5}{*}{$\begin{array}{c}3 \\
\text { Prairie } \\
\text { Street } \\
\text { With Board }\end{array}$} & 1 & 64 & & & & & & \\
\hline & 2 & 59 & & & & & & \\
\hline & 3 & 58 & 60 & 3 & 4 & 58 & 64 & 6 \\
\hline & 4 & 58 & & & & & & \\
\hline & 5 & 61 & & & & & & \\
\hline & 1 & 60 & & & & & & \\
\hline $\begin{array}{c}50 \\
\text { Droirio }\end{array}$ & 2 & 60 & & & & & & \\
\hline Street & 3 & 69 & 63 & 4 & 7 & 60 & 69 & 9 \\
\hline No Board & 4 & 66 & & & & & & \\
\hline & 5 & 60 & & & & & & \\
\hline & 1 & 38 & & & & & & \\
\hline 4 & 2 & 39 & & & & & & \\
\hline Vinton & 3 & 37 & 39 & 1 & 3 & 37 & 41 & 4 \\
\hline Street & 4 & 41 & & & & & & \\
\hline & 5 & 38 & & & & & & \\
\hline & 1 & 16 & & & & & & \\
\hline & 2 & 15 & & & & & & \\
\hline $\begin{array}{c}5 \\
2314\end{array}$ & 3 & 16 & 16 & 1 & 3 & 15 & 17 & 2 \\
\hline & 4 & 16 & & & & & & \\
\hline & 5 & 17 & & & & & & \\
\hline & 1 & 22 & & & & & & \\
\hline & 2 & 25 & & & & & & \\
\hline $\begin{array}{c}0 \\
\text { US } 231 \mathrm{~B}\end{array}$ & 3 & 23 & 23 & 1 & 5 & 22 & 25 & 3 \\
\hline & 4 & 22 & & & & & & \\
\hline & 5 & 23 & & & & & & \\
\hline
\end{tabular}


Table 4-10 Vendor D IRI Data and Summary Statistics for All Sites

\begin{tabular}{|c|c|c|c|c|c|c|c|c|}
\hline \multirow{2}{*}{$\begin{array}{c}\text { Site No } \\
\& \\
\text { Description }\end{array}$} & \multirow[b]{2}{*}{$\begin{array}{l}\text { Replicate } \\
\text { Measure }\end{array}$} & \multicolumn{7}{|c|}{ IRI (in/mi) } \\
\hline & & Values & Mean & $\begin{array}{l}\text { Std } \\
\text { Dev }\end{array}$ & $\begin{array}{c}\mathrm{COV} \\
(\%)\end{array}$ & Min & Max & Range \\
\hline \multirow{5}{*}{$\begin{array}{c}1 \\
\text { Covington } \\
\text { Street }\end{array}$} & 1 & 152 & \multirow{5}{*}{155} & \multirow{5}{*}{3} & \multirow{5}{*}{2} & \multirow{5}{*}{152} & \multirow{5}{*}{159} & \multirow{5}{*}{7} \\
\hline & 2 & 155 & & & & & & \\
\hline & 3 & 156 & & & & & & \\
\hline & 4 & 154 & & & & & & \\
\hline & 5 & 159 & & & & & & \\
\hline \multirow{5}{*}{$\begin{array}{c}2 \\
\text { I-65 } \\
\text { With Board }\end{array}$} & 1 & 82 & \multirow{5}{*}{84} & \multirow{5}{*}{4} & \multirow{5}{*}{5} & \multirow{5}{*}{80} & \multirow{5}{*}{91} & \multirow{5}{*}{11} \\
\hline & 2 & 84 & & & & & & \\
\hline & 3 & 81 & & & & & & \\
\hline & 4 & 91 & & & & & & \\
\hline & 5 & 80 & & & & & & \\
\hline \multirow{3}{*}{$\begin{array}{c}2 b \\
\text { I-65 } \\
\text { No Board } \\
\end{array}$} & 1 & 31 & \multirow{3}{*}{35} & \multirow{3}{*}{5} & \multirow{3}{*}{15} & \multirow{3}{*}{31} & \multirow{3}{*}{41} & \multirow{3}{*}{10} \\
\hline & 2 & 41 & & & & & & \\
\hline & 3 & 33 & & & & & & \\
\hline \multirow{5}{*}{$\begin{array}{c}3 \\
\text { Prairie } \\
\text { Street } \\
\text { With Board }\end{array}$} & 1 & 161 & & & & & & \\
\hline & 2 & 165 & & & & & & \\
\hline & 3 & 161 & 162 & 2 & 1 & 161 & 165 & 4 \\
\hline & 4 & 163 & & & & & & \\
\hline & 5 & 161 & & & & & & \\
\hline & 1 & 156 & & & & & & \\
\hline $\begin{array}{c}3 b \\
\text { Proirie }\end{array}$ & 2 & 155 & & & & & & \\
\hline $\begin{array}{l}\text { Prairie } \\
\text { Street }\end{array}$ & 3 & 157 & 154 & 3 & 2 & 149 & 157 & 8 \\
\hline So Bord & 4 & 149 & & & & & & \\
\hline & 5 & 154 & & & & & & \\
\hline & 1 & 103 & & & & & & \\
\hline 4 & 2 & 112 & & & & & & \\
\hline Vinton & 3 & 106 & 107 & 3 & 3 & 103 & 112 & 9 \\
\hline Street & 4 & 109 & & & & & & \\
\hline & 5 & 107 & & & & & & \\
\hline & 1 & 65 & & & & & & \\
\hline & 2 & 47 & & & & & & \\
\hline $\begin{array}{c}5 \\
\text { US } 231 \mathrm{~A}\end{array}$ & 3 & 49 & 52 & 7 & 14 & 47 & 65 & 18 \\
\hline & 4 & 50 & & & & & & \\
\hline & 5 & 49 & & & & & & \\
\hline & 1 & 61 & & & & & & \\
\hline & 2 & 60 & & & & & & \\
\hline $\begin{array}{c}6 \\
\text { IIS } 231 \mathrm{R}\end{array}$ & 3 & 60 & 60 & 1 & 1 & 59 & 61 & 2 \\
\hline & 4 & 60 & & & & & & \\
\hline & 5 & 59 & & & & & & \\
\hline
\end{tabular}


Table 4-11 Vendor D PI with a 0.2" Blanking Band and Summary Statistics for All Sites

\begin{tabular}{|c|c|c|c|c|c|c|c|c|}
\hline \multirow{2}{*}{$\begin{array}{c}\text { Site No } \\
\& \\
\text { Description }\end{array}$} & \multirow{2}{*}{$\begin{array}{c}\text { Replicate } \\
\text { Measure }\end{array}$} & \multicolumn{7}{|c|}{ PI - 0.2"(in/mi) } \\
\hline & & Values & Mean & $\begin{array}{l}\text { Std } \\
\text { Dev }\end{array}$ & $\begin{array}{c}\mathrm{COV} \\
(\%)\end{array}$ & Min & $\operatorname{Max}$ & Range \\
\hline \multirow{5}{*}{$\begin{array}{c}1 \\
\text { Covington } \\
\text { Street }\end{array}$} & 1 & 46 & \multirow{5}{*}{46} & \multirow{5}{*}{1} & \multirow{5}{*}{2} & \multirow{5}{*}{45} & \multirow{5}{*}{47} & \multirow{5}{*}{2} \\
\hline & 2 & 47 & & & & & & \\
\hline & 3 & 47 & & & & & & \\
\hline & 4 & 45 & & & & & & \\
\hline & 5 & 46 & & & & & & \\
\hline \multirow{5}{*}{$\begin{array}{c}2 \\
\text { I-65 } \\
\text { With Board }\end{array}$} & 1 & 14 & \multirow{5}{*}{14} & \multirow{5}{*}{2} & \multirow{5}{*}{11} & \multirow{5}{*}{13} & \multirow{5}{*}{17} & \multirow{5}{*}{4} \\
\hline & 2 & 17 & & & & & & \\
\hline & 3 & 13 & & & & & & \\
\hline & 4 & 13 & & & & & & \\
\hline & 5 & 14 & & & & & & \\
\hline \multirow{3}{*}{$\begin{array}{c}2 \mathrm{~b} \\
\mathrm{I}-65 \\
\text { No Board }\end{array}$} & 1 & 0 & \multirow{3}{*}{0} & \multirow{3}{*}{0} & \multirow{3}{*}{0} & \multirow{3}{*}{0} & \multirow{3}{*}{0} & \multirow{3}{*}{0} \\
\hline & 2 & 0 & & & & & & \\
\hline & 3 & 0 & & & & & & \\
\hline \multirow{5}{*}{$\begin{array}{c}3 \\
\text { Prairie } \\
\text { Street } \\
\text { With Board }\end{array}$} & 1 & 38 & & & & & & \\
\hline & 2 & 37 & & & & & & \\
\hline & 3 & 38 & 38 & 1 & 2 & 36 & 38 & 2 \\
\hline & 4 & 36 & & & & & & \\
\hline & 5 & 38 & & & & & & \\
\hline & 1 & 37 & & & & & & \\
\hline $\begin{array}{c}3 b \\
\text { Droirio }\end{array}$ & 2 & 37 & & & & & & \\
\hline Street & 3 & 37 & 37 & 1 & 2 & 36 & 37 & 1 \\
\hline $\begin{array}{l}\text { street } \\
\text { No Board }\end{array}$ & 4 & 37 & & & & & & \\
\hline & 5 & 36 & & & & & & \\
\hline & 1 & 16 & & & & & & \\
\hline 4 & 2 & 16 & & & & & & \\
\hline Vinton & 3 & 16 & 16 & 1 & 4 & 15 & 16 & 1 \\
\hline Street & 4 & 16 & & & & & & \\
\hline & 5 & 15 & & & & & & \\
\hline & 1 & 1 & & & & & & \\
\hline & 2 & 0 & & & & & & \\
\hline $\begin{array}{c}5 \\
2314\end{array}$ & 3 & 0 & 0 & 0 & 64 & 0 & 1 & 1 \\
\hline US $231 A$ & 4 & 0 & & & & & & \\
\hline & 5 & 0 & & & & & & \\
\hline & 1 & 3 & & & & & & \\
\hline & 2 & 2 & & & & & & \\
\hline $\begin{array}{c}0 \\
\mathrm{US}\end{array} 231 \mathrm{~B}$ & 3 & 3 & 3 & 0 & 15 & 2 & 3 & 1 \\
\hline US & 4 & 3 & & & & & & \\
\hline & 5 & 3 & & & & & & \\
\hline
\end{tabular}


Table 4-12 Vendor D PI with a 0.0" Blanking Band and Summary Statistics for All Sites

\begin{tabular}{|c|c|c|c|c|c|c|c|c|}
\hline \multirow{2}{*}{$\begin{array}{c}\text { Site No } \\
\& \\
\text { Description }\end{array}$} & \multirow{2}{*}{$\begin{array}{c}\text { Replicate } \\
\text { Measure }\end{array}$} & \multicolumn{7}{|c|}{ PI - 0.0"(in/mi) } \\
\hline & & Values & Mean & $\begin{array}{l}\text { Std } \\
\text { Dev }\end{array}$ & $\begin{array}{c}\mathrm{COV} \\
(\%)\end{array}$ & Min & $\operatorname{Max}$ & Range \\
\hline \multirow{5}{*}{$\begin{array}{c}1 \\
\text { Covington } \\
\text { Street }\end{array}$} & 1 & 79 & \multirow{5}{*}{79} & \multirow{5}{*}{0} & \multirow{5}{*}{1} & \multirow{5}{*}{79} & \multirow{5}{*}{80} & \multirow{5}{*}{1} \\
\hline & 2 & 80 & & & & & & \\
\hline & 3 & 79 & & & & & & \\
\hline & 4 & 79 & & & & & & \\
\hline & 5 & 80 & & & & & & \\
\hline \multirow{5}{*}{$\begin{array}{c}2 \\
\text { I-65 } \\
\text { With Board }\end{array}$} & 1 & 27 & \multirow{5}{*}{27} & \multirow{5}{*}{1} & \multirow{5}{*}{2} & \multirow{5}{*}{27} & \multirow{5}{*}{28} & \multirow{5}{*}{1} \\
\hline & 2 & 28 & & & & & & \\
\hline & 3 & 27 & & & & & & \\
\hline & 4 & 28 & & & & & & \\
\hline & 5 & 27 & & & & & & \\
\hline \multirow{3}{*}{$\begin{array}{c}2 \mathrm{~b} \\
\mathrm{I}-65 \\
\text { No Board }\end{array}$} & 1 & 7 & \multirow{3}{*}{8} & \multirow{3}{*}{1} & \multirow{3}{*}{12} & \multirow{3}{*}{7} & \multirow{3}{*}{9} & \multirow{3}{*}{2} \\
\hline & 2 & 9 & & & & & & \\
\hline & 3 & 9 & & & & & & \\
\hline \multirow{5}{*}{$\begin{array}{c}3 \\
\text { Prairie } \\
\text { Street } \\
\text { With Board }\end{array}$} & 1 & 57 & & & & & & \\
\hline & 2 & 60 & & & & & & \\
\hline & 3 & 57 & 57 & 2 & 3 & 56 & 60 & 4 \\
\hline & 4 & 57 & & & & & & \\
\hline & 5 & 56 & & & & & & \\
\hline & 1 & 59 & & & & & & \\
\hline $\begin{array}{c}3 b \\
\text { Droirio }\end{array}$ & 2 & 59 & & & & & & \\
\hline Street & 3 & 58 & 57 & 1 & 3 & 55 & 59 & 3 \\
\hline $\begin{array}{l}\text { street } \\
\text { No Board }\end{array}$ & 4 & 55 & & & & & & \\
\hline & 5 & 56 & & & & & & \\
\hline & 1 & 39 & & & & & & \\
\hline 4 & 2 & 39 & & & & & & \\
\hline Vinton & 3 & 39 & 39 & 1 & 2 & 38 & 40 & 2 \\
\hline Street & 4 & 40 & & & & & & \\
\hline & 5 & 38 & & & & & & \\
\hline & 1 & 18 & & & & & & \\
\hline & 2 & 17 & & & & & & \\
\hline $\begin{array}{c}5 \\
2314\end{array}$ & 3 & 18 & 17 & 0 & 2 & 17 & 18 & 1 \\
\hline US $231 A$ & 4 & 17 & & & & & & \\
\hline & 5 & 17 & & & & & & \\
\hline & 1 & 24 & & & & & & \\
\hline & 2 & 20 & & & & & & \\
\hline $\begin{array}{c}0 \\
\text { UIS } 231 \mathrm{~B}\end{array}$ & 3 & 20 & 22 & 2 & 9 & 20 & 24 & 4 \\
\hline & 4 & 24 & & & & & & \\
\hline & 5 & 22 & & & & & & \\
\hline
\end{tabular}


Table 4-13 INDOT Rip Van IRI Data and Summary Statistics for All Sites

\begin{tabular}{|c|c|c|c|c|c|c|c|c|}
\hline \multirow{2}{*}{$\begin{array}{c}\text { Site No } \\
\& \\
\text { Description }\end{array}$} & \multirow{2}{*}{$\begin{array}{l}\text { Replicate } \\
\text { Measure }\end{array}$} & \multicolumn{7}{|c|}{ IRI (in/mi) } \\
\hline & & Values & Mean & $\begin{array}{l}\text { Std } \\
\text { Dev }\end{array}$ & $\begin{array}{c}\mathrm{COV} \\
(\%)\end{array}$ & Min & Max & Range \\
\hline \multirow{5}{*}{$\begin{array}{c}1 \\
\text { Covington } \\
\text { Street }\end{array}$} & 1 & 169 & \multirow{5}{*}{167} & \multirow{5}{*}{2} & \multirow{5}{*}{1} & \multirow{5}{*}{163} & \multirow{5}{*}{169} & \multirow{5}{*}{6} \\
\hline & 2 & 163 & & & & & & \\
\hline & 3 & 168 & & & & & & \\
\hline & 4 & 167 & & & & & & \\
\hline & 5 & 169 & & & & & & \\
\hline \multirow{5}{*}{$\begin{array}{c}2 \\
\text { I-65 } \\
\text { With Board }\end{array}$} & 1 & 41 & \multirow{5}{*}{66} & \multirow{5}{*}{20} & \multirow{5}{*}{30} & \multirow{5}{*}{41} & \multirow{5}{*}{88} & \multirow{5}{*}{47} \\
\hline & 2 & 84 & & & & & & \\
\hline & 3 & 58 & & & & & & \\
\hline & 4 & 88 & & & & & & \\
\hline & 5 & 61 & & & & & & \\
\hline \multirow{3}{*}{$\begin{array}{c}2 b \\
\text { I-65 } \\
\text { No Board } \\
\end{array}$} & 1 & 57 & \multirow{3}{*}{46} & \multirow{3}{*}{15} & \multirow{3}{*}{33} & \multirow{3}{*}{36} & \multirow{3}{*}{57} & \multirow{3}{*}{21} \\
\hline & 2 & 36 & & & & & & \\
\hline & 3 & $\mathrm{n} / \mathrm{a}$ & & & & & & \\
\hline \multirow{5}{*}{$\begin{array}{c}3 \\
\text { Prairie } \\
\text { Street } \\
\text { With Board }\end{array}$} & 1 & 210 & & & & & & \\
\hline & 2 & 198 & & & & & & \\
\hline & 3 & 212 & 205 & 6 & 3 & 198 & 212 & 14 \\
\hline & 4 & 199 & & & & & & \\
\hline & 5 & 206 & & & & & & \\
\hline & 1 & 206 & & & & & & \\
\hline $3 b$ & 2 & 196 & & & & & & \\
\hline $\begin{array}{l}\text { Prairie } \\
\text { Street }\end{array}$ & 3 & 198 & 201 & 5 & 2 & 196 & 206 & 10 \\
\hline $\begin{array}{l}\text { Street } \\
\text { So Board }\end{array}$ & 4 & 201 & & & & & & \\
\hline & 5 & 206 & & & & & & \\
\hline & 1 & 124 & & & & & & \\
\hline 4 & 2 & 115 & & & & & & \\
\hline Vinton & 3 & 130 & 114 & 7 & 6 & 109 & 124 & 15 \\
\hline Street & 4 & 122 & & & & & & \\
\hline & 5 & $230^{*}$ & & & & & & \\
\hline & 1 & 69 & & & & & & \\
\hline & 2 & 68 & & & & & & \\
\hline $\begin{array}{c}5 \\
2314\end{array}$ & 3 & 65 & 67 & 2 & 3 & 65 & 69 & 4 \\
\hline & 4 & 66 & & & & & & \\
\hline & 5 & 65 & & & & & & \\
\hline & 1 & 76 & & & & & & \\
\hline & 2 & 77 & & & & & & \\
\hline 6 & 3 & 72 & 73 & 4 & 5 & 67 & 77 & 10 \\
\hline US 2318 & 4 & 67 & & & & & & \\
\hline & 5 & 72 & & & & & & \\
\hline
\end{tabular}

The reading marked with $*$ has been excluded as INDOT reported that constant speed was not maintained while profiling 


\subsubsection{Within Vendor Comparison}

All the lightweight profilers showed good consistency as far as the replicate measurements were concerned. Coefficients of variation for the IRI statistic were typically 5 percent or less with an occasional value of up to 15 percent. This suggests that all the lightweight profilers would provide good results if operated repeatedly at a particular site. These results were even more encouraging when one considers the fact that some test sites incorporated curves and steep grades. Hence these factors do not seem to have a significant effect on the repeatability of the profilers. Another impressive observation is that the repeatability was also very good at the sites where boards were placed in the wheel path(s) to simulate bridge abutments.

Another observation made is that PI with 0.2 inch blanking band is a poor choice of smoothness index. The reason is that in several cases the PI-0.2" index indicated perfectly smooth pavements. However when the PI-0.0" were compared for those cases measurable differences in roughness were observed. In the subsequent sections within vendor comparisons are discussed for PCC and HMA sites respectively.

\subsubsection{PCC Sites}

Table 4-14 is a summary of the observed smoothness measurements and summary statistics for the PCC sites. 
Table 4-14 Summary Smoothness Statistic for PCC sites

\begin{tabular}{|c|c|c|c|c|c|c|c|c|c|c|c|c|c|c|c|c|c|c|c|}
\hline \multirow{2}{*}{$\begin{array}{c}\text { Site number and } \\
\text { description } \\
\end{array}$} & \multirow{2}{*}{$\begin{array}{c}\text { Vendor } \\
\text { ID. }\end{array}$} & \multicolumn{6}{|c|}{ IRI $\quad$ (in/mi) } & \multicolumn{6}{|c|}{ PI 0.2" (in/mi) } & \multicolumn{6}{|c|}{ PI 0.0" (in/mi) } \\
\hline & & Mean & sD & $\mathrm{CV}(\%)$ & Min. & Max. & Range & Mean & $\mathrm{sD}$ & $\mathrm{CV}(\%)$ & Min & Max. & Range & Mean & $\mathrm{SD}$ & $\mathrm{CV}(\%)$ & Min. N & Max & Range \\
\hline \multirow{5}{*}{$\begin{array}{c}1 \\
\text { Convinton } \\
\text { (Heavy Broom) }\end{array}$} & $A$ & 107 & 5 & 4 & 100 & 111 & 11 & 8 & 1 & 18 & 6 & 9 & 3 & 27 & 3 & 12 & 21 & 29 & 8 \\
\hline & B & 152 & 3 & 2 & 149 & 155 & 6 & 44 & 2 & 4 & 41 & 45 & 4 & 76 & 1 & 1 & 75 & 77 & 2 \\
\hline & C & 154 & 1 & 1 & 153 & 155 & 2 & 49 & 0 & 1 & 49 & 49 & 1 & 83 & 1 & 1 & 82 & 84 & 2 \\
\hline & D & 155 & 3 & 2 & 152 & 159 & 7 & 46 & 1 & 2 & 45 & 47 & 1 & 79 & 0 & 1 & 79 & 80 & 2 \\
\hline & Rip Van & 167 & 2 & 1 & 163 & 169 & 6 & & & & & & & & & & & & \\
\hline \multirow{5}{*}{$\begin{array}{c}5 \\
\text { US } 231 \mathrm{~A} \\
\text { (Random Tining) }\end{array}$} & A & 65 & 0 & 0 & 65 & 65 & 0 & 0 & 0 & 224 & 0 & 1 & 1 & 13 & 1 & 12 & 11 & 15 & 4 \\
\hline & B & 44 & 2 & 3 & 43 & 46 & 4 & 0 & 0 & 78 & 0 & 0 & 0 & 19 & 1 & 7 & 18 & 21 & 3 \\
\hline & C & 43 & 1 & 1 & 43 & 44 & 1 & 0 & 0 & 135 & 0 & 1 & 1 & 16 & 1 & 3 & 15 & 17 & 1 \\
\hline & D & 52 & 7 & 14 & 47 & 65 & 18 & 0 & 0 & 64 & 0 & 1 & 1 & 17 & 0 & 2 & 17 & 18 & 1 \\
\hline & Rip Van & 67 & 2 & 3 & 65 & 69 & 4 & & & & & & & & & & & & \\
\hline \multirow{5}{*}{$\begin{array}{c}6 \\
\text { US 231B } \\
\text { (Random Tining) }\end{array}$} & $A$ & 77 & 5 & 7 & 70 & 83 & 13 & 1 & 1 & 70 & 0 & 2 & 2 & 17 & 2 & 10 & 15 & 20 & 5 \\
\hline & B & 55 & 1 & 3 & 54 & 55 & 1 & 3 & 0 & 15 & 3 & 4 & 1 & 23 & 1 & 3 & 23 & 24 & 1 \\
\hline & C & 57 & 1 & 2 & 56 & 59 & 3 & 2 & 0 & 10 & 2 & 3 & 1 & 23 & 1 & 5 & 22 & 25 & 3 \\
\hline & D & 60 & 1 & 1 & 59 & 61 & 2 & 3 & 0 & 15 & 2 & 3 & 1 & 22 & 2 & 9 & 20 & & 4 \\
\hline & Rip Van & 73 & 4 & 5 & 67 & 77 & 10 & & & & & & & & & & & & \\
\hline
\end{tabular}

As a site specific example, Figures 4-1 through 4-4 show the replicate IRI values reported by Vendors A through D for Site 1, respectively. Each bar indicates a single measurement (run number). Superimposed on each individual bar is a small solid bar with a center notch. The center notch indicates the vendor average (average of all five runs). The extremes of the small bar indicate the average plus and minus one standard deviation. Figures 4-5 through 4-8 and 4-9 through 4-12 represent similar data for PI with 0.0 inch and 0.2 inch blanking bands, respectively. These plots are typical examples and they illustrate the good repeatability observed for all smoothness indices at Site 1. 


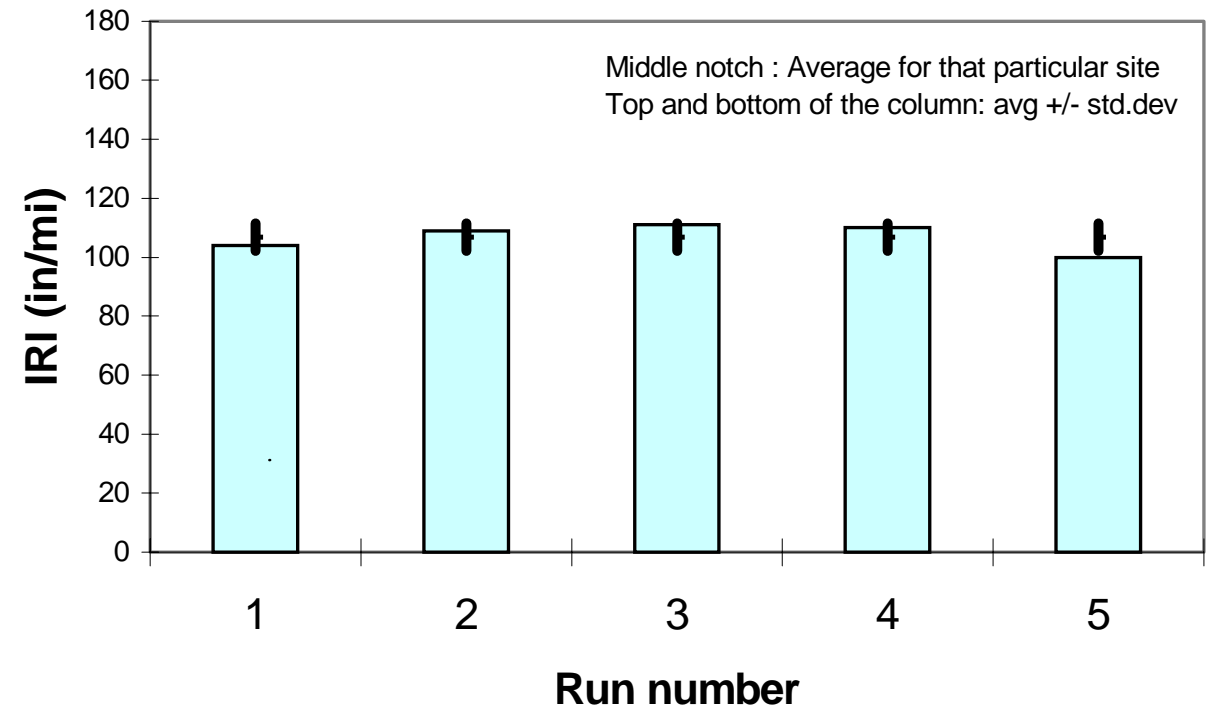

Figure 4-1 Replicate IRI measurements by Vendor $A$ at Site 1

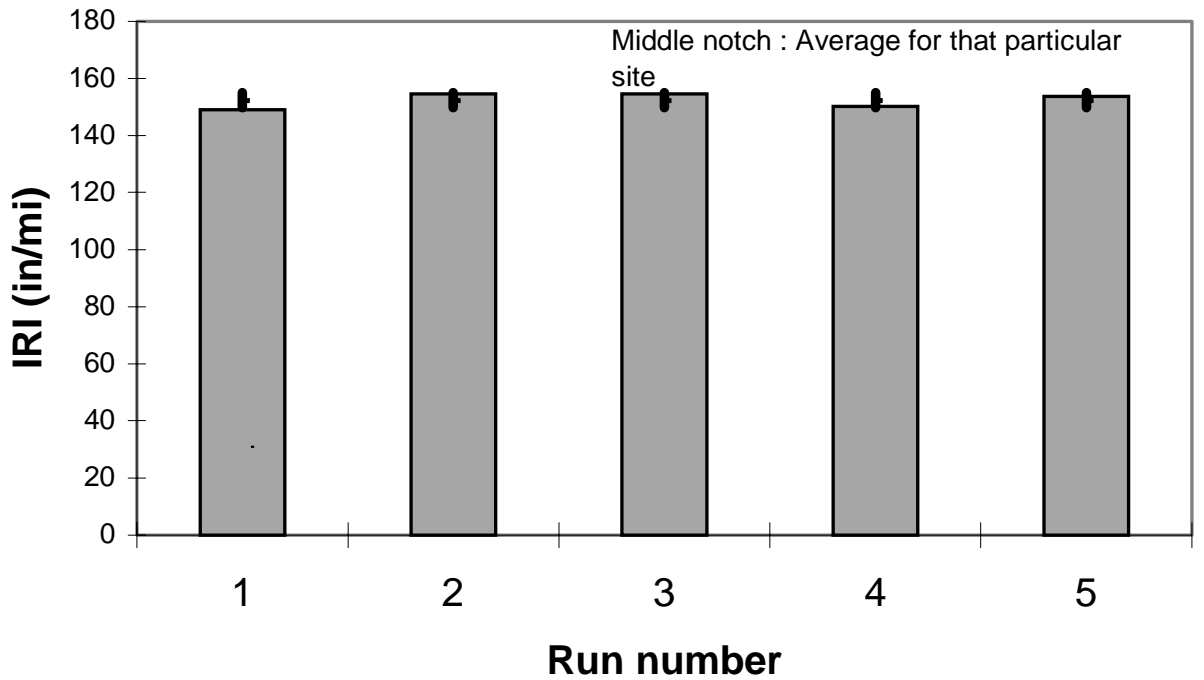

Figure 4-2 Replicate IRI measurements by Vendor B at Site 1 


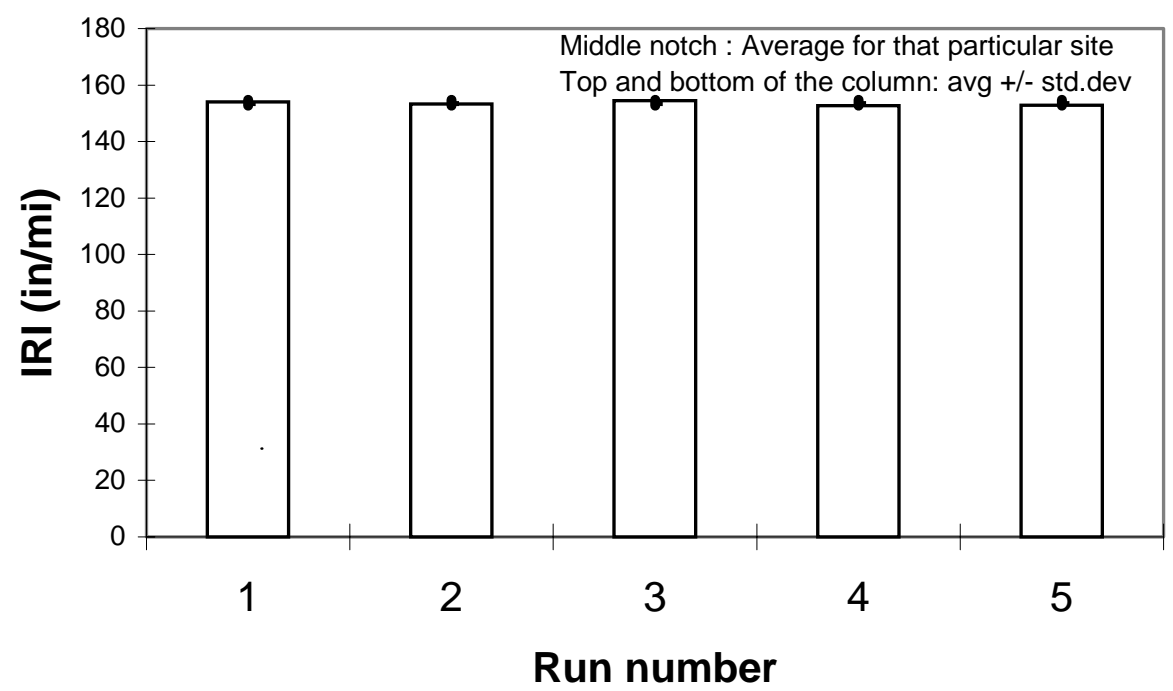

Figure 4-3 Replicate IRI measurements by Vendor C at Site 1

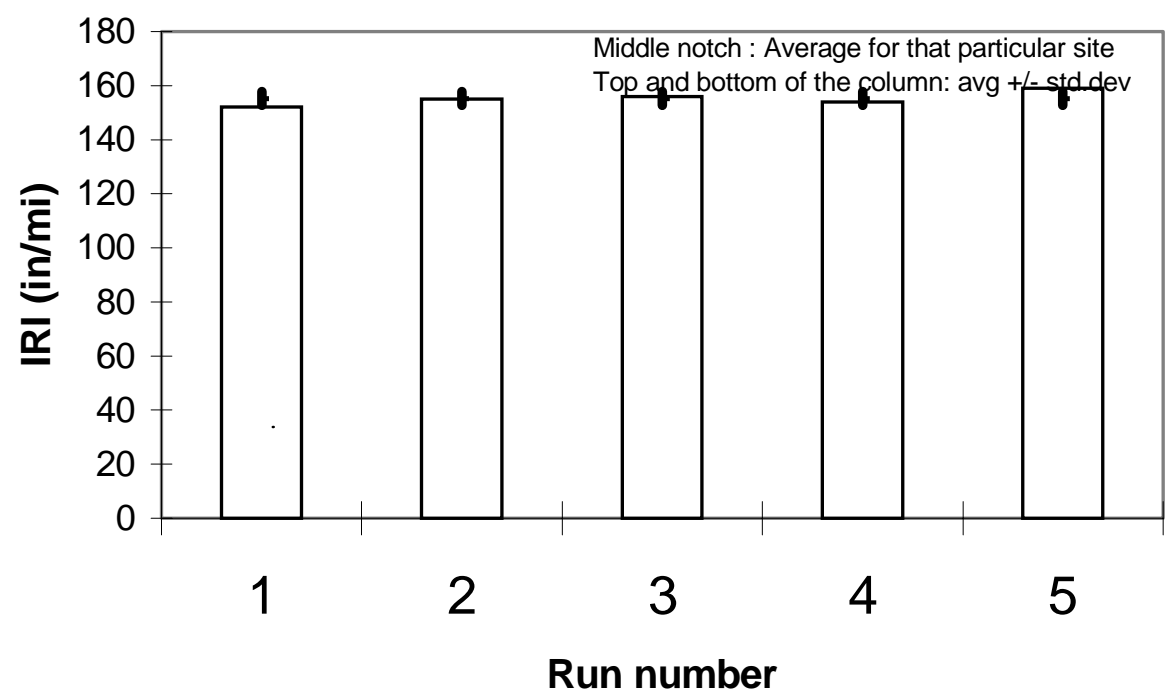

Figure 4-4 Replicate IRI measurements by Vendor D at Site 1 


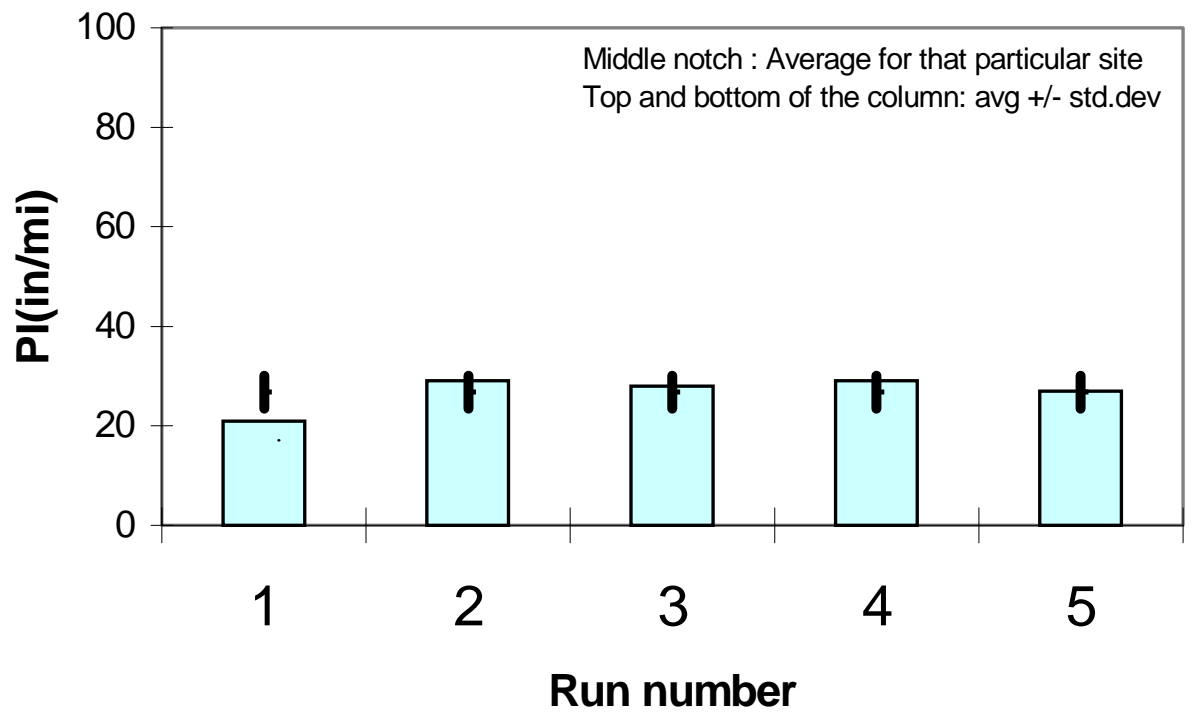

Figure 4-5 Replicate PI-0.0” measurements by Vendor A at Site 1

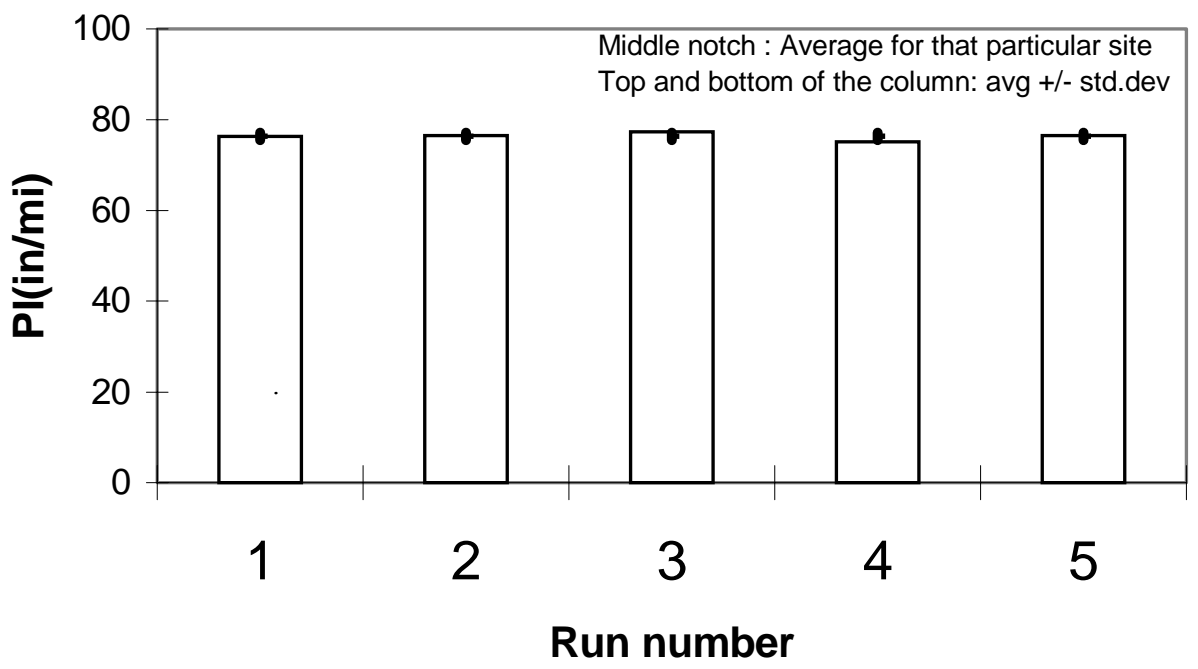

Figure 4-6 Replicate PI-0.0" measurements by Vendor B at Site 1 


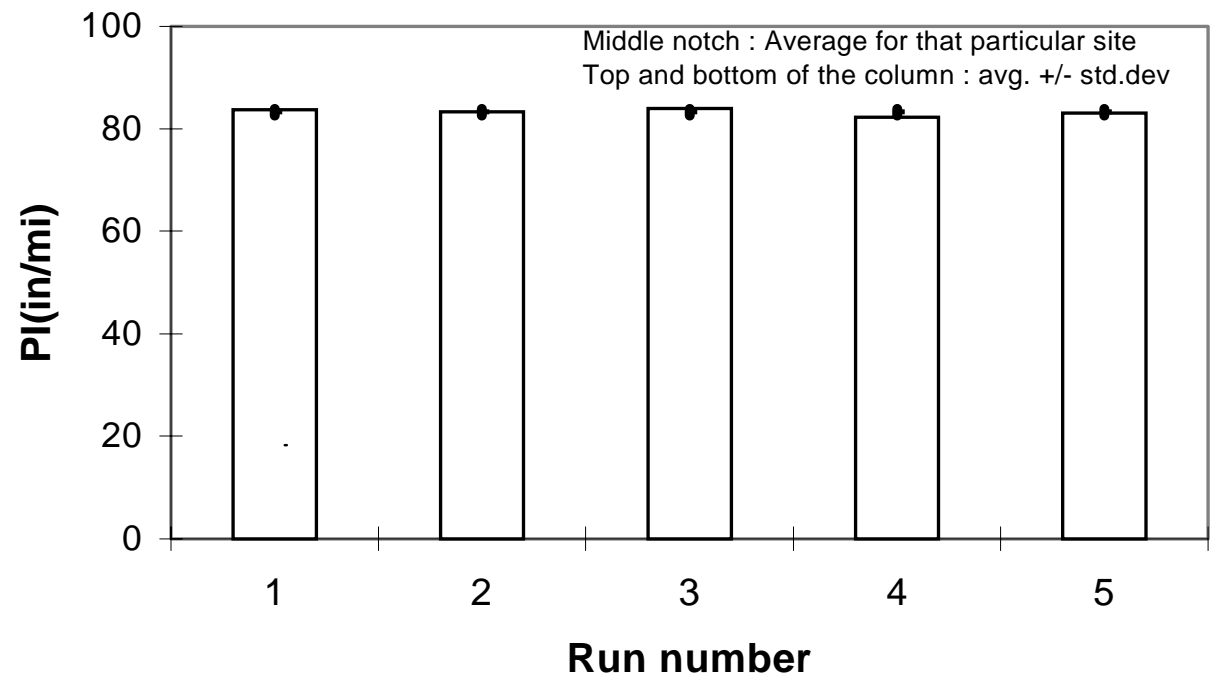

Figure 4-7 Replicate PI-0.0" measurements by Vendor C at Site 1

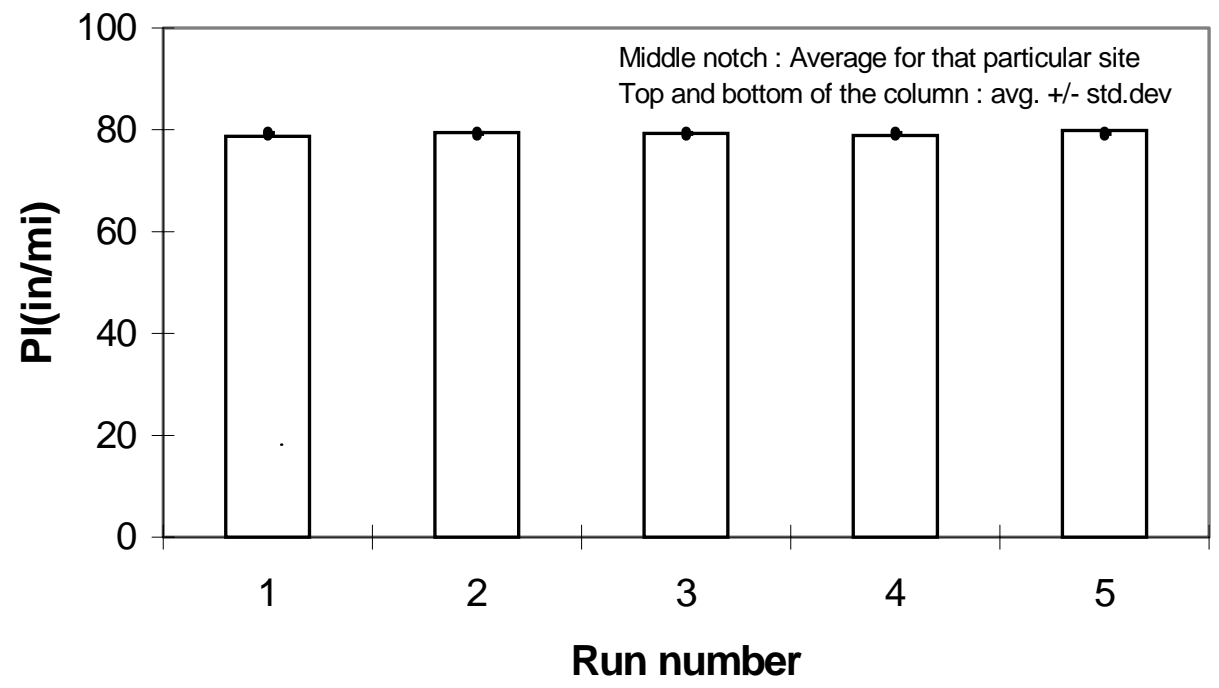

Figure 4-8 Replicate PI-0.0" measurements by Vendor D at Site 1 


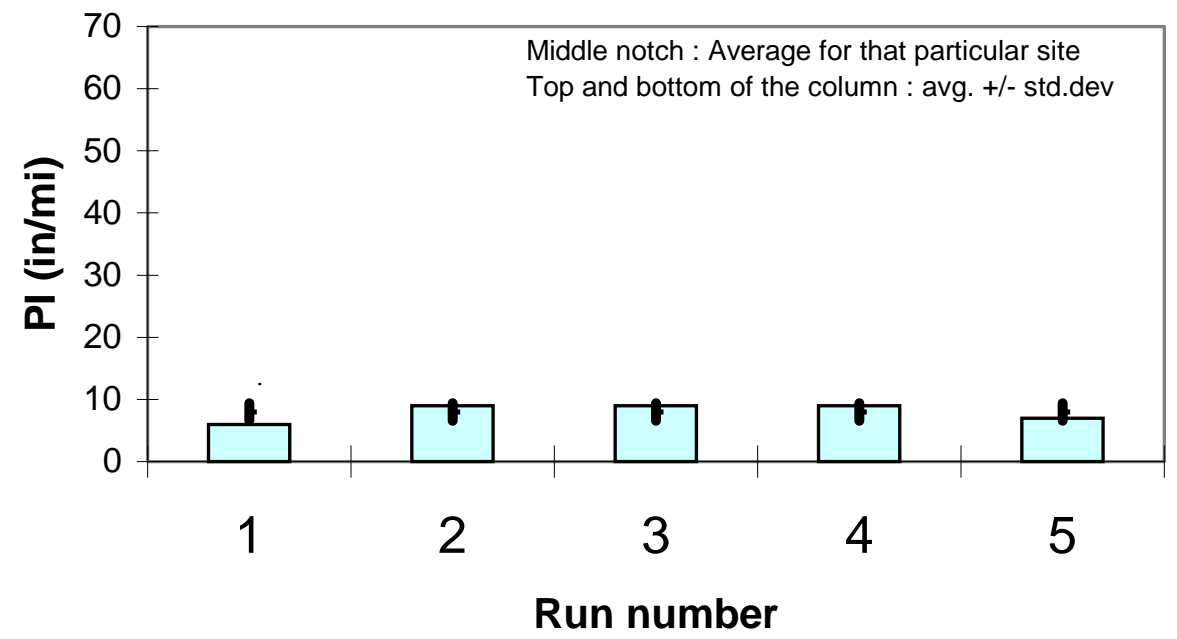

Figure 4-9 Replicate PI-0.2" measurements by Vendor A at Site 1

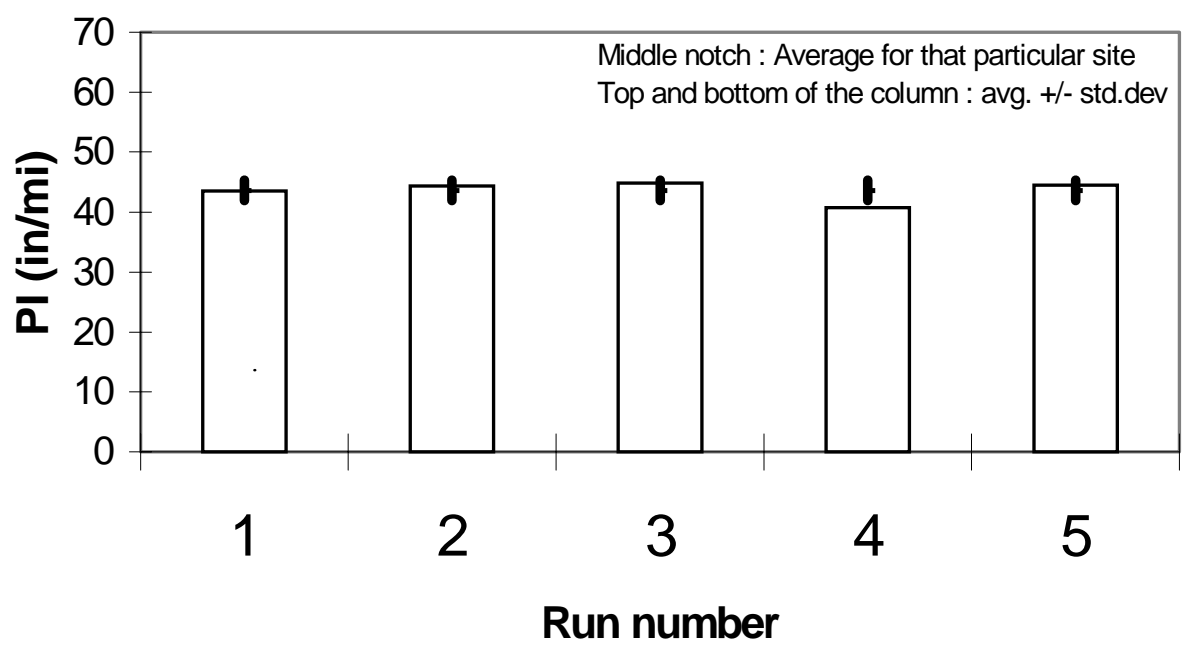

Figure 4-10 Replicate PI-0.2" measurements by Vendor B at Site 1 


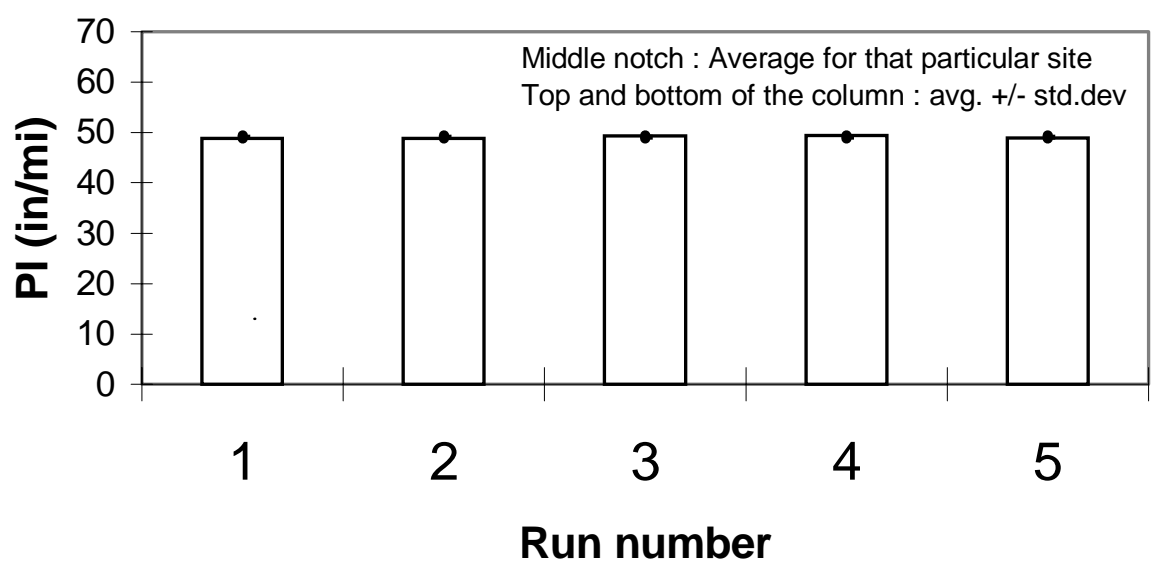

Figure 4-11 Replicate PI-0.2" measurements by Vendor C at Site 1

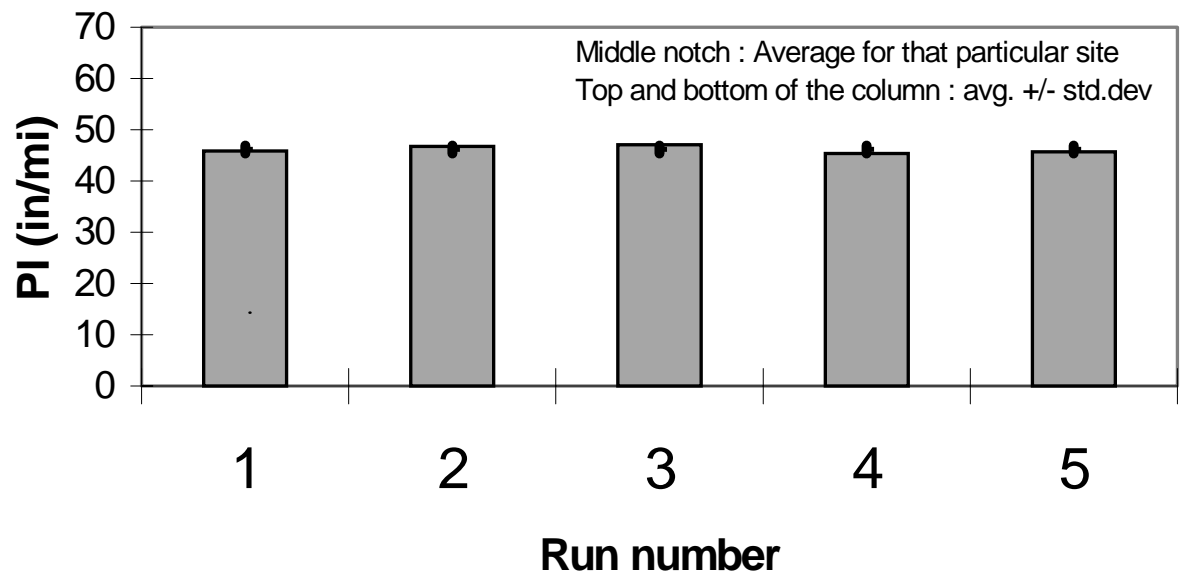

Figure 4-12 Replicate PI-0.2" measurements by Vendor D at Site 1

Covington Street (Site 1) was identified as the roughest among all the PCC sites by all profilers (reported IRI values ranged from 107 to $155 \mathrm{in} / \mathrm{mi}$ ). However all devices showed good consistency for the rough PCC pavement at this site. 
For the PI-0.0" blanking band index, standard deviations of around $1 \mathrm{in} / \mathrm{mi}$ were reported by vendors $\mathrm{B}, \mathrm{C}$ and $\mathrm{D}$ while vendor $\mathrm{A}$ reported a slightly higher standard deviation of $3.3 \mathrm{in} / \mathrm{mi}$ at Site 1. Similarly, all vendors reported low standard deviations for PI-0.2" for this site. One anomaly that appears in the data is the fact that Vendor A reported IRI and PI values of significantly lower magnitude than the other vendors.

The IRI, PI-0.0" and PI-0.2" plots by Vendor and Site for the US 231A and US 231B sites are presented in Appendices A, B, C and D respectively.

All of the profilers indicated that US231A (Site 5) was the smoothest site among the three PCC sites tested. Average IRI values ranged from 43 to $65 \mathrm{in} / \mathrm{mi}$ at this site, as indicated in Tables 4-14. The site incorporated essentially no grade and standard cross slope. No visual distress was observed at the site either. Vendor A showed exceptional consistency for this particular site, with all five replicate runs providing the same IRI value of $65 \mathrm{in} / \mathrm{mi}$. The other vendors showed good consistency with standard deviation of less than 2 percent with the exception of Vendor D which reported a somewhat higher standard deviation of $7 \mathrm{in} / \mathrm{mi}$.

For the PI-0.0" blanking band index, all vendors reported consistent replicate measurements with an average standard deviation of 1 percent or less for all vendors at Site 5. The smoothness indices were also similar in magnitude ranging from 13 to 19 in/mi. 
All of the vendors reported average values of $0 \mathrm{in} / \mathrm{mi}$ for the PI- 0.2 " index at Site 5. With this small average value, standard deviations and coefficients of variation are essentially meaningless. Comparing the average PI values with 0.0 and 0.2 inch blanking bands for this site provides an excellent example of the inadequacy of the 0.2 inch blanking band, with the 0.0 inch blanking band average PI values ranging from 13 to 19 inches/mile, as compared to no range at all with the 0.2 inch blanking band. The 0.2 inch blanking band indicates no roughness, while the 0.0 inch blanking band provides a measurable level of roughness.

US231B (Site 6) incorporated a slight downhill grade and a continuous curve throughout the section. Again no visual distress was observed at this site. Very low standard deviations (1in/mi) and coefficients of variation were reported for IRI by each vendor at this site with the exception of Vendor A which reported a standard deviation of $5 \mathrm{in} / \mathrm{mi}$.

Considering the PI-0.0" as the smoothness index, all the profilers also reported low standard deviations ( $<2 \mathrm{in} / \mathrm{mi})$ for this site. In fact all PI-0.0" values reported by all vendors were fairly close to each other with an average magnitude of 23 inches/mile, with the exception of Vendor A which reported an average of $17 \mathrm{in} / \mathrm{mi}$.

Good consistency was observed for the PI-0.2" data with all the standard deviations being less than $1 \mathrm{in} / \mathrm{mi}$. However the average values reported by all vendors were very small ranging from 0 to $4 \mathrm{in} / \mathrm{mi}$. 
Overall, all of the profilers showed good repeatability for the PCC sites. The primary issues identified through this review were the inadequacy of the PI-0.2" index and the observation that the magnitude of most index values reported by vendor $\mathrm{A}$ were either greater than or less than the other vendors.

\subsubsection{HMA Sites}

The test results associated with the HMA sites were presented in Tables 4-1 through 4-13 in the previous section. A summary of the results for the HMA sites is presented in Table 4-15.

Table 4-15 Summary Smoothness Statistic for the HMA sites

\begin{tabular}{|c|c|c|c|c|c|c|c|c|c|c|c|c|c|c|c|c|c|c|c|}
\hline \multirow{2}{*}{\begin{tabular}{|c|} 
Site number and \\
description
\end{tabular}} & \multirow{2}{*}{\begin{tabular}{|c|}
$\begin{array}{c}\text { Vendor } \\
\text { ID. }\end{array}$ \\
\end{tabular}} & \multicolumn{6}{|c|}{ IRI (in/mi) } & \multicolumn{6}{|c|}{ PI 0.2" (in/mi) } & \multicolumn{6}{|c|}{$\begin{array}{ll}\text { PI 0.0" } \quad(\mathrm{in} / \mathrm{mi}) \\
\end{array}$} \\
\hline & & Mean & Std Dev & Co.V(\%) & Min. & Max. & Range & Mean & Std.Dev & $\mathrm{CoV}(\%)$ & Min. & Max. & Range & Mean & Std.Dev & $\operatorname{CoV}(\%)$ & Min. & Max. & Range \\
\hline 2 & A & 218 & 24 & 11 & 183 & 240 & 57 & 52 & 5 & 9 & 46 & 56 & 10 & 68 & 2 & 3 & 65 & 69 & 4 \\
\hline \multirow{4}{*}{$\begin{array}{c}\mathrm{I}-65 \\
\text { (with board) }\end{array}$} & B & 91 & 4 & 5 & 86 & 96 & 10 & 14 & 1 & 10 & 13 & 17 & 4 & 34 & 2 & 7 & 32 & 38 & 6 \\
\hline & C & 32 & 0 & 1 & 31 & 32 & 1 & 1 & 1 & 39 & 1 & 2 & 1 & 11 & 1 & 8 & 9 & 11 & 2 \\
\hline & D & 84 & 4 & 5 & 80 & 91 & 11 & 14 & 2 & 11 & 13 & 17 & 4 & 27 & 1 & 2 & 27 & 28 & 2 \\
\hline & Rip Van & 66 & 20 & 30 & 41 & 88 & 47 & & & & & & & & & & & & \\
\hline \multirow{5}{*}{$\begin{array}{c}2 \mathrm{~b} \\
\mathrm{I}-65 \\
\text { (no board) }\end{array}$} & A & 59 & 1 & 2 & 58 & 60 & 2 & 0 & 0 & 0 & 0 & 0 & 0 & 5 & 1 & 11 & 5 & 6 & 1 \\
\hline & B & 39 & 1 & 3 & 38 & 41 & 2 & 0 & 0 & 0 & 0 & 0 & 0 & 14 & 2 & 11 & 13 & 16 & 3 \\
\hline & C & 31 & 1 & 4 & 30 & 32 & 2 & 0 & 0 & 0 & 0 & 0 & 0 & 7 & 0 & 4 & 6 & 7 & 0 \\
\hline & D & 35 & 5 & 15 & 31 & 41 & 10 & 0 & 0 & 0 & 0 & 0 & 0 & 8 & 1 & 12 & 7 & 9 & 2 \\
\hline & Rip Van & 46 & 15 & 33 & 36 & 57 & 21 & & & & & & & & & & & & \\
\hline \multirow{5}{*}{$\begin{array}{c}3 \\
\text { Prairie St. } \\
\text { (with board) }\end{array}$} & A & 148 & 5 & 4 & 141 & 155 & 14 & 34 & 2 & 7 & 32 & 37 & 5 & 36 & 2 & 6 & 33 & 39 & 6 \\
\hline & B & 150 & 3 & 2 & 146 & 154 & 8 & 43 & 1 & 3 & 42 & 45 & 3 & 61 & 1 & 1 & 60 & 62 & 2 \\
\hline & C & 171 & 9 & 5 & 166 & 187 & 20 & 49 & 4 & 9 & 45 & 55 & 10 & 60 & 3 & 4 & 58 & 64 & 7 \\
\hline & D & 162 & 2 & 1 & 161 & 165 & 4 & 38 & 1 & 2 & 36 & 38 & 2 & 57 & 2 & 3 & 56 & 60 & 4 \\
\hline & Rip Van & 205 & 6 & 3 & 198 & 212 & 14 & & & & & & & & & & & & \\
\hline \multirow{5}{*}{$\begin{array}{c}3 b \\
\text { Prairie St. } \\
\text { (no board) }\end{array}$} & A & 130 & 6 & 5 & 126 & 141 & 15 & 32 & 3 & 8 & 28 & 35 & 7 & 36 & 1 & 3 & 34 & 37 & 3 \\
\hline & B & 141 & 1 & 1 & 140 & 143 & 3 & 39 & 1 & 2 & 37 & 39 & 2 & 56 & 1 & 2 & 55 & 57 & 2 \\
\hline & C & 165 & 4 & 2 & 161 & 172 & 11 & 48 & 1 & 3 & 46 & 49 & 3 & 63 & 4 & 7 & 60 & 69 & 9 \\
\hline & D & 154 & 3 & 2 & 149 & 157 & 8 & 37 & 1 & 2 & 36 & 37 & 2 & 57 & 1 & 3 & 55 & 59 & 3 \\
\hline & Rip Van & 201 & 5 & 2 & 196 & 206 & 10 & & & & & & & & & & & & \\
\hline 4 & A & 92 & 4 & 5 & 87 & 98 & 11 & 18 & 3 & 15 & 14 & 20 & 6 & 23 & 1 & 2 & 23 & 24 & 1 \\
\hline \multirow[t]{4}{*}{ Vinton St. } & B & 101 & 3 & 3 & 95 & 102 & 7 & 16 & 1 & 7 & 14 & 17 & 3 & 37 & 2 & 5 & 36 & 40 & 4 \\
\hline & C & 102 & 1 & 1 & 100 & 103 & 3 & 19 & 1 & 7 & 17 & 20 & 3 & 39 & 1 & 3 & 37 & 41 & 3 \\
\hline & D & 107 & 3 & 3 & 103 & 112 & 9 & 16 & 1 & 4 & 15 & 16 & 2 & 39 & 1 & 2 & 38 & 40 & 2 \\
\hline & Rip Van & 114 & 7 & 6 & 109 & 124 & 15 & & & & & & & & & & & & \\
\hline
\end{tabular}


Approximately ten feet from the beginning and end of the I-65 site a $3 / 4$ inch thick plywood strip was placed transverse to the direction of testing to simulate a bridge abutment and provide a reference to assess the ability of the lightweight profilers to identify bumps. The plywood strips were simply nailed to the HMA pavement surface using several nails. The strips showed no signs of jarring loose during the testing. All of the vendors reported increases in all three smoothness indices due to the presence of the plywood strips, but the standard deviation and the coefficient of variation for the replicate measurements for each vendor were not significantly affected by the presence of the strips.

Based on review of the data from the five measurements per vendor at the I-65 site with the plywood strip in place, there was a significant difference in the IRI reported by the vendors. Vendor A reported very high values averaging $218 \mathrm{in} / \mathrm{mi}$ while Vendor C reported very low values averaging $32 \mathrm{in} / \mathrm{mi}$. The differences are clear from review of Figures 4-13 through 4-16. Vendors B and D reported similar values in the range of 84 to 91in/mi. However, all of the vendors with the exception of Vendor A, reported small standard deviations and coefficients of variation while those reported by Vendor A were significantly larger. This suggests that Vendor A may have a repeatability problem with IRI when bumps are present. This could very well be due to dynamics introduced to the profiler by the plywood strips that are not properly filtered out in the computation of IRI or perhaps the magnitude of the acceleration induced by the bumps exceeded the capacity of the accelerometer used on the device. 
Considering PI with a 0.0 inch blanking band and the plywood strips in place at the I-65 site, the same trend was observed as was seen in the IRI data. Once again Vendor A consistently reported very high values and Vendor $\mathrm{C}$ reported low values compared to the other two vendors. This is shown in Figures 4-17 through 4-20. However all of the vendors report similar standard deviations and coefficients of variation which were quite low. This suggests that the filtering/data analysis was responsible for the significant difference in repeatability relative to IRI for Vendor A, rather than an accelerometer problem.

Considering PI with a 0.2 inch blanking band and plywood strips in place at the I65 site, the same trends were observed as with the other indices. Once again Vendor A reported high PI values (average of $52 \mathrm{in} / \mathrm{mi}$ ) while Vendor $\mathrm{C}$ reported low single digit indices. Figures 4-21 through 4-24 show this along with the fact that the other two vendors showed good correlation. Under this condition, Vendor A reported a slightly higher standard deviation and coefficient of variation (5 and 9, respectively) than the other vendors which reported standard deviations of 1 or $2 \mathrm{in} / \mathrm{mi}$. 


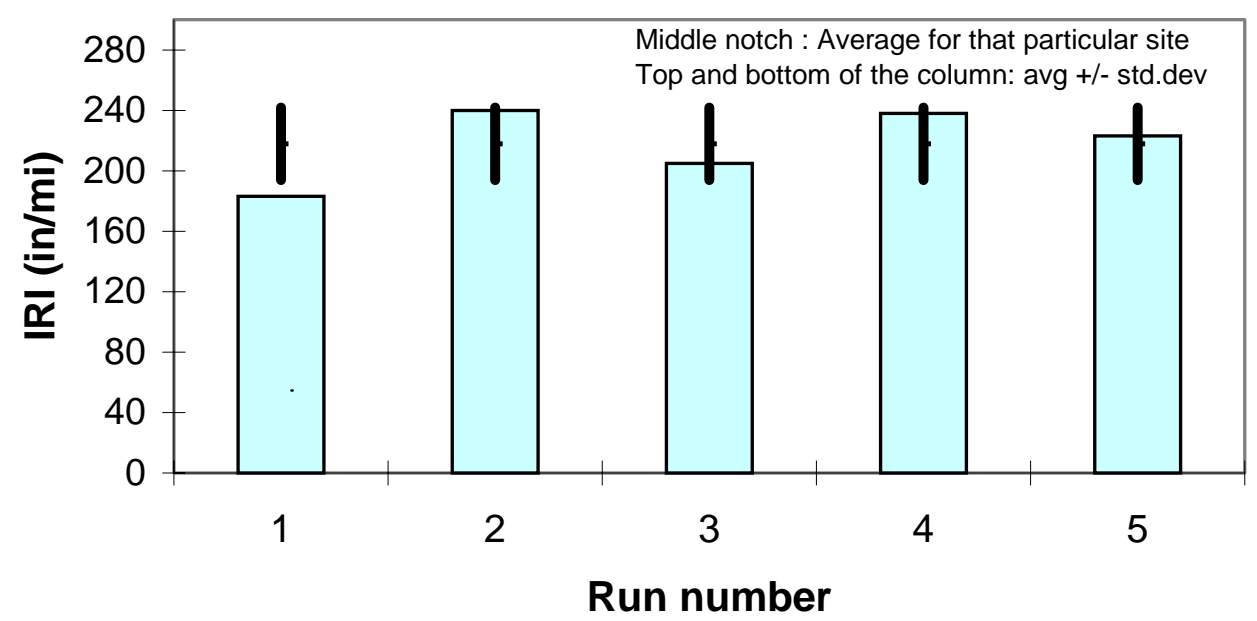

Figure 4-13 Replicate IRI measurements by Vendor A at Site 2 (I-65 with board)

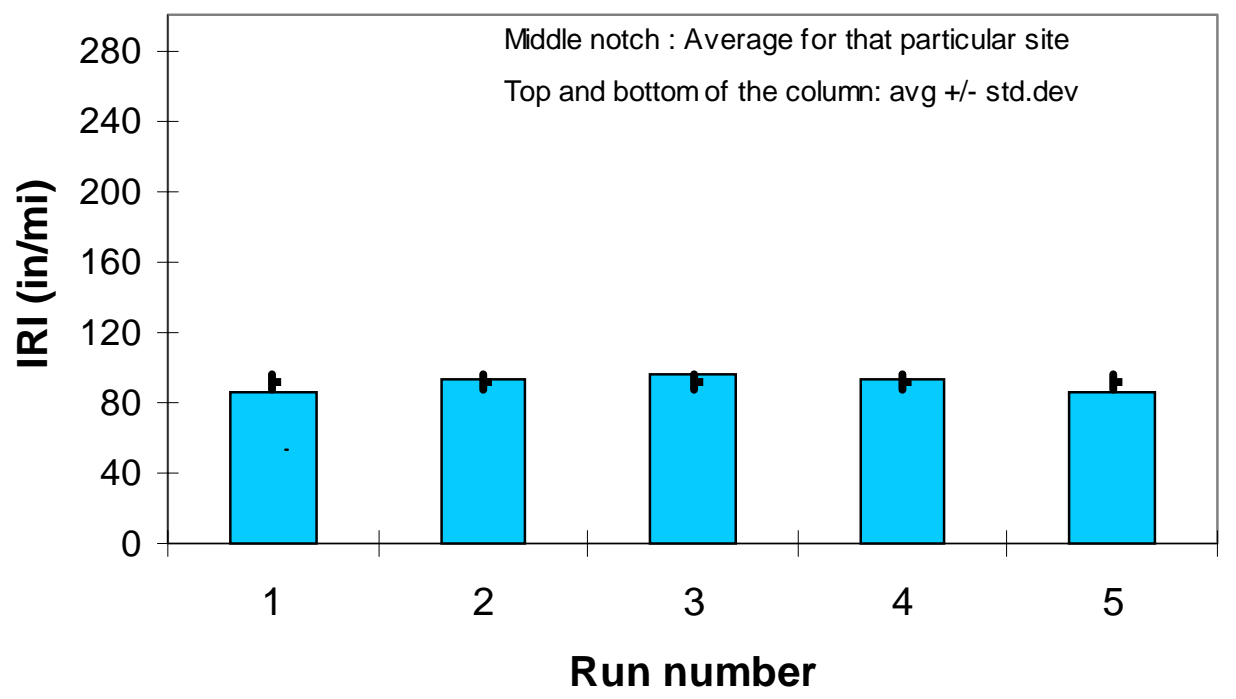

Figure 4-14 Replicate IRI measurements by Vendor B at Site 2 (I-65 with board) 


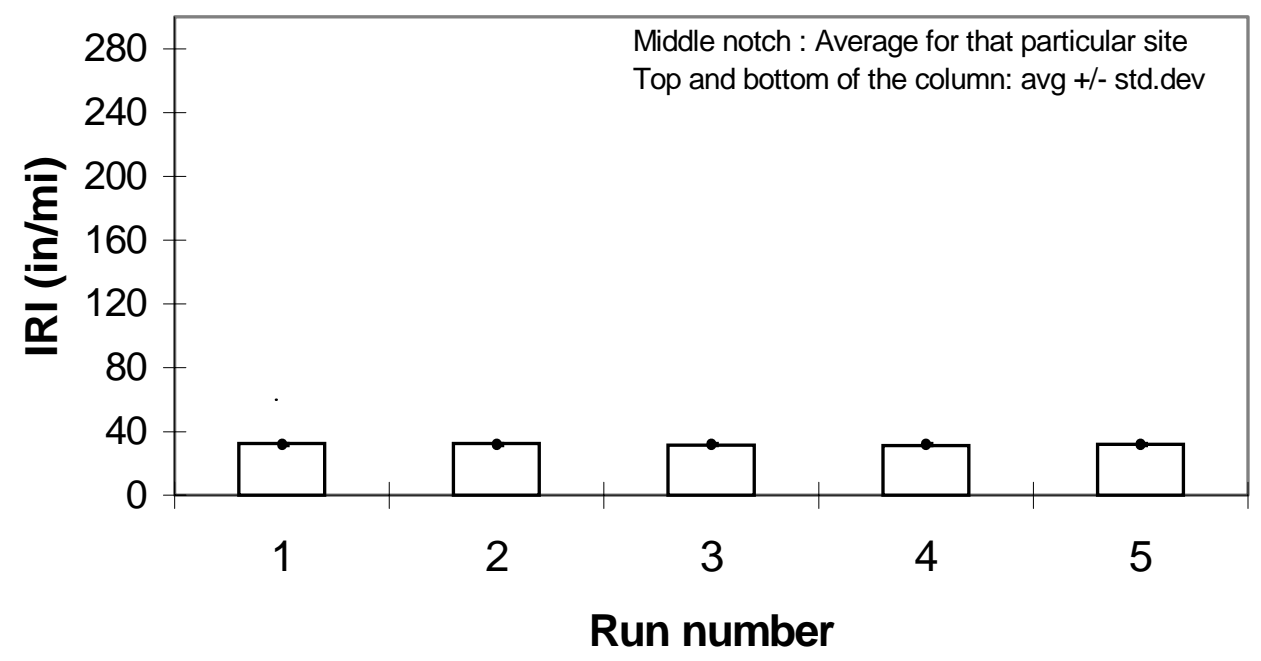

Figure 4-15 Replicate IRI measurements by Vendor C at Site 2 (I-65 with board)

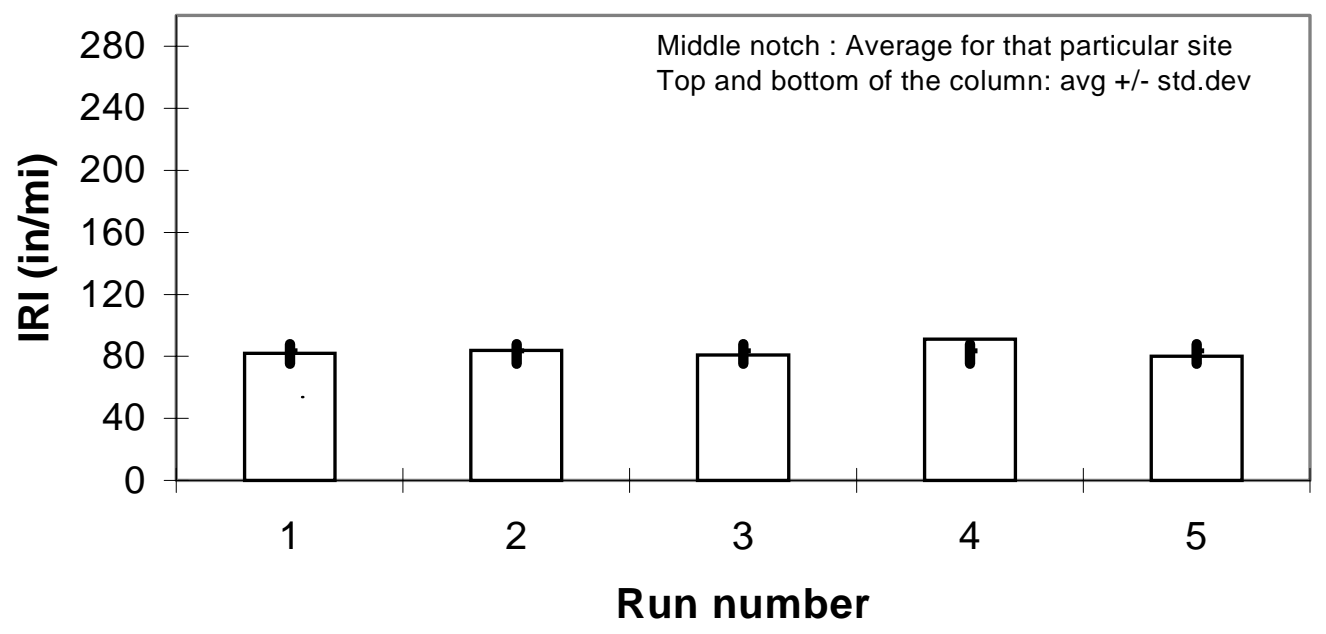

Figure 4-16 Replicate IRI measurements by Vendor D at Site 2 (I-65 with board) 


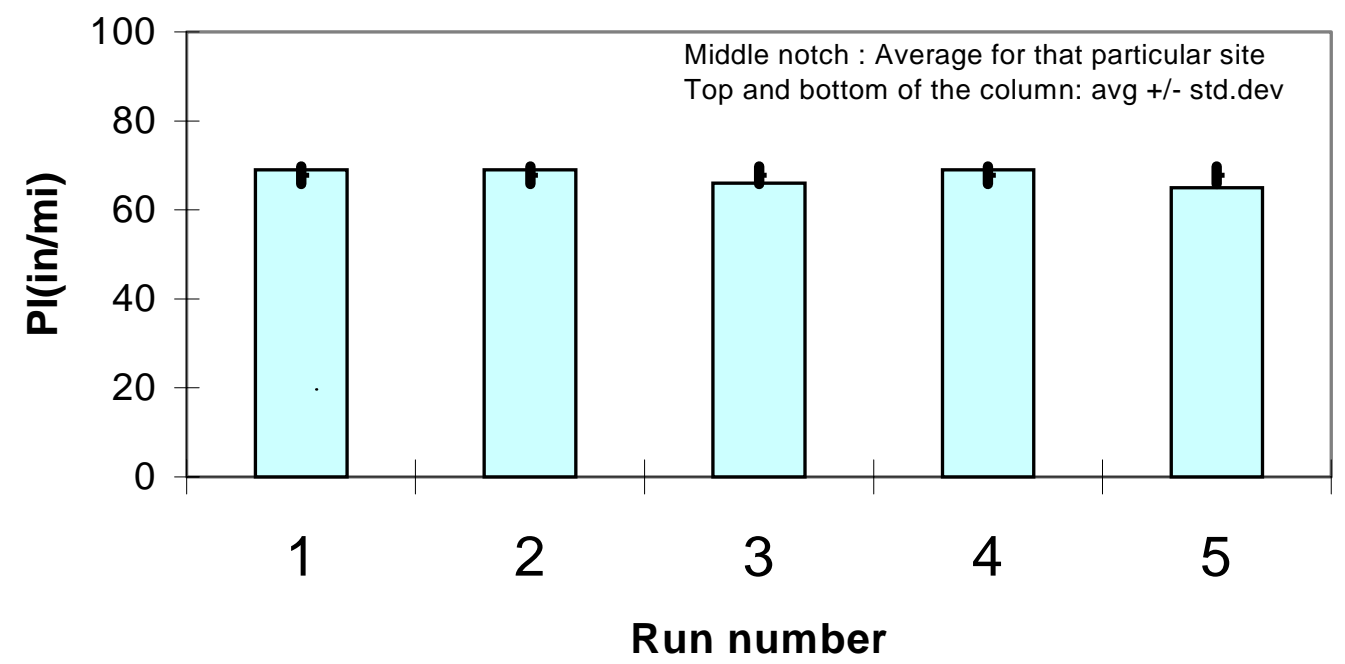

Figure 4-17 Replicate PI-0.0" measurements by Vendor A at Site 2 (I-65 with board)

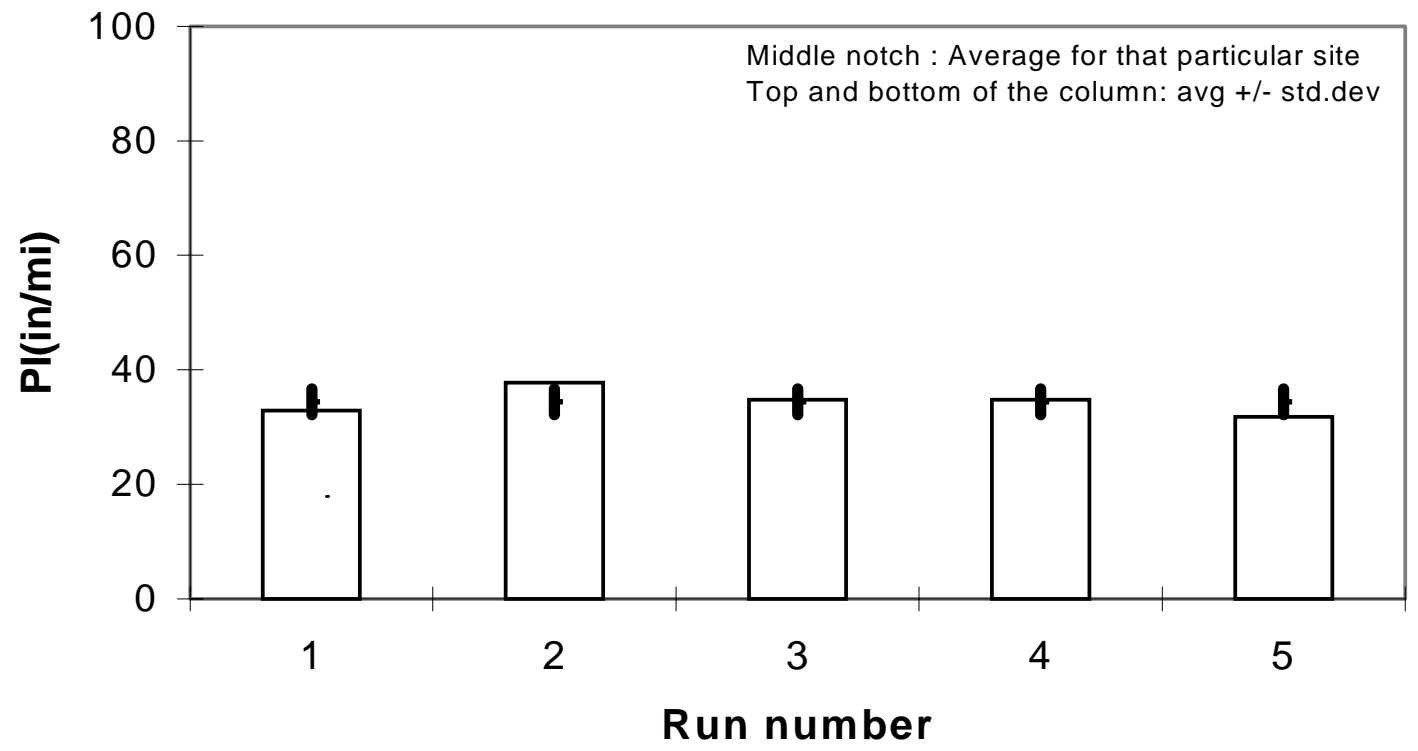

Figure 4-18 Replicate PI-0.0" measurements by Vendor B at Site 2 (I-65 with board) 


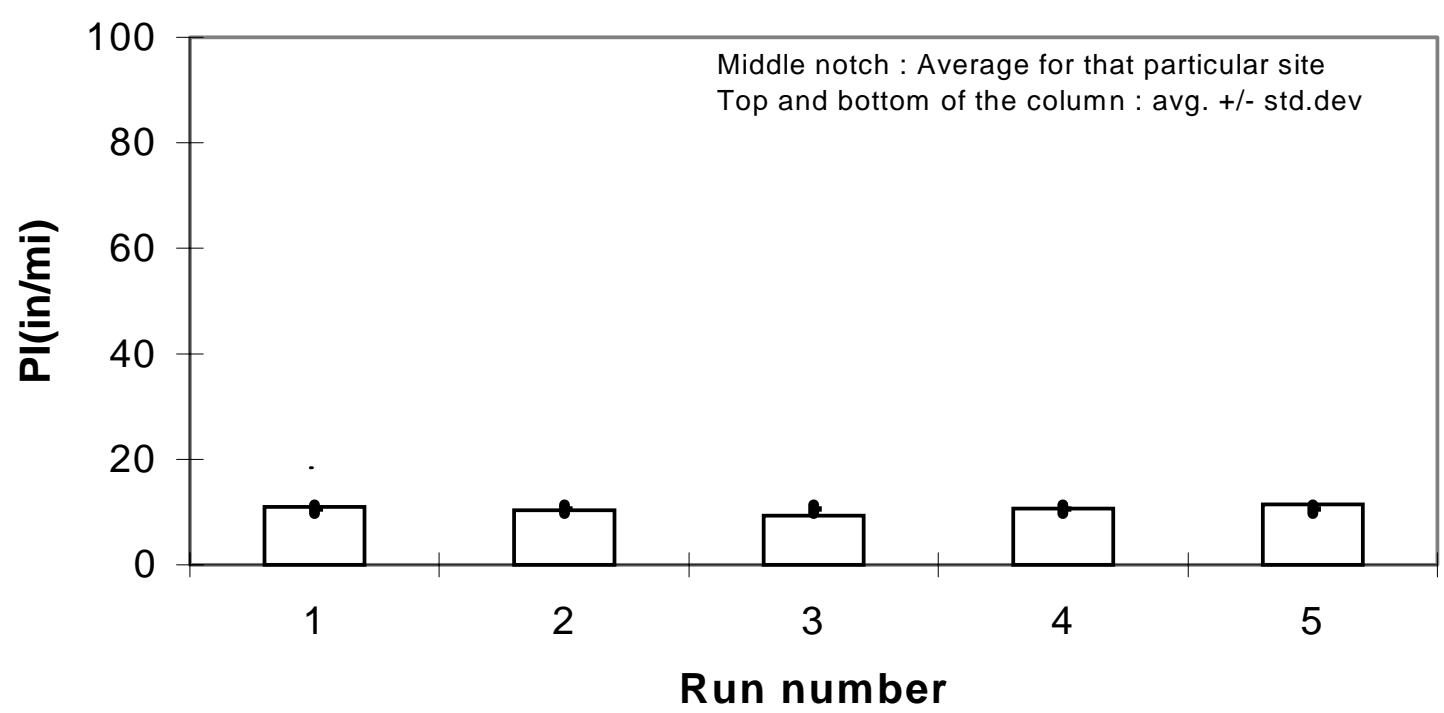

Figure 4-19 Replicate PI-0.0" measurements by Vendor C at Site 2 (I-65 with board)

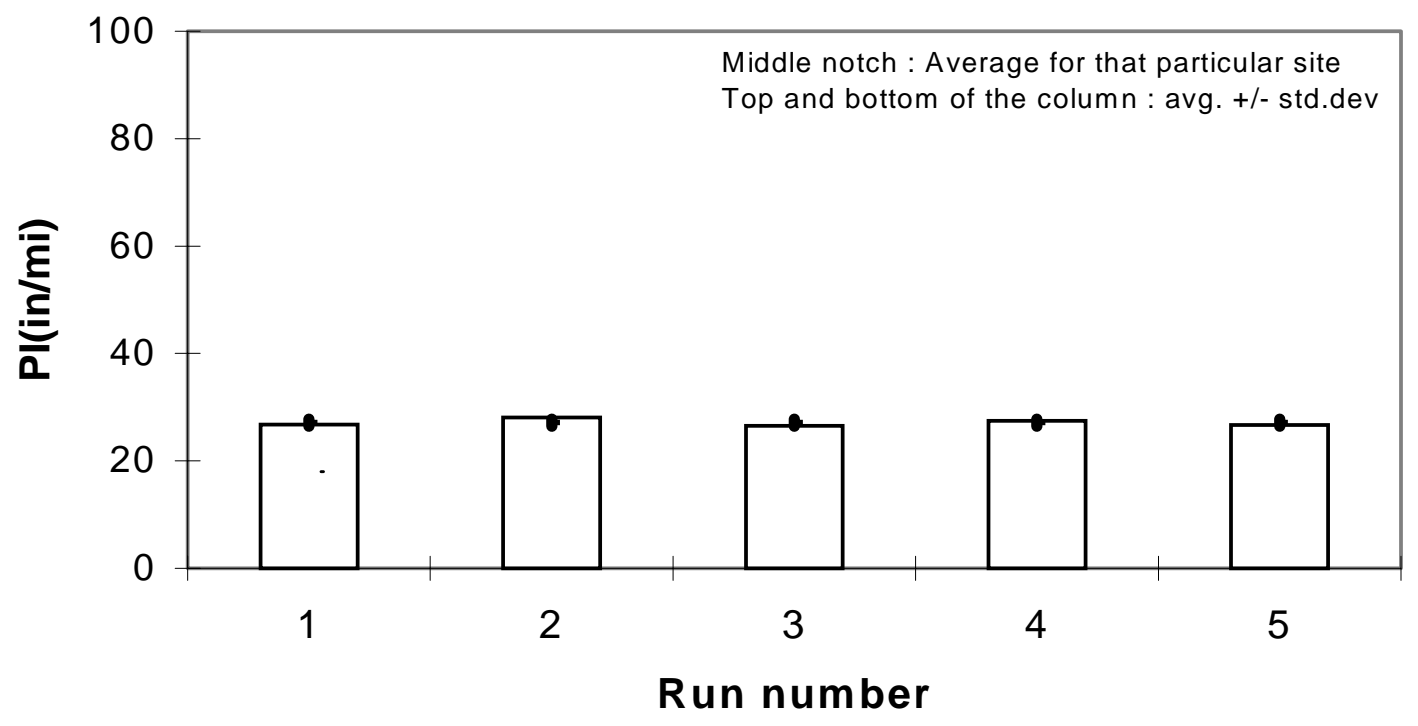

Figure 4-20 Replicate PI-0.0" measurements by Vendor D at Site 2 (I-65 with board) 


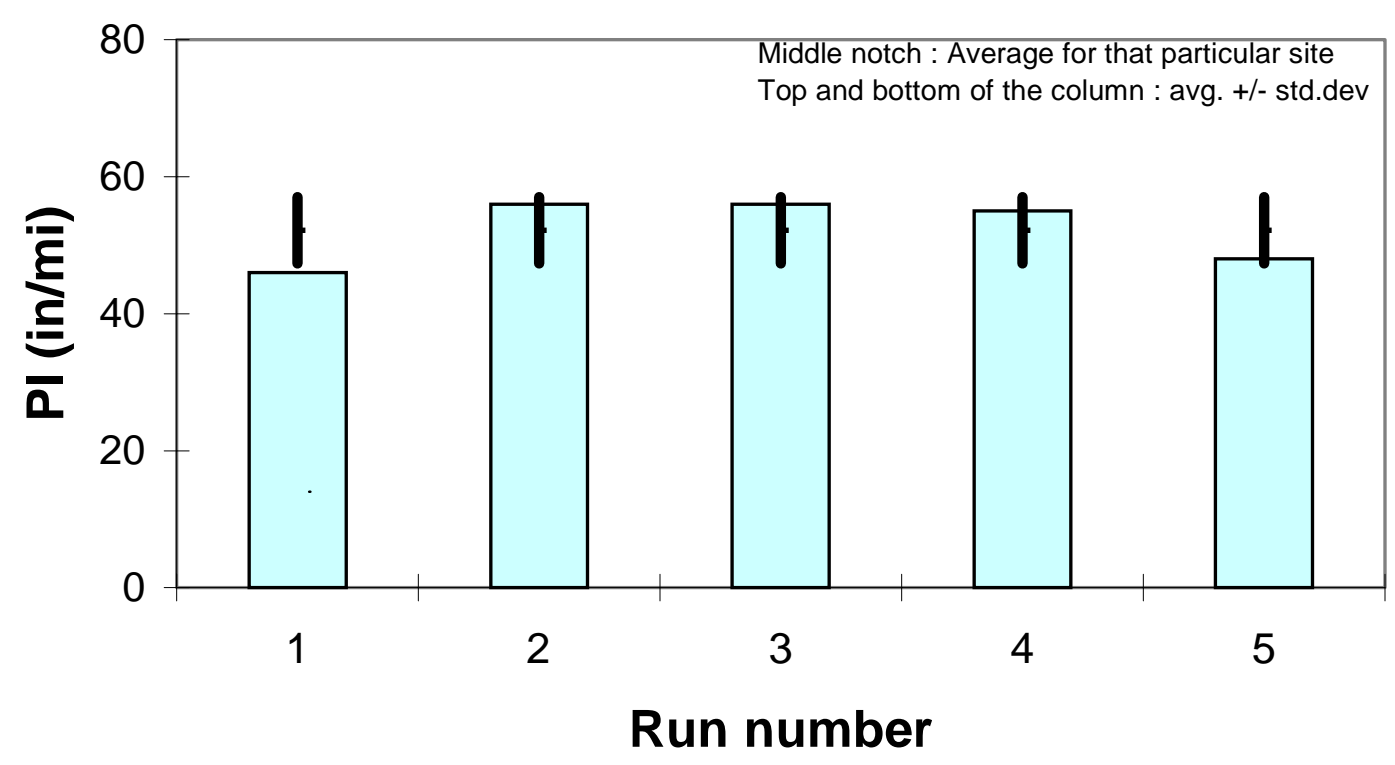

Figure 4-21 Replicate PI-0.2" measurements by Vendor A at Site 2 (I-65 with board)

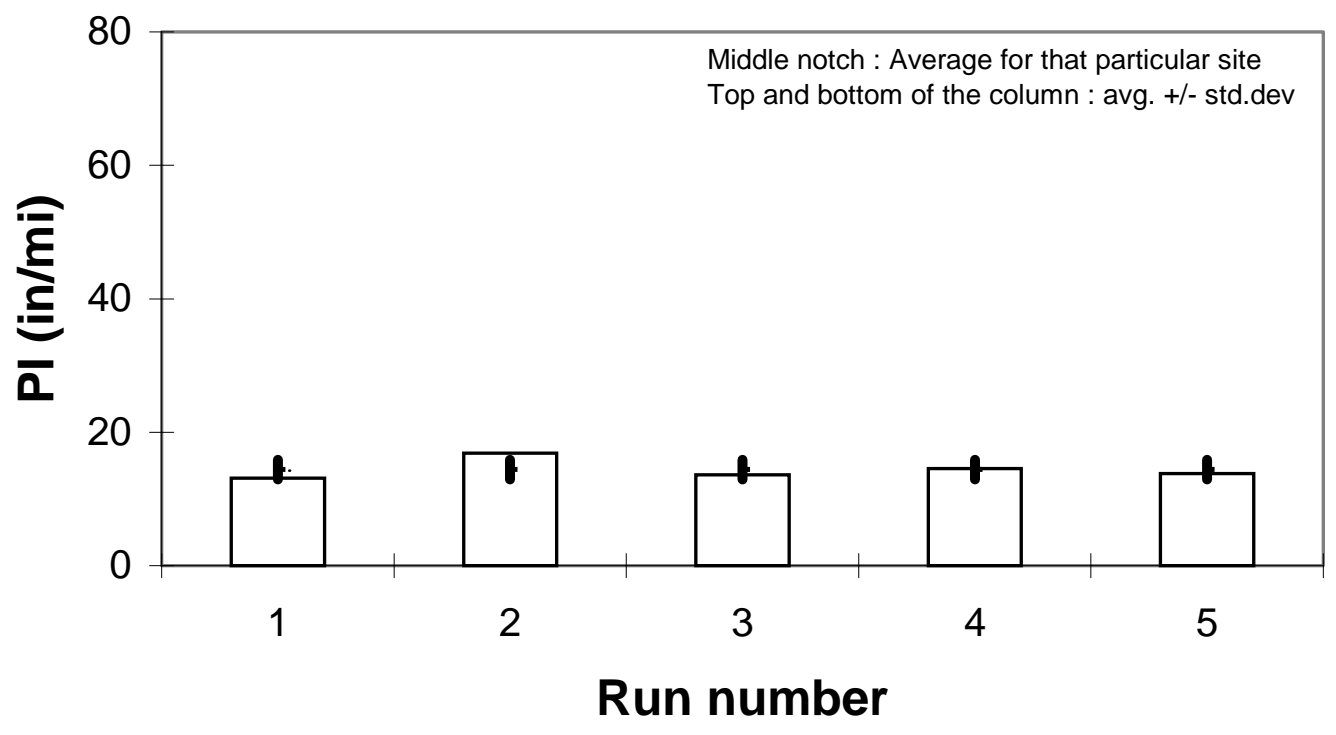

Figure 4-22 Replicate PI-0.2" measurements by Vendor B at Site 2 (I-65 with board) 


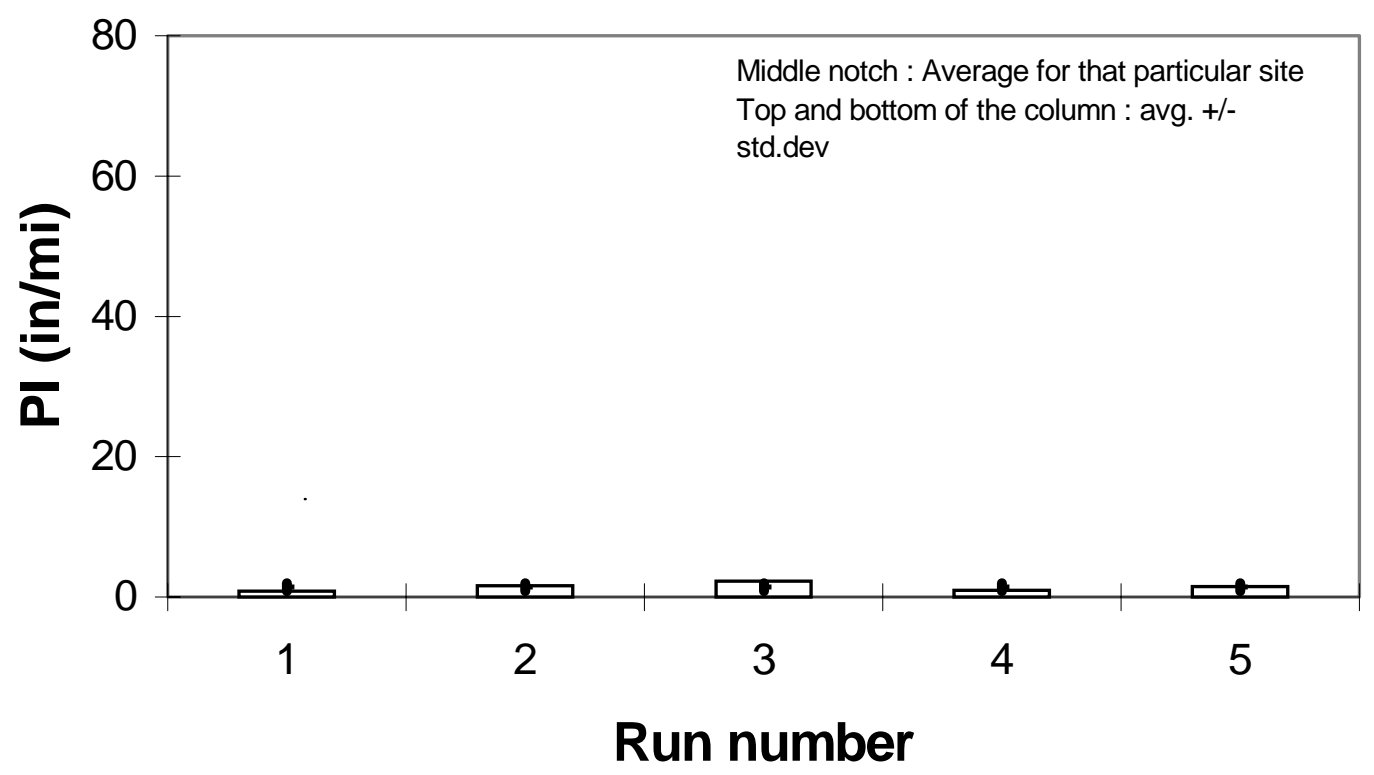

Figure 4-23 Replicate PI-0.2" measurements by Vendor C at Site 2 (I-65 with board)

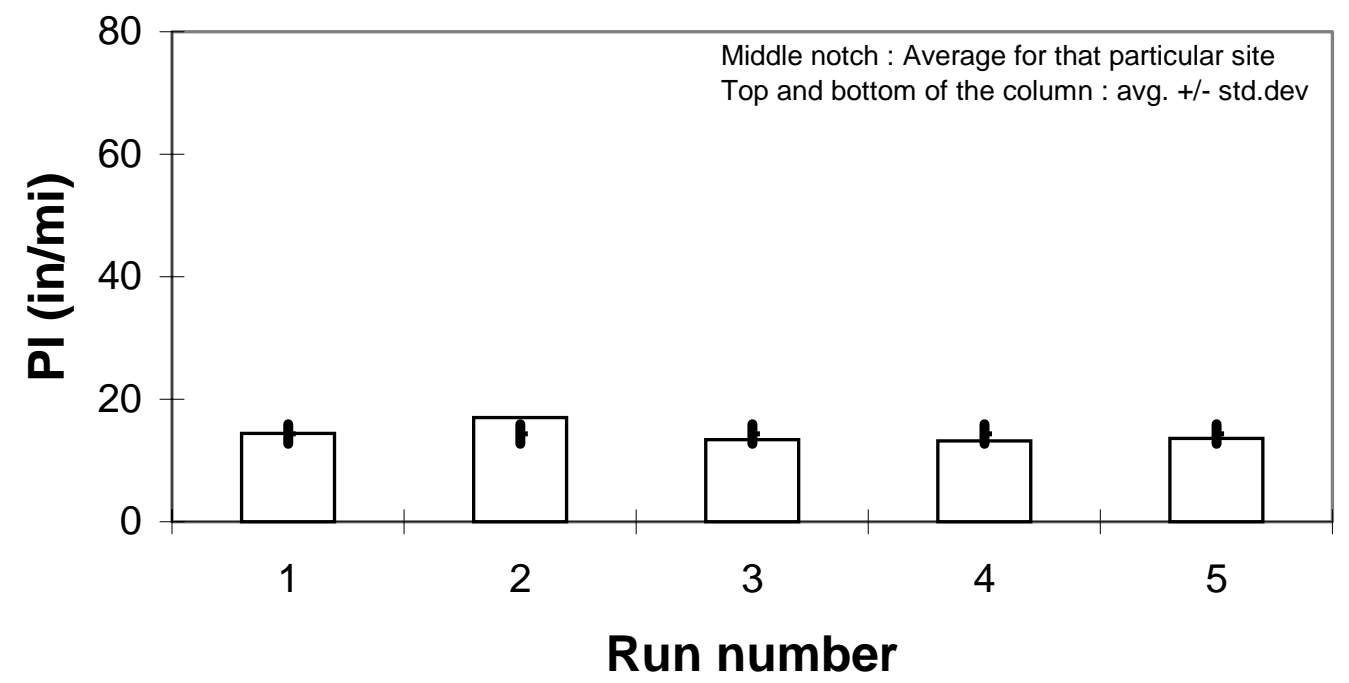

Figure 4-24 Replicate PI-0.2" measurements by Vendor D at Site 2 (I-65 with board) 
All the profilers identified the I-65 site (Site 2) without plywood strips as the smoothest among the three HMA sites considered in the experiment. Average IRI values reported by the individual vendors ranged from 31 to $59 \mathrm{in} / \mathrm{mi}$ with standard deviations ranging 1 to $5 \mathrm{in} / \mathrm{mi}$ at this site as shown in Figure 4-25 through Figure 4-28. All vendors reported average PI values with a 0.2 inch blanking band of $0 \mathrm{in} / \mathrm{mi}$. In fact, all vendors reported a value of $0 \mathrm{in} / \mathrm{mi}$ for each of five replicate measurements. Thus, they also reported standard deviations and coefficients of variation of zero with the 0.2 inch blanking band. Hence no figures have been provided for PI-0.2". Considering a blanking band of 0.0 inch however, they reported average PI values ranging from 5 to $14 \mathrm{in} / \mathrm{mi}$ with standard deviations ranging from 0 to 2 in/mi as shown in Figures 4-29 through 4-32. This again shows that measurable differences in smoothness identifiable with a 0.0 inch blanking band go undetected when a 0.2 inch blanking band is used.

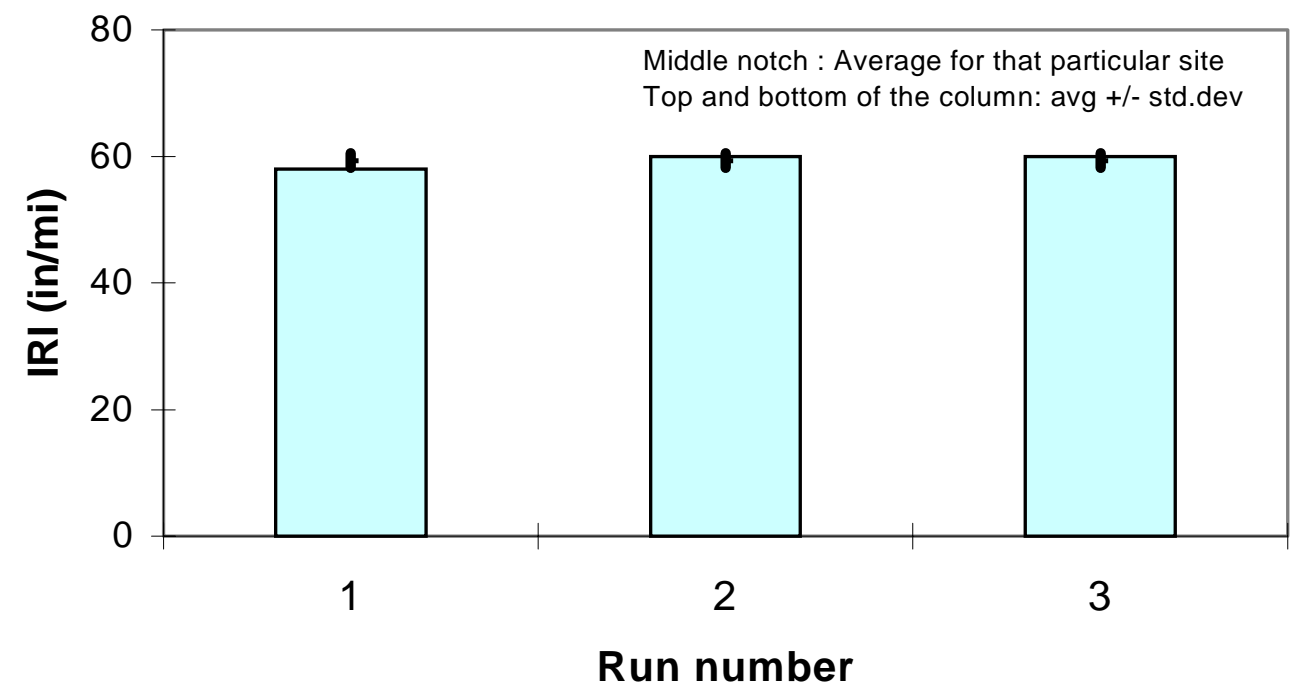

Figure 4-25 Replicate IRI measurements by Vendor $A$ at Site 2b (I-65 without board) 


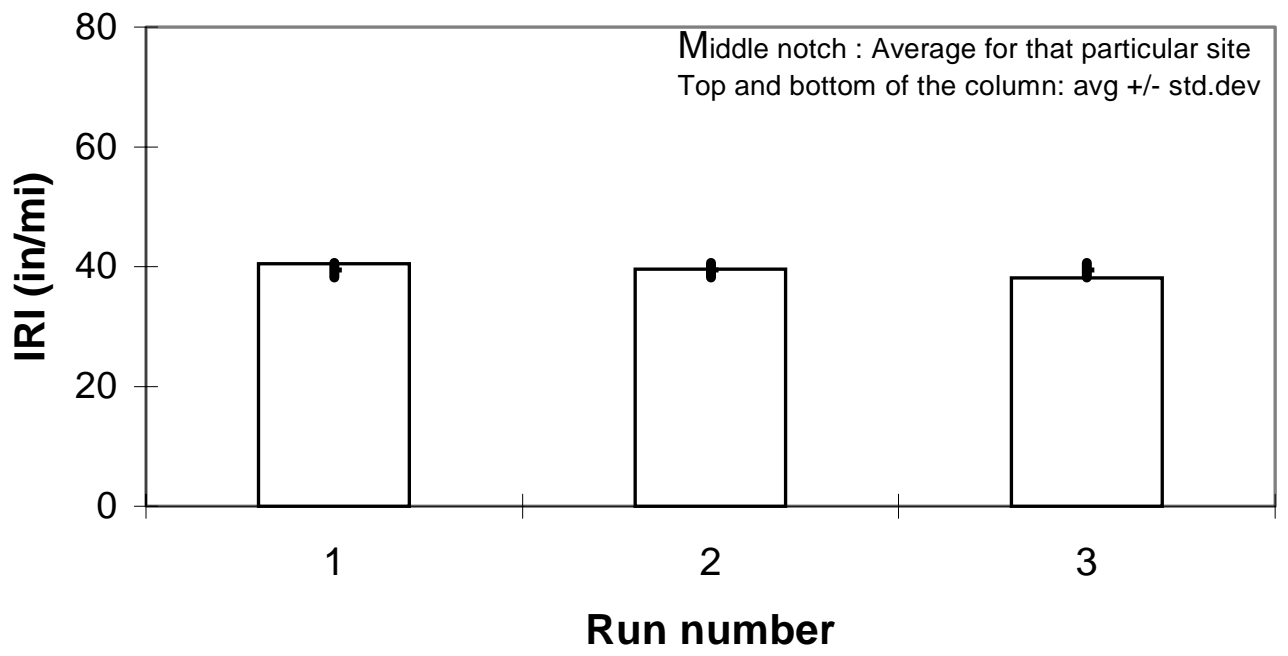

Figure 4-26 Replicate IRI measurements by Vendor B at Site 2b (I-65 without board)

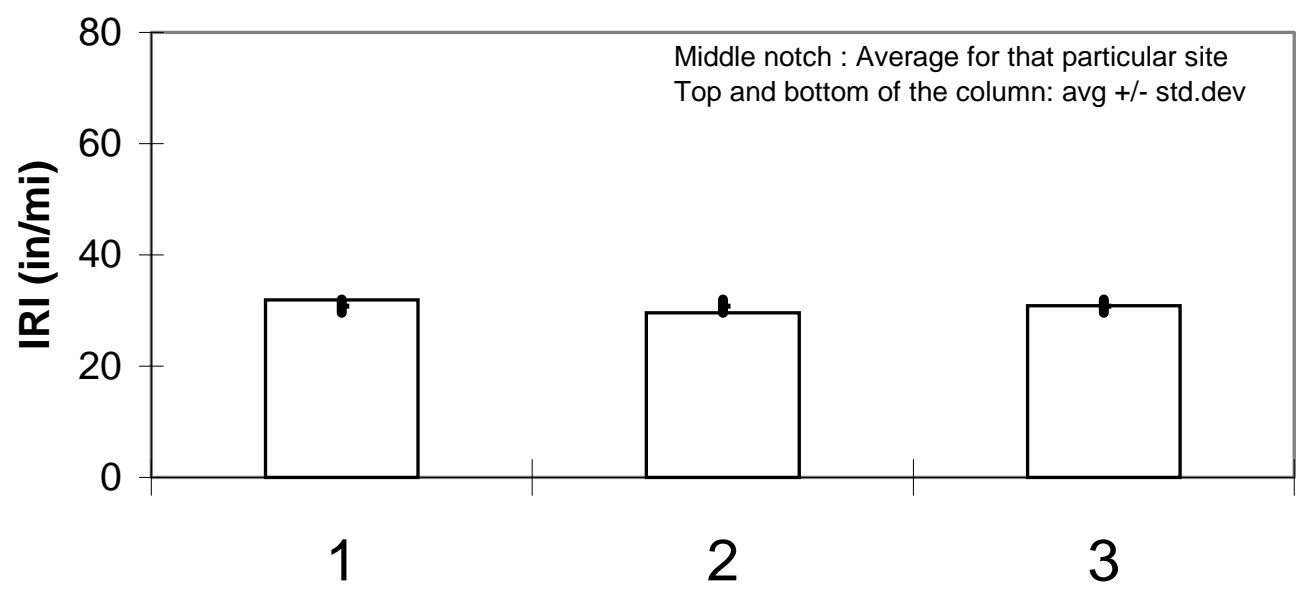

Run number

Figure 4-27 Replicate IRI measurements by Vendor C at Site 2b (I-65 without board) 


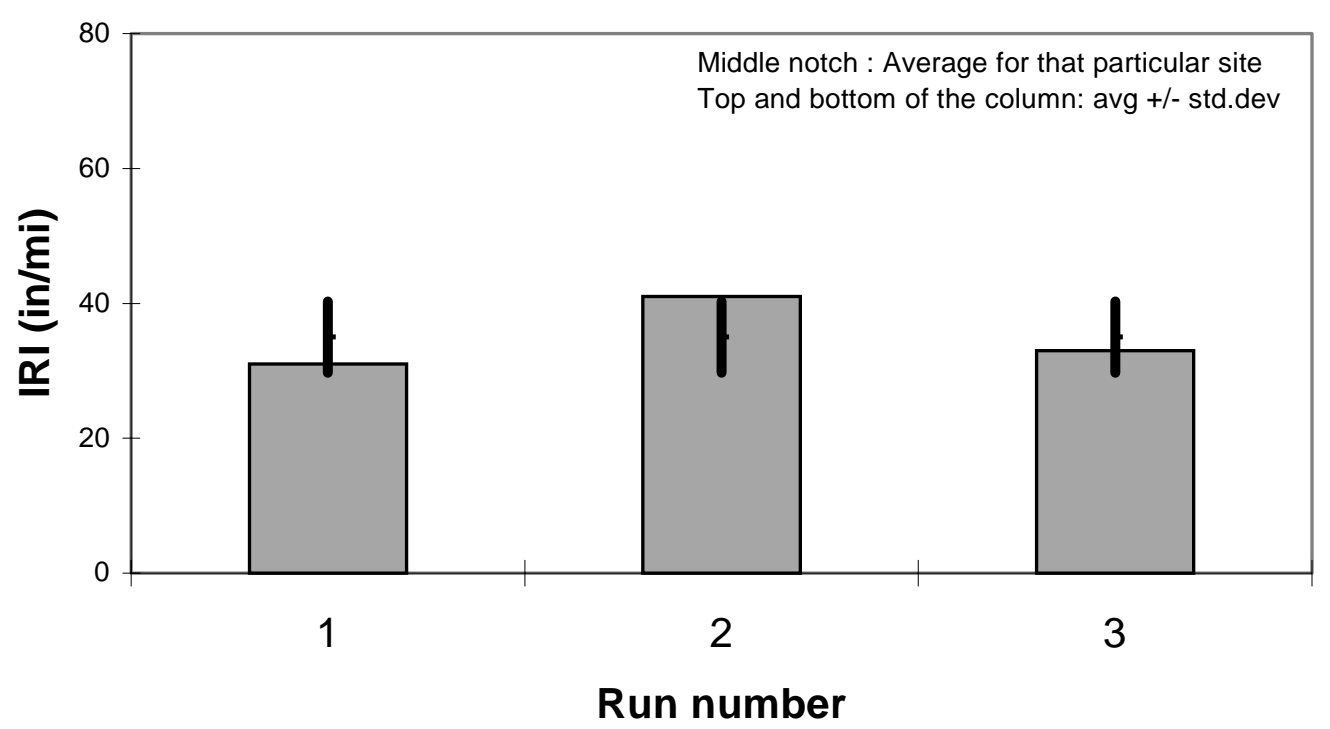

Figure 4-28 Replicate IRI measurements by Vendor D at Site 2b (I-65 without board)

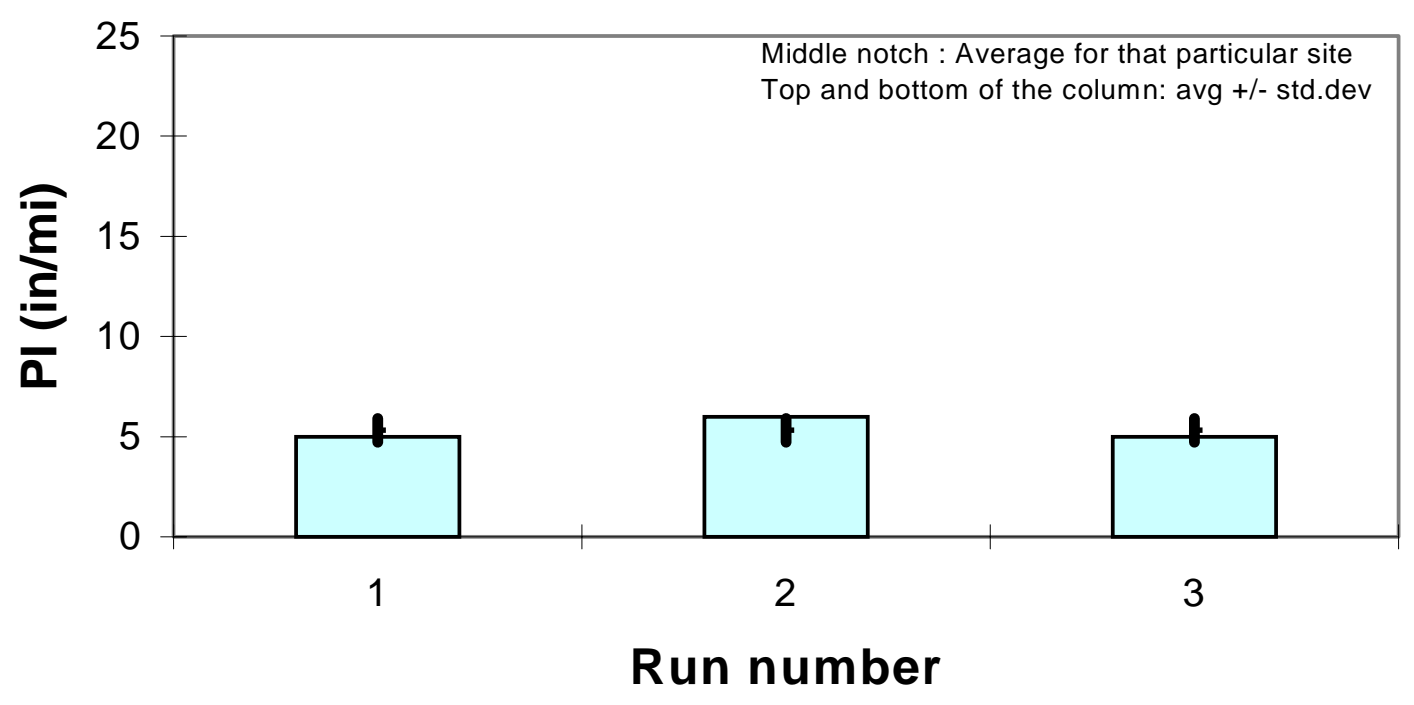

Figure 4-29 Replicate PI-0.0" measurements by Vendor A at Site 2b (I-65 without board) 


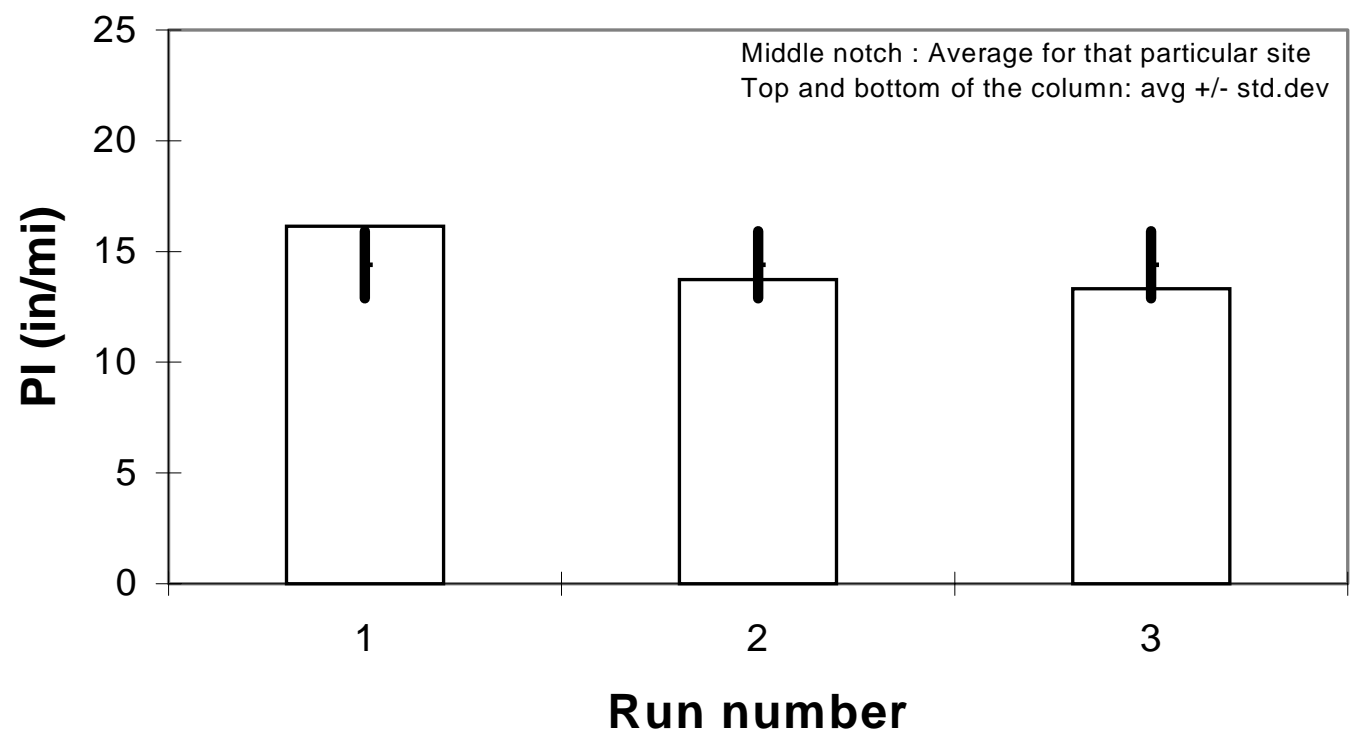

Figure 4-30 Replicate PI-0.0" measurements by Vendor B at Site 2b (I-65 without board)

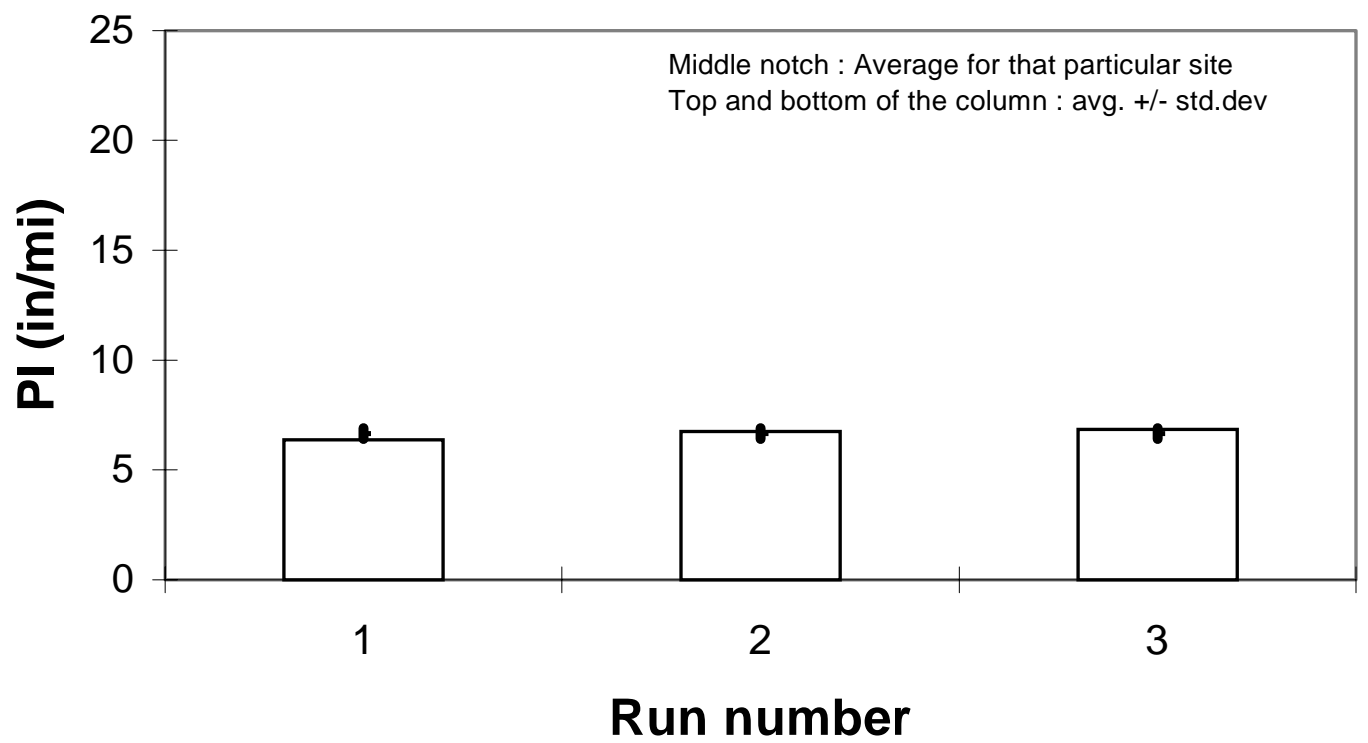

Figure 4-31 Replicate PI-0.0" measurements by Vendor C at Site 2b (I-65 without board) 


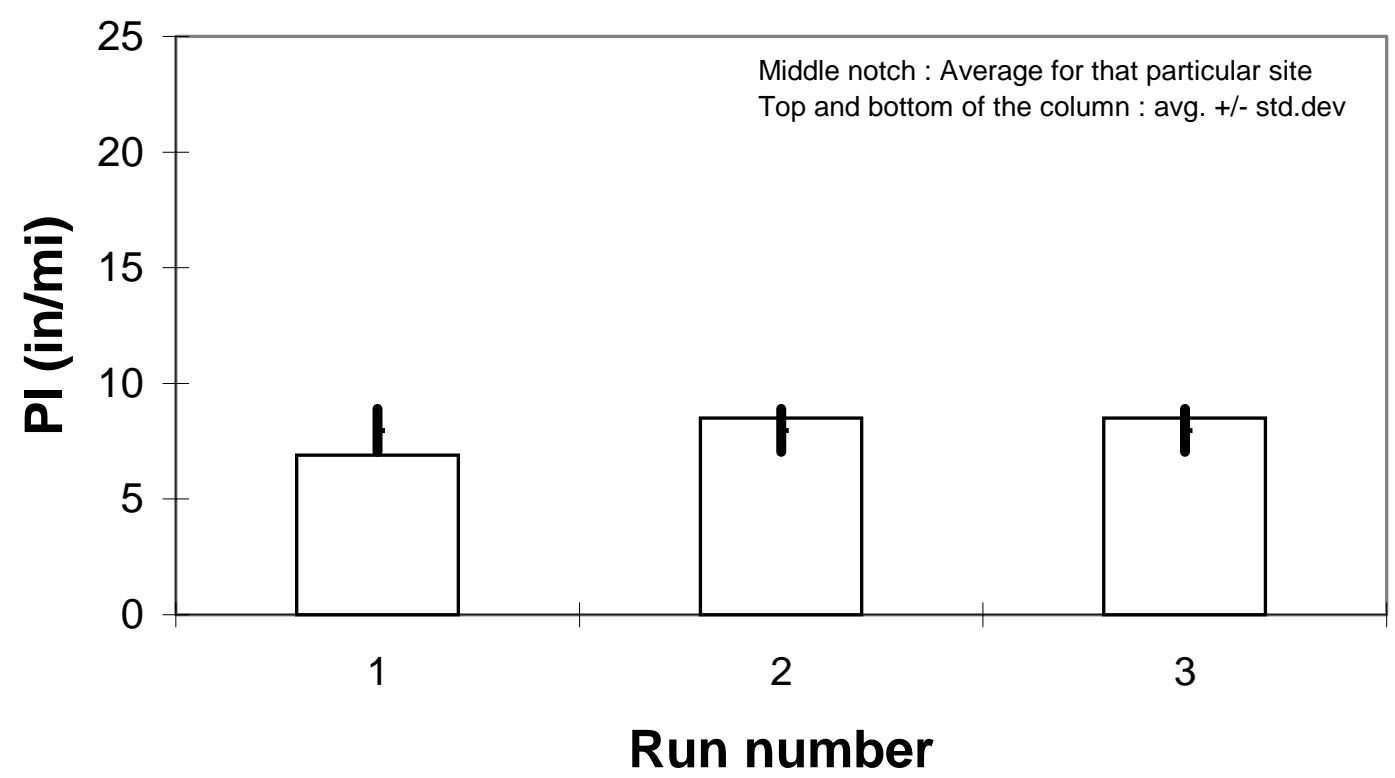

Figure 4-32 Replicate PI-0.0" measurements by Vendor D at Site 2b (I-65 without board)

The IRI, PI-0.0" and PI-0.2" plots by Vendor and site for the Prairie Street and Vinton Street sites are presented in Appendices A, B, C and D. All of the vendors reported the Prairie Street site (Site 3) to be the roughest HMA site. Similar to the I-65 site, measurements were made both with and without plywood strips at the Prairie Street site. However, due to the significant dynamics introduced by the $3 / 4$ " strips at the I- 65 site, 3/8" thick plywood strips were placed approximately ten feet from the beginning and end of the Prairie Street site. The dynamics introduced to the profilers due to these strips were nominal compared to those induced by the $3 / 4$ " strips. The thinner plywood strip did not significantly affect the smoothness indices or within vendor consistency. Reported average IRI values for the site ranged from 130 to $165 \mathrm{in} / \mathrm{mi}$ without the strips in place and only increased to 148 to $171 \mathrm{in} / \mathrm{mi}$ with the strips in place. Similarly, average 
reported PI values with a 0.0 inch blanking ranged from 36 to $63 \mathrm{in} / \mathrm{mi}$ without the strips in place and essentially were unchanged, averaging from 36 to $61 \mathrm{in} / \mathrm{mi}$ with the strips in place. Average PI's with a 0.2 inch blanking ranged from 32 to $48 \mathrm{in} / \mathrm{mi}$ without the strips in place and they only increased to 34 to $49 \mathrm{in} / \mathrm{mi}$ with the strips. Essentially no differences were observed in any of the indices and standard deviations and coefficients of variation were small and consistent. One anomaly in the test results is the fact that there was a significant increase in PI from the 0.2 inch to 0.0 inch blanking band, reported by all of the vendors averaging approximately $15 \mathrm{in} / \mathrm{mi}$, with the exception of Vendor A which only reported an average increase of $3 \mathrm{in} / \mathrm{mi}$.

This site incorporated significant uphill and downhill grades as well as a steep curve through the first two hundred feet. It also incorporated cross slope in excess of two percent. However these geometric features did not significantly impact within vendor consistency as indicated by low standard deviations and coefficients of variation. This suggests that cross slope, curves and grades do not have a significant effect on the within vendor performance for the lightweight profilers. However, it does not necessarily suggest that these geometric features do not impact the magnitude of the smoothness indices reported by the devices which could only be verified with a precision rod and level survey which was not performed at this site.

The Vinton Street site (Site 4) incorporated a continuous curve, constant downhill grade, and cross slope in excess of two percent. There was also a full width patch located approximately 150 feet from the start of the section. It should be noted that 
the patch was small and in excellent condition with no noticeable vertical deviations from the surrounding pavement at its perimeter. The observations at this site were similar to those made for the other HMA sites in that reported standard deviations and coefficients of variation were small. For all three smoothness indices, all vendors reported coefficients of variation of three percent or less with one exception. Vendor A reported a coefficient of variation of 14 percent for PI with a 0.2 inch blanking band. The average increase in PI moving from a blanking band of 0.2 inch to 0.0 inch was $20 \mathrm{in} / \mathrm{mi}$ for Vendors B, C, and D, but it was only 5 in/mi for Vendor A. The small reported standard deviations and the coefficients of variation again suggest that the geometrical features at the site did not significantly affect within vendor consistency.

Overall the test results suggest that all of the lightweight profilers provided good within vendor consistency at both PCC and HMA sites with the possible exception of Vendor A which showed inconsistency with the 3/4" thick plywood strips in place at the I65 site for IRI measurements and only a nominal differences in PI with 0.0 and 0.2 inch blanking bands at the Prairie and Vinton Street sites. A review of within vendor standard deviations was also conducted to assess within vendor consistency.

\subsubsection{Within Vendor Standard Deviations}

The simplest method of evaluating the repeatability (within vendor consistency) of the lightweight profilers is to evaluate the variance (standard deviation squared) associated with the replicate measurements made with the individual devices. A device that exhibits high variance would be less repeatable than another device that exhibited 
low variance. Standard deviation is a statistical term that is more familiar to most engineers. The same holds true for standard deviation, a device that exhibits high standard deviations would be less repeatable than another device that exhibited low standard deviations. Table 4-16 is a summary of observed standard deviations.

Table 4-16 Observed Standard Deviations

\begin{tabular}{|c|c|c|c|c|c|c|c|c|}
\hline \multirow{3}{*}{ Vendor } & \multicolumn{8}{|c|}{ IRI Standard Deviations } \\
\hline & \multicolumn{8}{|c|}{ Site } \\
\hline & 1 & $2(w b)$ & $2(\mathrm{nb})$ & $3(w b)$ & $3(\mathrm{nb})$ & 4 & 5 & 6 \\
\hline A & 5 & 24 & 1 & 5 & 6 & 4 & 0 & 5 \\
\hline $\mathrm{B}$ & 3 & 4 & 1 & 3 & 1 & 3 & 2 & 1 \\
\hline $\mathrm{C}$ & 1 & 0 & 1 & 9 & 4 & 1 & 1 & 1 \\
\hline \multirow[t]{2}{*}{$\mathrm{D}$} & 3 & 4 & 5 & 2 & 3 & 3 & 7 & 1 \\
\hline & \multicolumn{8}{|c|}{ PI-0.0" Standard Deviations } \\
\hline $\mathrm{A}$ & 3 & 2 & 1 & 2 & 1 & 1 & 1 & 2 \\
\hline $\mathrm{B}$ & 1 & 2 & 2 & 1 & 1 & 2 & 1 & 1 \\
\hline $\mathrm{C}$ & 1 & 1 & 0 & 3 & 4 & 1 & 1 & 1 \\
\hline \multirow[t]{2}{*}{$\mathrm{D}$} & 0 & 1 & 1 & 2 & 1 & 1 & 0 & 2 \\
\hline & \multicolumn{8}{|c|}{ PI-0.2" Standard Deviations } \\
\hline $\mathrm{A}$ & 1 & 5 & 0 & 2 & 3 & 3 & 0 & 1 \\
\hline $\mathrm{B}$ & 2 & 1 & 0 & 1 & 1 & 1 & 0 & 0 \\
\hline $\mathrm{C}$ & 0 & 1 & 0 & 4 & 1 & 1 & 0 & 0 \\
\hline $\mathrm{D}$ & 1 & 2 & 0 & 1 & 1 & 1 & 0 & 0 \\
\hline
\end{tabular}

(wb) indicates with plywood strips and (nb) indicates without plywood strips

The observed standard deviations are all fairly small and consistent both within and between the vendors with a few exceptions. Vendor A reported standard deviations of $24 \mathrm{in} / \mathrm{mi}$ for IRI and $5 \mathrm{in} / \mathrm{mi}$ for PI-0.2" at Site 2 with the thick plywood strips in place. Vendor C reported a standard deviation of 9in/mi for IRI at Site 3 with the thin plywood strips in place. Vendor D reported a standard deviation of $7 \mathrm{in} / \mathrm{mi}$ at Site 5 , which was a very smooth PCC pavement. Other than these few values which are relatively high, the 
repeatability (within vendor consistency) of the lightweight profiles appears to be favorable.

\subsubsection{Between Vendor Comparison}

A review of between vendor profiler performance is presented in this section. The five replicate IRI, PI-0.0", and PI-0.2" measurements reported by each lightweight profiler vendor at each site were averaged and compared. The average of the five IRI measurements obtained with the INDOT RIP Van at each site were also considered in the comparisons. Additionally, the California Profilograph and precision rod and level survey data obtained at Site 1 (Covington Street) are presented. The data was summarized in tables and figures, all of which are discussed. A statistical analysis (mean comparisons) of the between vendor test results is also presented. Similar to the previous within vendor comparison, graphical analysis is presented for two typical sites (Sites 1 and 2) and remainder of the figures are presented in Appendix D.

\subsubsection{PCC Sites}

Table 4-17 is a summary of the measured smoothness indices at the three PCC sites. The IRI data for Site 1 is presented in Figure 4-33. Vendor A reported a significantly lower average IRI compared to the other vendors at Site 1 . The average IRI obtained with the INDOT RIP Van was similar to the values reported by the other lightweight profilers (Vendors B, C and D) at this site. 
Table 4-17 Summary of Smoothness Indices for PCC Sites.

\begin{tabular}{|c|c|c|c|c|c|c|c|c|c|c|}
\hline \multirow{3}{*}{ Site } & \multirow{3}{*}{ Vendor } & \multicolumn{9}{|c|}{ Smoothness Index } \\
\hline & & \multicolumn{3}{|c|}{ IRI (in/mi) } & \multicolumn{3}{|c|}{ PI-0.2" (in/mi) } & \multicolumn{3}{|c|}{ PI-0.0" (in/mi) } \\
\hline & & Mean & $\begin{array}{l}\text { Std } \\
\text { Dev }\end{array}$ & $\mathrm{COV}$ & Mean & $\begin{array}{l}\text { Std } \\
\text { Dev }\end{array}$ & $\mathrm{COV}$ & Mean & $\begin{array}{l}\text { Std } \\
\text { Dev }\end{array}$ & $\mathrm{COV}$ \\
\hline \multirow{7}{*}{$\begin{array}{c}1 \\
\text { Covington } \\
\text { Street }\end{array}$} & $\bar{A}$ & 107 & 5 & 4 & 8 & 1 & 18 & 27 & 3 & 12 \\
\hline & B & 152 & 3 & 2 & 44 & 2 & 4 & 76 & 1 & 1 \\
\hline & $\mathrm{C}$ & 154 & 1 & 1 & 49 & 0 & 1 & 83 & 1 & 1 \\
\hline & $\mathrm{D}$ & 155 & 3 & 2 & 46 & 1 & 2 & 79 & 0 & 1 \\
\hline & INDOT RIP Van & 167 & 2 & 1 & $\mathrm{n} / \mathrm{a}$ & $\mathrm{n} / \mathrm{a}$ & $\mathrm{n} / \mathrm{a}$ & $\mathrm{n} / \mathrm{a}$ & $\mathrm{n} / \mathrm{a}$ & $\mathrm{n} / \mathrm{a}$ \\
\hline & $\begin{array}{l}\text { California } \\
\text { Profilograph }\end{array}$ & 188 & 2 & 1 & $\mathrm{n} / \mathrm{a}$ & $\mathrm{n} / \mathrm{a}$ & $\mathrm{n} / \mathrm{a}$ & $\mathrm{n} / \mathrm{a}$ & $\mathrm{n} / \mathrm{a}$ & $\mathrm{n} / \mathrm{a}$ \\
\hline & Rod and Level & 90 & $\mathrm{n} / \mathrm{a}$ & $\mathrm{n} / \mathrm{a}$ & $\mathrm{n} / \mathrm{a}$ & $\mathrm{n} / \mathrm{a}$ & $\mathrm{n} / \mathrm{a}$ & $\mathrm{n} / \mathrm{a}$ & $\mathrm{n} / \mathrm{a}$ & $\mathrm{n} / \mathrm{a}$ \\
\hline \multirow{5}{*}{$\begin{array}{c}5 \\
\text { US231 A }\end{array}$} & A & 65 & 0 & 0 & 0 & 0 & 224 & 13 & 1 & 12 \\
\hline & B & 44 & 2 & 3 & 0 & 0 & 78 & 19 & 1 & 7 \\
\hline & $\mathrm{C}$ & 43 & 1 & 1 & 0 & 0 & 135 & 16 & 1 & 3 \\
\hline & D & 52 & 7 & 14 & 0 & 0 & 64 & 17 & 0 & 2 \\
\hline & INDOT RIP Van & 67 & 2 & 3 & $\mathrm{n} / \mathrm{a}$ & $\mathrm{n} / \mathrm{a}$ & $\mathrm{n} / \mathrm{a}$ & $\mathrm{n} / \mathrm{a}$ & $\mathrm{n} / \mathrm{a}$ & $\mathrm{n} / \mathrm{a}$ \\
\hline \multirow{5}{*}{$\begin{array}{c}6 \\
\text { US231 B }\end{array}$} & A & 77 & 5 & 7 & 1 & 1 & 70 & 17 & 2 & 10 \\
\hline & B & 55 & 1 & 3 & 3 & 0 & 15 & 23 & 1 & 3 \\
\hline & $\mathrm{C}$ & 57 & 1 & 2 & 2 & 0 & 10 & 23 & 1 & 5 \\
\hline & $\mathrm{D}$ & 60 & 1 & 1 & 3 & 0 & 15 & 22 & 2 & 9 \\
\hline & INDOT RIP Van & 73 & 4 & 5 & $\mathrm{n} / \mathrm{a}$ & $\mathrm{n} / \mathrm{a}$ & $\mathrm{n} / \mathrm{a}$ & $\mathrm{n} / \mathrm{a}$ & $\mathrm{n} / \mathrm{a}$ & $\mathrm{n} / \mathrm{a}$ \\
\hline
\end{tabular}

The four California Profilograph traces obtained at Site 1 were digitized and used to calculate IRI values the using the RoadRuf software available online at the University of Michigan Transportation Research Institute (UMTRI) website. The average calculated IRI was slightly larger than the values reported by the lightweight profilers. The ROADRUF software was also used to calculate IRI from the rod and level survey data. The calculated IRI was significantly lower than the other reported values. This is very reasonable though because the sampling interval used for the rod and level survey was twelve inches which is substantially larger than the interval used by the lightweight profilers and most of the hand-tooled transverse joints in the pavement were bridged due to the large sampling interval. Reported standard deviations and coefficients of variation 
were quite low at this site also. This was the only site where both Rod and Level and California Profilograph testing were conducted.

Figures 4-34 and 4-35 represent the PI-0.0" and PI-0.2” data, respectively for Site 1. Vendor A also reported considerably lower values of PI-0.0" and PI-0.2" than the other vendors at this site. The standard deviations and coefficients of variation reported for PI0.0 " and PI-0.2" at Site 1 were also low with the exception of those reported by Vendor A.

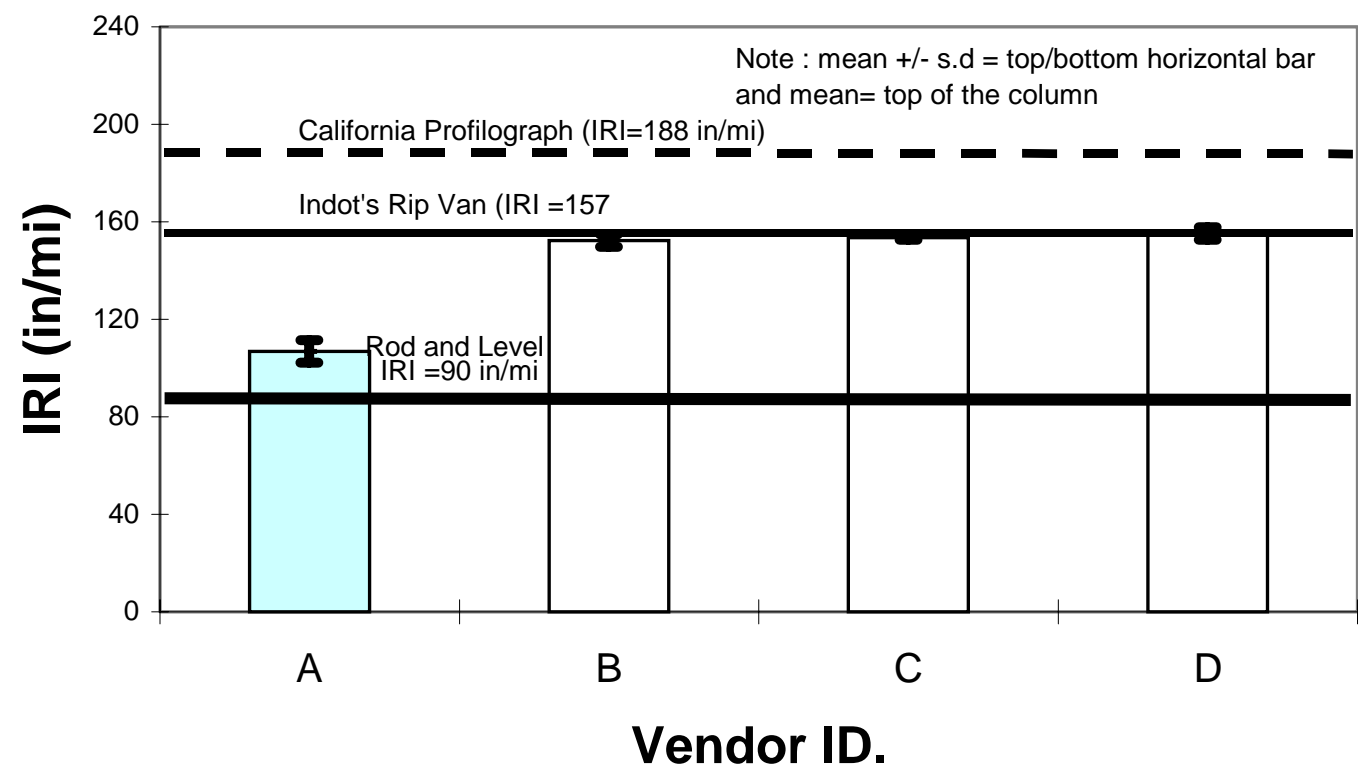

Figure 4-33 Between Vendor IRI Comparison for Covington Street (Site 1) 


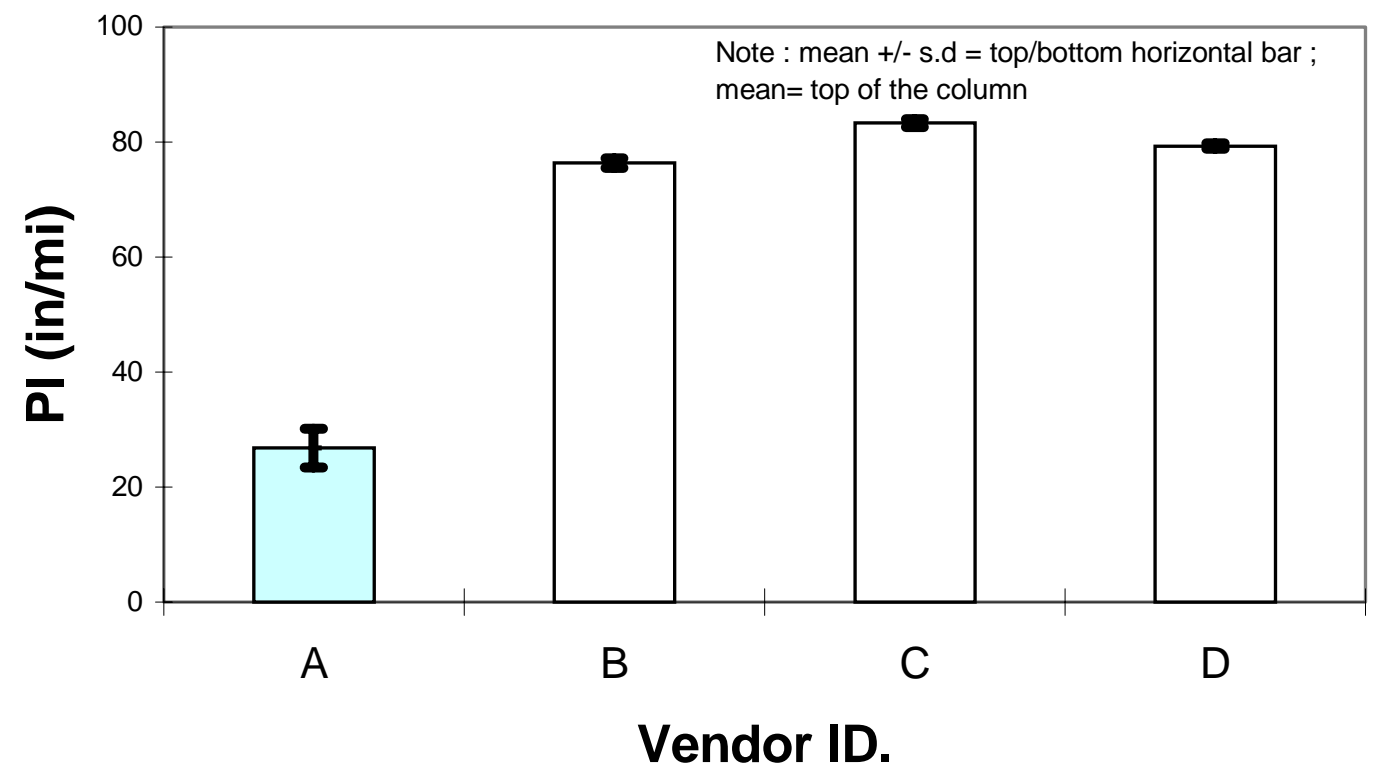

Figure 4-34 PI-0.0” Between Vendor Comparison for Covington Street (Site 1)

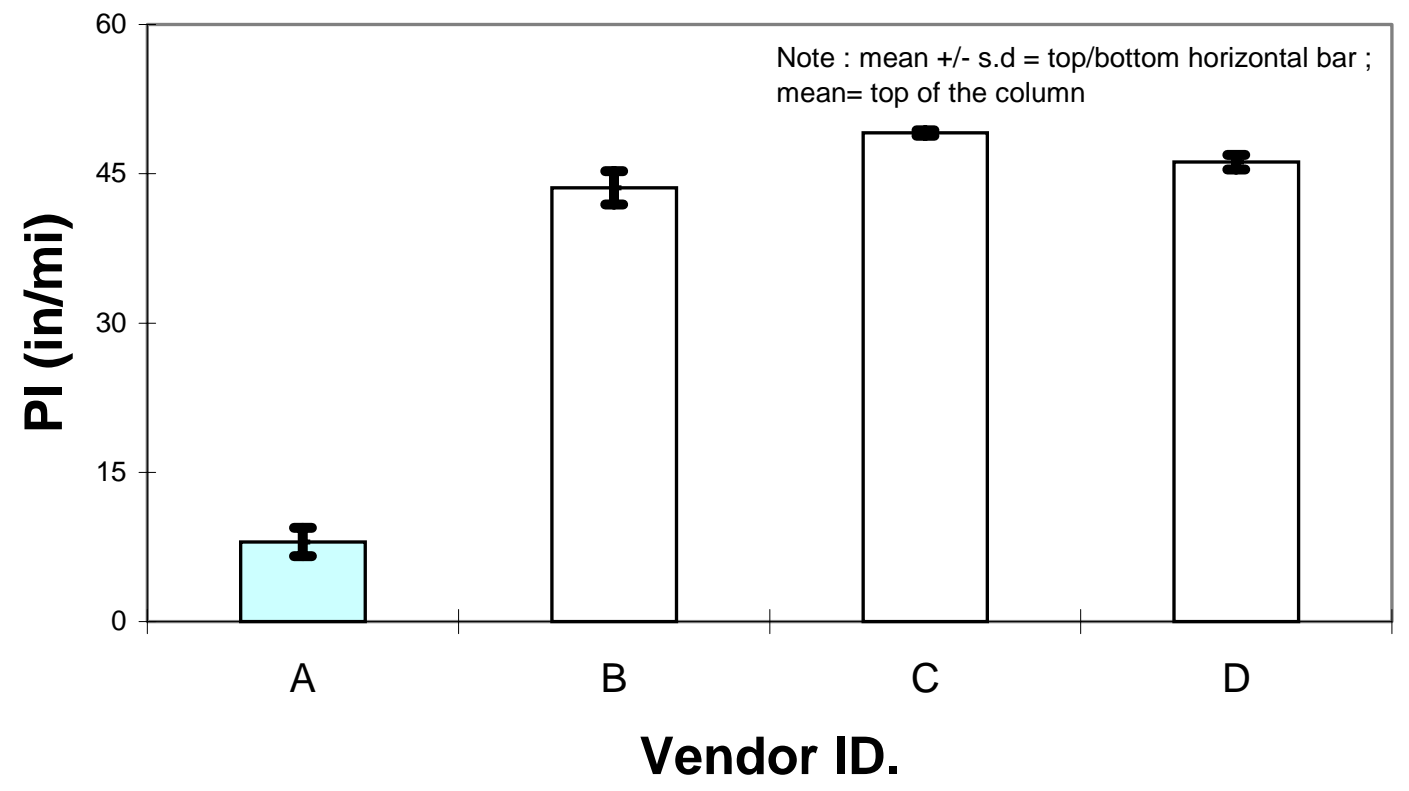

Figure 4-35 PI-0.2" Between Vendor Comparison for Covington St.(Site 1) 
The IRI, PI-0.0", and PI-0.2" plots for PCC Sites 5 and 6 are presented in Appendix E. Similar IRI values were reported by lightweight profiler Vendors B, C, and D for the US231A site (Site 5). Vendor A and the INDOT RIP Van reported similar values that were slightly larger. Reported standard deviations and coefficients of variation for IRI were very small for all of the Vendors with the exception of Vendor D.

Vendor A reported a slightly lower average value for PI-0.0" than the other vendors for Site 5 and all vendors reported small standard deviations. All of the vendors reported average values and standard deviations for PI-0.2" of 0in/mi for Site 5. Reported values of coefficient of variation are meaningless because they are inflated due to the very small measured values actually reported. This data highlights the inadequacy of using PI with a 0.2 inch blanking band.

Similar observations were made for the US231B site (Site 6). Vendor A reported a slightly lower average ( 1 versus 2 or $3 \mathrm{in} / \mathrm{mi}$ ) value for PI- 0.0 " than the other vendors for Site 6 and all vendors reported small standard deviations. All of the vendors reported similar average values and standard deviations for PI-0.2" for Site 6. Reported values of coefficient of variation are difficult to interpret because they may be inflated due to the very small measured values actually reported. 


\subsubsection{HMA Sites}

Table 4-18 is a summary of the smoothness indices measured at the three HMA sites.

Table 4-18 Summary of Smoothness Indices for HMA Sites.

\begin{tabular}{|c|c|c|c|c|c|c|c|c|c|c|}
\hline \multirow{3}{*}{ Site } & \multirow{3}{*}{ Vendor } & \multicolumn{9}{|c|}{ Smoothness Index } \\
\hline & & \multicolumn{3}{|c|}{ IRI (in/mi) } & \multicolumn{3}{|c|}{ PI-0.2" (in/mi) } & \multicolumn{3}{|c|}{ PI-0.0" (in/mi) } \\
\hline & & Mean & $\begin{array}{l}\text { Std } \\
\text { Dev }\end{array}$ & $\mathrm{COV}$ & Mean & $\begin{array}{l}\text { Std } \\
\text { Dev }\end{array}$ & $\mathrm{COV}$ & Mean & $\begin{array}{c}\text { Std } \\
\text { Dev }\end{array}$ & $\mathrm{COV}$ \\
\hline \multirow{5}{*}{$\begin{array}{c}2 \\
\text { I-65 With } \\
\text { Boards }\end{array}$} & A & 218 & 24 & 11 & 52 & 5 & 9 & 68 & 2 & 3 \\
\hline & B & 91 & 4 & 5 & 14 & 1 & 10 & 34 & 2 & 7 \\
\hline & $\mathrm{C}$ & 32 & 0 & 1 & 1 & 1 & 39 & 11 & 2 & 8 \\
\hline & $\mathrm{D}$ & 84 & 4 & 5 & 14 & 2 & 11 & 27 & 1 & 2 \\
\hline & INDOT RIP Van & 66 & 20 & 30 & $\mathrm{n} / \mathrm{a}$ & $\mathrm{n} / \mathrm{a}$ & $\mathrm{n} / \mathrm{a}$ & $\mathrm{n} / \mathrm{a}$ & $\mathrm{n} / \mathrm{a}$ & $\mathrm{n} / \mathrm{a}$ \\
\hline \multirow{5}{*}{$\begin{array}{c}2 \\
\text { I-65 } \\
\text { Without } \\
\text { Boards }\end{array}$} & A & 59 & 1 & 2 & 0 & 0 & 0 & 5 & 1 & 11 \\
\hline & B & 39 & 1 & 3 & 0 & 0 & 0 & 14 & 2 & 11 \\
\hline & $\mathrm{C}$ & 31 & 1 & 4 & 0 & 0 & 0 & 7 & 0 & 4 \\
\hline & $\mathrm{D}$ & 35 & 5 & 15 & 0 & 0 & 0 & 8 & 1 & 12 \\
\hline & INDOT RIP Van & 46 & 15 & 33 & $\mathrm{n} / \mathrm{a}$ & $\mathrm{n} / \mathrm{a}$ & $\mathrm{n} / \mathrm{a}$ & $\mathrm{n} / \mathrm{a}$ & $\mathrm{n} / \mathrm{a}$ & $\mathrm{n} / \mathrm{a}$ \\
\hline \multirow{5}{*}{$\begin{array}{c} \\
\text { Prairie } \\
\text { Street } \\
\text { With } \\
\text { Boards } \\
\end{array}$} & A & 148 & 5 & 4 & 34 & 2 & 7 & 36 & 2 & 6 \\
\hline & B & 150 & 3 & 2 & 41 & 1 & 3 & 61 & 1 & 1 \\
\hline & $\mathrm{C}$ & 171 & 9 & 5 & 49 & 4 & 9 & 60 & 3 & 4 \\
\hline & $\mathrm{D}$ & 162 & 2 & 1 & 38 & 1 & 2 & 57 & 2 & 3 \\
\hline & INDOT RIP Van & 205 & 6 & 3 & $\mathrm{n} / \mathrm{a}$ & $\mathrm{n} / \mathrm{a}$ & $\mathrm{n} / \mathrm{a}$ & $\mathrm{n} / \mathrm{a}$ & $\mathrm{n} / \mathrm{a}$ & $\mathrm{n} / \mathrm{a}$ \\
\hline \multirow{5}{*}{$\begin{array}{c}3 \\
\text { Prairie } \\
\text { Street } \\
\text { Without } \\
\text { Boards }\end{array}$} & A & 130 & 6 & 5 & 32 & 3 & 8 & 36 & 1 & 3 \\
\hline & B & 141 & 1 & 1 & 39 & 1 & 2 & 56 & 1 & 2 \\
\hline & $\mathrm{C}$ & 165 & 4 & 2 & 48 & 1 & 3 & 63 & 4 & 7 \\
\hline & $\mathrm{D}$ & 154 & 3 & 2 & 37 & 1 & 2 & 57 & 1 & 3 \\
\hline & INDOT RIP Van & 201 & 6 & 2 & $\mathrm{n} / \mathrm{a}$ & $\mathrm{n} / \mathrm{a}$ & $\mathrm{n} / \mathrm{a}$ & $\mathrm{n} / \mathrm{a}$ & $\mathrm{n} / \mathrm{a}$ & $\mathrm{n} / \mathrm{a}$ \\
\hline \multirow{5}{*}{$\begin{array}{c}4 \\
\text { Vinton } \\
\text { Street }\end{array}$} & A & 92 & 4 & 5 & 18 & 3 & 15 & 23 & 1 & 2 \\
\hline & $\mathrm{B}$ & 101 & 3 & 3 & 16 & 1 & 7 & 37 & 2 & 5 \\
\hline & $\mathrm{C}$ & 102 & 1 & 1 & 18 & 1 & 7 & 39 & 1 & 3 \\
\hline & D & 107 & 3 & 3 & 16 & 1 & 4 & 39 & 1 & 2 \\
\hline & INDOT RIP Van & 114 & 7 & 6 & $\mathrm{n} / \mathrm{a}$ & $\mathrm{n} / \mathrm{a}$ & $\mathrm{n} / \mathrm{a}$ & $\mathrm{n} / \mathrm{a}$ & $\mathrm{n} / \mathrm{a}$ & $\mathrm{n} / \mathrm{a}$ \\
\hline
\end{tabular}

Figure 4-36 represents the IRI data for the I-65 (Site 2b) with the plywood strips removed while the IRI data for Site 2 (I-65) with the thick plywood strips in place is presented in Figure 4-37. 
With the plywood strips in place, Vendor A reported a significantly higher average IRI compared to Vendors B and D while Vendor C reported a significantly lower average IRI compared to them. In fact the difference between the IRI values reported by Vendors $\mathrm{A}$ and $\mathrm{C}$ was approximately $185 \mathrm{in} / \mathrm{mi}$, which was unimaginably large. This suggests that the thick plywood strips imparted significant dynamics to some of the profilers adversely affecting their performance. The average IRI obtained with the INDOT Rip Van falls between these extremes. Inconsistency (high standard deviations) were reported by Vendor A and the INDOT Rip Van, especially the INDOT Rip Van as noted by the high coefficient of variation (30 percent).

The lightweight profilers showed better consistency at the I-65 site when the thick plywood strips were removed, as did the INDOT Rip Van. However, the average IRI reported by all of the lightweight profilers with the exception of Vendor A were similar. The RIP Van reported an average IRI that was greater than them and Vendor A reported an even greater value. 


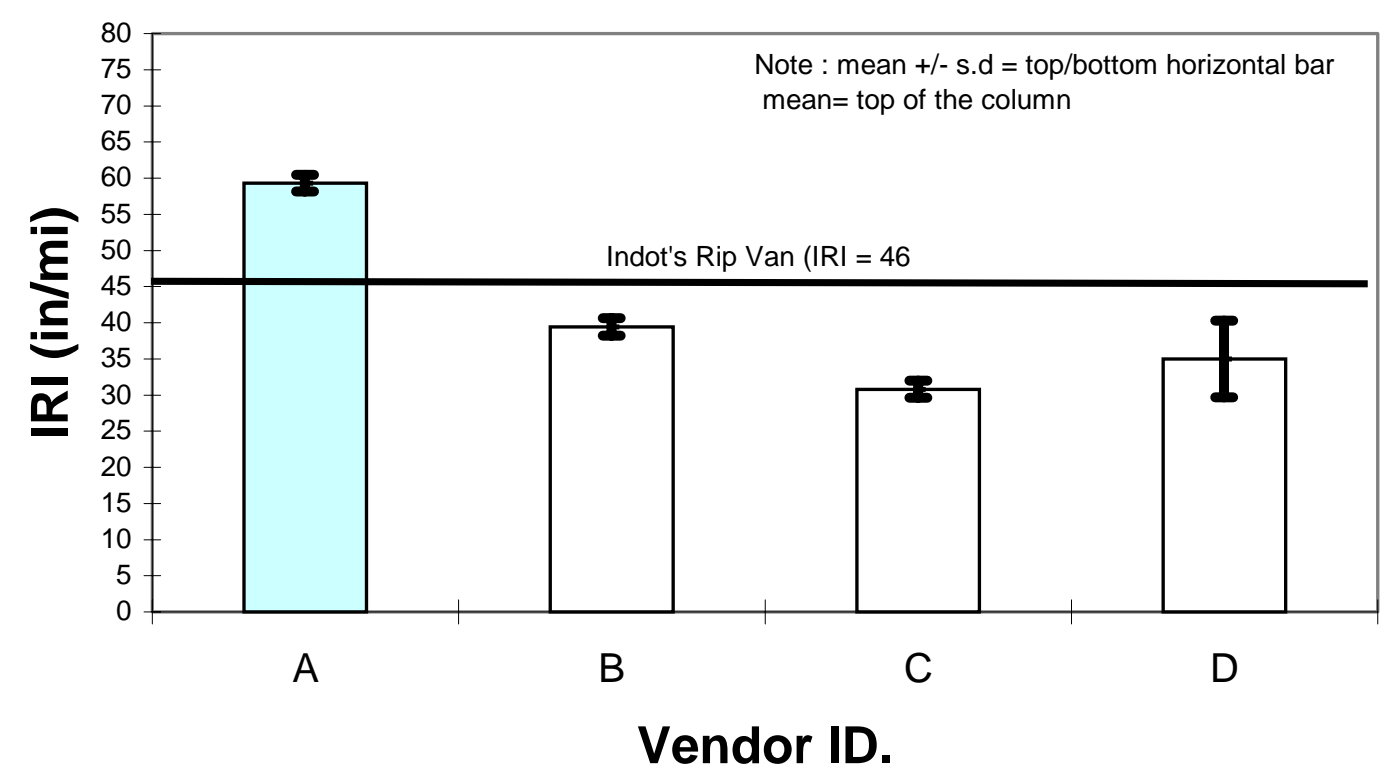

Figure 4-36 IRI Between Vendor Comparison for Site 2b (I-65 without board)

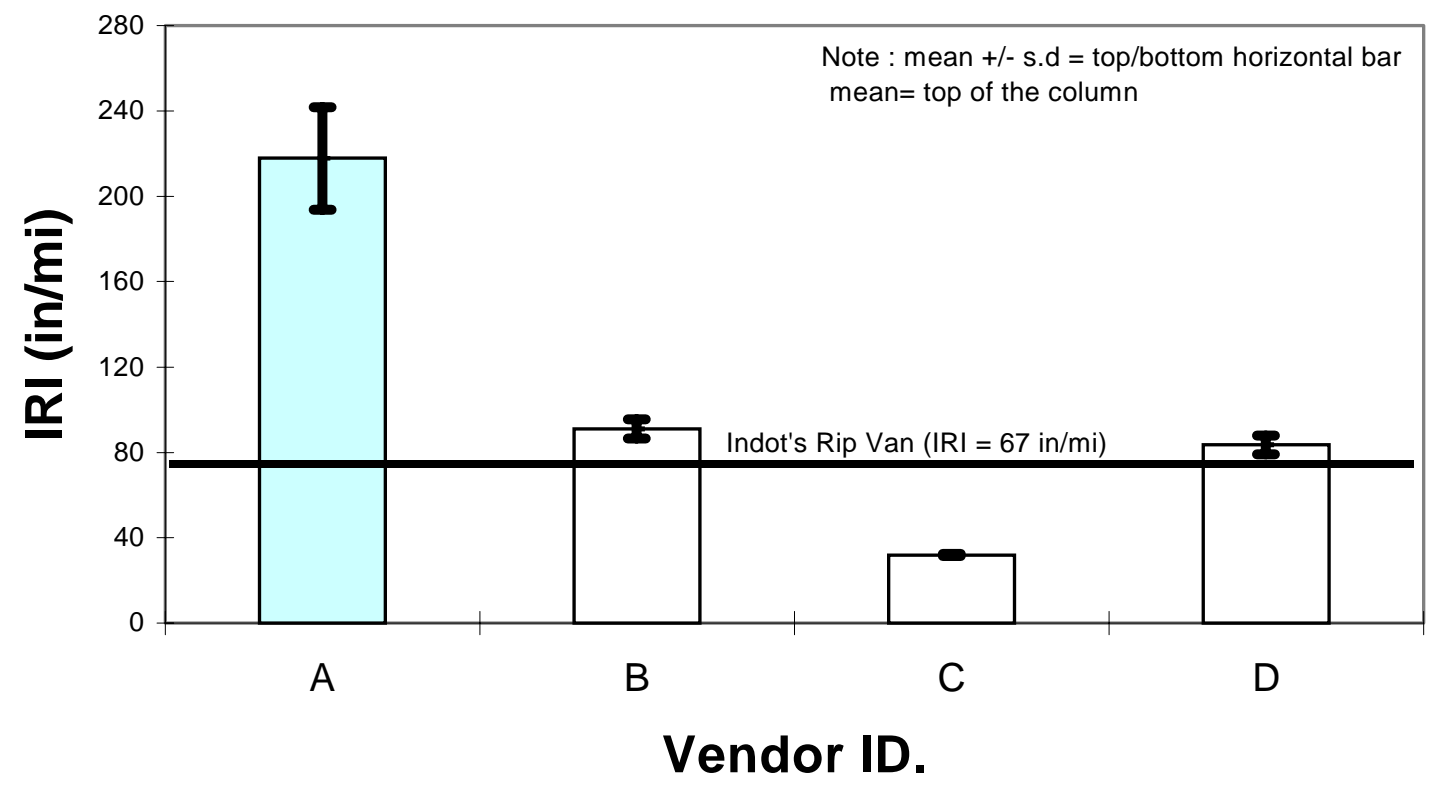

Figure 4-37 IRI Between Vendor Comparison for Site 2 (I-65 with board) 
The PI-0.0" data for the I-65 site with the thick plywood strips removed is presented in Figure 4-38 while that with the plywood strips in place is presented in Figure 4-39.

With the plywood strips in place, Vendors B and D reported similar PI-0.0" values, but Vendors $\mathrm{A}$ and $\mathrm{C}$ reported very high and low values relative to them. All of the devices exhibited similar consistency (low standard deviations). Very similar observations were made relative to reported PI-0.2" values. However, larger coefficients of variation were observed for all of the vendors. Again, profiler dynamics may have played a role in the inconsistency.

When the thick plywood strips were removed at the I-65 site a different trend was observed. Vendor A reported the lowest PI-0.0" value that was similar to Vendors C and D, but Vendor B reported a value which was double the other vendors. The data lacked consistency also in that fairly high coefficients of variation were reported by all vendors. All of the vendors reported average values and standard deviations of 0in/mi for PI-0.2" with the thick plywood strips removed. Hence the plot for between vendor comparison was not provided. Thus the data is very consistent, but the differences in actual pavement roughness are clearly masked by the large blanking band. 


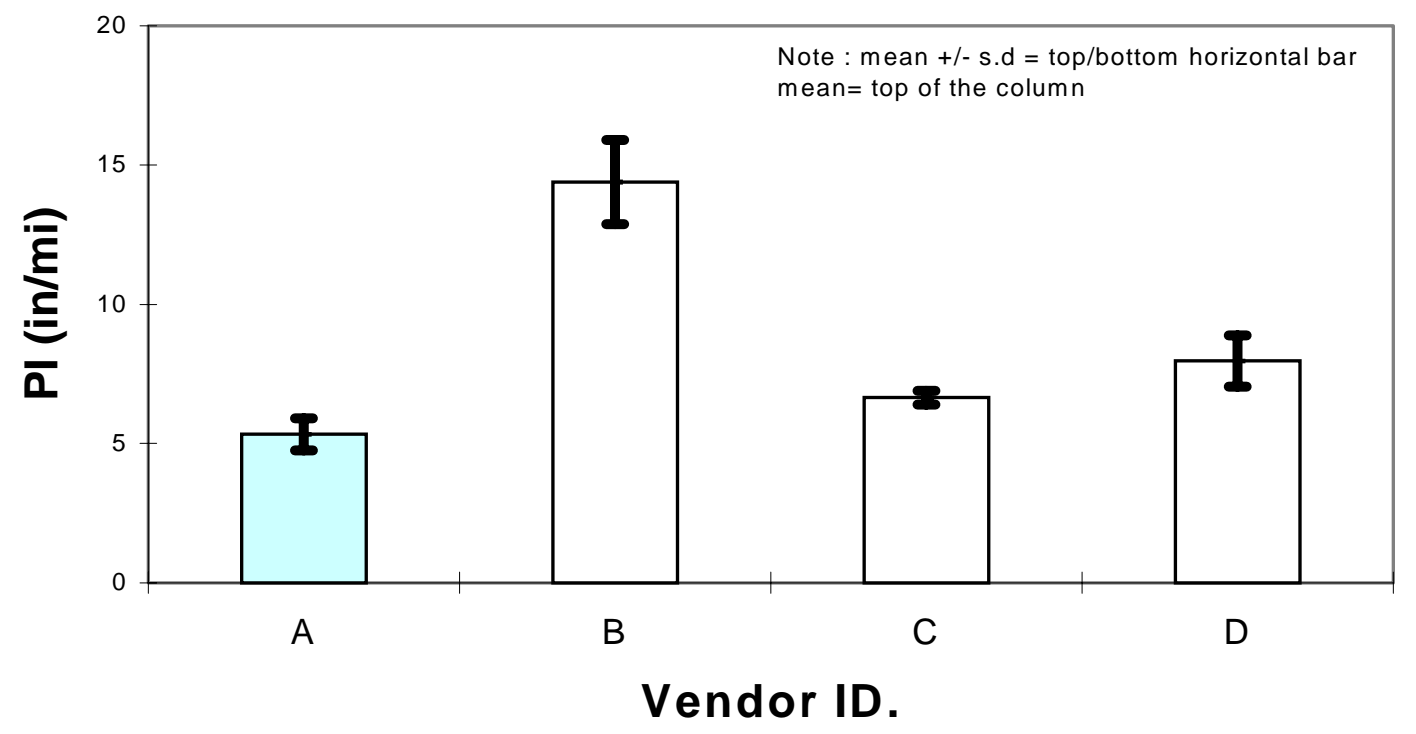

Figure 4-38 PI-0.0" Between Vendor Comparison for Site 2b (I-65 without board)

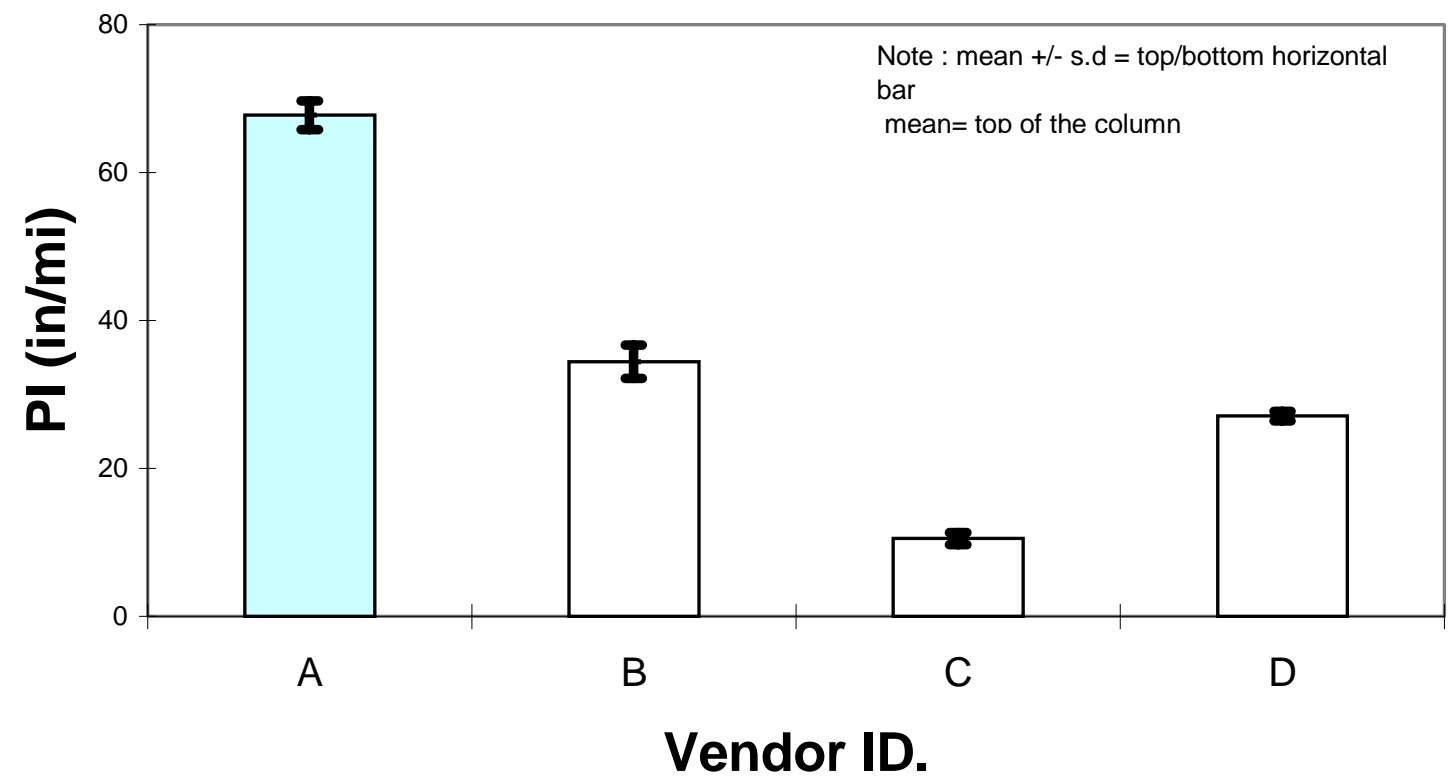

Figure 4-39 PI-0.0”' Between Vendor Comparison for Site 2 (I-65 with board) 


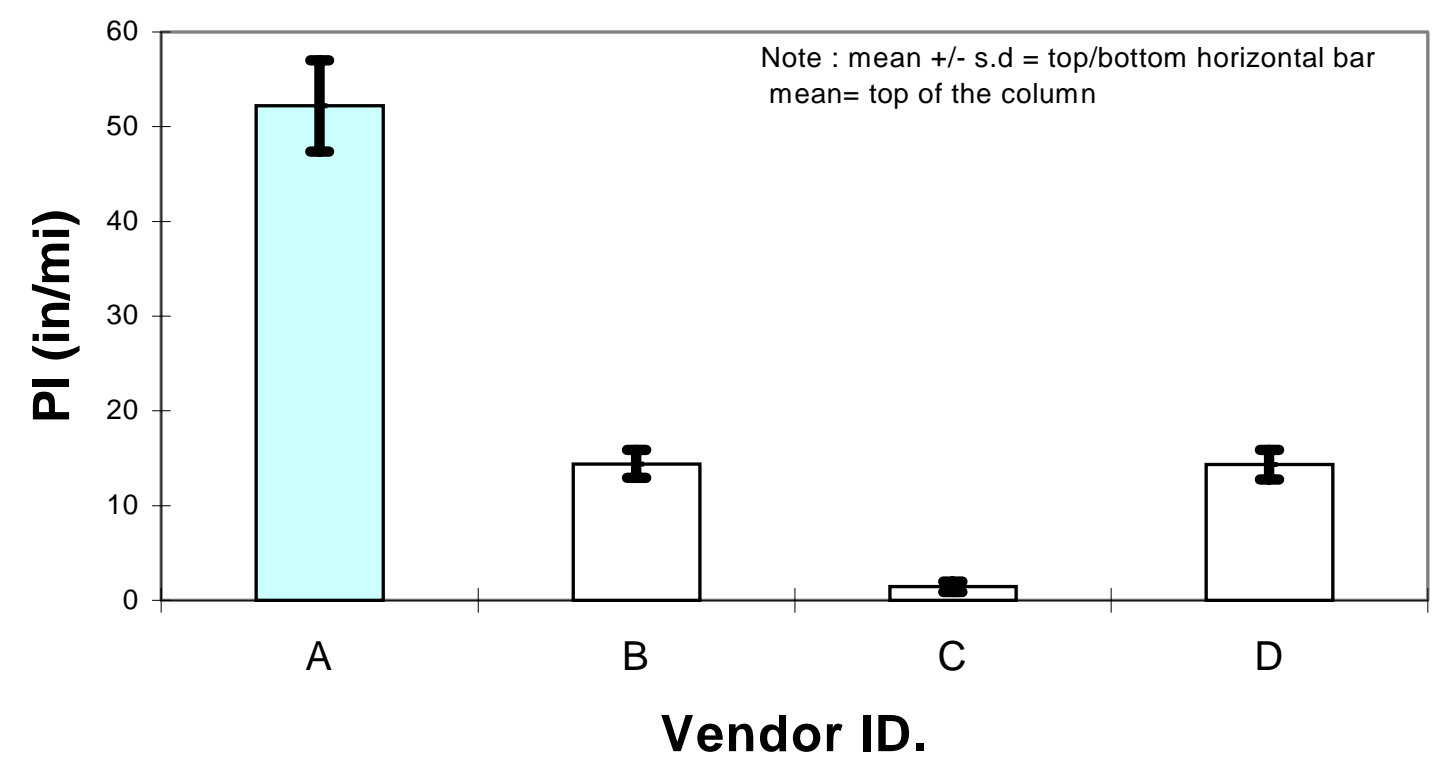

Figure 4-40 PI-0.2" Between Vendor Comparison for Site 2b (I-65 with board)

At the Prairie Street site (Site 3) with the thin plywood strips in place the average IRI values obtained with the lightweight profilers were fairly similar and consistent. The value obtained with the INDOT Rip Van was significantly higher however. When the plywood strips were removed similar observations were made. Interestingly, the average value reported by each vendor only dropped about five percent when the strips were removed. The data in Table 4-18 do not suggest that the geometric features at this site impacted the performance of the profilers.

Vendor A reported a significantly lower average PI- 0.0 " than the other vendors at the Prairie Street site with the thin plywood strips in place (36 versus 57 to $61 \mathrm{in} / \mathrm{mi}$ ) and all of the vendors showed consistency. Almost identical observations were made when the plywood strips were removed. When the PI-0.2" index is considered at this site with 
the boards in place Vendor A reported an average more similar to the other vendors (34 versus 38 to $49 \mathrm{in} / \mathrm{mi}$ ). Again, almost identical observations were made when the plywood strips were removed. All vendors also showed good consistency relative to PI. The data do not suggest that the geometric features or plywood strips at this site affected the performance of the profilers.

The average reported IRI were similar for all the lightweight profilers except Vendor A at the Vinton Street site (Site 4). Vendor A reported a slightly lower average IRI than the other Vendors and the INDOT Rip Van reported a slightly lower average then them. All devices showed good, similar consistency as noted by the low coefficients of variation reported (less than 6 percent).

Vendor A reported a significantly lower average PI-0.0" than the other profilers at this site and all vendors showed good consistency. All of the vendors reported similar average PI-0.2" values at this site. They did show less consistency, particularly Vendor A, for the 0.2 inch blanking band data. The data do not suggest that the geometric features at this site affected the performance of the profilers.

Overall the between vendor performance was not as good as expected. It also appears as though Vendor A had a tendency to report average IRI and PI values that were either much larger or smaller than the other vendors. However in some cases it reported values similar to the other devices, particularly for PI-0.2". The INDOT RIP Van did not provide results that were necessarily consistent with any of the lightweight profilers. 
Three tasks were performed to develop a better understanding of the reasons for the observed differences. A ranking was performed to determine if indeed Vendor A was consistently reporting values either higher or lower than the other vendors. This was followed by a statistical analysis (mean comparisons) of the data. And finally, the actual profiles generated by each vendor at each site were analyzed for potential differences.

\subsection{Smoothness Rankings by Vendor}

In order to determine whether one lightweight profiler consistently reported low or high values relative to all other vendors for a given smoothness index, rankings were performed. Within each site the average IRI, PI- 0.0 " and PI- 0.2 " reported by each vendor were ranked in order from lowest to highest, or in other words from smoothest to roughest. Thus the vendor that received a ranking of 1 for a given site had reported the lowest smoothness index value and the vendor that received a ranking of 4 for a given site had reported the highest smoothness index value for that site. The rankings are summarized in Tables 4-19 and 4-20. The rankings were performed based on pavement type such that differences could be observed relative to pavement type if they existed. Note that all vendors received a ranking of 1 for the I-65 site using PI with a 0.2 inch blanking band because they all reported PI values of $0 \mathrm{in} / \mathrm{mi}$ for that site. Tables 4.21 and 4.22 show the number and percentage of the time that each vendor obtained a given rank, respectively.

Based on the rankings in Tables 4.19 and 4.20 is it is evident that no particular Vendor consistently reported the lowest or highest smoothness index at all of the HMA or 
PCC sites. This implies that there is no consistent bias in the data and the differences in smoothness reported by the different vendors is to some degree due to the pavement condition. The reality is that the rankings appear to be random with the possible exception that Vendor A consistently ranks as either 4 or 1 with one exception. The data in Tables 4-21 and 4-22 show this most clearly.

Table 4-19 Ranking by Vendor for HMA Sites

\begin{tabular}{|c|c|c|c|c|c|c|}
\hline \multirow{3}{*}{ Index } & \multirow{3}{*}{ Vendor } & \multicolumn{5}{|c|}{ Site } \\
\hline & & $\begin{array}{c}\mathrm{I}-65 \\
\text { (no board) }\end{array}$ & $\begin{array}{c}\text { I-65 } \\
\text { (with board) }\end{array}$ & $\begin{array}{l}\text { Prairie Street } \\
\text { (no board) }\end{array}$ & $\begin{array}{l}\text { Prairie Street } \\
\text { (with board) }\end{array}$ & $\begin{array}{l}\text { Vinton } \\
\text { Street }\end{array}$ \\
\hline & & \multicolumn{5}{|c|}{ Ranking } \\
\hline \multirow{4}{*}{ IRI } & $\mathrm{A}$ & 1 & 1 & 4 & 4 & 4 \\
\hline & $\mathrm{B}$ & 2 & 2 & 3 & 3 & 3 \\
\hline & $\mathrm{C}$ & 3 & 4 & 1 & 1 & 2 \\
\hline & $\mathrm{D}$ & 4 & 3 & 2 & 2 & 1 \\
\hline \multirow{4}{*}{ PI-0.2 } & $\mathrm{A}$ & 1 & 1 & 4 & 4 & 2 \\
\hline & $\mathrm{B}$ & 2 & 1 & 2 & 2 & 3 \\
\hline & $\mathrm{C}$ & 4 & 1 & 1 & 1 & 1 \\
\hline & $\mathrm{D}$ & 3 & 1 & 3 & 3 & 4 \\
\hline \multirow{4}{*}{ PI-0.0 } & $\mathrm{A}$ & 1 & 4 & 4 & 4 & 4 \\
\hline & $\mathrm{B}$ & 2 & 1 & 1 & 3 & 3 \\
\hline & $\mathrm{C}$ & 4 & 3 & 2 & 1 & 2 \\
\hline & $\mathrm{D}$ & 3 & 2 & 3 & 2 & 1 \\
\hline
\end{tabular}


Table 4-20 Ranking by Vendor for PCC Sites

\begin{tabular}{|c|c|c|c|c|}
\hline \multirow{3}{*}{ Index } & \multirow{3}{*}{ Vendor } & \multicolumn{3}{|c|}{ Site } \\
\hline & & $\begin{array}{c}\mathrm{I}-65 \\
\text { (no board) }\end{array}$ & $\begin{array}{c}\text { I-65 } \\
\text { (with board) }\end{array}$ & $\begin{array}{l}\text { Prairie Street } \\
\text { (no board) }\end{array}$ \\
\hline & & & Ranking & \\
\hline \multirow{4}{*}{ IRI } & $\mathrm{A}$ & 4 & 1 & 1 \\
\hline & B & 3 & 3 & 4 \\
\hline & $\mathrm{C}$ & 2 & 4 & 3 \\
\hline & $\mathrm{D}$ & 1 & 2 & 2 \\
\hline \multirow{4}{*}{ PI- 0.2} & $\mathrm{~A}$ & 4 & 4 & 4 \\
\hline & B & 3 & 3 & 1 \\
\hline & C & 1 & 2 & 3 \\
\hline & $\mathrm{D}$ & 2 & 1 & 2 \\
\hline \multirow{4}{*}{ PI- 0.0} & $\mathrm{~A}$ & 4 & 4 & 4 \\
\hline & B & 3 & 1 & 1 \\
\hline & C & 1 & 3 & 2 \\
\hline & $\mathrm{D}$ & 2 & 2 & 3 \\
\hline
\end{tabular}

Table 4-21 Number of Times Each Received Each Rank

\begin{tabular}{|c|c|c|c|c|}
\hline \multirow{2}{*}{ Rank } & \multicolumn{5}{|c|}{ Vendor } \\
\cline { 2 - 5 } & A & B & C & D \\
\hline 1 & 6 & 5 & 8 & 4 \\
\hline 2 & 1 & 6 & 6 & 8 \\
\hline 3 & 0 & 11 & 4 & 1 \\
\hline 4 & 16 & 1 & 5 & \\
\hline
\end{tabular}

Table 4-22 Percentage of Times Each Received Each Rank

\begin{tabular}{|c|c|c|c|c|}
\hline \multirow{2}{*}{ Rank } & \multicolumn{4}{|c|}{ Vendor } \\
\cline { 2 - 5 } & A & B & C & D \\
\hline 1 & 26 & 22 & 35 & 17 \\
\hline 2 & 4 & 26 & 26 & 43 \\
\hline 3 & 0 & 48 & 17 & 35 \\
\hline 4 & 70 & 4 & 22 & 4 \\
\hline
\end{tabular}




\subsection{Statistical Analysis of Between Vendor Performance}

To compare the performance of the lightweight profilers on a one-to one basis Ftests and t-tests were conducted for all potential two-way combinations of the profilers. In other words, tests of equal variance and equal means were performed for each possible combination of vendors at each site for each of the three smoothness indices considered in the study. Six two-way combinations or one-to-one comparisons existed at each site per smoothness index. They were combinations of Vendor A and Vendor B, Vendor A and Vendor C, Vendor A and Vendor D, Vendor B and Vendor C, Vendor B and D, and finally Vendor C and Vendor D.

The statistical F-test is used to test for equality of two variances. The central hypothesis of the test is that the variances of the two data sets are equal. An F-value is calculated from the variances associated with each data set to be compared (eg. the five replicate IRI values reported by each of two vendors at a given site). The calculated Fvalue is compared to a critical F-value. If the observed F-value is greater than the critical F-value, at a selected level of significance $(\alpha)$, the variances of the data sets are considered to be significant, or different and the central hypothesis is rejected. If the calculated F-value is less than the critical F-value the central hypothesis is accepted. The maximum probability of wrongly rejecting the central hypothesis is termed alpha $(\alpha)$. For small data sets alpha should not be greater than five percent $(\alpha=0.05)$. With alpha equal to five percent, the chance that the variances may not be equal when the observed F-value is less than the critical F-value is five percent. In other words, one is ninety-five percent 
sure that the variances are equal. Critical F-values are determined from look-up tables of the F-distribution for given alpha levels and degrees of freedom.

A t-test is used to test for equality of means with small samples sizes. The central hypothesis of this test is that the means or averages of two data sets are equal. A t-value is calculated from the means of each data set, the variances associated with each data set to be compared, and the number of observations in each data set. The calculated t-value is compared to a critical t-value. If the observed t-value is greater than the critical t-value, at a selected level of significance $(\alpha)$, the means of the data sets are considered to be significant, or different and the central hypothesis is rejected. If the calculated t-value is less than the critical t-value the central hypothesis is accepted. Critical t-values are determined from look-up tables of the Student's t-distribution for given levels of alpha and degrees of freedom. The alpha concepts associated with the F-test hold true for the ttest also.

The F- and t-test results using a significance level of 5 percent $(\alpha=0.05)$ are summarized in Table 4-23 through 4-38. A "No" in the tables indicates unequal variances or unequal means and a "Yes" in the table indicates equal variances or equal means at the five percent significance level.

Equal variance was observed in 48 percent (23 of 48) of the comparisons for IRI. Thus unequal variance was observed in 52 percent of the comparisons. Equal variance was observed in 69 percent (33 of 48) of the comparisons for PI with the 0.0" blanking 
band (PI-0.0"). In the case of the PI with a 0.2 inch blanking band (PI-0.02") equal variance was observed in 49 percent (21 of 45) of the comparisons. Three tests could not be performed because of PI-0.2" standard deviation values of $0 \mathrm{in} / \mathrm{mi}$ were reported for a limited number of vendors at Sites 1 without the plywood strips and 5. All vendors reported 0in/mi for PI-0.02" for all measurements at site 2. Thus equal variance was observed for all vendors at this site.

The mean comparisons presented in Tables 4-31 through 4-38 provided somewhat discouraging results. Equal means were observed in only 23 percent (11 of 48) of the comparisons for IRI. Thus the average values of IRI reported by the individual vendors at a given site were statistically different for 77 percent of the observations. None of the vendors reported means that were the same statistically for IRI at Site 2 with the plywood strips, Site 3 without the plywood strips, or Site 6. The Vendor A average IRI was statistically different than all of the other vendors in three of the eight site comparisons (Site 2 without plywood, Site 4, and Site 5). The Vendor D average IRI was statistically different than all of the other vendors at Site 4 . The best correlation of reported average IRI values occurred at Sites 1 and 5 where three of six vendors reported statistically similar average IRI values.

Equal means were observed in only 25 percent (12 of 48) of the comparisons for PI-0.0". Thus the average values of PI-0.0" reported by the individual vendors at a given site were statistically different for 75 percent of the observations. None of the vendors reported means that were the same statistically for PI-0.0" at Site 1, Site 2 with the 
plywood strips, or Site 5. The Vendor A average PI-0.0" was statistically different than all of the other vendors in four of the eight site comparisons (Site 3 with plywood strips, Site 3 without plywood strips, Site 4, and Site 6). The best correlation of reported average PI-0.0" values occurred at Sites 4 and 6 where three of six vendor pairs reported statistically similar average IRI values.

Equal means were also observed in only 25 percent ( 12 of 48 ) of the comparisons for PI-0.2". Thus the average values of PI- 0.2 " reported by the individual vendors at a given site were statistically different for 75 percent of the observations. None of the vendors reported means that were the same statistically for PI-0.2" at Site 1, Site 3 with the plywood strips, or Site 3 without plywood strips. The Vendor A average PI-0.2" was statistically different than all of the other Vendors in only one of the eight site comparisons (Site 2 with plywood strips). The Vendor D average PI-0.2" was statistically different than all of the other vendors in one of the eight site comparisons also. This was observed at Site 2 with plywood strips. The best correlation of reported average PI-0.2" values occurred at Sites 2 and 5 where all six vendor pairs reported statistically similar average PI-0.2" values.

In more cases than not the reported Vendor A mean smoothness indices differed from the other vendors. However, in some cases the Vendor A data was similar to the Vendor C and D data while the Vendor B data differed from them. In other cases the Vendor B and D data were similar and both the Vendor and A and C data differed from 
it. While in other cases, all of the vendors data were similar. There is simply no consistency in the test results.

These results are very discouraging not only because of the poor correlation between vendors, but also because there was a lack of consistency between indices. A prime example of this existed at Site 5, a smooth PCC site, where the best between vendor correlation was observed for IRI. Three of six vendor pairs reported statistically similar mean IRI values. However, none of the vendors reported equal means for PI-0.0". On the other hand all of the vendors reported statistically similar means for PI-0.02". Another discourage finding was that between vendor correlation did not necessarily improve as roughness decreased depending on which smoothness statistic is considered.

It is clear from the analysis however that in situations where different brands of lightweight profilers were used for process control or quality control (QC) and quality assurance (QA) statistically different results would very likely be reported. This would certainly lead to disputes, which no agency likes to see exist or be caught in the middle of.

An analysis of the profiles generated by each vendor at each site was performed in an effort to develop an understanding of the potential reason(s) for the differences observed. 
Table 4-23 Summary of F-test results for equal variance for Site 1

\begin{tabular}{|c|c|c|c|c|c|c|c|c|c|c|c|c|c|c|c|}
\hline & \multicolumn{15}{|c|}{ Smoothness Statistic } \\
\hline & \multicolumn{5}{|c|}{ IRI } & & \multirow{2}{*}{\multicolumn{4}{|c|}{$\begin{array}{l}\text { PI-0.0" } \\
\text { Vendor }\end{array}$}} & & \multicolumn{4}{|c|}{ PI-0.02" } \\
\hline & \multicolumn{5}{|c|}{ Vendor } & & & & & & & & & & \\
\hline & & A & B & $\mathrm{C}$ & $\mathrm{D}$ & & $\mathrm{A}$ & $\mathrm{B}$ & $\mathrm{C}$ & $\mathrm{D}$ & & A & $\mathrm{B}$ & $\mathrm{C}$ & $\mathrm{D}$ \\
\hline \multirow{4}{*}{$\frac{\tilde{0}}{\frac{0}{0}}$} & A & & Yes & No & Yes & A & & No & No & No & A & & Yes & $*$ & Yes \\
\hline & $\mathrm{B}$ & Yes & & No & Yes & $\mathrm{B}$ & No & & Yes & Yes & $\mathrm{B}$ & Yes & & $*$ & Yes \\
\hline & $\mathrm{C}$ & No & No & & No & $\mathrm{C}$ & No & Yes & & Yes & $\mathrm{C}$ & * & $*$ & & No \\
\hline & $\mathrm{D}$ & Yes & Yes & No & & $\mathrm{D}$ & No & Yes & Yes & & $\mathrm{D}$ & Yes & Yes & No & \\
\hline
\end{tabular}

Table 4-24 Summary of F-test results for equal variance for Site 2 with Plywood Strips

\begin{tabular}{|c|c|c|c|c|c|c|c|c|c|c|c|c|c|c|c|}
\hline & \multicolumn{15}{|c|}{ Smoothness Statistic } \\
\hline & \multirow{2}{*}{\multicolumn{5}{|c|}{$\frac{\text { IRI }}{\text { Vendor }}$}} & & \multirow{2}{*}{\multicolumn{4}{|c|}{$\begin{array}{l}\text { PI-0.0" } \\
\text { Vendor }\end{array}$}} & & \multirow{2}{*}{\multicolumn{4}{|c|}{ PI-0.02" }} \\
\hline & & & & & & & & & & & & & & & \\
\hline & & $\bar{A}$ & $\mathrm{~B}$ & $\mathrm{C}$ & $\mathrm{D}$ & & $\bar{A}$ & $\mathrm{~B}$ & $\bar{C}$ & $\mathrm{D}$ & & $\bar{A}$ & $\mathrm{~B}$ & $\mathrm{C}$ & $\mathrm{D}$ \\
\hline \multirow{4}{*}{$\begin{array}{l}\frac{\tilde{0}}{0} \\
\frac{0}{0} \\
>\end{array}$} & $\bar{A}$ & & No & No & No & $\mathrm{A}$ & & & Yes & No & $\bar{A}$ & & No & No & No \\
\hline & B & No & & No & Yes & $\mathrm{B}$ & Yes & & No & No & B & No & & No & Yes \\
\hline & $\bar{C}$ & No & No & & No & $\mathrm{C}$ & Yes & No & & Yes & $\bar{C}$ & No & No & & No \\
\hline & $\bar{D}$ & No & Yes & No & & $\mathrm{D}$ & No & No & Yes & & $\bar{D}$ & No & Yes & No & \\
\hline
\end{tabular}

Table 4-25 Summary of F-test results for equal variance for Site 2 without Plywood Strips

\begin{tabular}{|c|c|c|c|c|c|c|c|c|c|c|c|c|c|c|c|}
\hline & \multicolumn{15}{|c|}{ Smoothness Statistic } \\
\hline & \multirow{2}{*}{\multicolumn{5}{|c|}{\begin{tabular}{|c|} 
IRI \\
Vendor
\end{tabular}}} & & \multirow{2}{*}{\multicolumn{4}{|c|}{$\begin{array}{l}\text { PI-0.0" } \\
\text { Vendor }\end{array}$}} & & \multirow{2}{*}{\multicolumn{4}{|c|}{$\begin{array}{l}\text { PI-0.02" } \\
\text { Vendor }\end{array}$}} \\
\hline & & & & & & & & & & & & & & & \\
\hline & & A & $\mathrm{B}$ & $\mathrm{C}$ & $\mathrm{D}$ & & A & $\mathrm{B}$ & $\mathrm{C}$ & $\mathrm{D}$ & & $\mathrm{A}$ & $\mathrm{B}$ & $\mathrm{C}$ & $\mathrm{D}$ \\
\hline \multirow{4}{*}{$\begin{array}{l}\overrightarrow{0} \\
\overrightarrow{0} \\
\overrightarrow{0}\end{array}$} & $\mathrm{~A}$ & & Yes & Yes & No & $\mathrm{A}$ & & Yes & Yes & Yes & $\mathrm{A}$ & & $* *$ & $* *$ & $* *$ \\
\hline & $\mathrm{B}$ & Yes & & Yes & Yes & $\mathrm{B}$ & Yes & & Yes & Yes & $\mathrm{B}$ & $* *$ & & $* *$ & $* *$ \\
\hline & $\mathrm{C}$ & Yes & Yes & & No & $\mathrm{C}$ & Yes & Yes & & Yes & $\mathrm{C}$ & $* *$ & $* *$ & & $* *$ \\
\hline & $\mathrm{D}$ & No & Yes & No & & $\mathrm{D}$ & Yes & Yes & Yes & & $\mathrm{D}$ & $* *$ & $* *$ & $* *$ & \\
\hline
\end{tabular}

Table 4-26 Summary of F-test results for equal variance for Site 3 with Plywood Strips

\begin{tabular}{|c|c|c|c|c|c|c|c|c|c|c|c|c|c|c|c|}
\hline & \multicolumn{15}{|c|}{ Smoothness Statistic } \\
\hline & \multirow{2}{*}{\multicolumn{5}{|c|}{\begin{tabular}{|l} 
IRI \\
Vendor
\end{tabular}}} & & \multirow{2}{*}{\multicolumn{4}{|c|}{$\begin{array}{l}\text { PI-0.0" } \\
\text { Vendor }\end{array}$}} & & \multirow{2}{*}{\multicolumn{4}{|c|}{$\begin{array}{l}\text { PI-0.02" } \\
\text { Vendor }\end{array}$}} \\
\hline & & & & & & & & & & & & & & & \\
\hline & & A & B & $\mathrm{C}$ & $\mathrm{D}$ & & A & B & $\mathrm{C}$ & $\mathrm{D}$ & & A & B & $\mathrm{C}$ & $\mathrm{D}$ \\
\hline \multirow{4}{*}{$\frac{8}{3}$} & $\mathrm{~A}$ & & Yes & Yes & No & $\mathrm{A}$ & & No & Yes & Yes & $\mathrm{A}$ & & Yes & Yes & No \\
\hline & B & Yes & & No & Yes & B & No & & No & Yes & B & Yes & & No & Yes \\
\hline & $\mathrm{C}$ & Yes & No & & No & $\mathrm{C}$ & Yes & No & & Yes & $\mathrm{C}$ & Yes & No & & No \\
\hline & $\mathrm{D}$ & No & Yes & No & & $\mathrm{D}$ & Yes & Yes & Yes & & $\mathrm{D}$ & No & Yes & No & \\
\hline
\end{tabular}


Table 4-27 Summary of F-test results for equal variance for Site 3 without Plywood Strips

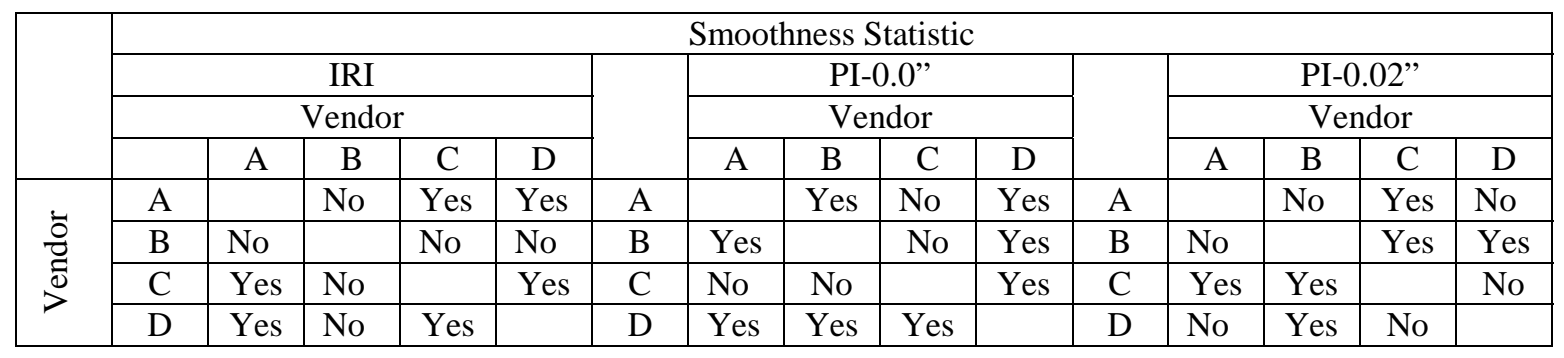

Table 4-28 Summary of F-test results for equal variance for Site 4

\begin{tabular}{|c|c|c|c|c|c|c|c|c|c|c|c|c|c|c|c|}
\hline & \multicolumn{15}{|c|}{ Smoothness Statistic } \\
\hline & \multirow{2}{*}{\multicolumn{5}{|c|}{$\begin{array}{c}\text { IRI } \\
\text { Vendor }\end{array}$}} & & \multirow{2}{*}{\multicolumn{4}{|c|}{$\begin{array}{l}\text { PI-0.0" } \\
\text { Vendor }\end{array}$}} & & \multirow{2}{*}{\multicolumn{4}{|c|}{ PI-0.02" }} \\
\hline & & & & & & & & & & & & & & & \\
\hline & & A & B & $\mathrm{C}$ & $\mathrm{D}$ & & A & $\mathrm{B}$ & $\mathrm{C}$ & $\mathrm{D}$ & & A & B & $\mathrm{C}$ & $\mathrm{D}$ \\
\hline \multirow{4}{*}{$\begin{array}{l}\overrightarrow{0} \\
\overrightarrow{0} \\
\overrightarrow{0}\end{array}$} & $\mathrm{~A}$ & & Yes & No & Yes & $\mathrm{A}$ & & No & No & Yes & $\mathrm{A}$ & & Yes & Yes & No \\
\hline & $\mathrm{B}$ & Yes & & Yes & Yes & $\mathrm{B}$ & No & & Yes & Yes & $\mathrm{B}$ & Yes & & Yes & No \\
\hline & $\mathrm{C}$ & No & Yes & & No & $\mathrm{C}$ & No & Yes & & Yes & $\mathrm{C}$ & Yes & Yes & & No \\
\hline & $\bar{D}$ & Yes & Yes & No & & $\mathrm{D}$ & Yes & Yes & Yes & & $\mathrm{D}$ & No & No & No & \\
\hline
\end{tabular}

Table 4-29 Summary of F-test results for equal variance for Site 5

\begin{tabular}{|c|c|c|c|c|c|c|c|c|c|c|c|c|c|c|c|}
\hline & & & & & & & Smoo & iness & atistic & & & & & & \\
\hline & & & IRI & & & & & PI- & & & & & & $02 ”$ & \\
\hline & & & Vendo & & & & & & & & & & & dor & \\
\hline & & $\mathrm{A}$ & B & $\mathrm{C}$ & $\mathrm{D}$ & & $\mathrm{A}$ & B & $\mathrm{C}$ & $\mathrm{D}$ & & $\mathrm{A}$ & B & $\bar{C}$ & $\mathrm{D}$ \\
\hline & $\mathrm{A}$ & & No & No & No & A & & Yes & Yes & No & A & & * & Yes & Yes \\
\hline$\frac{\overline{0}}{0}$ & B & No & & Yes & No & $\mathrm{B}$ & Yes & & Yes & Yes & $\mathrm{B}$ & * & & No & No \\
\hline లే & $\mathrm{C}$ & No & Yes & & No & $\mathrm{C}$ & Yes & Yes & & Yes & $\mathrm{C}$ & Yes & No & & Yes \\
\hline & $\mathrm{D}$ & No & No & No & & $\mathrm{D}$ & No & Yes & Yes & & $\mathrm{D}$ & Yes & No & Yes & \\
\hline
\end{tabular}

Table 4-30 Summary of F-test results for equal variance for Site 6

\begin{tabular}{|c|c|c|c|c|c|c|c|c|c|c|c|c|c|c|c|}
\hline & \multicolumn{15}{|c|}{ Smoothness Statistic } \\
\hline & \multicolumn{5}{|c|}{ IRI } & & \multirow{2}{*}{\multicolumn{4}{|c|}{$\frac{\text { PI-0.0" }}{\text { Vendor }}$}} & & \multirow{2}{*}{\multicolumn{4}{|c|}{$\begin{array}{l}\text { PI-0.02" } \\
\text { Vendor }\end{array}$}} \\
\hline & \multicolumn{5}{|c|}{ Vendor } & Vendor & & & & & & & & & \\
\hline & & $\bar{A}$ & $\mathrm{~B}$ & $\mathrm{C}$ & $\bar{D}$ & & $\mathrm{~A}$ & $\mathrm{~B}$ & $\bar{C}$ & $\mathrm{D}$ & & $\mathrm{A}$ & $\mathrm{B}$ & $\mathrm{C}$ & $\bar{D}$ \\
\hline \multirow{4}{*}{$\begin{array}{l}\tilde{0} \\
\frac{0}{0} \\
>0\end{array}$} & $\mathrm{~A}$ & & No & No & No & $\mathrm{A}$ & & No & Yes & Yes & $\mathrm{A}$ & & Yes & Yes & Yes \\
\hline & $\bar{B}$ & $\mathrm{No}$ & & Yes & Yes & $\mathrm{B}$ & No & & Yes & No & $\mathrm{B}$ & Yes & & Yes & Yes \\
\hline & $\mathrm{C}$ & $\mathrm{No}$ & Yes & & Yes & $\mathrm{C}$ & Yes & Yes & & Yes & $\mathrm{C}$ & Yes & Yes & & Yes \\
\hline & $\mathrm{D}$ & $\mathrm{No}$ & Yes & Yes & & $\mathrm{D}$ & Yes & No & Yes & & $\mathrm{D}$ & Yes & Yes & Yes & \\
\hline
\end{tabular}


Table 4-31 Summary of paired t-test results for equal means for Site 1

\begin{tabular}{|c|c|c|c|c|c|c|c|c|c|c|c|c|c|c|c|}
\hline & \multicolumn{15}{|c|}{ Smoothness Statistic } \\
\hline & \multicolumn{5}{|c|}{ IRI } & & \multirow{2}{*}{\multicolumn{4}{|c|}{$\begin{array}{l}\text { PI-0.0" } \\
\text { Vendor }\end{array}$}} & & \multicolumn{4}{|c|}{ PI-0.02" } \\
\hline & \multicolumn{5}{|c|}{ Vendor } & & & & & & & & & & \\
\hline & & $\mathrm{A}$ & $\mathrm{B}$ & $\mathrm{C}$ & $\mathrm{D}$ & & $\bar{A}$ & $\mathrm{~B}$ & $\mathrm{C}$ & $\mathrm{D}$ & & $\mathrm{A}$ & $\mathrm{B}$ & $\mathrm{C}$ & $\mathrm{D}$ \\
\hline \multirow{4}{*}{$\frac{\overline{0}}{\overline{0}}$} & A & & No & No & No & A & & No & No & No & A & & No & No & No \\
\hline & $\mathrm{B}$ & No & & Yes & No & $\mathrm{B}$ & No & & No & No & $\mathrm{B}$ & No & & No & No \\
\hline & $\mathrm{C}$ & No & Yes & & Yes & $\mathrm{C}$ & No & No & & No & $\bar{C}$ & No & No & & No \\
\hline & $\mathrm{D}$ & No & No & Yes & & $\mathrm{D}$ & No & No & No & & $\mathrm{D}$ & No & No & No & \\
\hline
\end{tabular}

Table 4-32 Summary of paired t-test results for equal means for Site 2 with Plywood Strips

\begin{tabular}{|c|c|c|c|c|c|c|c|c|c|c|c|c|c|c|c|}
\hline & \multicolumn{15}{|c|}{ Smoothness Statistic } \\
\hline & \multirow{2}{*}{\multicolumn{5}{|c|}{$\frac{\text { IRI }}{\text { Vendor }}$}} & & \multirow{2}{*}{\multicolumn{4}{|c|}{$\begin{array}{l}\text { PI-0.0" } \\
\text { Vendor }\end{array}$}} & & \multirow{2}{*}{\multicolumn{4}{|c|}{$\begin{array}{l}\text { PI-0.02" } \\
\text { Vendor }\end{array}$}} \\
\hline & & \multicolumn{3}{|c|}{ Vendor } & & & & & & & & & & & \\
\hline & & $\mathrm{A}$ & B & $\mathrm{C}$ & $\mathrm{D}$ & & A & B & $\mathrm{C}$ & $\mathrm{D}$ & & A & B & $\mathrm{C}$ & $\mathrm{D}$ \\
\hline \multirow{4}{*}{$\begin{array}{l}\overline{0} \\
\overline{0} \\
\overline{0} \\
>\end{array}$} & $\mathrm{A}$ & & No & No & No & $\mathrm{A}$ & & No & No & No & $\mathrm{A}$ & & No & No & No \\
\hline & $\mathrm{B}$ & No & & No & No & $\mathrm{B}$ & No & & No & No & $\mathrm{B}$ & No & & No & Yes \\
\hline & $\mathrm{C}$ & No & No & & No & $\mathrm{C}$ & No & No & & No & $\mathrm{C}$ & No & No & & No \\
\hline & $\mathrm{D}$ & No & No & No & & $\mathrm{D}$ & No & No & No & & $\mathrm{D}$ & No & Yes & No & \\
\hline
\end{tabular}

Table 4-33 Summary of paired t-test results for equal means for Site 2 without Plywood Strips

\begin{tabular}{|c|c|c|c|c|c|c|c|c|c|c|c|c|c|c|c|}
\hline & \multicolumn{15}{|c|}{ Smoothness Statistic } \\
\hline & \multicolumn{5}{|c|}{ IRI } & & \multirow{2}{*}{\multicolumn{4}{|c|}{$\begin{array}{l}\text { PI-0.0" } \\
\text { Vendor }\end{array}$}} & & \multirow{2}{*}{\multicolumn{4}{|c|}{$\begin{array}{l}\text { PI-0.02" } \\
\text { Vendor }\end{array}$}} \\
\hline & \multicolumn{5}{|c|}{ Vendor } & & & & & & & & & & \\
\hline & & $\mathrm{A}$ & B & $\mathrm{C}$ & $\mathrm{D}$ & & $\mathrm{A}$ & B & $\mathrm{C}$ & $\mathrm{D}$ & & $\mathrm{A}$ & $\mathrm{B}$ & $\mathrm{C}$ & $\mathrm{D}$ \\
\hline \multirow{4}{*}{$\begin{array}{l}\frac{0}{0} \\
\overline{0} \\
>\end{array}$} & $\mathrm{A}$ & & No & No & No & $\mathrm{A}$ & & No & Yes & No & $\mathrm{A}$ & & Yes & Yes & Yes \\
\hline & $\mathrm{B}$ & No & & No & Yes & $\mathrm{B}$ & No & & No & Yes & $\mathrm{B}$ & Yes & & Yes & Yes \\
\hline & $\mathrm{C}$ & No & No & & Yes & $\mathrm{C}$ & Yes & No & & No & $\mathrm{C}$ & Yes & Yes & & Yes \\
\hline & $\mathrm{D}$ & No & Yes & Yes & & $\mathrm{D}$ & No & Yes & No & & $\mathrm{D}$ & Yes & Yes & Yes & \\
\hline
\end{tabular}

Table 4-34 Summary of paired t-test results for equal means for Site 3 with Plywood Strips

\begin{tabular}{|c|c|c|c|c|c|c|c|c|c|c|c|c|c|c|c|}
\hline & \multicolumn{15}{|c|}{ Smoothness Statistic } \\
\hline & \multicolumn{5}{|c|}{ IRI } & & \multirow{2}{*}{\multicolumn{4}{|c|}{$\begin{array}{ll}\text { PI-0.0" } \\
\text { Vendor }\end{array}$}} & & \multicolumn{4}{|c|}{ PI-0.02" } \\
\hline & \multicolumn{5}{|c|}{ Vendor } & & & & & & & & & & \\
\hline & & A & B & $\mathrm{C}$ & $\mathrm{D}$ & & A & $\mathrm{B}$ & $\mathrm{C}$ & $\mathrm{D}$ & & A & B & $\mathrm{C}$ & $\mathrm{D}$ \\
\hline \multirow{4}{*}{$\begin{array}{l}0 \\
\overline{0} \\
\overline{0} \\
>\end{array}$} & $\mathrm{A}$ & & Yes & $\mathrm{No}$ & No & $\mathrm{A}$ & & No & No & No & $\mathrm{A}$ & & No & No & No \\
\hline & $\mathrm{B}$ & Yes & & & No & $\mathrm{B}$ & No & & Yes & No & $\mathrm{B}$ & No & & No & No \\
\hline & $\mathrm{C}$ & No & No & & Yes & $\mathrm{C}$ & No & Yes & & Yes & $\mathrm{C}$ & No & No & & No \\
\hline & $\mathrm{D}$ & No & No & Yes & & $\mathrm{D}$ & No & No & Yes & & $\mathrm{D}$ & No & No & No & \\
\hline
\end{tabular}


Table 4-35 Summary of paired t-test results for equal means for Site 3 without Plywood Strips

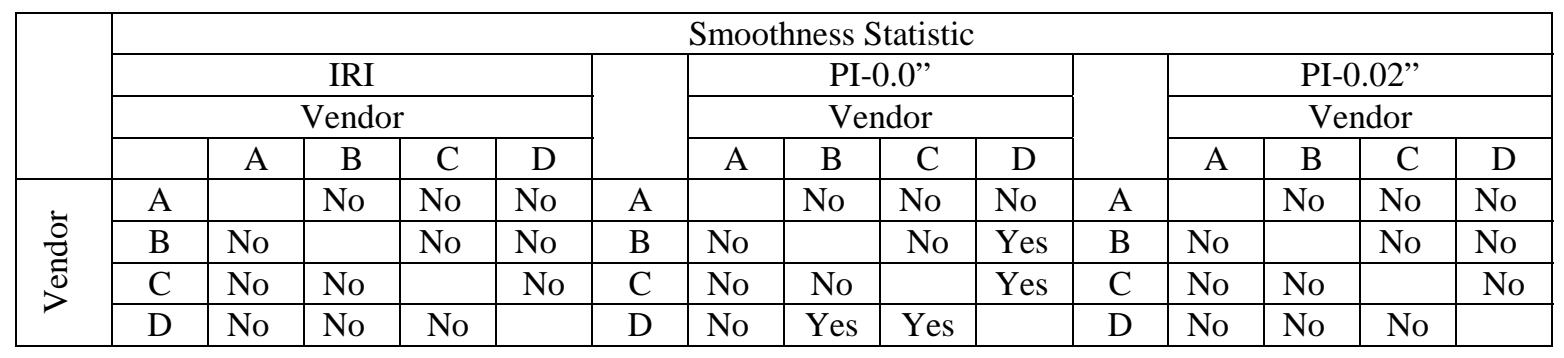

Table 4-36 Summary of paired t-test results for equal means for Site 4

\begin{tabular}{|c|c|c|c|c|c|c|c|c|c|c|c|c|c|c|c|}
\hline & \multicolumn{15}{|c|}{ Smoothness Statistic } \\
\hline & \multirow{2}{*}{\multicolumn{5}{|c|}{$\begin{array}{c}\text { IRI } \\
\text { Vendor }\end{array}$}} & & \multirow{2}{*}{\multicolumn{4}{|c|}{$\begin{array}{l}\text { PI-0.0" } \\
\text { Vendor }\end{array}$}} & & \multirow{2}{*}{\multicolumn{4}{|c|}{ PI-0.02" }} \\
\hline & & & & & & & & & & & & & & & \\
\hline & & A & B & $\mathrm{C}$ & $\mathrm{D}$ & & A & B & $\mathrm{C}$ & $\mathrm{D}$ & & A & B & $\mathrm{C}$ & $\mathrm{D}$ \\
\hline \multirow{4}{*}{$\frac{\overrightarrow{0}}{\bar{d}}$} & A & & No & No & No & A & & No & No & No & A & & Yes & Yes & Yes \\
\hline & $\mathrm{B}$ & No & & Yes & No & $\mathrm{B}$ & No & & Yes & Yes & $\mathrm{B}$ & Yes & & No & Yes \\
\hline & $\mathrm{C}$ & No & Yes & & No & $\mathrm{C}$ & No & Yes & & Yes & $\mathrm{C}$ & Yes & No & & No \\
\hline & $\mathrm{D}$ & No & No & No & & $\mathrm{D}$ & No & Yes & Yes & & $\mathrm{D}$ & Yes & Yes & No & \\
\hline
\end{tabular}

Table 4-37 Summary of paired t-test results for equal means for Site 5

\begin{tabular}{|c|c|c|c|c|c|c|c|c|c|c|c|c|c|c|c|}
\hline & \multicolumn{15}{|c|}{ Smoothness Statistic } \\
\hline & \multirow{2}{*}{\multicolumn{5}{|c|}{$\begin{array}{c}\text { IRI } \\
\text { Vendor }\end{array}$}} & & \multirow{2}{*}{\multicolumn{4}{|c|}{$\begin{array}{l}\text { PI-0.0" } \\
\text { Vendor }\end{array}$}} & & \multicolumn{4}{|c|}{ PI-0.02" } \\
\hline & & \multicolumn{4}{|c|}{ Vendor } & & & & & & & & & & \\
\hline & & $\mathrm{A}$ & $\mathrm{B}$ & $\mathrm{C}$ & $\mathrm{D}$ & & $\mathrm{A}$ & $\mathrm{B}$ & $\mathrm{C}$ & $\mathrm{D}$ & & $\mathrm{A}$ & $\mathrm{B}$ & $\mathrm{C}$ & $\mathrm{D}$ \\
\hline \multirow{4}{*}{$\begin{array}{l}\overline{0} \\
\overline{0} \\
\overline{0}\end{array}$} & $\mathrm{~A}$ & & No & No & No & $\mathrm{A}$ & & No & No & No & A & & Yes & Yes & Yes \\
\hline & $\mathrm{B}$ & No & & Yes & Yes & $\mathrm{B}$ & No & & No & No & $\mathrm{B}$ & Yes & & Yes & Yes \\
\hline & $\mathrm{C}$ & No & Yes & & Yes & $\mathrm{C}$ & No & No & & No & $\mathrm{C}$ & Yes & Yes & & Yes \\
\hline & $\mathrm{D}$ & No & Yes & Yes & & $\mathrm{D}$ & No & No & No & & $\mathrm{D}$ & Yes & Yes & Yes & \\
\hline
\end{tabular}

Table 4-38 Summary of paired t-test results for equal means for Site 6

\begin{tabular}{|c|c|c|c|c|c|c|c|c|c|c|c|c|c|c|c|}
\hline & \multicolumn{15}{|c|}{ Smoothness Statistic } \\
\hline & \multirow{2}{*}{\multicolumn{5}{|c|}{$\frac{\text { IRI }}{\text { Vendor }}$}} & & \multirow{2}{*}{\multicolumn{4}{|c|}{$\begin{array}{l}\text { PI-0.0" } \\
\text { Vendor }\end{array}$}} & & \multirow{2}{*}{\multicolumn{4}{|c|}{$\begin{array}{c}\text { PI-0.02" } \\
\text { Vendor }\end{array}$}} \\
\hline & & \multicolumn{4}{|c|}{ Vendor } & & & & & & & & & & \\
\hline & & $\mathrm{A}$ & $\mathrm{B}$ & $\mathrm{C}$ & $\mathrm{D}$ & & $\mathrm{A}$ & $\mathrm{B}$ & $\mathrm{C}$ & $\mathrm{D}$ & & $\mathrm{A}$ & $\mathrm{B}$ & $\mathrm{C}$ & $\mathrm{D}$ \\
\hline \multirow{4}{*}{$\begin{array}{l}\frac{0}{0} \\
\frac{0}{0}\end{array}$} & A & & No & No & No & A & & No & No & No & A & & No & No & No \\
\hline & $\mathrm{B}$ & No & & $\mathrm{No}$ & No & $\mathrm{B}$ & No & & Yes & Yes & $\mathrm{B}$ & No & & No & No \\
\hline & $\mathrm{C}$ & No & No & & No & $\mathrm{C}$ & No & Yes & & Yes & $\mathrm{C}$ & No & No & & Yes \\
\hline & $\mathrm{D}$ & No & No & No & & $\mathrm{D}$ & No & Yes & Yes & & $\mathrm{D}$ & No & No & Yes & \\
\hline
\end{tabular}




\section{$\underline{4.4}$ Profile Comparisons}

All smoothness indices are calculated from longitudinal profiles. If all inertial profiles produced similar profiles, then it would be expected that they would also produce similar smoothness indices. However, the devices may incorporate different hardware for measuring acceleration and horizontal and vertical distances. Sampling rates, sample sizes, and measurement precision may differ from device to device also. The output from the hardware must be processed to generate profiles. The processing includes averaging, filtering and finally applying algorithms to the processed measurements. The processing techniques and algorithms employed could result in different profiles being generated by different devices. Additionally, coefficients used in the algorithms may be calibrated for each individual device. All of this could lead to different devices generating slightly different profiles but fairly consistent smoothness indices. Therefore, both with and between vendor profile comparisons were conducted to assess the repeatability of the devices and to determine if some of the between vendor differences in smoothness indices identified in the previous section could be related to differences in the profiles generated by the different devices. Between vendor profile comparisons provide the necessary information to determine whether the processing used in computing the indices

or the actual profiles used to calculate the indices are the cause of potential differences in index values observed between vendors. 


\subsubsection{Within Vendor Profile Comparisons}

The objective of the within vendor profile comparisons was simply to determine whether the replicate profiles generated by each lightweight profiler at each site were consistent. The individual replicate profiles generated by all the devices at one PCC site (Site 1 - Covington Street) and one HMA site (Site 2 - I-65 both with and without plywood strips) are presented in this section and discussed as typical examples. The profiles generated at the other sites by each device are also discussed. The profile plots associated with these sites are presented in Appendix E.

\subsubsection{Vendor A Profile Comparisons}

The five profiles generated by Vendor A at Site 1 (Covington Street) are presented in Figure 4-41. Note that the five profiles are offset by two inches on the vertical scale (y-axis) to allow for visual observation of consistency. This is noted in the figure by the notes "Y $=\mathrm{X}$ is the baseline." The five replicate profiles appear to be similar and they all show a maximum bump of approximately 0.4 inch at the start of the test section. When portions of the profiles were magnified for closer inspection, it was observed that the Run 3 profile differed the most from the others. However the magnitude of difference in the shape of the profiles was small and the smoothness indices associated with this profile were consistent with the others. The PCC pavement at this site had handtooled transverse joints spaced approximately fourteen feet apart. The small closely spaced spikes on the profiles coincide with the fourteen foot joint spacing. 


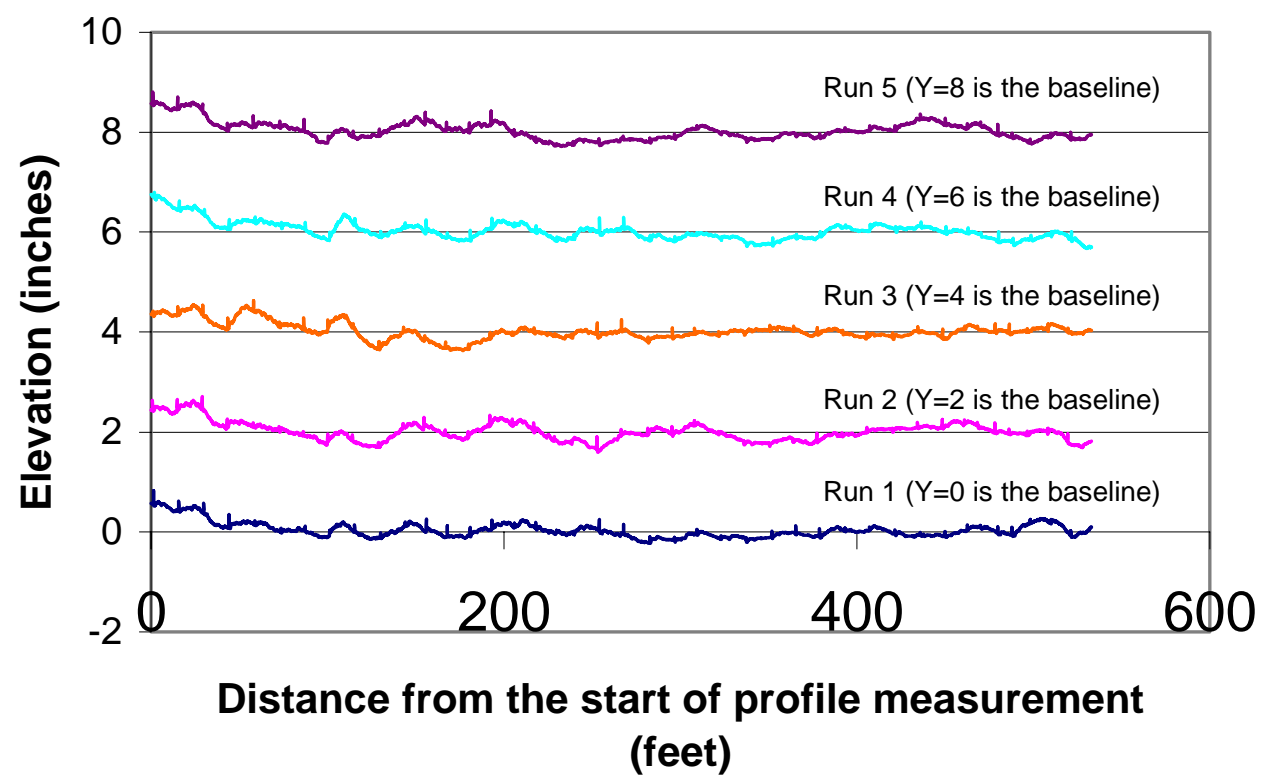

Fig 4-41 Profiles from Replicate Runs by Vendor A at Covington Street (Site 1)

At Site 2, I-65, five measurements were performed with the $3 / 4$ inch plywood strips in place and three were made without the strips. The profiles produced by Vendor A at this site are presented in Figure 4-42. The (wb) and (nb) notes on the figure indicate with and without plywood strips or boards, respectively. The measurements made with and without the plywood strips are clearly distinguishable on the figure. It is also clear from the figure that there is inconsistency within the profiles generated with the plywood in place. This is particularly noticeable in the magnitude of the large bumps centered around approximately 25 feet. It appears as though the plywood strip locations and magnitudes were identified at the beginning and end of the test section, but a large bump is present in the profiles following the strip at the start of the test section. Review of the profiles without the plywood strips clearly shows that no such bump existed. There is also 
considerable noise (small spikes) in the Run 5 profile. This, as well as the large bumps appearing at approximately 25 feet are likely due to the significant dynamics induces to the profiler by the plywood.

The magnitude of the large bump varies from 1.5 to 3 inches. These differences in the large bump magnitudes are reflected in the reported IRI values in that the IRI values associated with the small bumps (Runs 1 and 3) were approximately 35in/mi less than those associated with the large bumps (Runs 2, 4, and 5). Similar differences were not reported for the PI statistics however. This suggests that the processing used to calculate PI is either insensitive to such bumps, or more likely, the filtering used eliminated them.

The profiles generated without the plywood strips in place were very consistent and reflective of a very smooth pavement.

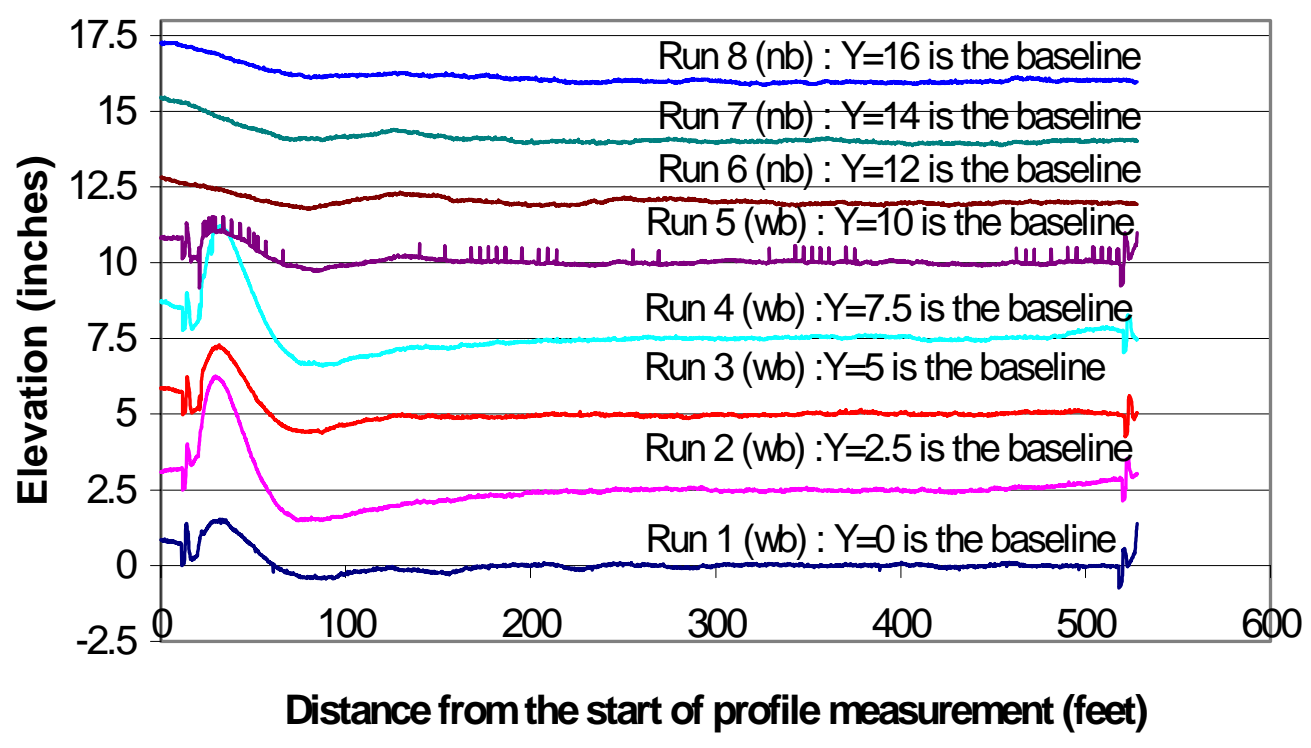

Figure 4-42 Profiles from Replicate Runs by Vendor A at I-65 (Site 2) with (wb) and without (nb) plywood strips 
The profiles associated with the Prairie Street, Vinton Street, and US231 sites are presented in Appendix E-1. The ten measurements (five each with and without 3/8 inch plywood strips) performed at Prairie Street (Site 3) by Vendor A generated similar profiles under each condition. Both the location and magnitude of the plywood strips were accurately identified. However, the profiles did not show large bumps following the thinner plywood strips. This may be due to the fact that the profiler dynamics induced by the thinner plywood strips were significantly less than those induce by the thick strips used at the I-65 site. As expected, the reported IRI values were slightly less when the plywood strips were removed. The reported PI values were essentially unaffected by the removal of the plywood strips.

The five profiles generated by Vendor A at Vinton Street (Site 4) were all very similar. There were unusual spikes in four of the profiles. Three had spikes at a distance of approximately 425 feet from the start of the section and one had a spike at a distance of approximately 275 feet from the start of the section. None of the other vendor profiles exhibited spikes or bumps/dips at these locations.

The five profiles generated by Vendor A at the US231A site (Site 5) were very consistent with the exception that spikes similar to those observed at the Covington Street site were observed somewhat randomly in the profiles. It is believed that the spikes occurred where joints were present in the PCC pavement. 
Similar observations were made for the five profiles generated by Vendor A at the US231B site (Site 6). Small differences were observed in the first fifty feet of the profiles, but beyond that they were very consistent. As with the other PCC sites, small spikes were observed at what are believed to have been joints. The standard deviation of the IRI values reported by Vendor A at this site was $5 \mathrm{in} / \mathrm{mi}$. At Site 5 , where similar profiles were observed but without the small differences in the first 50 feet of the section, the reported standard deviation on IRI was 0in/mi. Thus the differences in profile were reflected in reported IRI values.

In general, the profiles generated by Vendor A were very consistent with the exception of the small unexplained spikes in some of the profiles of HMA pavement at one site and the large bumps identified at the site with the thick plywood strips. The dynamics induced by the $3 / 4$ inch plywood strips were likely greater than the profiler could tolerate.

\subsubsection{Vendor B Profile Comparisons}

The profiles generated by Vendor B at the Covington Street site (Site 1) are presented in Figure 4-43. The profiles are all extremely consistent with the exception of the last 40 feet of the Run 4 profile which is relatively flat compared to the other profiles. This difference is not reflected in the reported IRI values for the site. It appears as though the hand-tooled joints in the PCC pavement which were observed in the Vendor A profiles were also observed in the Vendor B profiles. A maximum elevation change of 
about 2 inches is observed in all the profiles between a distance of 200 to 350 feet from the start of the profile measurements.

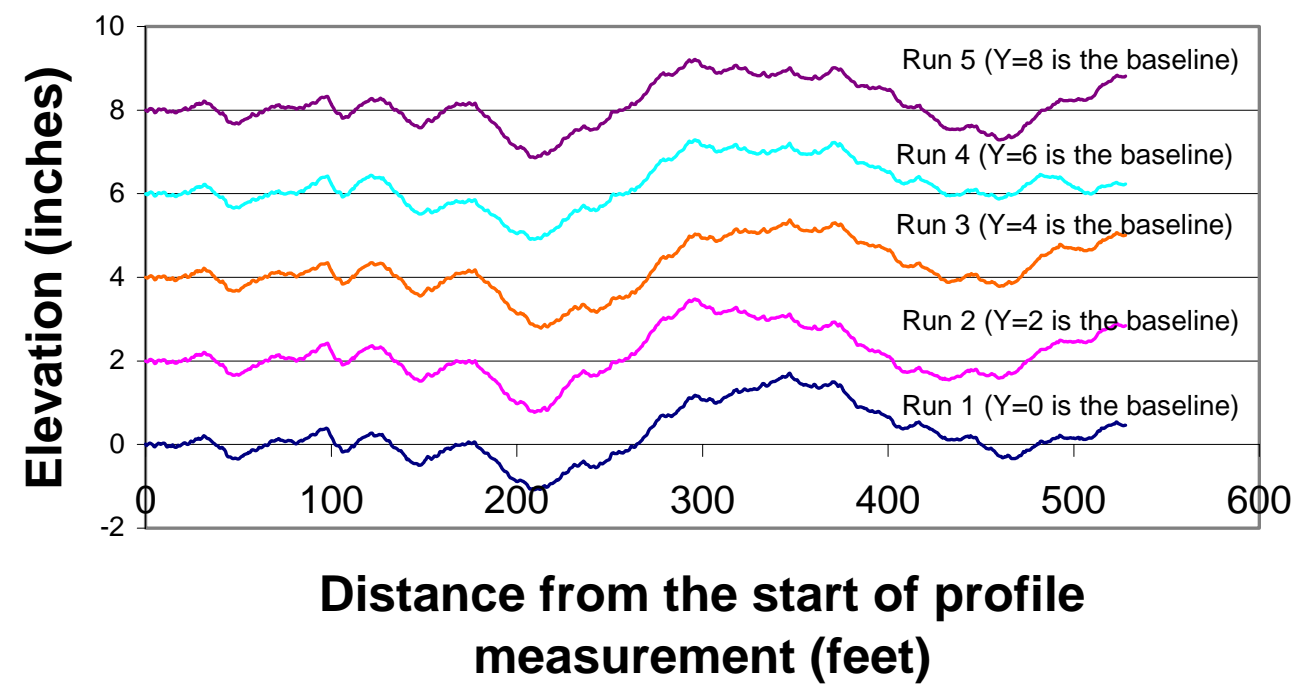

Figure 4-43 Profiles from Replicate Runs by Vendor B at Covington Street (Site 1)

The five profiles produced by Vendor B at the I-65 site with the $3 / 4$ inch plywood strips in place, as well as the three produced without the strips in place, were all consistent. All eight profiles are presented in Figure 4-44. The profiler correctly identified the locations and magnitude of the plywood strips at an approximate distance of 10 feet from the beginning and end of the test section. The IRI and PI statistics reported by the vendor all decreased when the strips were removed. 


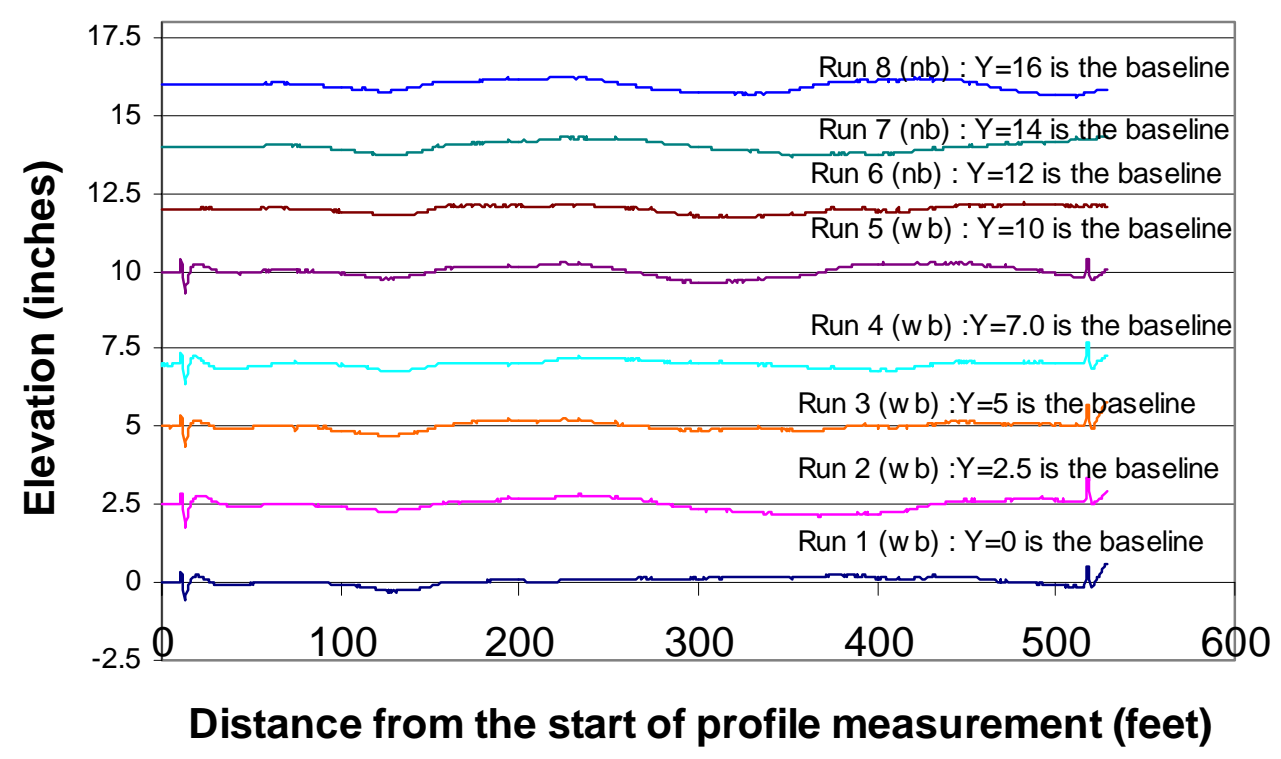

\section{Figure 4-44 Profiles from Replicate Runs by Vendor B at I-65 (Site 2) with (wb) and without plywood strips (Site 2b)}

The ten profiles produced by Vendor B at the Prairie Street site (Site 3) appear to be consistent. These profiles, as well as those associated with the Vinton Street and US231 sites are presented in Appendix E-2. However an unusual bump and/or dip of approximately 7 inches appear in all of the profiles. It is suspected that the accelerometer used by this device was adversely affected by the steep grade in combination with the curve and cross slope at the site. However, the reported smoothness statistics are similar to those reported by other vendors that did not generate unusual profiles at the site. The Vendor B profiles did reflect the thin plywood strips places near the beginning and end of the section. 
The five profiles generated by Vendor B at the Vinton Street site (Site 4) were very consistent, but they too show unusual bumps followed by a drop of approximately 7 inches in the last 150 feet of the profiles. It appears as though the geometric features of this site also had an impact on the accelerometer used by Vendor B. However the reported IRI and PI values for the site are consistent with those reported by the vendors which did do not appear to have been adversely impacted by the geometric features of the site.

Four of the five profiles generated by Vendor B at US231A (Site 5) were consistent. The first profile generated at this site shows a bump and/or dip of approximately 1 inch over a 100 foot distance which start approximately 50 feet into the test section. However, the bump/dip is not reflected in the reported smoothness statistics.

The five profiles produced by Vendor B at the US231B site (Site 6) were very consistent over the first 450 feet of the section, after which small differences were observed. The differences are not reflected in the reported smoothness statistics.

In general, the profiles generated by Vendor B were consistent with one exception in one profile observed at Site 5. However, it appears as though the geometric features at Sites 3 and 4 affected the performance of the accelerometer at these sites. The dynamics induced by the plywood strips at Sites 2 and 3 did not affect the performance of the profiler which accurately identified both the location and magnitude of the strips. 


\subsubsection{Vendor C Profile Comparisons}

Four of the five profiles generated by Vendor C at the Covington Street site (Site 1) are presented in Figure 4-45. One profile is missing due to a defective data file. The profiles are all extremely consistent as are the IRI and PI values reported for this site. The hand-tooled joints in the PCC pavement which were observed in the Vendor A and B profiles are also observed in the Vendor $\mathrm{C}$ profiles. A maximum elevation change of about 2 inches is observed in all of the profiles between a distance of 175 to 325 feet from the start of the profile measurements which is consistent with the other vendors.

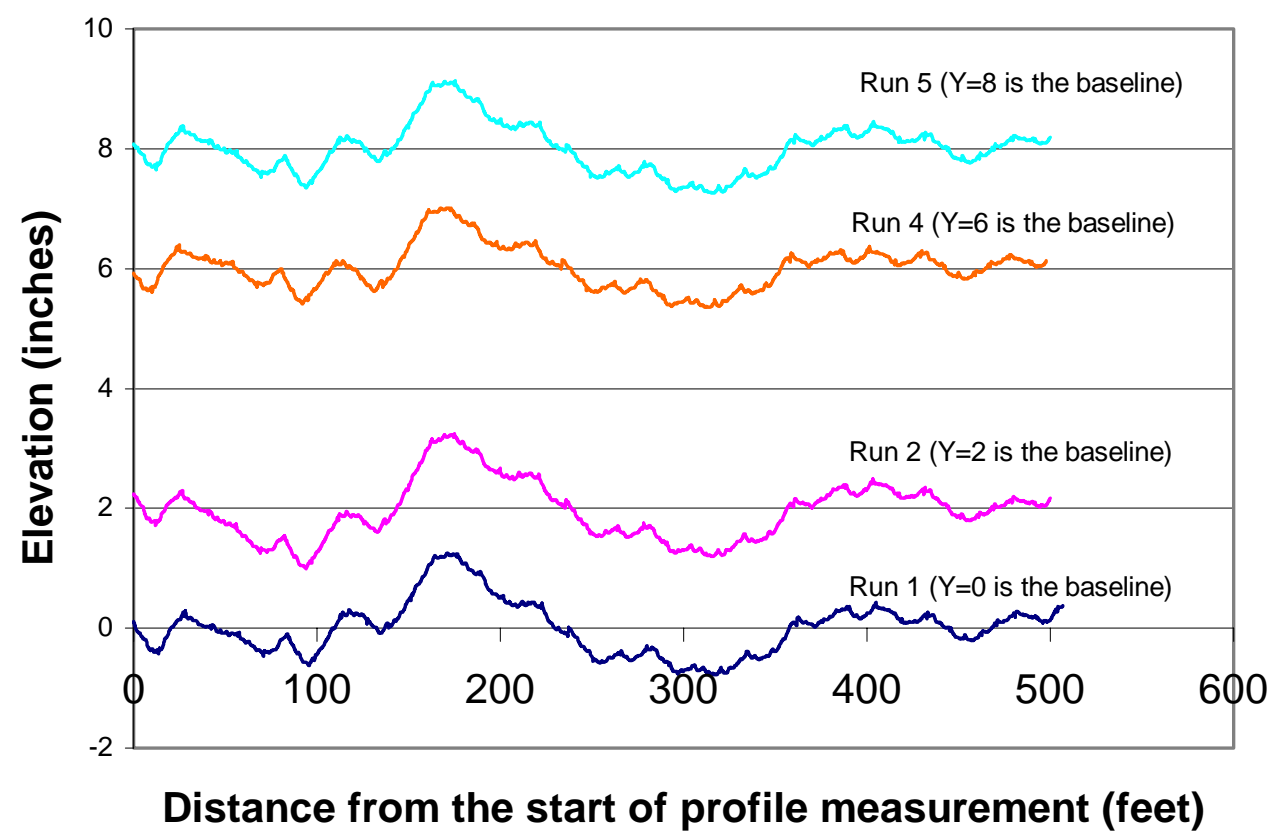

Figure 4-45 Profiles from Replicate Runs for Vendor C at Covington Street (Site 1) 
All the profiles generated by Vendor C at the I-65 Site (Site 2) both with and without the thick plywood strips in place appear to be very similar, as shown in Figure 446. This implied that the profiler was unable to identify the $3 / 4$ inch plywood strips placed in the wheel paths at the beginning and end of the test section. This is the only profiler among the four that did not identify the plywood strips. The reported IRI values for the site both with and without the strips in place were the same also. However small differences were reported in the PI data with and without the strips in place for this site.

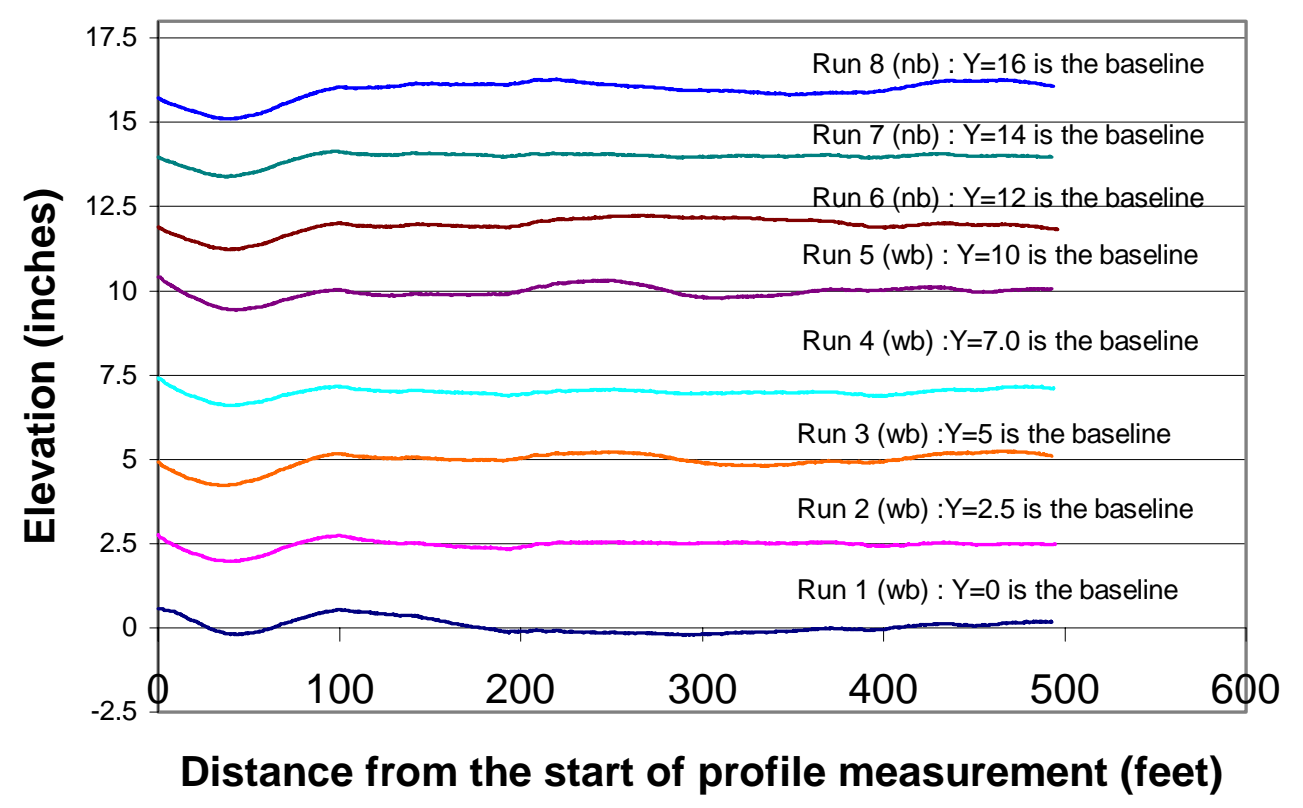

Figure 4-46 Profiles from Replicate Runs by Vendor C at I-65 (Site 2) with (wb) and without plywood strips (nb)

The ten profiles produced by Vendor C at the Prairie Street site (Site 3) were fairly consistent with the exception of a 75 foot portion starting approximately 275 feet 
from the beginning of the site. This is particularly noticeable in the Run 1 and Run 10 profiles. These profiles, as well as those associated with the Vinton Street and US231 sites are presented in Appendix E-3. An unusual bump and/or dip of approximately 7 inches appear in all of the profiles, much like those observed in the Vendor B profiles. It is suspected that the accelerometer used by this device was adversely affected by the steep grade in combination with the curve and cross slope at the site. However, the reported smoothness statistics are similar to those reported by other vendors that did not generate unusual profiles at the site. The Vendor $\mathrm{C}$ profiles did reflect the correct location and magnitude of the thin plywood strips placed near the beginning and end of the section.

Four of the five profiles generated by Vendor C at the Vinton Street site (Site 4) were very consistent. The one profile that differs from the others shows significantly less elevation change in the last 150 feet of the profile. All of the profiles show unusual bumps and dips through out the profiles. It appears as thought the geometric features of this site also had an impact on the accelerometer used by Vendor C. However the reported IRI and PI values for the site are fairly consistent with those reported by the vendors which did do not appear to have been adversely impacted by the geometric features of the site.

Four of the five profiles generated by Vendor C at US231A (Site 5) were consistent. The first profile generated at this site shows an increase in elevation of approximately 0.5 inch in the first 75 feet of the profile while all the other profile show a 
decrease in elevation of similar magnitude. However, this difference is not reflected in the reported smoothness indices.

The five profiles produced by Vendor C at the US231B site (Site 6) were very consistent and the reported smoothness index values reflect that.

In general, the profiles generated by Vendor $\mathrm{C}$ were consistent with the one of five or two of ten profile exceptions at Sites 3, 4, and 5. It appears as though the geometric features at Sites 3 and 4 affected the performance of the accelerometer at these sites. It also appears as though the dynamics induced by the plywood strips at Sites 2 affected the profiler performance because the thick plywood strips were not identified at this site. However, at Site 3 the location of the thin plywood strips were correctly identified.

\subsubsection{Vendor D Profile Comparisons}

The profiles generated by Vendor D at the Covington Street site (Site 1) are presented in Figure 4-47. The profiles are all extremely consistent. The hand-tooled joints in the PCC pavement which were observed in all of the other Vendors profiles were also observed in the Vendor D profiles. 


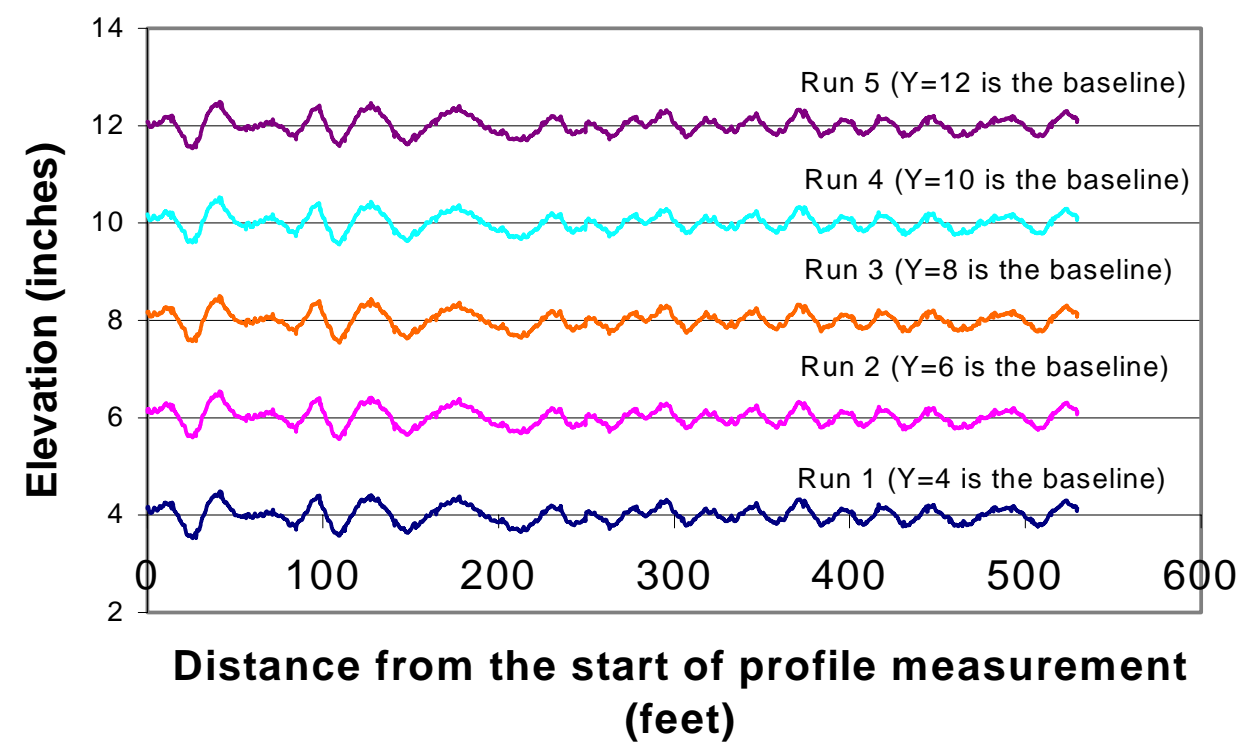

Figure 4-47 Profiles from Replicate Runs by Vendor D at Covington Street (Site 1)

All the profiles generated by the Vendor D at the I-65 Site (Site 2 and Site $2 b$ ) both with and without the thick plywood strips are extremely consistent as shown in Figure 4-48. The profiler accurately identified the location and magnitude of the $3 / 4$ inch plywood strips placed in the wheel paths at the beginning and end of the test section also. The reported IRI and PI values correctly reflect the plywood strips also in that they decrease when the strips were removed. The dynamics induced by the strips obviously did not affect the profiler performance. 


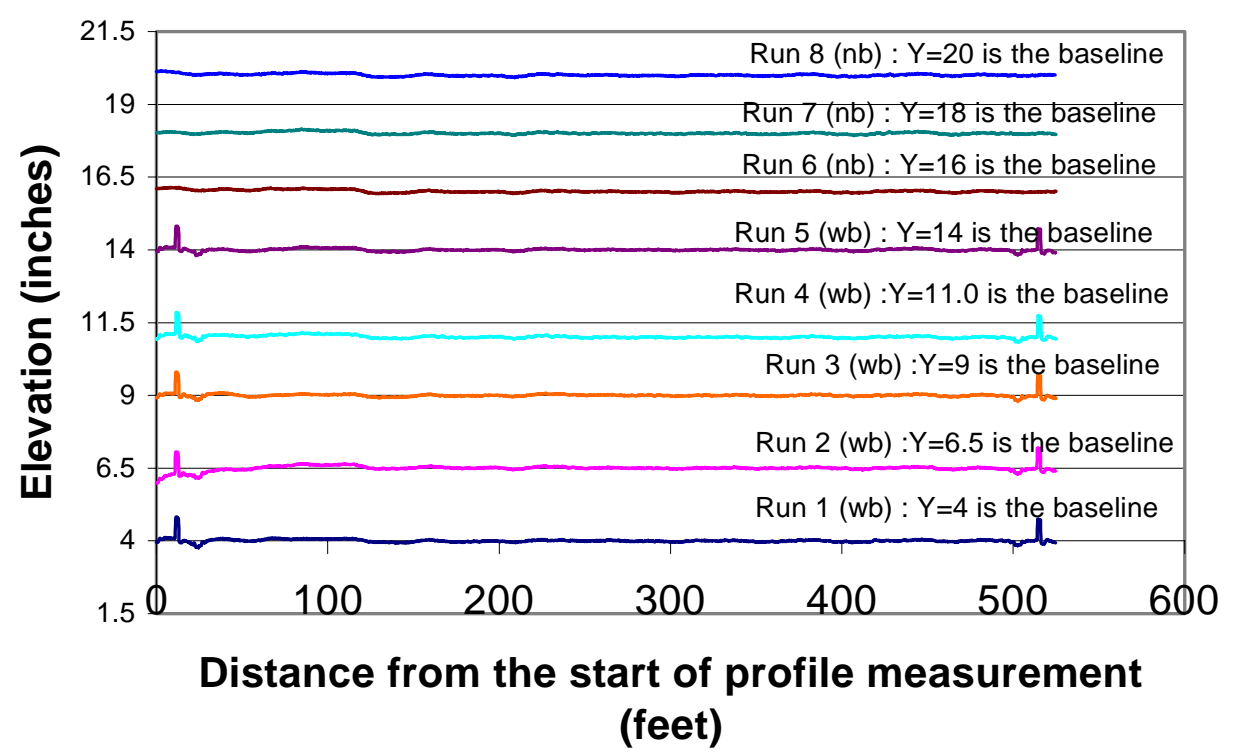

Figure 4-48 Profiles from Replicate Runs by Vendor D at I-65 (Site 2) with (wb) and without plywood strips (nb) (Site 2b)

The profiles generated by Vendor D at the Prairie Street, Vinton Street, and US231 sites are presented in Appendix E-4. The ten profiles produced by Vendor D at the Prairie Street site (Site 3) were also very consistent. The location and magnitude of the thin plywood strips used on this section were correctly identified in four of the five profiles generated with the strips in place. The geometric features at this site clearly did not adversely affect the device.

Four of the five profiles reported by Vendor D at the Vinton Street site (Site 4) were very consistent and the profiles do not show any unusual bumps and/or dips. One profile was simply an unexplained flat line. However, this is not reflected in the reported 
smoothness indices. It appears as thought the geometric features of this site did not adversely impact the accelerometer used by Vendor D either.

The five profiles produced by Vendor D at both of the US231 sites (Site 5 and 6) were very consistent. Strangely, one of the IRI values reported for Site 5 is approximately fifteen percent less than the other four reported values even though all five profiles appear to be nearly identical.

In general, the profiles generated by Vendor D were very consistent. They were the most consistent of all the vendors and they successfully identified the location and magnitude of both the thick and thin plywood strips used at Sites 2 and 3. The geometric features at Sites 3 and 4 did not affect the performance of the accelerometer used by Vendor D at these sites.

\subsubsection{Overall Within Vendor Profile Performance}

The within vendor profile comparisons are summarized in Table 4-39. All of the devices correctly identified the location and magnitude of the thin plywood strips. All of the devices with the exception of the Vendor $\mathrm{C}$ device correctly identified the location and magnitude of the thick plywood strips. It is important to note that the Vendor A profiles did show large fictitious bumps following the location of the thick plywood strips however. It is assumed this was a result of the dynamics induced into the profiler by the thick strips. Very unusual profiles were generated by Vendors B and C at the sites which incorporated steep grades and curves (geometric features). It is assumed that this was due 
to poor accelerometer performance under these conditions. Interestingly, the smoothness index values reported by these vendors at the sites with the geometric features were fairly consistent with those reported by the vendors with properly functioning accelerometers. An overall consistency ranking was assigned to each vendor based on these considerations, as is noted in the last column of Table 4-39. One thing that is alarming is that in several cases when individual profiles showed differences at a particular site the smoothness indices did not always reflect the differences, while in other cases when profiles appear to be similar, differences in smoothness indices were observed. This was observed for all vendors.

Table 4-39 Overall Within Vendor Profile Performance

\begin{tabular}{|c|c|c|c|c|}
\hline \multirow[t]{2}{*}{ Vendor } & \multicolumn{2}{|c|}{$\begin{array}{l}\text { Ability to Identify } \\
\text { Plywood Strips }\end{array}$} & \multirow{2}{*}{$\begin{array}{c}\text { Accelerometer Affected by } \\
\text { Geometric Features }\end{array}$} & \multirow{2}{*}{$\begin{array}{c}\text { Overall Consistency } \\
\text { Ranking }\end{array}$} \\
\hline & Location & Magnitude & & \\
\hline $\mathrm{A}$ & Yes & Yes & No & 3 \\
\hline B & Yes & Yes & Yes & 2 \\
\hline $\mathrm{C}$ & Yes* & Yes* & Yes & 4 \\
\hline $\mathrm{D}$ & Yes & Yes & No & 1 \\
\hline
\end{tabular}

- Only thin plywood strips were identified.

\subsubsection{Between Vendor Profile Comparisons}

In order to compare the profiles generated by each vendor, the five profiles generated by each vendor at each site were averaged and compared to each other. The profiles generated by the INDOT Rip Van were also included in the comparison. Additionally, at the Covington Street site the average vendor profiles were compared with the California Profilograph and the precision rod and level survey data collected at the site. 


\subsubsection{Covington Street (Site-1) Profile Comparison}

Figure 4-49 represents the average profile generated by each vendor along with the average California Profilograph trace and rod and level survey data. Each line on the figure represents the average of the five profiles measured by each vendor. The California Profilograph trace is the average of four measurements. The rod and level survey represents a single survey with a sampling interval of 12 inches $(0.3 \mathrm{~m})$, which is much greater than the interval used by the lightweight profilers.

The plot of the rod and level survey profile is much flatter than any of the profiles generated by the lightweight profilers. This may be partially due to the difference in sampling interval because the hand-tooled transverse joints were bridged with the rod and level survey. The profiles associated with all of the lightweight profilers appear to capture the hand-tooled joints and thus show undulations that are not present in the rod and level profile. However, the undulations are of significant magnitude when compared to the rod and level profile. The California Profilograph generated profile more closely resembles the rod and level profile than any of the lightweight profiler generated profiles. The Vendor A profile is the most similar to the rod and level of all the lightweight profilers, followed by the Vendor D profile. The Vendor B and C profiles both show significant deviations in elevation relative to the rod and level profile. The INDOT Rip Van profile shows approximately a six inch drop in elevation over the first 100 feet $(30 \mathrm{~m})$ of the test section and it deviates from the rod and level profile the most. 


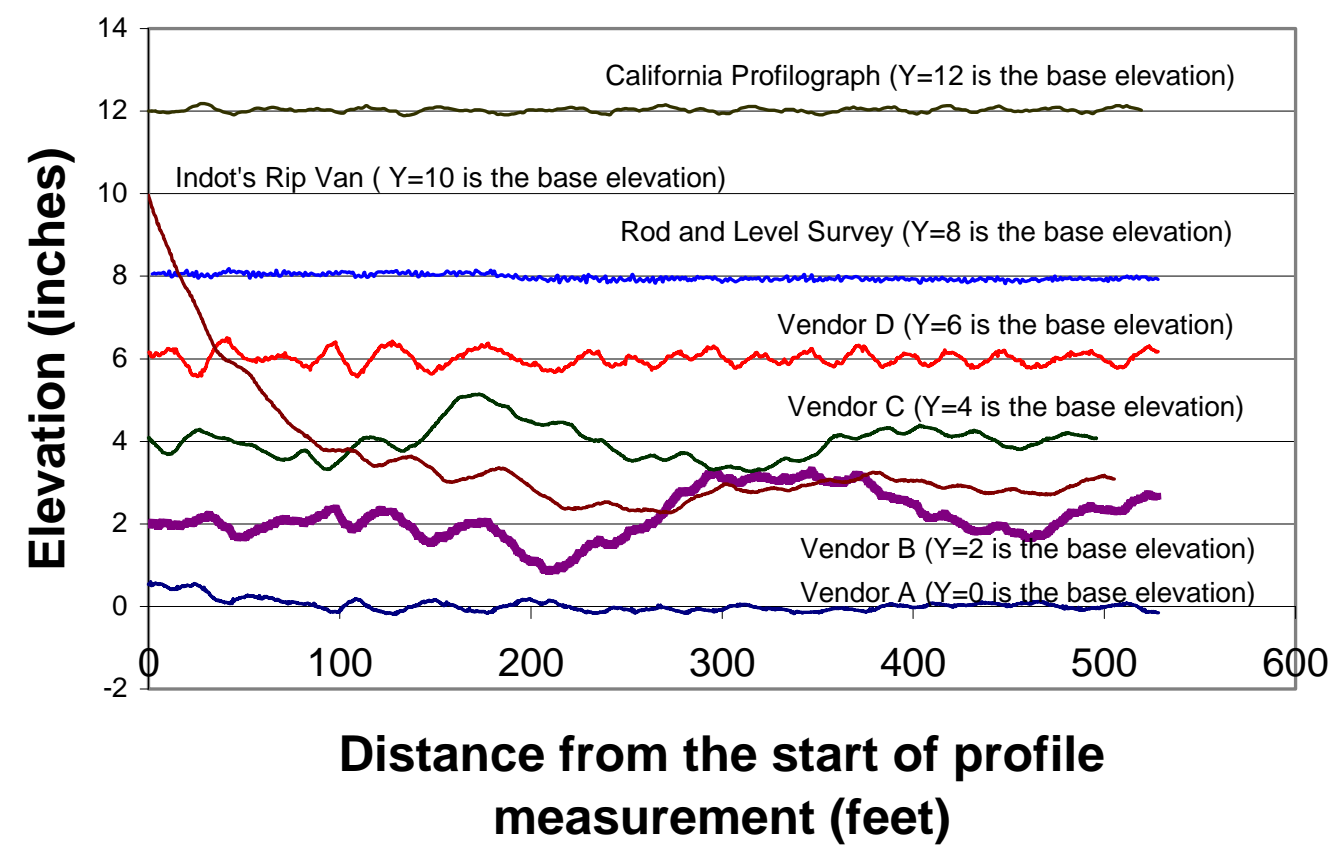

Figure 4-49 Between Vendor Profile Comparison for Covington Street (Site-1)

\subsubsection{I-65 (Site 2) with Plywood Strips Profile Comparison}

The average profiles generated by each lightweight profiler and the INDOT RIP Van at the I-65 site with the $3 / 4$ inch plywood strips in place are presented in Figure 4-50. Vendors A, B and D identified the strips at the correct locations. Vendor C and the Rip Van were unable to identify the strips. As previously noted, the dynamics induced by the bumps hampered the performance of both the Vendor $\mathrm{A}$ and $\mathrm{C}$ profilers. The plywood strips did not appear to induce significant dynamics to the INDOT RIP Van, so it is unclear whether or not they are part or all of the reason that the RIP Van did not identify the thick strips. The last 300 feet of all the profiles are actually fairly similar among the devices. 


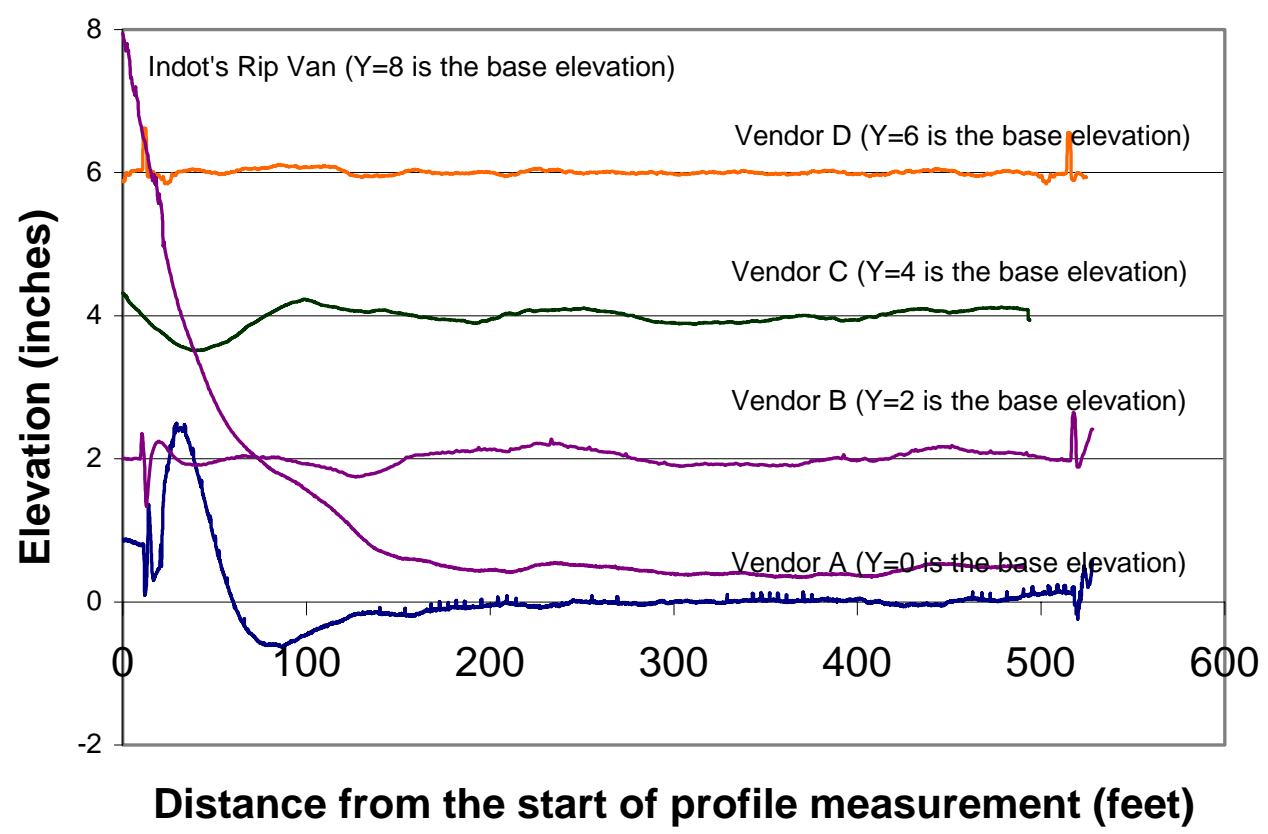

Fig 4-50 Between Vendors Profile Comparison for I-65 with Plywood Strips

(Site 2)

\subsubsection{I-65 (Site-2b) Without Plywood Strips Profile Comparison}

The average profiles generated with the thick plywood strips removed at the I-65 site are presented in Figure 4-51. Again, the last 300 feet of all of the profiles are similar. The Vendor B and D profiles are the most similar even though these vendors reported fairly different IRI and PI values for this site. The Vendor A and INDOT Rip Van profiles are similar in that they both show significant decreasing slopes in the first 100 feet of the section. Conversely, the Vendor $\mathrm{C}$ profile shows a significant dip in the first 100 feet. 


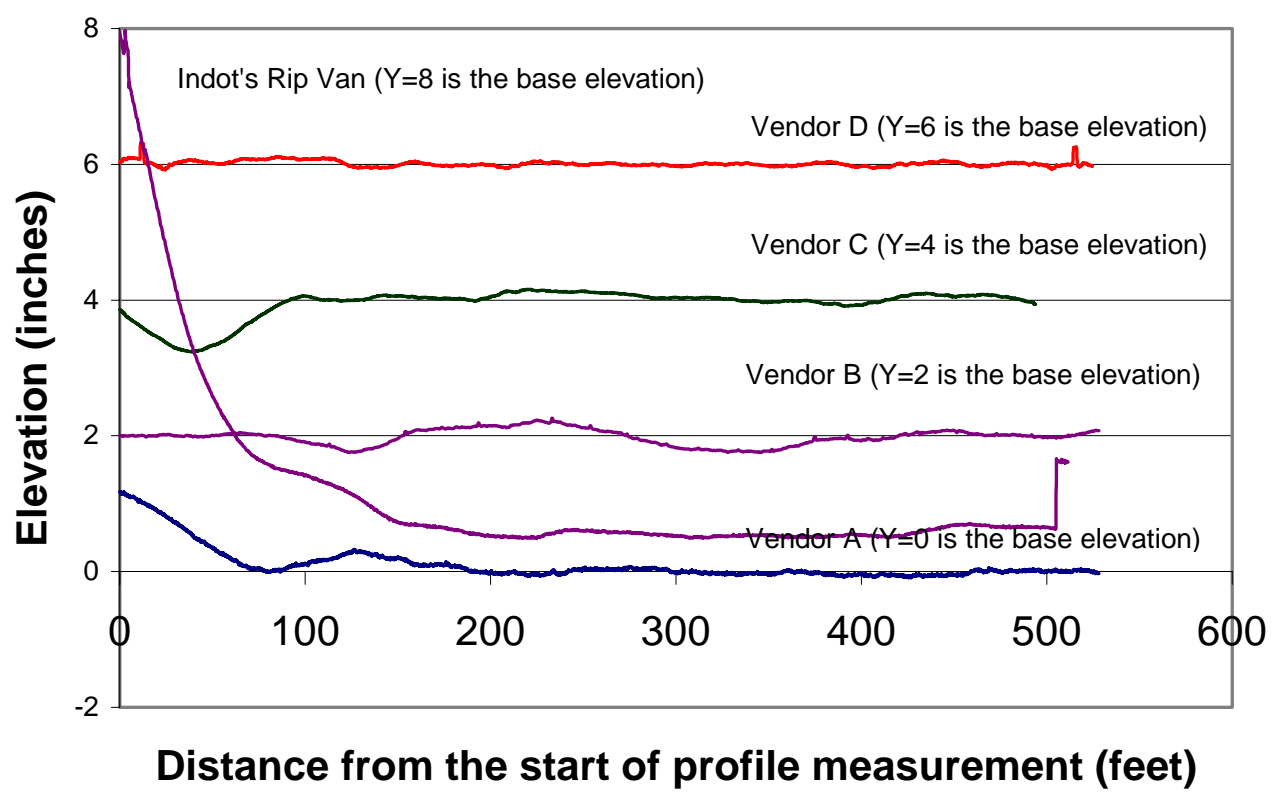

Figure 4-51 Between Vendors Profile Comparison for I-65 without Plywood Strips

(Site-2 without board)

\subsubsection{Prairie Street (Site-3) with Plywood Strips Profile Comparison}

The between vendor profile comparison plots for the Prairie Street, Vinton Street, and US231 sites are presented in Appendix F. As noted in the previous discussion of within vendor profile comparisons, the geometric features at the Prairie Street site significantly affected the performance of the Vendor B and D profilers resulting in very unusual profiles. However, these vendors reported IRI and PI values that were similar to those reported by Vendors A and D, neither of which were negatively impacted by the geometric features at the site. The average INDOT Rip Van profile is most similar to the Vendor B profile. It appears as though the performance of the accelerometer used on the van was also negatively impacted by the geometric features of the site. All of the 
lightweight profilers correctly identified the location of the thin plywood strips at the site even though this was masked to some degree when the replicate profiles provided by each vendor were averaged. The RIP Van profiles did not reflect the thin plywood strips. The Vendor A and D profiles appear to be the most similar and reasonable profiles for this site even though significantly different smoothness index values were reported by the vendor, particularly for PI- 0.0 ".

\subsubsection{Prairie Street (Site-3b) without Plywood Strips Profile Comparison}

The average profiles without the plywood strips in place at the Prairies Street site are very similar to the average profiles with the strips in place. Thus similar observations were made. The Vendor A and D profiles appear to be the most similar and reasonable profiles for this site even though significantly different smoothness index values were reported by the vendors. The Vendor B and C profiles, as well as the INDOT RIP Van profiles all show significant bumps and dips indicating that the performance of the accelerometers used by these devices were poor at this site due to geometric features.

\subsubsection{Vinton Street (Site 4) Profile Comparison}

The average profiles at the Vinton Street site show similar characteristics to those observed at the Prairie Street site. Again, similar observations were made. The Vendor A and $\mathrm{D}$ profiles appear to be the most similar and reasonable profiles for this site even though significantly different PI-0.0" values were reported by the vendors. The Vendor B and $\mathrm{C}$ profiles, as well as the INDOT RIP Van profiles all show significant bumps and 
dips indicating that the performance of the accelerometers used by these devices were poor at this site due to geometric features.

\subsubsection{US231A (Site 5) Profile Comparison}

The profiles generated by the lightweight profilers at the US231A site were all very similar. The PCC pavement was very smooth and the transverse joints in the PCC pavement were narrow sawn joints. The profiles do not show the undulations observed at the Covington Street site where the joints were hand-tooled. The lightweight profiler generated profiles all reflect the smoothness of this pavement. However, there were significant differences in the IRI values reported by the vendors for this site. The INDOT RIP Van exhibited the same significant decrease in elevation over the first 100 feet of the section as it did at all sites. Therefore, all of the profiles were similar at this site with the exception of the RIP Van profile. It could be stated that the profiles reflect a fairly good between vendor comparison for this site.

\subsubsection{US231B (Site 6) Profile Comparison}

The profiles generated by the lightweight profilers at the US231B site were all very similar. The PCC pavement was very smooth at this site, the joints were sawn, and the profiles reflect the smoothness of the pavement. This site did incorporate a slight downhill grade as well as a curve. However, these geometric features do not appear to have impacted the performance of the profilers. There were not significant differences in the IRI values reported among the vendors for this site, but there were some differences in both reported PI values. The INDOT RIP Van exhibited the same significant decrease 
in elevation over the first 100 feet of the section. Therefore, all of the profiles were similar at this site with the exception of the RIP Van profile and the profiles reflect a positive between lightweight profiler vendor comparison.

\subsubsection{Overall Between Vendor Profile Performance}

The between vendor profile comparisons showed that the different lightweight profilers generate somewhat similar profiles for smooth pavements, as was observed at Sites 2 without the plywood strips, 5 , and 6 . At rougher sites the profiles generated by the different devices exhibited greater differences. All of the lightweight profilers correctly identified the location and magnitude of thin plywood strips place in the wheel path. They all also correctly identified the location and magnitude of the thick plywood strips with the exception of the Vendor C. It is important to note that the Vendor A profiles did show large fictitious bumps following the location of the thick plywood strips however. It is assumed this was a result of the dynamics induced into the profiler by the strips. Very unusual profiles were generated by Vendors B and C at the sites which incorporated steep grades and curves (geometric features). It is assumed that this was due to poor accelerometer performance under these conditions. Interestingly, the smoothness index values reported by these vendors at the sites with the geometric features were fairly consistent with those reported by the vendors with properly functioning accelerometers. The profiles generated by the INDOT RIP Van were inconsistent with those generated by the lightweight profilers. 
Overall, the between vendor profile comparison was poor. This is the likely reason for the significant differences reported in smoothness indices between vendors. It is disturbing in some cases when different profilers showed differences in profiles at a particular site the smoothness indices did not always reflect the differences. Conversely, in some cases when the profiles appeared to be similar significant differences in smoothness indices were reported.

\section{$\underline{4.5 \text { Must Grind Locations }}$}

Almost all State smoothness specifications currently incorporate a bump provision. An additional feature of the lightweight profilers is the ability to identify must grind locations (bumps) given a maximum allowable bump specification. This is an excellent feature that is useful to the contractor for QC purposes and it also allows for inclusion of a bump parameter in specifications. A pavement may have a low IRI based on the whole section (e.g 0.1 inches/mile), but a small portion of it may have bumps, which would cause discomfort to motorists. Hence it would be very important that the bumps be identified and ground off.

Consistent with the current INDOT smoothness specification, a maximum bump of 0.3 inch was used to evaluate the performance of the lightweight profilers ( Section 401.18 “Indiana Smoothness Specification”). All the vendors except Vendor C provided must grind locations by station. It was very encouraging to see that all the must grind stations identified in replicate measurements for a particular vendor were similar. This implied that the repeatability in the identification of must grind locations was good for 
the lightweight profilers. A summary of the bump locations identified by each vendor at each site has been provided in Table 4-40. The must grind locations provided in the table incorporate the stations which have been identified by the profiler in more than one of its five replicate measurements at a particular site. The distances are not necessarily identical, but they are typically within one foot of each other. The Vendor A profiler detected significantly fewer must grind locations than the other two devices. The identified must grind locations for the other two devices were only similar.

Table 4-40 Comparison of Must Grind Locations identified by each Vendor

\begin{tabular}{|c|c|c|c|}
\hline \multirow{2}{*}{$\begin{array}{c}\text { Site number and } \\
\text { description }\end{array}$} & \multicolumn{3}{|c|}{ Must grind locations (in feet from the start of profile measurement) } \\
\hline & Vendor A & Vendor B & Vendor D \\
\hline $\begin{array}{c}1 \\
\text { Covington }\end{array}$ & no defects detected & $\begin{array}{l}33,35.5,98.6,96,123, \\
128.5,132.5,232.5,236.5, \\
297.5,348,372.5, \\
375.5,397,398.5,421.5, \\
418,448.5,494\end{array}$ & $\begin{array}{l}16.0,43.0,99.0, \\
130.0,233.0,299.0, \\
350.0,374.5,421.0, \\
451.0,527.5\end{array}$ \\
\hline $\begin{array}{c}2 \\
\text { I-65 (with board) }\end{array}$ & 34 & no defects detected & $13.5,19.5$ \\
\hline $\begin{array}{c}2 b \\
\text { I-65 (without board) }\end{array}$ & no defects detected & no defects detected & no defects detected \\
\hline $\begin{array}{c}3 \\
\begin{array}{c}3 \\
\text { Prairie Street } \\
\text { (with board) }\end{array}\end{array}$ & 26,314 & $\begin{array}{l}\text { 29,101.5,194.5,218, } \\
217,221.5,219,226.5,298 \\
.5,299.5,408.5,413, \\
501.5,518.5\end{array}$ & $\begin{array}{l}\text { 16.5, 76.5, 101.0, } \\
\text { 188.0, 211.0, 290.0, } \\
343.5,405.0\end{array}$ \\
\hline $\begin{array}{c}3 \mathrm{~b} \\
\text { Prairie Street (without } \\
\text { board) }\end{array}$ & 27,314 & $\begin{array}{l}29,101.5,194.5,218, \\
217,221.5,219,226.5, \\
298.5,299.5,408.5,413, \\
501.5,518.5\end{array}$ & $\begin{array}{l}16.5,100.5,188.0, \\
210.5,290.0,343.5 \text {, } \\
404.5,491.5\end{array}$ \\
\hline $\begin{array}{c}4 \\
\text { Vinton Street }\end{array}$ & no defects detected & 353,354 & 353.5 \\
\hline $\begin{array}{c}5 \\
\text { US } 231 \mathrm{~A}\end{array}$ & no defects detected & no defects detected & no defects detected \\
\hline $\begin{array}{c}6 \\
\text { US 231B }\end{array}$ & no defects detected & no defects detected & no defects detected \\
\hline
\end{tabular}


The repeatability performance of devices was good. As an example the must grind locations reported by Vendor D for each replicate measuremenst at each site are presented in Table 4-41 to illustrate this above fact. Vendors A and B also exhibited similar good repeatability performance. The values presented in the table are the distance to the must grind location from the start of the profile measurement.

As with the smoothness indices, the between vendor performance was poor. Based on the comparison of profiles presented in Section 4.4, this is not surprising. 
Table 4-41 Repeatability performance in the identification of must grind locations

for Vendor D

\begin{tabular}{|c|c|c|}
\hline Site & $\begin{array}{c}\text { Run } \\
\text { no }\end{array}$ & $\begin{array}{l}\text { Must Grind Locations } \\
\text { (in feet from the start of profile measurement) }\end{array}$ \\
\hline \multirow{5}{*}{$\begin{array}{c}1 \\
\text { Covington }\end{array}$} & 1 & $15.5,42.5,99,129,233,299,350,374.5,421,451.5$ \\
\hline & 2 & $15.5,43,99,129.5,233.5,299,350,375,421,451.5,527.5$ \\
\hline & 3 & $15.5,43,99,130,233,299.5,321.5,350,374.5,421,451$ \\
\hline & 4 & $16,43,99,130,233,299,350,374.5,421,451,527.5$ \\
\hline & 5 & $15.5,40,99,129.5,233.5,299,350,374.5,421,451,527.5$ \\
\hline \multirow{5}{*}{$\begin{array}{l}2 \\
\text { I-65 (with board) }\end{array}$} & 1 & $13.5,519.5$ \\
\hline & 2 & $13.5,519.5$ \\
\hline & 3 & $13.5,519.5$ \\
\hline & 4 & $13.5,520$ \\
\hline & 5 & $13.5,519$ \\
\hline \multirow{3}{*}{$\begin{array}{c}2 \mathrm{~b} \\
\mathrm{I}-65 \text { (no board) }\end{array}$} & 1 & No defects detected \\
\hline & 2 & No defects detected \\
\hline & 3 & No defects detected \\
\hline \multirow{5}{*}{$\begin{array}{c}3 \\
\text { Prairie Street (with board) }\end{array}$} & 1 & $17.0,82.0,101.0,188.0,210.0,289.5,343.5,405.0,494.0$ \\
\hline & 2 & $16.5,52.0,101.0,188.0,212.0,290.0,344.0,405.0$ \\
\hline & 3 & $15.5,76.5,100.5,187.5,210.5,344.0,405.0$ \\
\hline & 4 & $16.5,76.5,101.0,188.0,211.0,290.0,343.5,405.0$ \\
\hline & 5 & $16.5,76.5,102.0,187.5,210.5,342.5,404.5$ \\
\hline \multirow{5}{*}{$\begin{array}{c}3 \mathrm{~b} \\
\text { Prairie Street (no board) }\end{array}$} & 1 & $16.5,100.5,188.0,211.5,290.0,343.5,404.5$ \\
\hline & 2 & $17,101,188.0,211.5,242.0,290.0,344.0,405.0,492.0$ \\
\hline & 3 & $16.5,100.5,188,210.5,290,343.5,404.5,491.5$ \\
\hline & 4 & $16.5,76.5,101,187.5,210.5,341.0,404.5$ \\
\hline & 5 & $16.5,76.5,100.5,188.5,210.5,290,341.5,404.5$ \\
\hline \multirow{5}{*}{$\begin{array}{c}4 \\
\text { Vinton Street }\end{array}$} & 1 & 353.5 \\
\hline & 2 & 353.0 \\
\hline & 3 & 353.0 \\
\hline & 4 & 353.0 \\
\hline & 5 & 354.0 \\
\hline \multirow{5}{*}{$\begin{array}{c}5 \\
\text { US } 231 \mathrm{~A}\end{array}$} & 1 & No defects detected \\
\hline & 2 & No defects detected \\
\hline & 3 & No defects detected \\
\hline & 4 & No defects detected \\
\hline & 5 & No defects detected \\
\hline \multirow{5}{*}{$\begin{array}{c}6 \\
\text { US } 231 \mathrm{~B}\end{array}$} & 1 & No defects detected \\
\hline & 2 & No defects detected \\
\hline & 3 & No defects detected \\
\hline & 4 & No defects detected \\
\hline & 5 & No defects detected \\
\hline
\end{tabular}




\section{PRECISION ANALYSIS}

In order to develop a working specification three items must initially be established; specifically:

1. A definition of the pavement characteristic or property to be specified;

2. A technique and equipment to measure the characteristic or property (standard test method); and

3. The precision of the standard test method.

Relative to this study the pavement characteristics that could potential be specified are IRI, PI-0.0”, or PI-0.2". The formal standard test method specific to lightweight profilers does not currently exist, however a hybrid protocol was used for the field testing conducted. The precision of the test method used can be derived from the data generated during the field testing. This chapter provides details of the development of precision statements in accordance with the techniques specified by the American Society for Testing and Materials (ASTM) for each potential pavement characteristic (IRI, PI-0.0” and PI-0.2").

A description of the standard requirements of and procedures used to develop a precision statement are provided in subsequent sections. This is followed by the highlights of the development of five precision statements. 


\subsection{Test Method Precision}

When tests are performed on presumably identical materials under presumably identical circumstances it is not likely that identical results will be obtained. The difference in results is attributed to unavoidable random errors inherent in every test procedure. In other words, the factors that influence the outcome of a test cannot all be completely controlled. For practical interpretation of test results, this inherent variability must be accounted for. As an example, the difference between a test result and some specified value might be within that which can be expected due to unavoidable random error, in which case real deviation from the specified value has not been demonstrated. Similarly, the difference between test results on two batches of material will not indicate a fundamental quality difference if the difference is no greater than that which may be attributed to inherent variability in the test procedure.

Factors which may contribute to the variability associated with the application of a test method include: the operator; the equipment used; equipment calibration; and the environment. In the case of pavement smoothness measurements all of these factors may contribute to variability and the potential impact of each factor was discussed in Section 2.4 .

An ASTM precision statement normally contains elements of single-operator precision and multilaboratory precision (C 670-91a, “ Standard Practice for Preparing Precision and Bias Statements for Test Methods to Construction Materials”, Annual Book of ASTM Standards, Vol. 04.02.). Single-operator precision is a measure of the greatest 
difference between two results that would be considered acceptable when properly conducted repetitive determinations are made on the same material (site in the case of this study) by a single competent operator (vendor in the case of this study). Multilaboratory precision is a measure of the range (greatest difference between two test results) that would be considered acceptable when properly conducted determinations are made by two different operators in different laboratories (using different devices in the case of this study) on portions of a material (site) that are intended to be identical, or at least as nearly identical as possible. Single-operator precision is sometimes referred to as "repeatability" or within-laboratory precision, while multilaboratory precision is sometimes referred to as "reproducibility" or between-laboratory precision.

The fundamental statistic underlying all indexes of precision is the standard deviation of the population of measurements, termed the "one-sigma limit" and abbreviated "1S" (C 802-96, "Standard Practice for Conducting an Interlaboratory Test Program to Determine the Precision of Test Methods for Construction Materials”, Annual Book of ASTM Standards, Vol. 04.02.). It is an indication of the variability of a large group of individual test results obtained under similar conditions. One-sigma limits are determined for both single-operator and multilaboratory conditions. In some cases it is appropriate to use the coefficient of variation in place of the standard deviation as the fundamental statistic. The statistic is termed the "one-sigma limit in percents" and is abbreviated "1S\%." It is simply the standard deviation (1S) divided by the average of the measurements expressed as a percent. 
The "difference two-sigma limit" (D2S) or "difference two-sigma limit in percent" (D2S\%) has been selected as the appropriate index of precision for establishing the acceptable difference between two results. The indexes provide a maximum acceptable difference between two results on test portions of the same material. The D2S index equals the difference between two individual test results that would be equaled or exceeded in the long run in only 1 case of 20 (5 percent of the time) under the normal and correct operation of the test method. The D2S\% index equals the difference between two individual test results, expressed as a percentage of their average, that would be equaled or exceeded in the long run in only 1 case of 20 under the normal and correct operation of the test method. The D2S and D2S\% indexes are determined by multiplying the $1 \mathrm{~S}$ and $1 \mathrm{~S} \%$ indexes by a factor of $2 \sqrt{ } 2$.

Bias may be defined as systematic error inherent in the test method that contributes to the difference between a population mean of the measurements or test results and an accepted reference or true value. In all test methods, tolerances are placed on the accuracy of measuring equipment. All tests performed with a given set of equipment that has an error within the permitted tolerance will produce results with a small consistent bias, but the bias is not inherent in the test method, nor is it included in the bias statement for the method.

Two conditions that permit the bias of a test method to be established are:

1. A standard reference sample of known value has been measured by the test method; and 
2. The test method has been applied to a sample which has been compounded in a manner such that the true value of the property being measured is known.

Determining whether a potential reference sample is suitable for this purpose requires judgement. Rarely is there a reference material (value) available for a given test method; this is the case with the smoothness indices. When a reference is not available, that must be indicated in a precision statement along with a statement saying that no statement can be made on bias.

In order to be valid, precision indexes included in a precision statement as guides for the operator must be based on estimates of the precision of the test method obtained from a statistically designed series of tests. This is termed an "interlaboratory test program" by ASTM. ASTM C 802-96, "Conducting an Interlaboratory Test Program to Determine the Precision of Test Methods for Construction Materials," recommends that at least ten participating laboratories (vendors) be included, a minimum of 3 materials (sites) be used, and a minimum of 3 replicate determinations be made on each site (material) by each vendor (laboratory) (C 802-96, "Standard Practice for Conducting an Interlaboratory Test Program to Determine the Precision of Test Methods for Construction Materials", Annual Book of ASTM Standards, Vol. 04.02.). The experimental design was discussed in Section 3.2. Because only four vendors could participate in the experiment the number of sites and replicate measurements were increased to ensure that adequate degrees of freedom existed in the experiment to fulfill the minimum requirements established by ASTM. 
Recall that five replicate measurements were performed at each of six test sites by each of four vendors. This combination fulfills all the requirements of ASTM C 802-96. As a minimum, thirty degrees of freedom are necessary to obtain reliable estimates of precision. The experiment developed provided sixty [(4-1 vendors) $\mathrm{x}(6-1$ sites $) \times(5-1$ measurements/vendor/site)] degrees of freedom. The reality is even greater degrees of freedom were present in the situation where sites were tested with and without plywood strips present.

ASTM C 670-96, "Standard Practice for Preparing Precision and Bias Statements for Test Methods for Construction Materials," provides guidance in preparing precision and bias statements for ASTM test methods pertaining to certain construction materials. The standard also recommends forms for precision and bias statements. ASTM C 802-96 is the companion method that states minimum requirements for the interlaboratory test program which generates the data to be analyzed in accordance with ASTM C 670-96.

ASTM E 691-92, "Standard Practice for Conducting an Interlaboratory Study to Determine the Precision of a Test Method," is an ASTM standard that provides adequate information for formulating the precision statement of a test method. A PC-based software version of the standard is available that performs the calculation of the statistical terms needed to formulate a precision statement. The statistics generated may then be incorporated into the formats specified in ASTM C 670-96 and C 802-96. The ASTM E691-92 software was employed for all analyses presented in this report. 


\subsubsection{Application of Analysis of Variance (ANOVA) in Generating Precision Indices}

The treatment and analysis of interlaboratory study test results has three primary purposes:

1. To determine whether the data collected are adequately consistent to form the basis for a test method precision statement;

2. To investigate and act on any data considered to be inconsistent; and

3. To obtain the precision statistics from which the precision statement may be based. A one-way analysis of variance (within and between vendors) is conducted separately for each level (site) to obtain estimates of the precision statement. The underlying assumptions of an analysis of variance are:

1. Samples taken from the population under consideration are independent of one another;

2. Populations are normally distributed; and

3. Population standard deviations are equal (equal variance) (Neter, Wasserman, Nachtsheim and Kutner, 1996).

Because severely-outlying data may violate these assumptions and invalidate an analysis, it is necessary to examine variability of the data as a first step in the data analysis process.

The ASTM E 691-92 software was used to perform ANOVA. The software generates consistency statistics and provides the information necessary for formulation of precision statements. The first step in the analysis is to evaluate the consistency of the data. Both between and within vendor consistencies must be considered. The $h$-statistic is 
used to evaluate between-vendor consistency and the $k$-statistic is used to evaluate within vendor consistency.

The $h$-statistic is an indicator of how one vendor's average, for a particular site, compares with the average of all the other vendors, similar to the common $t$-statistic. The critical $h$-value used for comparisons is actually determined from an equation derived from an unpaired $t$-test. The critical $h$-value is calculated using published values of Student's $t$ at the 0.5 percent two tail significance level for the selected number of vendors.

The $k$-statistic is an indicator of how one vendor's within-vendor variability, under repeatability conditions, at a particular site, compares with all of the vendors combined. The $k$-statistic is similar to the common $F$ ratio and is actually a function of the $F$ statistic. A $k$-value larger than one indicates greater within vendor variability than the average for all laboratories. The critical $k$-value is calculated from the upper critical value of $F$ at the 0.5 percent significance level for the selected combination of number of test replicates and vendors.

The consistency statistics may be presented as a function of vendor or site by plotting. The $h$-statistic comparison will identify a vendor that is consistently reporting lower (negative) values compared to the other vendors reporting higher (positive) values or vice versa for a particular site or more likely for all sites. Data distributed on either side of zero are a sign of normal variability. The $k$-statistic comparison will identify a 
vendor that lacks within vendor precision relative to the other vendors, as indicated by consistently high $k$-values. The $h$ and $k$-statistics are evaluated by reviewing the plots by vendor and site concurrently. In some cases, review of a plot by vendor will show several $h$ or $k$-values near the critical value for a given vendor. Review of the corresponding plot by site may or may not show that for a given site the vendor is consistent with the other vendors. There is reason for concern when both plots by vendor and site show high values for a given vendor.

\subsubsection{Basis of Precision Statements}

Precision statements are based on two fundamental statistics, the repeatability $\left(\mathrm{s}_{\mathrm{r}}\right)$ and reproducibility $\left(\mathrm{S}_{\mathrm{R}}\right)$ standard deviations or single-operator and multilaboratory onesigma limits. The relationship between the average level of the measured property for different sites and the within and between laboratory standard deviations dictate the appropriate form of a precision statement. There are three main forms of this relationship:

1. The situation where the standard deviation is relatively constant over the range of sites;

2. The situation where the standard deviation has an approximately linear relationship with the average level and the coefficient of variation is relatively constant; and

3. The situation where the sites fall into two or more distinct groups within which either Form 1 or 2 holds approximately.

Coefficient of variation, expressed as a percent, is the ratio of standard deviation to the average times 100. The simplest method of determining the form of the 
relationship is to plot the standard deviations and coefficients of variation against the average levels measured. One of the reasons for plotting against the average levels measured is to determine whether a single statement is valid regardless of level or if statements for ranges of levels are required.

In the situation where the standard deviation is relatively constant over the range of materials (Form 1), the pooled within-laboratory standard deviation over all sites becomes the single-operator standard deviation or one-sigma limit (1S) and the pooled between-laboratory standard deviation becomes the multilaboratory standard deviation or one-sigma limit (1S) as described in ASTM C 670-96. In the situation where the standard deviation has an approximately linear relationship with the average level and the coefficient of variation is relatively constant (Form 2), the average within-laboratory coefficient of variation becomes the single-operator one-sigma limit in percent (1S\%) and the average between-laboratory coefficient of variation becomes the multilaboratory onesigma limit in percent (1S\%) as described in ASTM C 670-96. In the situation where the materials fall into two or more distinct groups within which either Form 1 or 2 approximately holds, the single-operator and multilaboratory one-sigma limits are established for each range using the appropriate form. If irregular or non-linear relations occur which do not fit any of the main forms, the largest estimate of standard deviation or coefficient of variation is used to establish the one-sigma limits. 


\subsubsection{Basis of Acceptable Range of Results}

Single-operator and multilaboratory acceptable range of results limits (difference two-sigma limits) are established at the 95 pecent reliability level by multiplying the repeatability and reproducibility standard deviations by $2 \sqrt{ } 2$ or 2.828 . The acceptable range of results limits are the most important components of a precision statement. They are what is referred to determine if differences in test results are due simply to variability associated with the test method used to measure them or due to true differences in quality.

\subsection{Development of Precision Statements}

Precision statements were developed for five cases. The first three cases are based on data obtained from the field tests conducted near West Lafayette, Indiana. The fourth case is based on data from a field test recently conducted in Connecticut using the same four lightweight profilers employed in Indiana. The last case is based on pooling of the data from the field tests conducted in both Indiana and Connecticut. For the first three cases (Indiana data) precision is assessed for IRI, PI-0.0”, and PI-0.2”. IRI was the only index considered in the testing conducted in Connecticut. Therefore, in the final two cases precision is only assessed for IRI.

\subsubsection{Case 1: Precision Based on Indiana Generated Data with Boards}

For this analysis all of the data generated in Indiana was considered. In other words, the data generated at the sites both with and without plywood strips (boards) in place were included in the analysis. Including the with and without board data effectively 
increased the degrees of freedom as the two data sets (with and without board for a given site) are treated as separate sites. All three smoothness indices were considered in the analysis also. The data employed is summarized in Table 5-1. The experiment exceeded the requirements of ASTM C802-96. One way ANOVA were conducted separately for the data associated with each site using the ASTM E691-92 software. The data were then summarized in the form of a precision statement in accordance with ASTM C670-96. Critical values of $\mathrm{h}$ - and k-statistic were chosen at the 0.5 percent significance level. The critical values of $\mathrm{h}$ and $\mathrm{k}$ were 1.49 and 1.66 , respectively for the conditions outlined in Table 5-1.

Table 5-1 Summary of Case 1 Precision Analysis Considerations

\begin{tabular}{|c|c|c|c|c|c|}
\hline $\begin{array}{c}\text { Number of } \\
\text { Vendors }\end{array}$ & $\begin{array}{c}\text { Number } \\
\text { of Sites }\end{array}$ & $\begin{array}{c}\text { Replicate } \\
\text { Measurements } \\
\text { per Site }\end{array}$ & $\begin{array}{c}\text { With Board } \\
\text { Data } \\
\text { Included? }\end{array}$ & $\begin{array}{c}\text { Smoothness } \\
\text { Statistics } \\
\text { Considered }\end{array}$ & $\begin{array}{c}\text { Total } \\
\text { Degrees } \\
\text { of } \\
\text { Freedom }\end{array}$ \\
\hline 4 & 8 & 5 & Yes & $\begin{array}{c}\text { IRI, PI-0.0”, } \\
\text { PI-0.2” }\end{array}$ & 84 \\
\hline (A, B, C, D) & 8 & 5 & &
\end{tabular}

\subsubsection{Analysis of IRI Data}

Figure 5-1 is a plot of the individual reported IRI values by each vendor at each site. The plot also includes the average IRI for all the vendors at each site. The only initial observation that can be made from the plot is that Vendor A reported significantly different IRI values at Site 2 than the other vendors and that there is significant range within the values reported by Vendor A for that site. Site 2 was the I-65 site with the $3 / 4$ " thick plywood strips in place approximately ten feet from the beginning and end of the 
test section which induced significant dynamics into the profilers. Figures 5-2 and 5-3 are the $\mathrm{h}$ and $\mathrm{k}$ plots by vendor and site, respectively for IRI. Figure 5-2 shows that the $\mathrm{h}$ values associated with Vendor A are typically the opposite in sign (+ vs. -) compared to the other vendors. Additionally, many of the $\mathrm{h}$ and $\mathrm{k}$ values for the different sites for Vendor A approach the critical $\mathrm{h}$ and $\mathrm{k}$ values. High $\mathrm{h}$ and $\mathrm{k}$ values are indicative of poor between and within vendor precision, respectively.

Review of Figure 5-3, the $\mathrm{h}$ and $\mathrm{k}$ plots by site, show that Vendor A has $\mathrm{h}$ values of exactly the opposite sign of those associated with Vendors B, C and D at five of the eight sites. Most of the Vendor A $\mathrm{h}$ values approach the critical $\mathrm{h}$ value also. The $\mathrm{k}$ plot by site shows that Vendor A has more $\mathrm{k}$ values approaching and exceeding the critical $\mathrm{k}$ value than the other vendors even though two k values associated with Vendor D exceed the critical value also. Based on review of the $\mathrm{h}$ and $\mathrm{k}$ plots it was concluded that Vendor A exhibited potential lack of both within and between vendor precision and Vendor D might exhibit potential lack of within vendor precision. This warranted further investigation into the reason for this observation.

Table 5-2 provides a summary of the statistics associated with a precision statement. Site averages as well as repeatability and reproducibility standard deviations and limits are presented by site. The repeatability and reproducibility standard deviations are all relatively small and consistent with the exception of those associated with Site 2 . It is clear from review of the Site $2 \mathrm{~b}$ data (I-65 without boards) that the boards induce greater dynamics than one or more of the profilers could tolerate. For most of the sites the 
reproducibility standard deviations are two to three times larger greater than the repeatability standard deviations as is typically expected with the exception of Sites 1 and 2. The Site 1 observation could possibly be due to the fact that it were the first site tested and it was a fairly rough pavement even though it were newly placed PCC. The reason for the high standard deviations associated with Site 2 is due to the significant difference and range in IRI reported by Vendor A when compared to the other vendors.

Figure 5-4 is a plot of 95 percent repeatability and reproducibility limits ( $\mathrm{r}$ and $\mathrm{R}$, respectively) versus average IRI by site. The $\mathrm{R}$ is much greater than the $\mathrm{r}$ in the case of the I-65 site with boards (Site 2). In fact the R for that site is a clearly an outlier, as the value of 223 is very high compared to the other sites. Review of Figure 5-1 provides the explanation for this as expected. Vendor A reported very high IRI values (220's in/mi) while Vendor C reported very low values (30’s in/mi).

The analysis discussed above suggests that the plywood strips at Site 2 induced profiler dynamics that were to great for at least one vendor. It also indicates that Vendor A may lack both within and between vendor precision. With recognition of these issues the within and between vendor precision for this data set are stated in the last two rows of Table 5-2 in bold. Because the observed standard deviations are relatively constant over the range of sites (Form 1) one-sigma (1S) and difference two sigma limits (D2S) are expressed rather than the one-sigma and difference two sigma limits in percent. Interpretation of the $1 \mathrm{~S}$ and D2S limits for within and between vendor acceptable range of results would be as follows: 
1. The within vendor acceptable range of two results equals $13.26 \mathrm{in} / \mathrm{mi}$. Therefore, the difference between two individual test results that would be equaled or exceeded in the long run in only 1 case of 20 (5\% of the time) under the normal and correct operation of the test using the same profiler and operator would be $13.3 \mathrm{in} / \mathrm{mi}$; and

2. The between vendor acceptable range of two results equals $60.8 \mathrm{in} / \mathrm{mi}$. Therefore, the difference between two individual test results that would be equaled or exceeded in the long run in only 1 case of 20 (5\% of the time) under the normal and correct operation of the test using two profilers would be $60.8 \mathrm{in} / \mathrm{mi}$. 
Table 5-2 Case 1 IRI Precision Statement Summary Statistics

\begin{tabular}{|c|c|c|c|c|c|}
\hline Sites & Site average & $\begin{array}{c}\text { Repeatability } \\
\text { standard } \\
\text { deviation (Sr) }\end{array}$ & $\begin{array}{c}\text { Reproducibility } \\
\text { standard } \\
\text { deviation (SR) }\end{array}$ & $\begin{array}{c}95 \% \\
\text { Repeatability } \\
\text { limit (r) }\end{array}$ & $\begin{array}{c}\text { Reproducibility } \\
\text { limit (R) }\end{array}$ \\
\cline { 2 - 6 } inches/mile & Inches/mile \\
\hline $\begin{array}{c}\text { Site 1 } \\
\text { Convington St. }\end{array}$ & 141.96 & 3.00 & 23.62 & 8.38 & 66.14 \\
\hline $\begin{array}{c}\text { Site 2 } \\
\text { I-65 with board }\end{array}$ & 106.08 & 12.39 & 79.76 & 34.71 & 223.31 \\
\hline $\begin{array}{c}\text { Site 2b } \\
\text { I-65 }\end{array}$ & 41.13 & 2.84 & 12.88 & 7.94 & 36.09 \\
\hline $\begin{array}{c}\text { Sith 3 } \\
\text { Prairie Street } \\
\text { with board }\end{array}$ & 157.74 & 5.40 & 12.15 & 15.12 & 34.03 \\
\hline $\begin{array}{c}\text { Site 3b } \\
\text { Prairie Street } \\
\text { without board }\end{array}$ & 147.69 & 4.05 & 15.62 & 11.34 & 43.75 \\
\hline $\begin{array}{c}\text { Site 4 } \\
\text { Vinton Street }\end{array}$ & 100.33 & 3.18 & 7.13 & 8.91 & 19.98 \\
\hline $\begin{array}{c}\text { Site 5 } \\
\text { US231 A }\end{array}$ & 51.06 & 3.76 & 10.65 & 10.53 & 29.81 \\
\hline $\begin{array}{c}\text { Site 6 } \\
\text { US 231B }\end{array}$ & 62.31 & 2.82 & 10.18 & 7.88 & 28.51 \\
\hline 1S & $\mathbf{4 . 6 9}$ & $\mathbf{2 1 . 5 0}$ & & $\mathbf{1 3 . 2 6}$ & $\mathbf{6 0 . 8 0}$ \\
\hline D2S
\end{tabular}

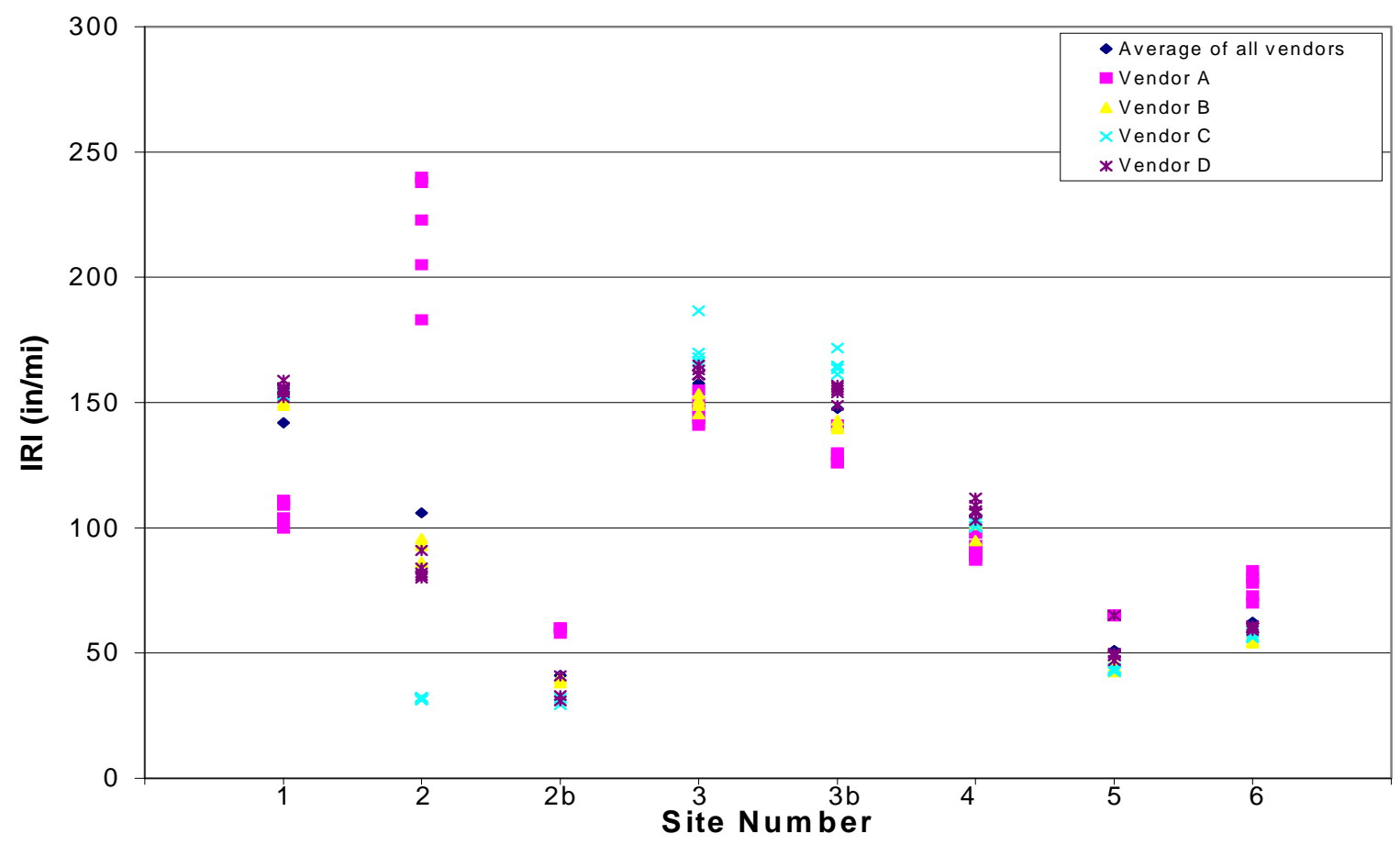

Figure 5-1 Case 1 Site Average Plot by Vendor for IRI 

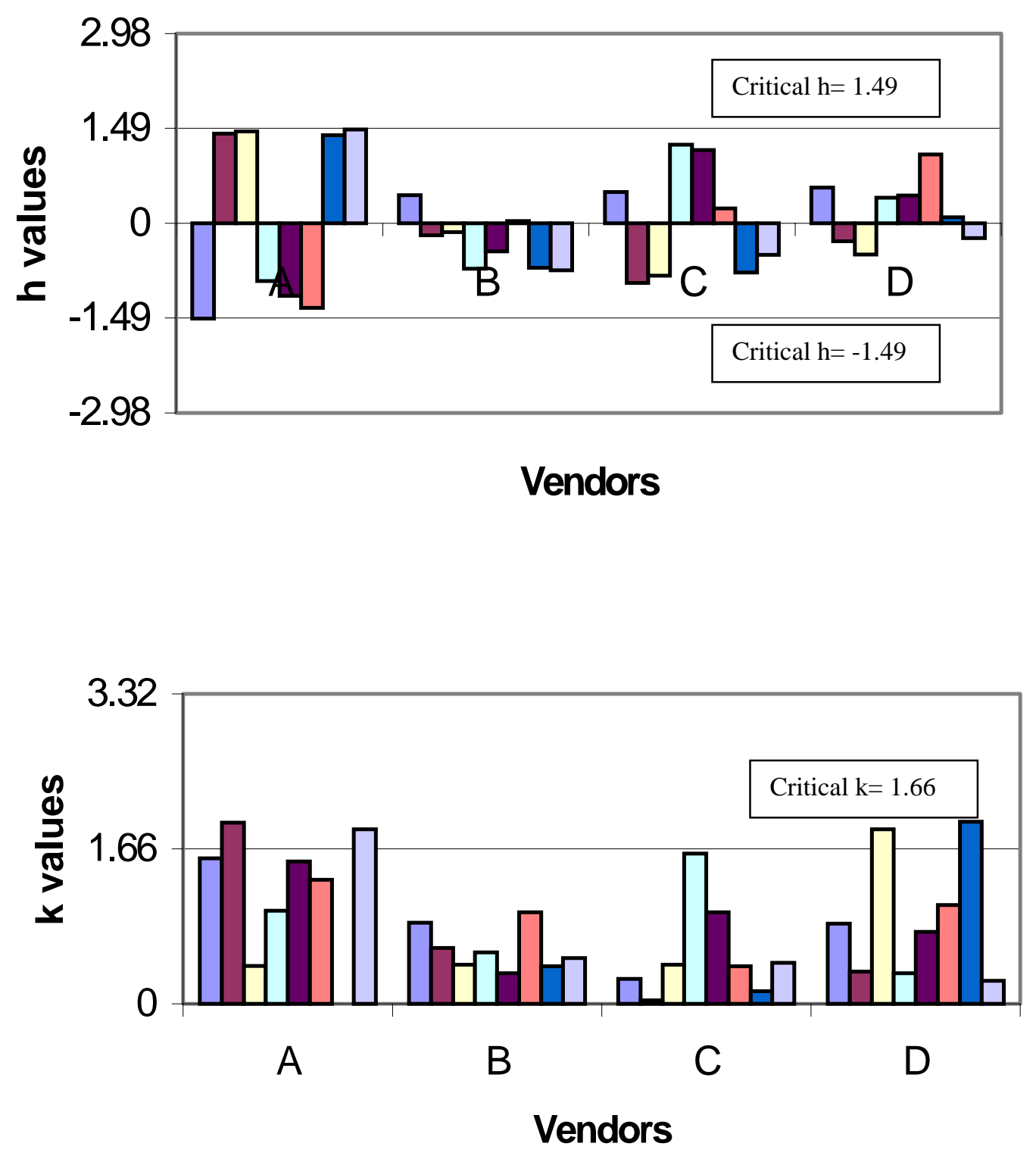

Figure 5-2 Case $1 \mathrm{~h}$ and $\mathrm{k}$ plots by Vendors for IRI Note: The 8 columns for each vendor represent the 6 sites (Site 2 and 3 with and without board). 

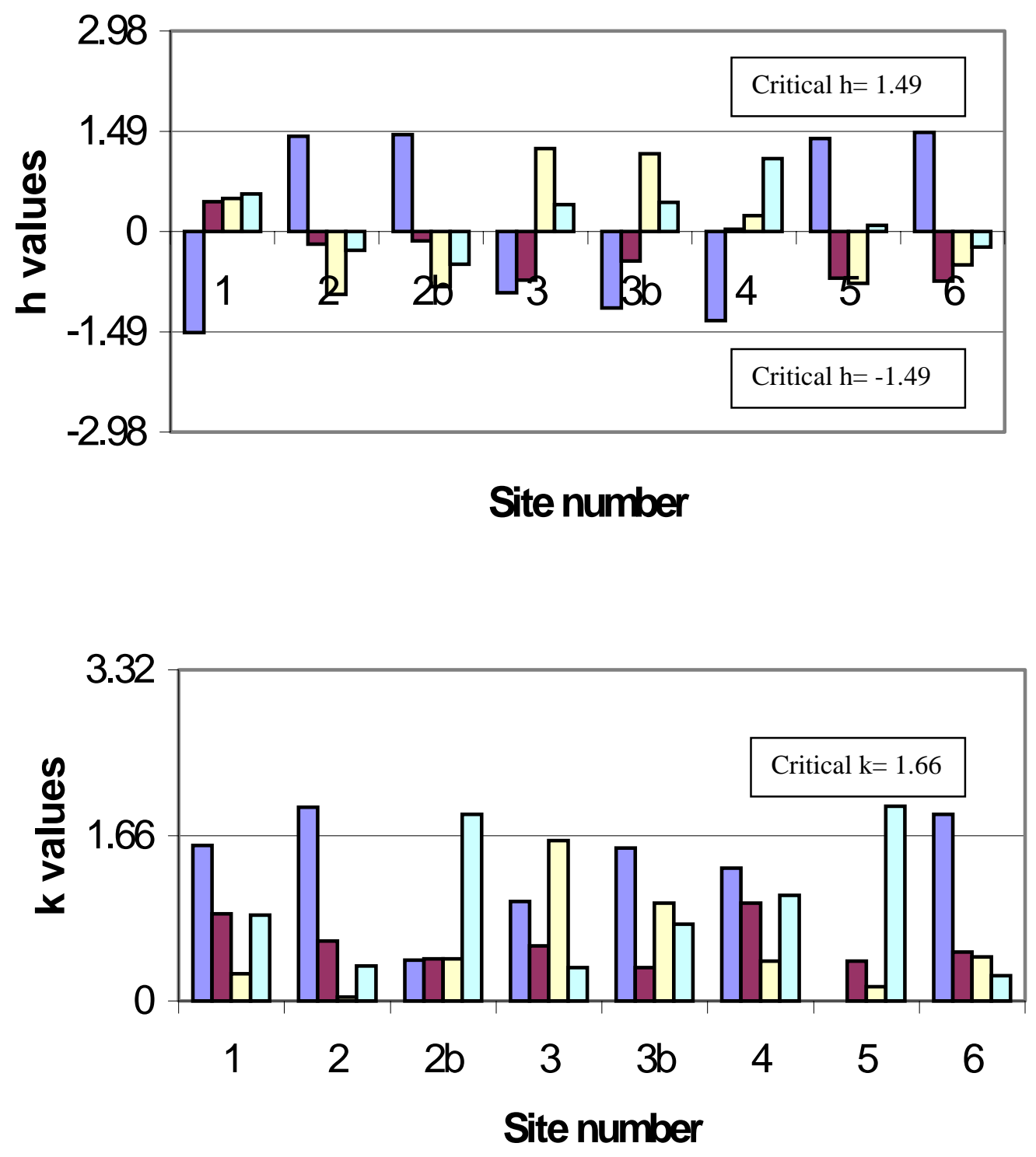

Figure 5-3 Case $1 \mathrm{~h}$ and $\mathrm{k}$ plots by Sites for IRI Note : The 4 columns for each site represent the 4 vendors. 


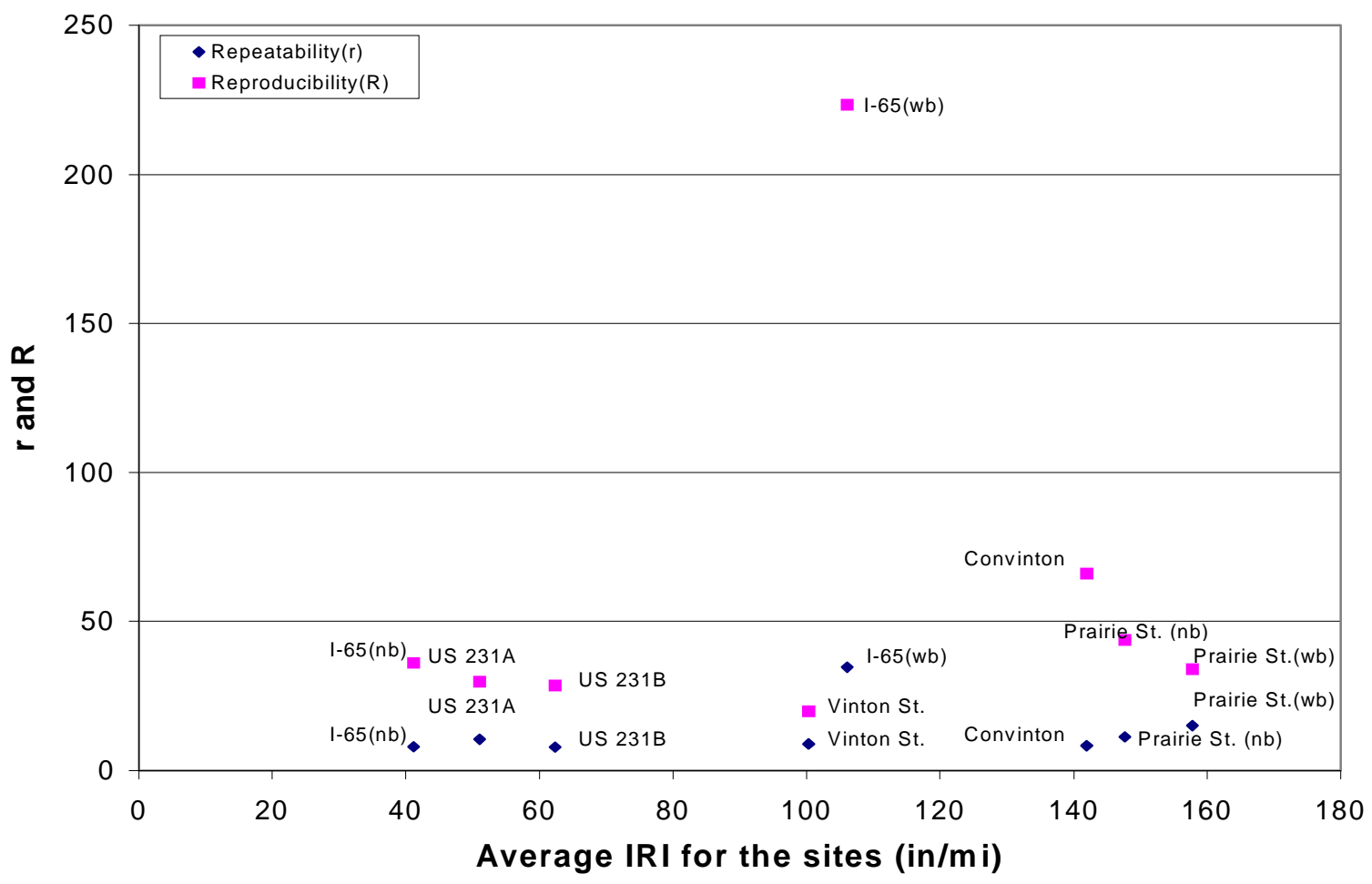

Figure 5-4 Case 1 Repeatability and Reproducibility Statistics by Site for IRI 


\subsubsection{Analysis of PI-0.0" Data}

Figure 5-5 is a plot of the individual reported PI-0.0" values by each vendor at each site. The plot also includes the average PI-0.0" for all the vendors at each site. An initial observation that can be made from the plot is that Vendor A reported significantly different PI-0.0" values than the other vendors at Sites 1, 2, 3, and 3b. Site 2 was the I-65 site with the $3 / 4$ " thick plywood strips in place approximately ten feet from the beginning and end of the test section which induced significant dynamics into the profilers. Figures 5-6 and 5-7 are the $\mathrm{h}$ and $\mathrm{k}$ plots by vendor and site, respectively for PI-0.0”. Figure 5-6 shows that the h values associated with Vendor A are typically the opposite in sign (+ vs. -) compared to the other vendors and many approach the critical h values of 1.49. One each of the Vendor A and Vendor $\mathrm{C} k$ values exceed the critical value of 1.66 , but they are fairly similar among the vendors. Review of Figure 5-7, the $\mathrm{h}$ and $\mathrm{k}$ plots by site, shows that Vendor A has h values of exactly the opposite sign of those associated with all the other vendors at six of eight sites and most of the Vendor A $h$ values approach the critical value. The $\mathrm{k}$ plot by site suggests similar within vendor precision for all of the vendors. Again Vendors $\mathrm{A}$ and $\mathrm{C}$ each have one site at which the critical $\mathrm{k}$ value is slightly exceeded and within vendor precision appears to be fairly consistent among the vendors. Based on review of the $\mathrm{h}$ and $\mathrm{k}$ plots it was concluded that Vendor A exhibited potential lack of between vendor precision.

Table 5-3 represents a summary of the statistics associated with a precision statement. Site averages as well as repeatability and reproducibility standard deviations and limits are presented by site. The repeatability standard deviations are all relatively 
small and consistent. A large range of reproducibility standard deviations were observed however. Review of Figure 5-5 suggests that this is likely due to the fact that Vendor A reported PI values either much smaller or much larger than the other vendors at several sites. This is also the likely reason that there is a lack consistent increase from repeatability to reproducibility standard deviations. As with the IRI data, both repeatability and reproducibility are improved when the boards were removed at Site 2 . Interestingly, only a nominal difference was observed at Site 3 when the boards were removed.

Figure 5-8 is a plot of 95 percent repeatability and reproducibility limits ( $\mathrm{r}$ and $\mathrm{R}$ ), respectively versus average PI- 0.0 " by site. The $\mathrm{R}$ is much greater than $\mathrm{r}$ in the case of the Covington Street (Site 1) and I-65 site with board (Site 2) sites. The difference is also large for the Prairie Street site both with and without the boards present (Sites 3 and 3b). At the Covington Street site Vendor A reported PI values of 20 inches/mile while all other vendors reported values in the 60 to $80 \mathrm{in} / \mathrm{mi}$ range. Just the opposite occurred at the I-65 with board site.

The analysis discussed above suggests that Vendor A lacked between vendor precision, particularly for rougher pavements. Even though the plywood strips at Site 2 induced significant dynamics to the profilers and improvements were observed at Site 2 when the boards were removed equal or greater differences in PI were observed at other sites where boards were not present (eg. Site 1). With recognition of these issues the within and between vendor precision for this data set are stated in the last two rows of 
Table 5-3 in bold. Because the observed standard deviations are relatively constant over the range of sites (Form 1) one-sigma (1S) and difference two sigma limits (D2S) are expressed rather than the one-sigma and difference two sigma limits in percent. Interpretation of $1 \mathrm{~S}$ and $\mathrm{D} 2 \mathrm{~S}$ limits for within and between vendor acceptable range of results would be as follows:

1. The within vendor acceptable range of two results equals $4.3 \mathrm{in} / \mathrm{mi}$. Therefore, the difference between two individual test results that would be equaled or exceeded in the long run in only 1 case of 20 (5\% of the time) under the normal and correct operation of the test using the same profiler and operator would be $4.3 \mathrm{in} / \mathrm{mi}$; and

2. The between vendor acceptable range of two results equals $32.7 \mathrm{in} / \mathrm{mi}$. Therefore, the difference between two individual test results that would be equaled or exceeded in the long run in only 1 case of 20 (5\% of the time) under the normal and correct operation of the test using two profilers would be $32.7 \mathrm{in} / \mathrm{mi}$. 
Table 5-3 Case 1 PI-0.0” Precision Statement Summary Statistics

\begin{tabular}{|c|c|c|c|c|c|}
\hline \multirow[t]{2}{*}{ Sites } & Site average & $\begin{array}{l}\text { Repeatability } \\
\text { standard } \\
\text { deviation }(\mathrm{Sr})\end{array}$ & $\begin{array}{c}\text { Reproducibility } \\
\text { standard } \\
\text { deviation (SR) }\end{array}$ & $\begin{array}{c}95 \% \\
\text { Repeatability } \\
\text { limit (r) }\end{array}$ & $\begin{array}{c}95 \% \\
\text { Reproducibility } \\
\text { limit }(\mathrm{R})\end{array}$ \\
\hline & Inches/mile & inches/mile & Inches/mile & Inches/mile & Inches/mile \\
\hline $\begin{array}{c}\text { Site } 1 \\
\text { Convington St. }\end{array}$ & 66.42 & 1.77 & 26.61 & 4.96 & 74.52 \\
\hline $\begin{array}{c}\text { Site } 2 \\
\text { I-65 with board }\end{array}$ & 34.93 & 1.59 & 24.01 & 4.44 & 67.22 \\
\hline $\begin{array}{c}\text { Site } 2 b \\
\text { I- } 65 \text { without board }\end{array}$ & 8.59 & 0.94 & 4.11 & 2.64 & 11.49 \\
\hline $\begin{array}{c}\text { Site } 3 \\
\text { Prairie Street } \\
\text { with board }\end{array}$ & 53.54 & 1.92 & 12.05 & 5.37 & 33.74 \\
\hline $\begin{array}{c}\text { Site 3b } \\
\text { Prairie Street } \\
\text { without board }\end{array}$ & 52.91 & 2.30 & 12.13 & 6.44 & 33.96 \\
\hline $\begin{array}{c}\text { Site } 4 \\
\text { Vinton Street }\end{array}$ & 34.59 & 1.25 & 7.57 & 3.49 & 21.18 \\
\hline $\begin{array}{c}\text { Site } 5 \\
\text { US231 A }\end{array}$ & 16.37 & 1.04 & 2.89 & 2.92 & 8.09 \\
\hline $\begin{array}{c}\text { Site } 6 \\
\text { US 231B }\end{array}$ & 21.42 & 1.45 & 3.04 & 4.06 & 8.52 \\
\hline \multicolumn{2}{|l|}{$1 \mathrm{~S}$} & 1.53 & 11.55 & & \\
\hline \multicolumn{4}{|l|}{ D2S } & 4.33 & 32.67 \\
\hline
\end{tabular}




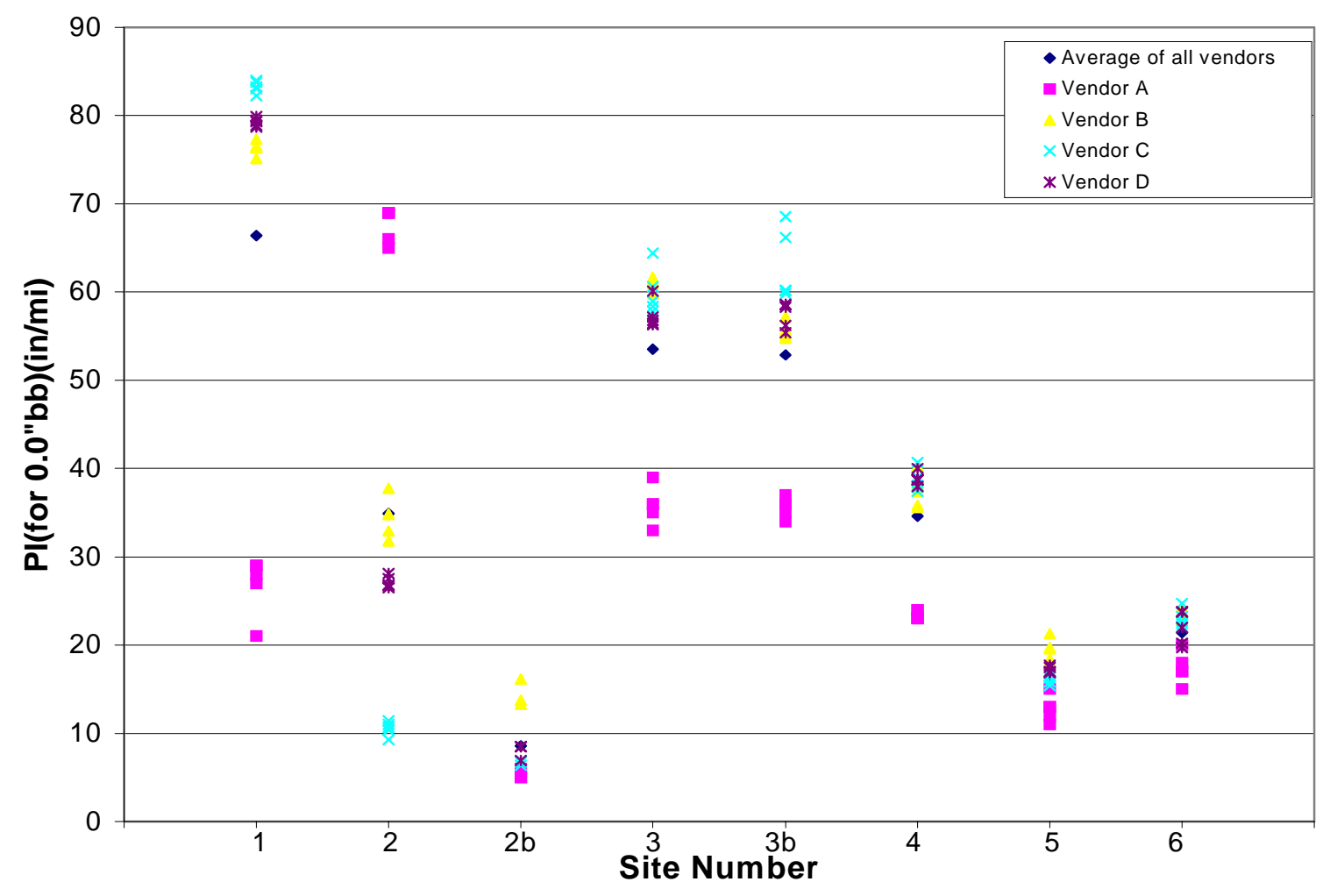

Figure 5-5 Case 1 Site Average Plot by Vendor for PI-0.0” 

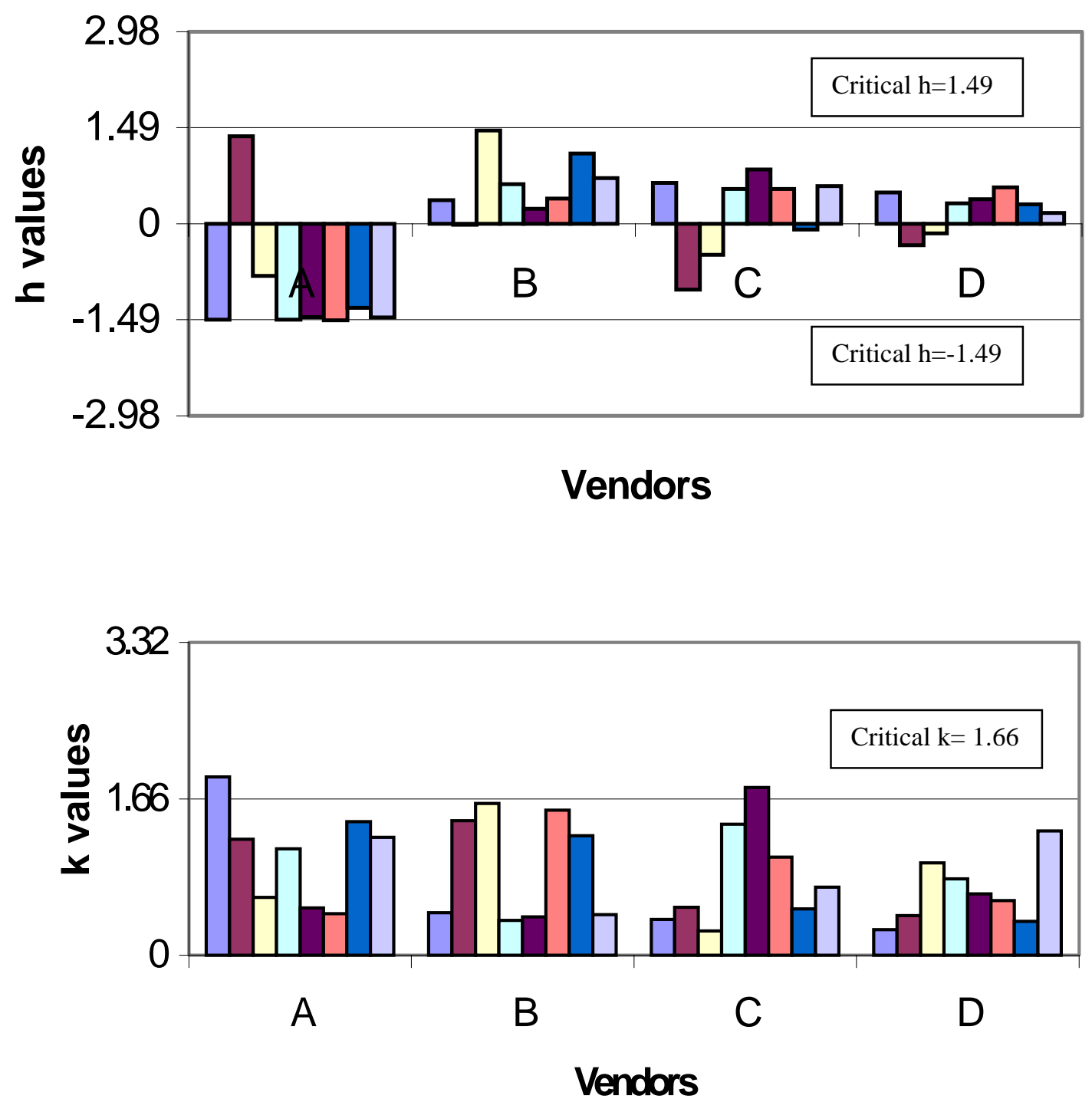

Figure 5-6 Case $1 \mathrm{~h}$ and k plots by Vendors for PI-0.0"

Note: The 8 columns for each vendor represent the 6 sites (Site 2 and 3 with and without board). 

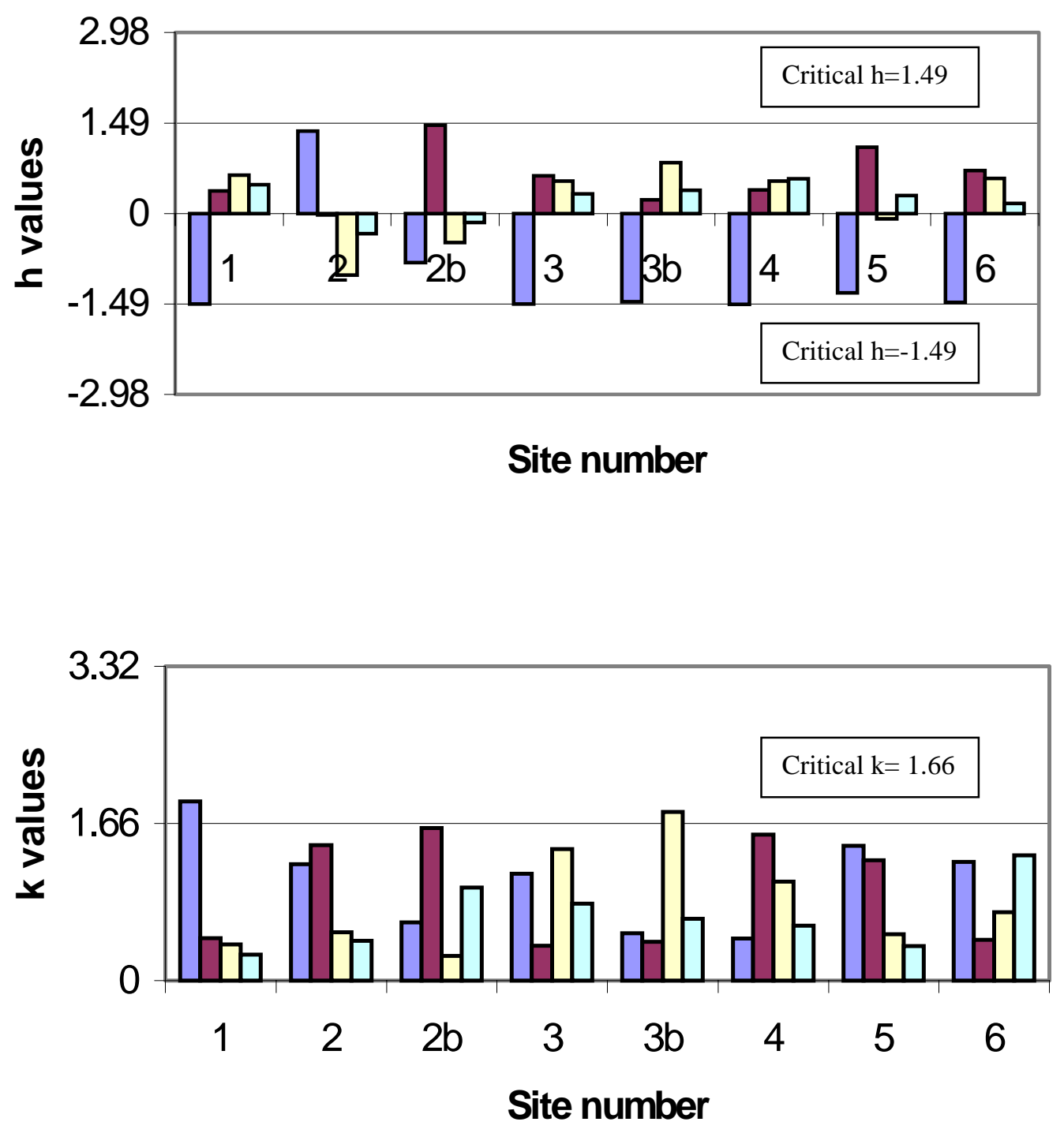

Figure 5-7 Case $2 \mathrm{~h}$ and $\mathrm{k}$ plots by vendors for PI-0.0" Note: The 4 columns for each site represent the 4 vendors. 


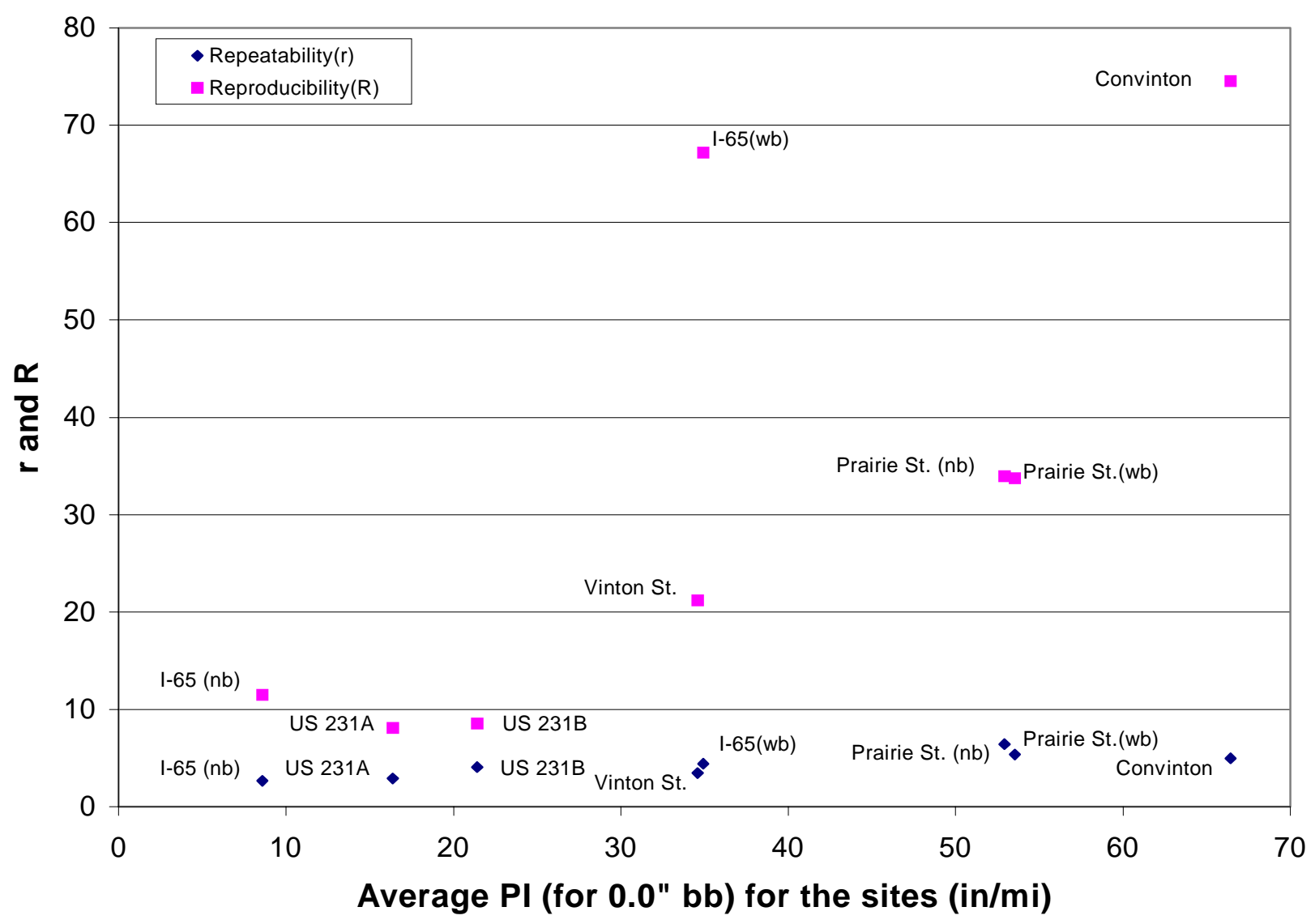

Figure 5-8 Case 1 Repeatability and Reproducibility Statistics by Site for PI-0.0" 


\subsubsection{Analysis of PI-0.2" Data}

Figure 5-9 is a plot of the individual reported PI-0.2" values by each vendor at each site. The plot also includes the average PI-0.2" for all the vendors at each site. Similar observations are made for the PI-0.2" data as to those that were made for the PI0.0 " data. Vendor A reported significantly different PI-0.2" values than the other vendors at Sites 1 and 2. It is worth noting that all vendors reported similar results for Sites 2 (without the boards), 5, and 6 which were all very smooth. Figures 5-10 and 5-11 are the $\mathrm{h}$ and $\mathrm{k}$ plots by vendor and site, respectively for PI-0.2". Figure 5-10 shows that the $\mathrm{h}$ values associated with Vendor A are opposite in sign (+ vs. -) at some sites compared to the other vendors and two of eight approach the critical $h$ values of 1.49 . Three of the Vendor A and one of the Vendor $\mathrm{C} \mathrm{k}$ values exceed the critical value of 1.66 , but they are fairly similar among the vendors. Review of Figure 5-11, the $\mathrm{h}$ and $\mathrm{k}$ plots by site, shows that Vendor A has h values of exactly the opposite sign of those associated with all the other vendors at three of eight sites. The $\mathrm{k}$ plot by site suggests similar within vendor precision for all of the vendors. Again Vendors A had three and Vendor C had one site at which the critical $\mathrm{k}$ value was slightly exceeded and within vendor precision appears to be fairly consistent among the vendors. Based on review of the $\mathrm{h}$ and $\mathrm{k}$ plots it was concluded that Vendor A exhibited potential lack of between vendor precision, although it was improved when compared to the PI-0.0" data.

Table 5-4 represents a summary of the statistics associated with a precision statement. Site averages as well as repeatability and reproducibility standard deviations 
and limits are presented by site. The repeatability standard deviations are all relatively small and consistent. A large range of reproducibility standard deviations were observed however. Review of Figure 5-9 suggests that this is likely due to the fact that Vendor A reported PI values either much smaller or much larger than the other vendors at Site 1 and 2 with the boards present. This is also the likely reason that there is a lack consistent increase from repeatability to reproducibility standard deviations. As with the IRI data, both repeatability and reproducibility are improved when the boards were removed at Site 2. If fact all of the vendors reported zero PI values. This is another example of the inadequacy of the 0.2 inch blanking bank.

Figure 5-12 is a plot of 95 percent repeatability and reproducibility limits ( $\mathrm{r}$ and $\mathrm{R}$, respectively) versus average PI- 0.2 " by site. The $\mathrm{R}$ is much greater than the $\mathrm{r}$ in the case of the Covington Street (Site 1) and I-65 site with board (Site 2) sites. The difference is also fairly large for the Prairie Street site both with and without the boards present (Sites 3 and 3b). At the Covington Street site Vendor A reported PI values less than $10 \mathrm{in} / \mathrm{mi}$ while all other vendors reported values in the 35 to $50 \mathrm{in} / \mathrm{mi}$ range. Just the opposite occurred at the I- 65 with boards site.

The analysis discussed above suggests that Vendor A lacks between vendor precision, particularly for rougher pavements. Even though the plywood strips at Site 2 induced significant dynamics to the profilers and improvements were observed at Site 2 when the boards were removed, equal or greater differences in PI were observed at other sites where boards were not present (eg. Site 1). With recognition of these issues the 
within and between vendor precision for this data set are stated in the last two rows of Table 5-4 in bold. Because the observed standard deviations are relatively constant over the range of sites (Form 1) one-sigma (1S) and difference two sigma limits (D2S) are expressed rather than the one-sigma and difference two sigma limits in percent. Interpretation of $1 \mathrm{~S}$ and D2S limits for within and between vendor acceptable range of results would be as follows:

1. The within vendor acceptable range of two results equals $3.7 \mathrm{in} / \mathrm{mi}$. Therefore, the difference between two individual test results that would be equaled or exceeded in the long run in only 1 case of 20 (5\% of the time) under the normal and correct operation of the test using the same profiler and operator would be 3.7in/mi; and

2. The between vendor acceptable range of two results equals $20.6 \mathrm{in} / \mathrm{mi}$. Therefore, the difference between two individual test results that would be equaled or exceeded in the long run in only 1 case of 20 (5\% of the time) under the normal and correct operation of the test using two profilers would be $20.6 \mathrm{in} / \mathrm{mi}$.

Application of the 0.2 inch blanking band actually made it appear as though the repeatability and reproducibility of the lightweight profilers was better than when the 0.0 inch blanking band were applied. This is due to the fact that low variability was associated with the smooth sites and this effectively reduced the $1 \mathrm{~S}$ and D2S limits.

The level of variability observed in the Case 1 data set is obviously too high for practical application in a smoothness specification. Therefore a second precision analysis was conducted. For this analysis, all of the vendors and sites were considered with the 
exception of the sites with the plywood strips in place. It was hoped that the Vendor A precision would be more in line with the other vendors under this condition. If not, then the Vendor A data would be dropped in a subsequent analyses. The Case 2 precision analysis is presented in the following section.

Table 5-4 Case 1 PI-0.2” Precision Statement Summary Statistics

\begin{tabular}{|c|c|c|c|c|c|}
\hline \multirow[t]{2}{*}{ Sites } & Site average & $\begin{array}{c}\text { Repeatability } \\
\text { standard } \\
\text { deviation }(\mathrm{Sr})\end{array}$ & $\begin{array}{c}\text { Reproducibility } \\
\text { standard } \\
\text { deviation (SR) }\end{array}$ & $\begin{array}{c}95 \% \\
\text { Repeatability } \\
\text { limit (r) }\end{array}$ & $\begin{array}{c}95 \% \\
\text { Reproducibility } \\
\text { limit (R ) }\end{array}$ \\
\hline & Inches/mile & inches/mile & Inches/mile & Inches/mile & Inches/mile \\
\hline $\begin{array}{c}\text { Site } 1 \\
\text { Convington St. }\end{array}$ & 36.71 & 1.17 & 19.30 & 3.27 & 54.03 \\
\hline $\begin{array}{c}\text { Site } 2 \\
\text { I-65 with board }\end{array}$ & 20.59 & 2.65 & 22.07 & 7.42 & 61.79 \\
\hline $\begin{array}{c}\text { Site } 2 b \\
\text { I- } 65 \text { without board }\end{array}$ & 0 & 0 & 0 & 0 & 0 \\
\hline $\begin{array}{c}\text { Site } 3 \\
\text { Prairie Street } \\
\text { With board }\end{array}$ & 40.77 & 2.56 & 6.77 & 7.16 & 18.97 \\
\hline $\begin{array}{c}\text { Site 3b } \\
\text { Prairie Street } \\
\text { without board }\end{array}$ & 38.75 & 1.52 & 6.73 & 4.26 & 18.86 \\
\hline $\begin{array}{c}\text { Site } 4 \\
\text { Vinton Street }\end{array}$ & 16.99 & 1.61 & 1.98 & 4.52 & 5.55 \\
\hline $\begin{array}{c}\text { Site } 5 \\
\text { US231 A }\end{array}$ & 0.24 & 0.31 & 0.31 & 0.87 & 0.87 \\
\hline $\begin{array}{c}\text { Site } 6 \\
\text { US 231B }\end{array}$ & 2.45 & 0.55 & 1.05 & 1.53 & 2.93 \\
\hline \multicolumn{2}{|l|}{$1 \mathrm{~S}$} & 1.29 & 7.27 & & \\
\hline \multicolumn{4}{|l|}{ D2S } & 3.67 & 20.58 \\
\hline
\end{tabular}




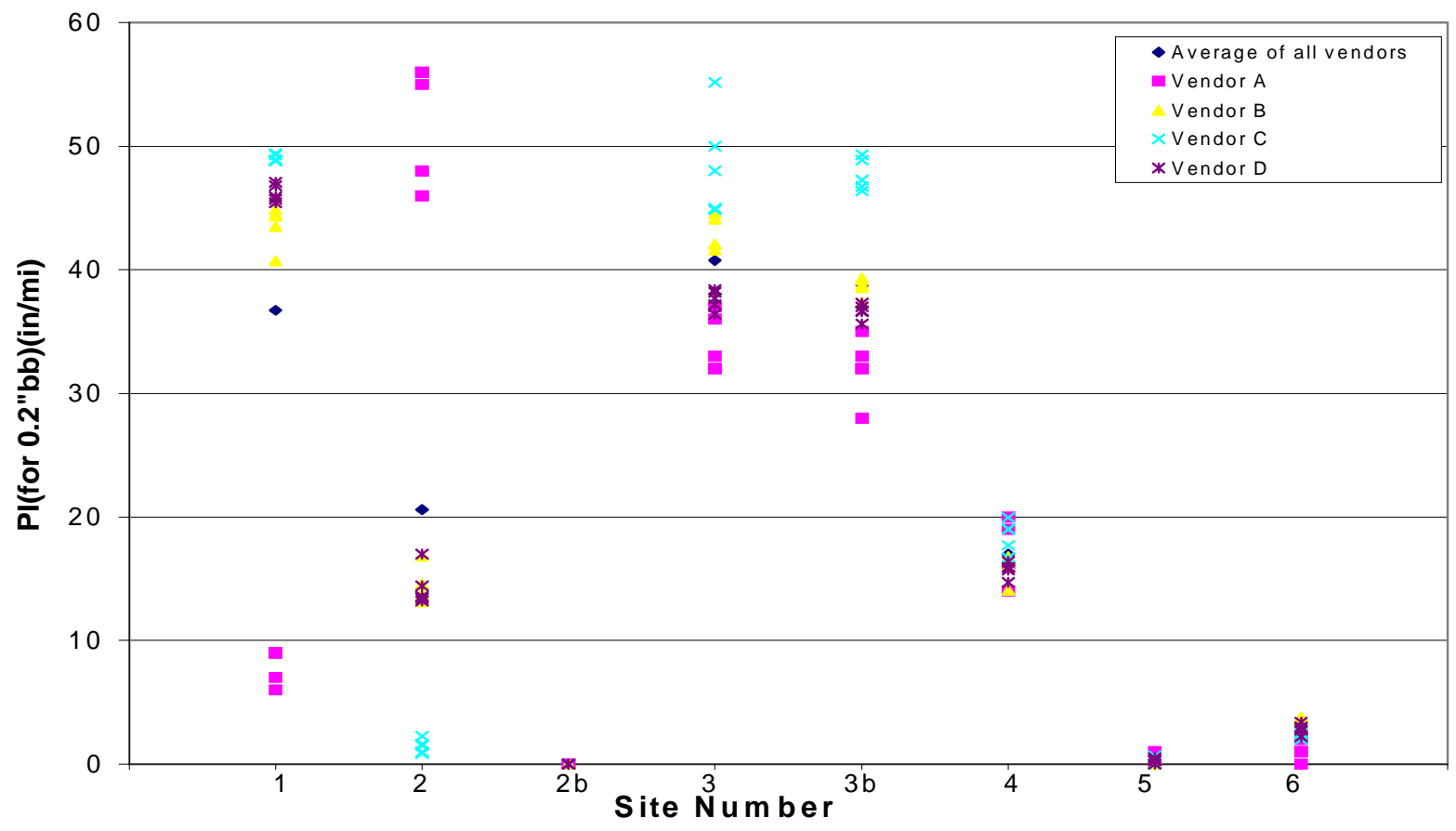

Figure 5-9 Case 1 Site Average Plot by Vendor for PI-0.2" 

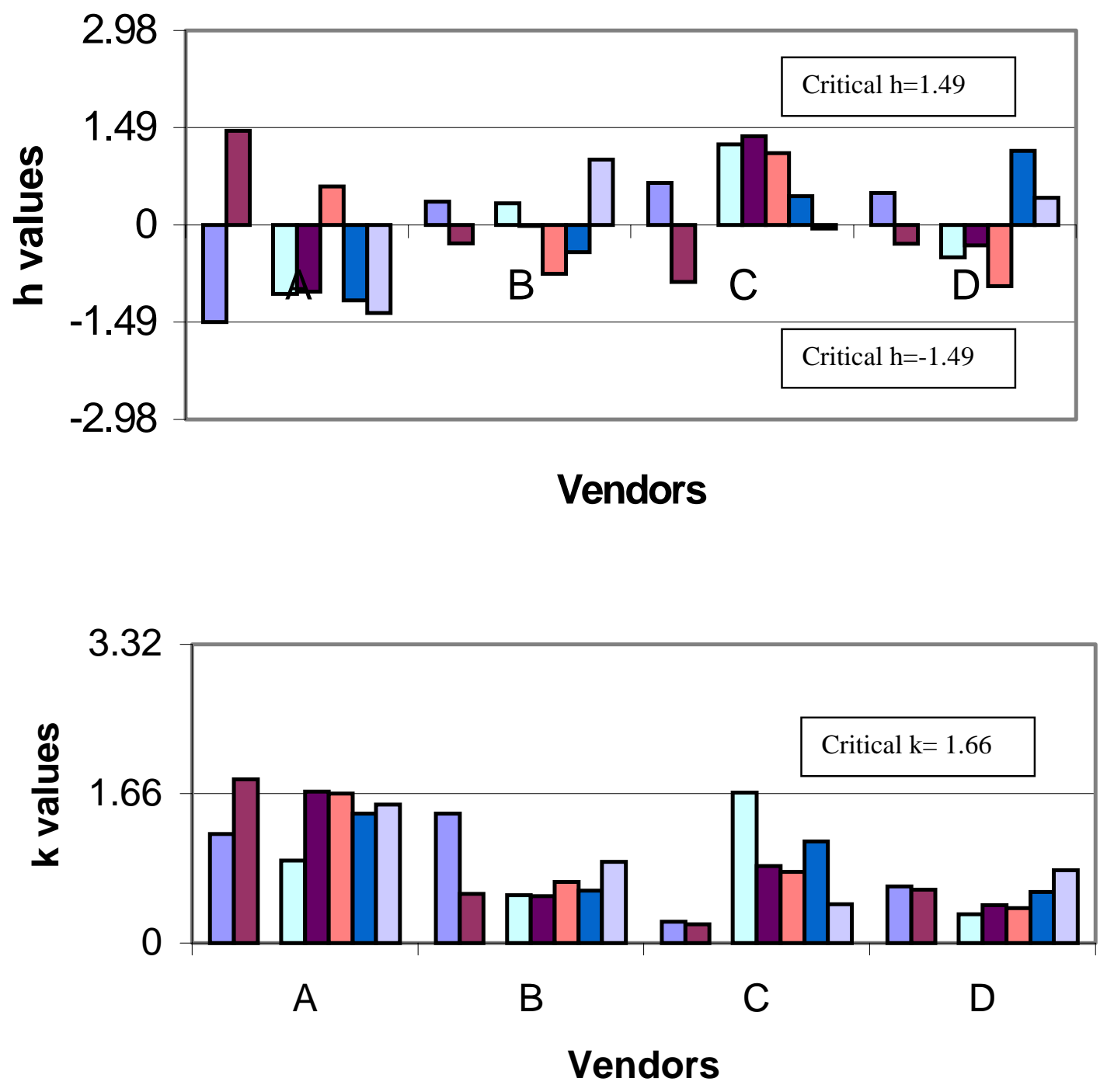

Figure 5-10 Case $1 \mathrm{~h}$ and k plots by Vendors for PI-0.2" Note: The 8 columns for each vendor represent the 6 sites (Site 2 and 3 with and without board). 

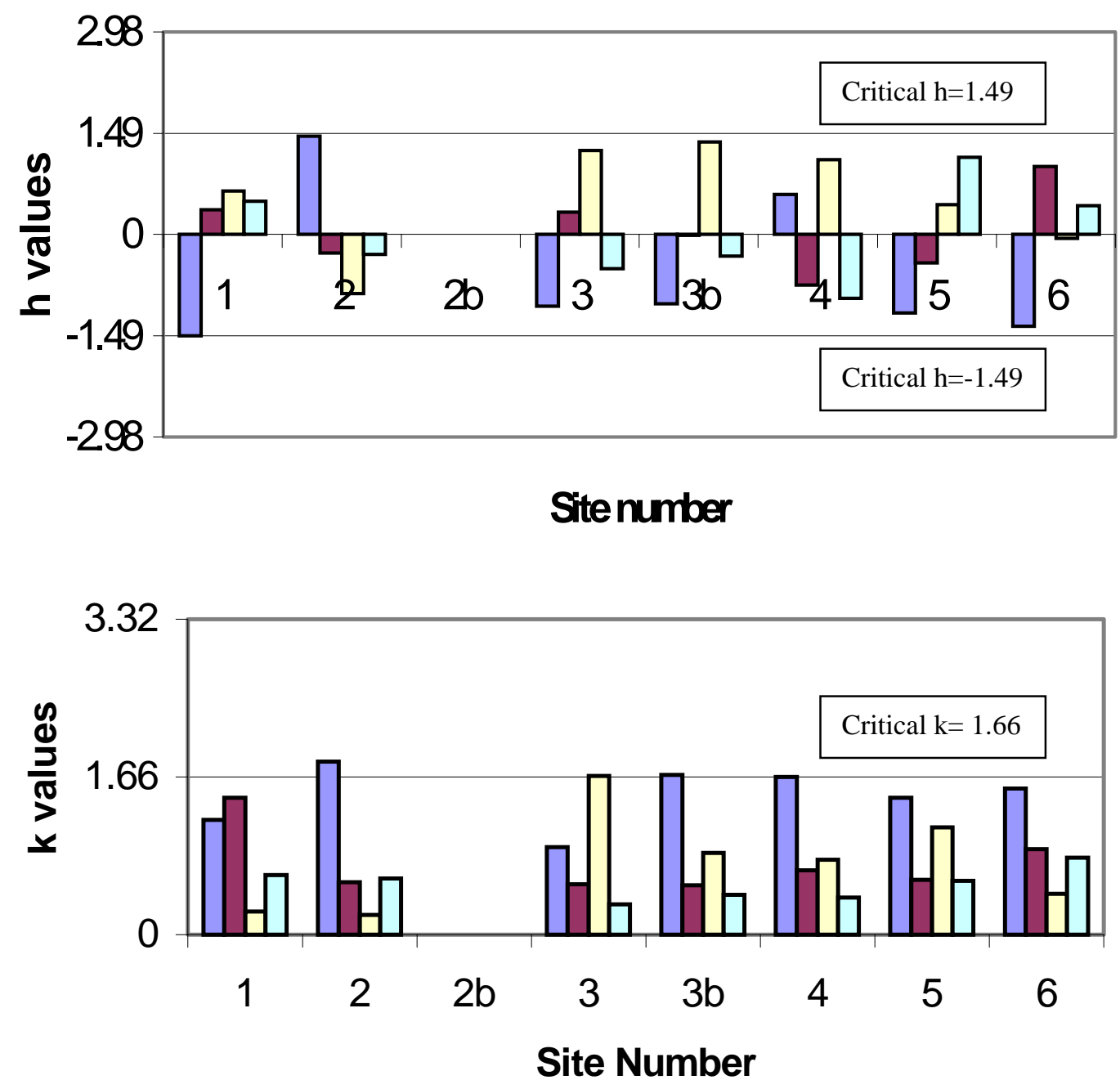

Figure 5-11 Case $1 \mathrm{~h}$ and k plots by Sites for PI-0.2"

Note: The 4 columns for each site represent the 4 vendors . 


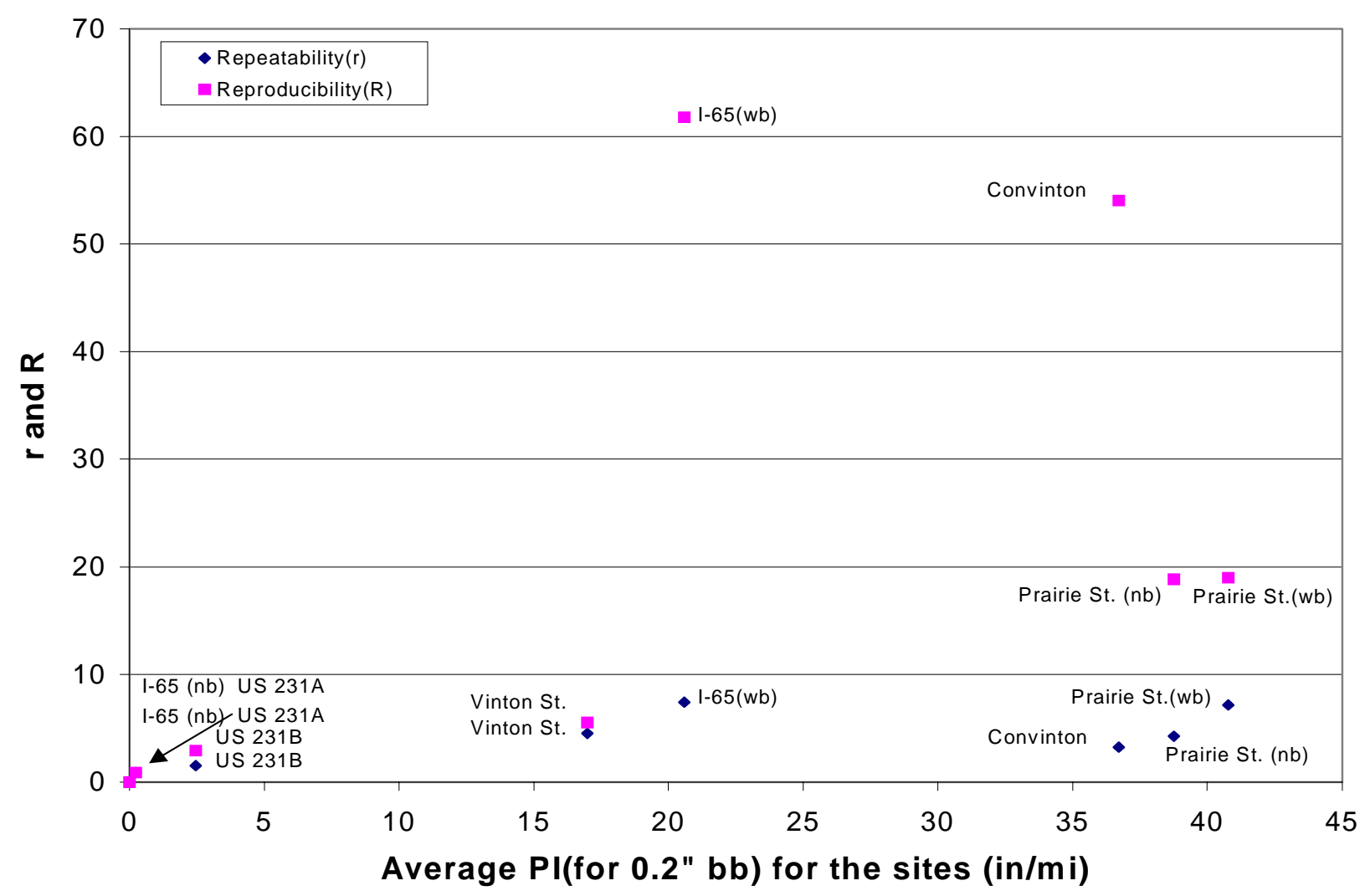

Figure 5-12 Case 1 Repeatability and Reproducibility Statistics by Site for PI-0.2” 


\subsubsection{Case 2: Precision Based on Indiana Generated Data without Boards}

For this analysis the data generated in Indiana, at the sites without plywood strips (boards) in place were considered in the analysis. The with board data was excluded because significantly high standard deviations were associated with Site 2 with boards present, which affected the overall consistency of the data. All three smoothness indices were considered in the analysis. The data employed is summarized in Table 5-5. The experiment exceeded the minimum requirements of ASTM C802-96. One way ANOVA were conducted separately for the data associated with each site using the ASTM E69192 software. The data were then summarized in the form of a precision statement in accordance with ASTM C670-96. Critical values of h- and k-statistic were chosen at the 0.5 percent significance level. The critical values of $\mathrm{h}$ and $\mathrm{k}$ were 1.49 and 1.66, respectively for the conditions outlined in Table 5-5.

Table 5-5 Summary of Case 2 Precision Analysis Considerations

\begin{tabular}{|c|c|c|c|c|c|}
\hline $\begin{array}{c}\text { Number of } \\
\text { Vendors }\end{array}$ & $\begin{array}{c}\text { Number } \\
\text { of Sites }\end{array}$ & $\begin{array}{c}\text { Replicate } \\
\text { Measurements } \\
\text { per Site }\end{array}$ & $\begin{array}{c}\text { With Board } \\
\text { Data } \\
\text { Included? }\end{array}$ & $\begin{array}{c}\text { Smoothness } \\
\text { Statistics } \\
\text { Considered }\end{array}$ & $\begin{array}{c}\text { Total } \\
\text { Degrees } \\
\text { of } \\
\text { Freedom }\end{array}$ \\
\hline $\begin{array}{c}4 \\
\text { (A, B, C, D) }\end{array}$ & 6 & 5 & No & $\begin{array}{c}\text { IRI, PI-0.0”, } \\
\text { PI-0.2” }\end{array}$ & 60 \\
\hline
\end{tabular}

\subsubsection{Analysis of IRI Data}

Figure 5-13 is a plot of the individual reported IRI values by each vendor at each site. The plot also includes the average IRI for all the vendors at each site. Plots show that 
reported IRI values were more consistent among vendors when the data associated with the sites with boards were removed. The within and between vendor consistency is much better in this case than that observed with all vendors and all sites (including the sites with board). Figures 5-14 and 5-15 are the $\mathrm{h}$ and $\mathrm{k}$ plots by vendor and site, respectively for IRI. Figure 5-14 shows that the $\mathrm{h}$ values associated with Vendor A are typically the opposite in sign (+ vs. -) compared to the other vendors. Additionally, many of the $\mathrm{h}$ and $\mathrm{k}$ values for the different sites for vendor A approach the critical $\mathrm{h}$ and $\mathrm{k}$ values. High $\mathrm{h}$ and $\mathrm{k}$ values are indicative of poor between and within vendor precision, respectively. Review of Figure 5-15, the $\mathrm{h}$ and $\mathrm{k}$ plots by site, show that Vendor A reported $\mathrm{h}$ values of exactly the opposite sign of those associated with Vendors B, C and D at five of the six sites. Most of Vendor A's, h values approach the critical h value also. The k plot by site shows that Vendor A has more $\mathrm{k}$ values approaching and exceeding the critical $\mathrm{k}$ value than the other vendors even though two of $k$ values associated with Vendor D exceed the critical value also. Based on review of the $\mathrm{h}$ and $\mathrm{k}$ plots it was concluded that Vendor A exhibited potential lack of both within and between vendor precision and Vendor D might exhibit potential lack of within vendor precision.

Table 5-6 provides a summary of the statistics associated with a precision statement. Site averages as well as repeatability and reproducibility standard deviations and limits are presented by site. The repeatability and reproducibility standard deviations are all relatively small and consistent. For most of the sites the reproducibility standard deviations are two to three times larger greater than the repeatability standard deviations with the exception of Sites 1 and 2. The Site 1 observation could be possibly due to the 
fact that it were the first site tested and it was a fairly rough pavement even though it was newly placed PCC. The reason for the high standard deviations associated with Site 2 is due to both the significant difference and range in IRI reported by Vendor A when compared to the other vendors.

Figure 5-16 is a plot of 95 percent repeatability and reproducibility limits ( $\mathrm{r}$ and $R$, respectively) versus average IRI by site. The $r$ and $R$ values for this case do not suggest any clear outliers with the possible exception of Site 1, which shows that the profilers reported more consistent results without the boards in place compared to the previous case (Case 1, considering all sites with and without board).

The analysis discussed above suggests that Vendor A may lack both within and between vendor precision. However both the between and within vendor performance was improved compared to the previous case (Case 1) when all the vendors and all sites were considered (including the data from the sites with board). With recognition of the above stated facts, the within and between vendor precision for this data set are stated in the last two rows of Table 5-6 in bold. Because the observed standard deviation is relatively constant over the range of sites (Form 1) one-sigma (1S) and difference two sigma limits (D2S) are expressed rather than the one-sigma and difference two sigma limits in percent. Interpretation of the $1 \mathrm{~S}$ and D2S limits for within and between vendor acceptable range of results would be as follows:

1. The within vendor acceptable range of two results equals $9.2 \mathrm{in} / \mathrm{mi}$. Therefore, the difference between two individual test results that would be equaled or exceeded in 
the long run in only 1 case of 20 (5\% of the time) under the normal and correct operation of the test using the same profiler and operator would be $9.2 \mathrm{in} / \mathrm{mi}$; and

2. The between vendor acceptable range of two results equals $37.4 \mathrm{in} / \mathrm{mi}$. Therefore, the difference between two individual test results that would be equaled or exceeded in the long run in only 1 case of 20 (5\% of the time) under the normal and correct operation of the test using two profilers would be $37.4 \mathrm{in} / \mathrm{mi}$.

Table 5-6 Case 2 IRI Precision Statement Summary Statistics

\begin{tabular}{|c|c|c|c|c|c|}
\hline Sites & Site average & $\begin{array}{l}\text { Repeatability } \\
\text { standard } \\
\text { deviation }(\mathrm{Sr})\end{array}$ & $\begin{array}{c}\text { Reproducibility } \\
\text { standard } \\
\text { deviation (SR) }\end{array}$ & $\begin{array}{c}95 \% \\
\text { Repeatability } \\
\text { limit (r) }\end{array}$ & $\begin{array}{c}95 \% \\
\text { Reproducibility } \\
\text { limit }(\mathrm{R})\end{array}$ \\
\hline & inches/mile & inches/mile & inches/mile & inches/mile & inches/mile \\
\hline $\begin{array}{c}\text { Site } 1 \\
\text { Covington St. }\end{array}$ & 141.96 & 2.99 & 23.62 & 8.39 & 66.14 \\
\hline $\begin{array}{c}\text { Site } 2 b \\
\text { I-65 without board }\end{array}$ & 41.13 & 2.84 & 12.89 & 7.94 & 36.09 \\
\hline $\begin{array}{c}\text { Site } 3 \mathrm{~b} \\
\text { Prairie Street } \\
\text { without board }\end{array}$ & 147.69 & 4.05 & 15.62 & 11.34 & 43.75 \\
\hline $\begin{array}{c}\text { Site } 4 \\
\text { Vinton Street }\end{array}$ & 100.33 & 3.18 & 7.14 & 8.91 & 19.98 \\
\hline $\begin{array}{c}\text { Site } 5 \\
\text { US231 A }\end{array}$ & 51.06 & 3.76 & 10.65 & 10.53 & 29.81 \\
\hline $\begin{array}{c}\text { Site } 6 \\
\text { US 231B }\end{array}$ & 62.32 & 2.82 & 10.18 & 7.88 & 28.51 \\
\hline $1 S$ & & 3.27 & 13.35 & & \\
\hline D2S & & & & 9.16 & 37.38 \\
\hline
\end{tabular}




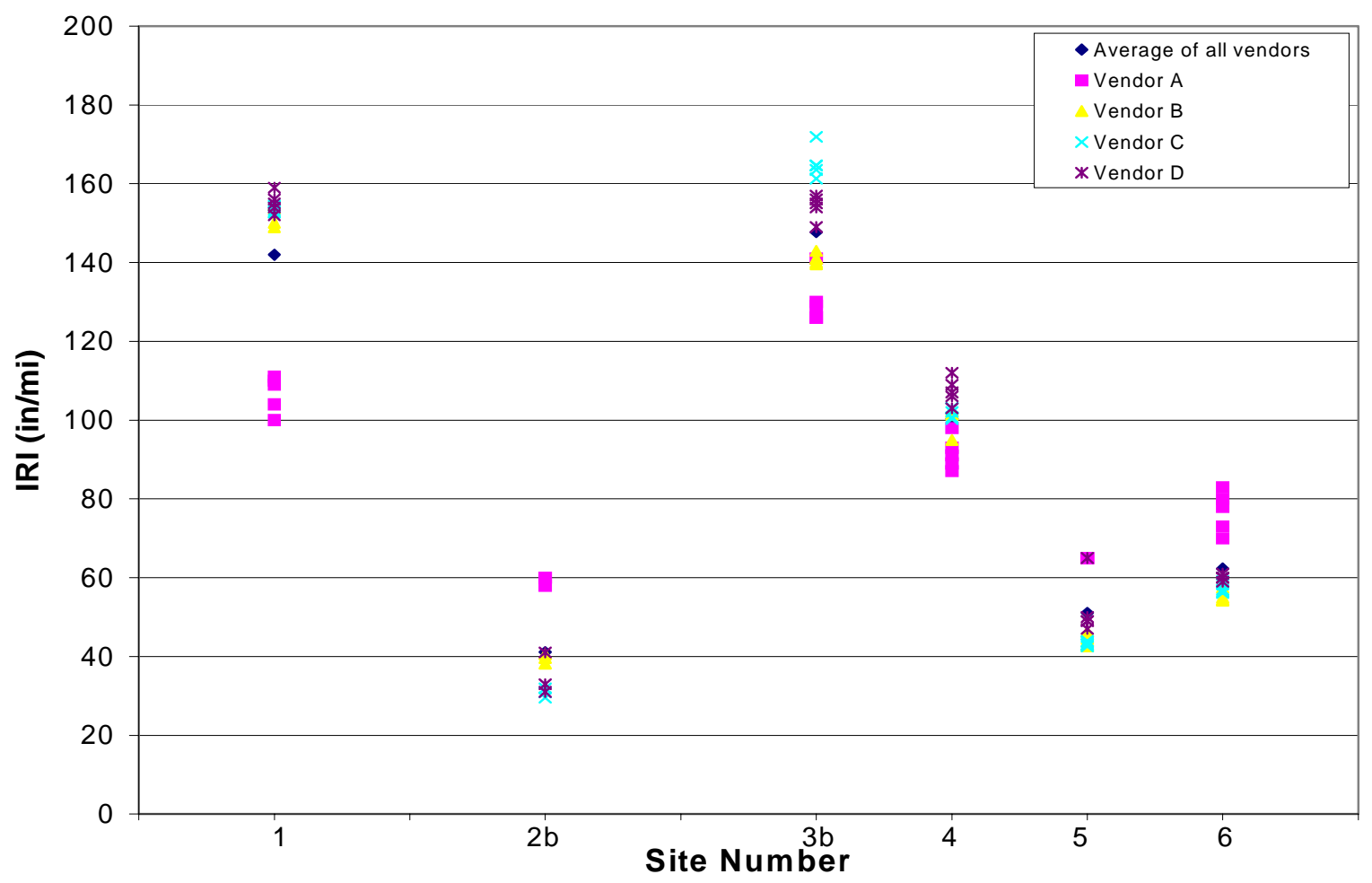

Figure 5-13 Case 2 Site Average Plot by Vendor for IRI 

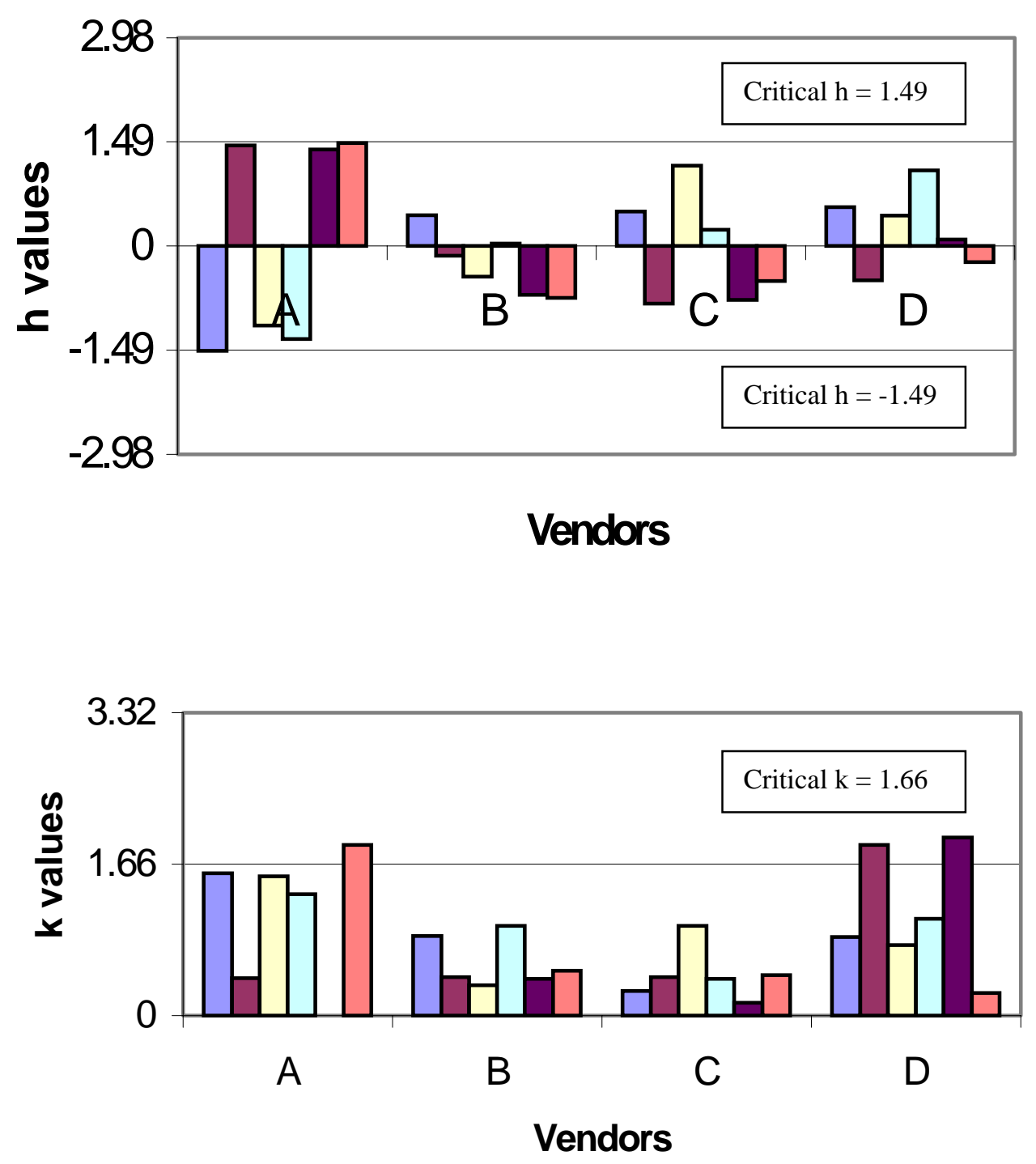

Figure 5-14 Case $2 \mathrm{~h}$ and $\mathrm{k}$ plots by Vendors for IRI

Note: The 6 columns for each vendor represent the 6 sites . 

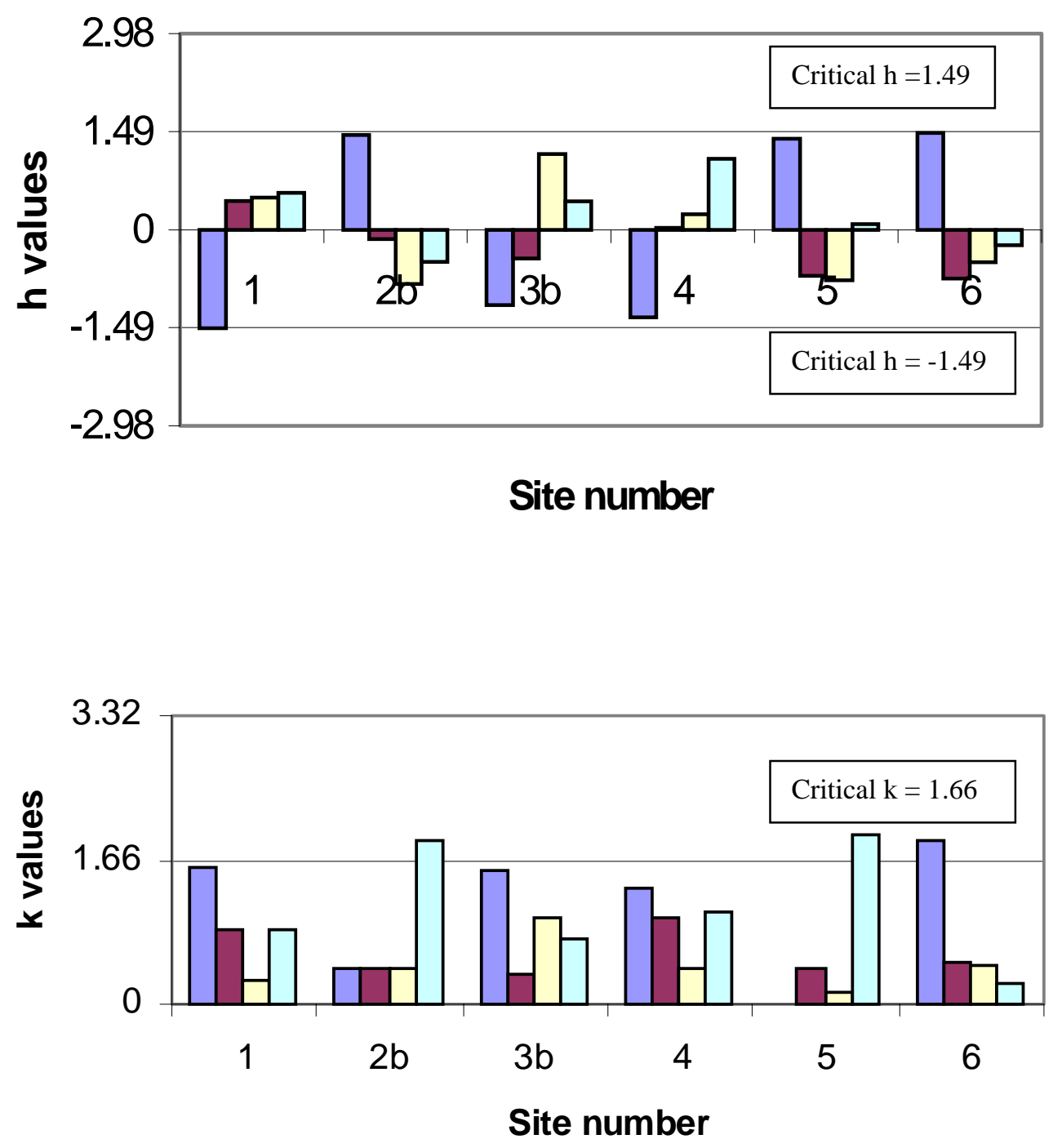

Figure 5-15 Case $2 \mathrm{~h}$ and $\mathrm{k}$ plots by Sites for IRI Note : The 4 columns for each site represent the 4 vendors. 


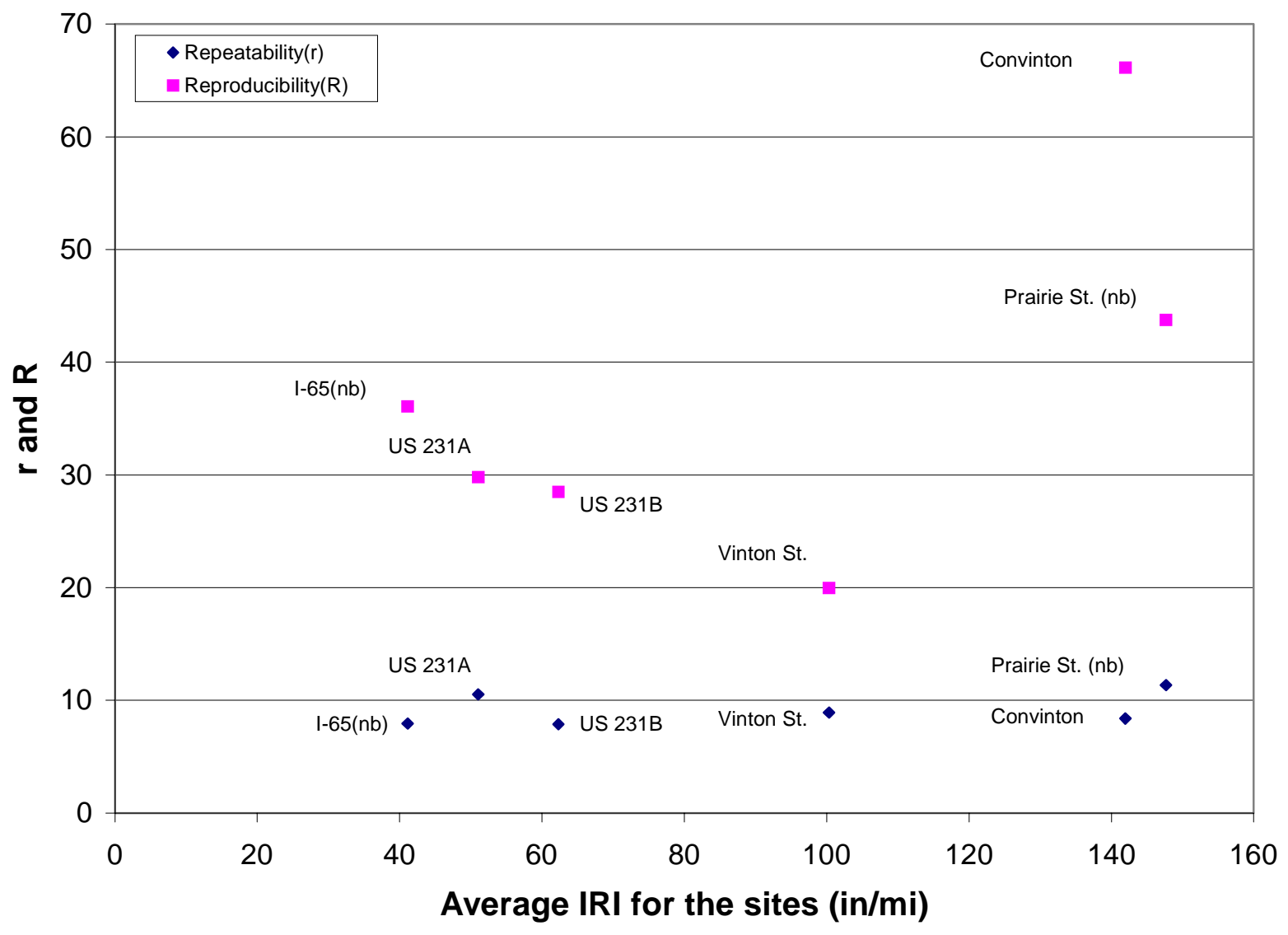

Figure 5-16 Case 2 Repeatability and Reproducibility Statistics by Site for IRI 


\subsubsection{Analysis of PI-0.0" Data}

Figure 5-17 is a plot of the individual reported PI-0.0" values by each vendor at each site. The plot also includes the average PI-0.0" for all the vendors at each site. The plot shows that Vendor A reported significantly lower PI-0.0" values than the other vendors at Sites 1 and $3 b$. Site 1 was a PCC site while Site $3 b$ was a HMA site and both were rougher compared to the other sites used in the field tests. Figures 5-18 and 5-19 are the $\mathrm{h}$ and $\mathrm{k}$ plots by vendor and site, respectively for PI-0.0". Figure 5-18 shows that the $\mathrm{h}$ values associated with Vendor A are typically opposite in sign (+ vs. -) compared to the other vendors and many approach the critical h values of 1.49 . One each of the Vendor A and Vendor $\mathrm{C} \mathrm{k}$ values exceed the critical value of 1.66 , but they are fairly similar among the vendors. Review of Figure 5-19, the $\mathrm{h}$ and $\mathrm{k}$ plots by site, shows that Vendor A reported $h$ values of exactly the opposite sign of those associated with all the other vendors at four of six sites and most of the Vendor A h-values approach the critical value. The k plot by site suggests similar within vendor precision for all of the vendors. Again Vendors $\mathrm{A}$ and $\mathrm{C}$ each have one site at which the critical $\mathrm{k}$ value was slightly exceeded and within vendor precision appears to be fairly consistent among the vendors. Based on review of the $\mathrm{h}$ and $\mathrm{k}$ plots it was concluded that Vendor A exhibited potential lack of between vendor precision.

Table 5-7 represents a summary of the statistics associated with a precision statement. Site averages as well as repeatability and reproducibility standard deviations and limits are presented by site. The repeatability standard deviations are all relatively small and consistent. However a large range of reproducibility standard deviations was 
observed. Review of Figure 5-17 suggests that this is likely due to the fact that Vendor A reported PI values much smaller than the other vendors at several sites. This is also the likely reason that there is a lack of consistent increase from repeatability to reproducibility standard deviations. As with the IRI data, both repeatability and reproducibility were improved when the boards were removed at Site 2. Interestingly, only a nominal difference was observed at Site 3 when the boards were removed. The plywood strips placed at Site 3 were 3/8" thick while those used at Site 2 were 3/4" thick. This must be taken into consideration while investigating the difference in behavior at the two sites where plywood strips were placed.

Figure 5-20 is a plot of 95 percent repeatability and reproducibility limits ( $\mathrm{r}$ and $R$, respectively) versus average PI- 0.0 " by site. The $R$ is much greater than the $r$ in the case of Covington Street (Site 1), thus it appears to be a potential outlier. The difference is also large for the Prairie Street site (Site 3b). At the Covington Street site, Vendor A reported PI values in the 20-30in/mi range while all other vendors reported values in the 60 to 80in/mi range. It can be concluded from Figure 5-8 and Figure 5-20 that the R value at Covington Street is large compared to the other vendors, but it is lesser than that at I-65 with the boards (Site 2). The maximum between vendor inconsistency among all the PCC sites was observed for Covington Street where hand-tooled transverse joints were present.

The analysis discussed above suggests that Vendor A lacked between vendor precision, particularly for rougher pavements. However both the between and within vendor performance was improved slightly compared to the previous case (Case 1) when 
all the vendors and all sites were considered (including the data from the sites with boards). With recognition of these issues the within and between vendor precision for this data set are stated in the last two rows of Table 5-7 in bold. Because the observed standard deviation is relatively constant over the range of sites (Form 1) one-sigma (1S) and difference two sigma limits (D2S) are expressed rather than one-sigma and difference two sigma limits in percent. Interpretation of $1 \mathrm{~S}$ and D2S limits for within and between vendor acceptable range of results would be as follows:

1. The within vendor acceptable range of two results equals $4.1 \mathrm{in} / \mathrm{mi}$. Therefore, the difference between two individual test results that would be equaled or exceeded in the long run in only 1 case of 20 (5\% of the time) under the normal and correct operation of the test using the same profiler and operator would be $4.1 \mathrm{in} / \mathrm{mi}$; and

2. The between vendor acceptable range of two results equals $26.3 \mathrm{in} / \mathrm{mi}$. Therefore, the difference between two individual test results that would be equaled or exceeded in the long run in only 1 case of 20 (5\% of the time) under the normal and correct operation of the test using two profilers would be $26.3 \mathrm{in} / \mathrm{mi}$. 
Table 5-7 Case 2 PI-0.0” Precision Statement Summary Statistics

\begin{tabular}{|c|c|c|c|c|c|}
\hline Sites & Site average & $\begin{array}{l}\text { Repeatability } \\
\text { standard } \\
\text { deviation }(\mathrm{Sr})\end{array}$ & $\begin{array}{c}\text { Reproducibility } \\
\text { standard } \\
\text { deviation (SR) }\end{array}$ & $\begin{array}{c}95 \% \\
\text { Repeatability } \\
\text { limit (r) }\end{array}$ & $\begin{array}{c}95 \% \\
\text { Reproducibility } \\
\text { limit (R ) }\end{array}$ \\
\hline & inches/mile & inches/mile & inches/mile & inches/mile & inches/mile \\
\hline $\begin{array}{c}\text { Site } 1 \\
\text { Covington }\end{array}$ & 66.42 & 1.77 & 26.61 & 4.96 & 74.52 \\
\hline $\begin{array}{c}\text { Site } 2 \mathrm{~b} \\
\text { I-65 without board }\end{array}$ & 8.59 & 0.94 & 4.11 & 2.64 & 11.49 \\
\hline $\begin{array}{c}\text { Site 3b } \\
\text { Prairie Street } \\
\text { without board }\end{array}$ & 52.91 & 2.3 & 12.13 & 6.44 & 33.96 \\
\hline $\begin{array}{c}\text { Site } 4 \\
\text { Vinton Street }\end{array}$ & 34.59 & 1.25 & 7.57 & 3.49 & 21.18 \\
\hline $\begin{array}{c}\text { Site } 5 \\
\text { US231 A }\end{array}$ & 16.37 & 1.04 & 2.89 & 2.92 & 8.09 \\
\hline $\begin{array}{c}\text { Site } 6 \\
\text { US 231B }\end{array}$ & 21.42 & 1.45 & 3.04 & 4.06 & 8.52 \\
\hline \multicolumn{2}{|l|}{$1 \mathrm{~S}$} & 1.46 & 9.39 & & \\
\hline \multicolumn{4}{|l|}{ D2S } & 4.08 & 26.29 \\
\hline
\end{tabular}

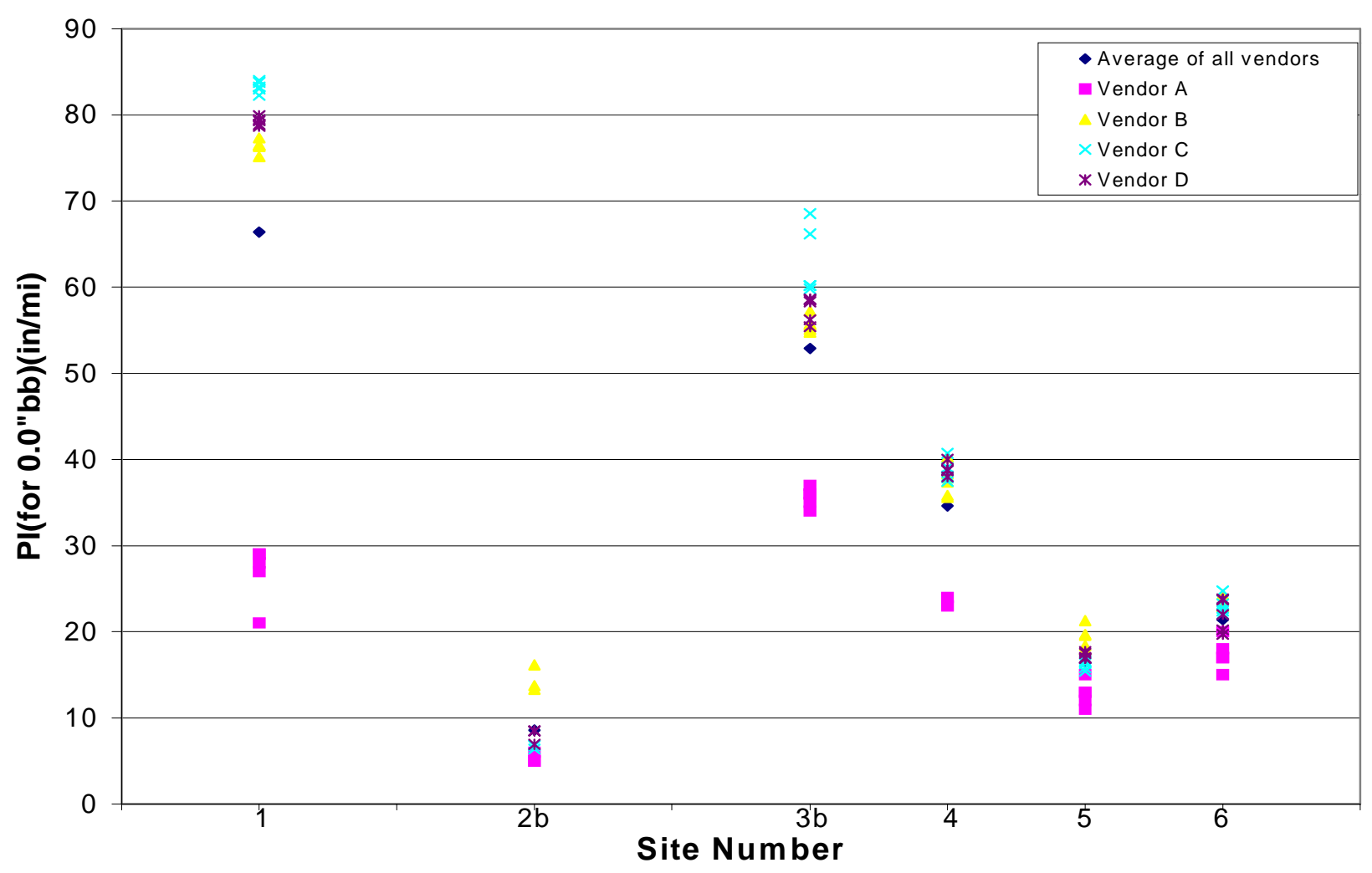

Figure 5-17 Case 2 Site Average Plot by Vendor for PI-0.0” 

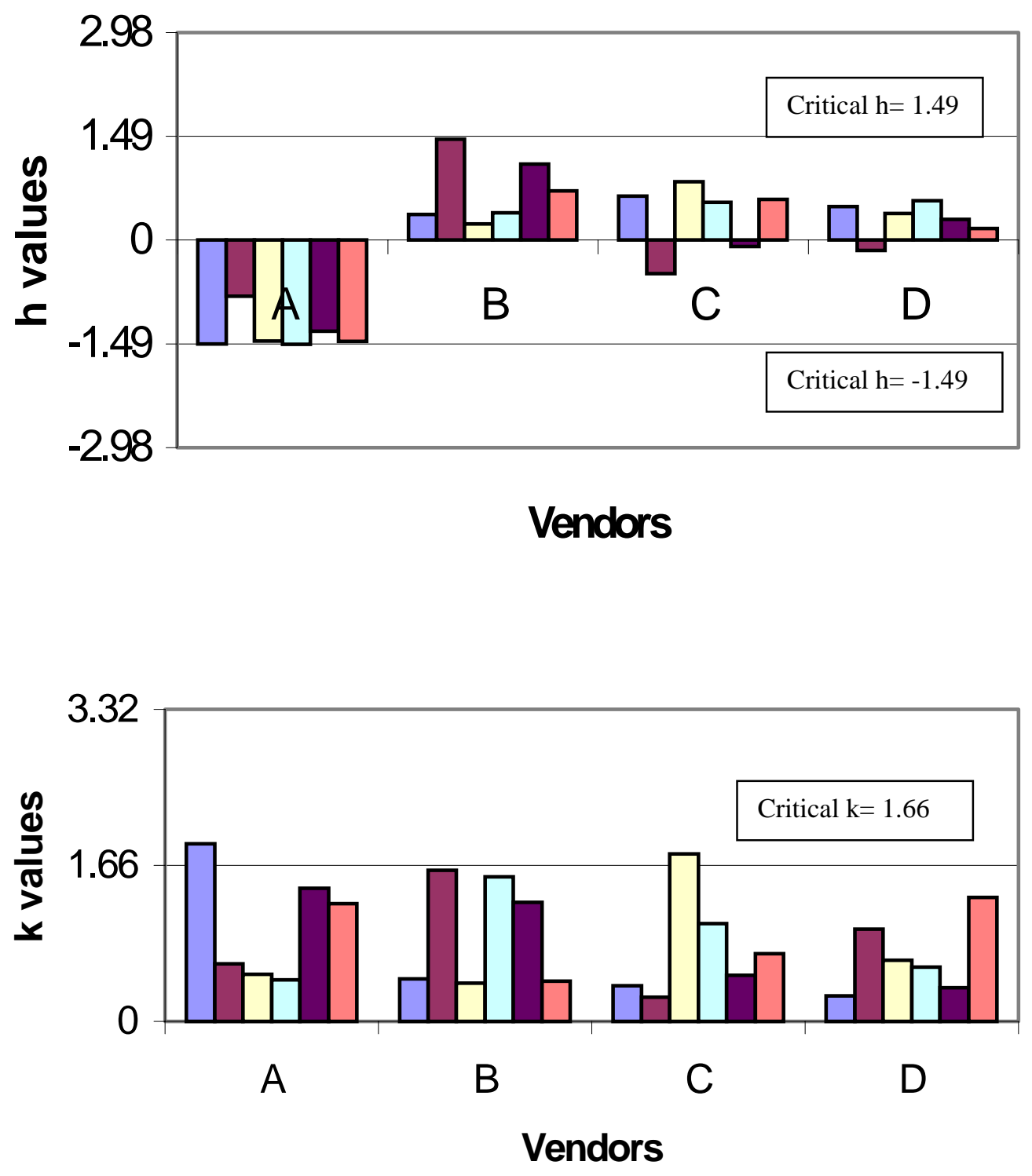

Figure 5-18 Case $2 \mathrm{~h}$ and $\mathrm{k}$ plots by Vendors for PI-0.0" Note : The 6 columns for each vendor represent the 6 sites . 

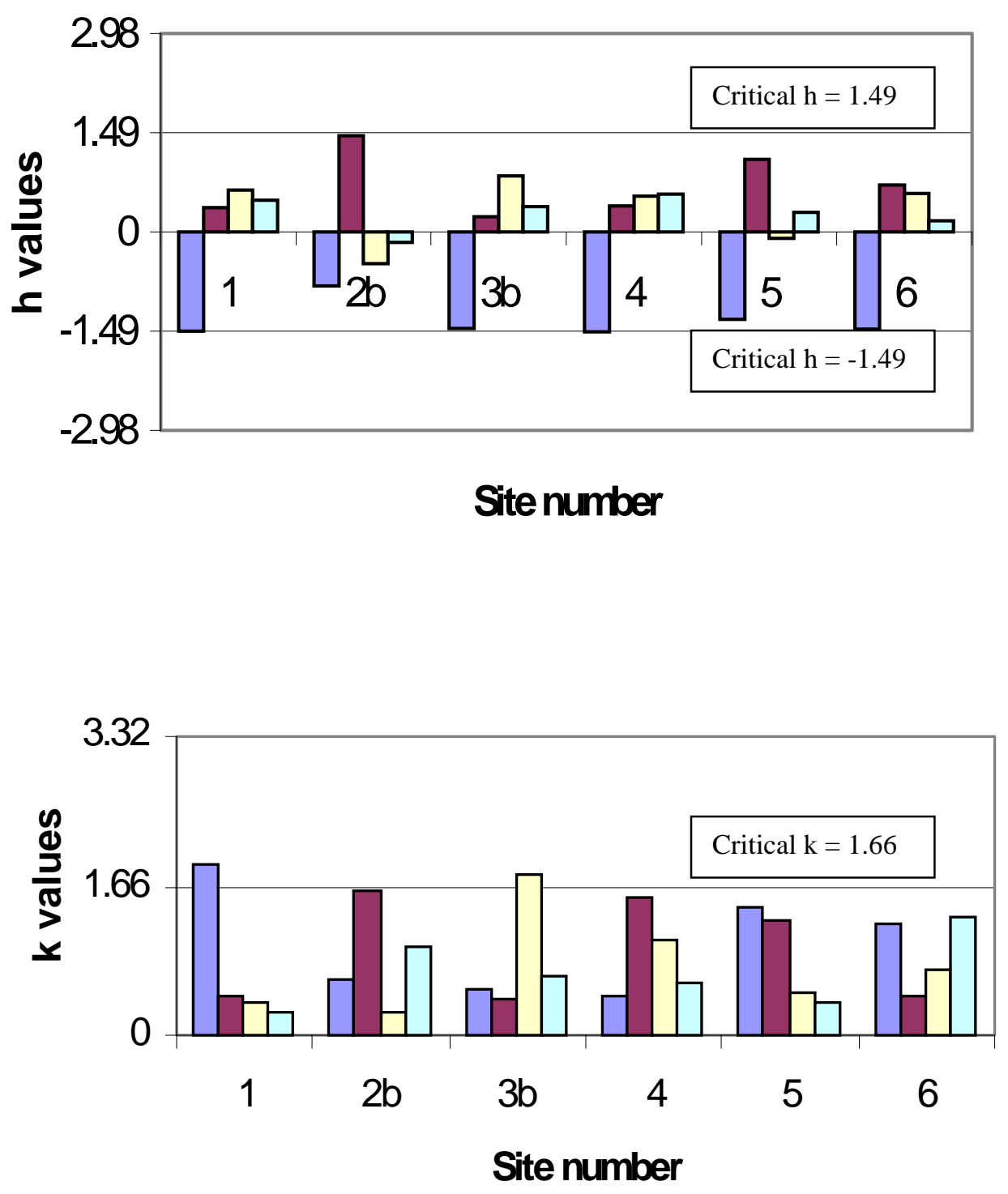

Figure 5-19 Case $2 \mathrm{~h}$ and k plots by Sites for PI-0.0"

Note: The 4 columns for each site represent the 4 vendors . 


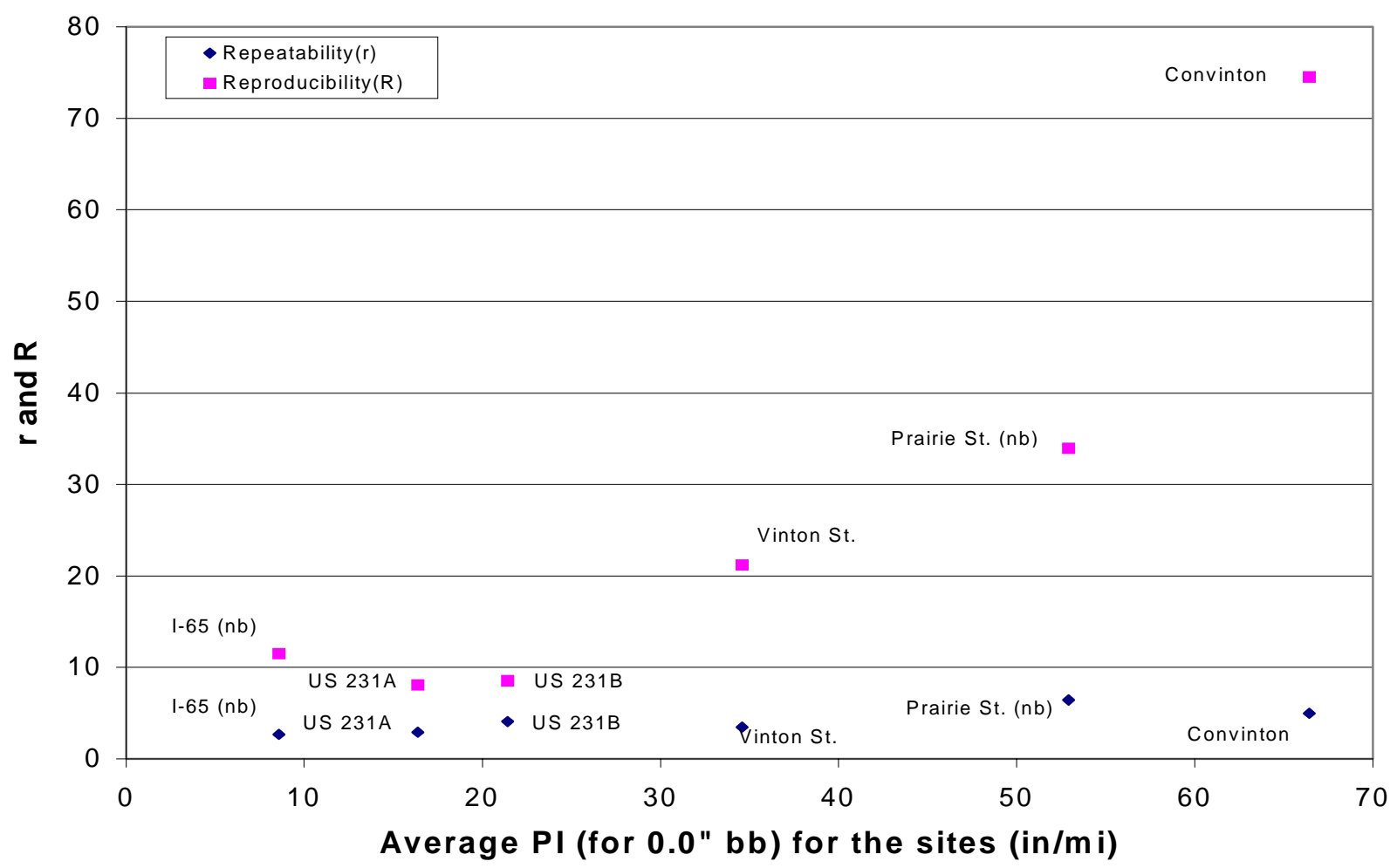

Figure 5-20 Case 2 Repeatability and Reproducibility Statistics by Site for PI-0.0" 


\subsubsection{Analysis of PI-0.2' Data}

Figure 5-21 is a plot of the individual reported PI-0.2" values by each vendor at each site. The plot also includes the average PI-0.2" for all the vendors at each site. Similar observations are made for the PI-0.2" data as to those that were made for the PI0.0" data. Vendor A reported significantly different PI-0.2" values than the other vendors at Site 1. It is worth noting that all vendors reported similar results for Sites 2 (without the boards), 5, and 6 which were all very smooth. Figures 5-22 and 5-23 are the $\mathrm{h}$ and $\mathrm{k}$ plots by vendor and site, respectively for PI-0.2”. Figure 5-22 shows that the $\mathrm{h}$ values associated with Vendor A are opposite in sign (+ vs. -) at some sites compared to the other vendors and two of six approach the critical h values of 1.49. Two of the Vendor A and one of the Vendor $\mathrm{C}$ k-values approach or exceed the critical value of 1.66 , but they are fairly similar among the vendors. Review of Figure 5-23, the $\mathrm{h}$ and $\mathrm{k}$ plots by site, shows that Vendor A has h values of exactly the opposite sign of those associated with all the other vendors at one of six sites. The $\mathrm{k}$ plot by site suggests good within vendor precision for all of the vendors, with the exception of Vendor A. Again there were two sites at which the critical $\mathrm{k}$ value was slightly exceeded by Vendor A. Based on review of the $\mathrm{h}$ and $\mathrm{k}$ plots, it was concluded that Vendor A exhibited potential lack of both within and between vendor precision, although it is improved when compared to the PI-0.0" data.

Table 5-8 represents a summary of the statistics associated with a precision statement. Site averages as well as repeatability and reproducibility standard deviations 
and limits are presented by site. The repeatability standard deviations are all relatively small and consistent. Some reproducibility standard deviations were however significantly larger than the repeatability standard deviations. Review of Figure 5-21 suggests that this is likely due to the fact that Vendor A reported PI values much smaller than the other vendors at Site 1. As with the IRI data, both repeatability and reproducibility were improved when the boards were removed at Site 2. If fact all of the vendors reported $0 \mathrm{in} / \mathrm{mi}$ PI values. This is another example of the inadequacy of the 0.2 inch blanking band.

Figure 5-24 is a plot of 95 percent repeatability and reproducibility limits ( $\mathrm{r}$ and $\mathrm{R}$, respectively) versus average PI- 0.2 " by site. The $\mathrm{R}$ is much greater than the $\mathrm{r}$ in the case of the Covington Street (Site 1). The difference is also fairly large for the Prairie Street site without the boards present (Sites 3b). At the Covington Street site Vendor A reported PI values of less than 10in/mi while all other vendors reported values in the 35 to 50in/mi range while at Prairie Street the reported PI values for Vendor A and Vendor C by approximately $20 \mathrm{in} / \mathrm{mi}$.

The analysis discussed above suggests that Vendor A lacks between vendor precision, particularly for rougher pavements. With recognition of the above stated facts, the within and between vendor precision for this data set are stated in the last two rows of Table 5-8 in bold. Because the observed standard deviation is relatively constant over the range of sites (Form 1) one-sigma (1S) and difference two sigma limits (D2S) are expressed rather than the one-sigma and difference two sigma limits in percent. 
Interpretation of the $1 \mathrm{~S}$ and $\mathrm{D} 2 \mathrm{~S}$ limits for within and between vendor acceptable range of results limits would be as follows:

1. The within vendor acceptable range of two results equals $2.4 \mathrm{in} / \mathrm{mi}$. Therefore, the difference between two individual test results that would be equaled or exceeded in the long run in only 1 case of 20 (5\% of the time) under the normal and correct operation of the test using the same profiler and operator would be $2.4 \mathrm{in} / \mathrm{mi}$; and

2. The between vendor acceptable range of two results equals $13.7 \mathrm{in} / \mathrm{mi}$. Therefore, the difference between two individual test results that would be equaled or exceeded in the long run in only 1 case of 20 (5\% of the time) under the normal and correct operation of the test using two profilers would be $13.7 \mathrm{in} / \mathrm{mi}$.

Application of the 0.2 inch blanking band actually made it appear as though the repeatability and reproducibility of the lightweight profilers was better than when the 0.0 inch blanking band were applied. This is due to the fact that low variability was associated with the smooth sites and this effectively reduced the $1 \mathrm{~S}$ and D2S limits.

The level of variability observed in the Case 2 data set was lower than that observed in the Case 1 data set, where the with boards data were considered. However Vendor A showed significant inconsistency relative to the other vendors at many sites, which in turn affected the overall precision estimates for the profilers. Hence another analysis was conducted with the Vendor A data excluded. This analysis is presented in the next section and has been identified as Case 3. The board data was considered in this case, in order to 
obtain an indication of the precision of profilers B, C and D over the largest possible range of pavement roughness.

Table 5-8 Case 2 PI-0.2” Precision Statement Summary Statistics

\begin{tabular}{|c|c|c|c|c|c|}
\hline Sites & Site average & $\begin{array}{c}\text { Repeatability } \\
\text { standard } \\
\text { deviation (Sr) }\end{array}$ & $\begin{array}{c}\text { Reproducibility } \\
\text { standard } \\
\text { deviation (SR) }\end{array}$ & $\begin{array}{c}95 \% \\
\text { Repeatability } \\
\text { limit (r) }\end{array}$ & $\begin{array}{c}95 \% \\
\text { Reproducibility } \\
\text { limit (R) }\end{array}$ \\
\hline $\begin{array}{c}\text { Site 1 } \\
\text { Covington }\end{array}$ & inches/mile & inches/mile & inches/mile & inches/mile & inches/mile \\
\hline $\begin{array}{c}\text { Site 2b } \\
\text { I-65 without board }\end{array}$ & 0 & 1.17 & 19.3 & 3.27 & 54.03 \\
\hline $\begin{array}{c}\text { Site 3b } \\
\text { Prairie Street } \\
\text { without board }\end{array}$ & 38.75 & 1.52 & 6.73 & 4.26 & 0 \\
\hline $\begin{array}{c}\text { Site4 } \\
\text { Vinton Street }\end{array}$ & 16.99 & 1.61 & 1.98 & 4.52 & 5.55 \\
\hline $\begin{array}{c}\text { Site 5 } \\
\text { US231 A }\end{array}$ & 0.24 & 0.31 & 0.31 & 0.87 & 0.87 \\
\hline $\begin{array}{c}\text { Site 6 } \\
\text { US 231B }\end{array}$ & 2.45 & 0.55 & 1.05 & 1.53 & 2.93 \\
\hline 1S $\quad$ & $\mathbf{0 . 8 6}$ & $\mathbf{4 . 9 0}$ & & $\mathbf{2 . 4 1}$ & $\mathbf{1 3 . 7 1}$ \\
\hline D2S
\end{tabular}




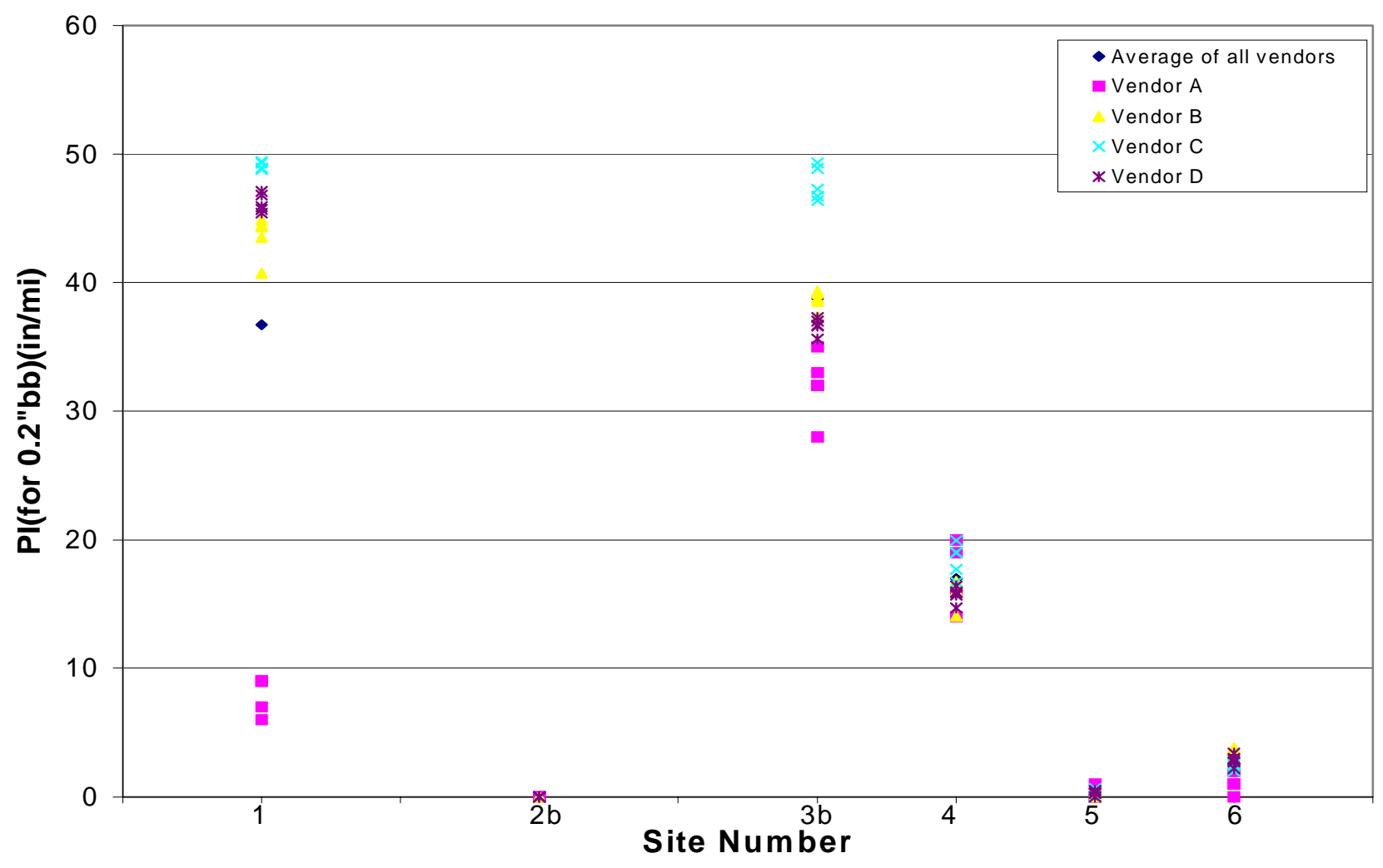

Figure 5-21 Case 2 Site Average Plot by Vendor for PI-0.2" 

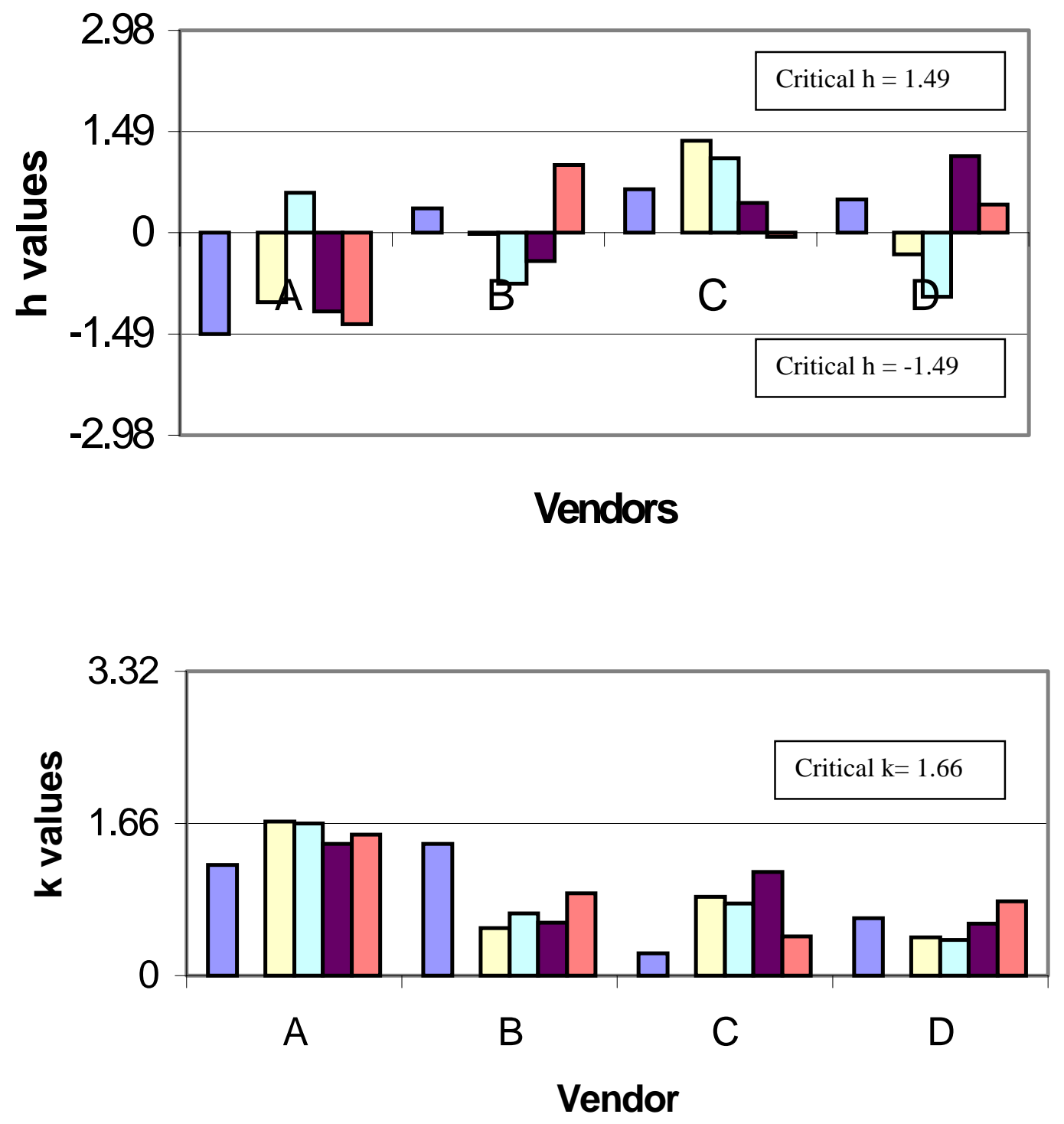

Figure 5-22 Case $2 \mathrm{~h}$ and $k$ plots by Vendors for PI-0.2" Note: The 6 columns for each vendor represent the 6 sites. 

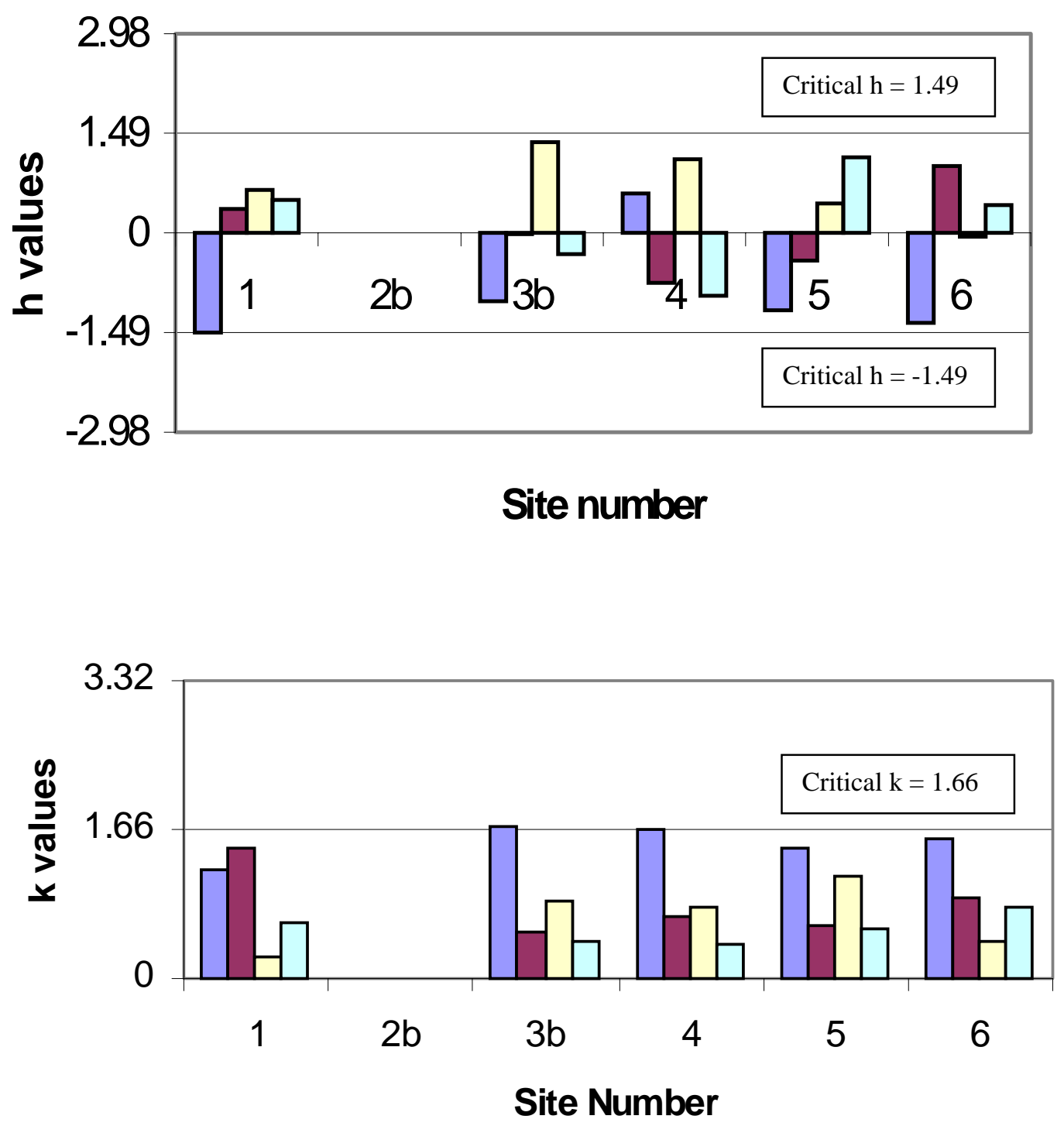

Figure 5-23 Case $2 \mathrm{~h}$ and $\mathrm{k}$ plots by Sites for PI-0.2”

Note : The 4 columns for each site represent the 4 vendors . 


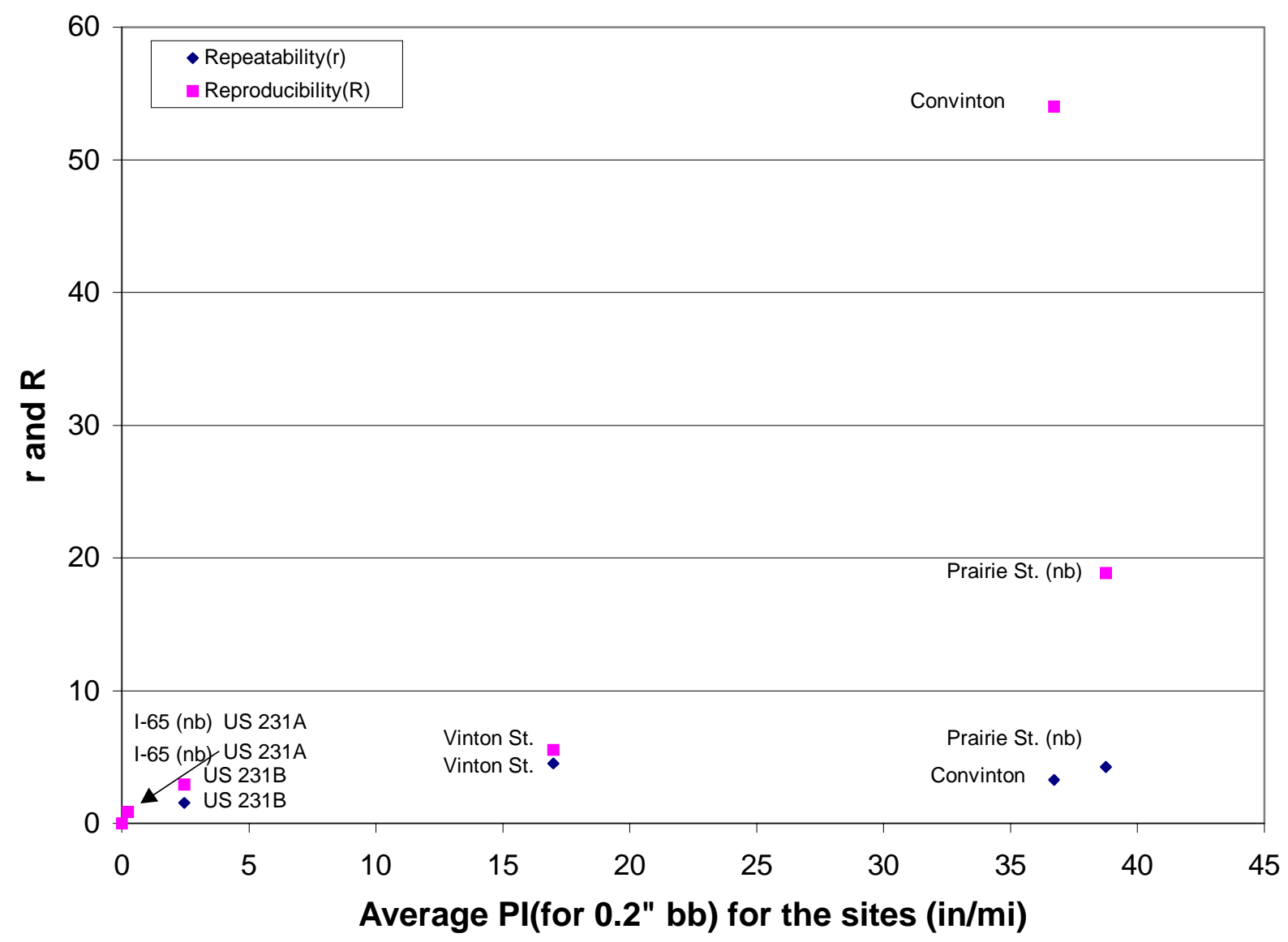

Figure 5-24 Case 2 Repeatability and Reproducibility Statistics by Site for

\section{PI-0.2”}

\subsubsection{Case 3: Precision Based on Indiana Generated Data with Boards Excluding Vendor A}

For this analysis all of the data considered were generated in Indiana and the data generated by Vendor A was excluded. However the data generated with the plywood strips (or boards in place) were included. Including the with and without board data effectively increases the degrees of freedom as the two data sets are treated as separate 
sites. All three smoothness indices were considered in this analysis. The data employed is summarized in Table 5-9. The experiment still met the requirements of ASTM C802-96. One way ANOVA was conducted separately for the data associated with each site using the ASTM E691-92 software. The data were then summarized in the form of a precision statement in accordance with ASTM C670-96. Critical values of h- and k-statistics were chosen at the 0.5 percent significance level. The critical values of $h$ and $\mathrm{k}$ were 1.15 and 1.56, respectively for the conditions outlined in Table 5-9.

Table 5-9 Summary of Case 3 Precision Analysis Considerations

\begin{tabular}{|c|c|c|c|c|c|}
\hline $\begin{array}{c}\text { Number of } \\
\text { Vendors }\end{array}$ & $\begin{array}{c}\text { Number } \\
\text { of Sites }\end{array}$ & $\begin{array}{c}\text { Replicate } \\
\text { Measurements } \\
\text { per Site }\end{array}$ & $\begin{array}{c}\text { With Board } \\
\text { Data } \\
\text { Included? }\end{array}$ & $\begin{array}{c}\text { Smoothness } \\
\text { Statistics } \\
\text { Considered }\end{array}$ & $\begin{array}{c}\text { Total } \\
\text { Degrees } \\
\text { of } \\
\text { Freedom }\end{array}$ \\
\hline 3 & 8 & 5 & Yes & $\begin{array}{c}\text { IRI, PI-0.0", } \\
\text { PI-0.2” }\end{array}$ & 56 \\
\hline (B, C, D) & 8 & 5 & &
\end{tabular}

\section{$\underline{\text { 5.2.3.1 Analysis of IRI Data }}$}

Figure 5-25 is a plot of the individual reported IRI values by each vendor (B,C and D) at each site. The plot also includes the average IRI for all the vendors (B,C, and D) at each site. The plot shows that Vendor $\mathrm{C}$ reported significantly low IRI values at Site 2 with boards than the other vendors. Site 2 was the I-65 site with the $3 / 4$ " thick plywood strips in place approximately ten feet from the beginning and end of the test section which induced significant dynamics into the profilers. Figures 5-26 and 5-27 are the $\mathrm{h}$ and $\mathrm{k}$ plots by vendor and site, respectively for IRI. Figure 5-26 shows that the $\mathrm{h}$ values associated with Vendor D differ in sign (+ vs. -) from that of other vendors at four 
sites. Review of Figure 5-27, the $\mathrm{h}$ and $\mathrm{k}$ plots by site, show that Vendor D reported $\mathrm{h}$ values of exactly the opposite sign of those associated with Vendors B, C and D at four of the eight sites. Many of the Vendor D h values also approach the critical $h$ value. This is also true for the other vendors too however. The $\mathrm{k}$ plot by site shows that Vendor D has $\mathrm{k}$ values at two sites exceeding the critical $\mathrm{k}$ value. But the within vendor precision appears to be fairly similar among the vendors. Based on review of the $\mathrm{h}$ and $\mathrm{k}$ plots it might be concluded that Vendor D exhibited potential lack of within vendor precision. However from the analysis of the previous two cases (Case 1 and 2), it can also be concluded that the lack of precision associated with Vendor D is less than was observed for Vendor A. Precision analysis always highlights the vendors with a potential lack of between and within vendor consistency. Hence when Vendor A was excluded, the lack of within vendor consistency observed for Vendor D compared to the other vendors became more evident.

Table 5-10 provides a summary of the statistics associated with a precision statement. Site averages as well as repeatability and reproducibility standard deviations and limits are presented by site. It is important to note the impact of removing the Vendor A data on the reproducibility standard deviation for Site 1 . The repeatability and reproducibility standard deviations are all relatively small and consistent with the exception of those associated with Site 2. It is clear from review of the Site $2 b$ data (I-65 without boards) that the boards induced greater dynamics than one or more of the profilers could tolerate. For most of the sites the reproducibility standard deviations are one to two times greater than the repeatability standard deviations with the exception of 
Site 2. The reason for the high standard deviations associated with Site 2 is due to the significant difference in IRI reported by Vendor C when compared to the other vendors.

Figure 5-28 is a plot of 95 percent repeatability and reproducibility limits ( $\mathrm{r}$ and $R$, respectively) versus average IRI by site. The $R$ value is much greater than $r$ in the case of the I-65 site with board (Site 2). However it is not as high as that observed in Case 1( IRI value of $91 \mathrm{in} / \mathrm{mi}$ versus $223 \mathrm{in} / \mathrm{mi}$ in Case 1). Vendor $\mathrm{C}$ reported a relatively low value in the range of $30-40 \mathrm{in} / \mathrm{mi}$ due to which the $\mathrm{R}$ value for this site is relatively high.

The analysis discussed above suggests that the plywood strips at Site 2 induced profiler dynamics that were too great for some vendors. With recognition of the above mentioned facts the within and between vendor precision for this data set are stated in the last two rows of Table 5-10 in bold. Because the observed standard deviations are relatively constant over the range of sites (Form 1) one-sigma (1S) and difference two sigma limits (D2S) are expressed rather than the one-sigma and difference two sigma limits in percent. Interpretation of $1 \mathrm{~S}$ and $\mathrm{D} 2 \mathrm{~S}$ limits for within and between vendor acceptable range of results limits would be as follows:

1. The within vendor acceptable range of two results equals $9.0 \mathrm{in} / \mathrm{mi}$. Therefore, the difference between two individual test results that would be equaled or exceeded in the long run in only 1 case of 20 (5\% of the time) under the normal and correct operation of the test using the same profiler and operator would be $9.0 \mathrm{in} / \mathrm{mi}$; and

2. The between vendor acceptable range of two results equals $27.2 \mathrm{in} / \mathrm{mi}$. Therefore, the difference between two individual test results that would be equaled or exceeded in 
the long run in only 1 case of 20 (5\% of the time) under the normal and correct operation of the test using two profilers would be $27.2 \mathrm{in} / \mathrm{mi}$.

Table 5-10 Case 3 IRI Precision Statement Summary Statistics

\begin{tabular}{|c|c|c|c|c|c|}
\hline Sites & Site average & $\begin{array}{l}\text { Repeatability } \\
\text { standard } \\
\text { deviation }(\mathrm{Sr})\end{array}$ & $\begin{array}{c}\text { Reproducibility } \\
\text { standard } \\
\text { deviation (SR) }\end{array}$ & $\begin{array}{c}95 \% \\
\text { Repeatability } \\
\text { limit } \AA\end{array}$ & $\begin{array}{c}95 \% \\
\text { Reproducibility } \\
\text { limit (R ) }\end{array}$ \\
\hline & inches/mile & inches/mile & inches/mile & inches/mile & inches/mile \\
\hline $\begin{array}{c}\text { Site } 1 \\
\text { Covington } \\
\end{array}$ & 153.68 & 2.17 & 2.41 & 6.09 & 6.76 \\
\hline $\begin{array}{c}\text { Site } 2 \\
\text { I-65 with board }\end{array}$ & 68.85 & 3.61 & 32.36 & 10.11 & 90.6 \\
\hline $\begin{array}{c}\text { Site } 2 \mathrm{~b} \\
\mathrm{I}-65 \text { without board }\end{array}$ & 35.06 & 3.21 & 5.18 & 8.98 & 14.50 \\
\hline $\begin{array}{c}\text { Site } 3 \\
\text { Prairie Street } \\
\text { with board }\end{array}$ & 161.11 & 5.4 & 11.89 & 15.1 & 33.30 \\
\hline $\begin{array}{c}\text { Site } 3 \mathrm{~b} \\
\text { Prairie Street } \\
\text { without board }\end{array}$ & 153.46 & 3.02 & 12.42 & 8.46 & 34.79 \\
\hline $\begin{array}{c}\text { Site } 4 \\
\text { Vinton Street }\end{array}$ & 103.24 & 2.75 & 4.41 & 7.69 & 12.36 \\
\hline $\begin{array}{c}\text { Site } 5 \\
\text { US231 A }\end{array}$ & 46.42 & 4.34 & 6.21 & 12.16 & 17.4 \\
\hline $\begin{array}{c}\text { Site } 6 \\
\text { US 231B }\end{array}$ & 57.49 & 1.15 & 2.68 & 3.23 & 7.51 \\
\hline \multicolumn{2}{|l|}{$1 \mathrm{~S}$} & 3.21 & 9.7 & & \\
\hline \multicolumn{4}{|l|}{ D2S } & 8.98 & 27.15 \\
\hline
\end{tabular}




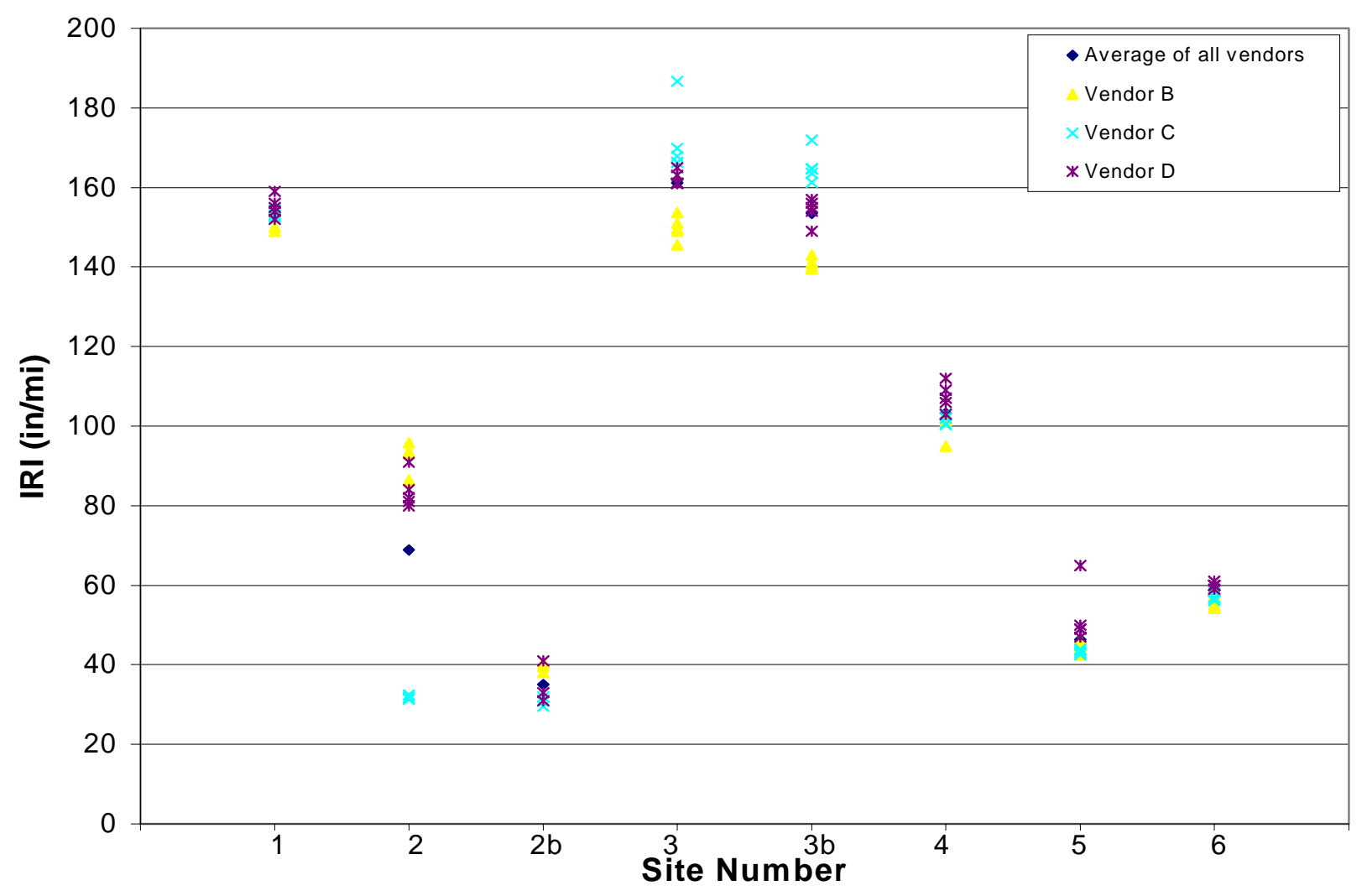

Figure 5-25 Case 3 Site Average Plot by Vendor for IRI 


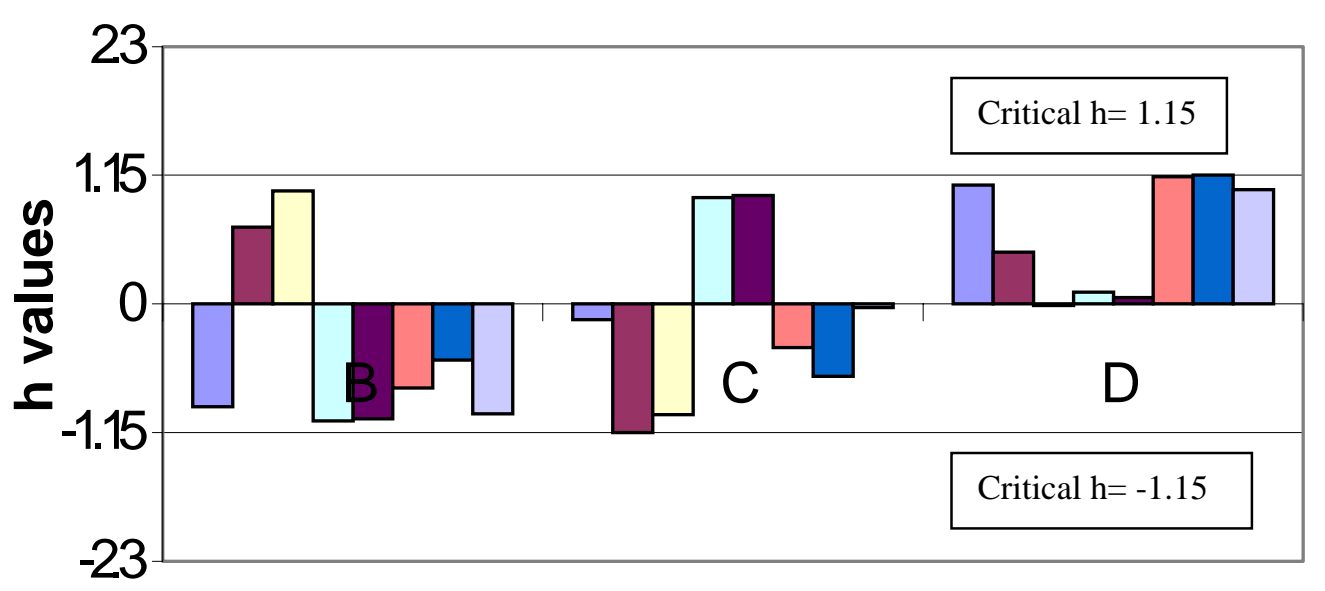

Vendars

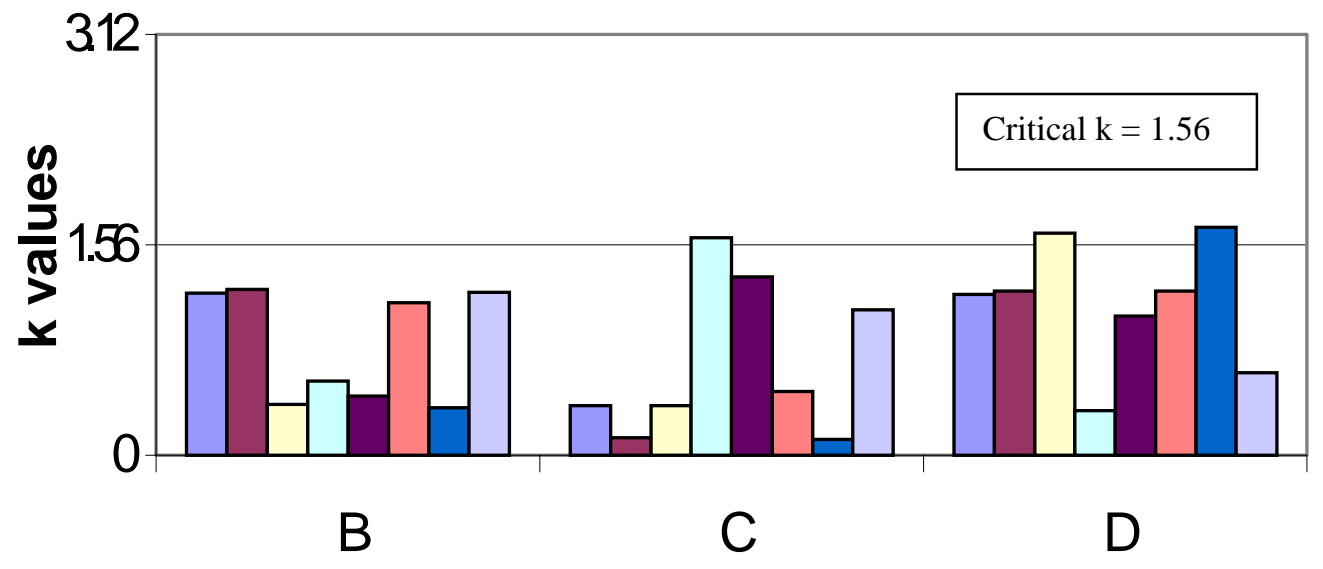

Vendors

Figure 5-26 Case $3 \mathrm{~h}$ and $\mathrm{k}$ plots by Vendors for IRI

Note : The 8 columns for each vendor represent the 6 sites (Site 2 and Site 3-with and without board) 

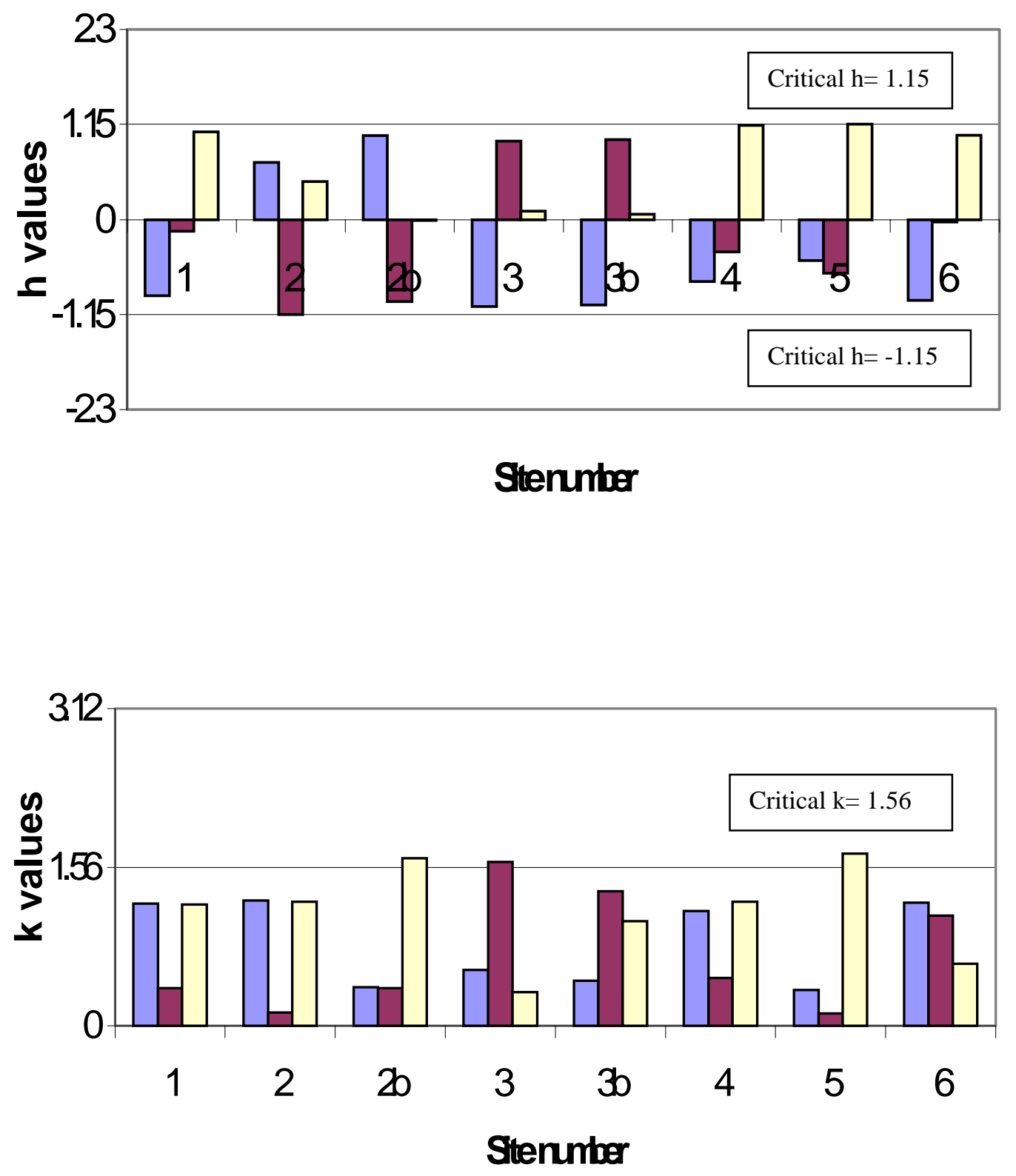

Figure 5-27 Case $3 \mathrm{~h}$ and $\mathrm{k}$ plots by Sites for IRI Note: The 3 columns for each site represent the 3 vendors $B, C$ and $D$. 


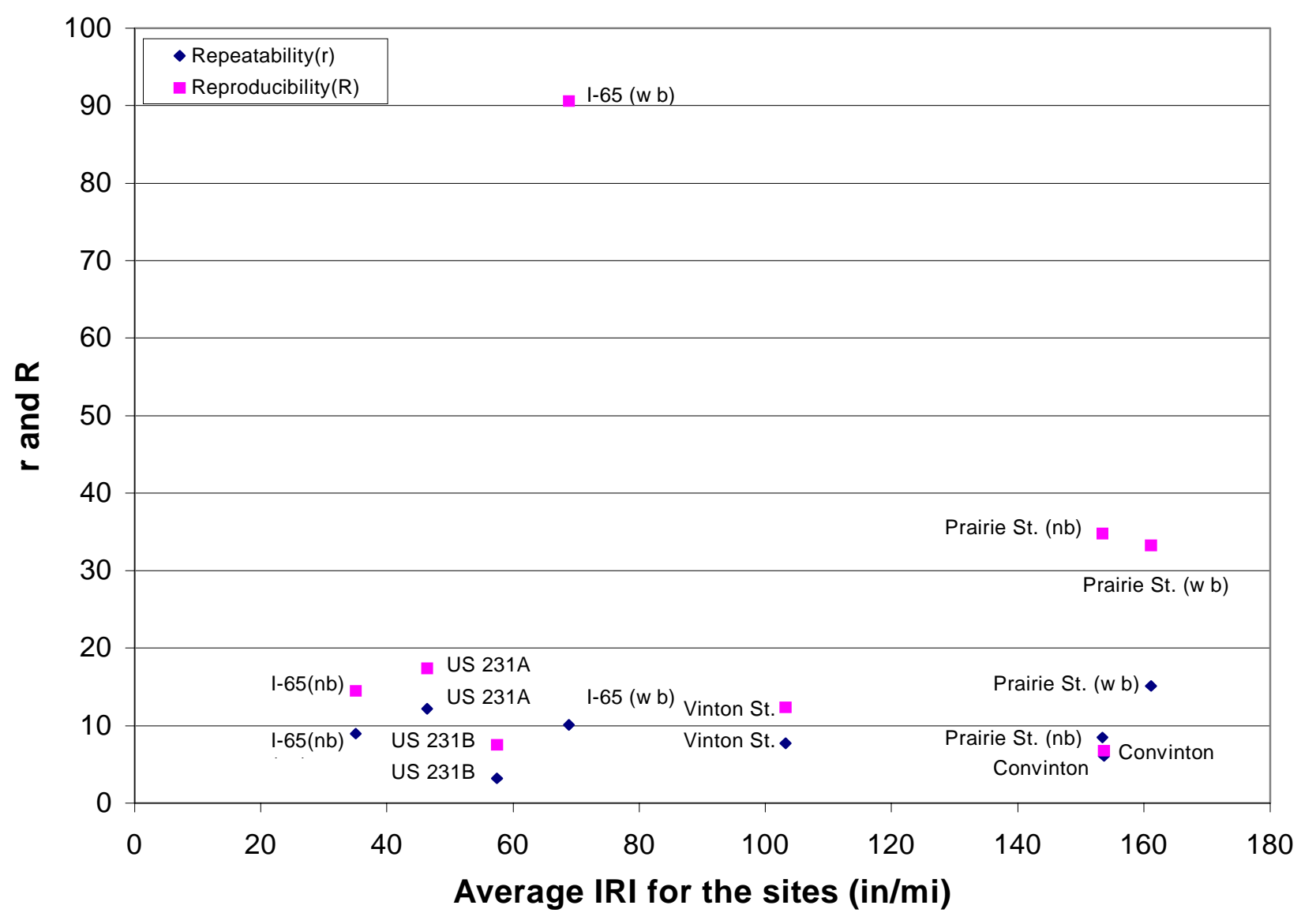

Figure 5-28 Case 3 Repeatability and Reproducibility Statistics by Site for IRI

\subsubsection{Analysis of PI-0.0"' Data}

Figure 5-29 is a plot of the individual reported PI-0.0" values by each vendor at each site. The plot also includes the average PI-0.0" for all the vendors at each site. Plot shows considerable scatter of PI values at Site 2 with the boards in place. Site 2 was the I-65 site with the $3 / 4$ " thick plywood strips in place approximately ten feet from the beginning and end of the test section which induced significant dynamics into the profilers. Figures 5-30 and 5-31 are the $\mathrm{h}$ and $\mathrm{k}$ plots by vendor and site, respectively for PI-0.0". Figure 5-30 shows fairly well distributed h values even though several approach the critical h value of 1.15 . Two of the Vendor B $\mathrm{k}$ values approach or exceed the critical 
value of 1.56, but they are fairly similar among the vendors. Review of Figure 5-31, the $\mathrm{h}$ and $\mathrm{k}$ plots by site, again shows fairly well distributed $\mathrm{h}$ and $\mathrm{k}$ values. The $\mathrm{k}$ plot by site suggests similar within vendor precision. Again the critical k value is slightly exceeded by Vendor B at two sites and Vendor $\mathrm{C}$ at one site and within vendor precision appears to be fairly consistent among the vendors. Based on review of the $\mathrm{h}$ and $\mathrm{k}$ plots it is concluded that both within and between vendor consistency is fairly good. This is in sharp contrast with the analysis of IRI where Vendor D was identified to exhibit potential lack of within vendor consistency.

Table 5-11 represents a summary of the statistics associated with a precision statement. Site averages as well as repeatability and reproducibility standard deviations and limits are presented by site. The repeatability standard deviations are all relatively small and consistent. The reproducibility standard deviation was larger than the repeatability standard deviation but was much smaller than that observed for the other two cases (Cases 1 and 2). Review of Figure 5-29 suggests that this is likely due to the fact that Vendor B reported PI values larger than the Vendors C and D at Site 2 with the boards in place (Site 2). As with the IRI data, both repeatability and reproducibility are improved when the boards were removed at Site 2. Interestingly, only a nominal difference was observed at Site 3 when the boards were removed.

Figure 5-32 is a plot of 95 percent repeatability and reproducibility limits ( $\mathrm{r}$ and $\mathrm{R}$, respectively) versus average PI-0.0" by site. The $\mathrm{R}$ is much greater than the $\mathrm{r}$ in the 
case of I-65 site with board (Site 2) as Vendor B reported high PI-0.0" values relative to the other vendors at this site.

The analysis discussed above suggests that Vendor B lacked between vendor precision, in comparison to Vendors C and D. However the lack of precision is much less than that observed for Vendor A in Cases 1 and 2. This is evident from the improvement in repeatability and reproducibility standard deviations observed for this case compared to Cases 1 and 2. Plywood strips at Site 2 induced significant dynamics to the profilers and improvements were observed at Site 2 when the boards were removed. With recognition of the potential lack of between vendor precision (Vendor B), the within and between vendor precision for this data set are stated in the last two rows of Table 5-11 in bold. Because the observed standard deviations are relatively constant over the range of sites (Form 1) one-sigma (1S) and difference two sigma limits (D2S) are expressed rather than one-sigma and difference two sigma limits in percent. Interpretation of the $1 \mathrm{~S}$ and D2S limits for within and between vendor acceptable range of results limits would be as follows:

1. The within vendor acceptable range of two results equals $3.9 \mathrm{in} / \mathrm{mi}$. Therefore, the difference between two individual test results that would be equaled or exceeded in the long run in only 1 case of 20 (5\% of the time) under the normal and correct operation of the test using the same profiler and operator would be 3.9in/mi; and

2. The between vendor acceptable range of two results equals $11.1 \mathrm{in} / \mathrm{mi}$. Therefore, the difference between two individual test results that would be equaled or exceeded in 
the long run in only 1 case of 20 (5\% of the time) under the normal and correct operation of the test using two profilers would be $11.1 \mathrm{in} / \mathrm{mi}$.

Table 5-11 Case 3 PI-0.0” Precision Statement Summary Statistics

\begin{tabular}{|c|c|c|c|c|c|}
\hline Sites & Site average & $\begin{array}{l}\text { Repeatability } \\
\text { standard } \\
\text { deviation }(\mathrm{Sr})\end{array}$ & $\begin{array}{c}\text { Reproducibility } \\
\text { standard } \\
\text { deviation (SR) }\end{array}$ & $\begin{array}{c}95 \% \\
\text { Repeatability } \\
\text { limit (r) }\end{array}$ & $\begin{array}{c}95 \% \\
\text { Reproducibility } \\
\text { limit (R) }\end{array}$ \\
\hline & inches/mile & inches/mile & inches/mile & inches/mile & inches/mile \\
\hline $\begin{array}{c}\text { Site } 1 \\
\text { Covington }\end{array}$ & 79.63 & 0.67 & 3.53 & 1.87 & 9.87 \\
\hline $\begin{array}{c}\text { Site } 2 \\
\text { I-65 with board }\end{array}$ & 24.04 & 1.44 & 12.3 & 4.04 & 34.44 \\
\hline $\begin{array}{c}\text { Site } 2 b \\
\text { I-65 without board }\end{array}$ & 9.67 & 1.04 & 4.24 & 2.90 & 11.88 \\
\hline $\begin{array}{c}\text { Site } 3 \\
\text { Prairie Street } \\
\text { with board }\end{array}$ & 59.46 & 1.83 & 2.48 & 5.12 & 6.94 \\
\hline $\begin{array}{c}\text { Site } 3 b \\
\text { Prairie Street } \\
\text { without board }\end{array}$ & 58.68 & 2.57 & 4.46 & 7.20 & 12.48 \\
\hline $\begin{array}{c}\text { Site } 4 \\
\text { Vinton Street }\end{array}$ & 38.32 & 1.40 & 1.45 & 3.93 & 4.07 \\
\hline $\begin{array}{c}\text { Site } 5 \\
\text { US231 A }\end{array}$ & 17.56 & 0.85 & 1.82 & 2.37 & 5.09 \\
\hline $\begin{array}{c}\text { Site } 6 \\
\text { US 231B }\end{array}$ & 22.76 & 1.30 & 1.40 & 3.65 & 3.92 \\
\hline \multicolumn{2}{|l|}{$1 \mathrm{~S}$} & 1.39 & 3.96 & & \\
\hline \multicolumn{4}{|l|}{ D2S } & 3.88 & 11.09 \\
\hline
\end{tabular}




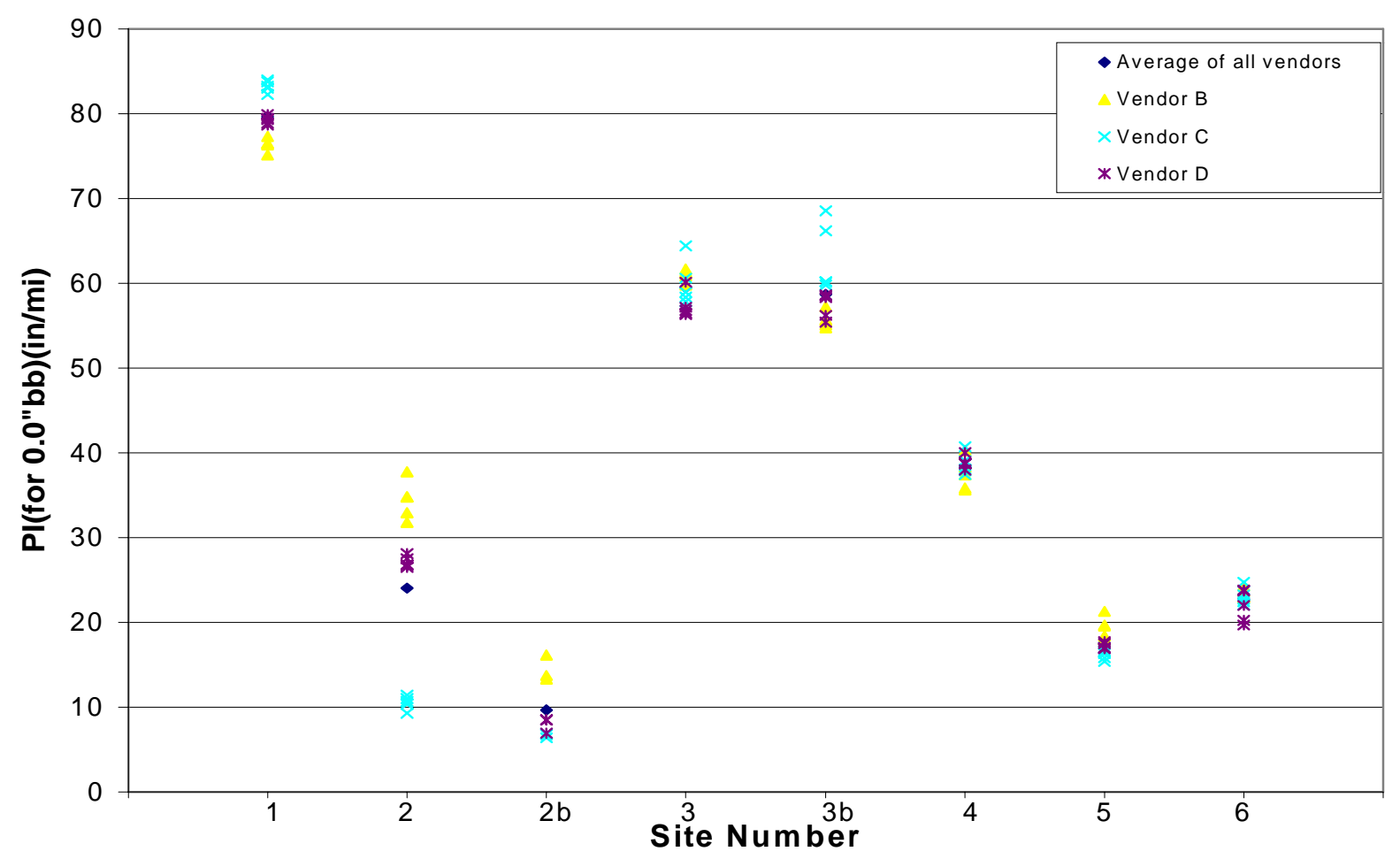

Figure 5-29 Case 3 Site Average Plot by Vendor for PI-0.0” 

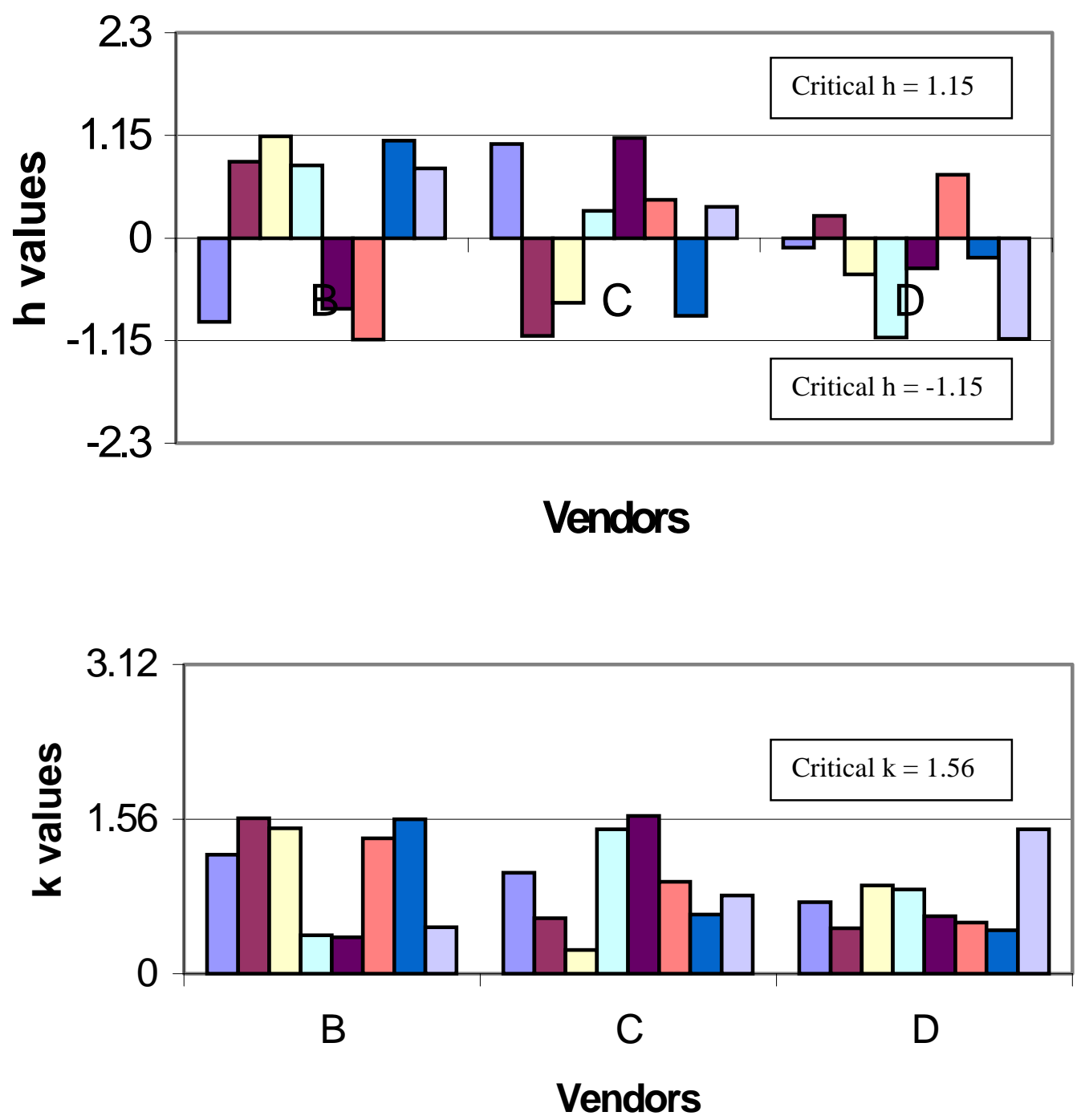

Figure 5-30 Case $3 \mathrm{~h}$ and k plots by Vendors for PI-0.0"

Note: The 8 columns for each vendor represent the 6 sites (site 2 and 3 with and without board). 

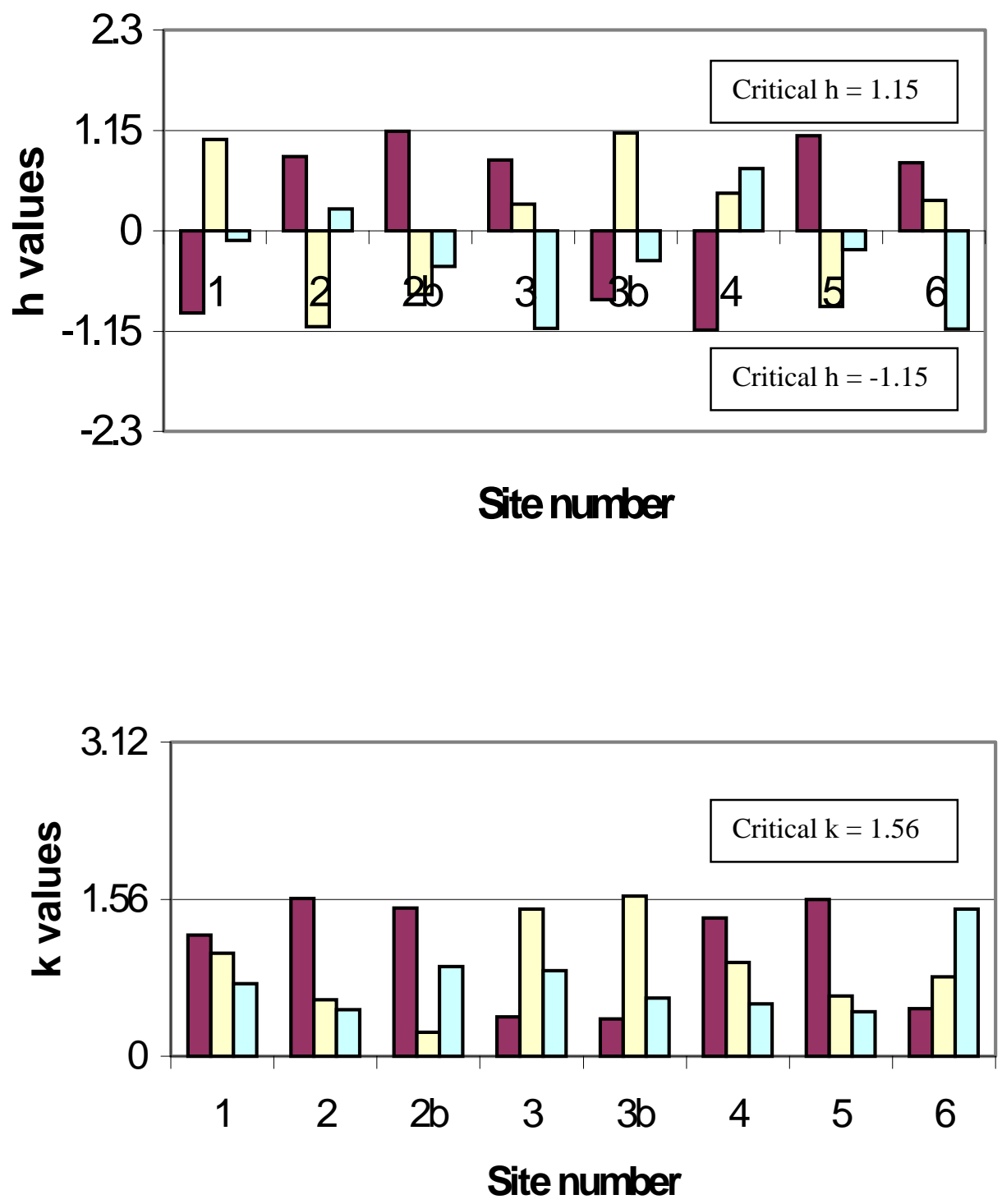

Figure 5-31 Case $3 \mathrm{~h}$ and k plots by Sites for PI-0.0"

Note : The 3 columns for each site represent the 3 vendors . 


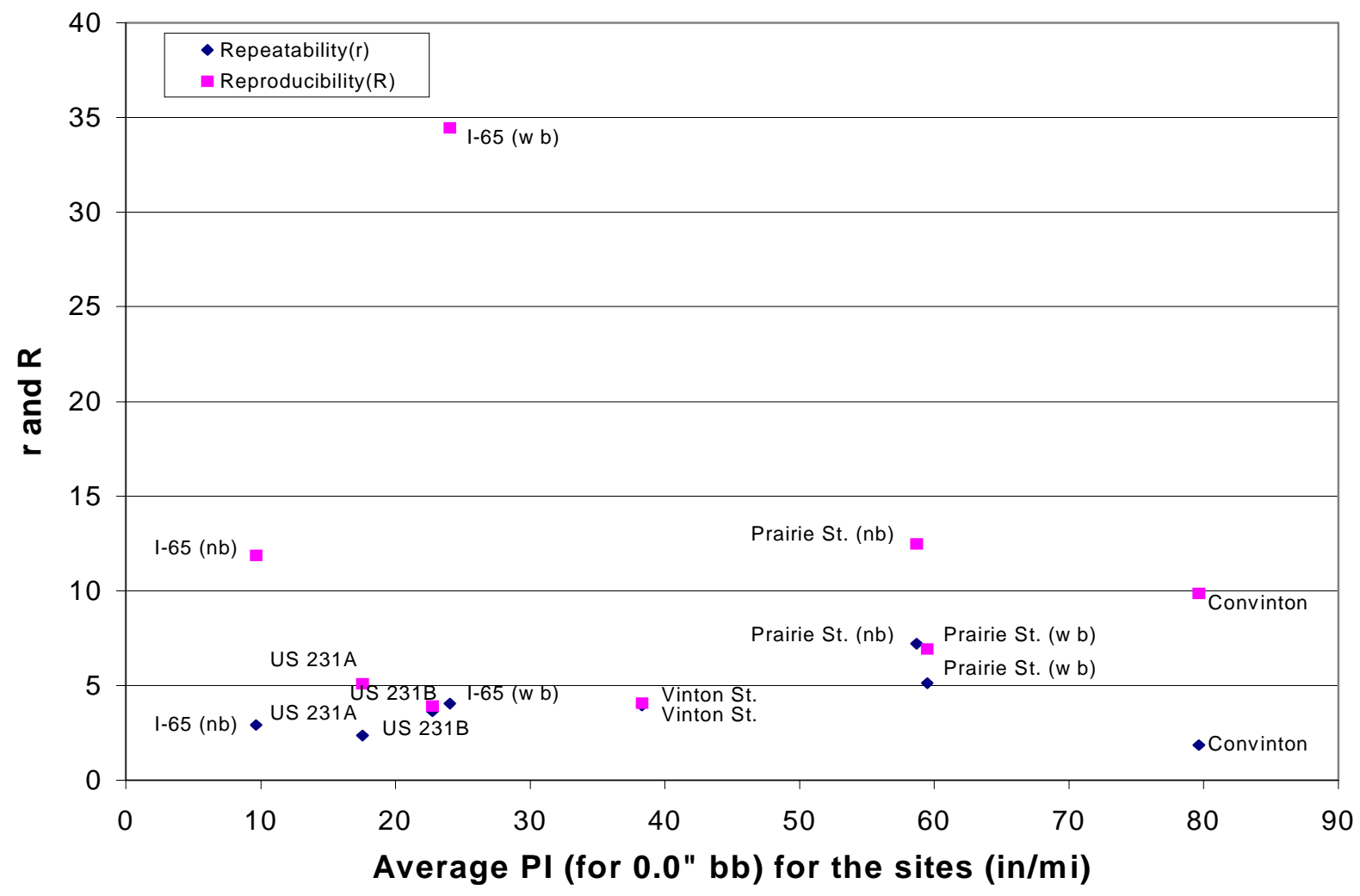

Figure 5-32 Case 3 Repeatability and Reproducibility Statistics by Site for PI-0.0"

\subsubsection{Analysis of PI-0.2" Data}

Figure 5-33 is a plot of the individual reported PI-0.2" values by each vendor at each site. The plot also includes the average PI-0.2" for all the vendors at each site. Similar observations are made for the PI-0.2" data as to those that were made for the PI0.0" data. Vendor C reported significantly different PI-0.2" values than the other vendors at Sites 2 with boards (Site 2) and Site 3 with boards (Site 3). It is worth noting that all vendors reported similar results for Sites 2 (without the boards), 5, and 6 which were all very smooth. Figures 5-34 and 5-35 are the $\mathrm{h}$ and $\mathrm{k}$ plots by vendor and site, respectively for PI-0.2". Figure 5-34 shows that the h values associated with Vendor $\mathrm{C}$ are opposite in sign (+ vs. -) at several sites compared to the other vendors and three of eight approach 
the critical $h$ values of 1.15 . One of the Vendor $\mathrm{C}$ and one of the Vendor $\mathrm{B} \mathrm{k}$ values exceed the critical value of 1.56 , but they are fairly similar among the vendors. Review of Figure 5-35, the $\mathrm{h}$ and $\mathrm{k}$ plots by site, shows that Vendor $\mathrm{C}$ has $\mathrm{h}$ values of exactly the opposite sign of those associated with the other vendors at five of eight sites. The k plot by site suggests similar within vendor precision for all of the vendors. Again Vendors A and $\mathrm{C}$ had one site each at which the critical $\mathrm{k}$ value was slightly exceeded. Within vendor precision appears to be fairly consistent among the vendors. Based on review of the $\mathrm{h}$ and $\mathrm{k}$ plots, it was concluded that Vendor $\mathrm{C}$ exhibited potential lack of between vendor precision, compared to vendors B and D. This is in contrast to the analyses of IRI and PI-0.0" where Vendors D and B exhibited lack of within vendor consistency, respectively.

Table 5-12 represents a summary of the statistics associated with a precision statement. Site averages as well as repeatability and reproducibility standard deviations and limits are presented by site. The repeatability standard deviations are all relatively small and fairly consistent. Reproducibility standard deviations were larger than the repeatability standard deviations but they were significantly smaller than observed for Cases 1 and 2. A significantly higher reproducibility standard deviation was observed for the I-65 site with the boards in place due to the very low PI values reported by Vendor C relative to the other vendors. However both the repeatability and reproducibility standard deviations improved when the board was removed. In fact at Site 2 without the boards, all the vendors reported zero PI values. Figure 5-36 is a plot of 95 percent repeatability and reproducibility limits ( $\mathrm{r}$ and $\mathrm{R}$, respectively) versus average PI-0.2" by site. The $\mathrm{R}$ is 
much greater than the $\mathrm{r}$ in the case of the I-65 site with boards (Site 2) due to the low PI values reported by Vendor C.

The analysis discussed above suggests that Vendor $\mathrm{C}$ potentially lacks between vendor precision when compared to Vendors B and D. However when the three cases (Case 1, 2, and 3) are contrasted, it is apparent that it is much similar to the other devices than Vendor A. The plywood strips at Site 2 induced significant dynamics to the profilers and improvements in precision were observed at Site 2 when the boards were removed. With recognition of these issues the within and between vendor precision for this data set are stated in the last two rows of Table 5-12 in bold. Because the observed standard deviations are relatively constant over the range of PI values (Form 1) one-sigma (1S) and difference two sigma limits (D2S) are expressed rather than the one-sigma and difference two sigma limits in percent. Interpretation of the $1 \mathrm{~S}$ and D2S limits for within and between vendor acceptable range of results limits would be as follows:

1. The within vendor acceptable range of two results equals $2.7 \mathrm{in} / \mathrm{mi}$. Therefore, the difference between two individual test results that would be equaled or exceeded in the long run in only 1 case of 20 (5\% of the time) under the normal and correct operation of the test using the same profiler and operator would be $2.7 \mathrm{in} / \mathrm{mi}$; and

2. The between vendor acceptable range of two results equals $8.8 \mathrm{in} / \mathrm{mi}$. Therefore, the difference between two individual test results that would be equaled or exceeded in the long run in only 1 case of 20 (5\% of the time) under the normal and correct operation of the test using two profilers would be $8.8 \mathrm{in} / \mathrm{mi}$. 
Application of the 0.2 inch blanking band actually made it appear as though the repeatability and reproducibility of the lightweight profilers was better than when the 0.0 inch blanking band were applied. This is due to the fact that low variability was associated with the smooth sites and this effectively reduced the $1 \mathrm{~S}$ and D2S limits.

The level of variability observed in the Case 3 data set was the lowest among the three cases considered. The between vendor standard deviations at the sites with boards were high. Overall the within vendor precision for all the vendors was good. The between vendor precision was poor even though it improved when smoother pavements and sites without bumps were considered.

A similar test of lightweight profilers was recently conducted in Connecticut (Larsen D., December 1999) . The same vendors, which participated in the field tests in Indiana, also participated in the testing in Connecticut. However one additional lightweight profiler manufactured by Trigg Industries participated in the field tests conducted in Connecticut. A precision analysis of the data generated from the field tests in Connecticut was carried out in order to obtain an indication of whether the lightweight profiler performance observed in Indiana was consistent with the performance observed in Connecticut. Ten measurements were made at each site by each vendor in the Connecticut study. Five of the measurements per site and vendor were randomly selected and used to develop a precision statement based on the Connecticut data. The analysis of this Connecticut data represents a fourth precision analysis (Case 4) and it is presented in the following section. 
Table 5-12 Case 3 PI-0.2” Precision Statement Summary Statistics

\begin{tabular}{|c|c|c|c|c|c|}
\hline Sites & Site average & $\begin{array}{l}\text { Repeatability } \\
\text { standard } \\
\text { deviation }(\mathrm{Sr})\end{array}$ & $\begin{array}{c}\text { Reproducibility } \\
\text { standard } \\
\text { deviation (SR) }\end{array}$ & $\begin{array}{c}95 \% \\
\text { Repeatability } \\
\text { limit @ }\end{array}$ & $\begin{array}{c}95 \% \\
\text { Reproducibility } \\
\text { limit (R ) }\end{array}$ \\
\hline & inches/mile & inches/mile & inches/mile & inches/mile & inches/mile \\
\hline $\begin{array}{c}\text { Site } 1 \\
\text { Covington }\end{array}$ & 46.28 & 1.07 & 2.90 & 3.00 & 8.11 \\
\hline $\begin{array}{cl}\text { Site } 2 \\
\text { I-65 } & \text { with board }\end{array}$ & 10.05 & 1.28 & 7.55 & 3.58 & 21.14 \\
\hline $\begin{array}{c}\text { Site } 2 b \\
\text { I-65 without board }\end{array}$ & 0 & 0 & 0 & 0 & 0 \\
\hline $\begin{array}{c}\text { Site3 } \\
\text { Prairie Street } \\
\text { with board }\end{array}$ & 43.02 & 2.63 & 6.00 & 7.35 & 16.79 \\
\hline $\begin{array}{c}\text { Site 3b } \\
\text { Prairie Street } \\
\text { without board }\end{array}$ & 40.5 & 0.96 & 5.97 & 2.68 & 16.71 \\
\hline $\begin{array}{c}\text { Site } 4 \\
\text { Vinton Street }\end{array}$ & 16.72 & 1.03 & 1.79 & 2.89 & 5.00 \\
\hline $\begin{array}{c}\text { Site } 5 \\
\text { US 231A } \\
\end{array}$ & 0.25 & 0.25 & 0.25 & 0.70 & 0.70 \\
\hline $\begin{array}{c}\text { Site } 6 \\
\text { US 231B }\end{array}$ & 2.87 & 0.40 & 0.61 & 1.13 & 1.71 \\
\hline \multicolumn{2}{|l|}{$1 S$} & 0.95 & 3.13 & & \\
\hline \multicolumn{4}{|l|}{ D2S } & 2.67 & 8.77 \\
\hline
\end{tabular}




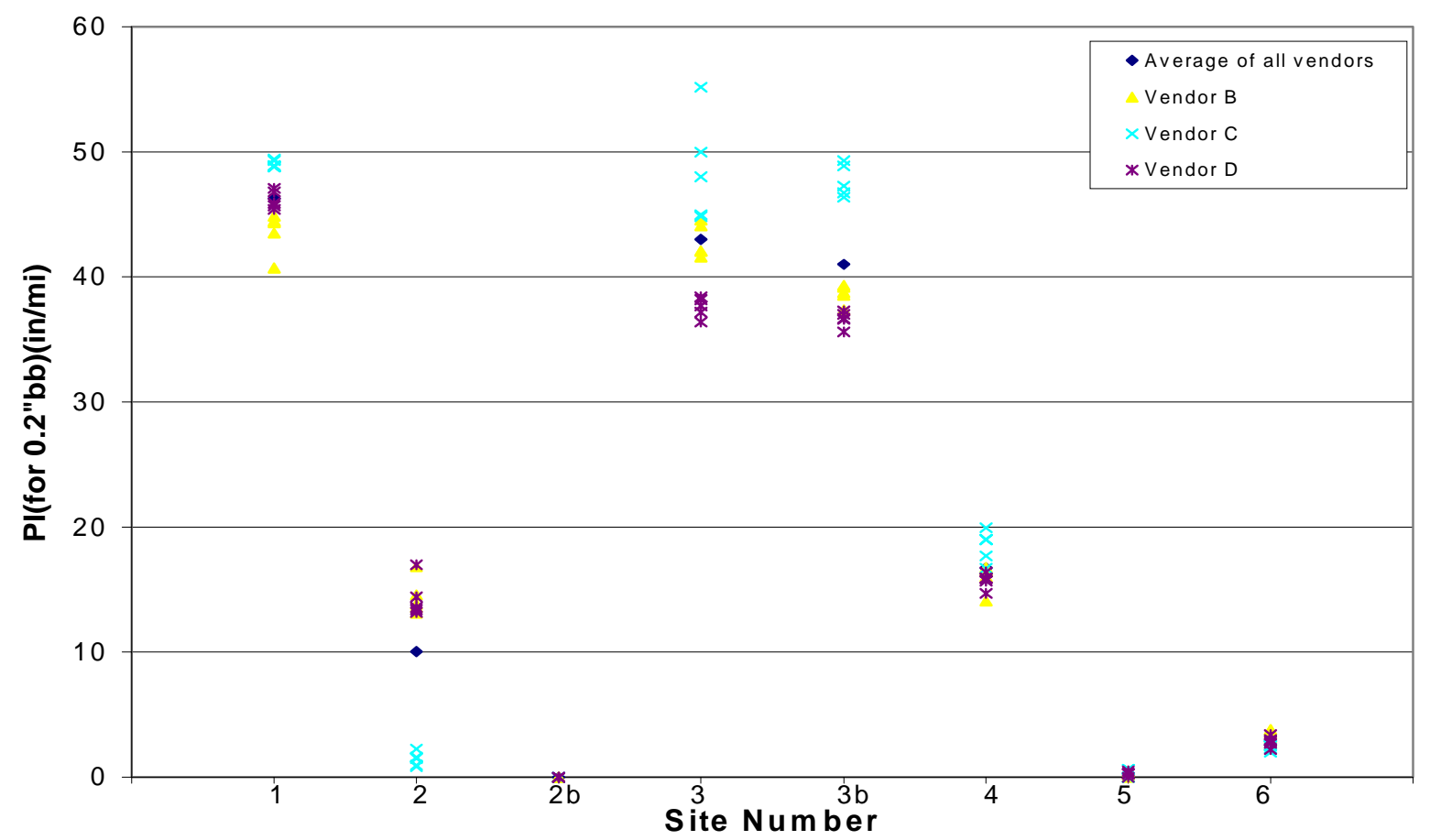

Figure 5-33 Case 3 Site Average Plot by Vendor for PI-0.2" 

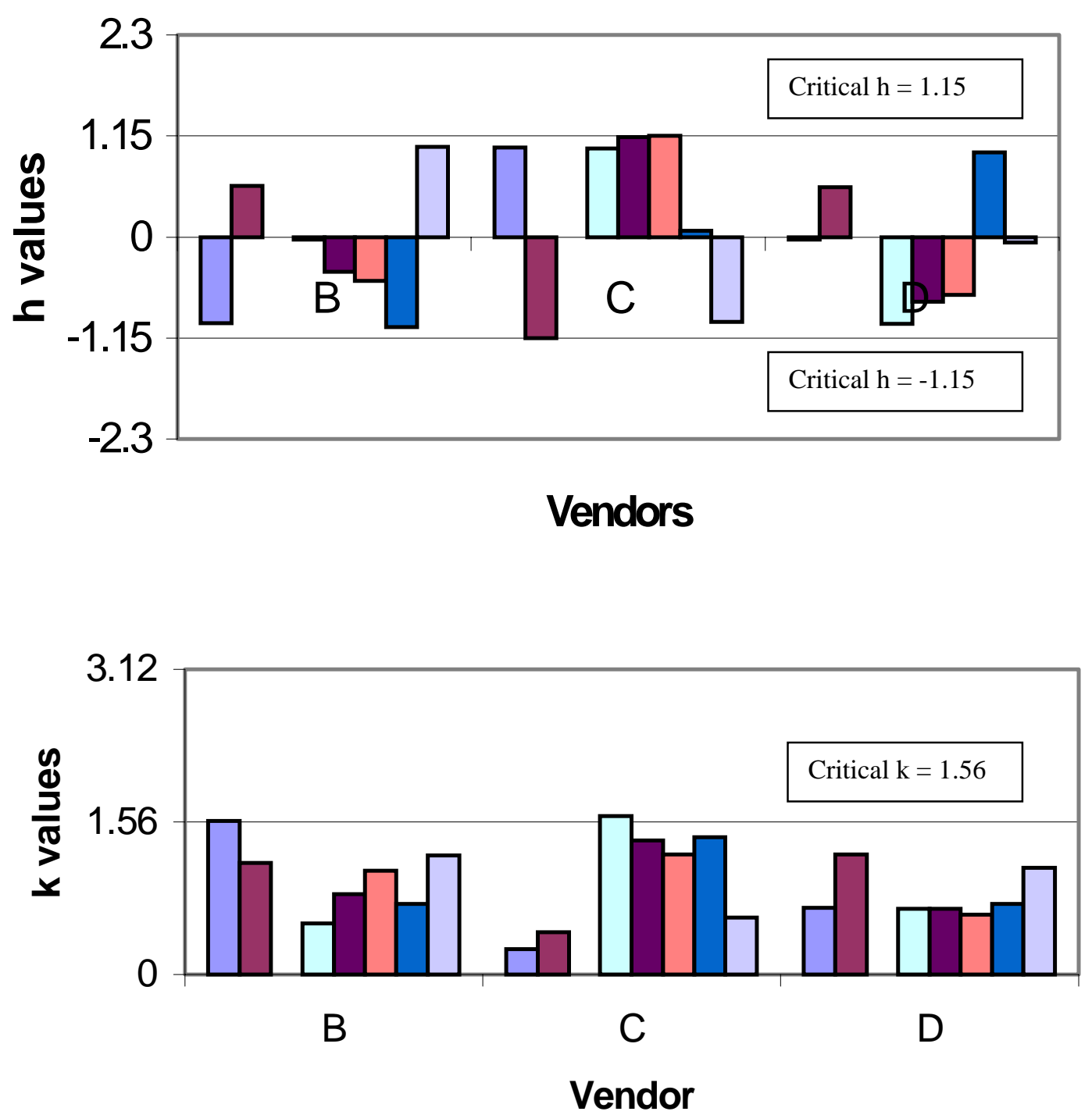

Figure 5-34 Case $3 \mathrm{~h}$ and $k$ plots by Vendors for PI-0.2" Note: The 8 columns for each vendor represent the 6 sites (Site 2 and 3 with and without board). 

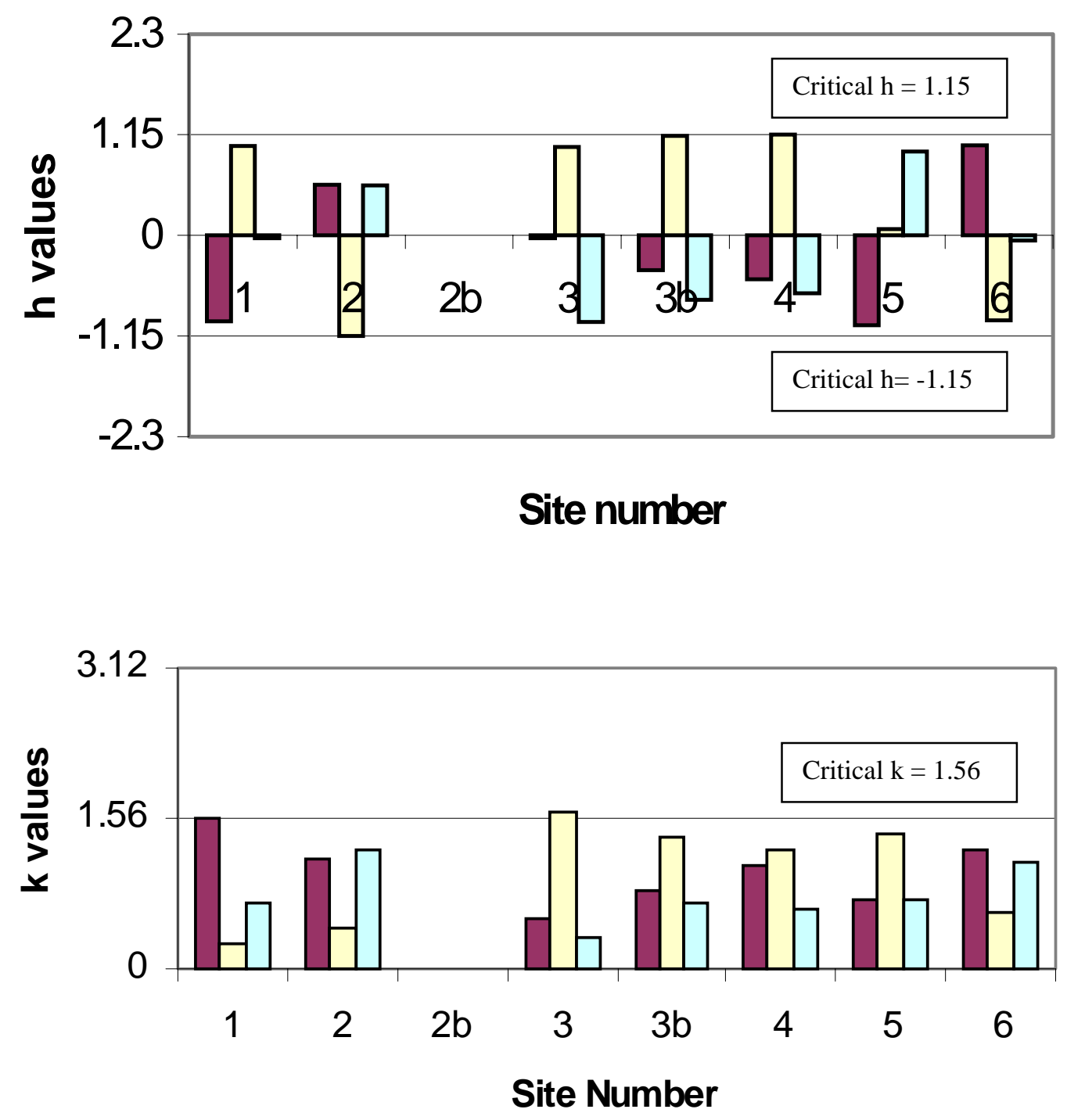

Figure 5-35 Case $3 \mathrm{~h}$ and $\mathrm{k}$ plots by vendors for PI-0.2"

Note: The 3 columns for each site represent the 3 vendors . 


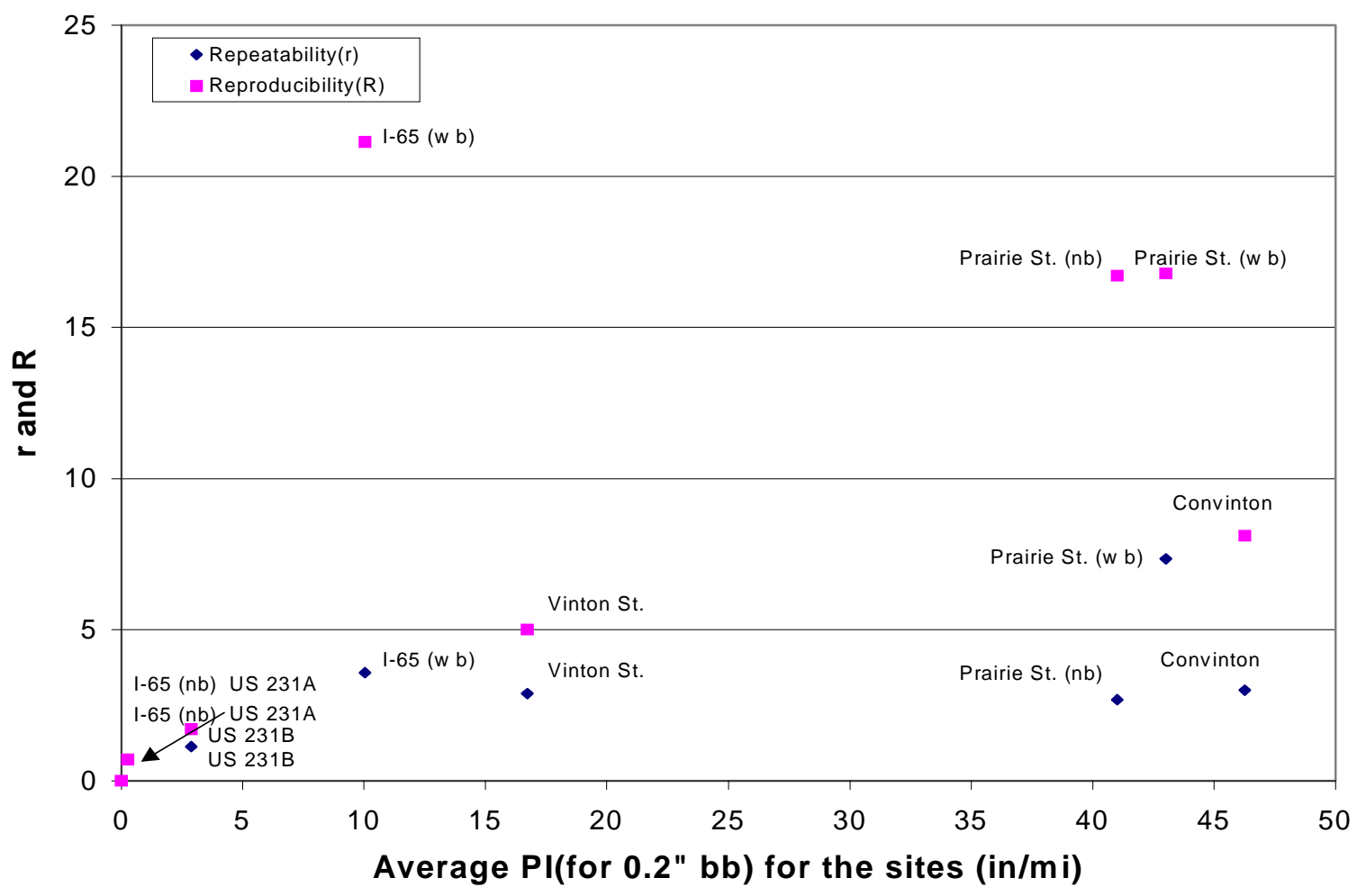

Figure 5-36 Case 3 Repeatability and Reproducibility Statistics by Site for PI-0.0”

\subsubsection{Case 4: Precision Based on Connecticut Generated Data}

For this analysis all of the data considered were generated in Connecticut. Five lightweight profilers, including the same four that operated in the field tests in Indiana and a profiler manufactured by Trigg Industries were considered. The four common vendors have been identified with the same set letters (A, B, C and D). The Trigg device was identified as Vendor E. Tests were conducted at three HMA sites and five of the ten measurements made per site were randomly selected for analysis. There were no measurements made with boards. IRI was the only index considered in the Connecticut study and it was measured in both wheel paths. The left wheel path IRI was chosen in order to maintain consistency with the data generated in Indiana. The data employed is 
summarized in Table 5-13. One way ANOVA was conducted separately for the data associated with each site using the ASTM E691-92 software. The data were then summarized in the form of a precision statement in accordance with ASTM C670-96. Critical values of $\mathrm{h}$ - and $\mathrm{k}$-statistic were chosen at the 0.5 percent significance level. The critical values of $\mathrm{h}$ and $\mathrm{k}$ were 1.75 and 1.71 , respectively for the conditions outlined in Table 5-13.

Table 5-13 Summary of Case 4 Precision Analysis Considerations

\begin{tabular}{|c|c|c|c|c|c|}
\hline $\begin{array}{c}\text { Number of } \\
\text { Vendors }\end{array}$ & $\begin{array}{c}\text { Number } \\
\text { of Sites }\end{array}$ & $\begin{array}{c}\text { Replicate } \\
\text { Measurements } \\
\text { per Site }\end{array}$ & $\begin{array}{c}\text { With Board } \\
\text { Data } \\
\text { Included? }\end{array}$ & $\begin{array}{c}\text { Smoothness } \\
\text { Statistics } \\
\text { Considered }\end{array}$ & $\begin{array}{c}\text { Total } \\
\text { Degrees } \\
\text { of } \\
\text { Freedom }\end{array}$ \\
\hline $\begin{array}{c}5 \\
(\mathrm{~A}, \mathrm{~B}, \mathrm{C}, \mathrm{D}, \mathrm{E})\end{array}$ & 3 & 5 & No & IRI & 32 \\
\hline
\end{tabular}

\subsubsection{Analysis of Data}

Figure 5-37 is a plot of the individually reported IRI values by each vendor at each site. The plot also includes the average IRI for all the vendors at each site. The plot shows that the data were more or less clustered at each site with the exception of Section 3 where the Vendors B and C data showed some scatter. Figures 5-38 and 5-39 are the $\mathrm{h}$ and $\mathrm{k}$ plots by vendor and site. Figure 5-38 does not show any significant trend in terms of sign (+ vs. -). Only one $\mathrm{h}$ value for Vendor $\mathrm{D}$ approached the critical value, and Vendor C had one value that exceeded the critical k value. Review of Figure 5-39, the h and $\mathrm{k}$ plots by site, also show a reasonable distribution of the $\mathrm{h}$ and $\mathrm{k}$ values, with only Vendor $\mathrm{C}$ a potential for inconsistency between Vendors at Section 3. In other words, 
both within and between vendor consistency appear to be reasonable based on the Indiana data.

Table 5-14 provides a summary of the statistics associated with a precision statement. Site averages as well as repeatability and reproducibility standard deviations and limits are presented by site. The repeatability and reproducibility standard deviations were all small. Interestingly, as the site average IRI increased, the standard deviations unexpectedly decreased.

Figure 5-40 is a plot of 95 percent repeatability and reproducibility limits ( $\mathrm{r}$ and R) respectively, versus average IRI by site. The $R$ value was slightly larger than the $r$ value at all the sites, with the maximum $\mathrm{r}$ value of $21.8 \mathrm{in} / \mathrm{mi}$ and $\mathrm{R}$ value of $22.7 \mathrm{in} / \mathrm{mi}$ observed at Section 3. The $\mathrm{r}$ value at Section 3 was large compared to the other two sites but the difference in $\mathrm{r}$ and $\mathrm{R}$ at this site was smaller than observed at the other sites. In other words, within vendor precision was poor at this site, but between vendor precision was very good considering the poor within vendor precision.

With recognition of this the within and between vendor precision for this data set are stated in the last two rows of Table 5-14 in bold. Because the observed standard deviations were relatively constant over the range of sites (Form 1) one-sigma (1S) and difference two sigma limits (D2S) are expressed rather than the one-sigma and difference two sigma limits in percent. Interpretation of $1 \mathrm{~S}$ and D2S limits for within and between vendor acceptable range of results limits would be as follows: 
1. The within vendor acceptable range of two results equals $12.5 \mathrm{in} / \mathrm{mi}$. Therefore, the difference between two individual test results that would be equaled or exceeded in the long run in only 1 case of 20 (5\% of the time) under the normal and correct operation of the test using the same profiler and operator would be $12.5 \mathrm{in} / \mathrm{mi}$; and

2. The between vendor acceptable range of two results equals $19.1 \mathrm{in} / \mathrm{mi}$. Therefore, the difference between two individual test results that would be equaled or exceeded in the long run in only 1 case of 20 (5\% of the time) under the normal and correct operation of the test using two profilers would be $19.1 \mathrm{in} / \mathrm{mi}$.

Table 5-15 represents a comparison of IRI precision statistics for Cases 1 through 4. The data showed similar within vendor precision for the tests conducted in both states as noted by the repeatability standard deviations and limits ( $\mathrm{Sr}$ and $\mathrm{r}$ ). The reproducibility standard deviations and limits also show similar between vendor precision when the range of site average IRI values are considered. The range of average IRI for the Connecticut sites was much narrower than was observed in Indiana.

A precision statement was developed for IRI using the data generated in Indiana and Connecticut. The precision analysis of the combined data (Case 5) is presented in the next section. Pooling the data provides a more powerful indication of both within and between vendor performance of the lightweight profilers. 
Table 5-14 Case 4 IRI Precision Statement Summary Statistics

\begin{tabular}{|c|c|c|c|c|c|}
\hline Sites & Site average & $\begin{array}{c}\text { Repeatability } \\
\text { standard } \\
\text { deviation }(\mathrm{Sr})\end{array}$ & $\begin{array}{c}\text { Reproducibility } \\
\text { standard } \\
\text { deviation (SR) }\end{array}$ & $\begin{array}{c}95 \% \\
\text { Repeatability } \\
\text { limit ( } \mathrm{r} \text { ) }\end{array}$ & $\begin{array}{c}95 \% \\
\text { Reproducibility } \\
\text { limit (R) }\end{array}$ \\
\hline & inches/mile & inches/mile & inches/mile & inches/mile & inches/mile \\
\hline Section 1 & 71.92 & 3.12 & 7.98 & 8.74 & 22.34 \\
\hline Section 2 & 91 & 2.47 & 4.36 & 6.93 & 12.2 \\
\hline Section 3 & 59.04 & 7.77 & 8.09 & 21.76 & 22.66 \\
\hline D1S & & 4.45 & 6.81 & & \\
\hline D2S & & & & 12.48 & 19.07 \\
\hline
\end{tabular}

Table5-15 Comparision of Case 1 through 4 IRI Precision Statistics

\begin{tabular}{|c|c|c|c|c|c|}
\hline \multirow{2}{*}{ Case } & \multicolumn{4}{|c|}{ IRI Precision } & \multirow{2}{*}{$\begin{array}{c}\text { Site Average } \\
\text { Range } \\
\text { (in/mi) }\end{array}$} \\
\cline { 2 - 5 } & Repeatability & Reproducibility & Repeatability & Reproducibility & $41-148$ \\
\hline 1 & 4.7 & 21.5 & 13.3 & 60.8 & $41-142$ \\
\hline 2 & 3.3 & 13.4 & 9.2 & 37.4 & $35-154$ \\
\hline 3 & 3.2 & 9.7 & 9.0 & 27.2 & $59-91$ \\
\hline 4 & 4.5 & 6.8 & 12.5 & 19.1 & D2S \\
\hline
\end{tabular}




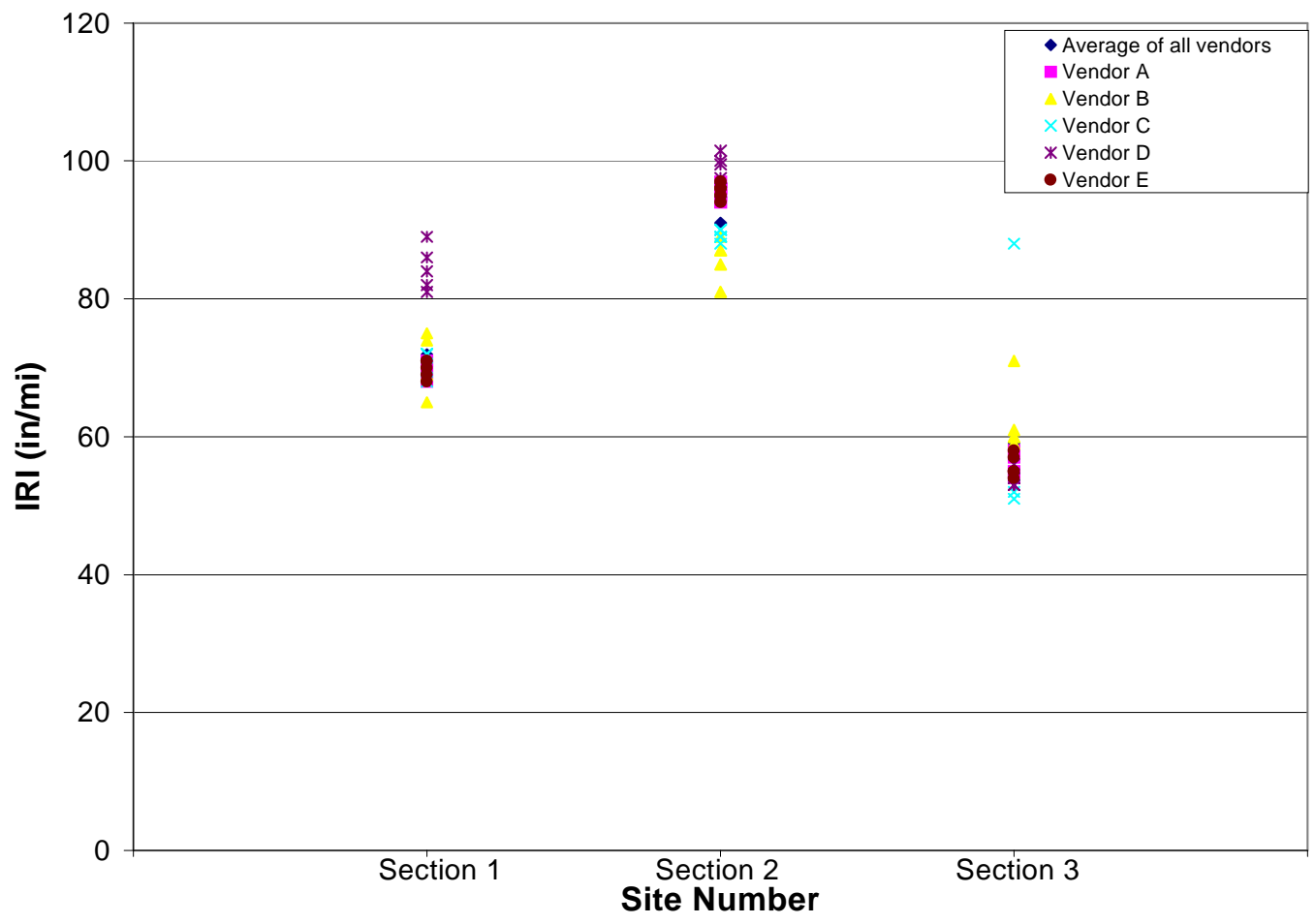

Figure 5-37 Case 4 Site Average Plot by Vendor for IRI 

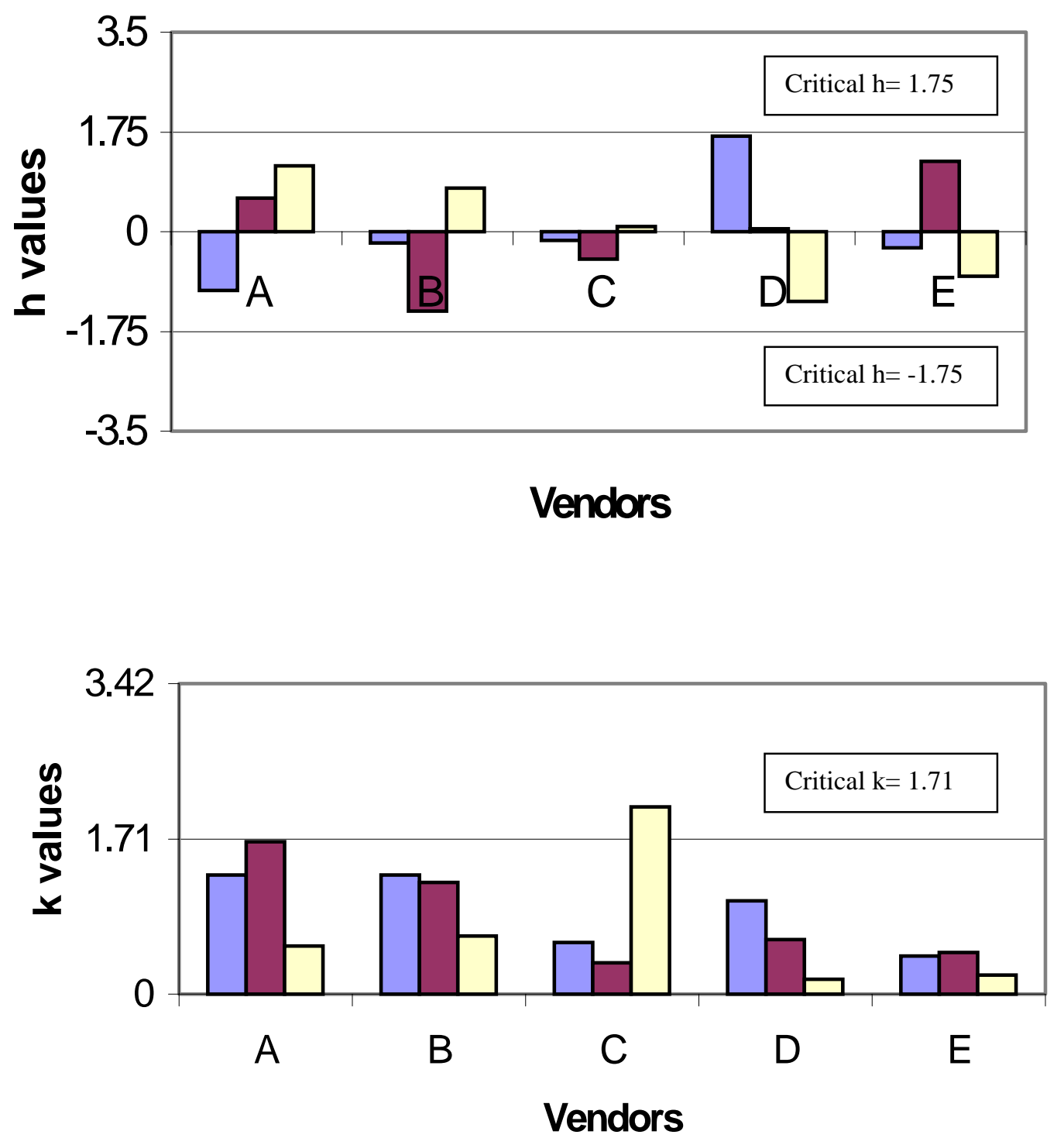

Figure 5-38 Case $4 \mathrm{~h}$ and $\mathrm{k}$ plots by Vendors for IRI

Note : The 3 columns for each vendor represent the 3 sites from Connecticut field tests 

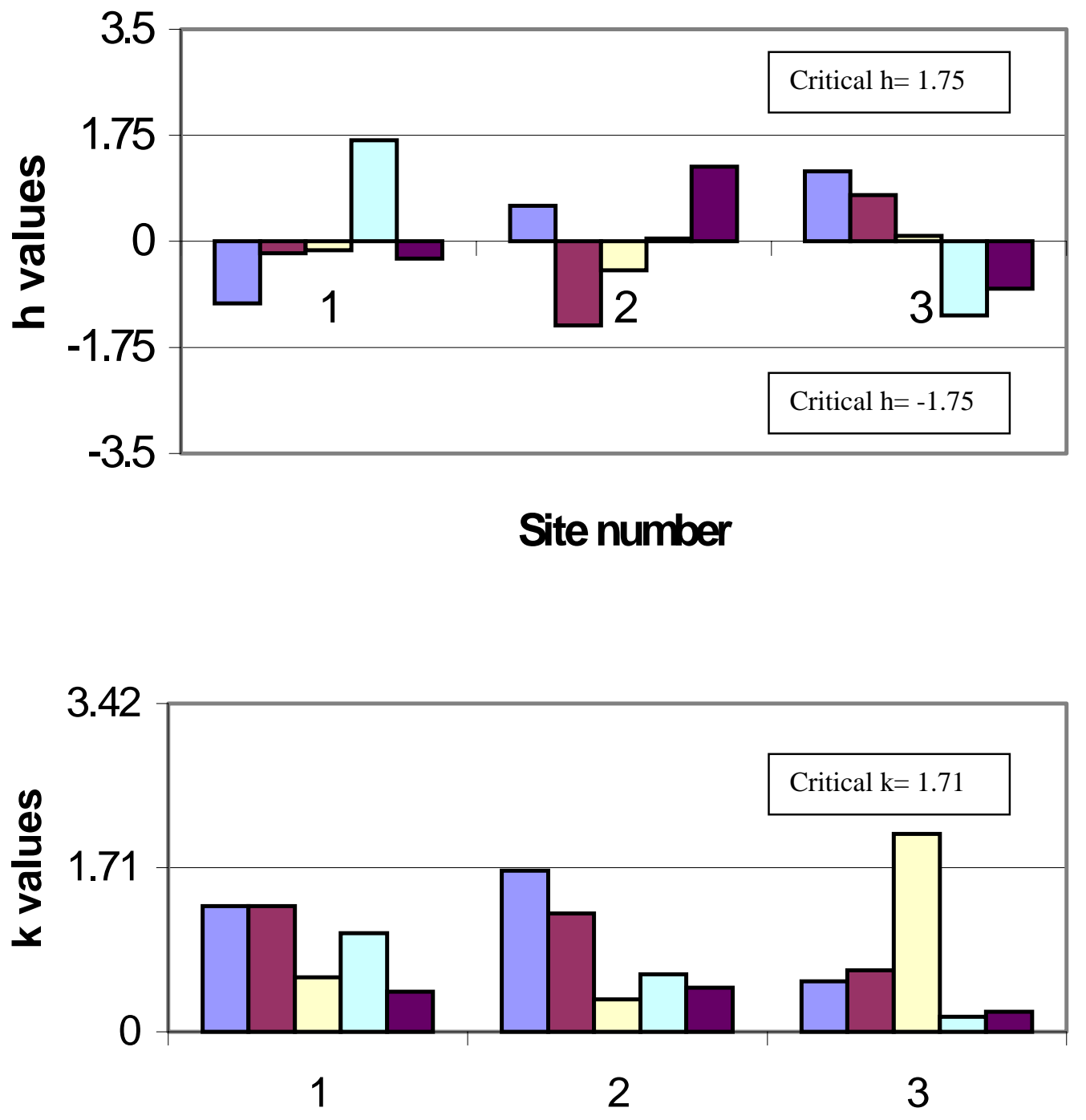

Site number

Figure 5-39 Case $4 \mathrm{~h}$ and $\mathrm{k}$ plots by Sites for IRI

Note : The 5 columns for each site represent the 5 vendors (There was one additional vendor in Connecticut, identified as Vendor E) . 


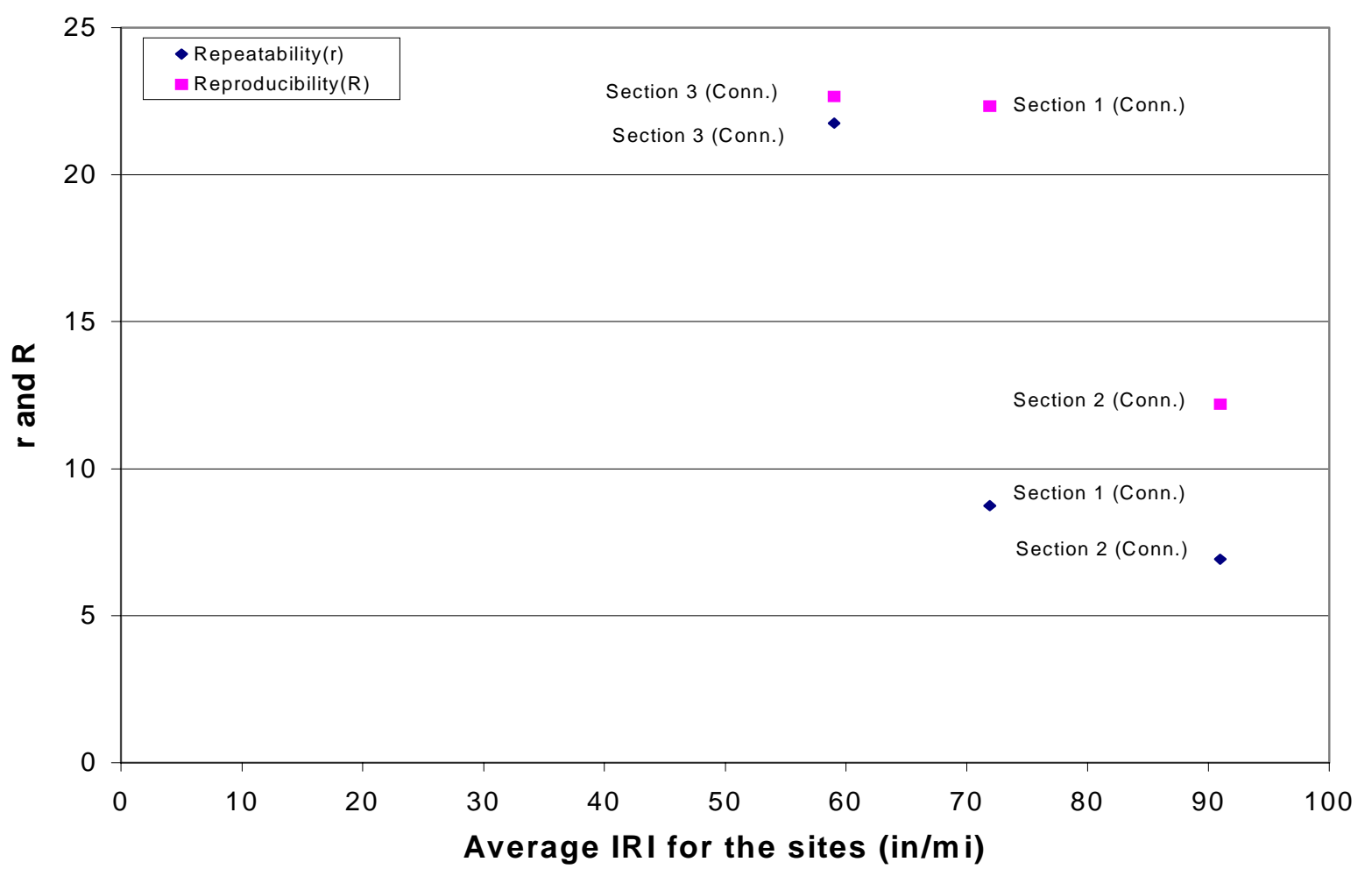

Figure 5-40 Case 4 Repeatability and Reproducibility Statistics by Site for

\section{IRI}

\subsubsection{Case 5: Precision Based on Pooled Indiana and Connecticut Data}

Four vendors were common in both Indiana and Connecticut field tests. The data generated by the four common lightweight profilers, at the three sections in Connecticut, was combined with the without boards data generated at the six sites in Indiana. IRI was the only index considered simply because PI-0.0" and PI-0.2" data were not collected in Connecticut. The data employed is summarized in Table 5-16 and reflects ninety six degrees of freedom. The experiment exceeded the requirements of ASTM C802-96. One 
way ANOVA was conducted separately for the data associated with each site using the ASTM E691-92 software. The data were then summarized in the form of a precision statement in accordance with ASTM C670-96. Critical values of h- and k-statistic were chosen at the 0.5 percent significance level. The critical values of $h$ and $k$ were 1.49 and 1.66, respectively for the conditions outlined in Table 5-16.

Table 5-16 Summary of Case 5 Precision Analysis Considerations

\begin{tabular}{|c|c|c|c|c|c|}
\hline $\begin{array}{c}\text { Number of } \\
\text { Vendors }\end{array}$ & $\begin{array}{c}\text { Number } \\
\text { of Sites }\end{array}$ & $\begin{array}{c}\text { Replicate } \\
\text { Measurements } \\
\text { per Site }\end{array}$ & $\begin{array}{c}\text { With Board } \\
\text { Data } \\
\text { Included? }\end{array}$ & $\begin{array}{c}\text { Smoothness } \\
\text { Statistics } \\
\text { Considered }\end{array}$ & $\begin{array}{c}\text { Total } \\
\text { Degrees } \\
\text { of } \\
\text { Freedom }\end{array}$ \\
\hline $\begin{array}{c}4 \\
(\mathrm{~A}, \mathrm{~B}, \mathrm{C}, \mathrm{D})\end{array}$ & 9 & 5 & No & IRI & 96 \\
\hline
\end{tabular}

\subsubsection{Analysis of Data}

Figure 5-41 is a plot of the individual reported IRI values by each vendor at each site. The plot also includes the average IRI for all the vendors at each site. The plot shows that Vendor A reported significantly lower IRI values than the other vendors at the Covington street site (Site 1). However the rest of the data are fairly closely clustered at other sites. Figures 5-42 and 5-43 are the $\mathrm{h}$ and $\mathrm{k}$ plots by vendor and site. Figure 5-42 shows that the $\mathrm{h}$ values associated with Vendor $\mathrm{A}$ are higher on average than the other vendors. However only one Vendor A and one Vendor $\mathrm{C} \mathrm{k}$ value exceed the $\mathrm{k}$ critical value while two Vendor $\mathrm{D}$ values exceed it. Three Vendor $\mathrm{A} h$ values approach the critical $h$ value. 
Review of Figure 5-43, the $\mathrm{h}$ and $\mathrm{k}$ plots by site, show that Vendor $\mathrm{A}$ has $\mathrm{h}$ values of exactly the opposite sign of those associated with Vendors B, C and D at only three of the nine sites. However none of the $\mathrm{h}$ values exceed the critical $\mathrm{h}$. The $\mathrm{k}$ plot by site shows that Vendor D has two k values, while Vendors $\mathrm{C}$ and $\mathrm{A}$ had one $\mathrm{k}$ value each approaching and exceeding the critical $\mathrm{k}$ value. Based on review of the $\mathrm{h}$ and $\mathrm{k}$ plots it was concluded that all the vendors exhibited similar within and between vendor consistency. Only Vendor A showed signs of potential lack of between vendor precision. However because none of the $h$ values associated with Vendor A exceeded the critical $h$ value, the problem was not significant.

Table 5-17 provides a summary of the statistics associated with a precision statement. Site averages as well as repeatability and reproducibility standard deviations and limits are presented by site. The repeatability standard deviations were all relatively small and consistent with the exception of Connecticut Section 3 site where a high repeatability standard deviation of $8.65 \mathrm{in} / \mathrm{mi}$ was observed. The reproducibility standard deviations are similar at all the sites except the Covington Street site, where a high reproducibility standard deviation of $66.05 \mathrm{in} / \mathrm{mi}$ was observed. Figure 5-44 is a plot of 95 percent repeatability and reproducibility limits ( $\mathrm{r}$ and $\mathrm{R}$ ) respectively, versus average IRI by site. The observed $\mathrm{R}$ is much greater than the $\mathrm{r}$ in the case of the Covington Street site. This was the new, but rough PCC site with hand-tooled transverse joints. Review of Figure 5-41 provides the explanation for this. Vendor A reported very high IRI values (220’s in/mi) while Vendor C reported very low values (30’s in/mi). 
The analysis discussed above suggests that with the exception of the Covington street site, the vendors provided similar within and between vendor performance. With recognition of this issue the within and between vendor precision for this data set are stated in the last two rows of Table 5-17 in bold. Because the observed standard deviations are relatively constant over the range of sites (Form 1) one-sigma (1S) and difference two sigma limits (D2S) are expressed rather than the one-sigma and difference two sigma limits in percent. Interpretation of the $1 \mathrm{~S}$ and $\mathrm{D} 2 \mathrm{~S}$ limits for within and between vendor acceptable range of results limits would be as follows:

1. The within vendor acceptable range of two results equals $10.7 \mathrm{in} / \mathrm{mi}$. Therefore, the difference between two individual test results that would be equaled or exceeded in the long run in only 1 case of 20 (5\% of the time) under the normal and correct operation of the test using the same profiler and operator would be $10.7 \mathrm{in} / \mathrm{mi}$; and

2. The between vendor acceptable range of two results equals $31.7 \mathrm{in} / \mathrm{mi}$. Therefore, the difference between two individual test results that would be equaled or exceeded in the long run in only 1 case of 20 (5\% of the time) under the normal and correct operation of the test using two profilers would be $31.7 \mathrm{in} / \mathrm{mi}$. 
Table 5-17 Case 5 IRI Precision Statement Summary Statistics

\begin{tabular}{|c|c|c|c|c|c|}
\hline Sites & Site average & $\begin{array}{c}\text { Repeatability } \\
\text { standard } \\
\text { deviation }(\mathrm{Sr})\end{array}$ & $\begin{array}{c}\text { Reproducibility } \\
\text { standard } \\
\text { deviation (SR) }\end{array}$ & $\begin{array}{c}95 \% \\
\text { Repeatability } \\
\text { limit }(r)\end{array}$ & $\begin{array}{c}95 \% \\
\text { Reproducibility } \\
\text { limit (R ) }\end{array}$ \\
\hline & inches/mile & inches/mile & inches/mile & inches/mile & inches/mile \\
\hline $\begin{array}{c}\text { Section 1 } \\
\text { (Connecticut) }\end{array}$ & 72.45 & 3.43 & 9.06 & 9.60 & 25.36 \\
\hline $\begin{array}{c}\text { Section } 2 \\
\text { (Connecticut) }\end{array}$ & 89.85 & 2.71 & 3.98 & 7.58 & 11.14 \\
\hline $\begin{array}{c}\text { Section } 3 \\
\text { (Connecticut) }\end{array}$ & 59.85 & 8.65 & 8.85 & 24.22 & 24.79 \\
\hline $\begin{array}{c}\text { Site } 1 \\
\text { Covington }\end{array}$ & 141.91 & 2.95 & 23.59 & 8.27 & 66.05 \\
\hline $\begin{array}{c}\text { Site } 2 b \\
\text { I- } 65 \text { without board }\end{array}$ & 41.13 & 2.84 & 12.89 & 7.94 & 36.09 \\
\hline $\begin{array}{c}\text { Site } 3 b \\
\text { Prairie Street } \\
\text { without board } \\
\end{array}$ & 147.69 & 4.05 & 15.62 & 11.34 & 43.75 \\
\hline $\begin{array}{c}\text { Site } 4 \\
\text { Vinton Street }\end{array}$ & 100.33 & 3.18 & 7.14 & 8.91 & 19.98 \\
\hline $\begin{array}{c}\text { Site } 5 \\
\text { US231 A }\end{array}$ & 51.06 & 3.76 & 10.65 & 10.53 & 29.81 \\
\hline $\begin{array}{c}\text { Site } 6 \\
\text { US 231B }\end{array}$ & 62.32 & 2.82 & 10.18 & 7.88 & 28.51 \\
\hline $1 S$ & & 3.82 & 11.33 & & \\
\hline D2S & & & & 10.70 & 31.72 \\
\hline
\end{tabular}




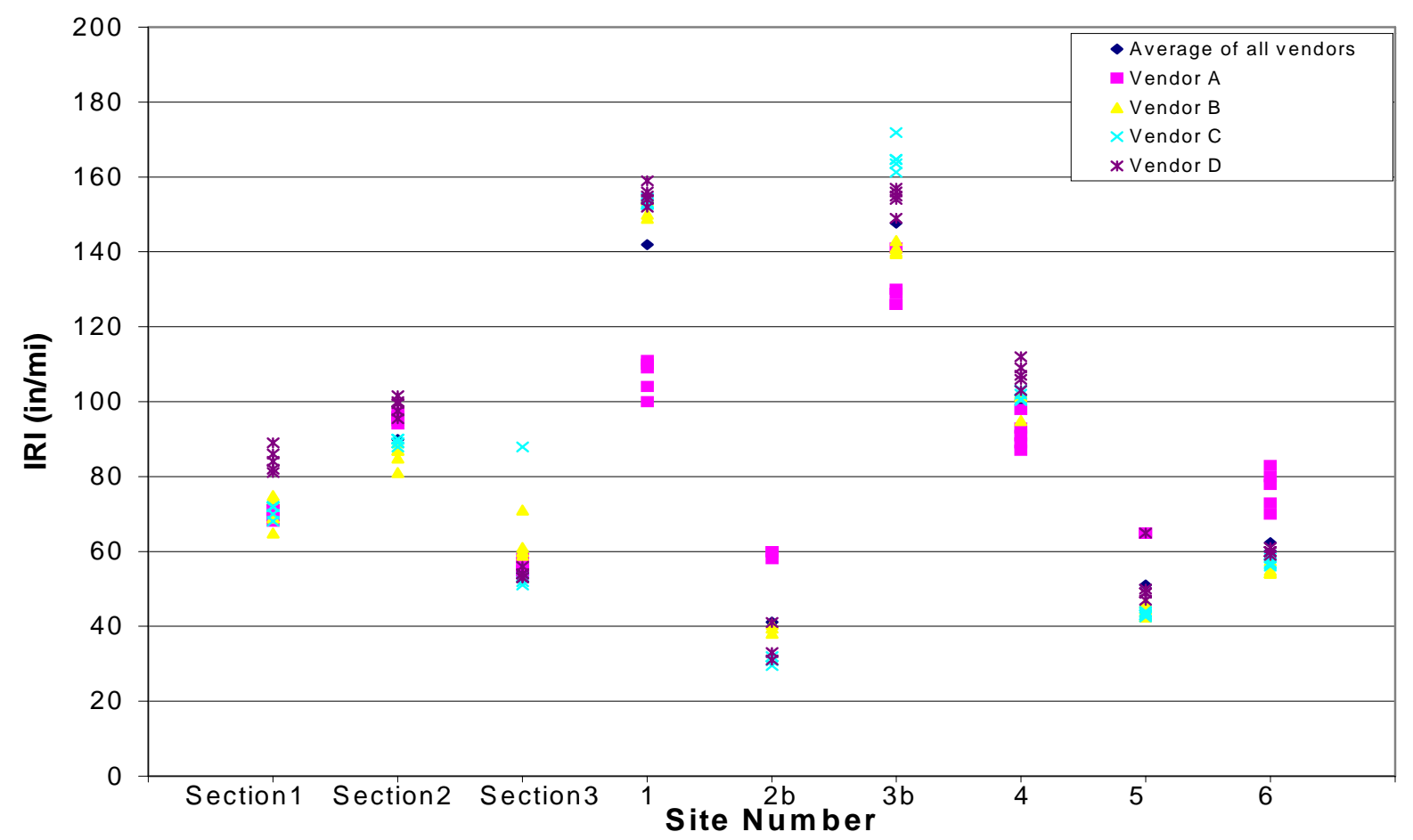

Figure 5-41 Case 5 Site Average Plot by Vendor for IRI 

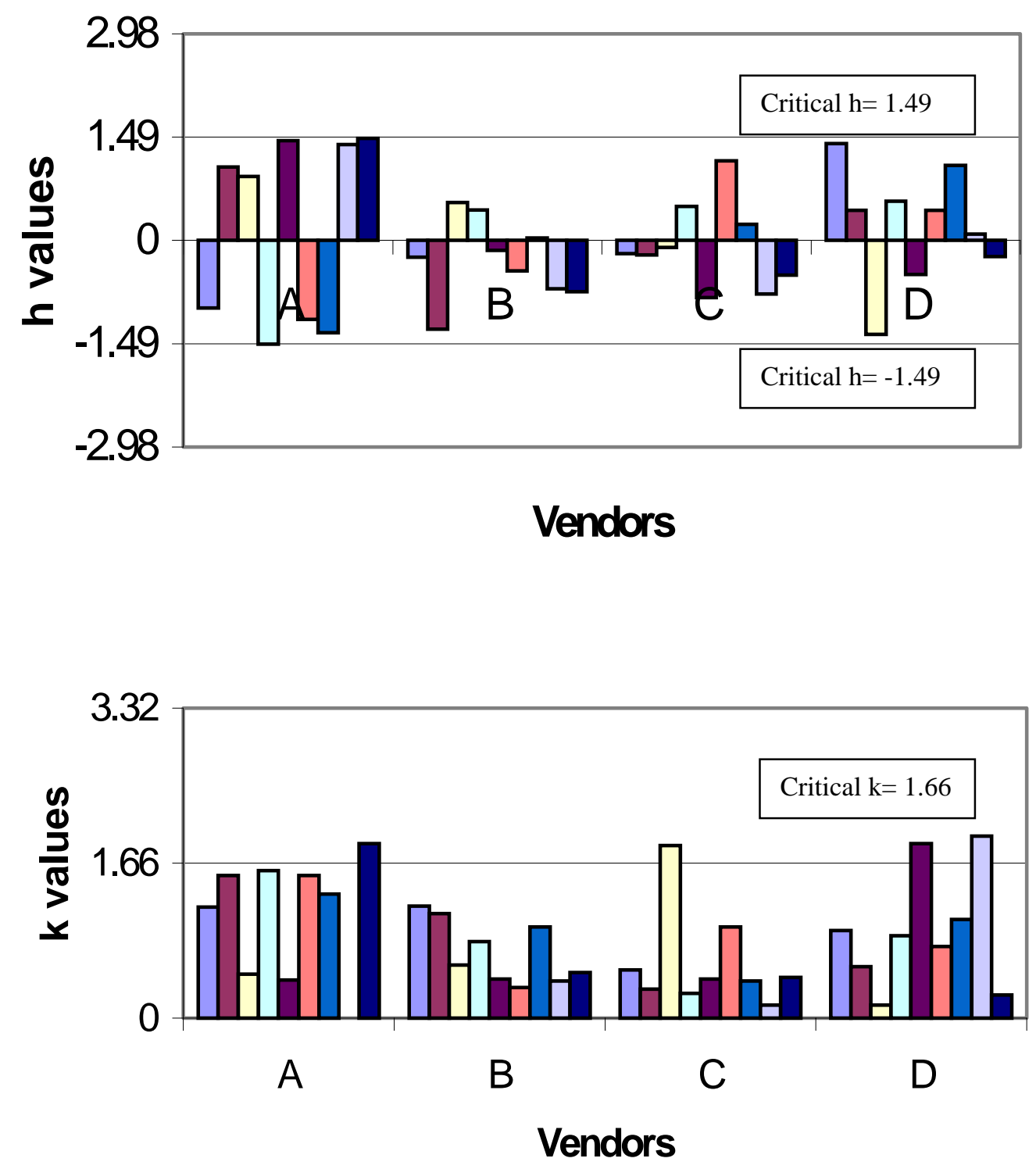

Figure 5-42 Case $5 \mathrm{~h}$ and $\mathrm{k}$ plots by Vendors for IRI

Note: The 9 columns for each vendor represent the 9 sites; first 3 are from Connecticut and last six from Indiana field tests . 

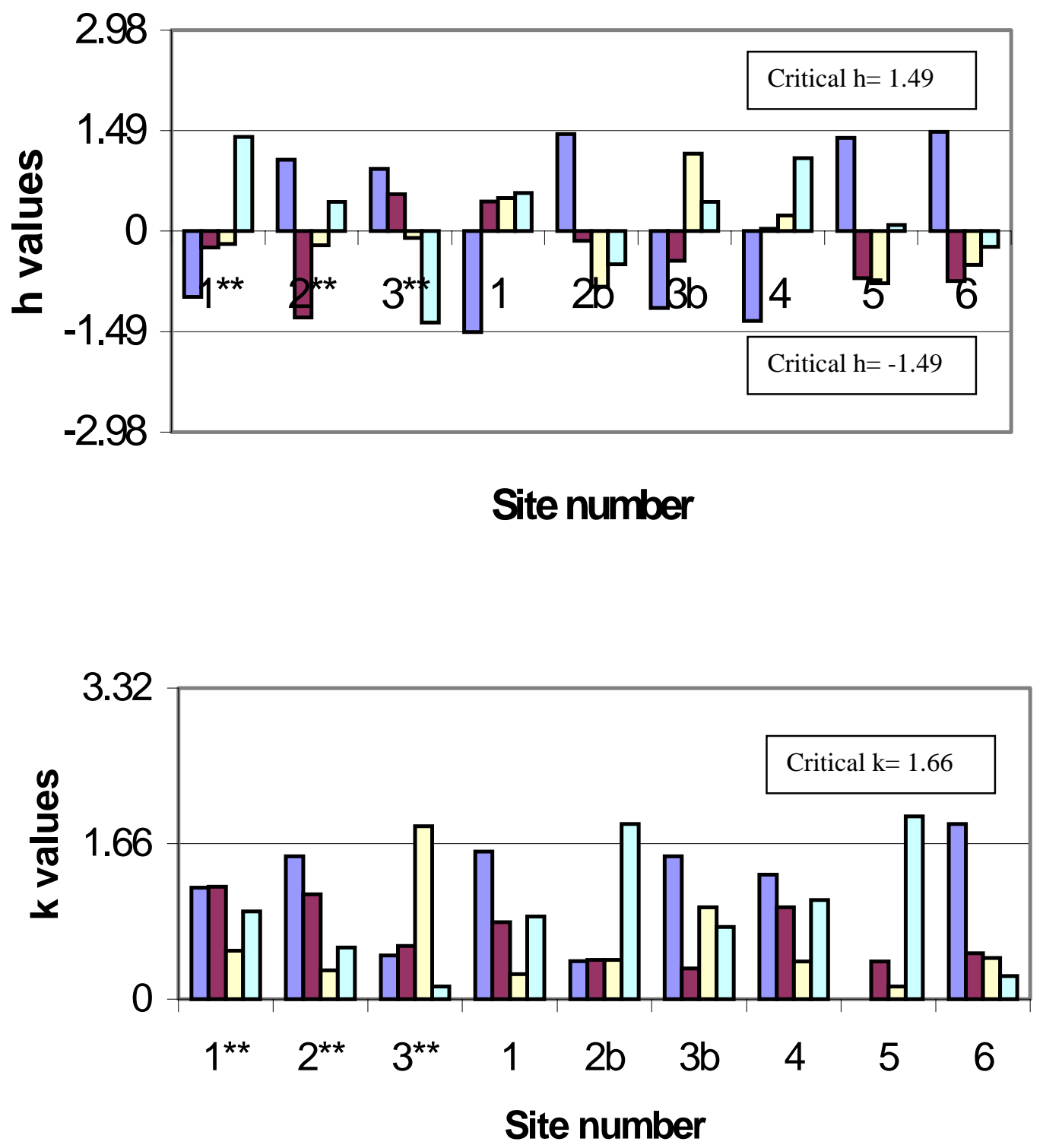

.Figure 5-43 Case $5 \mathrm{~h}$ and $\mathrm{k}$ plots by Sites for IRI

Note: The 4 columns for each site represent the 4 vendors. The sites marked with ** are Connecticut sites and the sites without $* *$ are Indiana sites. 


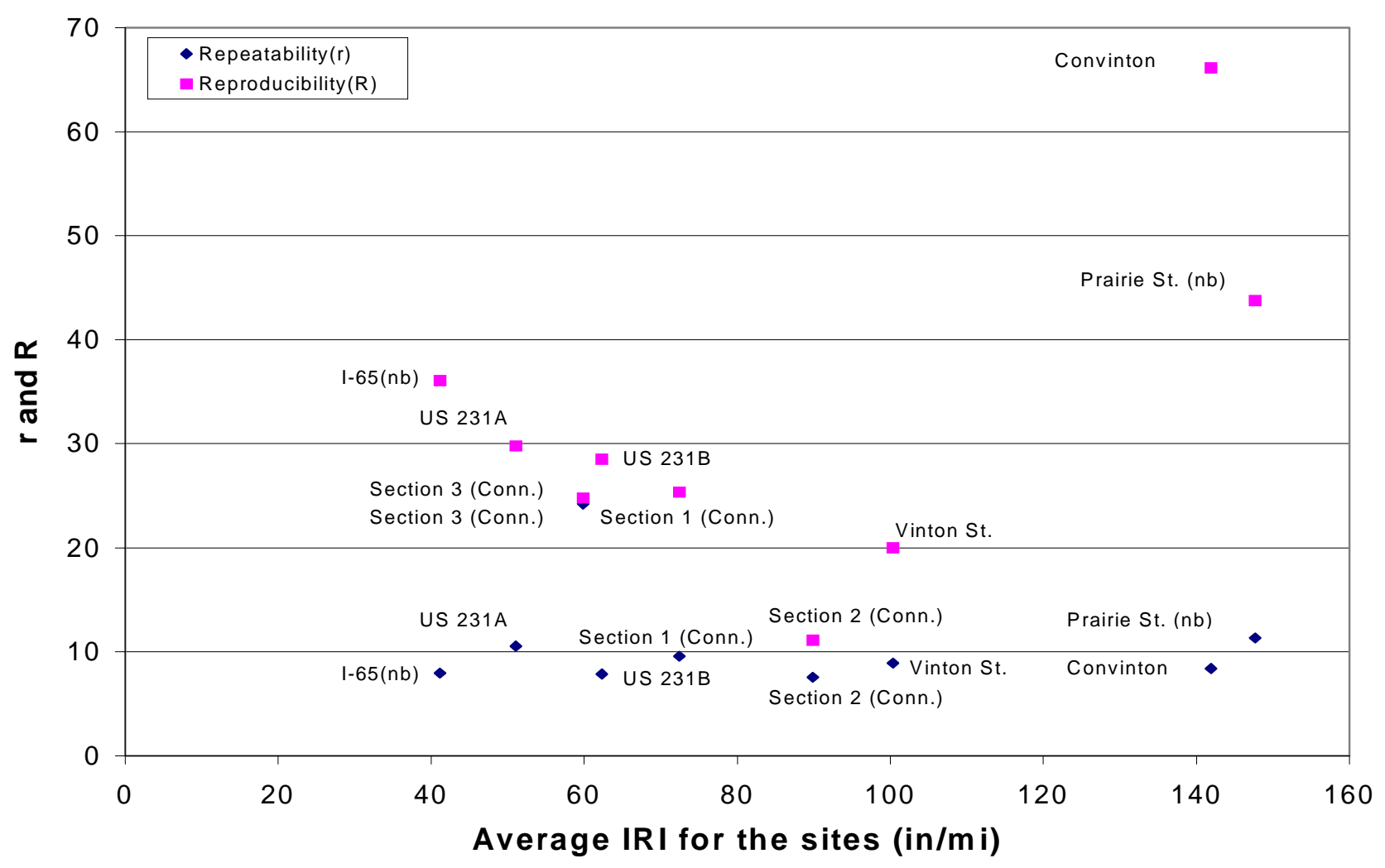

Figure 5-44 Case 5 Repeatability and Reproducibility Statistics by Site for IRI

\subsection{Comparision of Developed Statistics}

The IRI precision statistics for all five cases are summarized in Table 5-18.

Table5-18 Comparision of Case 1 through 5 IRI Precision Statistics

\begin{tabular}{|c|c|c|c|c|c|}
\hline \multirow{2}{*}{ Case } & \multicolumn{4}{|c|}{ IRI Precision } & \multirow{2}{*}{$\begin{array}{c}\text { Site Average } \\
\text { Range } \\
\text { (in/mi) }\end{array}$} \\
\cline { 2 - 5 } & Repeatability & Reproducibility & Repeatability & Reproducibility & $41-148$ \\
\hline 1 & 4.7 & 21.5 & 13.3 & 60.8 & $41-142$ \\
\hline 2 & 3.3 & 13.4 & 9.2 & 37.4 & $35-154$ \\
\hline 3 & 3.2 & 9.7 & 9.0 & 27.2 & $59-91$ \\
\hline 4 & 4.5 & 6.8 & 12.5 & 19.1 & $41-142$ \\
\hline 5 & 3.8 & 11.3 & 10.7 & 31.7 & \\
\hline
\end{tabular}

Similarly PI-0.0" and PI-0.2" summaries are presented in Tables 5-19 and 5-20. 
Table5-19 Comparision of Case 1 through 3 PI-0.0" Precision Statistics

\begin{tabular}{|c|c|c|c|c|c|}
\hline \multirow{2}{*}{ Case } & \multicolumn{4}{|c|}{ PI-0.0" Precision } & \multirow{2}{*}{$\begin{array}{c}\text { Site Average } \\
\text { Range } \\
\text { (in/mi) }\end{array}$} \\
\cline { 2 - 5 } & \multicolumn{2}{|c|}{ 1S } & \multicolumn{2}{c|}{ D2S } & $8-67$ \\
\hline 1 & 1.5 & 11.6 & 4.3 & 32.7 & $8-67$ \\
\hline 2 & 1.5 & 9.4 & 4.0 & 26.3 & $9-80$ \\
\hline 3 & 1.4 & 4.0 & 4.0 & 11.1 & \\
\hline
\end{tabular}

Table5-20 Comparision of Case 1 through 5 PI-0.2" Precision Statistics

\begin{tabular}{|c|c|c|c|c|c|}
\hline \multirow{2}{*}{ Case } & \multicolumn{5}{|c|}{ PI-0.2" Precision Statement Statistics } \\
\cline { 2 - 6 } & $\mathrm{Sr}$ & $\mathrm{SR}$ & $\mathrm{r}$ & $\mathrm{R}$ & Site average range (in/mi) \\
\hline 1 & 1.3 & 7.3 & 3.7 & 20.6 & $0-41$ \\
\hline 2 & 0.9 & 4.9 & 2.4 & 13.7 & $0-39$ \\
\hline 3 & 1.0 & 3.1 & 2.7 & 8.8 & $0-46$ \\
\hline
\end{tabular}

The data shows that both within and between vendor precision was best at smooth sites, regardless of smoothness index. Individual vendor repeatability was good. Unfortunately, the observed reproducibility was poor.

As summarized in Table 5-18 through 5-20, the observed repeatability and reproducibility limits $1 \mathrm{~S}$ and D2S were large for all three smoothness indices. They are actually large enough, that they suggest the use of lightweight profilers for specification purposes would be inappropriate until the technology is refined to a point that the reproducibility of the devices is improved. It simply would not be possible to enforce smoothness specifications based on the use of lightweight profilers that provided the poor reproducibility in this study. 


\section{Smoothness Specifications}

One of the objectives of this study was to develop a draft smoothness specification that relied on the use of lightweight profilers for INDOT. The poor reproducibility of the profilers reported in Section 4, as well as the precision analysis presented in Section 5 clearly suggest that the lightweight profiler technology requires some refinement prior to implementation of smoothness specifications which rely on lightweight profilers, at least some of those evaluated in this study. Some State DOTs are currently in the process of developing and implementing smoothness specifications that do rely on lightweight profilers. It is possible they have not yet had the opportunity to assess the reproducibility of the devices, which they will likely face after the first construction season in which different profilers are employed for QC and QA smoothness testing.

A review of the available literature focusing on surveys of smoothness specification use, measurement techniques, and payment methods was conducted to assess the industry trends in these areas. Requests were made for copies of proposed specifications from states that were identified as developing or implementing new specifications based on lightweight profilers and/or IRI. Recently completed research focusing on the appropriateness of different indices for smoothness specifications and critical aspects of specifications that rely on inertial profilers were also reviewed. A brief review of this literature is presented in the following section along with a review of specifications obtained from other states. Available specifications were then applied to 
the data generated in the field study associated with this project to assess there applicability in Indiana. This is reported followed by a draft specification based on the analysis of the data and the available literature.

\subsection{Smoothness Specification Literature}

Many state highway agencies are now implementing or using quality control/quality assurance (QC/QA) specifications to improve pavement quality. However the profile index (PI) smoothness statistic obtained with the profilograph is still being considered as the basis for the smoothness specifications in most states (Larsen D., December 1999). The 1987 AASHTO Rideability survey indicated that more highway agencies were employing smoothness specifications for rigid pavements than for flexible pavements (Smith, K.L, Smith, L.D., Evans T.E, Hoerner T.E., Darter M.I., March 1997). A survey conducted by researchers at the University of Wisconsin Madison as part of NCHRP Project 10-39A that involved reviewing QC/QA specifications for HMA pavements summarized responses from forty two State DOTs and specifications from thirty six of them (Russell, J.S. et al. 1998). The survey results showed that twelve states used California Profilographs, six used a regular straightedge, two used a rolling straightedge, three used a profilometer and one used a Mays Ride Meter for measuring smoothness at that time. PI was used as the index of smoothness by sixteen states while the rest used surface variations to identify roughness in pavements. The sixteen states with specifications using PI as the measure of smoothness also incorporated pay factor adjustments. One each of the state specifications based on a rolling straightedge and a 
Mays Meter also incorporated pay factor adjustments while more than half the states surveyed did not use pay factor adjustments as part of their smoothness specifications.

A survey, conducted by the South Carolina DOT in 1999 of over fifty states with responses from thirty-four, revealed that three states are using lightweight profilers for HMA construction (Baus, R.L, and Hong, W., November 1999). The states using the lightweight profilers are Michigan, Pennsylvania, and Texas. The survey also revealed that Connecticut, Maine, Vermont and Virginia are all using IRI as the basis for their smoothness specifications rather than PI. Finally, the survey indicated that twenty-two states surveyed incorporate pay adjustment factors in their construction specifications. The Pennsylvania DOT has indicated it is planning on using lightweight profilers and specifications based on IRI in the coming construction season also. (Pennsylvania DOT, 1999).

These surveys and reports suggest that the current industry trends include increased use of QC/QA smoothness specifications, increased use of lightweight profilers, increased use of IRI rather than PI as an index of smoothness, and the use of pay adjustment factors as tools for improving pavement smoothness. In light of these trends and considering the potential advantages of using lightweight profilers, a review of the critical aspects associated with a smoothness specification based on an inertial profiler was conducted. 


\subsection{Critical Aspects of Smoothness Specifications Based on the Use of Inertial}

\section{$\underline{\text { Profilers }}$}

A brief review of the literature was conducted to determine critical aspects of a smoothness specification based on the use of inertial profilers. The literature revealed the following critical aspects:

1. Choice of smoothness indices;

2. Choice of wheel path(s);

3. Section length; and

4. Number of replicate measurements required per section.

Each item is discussed in the following subsections.

\subsubsection{Choice of Smoothness Indices}

A recent NCHRP study showed that IRI correlates well with user response for HMA pavements, but not as well as for PCC pavements. (Smoothness Specification for Pavements, Final Report, March 1997). In fact reported $\mathrm{R}^{2}$ values for correlation between IRI and user response for HMA pavements were as high as 0.90 , while they were typically about 0.60 for PCC pavements. The poor correlation for PCC pavements was explained as the result of using an effective half car roughness index, which attenuated the short wavelength content of the profile and affected the correlation. (Sayers and Karamihas, 1996). It was suggested that the reason for the weaker correlation was because PCC pavements generally contain shorter wavelength roughness components than HMA pavements (Sayers, 1989). Conversely, it was suggested that the good 
correlation for HMA pavements was due to the fact that the wavelength content commonly found in HMA pavements are attenuated by the IRI algorithm.

The PI smoothness statistic attenuates longer pavement wavelengths and amplifies short wavelength. (Darlington, 1995). Hence in PCC pavements, PI has correlated fairly well with user response and the correlation has been found to improve with decreasing blanking band width (Walker and Lin, 1988). In fact reducing or eliminating the blanking band should make the PI more sensitive to the short wavelength oscillations that can occur in paving operations, thus resulting in stronger correlation with user response. Based on this, PI with a 0.0 inch blanking band (PI-0.0”) was recommended as a good index for measuring PCC pavement smoothness.

The final recommendation in the 1997 NCHRP Smoothness Specifications for Pavements Report was to retain PI as the smoothness index for both PCC and HMA pavements, but with the banking band reduced to 0.0 inch. It was further suggested that IRI provided another opportunity to develop profile-based smoothness specifications but that it should be used cautiously.

\subsubsection{Choice of Wheel Path}

Individual wheel path measurements of smoothness provide a direct indication of true pavement features, as opposed to the average of measurements obtained in two wheel paths. Hence, Sayers has suggested choosing a single wheel path instead of dual wheel paths. (Sayers, 1995). An investigation of the differences between measurements 
obtained in two wheel paths revealed that the difference was not statistically significant. (Hajek, Kazmierowski, Musgrove, 1998). The study also suggested that the right wheel path was typically rougher than the left wheel path and should be used in specifications.

\subsubsection{Section Length}

Sayers has recommended a 0.1 mile $(528 \mathrm{ft}$ or $160 \mathrm{~m})$ moving IRI base-length for measuring the overall quality of a pavement. (Sayers, 1990). Most state smoothness specifications currently use this distance as the standard section or lot length for pavement already. Hence the 0.1 mile (528ft or $160 \mathrm{~m}$ ) section length appears to be appropriate.

\subsubsection{Number of Replicate Measurements}

The repeatability of the lightweight profilers evaluated in this study was good regardless of the observed level of roughness. However several of the F-tests resulted in significance, which suggests that replicate measurements should be conducted. In recognition of the fact that replicate testing takes time and thus money, but considering the fact that the lightweight profilers operate at significantly high speeds than a California Profilograph it is recommended that three measurements be made and averaged.

\subsection{Review of Smoothness Specifications}

As previously stated, smoothness specifications were requested from states seriously considering the option of moving to specifications based on lightweight profilers and/or IRI rather than PI. The specifications received were reviewed in the 
following subsections. Rigid (PCC) and flexible (HMA) pavement smoothness specifications have been reviewed separately. The items discussed for each specification include the smoothness index employed, a brief description of the test method employed, and pay factors. The material presented has essentially been extracted directly (word for word) from specifications. This is not an attempt to plagiarize, but rather to retain the meaning as stated in the complete specifications.

\subsubsection{Rigid Pavement Smoothness Specifications}

Rigid pavement smoothness specifications were obtained from Connecticut, Georgia, Kansas and Pennsylvania DOT's. The specifications are reviewed in the subsequent subsections.

\subsubsection{Connecticut DOT - Rigid Pavement Smoothness Specification}

Profile Index (PI) as determined by the California type profilograph is used as the measurement criteria, to determine the smoothness of rigid pavements.

\subsection{Test Method}

Pavement profiles will be taken $1 \mathrm{~m}$ from and parallel to each edge of pavement for pavements placed at a $3.7 \mathrm{~m}$ width or less. When pavement is placed at a width greater than $3.7 \mathrm{~m}$, the profile will be taken $1 \mathrm{~m}$ from and parallel to each edge and at the approximate locations of the longitudinal joint. The pavement is evaluated in $150 \mathrm{~m}$ sections. 
The finished surface of all mainline pavements (all pavements for traffic lanes and climbing lanes, but excluding concrete base, acceleration lanes and deceleration lanes, and all taper sections, pavement widening, shoulders, and side street returns) shall be tested. Pavement on horizontal curves having a baseline radius of curvature of 6 degrees or greater and super elevation transitions of such curves will however be excluded.

Pavements not considered as mainline pavements, should be tested using a $3 \mathrm{~m}$ straightedge, placed both transversely and longitudinally to the centerline at sufficient intervals to check the surface profile.

\subsection{Pay Factors}

Payment to the Contractor will be based on the average PI per $150 \mathrm{~m}$ section according to the following table:

\section{Table 6.1: Pay Factor Adjustment}

\begin{tabular}{|c|c|}
\hline Average Profile Index -0.2 'bb & Contract Unit Price Adjustment \\
\hline (mm per km per 150m section) & Percent of pavement unit bid price \\
\hline $0-95$ & 106 \\
$95-125$ & 104 \\
$125-160$ & 102 \\
$160-190$ & 100 \\
$190-220$ & 98 \\
$220-250$ & 96 \\
$250-285$ & 94 \\
$285-315$ & 92 \\
over 315 & \\
\hline
\end{tabular}

This unit bid price adjustment will apply to the total area of the $150 \mathrm{~m}$ long section for the lane width represented by the profile (usually $3.7 \mathrm{~m}$ ). No payment will be made for any pavement which has an average PI in excess of $315 \mathrm{~mm} / \mathrm{km}$, until any corrective work 
has been completed and the pavement re-profiled to verify that the average PI has been reduced to $315 \mathrm{~mm} / \mathrm{km}$ or less.

Within each $150 \mathrm{~m}$ section, high points having deviation in excess of $13 \mathrm{~mm}$, will be removed by the contractor. Thus the two major points to be kept in mind by the contractor are:

- Profile Index less than $315 \mathrm{~mm} / \mathrm{km}$

- Surface deviations less than $13 \mathrm{~mm}$.

\subsubsection{Georgia DOT - Rigid Pavement Smoothness Specification}

Profile Index as measured by a Rainhart (Model 860) Profilograph will be used for measuring the smoothness of rigid pavements. Prior to use, the equipment has to be evaluated and certified at the Georgia DOT Materials and Research Division.

\subsection{Test Method}

Pavement profiles shall be taken four feet from and parallel to the new pavement edges for pavements greater than sixteen feet in width, and up to twenty four feet in width. Pavements six to sixteen feet in width shall be tested parallel to and at the centerline of the pavement section. The profile index value shall be evaluated for each 0.25 miles segment. 


\subsection{Smoothness Requirement}

The mainline riding surface shall produce a profile index value not exceeding 7.0 inches per mile on each 0.25 miles segment of each travel lane. If the profile index exceeds 7.0 inches per mile for any segment, the paving operation has to be suspended and corrective action (as approved by the Engineer) taken. Individual bumps or depressions exceeding the blanking band by more than 0.2 inches shall have to be corrected at no additional cost of the department.

Ramps, acceleration and deceleration lanes shall be tested to produce an average profile index value not exceeding 12 inches/mile for the entire section length. In addition the bumps exceeding 0.2 inches have to be corrected.

All correction will be performed at no additional expense to the Department. Retesting will be done after correction and the Engineer will verify the results. If the engineer determines the contractor's test results to be inaccurate, the contractor will be charged at the rate of $\$ 250.00$ for each trace mile inaccurately reported, with a minimum charge of $\$ 500.00$.

\subsubsection{Kansas DOT - Rigid Pavement Smoothness Specification}

Profile Index (PI with 0.0" blanking band), as measured by the California Profilograph, is used as the criteria to determine the smoothness of pavements. Other types of profilographs may be used but they have to be approved by KDOT's Bureau of 
Materials and Research, and have to be certified according to Kansas Test Method KT46.

\subsection{Test Method}

The pavement is divided into sections. A pavement section is defined as a continuous area of finished pavement 0.1 mile in length and 12' (nominal) in width. A partial section resulting from an interruption of the continuous pavement surface is subject to the same evaluation as a whole section.

Profilograph testing is carried out on the finished surface of the mainline pavements, side roads, auxiliary lanes, and ramps. Excluded from profilograph testing are:

- Bridge Decks

- Acceleration and Deceleration lanes

- Shoulders

- Pavement on horizontal curves with a centerline radius of curvature of less than 1000' and pavement within the super-elevation transition of such curves

- Individual sections of pavements less than 50' in length

- The first and last $15^{\prime}$ of a pavement section Smoothness will be determined using a 10' straight edge on these excluded sections. The variation of the surface from the testing edge of the straight edge shall not exceed 1/8" between any two contacts, longitudinal or transverse. 


\subsection{Pay Factors}

Pay adjustments will be based on the initial average profile index determined for the sections prior to performing any corrective work. However if the contractor elects to remove and replace the sections, the contractor will be paid price adjustment corresponding to the initial PI obtained on the pavement sections after replacement. The pay adjustments are made according to the following table:

\section{Table 6.2: Schedule for Adjusted Payment}

\begin{tabular}{|c|c|c|}
\hline $\begin{array}{c}\text { Average PI in in/mile per 0.1 mile } \\
\text { section }\end{array}$ & Avg. PI in in/mi per 0.1 mile section & $\begin{array}{c}\text { Contract price adjustment per } 0.1 \\
\text { mile section per lane }\end{array}$ \\
\hline (greater than $45 \mathrm{mph})$ & $(45 \mathrm{mph}$ or less $\&$ ramps $)$ & $+\$ 1200$ \\
6.0 or less & & $+\$ 1000$ \\
6.0 to 10.0 & 15.0 or less & $+\$ 750$ \\
10.1 to 15.0 & 15.1 to 25.0 & $+\$ 500$ \\
& & $+\$ 370$ \\
15.1 to 18.0 & 25.1 to 45.0 & $+\$ 0.00$ \\
18.1 to 30.0 & 45.1 to 65.0 & $+\$ 0.00^{*}$ \\
30.1 to 40.0 & 65.1 or $\mathrm{more}$ & $-\$ 750.00^{*}$ \\
\hline 40.1 or $\mathrm{more}$ & *Correct to $45.0 \mathrm{in} / \mathrm{mile}$ & \\
\hline
\end{tabular}

Within each 0.1 mile section, all areas representing high points (bumps), with a deviation in excess of 0.3 inches in a length of $25.0 \mathrm{ft}$. or less, shall be corrected by the contractor regardless of the PI value. Each individual profilograph trace will be evaluated and not the average of the multiple traces, to determine the areas where corrective action is required. All corrective work will be done at the contractor's expense.

The Engineer may perform profilograph testing for monitoring and comparison purposes. The Engineer may test the entire length of the project if he determines that the 
contractor certified results are inaccurate, and the contractor will be charged $\$ 400 / \mathrm{mile}$, per profile track, with a minimum charge of $\$ 800.00$.

Pavements which are hand finished will not be subject to price adjustment. However the area is to be profiled and corrected if necessary to meet the specifications (i.e. $25 \mathrm{in} / \mathrm{mile}$ or less for roadways with a posted speed $>45 \mathrm{mph}$ and $45 \mathrm{in} / \mathrm{mile}$ or less for roadways with a posted speed $\leq 45 \mathrm{mph}$ ).

Daily monitoring is also carried out. If the days average PI exceeds $40 \mathrm{in} / \mathrm{mile}$ (65.0 in/mile on roadways with a posted speed of $45 \mathrm{mph}$ or less), the paving operation will be suspended until corrective action is taken by the contractor.

\subsubsection{Pennsylvania DOT - Rigid Pavement Smoothness Specification}

Profile Index (PI with 0.0" blanking band) and International Roughness Index (IRI) are used.(Average of the two wheel paths for each lot is the PI of the lot and similar reporting is also followed for IRI ). Must Grind areas are also recorded and are reported to the nearest $1 \mathrm{~mm}(0.05$ "). A $10 \mathrm{~mm}(0.4$ ") deviation of the pavement surface recorded from a chord representing $7.5 \mathrm{~m}\left(25^{\prime}\right)$ on the longitudinal scale is considered as a must grind point. 


\subsection{Test Method}

Pavement profiles shall be taken in both wheel paths. The first profile shall be approximately $1 \mathrm{~m}\left(3^{\prime}\right)$ from and parallel to the outside edge of pavement and the second profile shall be approximately $1.75 \mathrm{~m}\left(5.75^{\prime}\right.$ ) from the first profile (or as directed by the Project Engineer). Sampling areas shall be designated as lots and the excluded areas (i.e the area not included in the measurement of profile used to determine lot payment) shall be defined and measured separately. A full lot is defined as a single lane of pavement which is $12^{\prime}$ or greater in width and having a length of $528^{\prime}$. A partial lot can also be measured which is $12^{\prime}$ or greater in width but has a length less than 528 '. The partial lots are evaluated as a percentage of the full lot. Lots will start at the beginning of the paving and progress continuously throughout the entire construction. Lots will be specific to an individual pavement lane or ramp and are separated into two categories based on whether they are measured with high speed (Type 1) or with other equipment (Type 2).

\subsection{Calibration}

The operational system software shall allow the operator to perform a distance sensor calibration and save the calculated factor to be used for calculations later on. A 5, per mile accuracy is required. The calibration software shall also allow the operator to perform a profile system calibration, which will be stored and used later on in calculation. 


\subsection{Pay Factors}

Acceptance relative to pavement surface tolerance is determined on a lot-by-lot basis. Evaluation is done to determine compliance with the following specification requirements.

Table 6.3: Schedule A-Ride Quality Index ( 5 Contiguous Lots Uncorrected)

\begin{tabular}{|c|c|c|}
\hline $\begin{array}{c}\text { Type 1 } \\
\text { High Speed }\end{array}$ & $\begin{array}{c}\text { Type 2 } \\
\text { Other than High Speed }\end{array}$ & Characteristic Percentage (Pp) \\
\hline Inches/mile/lot & Inches/mile/lot & $107 \%$ \\
\hline 18 & 20 & $105 \%$ \\
24 & 28 & $103 \%$ \\
30 & 36 & $101 \%$ \\
36 & 42 & Corrective Action Required \\
\hline
\end{tabular}

Table 6.4: Schedule B-Ride Quality Index (Uncorrected or Corrected less than 5 m per lot)

\begin{tabular}{|c|c|c|}
\hline $\begin{array}{c}\text { Type 1 } \\
\text { High Speed }\end{array}$ & $\begin{array}{c}\text { Type 2 } \\
\text { Other than High Speed }\end{array}$ & Characteristic Percentage (Pp) \\
\hline Inches/mile/lot & Inches/mile/lot & $106 \%$ \\
24 & 20 & $104 \%$ \\
30 & 28 & $102 \%$ \\
36 & 36 & $100 \%$ \\
36 & 42 & Corrective Action Required \\
\hline
\end{tabular}

Table 6.5: Schedule C-Ride Quality Index (Corrected $5 m$ or more per lot)

\begin{tabular}{|c|c|c|}
\hline $\begin{array}{c}\text { Type 1 } \\
\text { High Speed }\end{array}$ & $\begin{array}{c}\text { Type 2 } \\
\text { Other than High Speed }\end{array}$ & Characteristic Percentage (Pp) \\
\hline Inches/mile/lot & Inches/mile/lot & $104 \%$ \\
24 & 20 & $102 \%$ \\
30 & 28 & $101 \%$ \\
36 & 36 & $100 \%$ \\
36 & 42 & Corrective Action Required \\
\hline
\end{tabular}


The Characteristic Percentage will be based on the ride quality index measured after the completion of all corrective work (includes the must grind high points or low points).

The lot payment is determined in accordance with the following formula :

\section{Lot Payment $=($ Contract Price per Lot $)[(P p-100) / 100]$}

Where $\mathrm{Pp}=$ Characteristic percentage for Surface Tolerance

No payment will be allowed for defective payment left in place. Remedial action plans should be submitted before the corrective action is carried out and the pavement retested to verify that corrections have produced a ride quality index which meets the minimum requirements specified in the table. Equipment and labor required for testing of the pavement surface is incidental to the items of concrete paving.

\subsubsection{Smoothness Specification of Flexible Pavements}

Flexible pavement smoothness specifications were obtained from Arizona, Connecticut, Florida, Kansas, Virginia and Pennsylvania DOT's. A draft specification based on the use of lightweight profilers was also obtained from Texas DOT. The specifications are reviewed in the subsequent subsections. 


\subsubsection{Arizona DOT - Flexible Pavement Smoothness Specification}

Mays-Meter (in inches/mile) is the smoothness measurement criteria used to evaluate the pavements. General Motors Research (GMR) type profilometer or MaysMeter may be used for measurement. GMR type profilometer values will be reduced to Mays-Meter values (inches/mile) using the model defined in NCHRP Report \#228, "Calibration of Response-Type Road Roughness Measuring Systems" (Page 25 of the report). The correlation coefficient of the equation is around 0.92 .

\subsection{Test Method}

Testing will be done on mainline traffic lanes only and will include the full length of the pavement placed under contract. Distress lanes, shoulders, ramps, tapers, crossroads and frontage roads will not be tested. Testing will also not be performed on any portions that cannot be made safe for testing at the design speed or any lanes less than 0.3 miles in length. The measuring device is driven over the area to be tested and measurements are taken for each 0.1 mile increment. Three replicate measurements for each 0.1 mile increment are obtained and the average is obtained to the nearest $0.1 \mathrm{in} / \mathrm{mile}$. The GMR-type Profilometer will operate at constant speed 2 mph (between 30 and 50 mph), and Mays-Meter will do so between $502 \mathrm{mph}$. Testing will not be done when the ambient air temperature is less than 40 degrees $\mathrm{F}$ or during rain or other weather conditions as determined inclement by the Engineer. 
For projects where the pavement is removed and replaced to grade, followed by an asphaltic concrete friction course (ACFC) overlay, no smoothness measurements are required in the following areas:

- Pavement placed within $35^{\prime}$ of the termini of the project

- Pavement placed within 35' of the approaches and departures for bridge structures not being overlain as part of the project

For project where pavement is removed and replaced to grade, followed by an overlay, followed by an ACFC overlay, no smoothness measurements will be made for the following areas:

- Pavement placed within 100' of the termini of the project

- Pavement placed within 100' of the approaches and departures for bridge structures not being overlain as part of the project

Bridges and their approaches which are overlain with this project will be subject to the smoothness requirements.

\subsection{Pay Factors}

An Incentive/Disincentive value (determined for every 0.1 lane mile) will be added to or subtracted from the contract based on the following formulas:

When Actual Smoothness (AS) $<$ XX:

Incentive Value $=((\mathrm{XX}-\mathrm{AS}) /(\mathrm{XX}+2)) *($ Incentive Base for the contract for each 0.1lane mile)

When Actual Smoothness (AS) > YY: 
Disincentive Value $=((\mathrm{YY}-\mathrm{AS}) /(\mathrm{XX}+2)) *($ Disincentive Base for the contract for each 0.1 lane mile)

The limits XX and YY are specific to a particular project but generally lie between 33 and 45 .

The AS will be determined in accordance with Arizona Texas Method 829. $\$ 2500$ can be taken to be a sample Incentive Base for a 0.1 lane mile increment and $\$ 1000$ can be taken to a sample Disincentive Base for a 0.1 lane mile increment. The total Incentive/Disincentive Value is the summation of the Incentive/Disincentive Value for the respective 0.1 mile segments.

Any 0.1 lane mile increment having an Actual Smoothness (AS) equal to or greater than the Correction Value (which is specific to a contract; sample value $=100$ inch/mile) shall be repaired at contractor's cost. The pavement will be re-tested after the repair is over.

In addition to these smoothness requirements, the pavement surface shall not vary more than $1 / 8$ inch from the lower edge of a $10^{\prime}$ straightedge when the straightedge is placed parallel to the centerline of the roadway. 


\subsubsection{Connecticut DOT - Flexible Pavement Smoothness Specification}

International Roughness Index (IRI), determined by ARAN (Automatic Road Analyzer) is the measurement criteria used to evaluate the pavements. The ARAN has the capability to measure longitudinal profile in each wheel path simultaneously. The IRI calculated from each wheel path for each 0.1 mile segment will be averaged to determine the IRI value for that segment. This provision is applicable to projects with a minimum of 2 courses of HMA in which the compacted depth of each is 1.5 " or greater.

\subsection{Test Method}

The final pavement surface will be divided into 0.1 mile (160m) segments representing the total lane miles of the project (which is equal to the miles of resurfacing multiplied by the number of lanes being evaluated). The final segment will include any remaining portion of the segment not equaling 0.1 miles. ARAN will operate at $48 \mathrm{miles} / \mathrm{hr}(77 \mathrm{~km} / \mathrm{hr})$ while operating over the specified segment. There would be a trial evaluation consisting of 1 run in each direction of travel over the specified segment. IRI computed from the trial run will serve as a guide to the Contractor in evaluating his current level of conformance with the smoothness specifications.

Bridge decks will be evaluated if and only if they are included as part of the project otherwise, profile testing will be suspended 0.02 miles prior to the first expansion joint and after the last expansion joint on the bridge decks. Climbing and operational lanes less than 0.4 miles in length, acceleration and deceleration lanes, shoulders and 
pavements on horizontal curves which have a 900' or less centerline radius of curvature will not be evaluated.

No testing shall be conducted during rain and under other conditions deemed inclement by the Engineer.

\subsection{Pay Factors}

Payment to the contractor shall be based on the IRI according to the following table. The percent adjustment will be applied to payment(s) for the total quantity of HMA surface course, excluding ramps.

Table 6.6: Schedule for Payment

\begin{tabular}{|c|c|c|}
\hline IRI (inches per mile) & IRI (meters per kilometer) & Percent Adjustment \\
\hline$<50$ & $<0.79$ & +10 \\
\hline $51-60$ & $0.8-0.95$ & +05 \\
\hline $61-80$ & $0.96-1.26$ & 0 \\
\hline $81-100$ & $1.27-1.58$ & -05 \\
\hline $101-110$ & $1.59-1.74$ & -10 \\
\hline $111-120$ & $1.75-1.89$ & -25 \\
\hline$>120$ & $>1.89$ & -50 \\
\hline
\end{tabular}

All values in the English system will be rounded to the nearest whole number and all values in the metric system will be rounded to the nearest hundredth.

Positive adjustments for rideabiltiy shall not be made for those areas reviewed and determined by the Engineer to be defective. 


\subsubsection{Florida DOT - Flexible Pavement Smoothness Specification}

Unit Price adjustments will be based on the Ride Number(RN) as established by a Laser Profiler. The RN will be determined in accordance with ASTM Standard E 1489.

\subsection{Test Method}

Pavements will be tested in lots of 0.1 mile. Only those lots actually tested in accordance with this Article will be eligible for price adjustments. Acceleration and deceleration lanes, storage lanes or turns, cross-overs, shoulders, signalized intersections and ramps are excluded from testing. Additionally $1000 \mathrm{ft}$ of roadway before and $500 \mathrm{ft}$ after the sections to be tested are excluded from price adjustment under this Article.

Profiling will begin and end within 500ft from each bridge approach pavement or existing pavement that is joined by the new pavement.

\subsection{Pay Factors}

Payment for smoothness will be determined in accordance with the following:

\section{Table 6.7: Pay Factor Adjustment}

\begin{tabular}{|c|c|}
\hline Ride Number $(\mathrm{RN})$ & Unit Price Adjustment \\
\hline $\mathrm{RN} \geq 4.47$ & \$600 per LOT \\
\hline $4.45 \leq \mathrm{RN}<4.47$ & \$300 per LOT \\
\hline $4.43 \leq \mathrm{RN}<4.45$ & \$100 per LOT \\
\hline
\end{tabular}


In no case will the pavement be re-tested once the smoothness of the LOT is determined. Total unit price adjustment will be limited to $2 \%$ of the original total contract amount.

\subsubsection{Kansas DOT - Flexible Pavement Smoothness Specification}

Profile Index (PI with 0.0" blanking band), as measured by the California Profilograph, is used as the criteria to determine the smoothness of pavements. Other types of profilograph may be used but they have to be approved by KDOT's Bureau of Materials and Research, and have to be certified according to Kansas Test Method KT46.

\subsection{Test Method}

The pavement is divided into sections. A pavement section is defined as a continuous area of finished pavement 0.1 mile in length and 12' (nominal) in width. A partial section resulting from an interruption of the continuous pavement surface is subject to the same evaluation as a whole section.

Profilograph testing is carried out on the finished surface of the mainline pavements, side roads, auxiliary lanes, and ramps. Excluded from profilograph testing are:

- Bridge Decks

- Acceleration and Deceleration lanes

- Shoulders

- Side Roads less than 0.1 mile in length 
- Pavement on horizontal curves with a centerline radius of curvature of less than 1000' and pavement within the super-elevation transition of such curves

- Individual sections of pavements less than 50' in length

- County secondary projects

- Federal aid urban projects with posted speeds of $40 \mathrm{mph}$ or less

- Existing roadways that are surfaced with less than 4" of virgin or hot recycled bituminous pavement which is placed in one lift

- Projects less than 0.5 mile in length

- The first and last $15^{\prime}$ of a pavement section

Smoothness will be determined using a 10' straight edge on these excluded sections.

The following bituminous surfaces will be profiled, and corrected if necessary, but are not eligible for pay adjustments:

- Existing roadways that are milled, then surfaced with less than 4" of HMA

- Existing roadways that are surfaced with less than 4" of HMA which is placed in two lifts

- Existing roadways that are cold recycled, then surfaced with less than 4" of HMA

\subsection{Pay Factors}

Pay adjustments will be based on the initial average profile index, determined for the sections prior to performing any corrective work. However if the contractor elects to remove and replace the sections, the contractor will be paid price adjustment 
corresponding to the initial PI obtained on the pavement sections after replacement. The pay adjustments are made according to the following table:

\section{Table 6.8: Schedule for Adjusted Pavement}

\begin{tabular}{|c|c|}
\hline Average Profile Index & Contract Price Adjustment \\
\hline (in/mile per lane per 0.1 mile section) & (per 0.1 mile section per lane) \\
\hline 7.0 or less & $+\$ 152.00$ \\
7.1 to 10.0 & $+\$ 76.00$ \\
10.1 to 30.0 & 0.00 \\
30.1 to 40.0 & $0.00^{*}$ \\
40.1 or more & $-\$ 203.00^{*}$ \\
\hline
\end{tabular}

*Correct to $30 \mathrm{in} / \mathrm{mile}$ (40 in/mile for ramps)

All areas within each section having high or low points (bumps or dips) with deviations in excess of 0.4 " inches in a length of $25^{\prime}$ or less shall be corrected regardless of the PI value. Each individual profilograph trace is evaluated and not the average of the multiple traces, to determine the areas where corrective action is required.

Pavement surfaces having an initial PI greater than 30in/mile per section (40 in/mile for ramps) on an individual trace, shall be corrected to reduce the PI below the limits specified. Corrections have to be performed by the contractor at his own cost.

The Engineer may perform profilograph testing for monitoring and comparison purposes. The Engineer may test the entire length of the project if he determines that the contractor certified results are inaccurate, and the contractor will be charged $\$ 400 / \mathrm{mile}$, per profile track, with a minimum charge of $\$ 800$. 
Daily monitoring is also performed. If the day's average PI exceeds 40in/mile, the paving operation will be suspended until corrective action is taken by the contractor.

\subsubsection{Virginia DOT - Flexible Pavement Smoothness Specification}

International Roughness Index (IRI) is the measurement used for ride quality acceptance. The lowest site average produced by a minimum of 2 test runs using a South Dakota style road profiling device (and reported for each lane) is used for measuring ride quality. Both the wheel paths are measured. The test has to be conducted within 14 calendar days of completion of the final surface course over the designated section.

\subsection{Test Method}

Each 0.1 mile section of each travel lane of the overlay will be subjected to test. Only the last 0.01 mile section before a bridge, the first 0.01 mile section after a bridge and the beginning and end 0.01 mile sections of the overlay will not be subject to a pay adjustment. Areas that have been excluded from testing by the profiler, will be tested using a 10’ straightedge.

\subsection{Pay Factors}

The following two tables provide the acceptance quality of pavement based on the finished rideability for interstate and primary roadways. Pay adjustments will be applied to the theoretical tonnage of the surface mix asphalt material for the lane width and section tested (generally 12' wide and 528' long) based on testing prior to corrective action directed by the Engineer. 


\section{Table 6.9: Interstate System}

\begin{tabular}{|c|c|}
\hline International Roughness Index after completion & Pay adjustment \\
\hline (inches/mile) & (Percent Pavement Unit Price) \\
\hline 45.0 and under & 104 \\
$45.1-50.0$ & 103 \\
$50.1-55.0$ & 102 \\
$55.1-60.0$ & 101 \\
$60.1-70.0$ & 100 \\
$70.1-80.0$ & 98 \\
$80.1-90.0$ & 95 \\
$90.1-100.0$ & 90 \\
Over 100.0 & Subject to Corrective Action \\
\hline
\end{tabular}

Table 6.10 :Primary System

\begin{tabular}{|c|c|}
\hline International Roughness Index after completion & Pay adjustment \\
\hline (inches/mile) & (Percent Pavement Unit Price) \\
\hline 55.0 and under & 104 \\
$55.1-60.0$ & 102 \\
$60.1-65.0$ & 101 \\
$65.1-70.0$ & 100 \\
$70.1-80.0$ & 98 \\
$80.1-90.0$ & 95 \\
$90.1-100.0$ & 90 \\
$100.1-110.0$ & Subject to Corrective Action \\
Over 110.0 & \\
\hline
\end{tabular}

In addition any 0.01 mile of a 0.1 mile section that has an IRI which exceeds 110inches/mile (Interstate) or 120inches/mile (Primary) will also be subject to correction as directed by the Engineer regardless of the IRI average over that 0.1 mile section. This 0.1mile section will not receive any incentive payment. The Engineer has the option of applying up to the minimum percent payment to any 0.1 mile section subject to corrective action or to any 0.1 mile section containing a 0.01 mile section subject to corrective action. 
The variation of the surface from the testing edge of the straightedge between any two contacts with the surface shall not be more than 0.25 ". Humps and depressions exceeding the specified tolerance shall be subject to correction at no additional cost of the Department. In order to produce a uniform cross section, the Engineer may require correction to the adjoining traffic lanes and shoulders at no cost to the Department.

When corrections are made, the pavement will be re-tested to verify that corrections have produced the acceptable ride surface. No incentives will be provided for sections on which corrective actions have been required. Re-testing of a corrected pavement will be at the contractor's expense and pay disincentives or corrections may be required based on the re-tested IRI measurements.

Initial smoothness of the pavement also plays an important role in determining the payment received by the contractor. If the contractor is able to improve the ride quality of the pavement by $25 \%$, regardless of the final IRI after rehabilitation, he will not receive a disincentive.

\subsubsection{Virginia DOT - New Flexible Pavement Smoothness Specification}

International Roughness Index (IRI) is the measurement used for ride quality acceptance. The lowest site average produced by a minimum of 2 test runs using a South Dakota style road profiling device (and reported for each lane) is used for measuring ride quality. Both the wheel paths are measured. The test has to be conducted within 14 calendar days of completion of the final surface course over the designated section. 


\subsection{Test Method}

Each 0.1 mile section of each travel lane of the overlay will be subjected to test. Only the last 0.01 mile section before a bridge, the first 0.01 mile section after a bridge and the beginning and end 0.01 mile sections of the overlay will not be subject to a pay adjustment. Areas that have been excluded from testing by the profiler, will be tested using a 10' straightedge. Pavement smoothness will be determined by a profiler on designated lanes at a design speed of 40 miles/hr or higher as specified. Intersections, urban areas, transition lanes and pavement within $25^{\prime}$ of bridge approach slabs will be tested using a straightedge.

\subsection{Pay Factors}

The following table provides the acceptance quality rating scale of pavement based on the final rideability determination. The pay incentive/disincentive schedule will be applied to the final surface area of the surface course. The surface area in sq. yards, shall be calculated based on the tested section length and lane width.

Table 6.11: Pay Factor Adjustment

\begin{tabular}{|c|c|}
\hline International Roughness Index & Incentive/Disincentive Pay adjustment \\
\hline (inches/mile) & (dollars per square yard) \\
\hline 50.0 and under & 0.16 incentive \\
$50.1-55.0$ & 0.13 incentive \\
$55.1-60.0$ & 0.10 incentive \\
$60.1-70.0$ & 0.00 \\
$70.1-80.0$ & 0.03 disincentive \\
$80.1-90.0$ & 0.10 disincentive \\
$90.1-100.0$ & 0.17 disincentive \\
Over 100.0 & Subject to Corrective Action \\
\hline
\end{tabular}


In addition any 0.01 mile of a 0.1 mile section that has an IRI which exceeds 110inches/mile will also be subject to correction as directed by the Engineer regardless of the IRI average over that 0.1 mile section. This 0.1 mile section will not receive any incentive payment. The Engineer has the option of applying up to the minimum percent payment to any 0.1 mile section subject to corrective action or to any 0.1 mile section containing a 0.01 mile section subject to corrective action.

The variation of the surface from the testing edge of the straightedge between any two contacts with the surface shall not be more than 0.25 ". Humps and depressions exceeding the specified tolerance shall be subject to correction at no additional cost of the department. In order to produce a uniform cross section, the Engineer may require correction to the adjoining traffic lanes and shoulders at no cost to the Department.

When corrections are made, the pavement will be re-tested to verify that corrections have produced the acceptable ride surface. No incentives will be provided for sections on which corrective actions have been required. Re-testing of a corrected pavement will be at the contractors expense and pay disincentives or corrections may be required based on the re-tested IRI measurements.

\subsubsection{Pennsylvania DOT - Flexible Pavement Smoothness Specification}

Profile Index, in inches/mile (with a 0.0" blanking band) has been identified as the index used for determining the ride quality of the pavement. Measurements will be carried out in both the wheel paths and its average will be used. 


\subsection{Test Method}

Pavements will be tested in lots of 0.1 mile (528 feet). The pavement profile will be determined for each lot according to the test procedure PTM no. 424 or PTM no. 428. Lots will start from the beginning of paving and continue till the end of the paving operation. Areas such as joints at the beginning and end of paving, and bridge approaches will be excluded from ride quality payment. These areas will be tested using a 10' straightedge and any irregularities greater than $3 / 16$ " have to be corrected.

\subsection{Pay Factors}

Profile Index is determined for each pavement lot and its compliance to Table 6.12 is determined. Corrective action like diamond grinding, removal and replacement etc. have to be carried out if the pavement is not found to adhere to the specifications. There will be no payment for lots, corrected by diamond grinding, including lots ground for correction of individual high and low points. However for lots where removal and replacement have been carried out, payment will be determined from Table 6.12, corresponding to the PI obtained for the lot after corrective action has been employed.

Table 6.12 Pay Factors

\begin{tabular}{|c|c|}
\hline Average Profile index (inches/mile) per lot & Payment \\
\hline 10.0 or less & $+\$ 300 /$ lot \\
10.1 to 15.0 & $+\$ 150 /$ lot \\
15.1 to 20.0 & $+\$ 75 /$ lot \\
20.1 to 25.0 & $0 /$ lot \\
25.1 to 36.0 & $-\$ 150 /$ lot \\
36.1 or more & must correct to 25 inches/mile or less \\
\hline
\end{tabular}




\subsubsection{Draft of the Lightweight Profiler Equipment Smoothness Specification}

\section{prepared by the Texas Department of Transportation}

The smoothness specification consists of certification tests, which the profiler must pass. Two methods are used to evaluate the profiler precision. The repeatability of the profiler is determined by elevation measurements measured by the profiler at about 6 " reporting intervals. Secondly IRI and PSI are used as ride statistics to determine the profiler accuracy.

\subsection{Test Method}

The tests will be conducted on a 0.1 mile section. There will be two types of facility - smooth and medium-smooth sections which have to be approved by the Texas Department of Transportation. Twelve repeat runs have to be made on the designated wheel-path of each test section, in the prescribed direction of measurement. The test wheel-path on the smooth sections shall have an IRI ( $\leq 63$ in/mile), while the corresponding wheel-path on the medium-smooth section shall have an IRI within the range of 95 to $114 \mathrm{in} / \mathrm{mile}$.

Two types of profiler measurement are acceptable. If there are sensors on both sides, (spacing between the wheel path sensors being 65 inches) then profile data will be collected on both the wheel paths, else they will be collected on a prescribed wheel path. However for a given section, the same wheel-path(s) have to be measured for all runs. 


\subsection{Equipment Repeatability}

All possible unique combinations of ten runs (from the twelve runs tested) have to be considered for the repeatability measurement. All the runs have the same reporting interval. At each reporting interval, the standard deviation of the elevation measurements, for any combination of the ten repeat runs have to be computed. For each combination of ten runs, the average of the standard deviation at the different reporting intervals will be determined. The lowest average standard deviation corresponding to the best combination of ten profile measurements, will be used to establish the repeatability of the profiler. To pass the repeatability test, these average standard deviations (corresponding to the best combination of ten out of twelve profile measurements) must not exceed thirty mils $(0.8 \mathrm{~mm})$.

\subsection{Verification of Equipment Accuracy}

Static methods like Rod and Level Survey, Dipstick and other suitable devices that provide true profiles and meet the requirements for a Class I survey given in ASTM E1364 shall be used to determine the accuracy of the profiler and at least three repeat measurements have to carried out. The reference profiles shall have the same reporting interval and the same type of filter (200 ft high pass filter) as the lightweight profiler. To evaluate the accuracy, the average profile from the best combination of ten repeat runs, is identified by the repeatability test (previously described). The average filtered reference profile has to be developed from the three repeat runs. Differences between the average test profile and the average filtered reference profile are then calculated at each of the reporting interval. The average of these differences $\left(\mu_{1}\right)$, as well as the average of the 
absolute differences $\left(\mu_{2}\right)$ are computed to establish the accuracy of the lightweight profiler. This average difference is a measure of the bias in the data from a given profiler and also gives us an indication of the degree of agreement between the test and the reference profiles. To pass the test, the average of the point to point differences, $\mu_{1}$, must be within \pm 6 mils $(0.15 \mathrm{~mm})$ and the average of the absolute differences, $\mu_{2}$, must not be greater than 60 mils $(1.52 \mathrm{~mm})$ for all sets of statistics determined.

\subsection{Verification of Computed Ride Statistics}

The IRI and PSI are computed using the profiles that make up the best combination of ten out of twelve runs as determined from the repeatability test described previously. For each wheel-path, the standard deviation of IRI and PSI are computed. To pass the repeatability test, each standard deviation of the IRI shall not exceed $2.5 \mathrm{in} / \mathrm{mile}$ and the standard deviation of the PSI shall be less than or equal to 0.05 .

For reproducibility measurement, the accuracy of the IRI and PSI from the test data are compared with the average IRI and PSI from the unfiltered reference profiles. The average difference between the average IRI's must not exceed 13in/mile and the average PSI's must be less than 0.25 for each test section. Both these have to verified for determining the accuracy of the profilers.

\section{$\underline{\text { 6.4 Pay Factors }}$}

Where possible, the state smoothness specifications reviewed were applied to the data generated with the lightweight profilers at each of the Indiana field sites. The site 
average IRI or PI for all vendors at each site was used in the specifications. The purpose of this exercise was to obtain an indication of the impact of the pay factors present in each specification. Tables 6.13 and 6.14 provide a summary of the payment that would be expected under each specification for each site. The terms large and small bonus or penalty in the tables are based on the maximum bonus or penalty the specification offered. If the bonus or penalty was more than 50 percent of the maximum possible bonus or penalty, it was regarded as large. If it were less than 50 percent it was referred to as small.

The data in Table 6.13 shows that the very rough Covington Street site would have resulted in a large penalty under the Kansas specification, corrective action under the Pennsylvania specification and no action under the Connecticut specification. The very smooth US231A site would have resulted in a small bonus under the Connecticut specifications and large bonuses under the Pennsylvania and Kansas specifications. The slightly rougher US231B site would have resulted in no action under all three specifications. The difference in PI of 5in/mi (21 versus 16) between the US231A and US231B sites made a significant difference the payment that would have been received, particularly under the Pennsylvania and Kansas specifications (no action versus large bonus). The Pennsylvania and Kansas specifications which are based on PI appear to be more appropriate than the Connecticut specification which is based on IRI for the PCC sites after conducting field surveys of the sites. 
Table 6.13 Payment Based on Various State Specifications for Rigid Pavements

\begin{tabular}{|c|c|c|c|c|c|}
\hline Site & $\begin{array}{c}\text { PI-0.2”bb } \\
\text { (inches/mile) }\end{array}$ & $\begin{array}{c}\text { PI-0.0" bb* } \\
\text { (inches/mile) }\end{array}$ & $\begin{array}{c}\text { Connecticut } \\
\text { specification }\end{array}$ & $\begin{array}{c}\text { Pennsylvania } \\
\text { specification }\end{array}$ & $\begin{array}{c}\text { Kansas } \\
\text { specification }\end{array}$ \\
\hline Convington & 37 & 66 & Large Penalty & Correction & Large penalty \\
\hline US 231A & 0 & 16 & Large bonus & Large bonus & Large bonus \\
\hline US 231B & 2 & 21 & Large bonus & No action & No action \\
\hline
\end{tabular}

* bb implies blanking band

The data in Table 6.14 shows that the very smooth I-65 site would have resulted in small bonuses under the Connecticut, Virginia, and Kansas specification and a large bonus under the Pennsylvania specification. The rough Prairie Street site would have resulted in large penalties under the Connecticut and Kansas specifications and corrective action under the Virginia and Pennsylvania specifications. The Vinton Street site would have resulted in small penalties under the Connecticut and Virginia specifications which is contrasted with a large penalty and no action under the Pennsylvania and Kansas specifications, respectively. The exact same observations are made for the I-65 site with the boards in place. Finally, the Prairie Street site with the boards in place resulted in payments identical to those observed for this site when the boards were not in place. This was expected however because the smoothness indices were unaffected by the presence of the boards. The payments appear reasonable for the I- 65 without boards site for all the specifications, and the Virginia specification appears to be most appropriate based on surveys of the sites, while the Kansas specifications appears to be least appropriate. The Virginia specification relied on IRI while the Kansas specification relied on PI. 
Table 6.14 Payment Based on Various State Specifications for Flexible Pavements

\begin{tabular}{|c|c|c|c|c|c|c|}
\hline Site & $\begin{array}{c}\text { IRI } \\
\text { (inches/mile) }\end{array}$ & $\begin{array}{c}\text { PI-0.0" bb* } \\
\text { (inches/mile) }\end{array}$ & $\begin{array}{c}\text { Connecticut } \\
\text { specification }\end{array}$ & $\begin{array}{c}\text { Virginia } \\
\text { specification }\end{array}$ & $\begin{array}{c}\text { Pennsylvania } \\
\text { specification }\end{array}$ & $\begin{array}{c}\text { Kansas } \\
\text { specification }\end{array}$ \\
\hline $\begin{array}{c}\text { I-65 } \\
\text { (without board) }\end{array}$ & 41 & 9 & Small bonus & Small bonus & Large bonus & Small bonus \\
\hline $\begin{array}{c}\text { Prairie Street } \\
\text { (without board) }\end{array}$ & 148 & 53 & $\begin{array}{c}\text { Large } \\
\text { Penalty }\end{array}$ & $\begin{array}{c}\text { Correction } \\
\text { required }\end{array}$ & $\begin{array}{c}\text { Correction } \\
\text { Required }\end{array}$ & $\begin{array}{c}\text { Large } \\
\text { penalty }\end{array}$ \\
\hline Vinton Street & 100 & 35 & $\begin{array}{c}\text { Small } \\
\text { penalty }\end{array}$ & $\begin{array}{c}\text { Small } \\
\text { penalty }\end{array}$ & $\begin{array}{c}\text { Large } \\
\text { Penalty }\end{array}$ & No action \\
\hline $\begin{array}{c}\text { I-65 } \\
\text { (with board) }\end{array}$ & 106 & 35 & $\begin{array}{c}\text { Small } \\
\text { penalty }\end{array}$ & $\begin{array}{c}\text { Small } \\
\text { penalty }\end{array}$ & $\begin{array}{c}\text { Large } \\
\text { Penalty }\end{array}$ & No action \\
\hline $\begin{array}{c}\text { Prairie Street } \\
\text { (with board) }\end{array}$ & 158 & 54 & $\begin{array}{c}\text { Large } \\
\text { penalty }\end{array}$ & $\begin{array}{c}\text { Correction } \\
\text { required }\end{array}$ & $\begin{array}{c}\text { Correction } \\
\text { required }\end{array}$ & $\begin{array}{c}\text { Large } \\
\text { penalty }\end{array}$ \\
\hline
\end{tabular}

This comparison coupled with actual physical surveys of the pavements in Indiana assisted with the development of pay factors for a proposed draft guide specification for Indiana.

\subsection{Draft Guide Pavement Smoothness Specification Based on the Use of}

\section{Lightweight Non-Contact Profilers}

The draft specification presented in this specification should be viewed only as a preliminary draft specification that should be shared with industry for comment prior any attempts to implement it.

\subsubsection{Test Method}

The ride quality acceptance will be based on the smoothness indices, (IRI and PI0.0"blanking band) as measured by a lightweight non-contact profiler (LWP) meeting the Class 1 requirements of ASTM E950. The reported smoothness index shall be the average of three measurements. The smoothness value and the pavement profile must be 
reported for each of these measurements. Profile Index with a 0.0 " blanking band will be used as the index for evaluating rigid pavements. International Roughness Index will be the measurement criteria for evaluating the smoothness of flexible pavements.

The pavement will be evaluated in $0.1 \mathrm{mile}(160 \mathrm{~m})$ sections and the profile measurements shall be conducted in the right wheel path.

Profiling with lightweight non-contact profilers shall be conducted on the finished surface of the mainline pavements, side roads and auxiliary lanes. The following areas will be excluded from profile measurement:

- Bridge Decks

- Acceleration and deceleration lanes

- Ramps

- Shoulders

- Side roads less than 0.1 mile in length

- Pavements on horizontal curves with a centerline radius of curvature of less than 1000 feet and pavement within the super-elevation transition of such curves

- Signalized intersection

- Areas of pavement widening

- All taper sections

- Projects less than 0.5 mile in length

- The first and last 15 feet of a pavement section 
For pavements approaching bridges, profiling will begin and end within 500 feet $(167 \mathrm{~m})$ from each bridge approach. Only those sections of $0.1 \mathrm{mile}(160 \mathrm{~m})$ profiled with the lightweight profiler will be eligible for price adjustments.

Smoothness for the excluded sections will be determined using a 10 foot $(3 \mathrm{~m})$ straight edge, placed both transversely and longitudinally to the centerline. All bumps greater than 0.3 inch $(8 \mathrm{~mm})$ between any two contact points of the straight edge, shall be repaired by the contractor at no cost of the Department.

\subsubsection{Calibration and Verification}

Proof of accelerometer, laser, and distance measurement calibration or verification in accordance with specific profiler manufacturer recommendations shall be provided with all submitted measurement results.

\subsubsection{Pay Factors}

Payment to the contractor will be based on the smoothness indices obtained for each $0.1 \mathrm{mile}(160 \mathrm{~m})$ of the section prior to performing any corrective work in accordance with Table 6.15 or 6.16 . The unit bid price adjustment will apply to the total area of the $0.1 \mathrm{mile}(160 \mathrm{~m})$ long section for the lane width represented by the profile. However if the contractor elects to remove and replace sections, the contractor will be made price adjustments corresponding to the initial smoothness indices obtained on the pavement sections after placement. 
Table 6.15 Rigid Pavement Pay Factor Adjustments

\begin{tabular}{|c|c|c|}
\hline $\begin{array}{c}\text { Profile Index (PI) with 0.0" } \\
\text { blanking band (inches/mile) per } \\
0.1 \text { mile section per lane }\end{array}$ & $\begin{array}{c}\text { Profile Index (PI)with 0.0" } \\
\text { blanking band (inches/mile) per } \\
0.1 \text { mile section per lane }\end{array}$ & $\begin{array}{c}\text { Contract Unit Price Adjustment per } \\
0.1 \text { mile section per lane }\end{array}$ \\
\hline (for traffic speed >45miles/hr) & (for traffic speed <=45 miles/hr) & Percent of pavement unit bid price \\
\hline$<=6$ & $<=15.0$ & $105 \%$ \\
\hline $6.1-10.0$ & & $104 \%$ \\
\hline $10.1-15$ & $15.1-20.0$ & $103 \%$ \\
\hline $15.1-20$ & $20.1-25.0$ & $102 \%$ \\
\hline $20.1-25.0$ & $25.1-30.0$ & $101 \%$ \\
\hline $25.1-30.0$ & $30.1-40.0$ & 0.0 \\
\hline $30.1-36.0$ & $40.1-45.0$ & 0.0 \\
\hline$>36.0 * *$ & $>45.0 * *$ & Subject to correction* \\
\hline$*$ Correct to 25 inches $/$ mile & $*$ Correct to 40 inches/mile & \\
\hline \multicolumn{2}{|c|}{$* *$ Must be corrected before any payment is received } \\
\hline
\end{tabular}

Table 6.16 Flexible Pavement Pay Factor Adjustments

\begin{tabular}{|c|c|}
\hline $\begin{array}{c}\text { International Roughness Index (IRI) per 0.1 mile } \\
\text { section per lane }\end{array}$ & $\begin{array}{c}\text { Contract Unit Price Adjustment per 0.1 mile section } \\
\text { per lane }\end{array}$ \\
\hline (inches/mile) & Percent of pavement unit bid price \\
\hline$<=45.0$ & $105 \%$ \\
\hline $45.1-50.0$ & $104 \%$ \\
\hline $50.1-55.0$ & $103 \%$ \\
\hline $55.1-60.0$ & $102 \%$ \\
\hline $60.1-65.0$ & $101 \%$ \\
\hline $65.1-70.0$ & $100 \%$ \\
\hline $70.1-80.0$ & $98 \%$ \\
\hline $80.1-90.0$ & $96 \%$ \\
\hline $90.1-100.0$ & $92 \%$ \\
\hline$>100$ & *Subject to Corrective Action \\
\hline$*$ Correct to at least 100 inches/mile to be eligible for payment \\
\hline
\end{tabular}

Must grind areas shall also be recorded and reported by the lightweight profiler. All areas having a high point deviation in excess of 0.3inch shall have to be corrected regardless of the index value for the whole section. Each individual profile trace will be evaluated and not the average of the multiple traces, to determine the areas where corrective action is required. All corrective work will be done at the contractor's expense. 
No payment will be allowed for defective pavements left in place. Remedial action plans will be submitted to the Engineer and approved, before any corrective action is carried out.

The pavement will be re-tested to verify that the corrections performed have resulted in a pavement that meets the minimum requirements as specified in the Tables 6.15 or 6.16. Equipment and labor required for re-testing the pavement surface are incidental to the items of concrete paving. The Engineer may perform testing for monitoring and comparison purposes. In fact the Engineer may test the entire length of the project if he/she determines that the contractor certified results are inaccurate, and the contractor will be charged $\$ 400$ per mile, with a minimum charge of $\$ 800$.

However a special provision will be applied when the pavement lane initially is very rough. No disincentive or rejection of payment will be made on the completed surface which indicates a 25 percent or greater improvement in ride quality but still does not meet the minimum smoothness level required to be eligible for payment.

\subsection{Assessment of the Proposed Guide Specification}

The precision analysis conducted in Section 5 served as the basis for verification of the proposed smoothness specification. Coefficient of variation for the repeatability and reproducibility statistics were calculated from the field test data of Indiana. All vendors, all sites, and all replicates were considered but only the without boards data were evaluated. IRI and PI-0.0" blanking band were employed since they were the two 
indices which were in contention for being selected as the smoothness indices for the specification. One important fact must be recognized is that the coefficient of variation for the reproducibility values were high because of the unsatisfactory between vendor performance.

The data are provided in Tables 6.17 and 6.18. The data suggests that IRI is most consistent (less variable) for HMA pavements and PI-0.0" is most consistent for PCC pavements as noted by the reported coefficients of variation. This supports the recommendation that IRI should be used for measurement of HMA pavements and PI0.0" should be used for measurement PCC pavements smoothness.

Table 6.17 Comparison of Repeatability Statistics of Indiana Test Sites

\begin{tabular}{|c|c|c|c|c|c|c|c|}
\hline \multirow{3}{*}{ Type } & \multirow{3}{*}{$\begin{array}{c}\text { Site } \\
\text { description }\end{array}$} & \multicolumn{6}{|c|}{ Repeatability Statistic } \\
\hline & & \multicolumn{3}{|c|}{$\begin{array}{c}\text { IRI } \\
\text { (inches/mile) }\end{array}$} & \multicolumn{3}{|c|}{$\begin{array}{l}\text { PI-0.0" blanking band } \\
\text { (inches/mile) }\end{array}$} \\
\hline & & Avg. & $\mathrm{SD}(\mathrm{Sr})$ & $\mathrm{CV}(\%)$ & Avg. & $\mathrm{SD}(\mathrm{Sr})$ & $\mathrm{CV}(\%)$ \\
\hline \multirow{3}{*}{ HMA } & $\mathrm{I}-65$ & 41 & 3 & 7.3 & 9 & 1 & 11.1 \\
\hline & Prairie St. & 148 & 4 & 2.7 & 53 & 2 & 3.8 \\
\hline & Vinton St. & 100 & 3 & 3 & 35 & 1 & 2.9 \\
\hline \multirow{3}{*}{ PCC } & Convington & 142 & 3 & 2.1 & 66 & 2 & 3 \\
\hline & US 231A & 51 & 4 & 7.8 & 16 & 1 & 6.2 \\
\hline & US 231B & 62 & 3 & 4.8 & 21 & 1 & 4.8 \\
\hline
\end{tabular}

Note : Avg. implies site average and standard deviation (SD) is the repeatability standard deviation. CV means coefficient of variation calculated from the above two statistic. 
Table 6.18 Comparison of Reproducibility Statistic for Indiana Test Sites

\begin{tabular}{|c|c|c|c|c|c|c|c|}
\hline \multirow{3}{*}{ Type } & \multirow{3}{*}{$\begin{array}{c}\text { Site } \\
\text { description }\end{array}$} & \multicolumn{6}{|c|}{ Repoducibility Statistic } \\
\hline & & \multicolumn{3}{|c|}{$\begin{array}{c}\text { IRI } \\
\text { (inches/mile) }\end{array}$} & \multicolumn{3}{|c|}{$\begin{array}{l}\text { PI-0.0" blanking band } \\
\text { (inches/mile) }\end{array}$} \\
\hline & & Avg. & $\mathrm{SD}(\mathrm{Sr})$ & $\mathrm{CV}(\%)$ & Avg. & $\mathrm{SD}(\mathrm{Sr})$ & $\mathrm{CV}(\%)$ \\
\hline \multirow{3}{*}{ HMA } & I-65 & 41 & 13 & 31.7 & 9 & 4 & 44.4 \\
\hline & Prairie St. & 148 & 16 & 10.8 & 53 & 12 & 22.6 \\
\hline & Vinton St. & 100 & 7 & 7 & 35 & 8 & 22.9 \\
\hline \multirow{3}{*}{ PCC } & Convington & 142 & 24 & 16.9 & 66 & 27 & 40.9 \\
\hline & US 231A & 51 & 11 & 21.6 & 16 & 3 & 18.7 \\
\hline & US 231B & 62 & 10 & 16.1 & 21 & 3 & 14.3 \\
\hline
\end{tabular}

Note : Avg. implies site average and standard deviation (SD) is the reproducibility standard deviation. CV means coefficient of variation calculated from the above two statistic.

The proposed specification was applied to the average values of IRI for the HMA pavements and PI-0.0" for the PCC pavements tested in Indiana. Table 6.19 is a summary of the bonus schedule that resulted from the application of the proposed specification. It can not be over emphasized that the sites tested were either very smooth or very rough.

Table 6.19 Bonus Schedule Based on Proposed Specification

\begin{tabular}{|c|c|c|c|c|c|}
\hline $\begin{array}{c}\text { Type } \\
\text { of site }\end{array}$ & Site description & \multicolumn{2}{|c|}{$\begin{array}{c}\text { IRI } \\
\text { (inches/mile) }\end{array}$} & \multicolumn{2}{c|}{$\begin{array}{c}\text { PI-0.0" blanking band } \\
\text { (inches/mile) }\end{array}$} \\
\hline & & Average & Bonus paid & Average & Bonus paid \\
\hline HMA & I-65 with board & 106 & No & 35 & \\
& I-65 without board & 41 & Yes & 9 & \\
\hline & Prairie St. with board & 158 & No & 54 & \\
\hline & Prairie St. without board & 148 & No & 53 & \\
\hline & Vinton St. & 100 & No & 35 & \\
\hline PCC & Convinton & 142 & & 66 & No \\
\hline & US 231A & 51 & & 16 & Yes \\
\hline & U 231B & 62 & & 21 & Yes \\
\hline
\end{tabular}

The data in Table 6.19 indicates that a bonus would have been paid according to the specification at the I-65 and US 231A and US 231B sites. All three sites were very 
smooth, thus the proposed specification appears to be reasonable. It is very important to note that the pavements evaluated were either very smooth or very rough, thus this assessment should be viewed with caution. 


\section{Summary and Conclusions}

Pavement smoothness was recently identified as the most significant factor the motoring public uses to judge the quality of our Nation's roadways. A new generation of lightweight profilers has recently been developed which have the potential of providing nearly instantaneous smoothness measurements providing contractors with a tool to spot quality control issues more promptly and cost effectively. This could ultimately translate into more rapidly constructed smoother pavements for motorists. A team of INDOT and Purdue University researchers undertook a cooperative effort to evaluate the new generation of profilers through a JTRP research project with partial support from a FHWA initiative. The objective of the research was to field test and evaluate various state-of-the-art lightweight non-contact profilers, which would provide the data from which end result guide smoothness specifications could be developed for QC/QA purposes. The project was focused directly on one of the primary objectives of the INDOT Strategic Plan, improving pavement smoothness.

Several lightweight profilers along with conventional tools were used to measure the roughness of three each HMA and PCC pavements during June of 1999. Four lightweight profilers and the INDOT RIP Van performed five replicate measurements at each site. California Profilograph tests and a precision rod and level survey were also conducted at one site for comparison with the lightweight profilers. Each test section was 0.1 miles $(160 \mathrm{~m})$ in length and several incorporated curves and grades such that the sensitivity of the profilers to such geometric features could also be assessed. At two of 
the test sites boards were placed in the test wheel path to simulate bumps and/or bridge abutments, again to assess the response of the profilers to such features.

Consultants currently performing smoothness measurements on INDOT projects and contractors were encouraged to observe/participate in the field evaluation. A field open house was held during one day of the testing and was attended by over forty INDOT, FHWA, consultant, contractor, and producer personnel. This provided excellent exposure of the new technology and demonstrated the willingness of INDOT to involve industry in the development of specifications that would ultimately provide for equitable payment based on contractor performance. The objective was to keep all those that would possibly be affected by a new specification abreast of the project, thus fostering a partnering atmosphere.

All of the participating lightweight profiler vendors were able to provide the data requested by the research team. Analysis of the collected field test data showed that the quality of data was good. Three smoothness indices were considered in the data analysis, specifically IRI and PI with both 0.0 and 0.2 inch blanking bands. The data clearly showed that PI with a 0.2 inch blanking band is a poor index for measuring pavement smoothness when compared to PI with a 0.0 inch blanking band. The reason for this is that reported PI values with the 0.2 inch blanking band at several sites were $0 \mathrm{in} / \mathrm{mi}$. However when observed values of PI with a 0.0 inch blanking band for the same site were reviewed measurable differences in smoothness were observed. It was also observed that the differences in PI with the 0.2 and 0.0 inch blanking were inconsistent which 
suggests again that true differences in the physical pavement were masked by the 0.2 inch blanking band.

Statistical analysis of the field generated data showed that the individual lightweight profilers exhibited good consistency or repeatability over the large range of roughness observed at the test sites. In other words, good within vendor precision was observed. The data did not suggest that the boards used at two sites or the geometric features at any of the sites impacted the performance of the profilers. However, the analysis revealed that the profilers exhibited poor reproducibility or between vendor consistency. In fact, paired t-tests (mean comparisons) of all possible one-to-one vendor combinations, totaling 144 tests, showed that the different devices only reported similar average values for individual test sites approximately 25 percent of the time, regardless of the smoothness index considered. This could also be stated as the profilers reported statistically different means in 75 percent of the comparisons for IRI and PI. The data generated with the INDOT Rip Van did not correlate well with any of the lightweight profilers. Similar findings were observed in a recent Connecticut DOT evaluation of the same lightweight profilers that focused on IRI. This finding was very disturbing and led to an analysis of the actual profiles generated by each profiler at each site.

The actual profiles generated by each lightweight profiler were compared among themselves and between profilers. The comparisons again showed good individual profiler repeatability, but they did reveal the fact that the performance of some profilers was adversely affected by the boards used at one site and the geometric features at two 
sites. The between vendor profile comparisons showed very poor reproducibility. Because smoothness indices (IRI and PI) are determined from profiles this provided an explanation for the poor IRI and PI reproducibility. The INDOT RIP Van, California Profilograph and precision rod and level survey data were included in the analysis. The California Profilograph profile was more similar to the rod and level survey data than the lightweight profilers. However, the lightweight profilers identified hand-tooled transverse joints in the pavement that the other techniques were insensitive to. The INDOT Rip Van Profiles were not similar to any of the other profiles.

A nice feature of the lightweight profilers is the ability to provide bump identification or must grind locations given a specified maximum allowable bump. Within and between lightweight profiler comparisons of this feature were also conducted. Only three of the four profiler vendors provided must grind location data, but the previously analyzed profiles essentially provided data similar data for the fourth device. All of the devices were capable of identifying the location and magnitude of the thin boards used at one site and three of four also identified the location and magnitude of thick boards used at another site. The thick boards did induce significant dynamics to several of the profilers and the data suggests that the performance of the accelerometers used by two of the vendors were adversely affected by the dynamics.

With recognition of the poor reproducibility observed, precision statements were developed in accordance with ASTM standards for IRI under five cases and PI under three cases in an effort to establish overall estimates of repeatability and reproducibility 
for the lightweight profilers. The data generated in Indiana were used in the first three cases that considered both IRI and PI with 0.0 and 0.2 inch blanking bands. In the first case all of the data generated were analyzed. In the second case all of the data with the exception of the data obtained with boards placed in the wheel paths were analyzed. These efforts revealed good repeatability and poor reproducibility as expected. They also revealed the fact that one of the profilers exhibited a tendency to report smoothness values of much larger or small magnitude than the other vendors for both IRI and PI at several sites. Thus for the third case the data associated with this profiler were excluded. As expected, only a nominal improvement in repeatability was observed, but a significant improvement in reproducibility was observed for both IRI and PI.

The data generated in a recent Connecticut DOT evaluation of the same lightweight profilers was obtained and a precision statement was generated with it for IRI. This data resulted in similar estimates of repeatability and reproducibility to those observed with the Indiana generated data. In order to improve the power of the estimates of precision for IRI, the Indiana and Connecticut data were pooled and a fifth precision statement was developed. This effort resulted in precision estimates similar to those observed in Cases 2 through 4.

Unfortunately, the precision analysis revealed that the lightweight profiler technology needs to be further refined in order to develop smoothness specifications which rely upon their use, at least those evaluated in Indiana and Connecticut. The profilers evaluated are actually the only devices commercially available at this point in 
time. The reason refinements are needed is that both the repeatability in most cases and reproducibility limits in all cases observed for IRI and PI both exceed the increments in smoothness used in most specifications today. For example, the states using IRI as the measure of smoothness are typically using increments of 5 to $10 \mathrm{in} / \mathrm{mi}$ for pay factor adjustments (eg. $98 \%$ payment is made for IRI between 80 and $90 \mathrm{in} / \mathrm{mi}, 100 \%$ payment is made for IRI between 70 and $80 \mathrm{in} / \mathrm{mi}, 101 \%$ payment is made for IRI between 65 and 70in/mi, etc.). The observed reproducibility limit for IRI with the Indiana and Connecticut data pooled was $31.8 \mathrm{in} / \mathrm{mi}$. This means that based on the performance of the profilers evaluated, the difference in IRI reported by two different vendors at a single site could be up to $31.8 \mathrm{in} / \mathrm{mi}$ simply due to the inherent variability of the test. This value $(31.8 \mathrm{in} / \mathrm{mi})$ is obviously much larger than the 5 to $10 \mathrm{in} / \mathrm{mi}$ increments currently in specifications. The observed repeatability limit for the pooled data was $10.7 \mathrm{in} / \mathrm{mi}$. This suggests that if the same device and operator perform replicate measurements at the same site the difference in IRI reported for the two measurements could be up to $10.7 \mathrm{in} / \mathrm{mi}$ simply due to the variability of the test. This difference in itself is greater than the limits used in specifications for payment. Similar observations were also made when PI was considered.

What the precision statements as well as the previously mentioned statistical analysis of means actually indicate is that the incremental pay adjustment steps used in specifications could very likely lead to situations where one lightweight would indicate that a significant penalty should be imposed while another would indicate that a significant bonus should be paid. The data also suggest that the variability observed for 
the profilers evaluated could very easily lead to disputes in QC/QA situation when different brands of profilers were used by different parties. The disputes could also occur if the same brand of profiler were used by both parties. This means the disputes between the state and the contractor, as well as the contractor and his/her subcontractor could easily develop which none of the potentially involved parties like to see occur.

Even though the statistical analysis and precision statement tasks revealed unacceptable inconsistency among the lightweight profilers a smoothness specification was drafted which would rely on the used of the lightweight profilers. The literature as well as several state DOT smoothness specifications were reviewed in this process. These information revealed an increasing trend in the use of smoothness specifications, an increasing trend in the use of IRI rather than PI as the index of smoothness, and an increasing trend in the use of ASTM Class 1 inertial profilers (both lightweight and high speed) for smoothness measurement. A review of the literature relative to critical specification aspects including smoothness index, test wheel path, number of replicate measures required, and pay adjustment factors was also performed.

The developed draft relies on IRI as the measure of smoothness for HMA pavements. For PCC pavements PI with a 0.0 inch blanking band is employed. It also incorporates pay adjustment factors for both pavement types. The data generated as part of the lightweight profiler evaluation was substituted into several state smoothness specifications and reviewed in light of inspections of the field sites tests. Based on this pay adjustment factors were proposed for the Indiana specification. The proposed 
specification (pay adjustment factors) was then applied to the field test data generated in Indiana. The payment based on this process appeared to be reasonable for the sites considered. It is important to note however that the sites evaluated were either very smooth or very rough. Therefore the proposed draft specification should be viewed with caution.

\subsection{Recommendations}

Based on the data generated through the field evaluation and analyses conducted of it, the following recommendations are made:

1. INDOT should retain the use of its current smoothness specification until further refinements are made to the currently available lightweight profilers to improve reproducibility;

2. INDOT should seriously consider changing the current smoothness specification measurement from PI with a 0.2 inch blanking band to PI with a 0.0 inch blanking band;

3. The results of concurrent FHWA sponsored field evaluations of lightweight profilers in other states should be obtained and closely reviewed when they are published to determine whether similar lightweight profiler performance is exhibited in them;

4. The FHWA summary report based on the concurrent studies should be obtained and closely reviewed once published;

5. The FHWA Smoothness Expert Task Group has been given the task of developing an AASHTO Guide Specification based on the use lightweight profilers which should be closely review upon completion; 
6. When these reports and the AASHTO Guide Specification are completed the data generated in this study as well as the proposed draft specification should be thoroughly evaluated relative to them;

7. INDOT should participate in future efforts to refine the lightweight profiler technology due to the significant potential benefits the devices offer;

8. When the lightweight profiler technology is refined to improve reproducibility INDOT should re-evaluate the devices and refine the proposed draft specification based on this evaluation and implement it on a trial basis in the first possible construction season;

9. Prior to implementing the use of lightweight profilers in Indiana a standard INDOT test method should be developed;

10. Prior to implementing the use of lightweight profilers in Indiana a procedure for certifying the devices, similar to the one currently used for California Profilograph certification should be developed; and

11. This report should be distributed to INDOT personnel working to achieve one of INDOTs Strategic Plan objectives of improving pavement smoothness.

\subsection{Implementation}

The findings of this research should be implemented as soon as practically possible. The implementation would require that an implementation study be initiated in which personnel are assigned to monitor developments and review forthcoming state and FHWA reports on concurrent evaluations in other states. They should also obtain and 
review the forthcoming AASHTO Guide Specification in light of the evaluation and finding stated in this report.

The current INDOT smoothness specification should be modified to incorporate PI with a 0.0 inch blanking band, rather than a 0.2 inch blanking band. This should be done based on the pay factor adjustments in the proposed draft specification presented in Section 6 of this report, the other specifications reviewed in this report, and through interaction with industry in the process. The modified specification should then be evaluated on a trial basis on several projects in the coming construction season for informational purposes rather than payment. The data generated through this process should then be used to validate or further refine the proposed pay factor adjustments.

INDOT should support and cooperate in future efforts to refine the lightweight profiler technology. It should also make every effort possible to implement the use of lightweight profilers for smoothness measurements once the technology is refined. This may even dictate the investment in one or more lightweight profilers in the near future. 


\section{BIBLIOGRAPHY}




\section{BIBLIOGRAPHY}

ASTM E 691-92, "Standard Practice for Conducting an Interlaboratory Study to Determine the Precision of a Test Method", Annual Book of ASTM Standards, Vol. 14.02 .

ASTM C 670-91a, " Standard Practice for Preparing Precision and Bias Statements for Test Methods to Construction Materials", Annual Book of ASTM Standards, Vol. 04.02.

ASTM C 802-96, "Standard Practice for Conducting an Interlaboratory Test Program to Determine the Precision of Test Methods for Construction Materials," Annual Book of ASTM Standards, Vol. 04.02.

ASTM C 1067-87 (Reapproved 1993), "Standard Practice for Conducting a Ruggedness or Screening Program for Test Methods for Construction Materials", Annual Book of ASTM Standards, Vol. 04.02.

ASTM E 177-90a, "Standard Practice for Use of the Terms Precision and Bias in ASTM Test Methods", Annual Book of ASTM Standards, Vol. 14.02.

ASTM E 950-94, "Standard Test Method for Measuring the Longitudinal Profile of Traveled Surfaces with an Accelerometer Established Inertial Profiling Reference", Annual Book of ASTM Standards, Vol. 04.03, 1998, pp. 726-730.

Neter, J., Kutner, M.H., Nachtsheim, C.J., Wasserman, W., “Applied Linear Statistical Models," $4^{\text {th }}$ edition, 1996.

Federal Highway Administration, NCHRP Report no. 10-47, “Guidelines for Longitudinal Pavement Profile Measurement”, FHWA Draft Report, 1999.

Smith K.L, Smith K.D, Evans L.D., Hoerner T.E, Darter M.I and Woodstrom J.H, "Smoothness Specifications for Pavements", Final Report prepared for NCHRP, Transportation Research Board and National Research Council, March 1997. 
Hajek J.J, Kazmierowski T.J and Musgrove G., "A Switch to the International Roughness Index", Proceedings, $77^{\text {th }}$ Annual Meeting, Transportation Research Board, January 1998, Washington D.C, January 1998.

Ksaibati Khaled, Mcnamara Ronald, Miley William and Armaghani Jamshid, "Evaluating the Ride Number as Pavement Management Roughness Index," $78^{\text {th }}$ Annual Meeting, Transportation Research Board, Washington D.C, January 1999.

Ksaibati Khaled, Kercher Keith, Gulen Sedat, White Thomas D., Hooker Gordon, Law Jeff, "Evaluation of the ProRut System in Indiana", Publication no: FHWA-TS-89-030, September 1989.

Mcghee Kevin K., "Measuring, Achieving, and Promoting Smoothness of Virginia's Asphalt Overlays", Final Report, Virginia Transportation Research Council 99-R19, Charlottesville, VA, April 1999.

Mcghee Kevin K. and Hughes Charles S., "Development of a Pavement Smoothness Specification with a Variability Component", Virginia Transportation Research Council, Charlottesville, VA, 1999.

Ksaibati Khaled, Miley William, Mcnamara Ronald and Armaghani Jamshid, "The Development of Florida Smoothness Specifications for Flexible Pavements", Paper prepared for Annual Meeting of Transportation Research Board, August 1998.

Fernando Emmanuel G., Leong Sut Ian Carolyn, "Profile Equipment Evaluation" Texas Transportation Institute, Texas A\&M University System, College Station, Texas, December 1997.

Russel Jeffrey S., Hanna Awad S., Bahia Hussain U., Robert L., Jung Galadriel S. Jung , "Summary of Current QC/QA Practices for Hot-Mix Asphalt Construction", $77^{\text {th }}$ Annual Meeting, Transportation Research Board, Washington D.C, January 1998.

Kulakowski, B.T., " Development of Procedures for the Calibration of Profilographs," FHWA Final Report, February 1989. 
Janoff, M.S., " The Relationship between Initial Pavement Smoothness and Long-Term Pavement Performance", TRB Committee A2B07, Washington, DC. January, 1990.

Woodstrom, J.H., “ Measurements, Specifications, and Achievement of Smoothness for Pavement Construction”, NCHRP Report 167, 1990.

Budwig, J.L. "Bituminous Pavement Smoothness : Statistically Based Approach to Acceptance", Transportation Research Record 1544, TRB, National Research Council, Washington D.C., 1996, pp. 125-144.

Sayers, M.W., " On the Calculation of IRI from Longitudinal Road Profile", TRB Paper No. 95-0842, Presented at the Transportation Research Board, $74^{\text {th }}$ Annual Meeting, 1995.

Gillespie, T.D., " Everything You Always Wanted to Know About the IRI, But Were Afraid to Ask! ” Presented to the Road Profile Users Group Meeting, 1992.

Paterson, W. "International Roughness Index: Relationship to the Other Measures of Roughness and Ride Quality", Transportation Research Record 1084,1986.

Sayers, M.W., " Development, Implementation, and Application of The Reference Quarter-Car Simulation”, ASTM Special Technical Publication 884, 1985.

Ksaibati, K., Staigle, R., Adkins, T., “ Pavement Construction Smoothness Specifications in the United States", Transportation Research Board, Washington, D.C, 1995.

Bohdan T. Kulakowski and James C. Wambold, "Development of Procedures for the Calibration of Profilographs." Report No. FHWA-RD-89-110, Pennsylvania Transportation Institute, August 1989.

Larry A. Scofield, Sylvester A. Kalavela, and Mary R. Anderson, " Evaluation of the California Profilograph," $71^{\text {st }}$ Annual Meeting of the Transportation Research Board, Washington D.C., January 1992. 


\section{APPENDICES}


APPENDIX A - IRI Comparison-Within Vendors 


\section{APPENDIX A - IRI Comparison-Within Vendors}

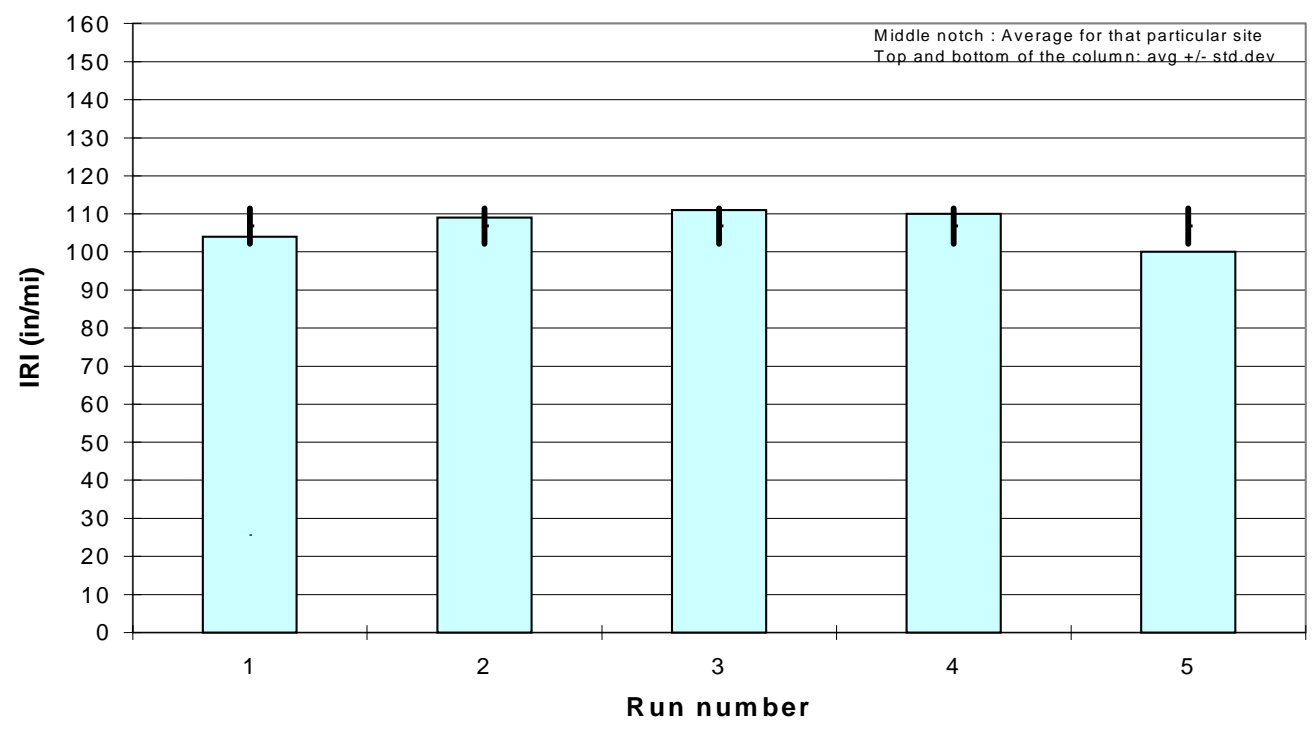

Figure A-1 IRI for replicate runs at Covington (Site 1) of Vendor A

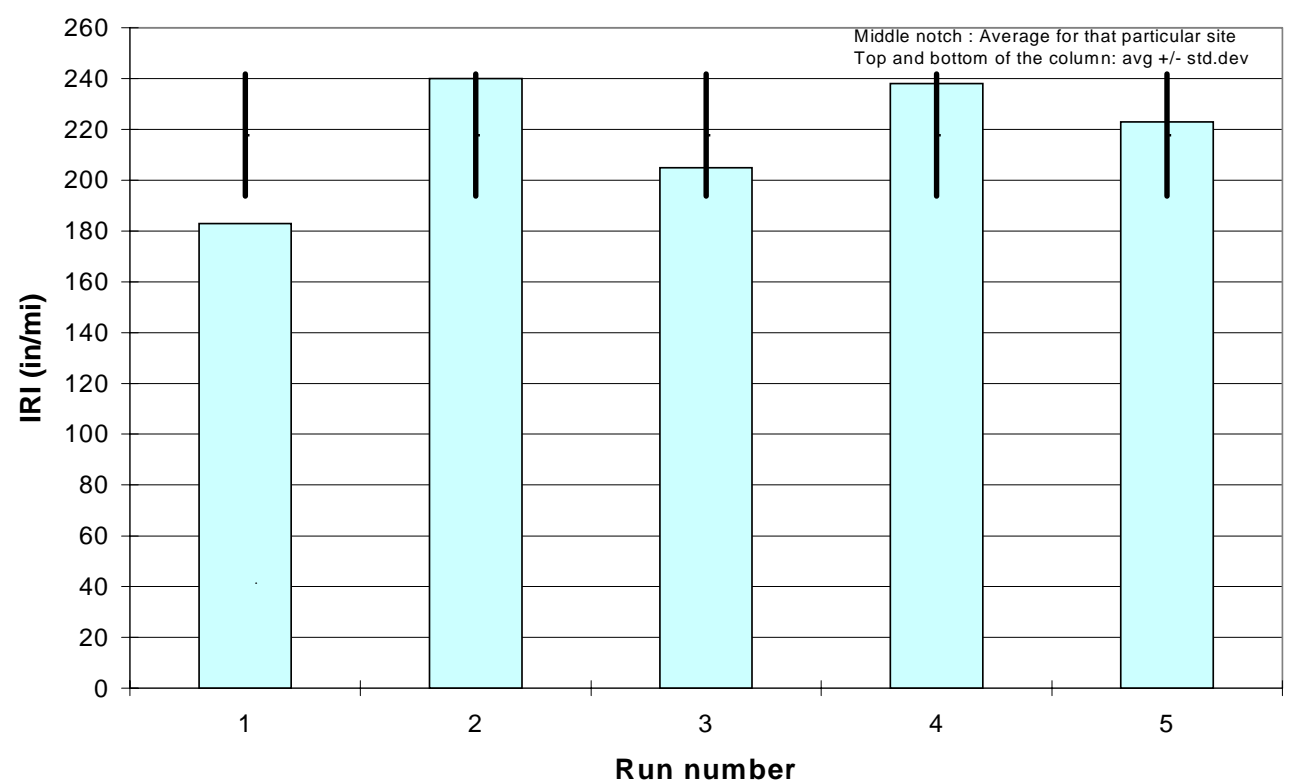

Figure A-2 IRI for replicate runs at I-65 (Site 2 with board) of Vendor A 


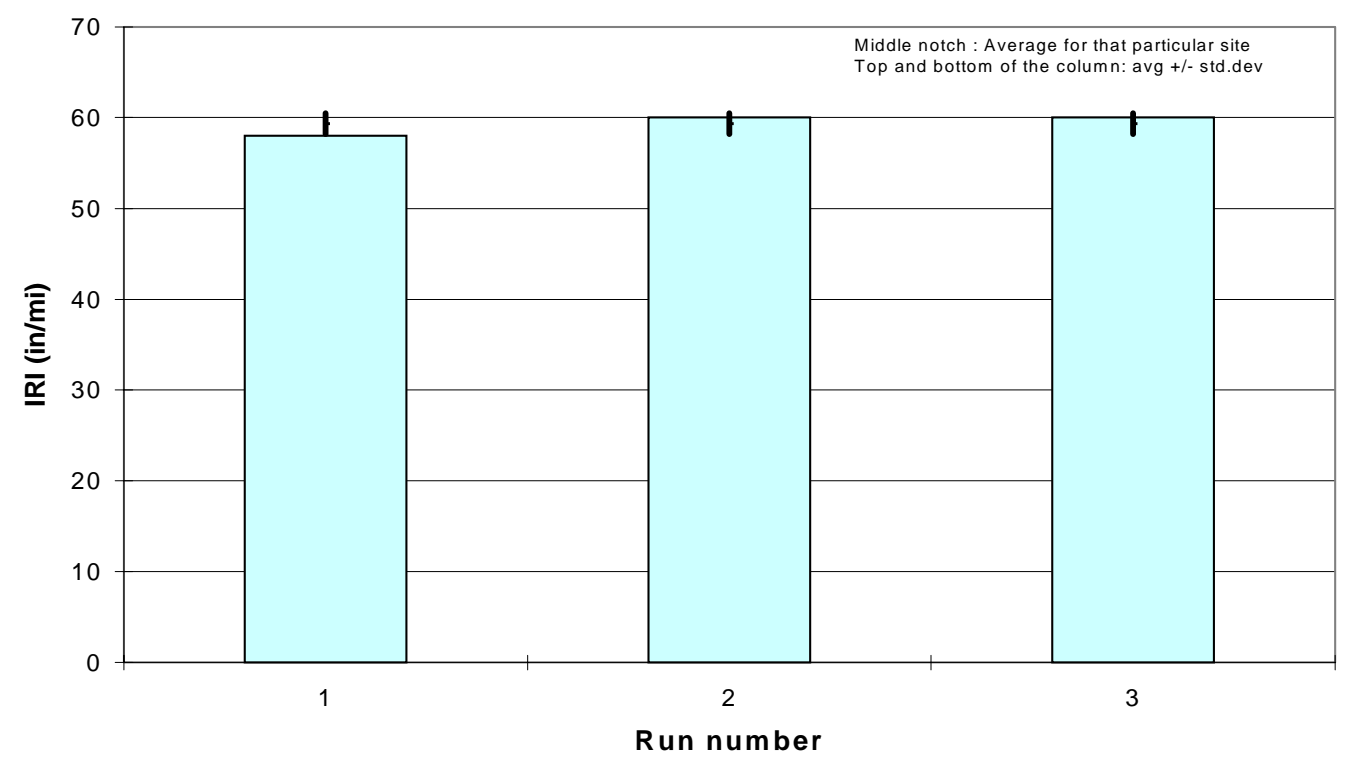

Figure A-3 IRI for replicate runs at I-65 (Site 2 without board) of Vendor A

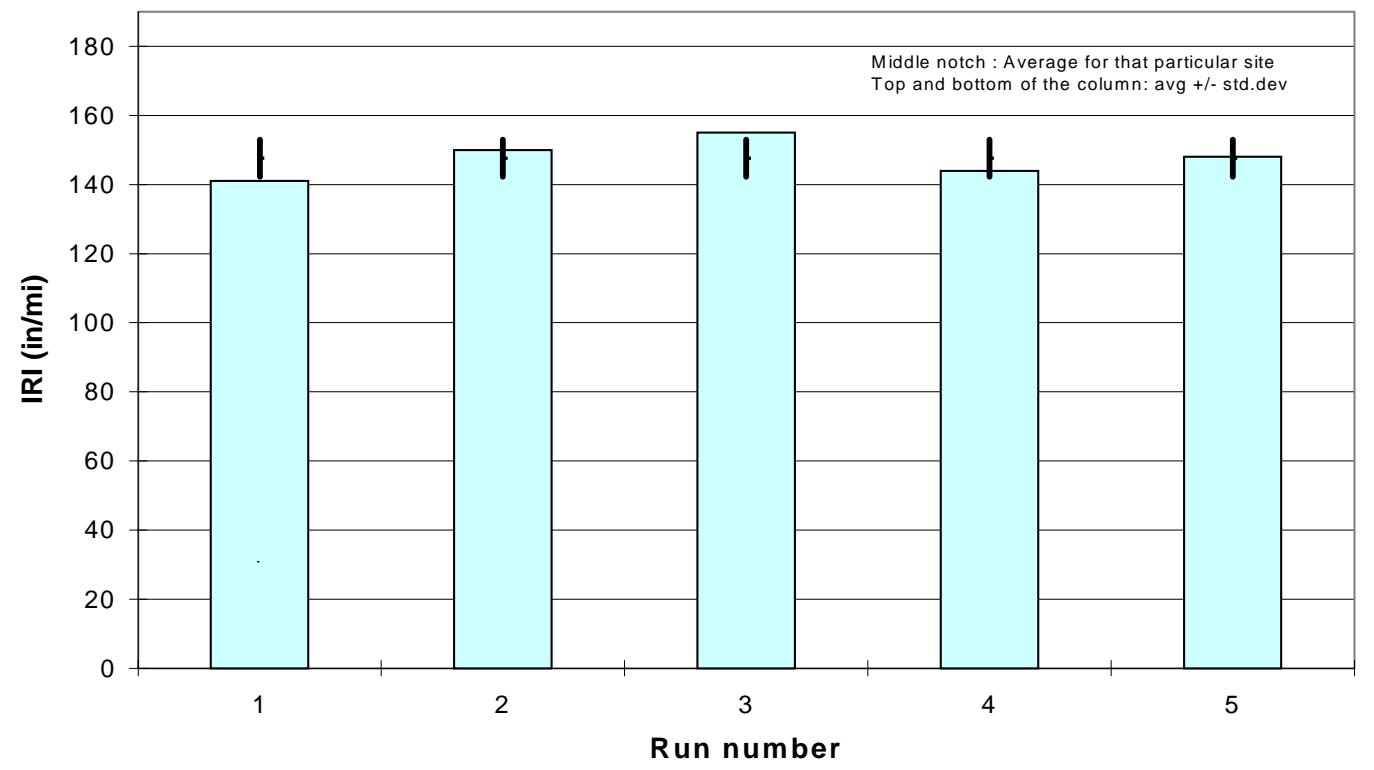

Figure A-4 IRI for replicate runs at Prairie Street (Site 3 with board) of Vendor A 


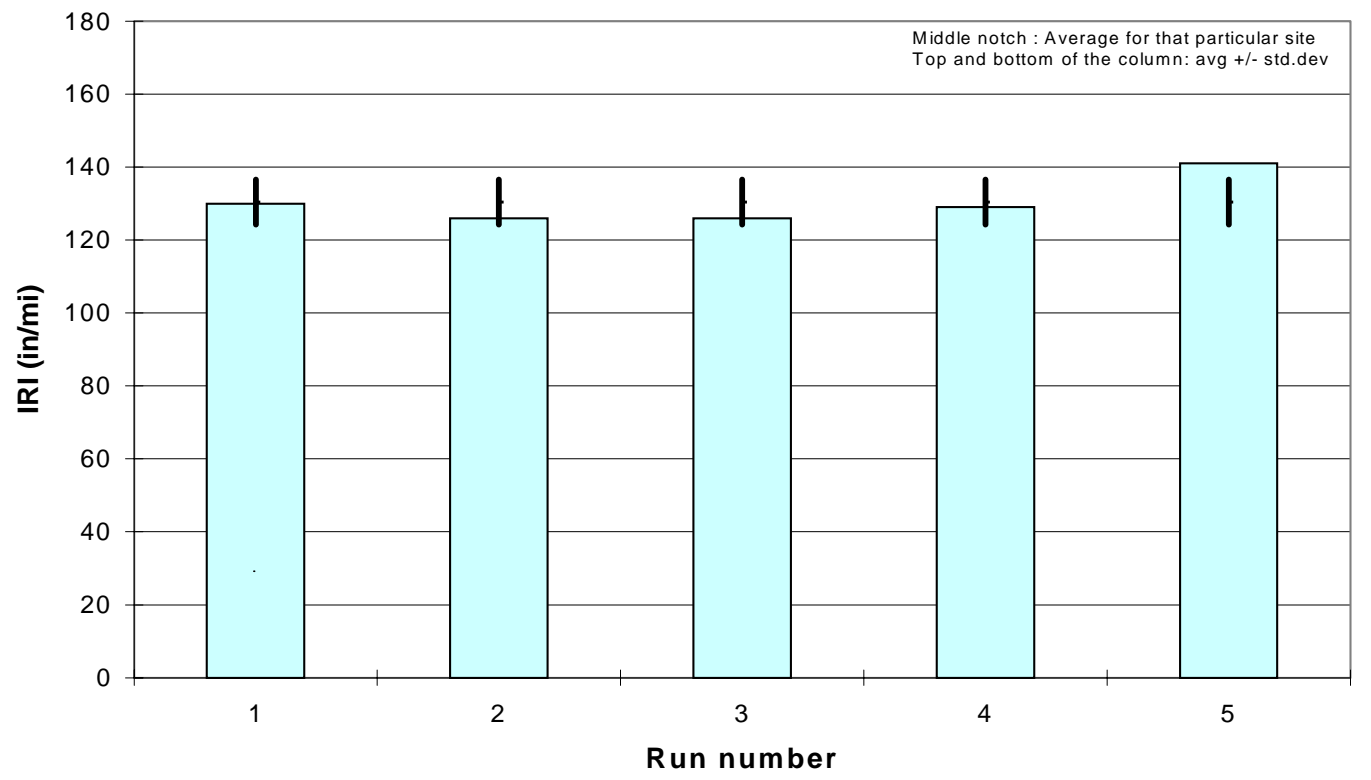

FigureA-5 IRI for replicate runs at Prairie Street (Site 3 without board) of Vendor A

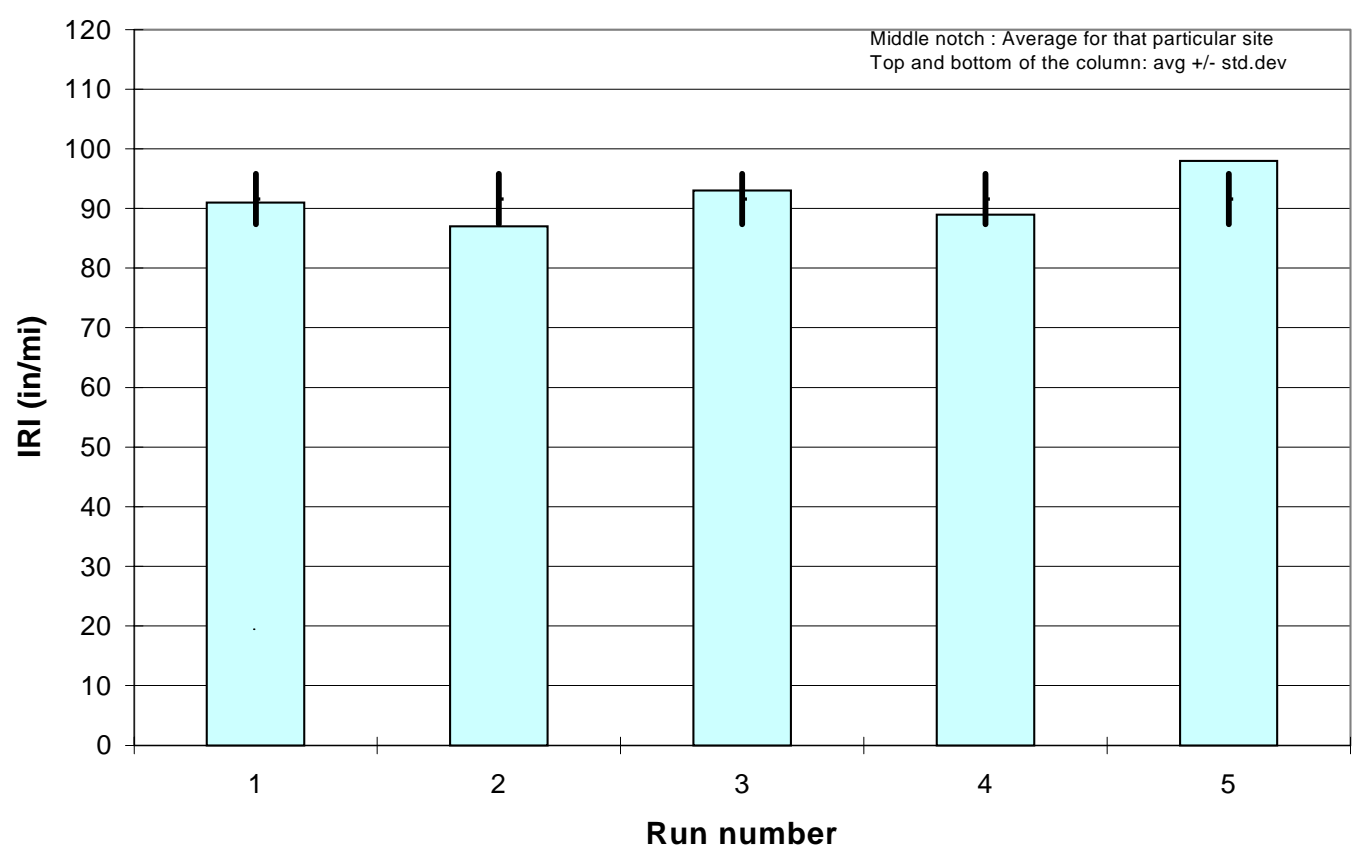

Figure A-6 IRI for replicate runs at Vinton Street (Site 4) of Vendor A 


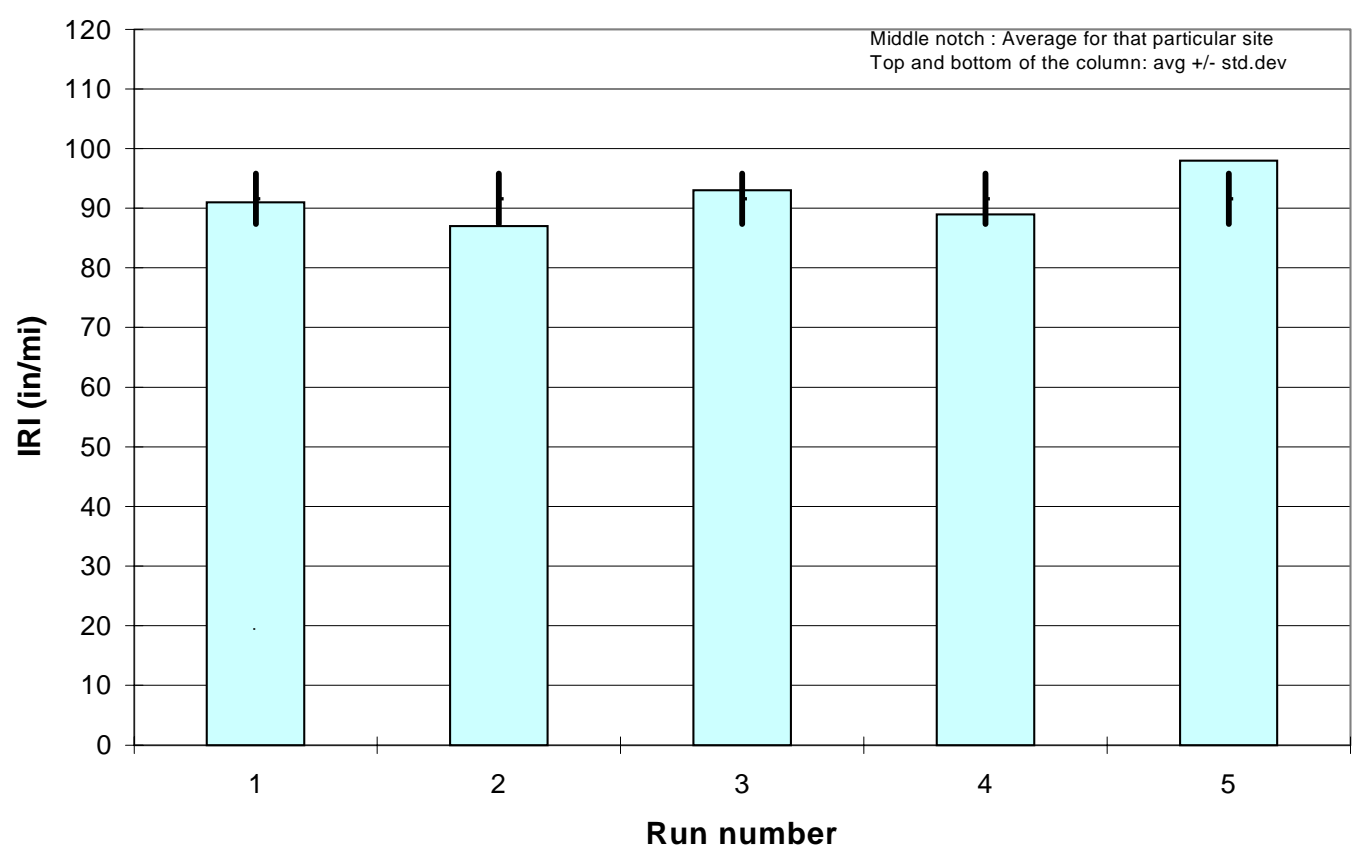

Figure A-7 IRI for replicate runs at US 231A (Site 5) of Vendor A

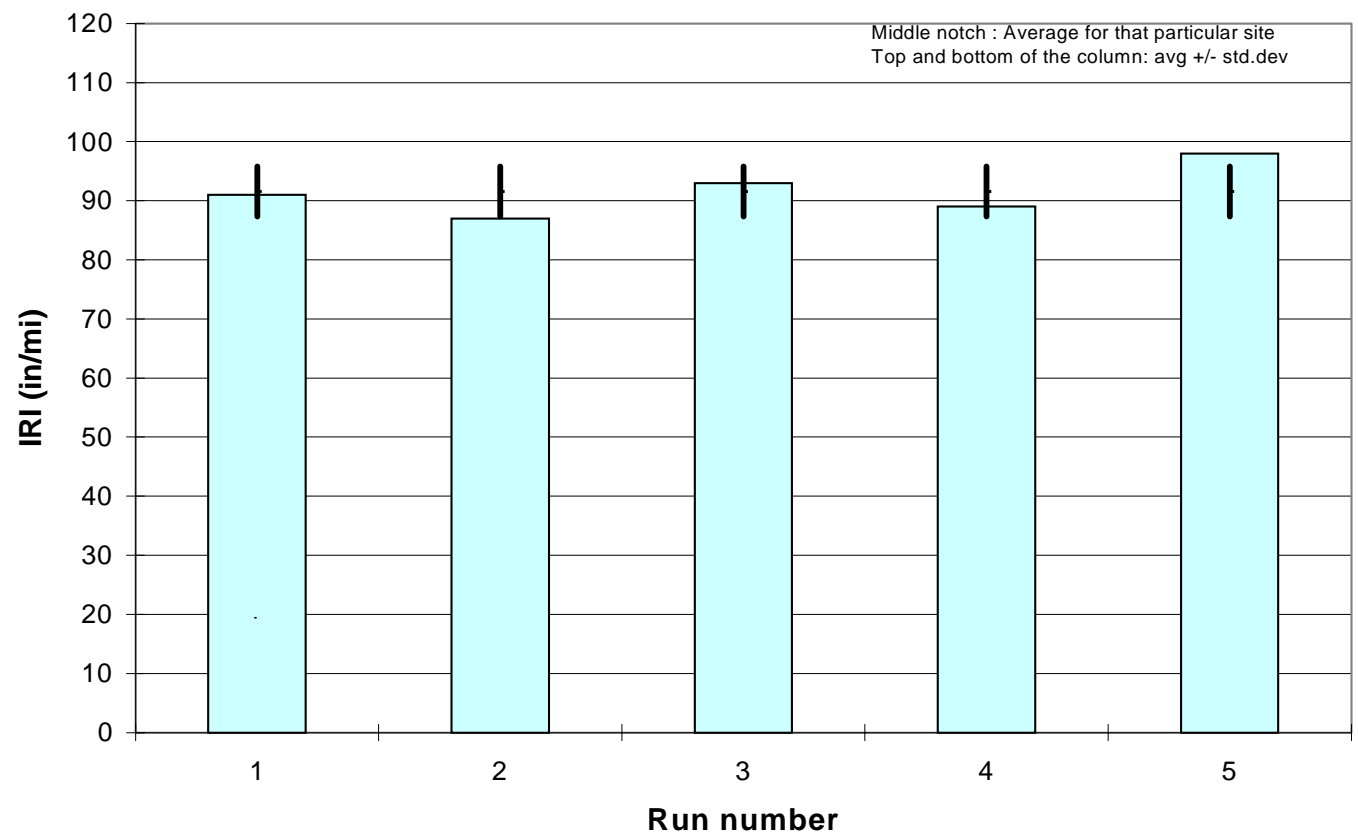

Figure A-8 IRI for replicate runs at US 231B (Site 6) of Vendor A 


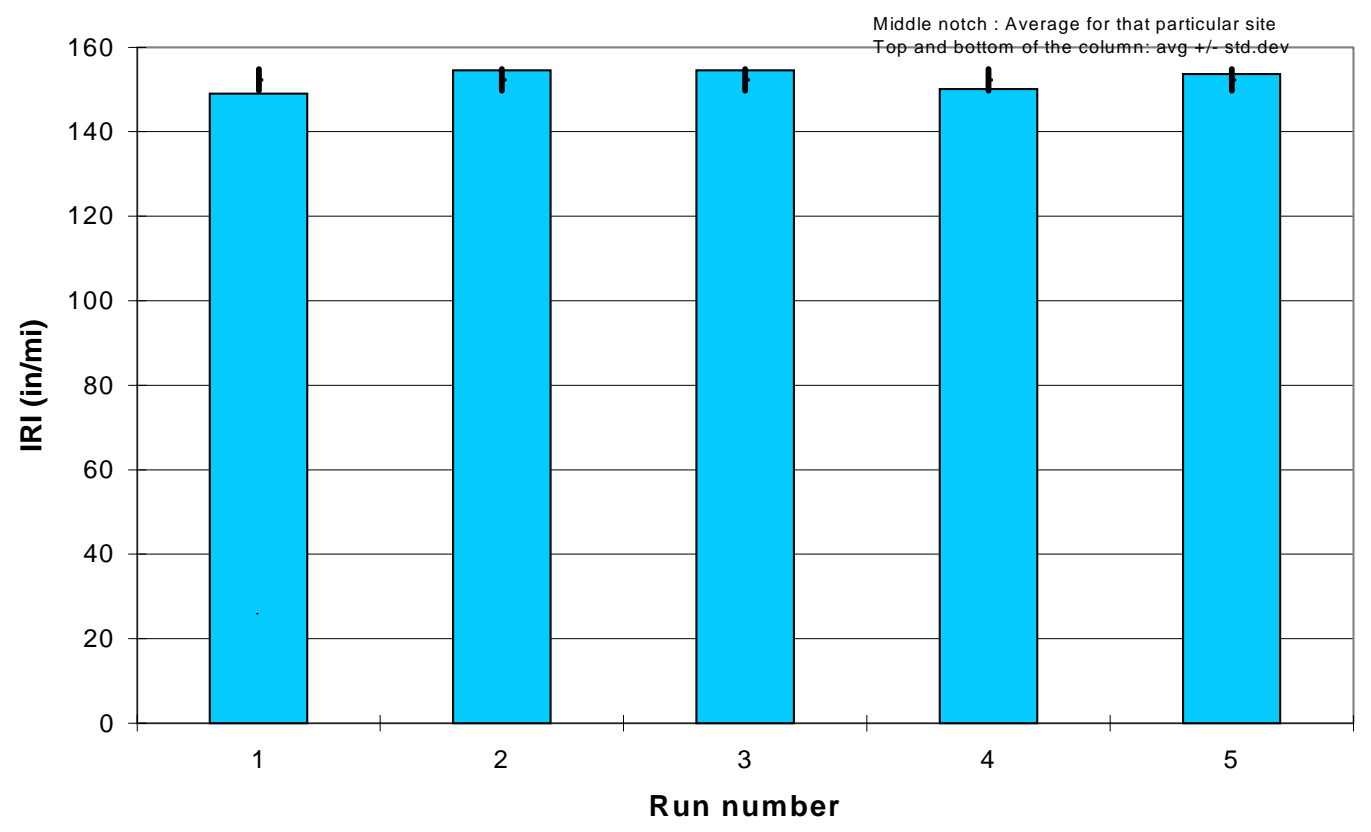

Figure A-9 IRI for replicate runs at Covington (Site 1) of Vendor B

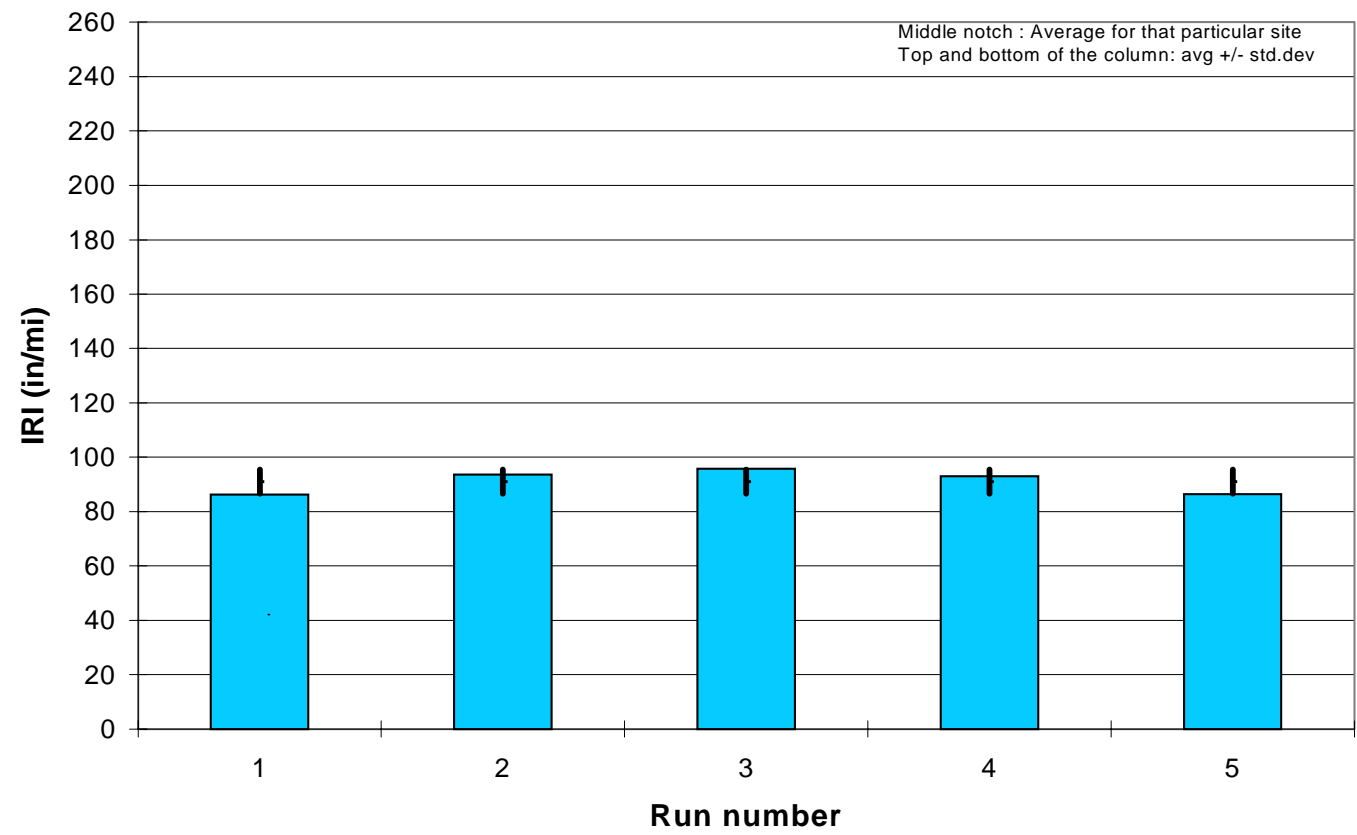

Figure A-10 IRI for replicate runs at I-65 (Site 2 with board) of Vendor B 


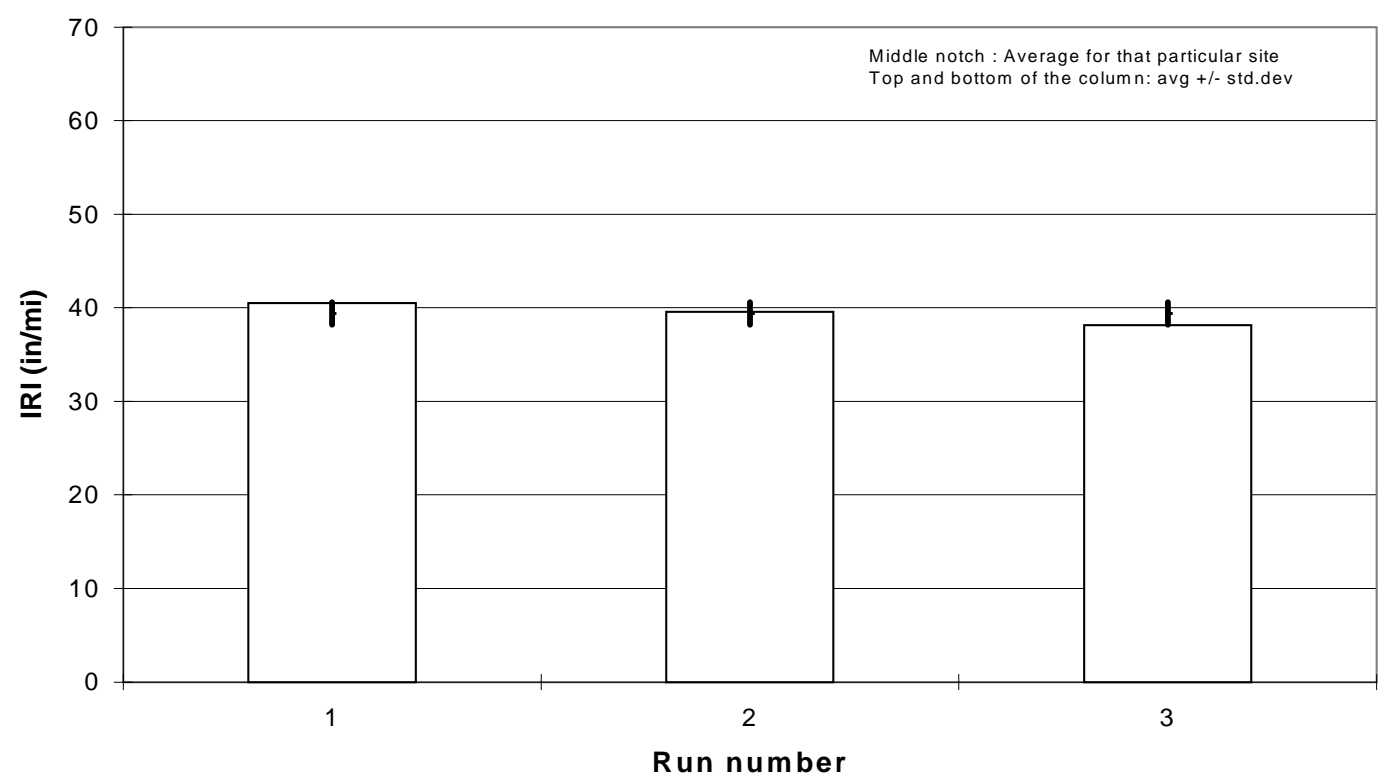

Figure A-11 IRI for replicate runs at I-65 (Site 2 without board) of Vendor B

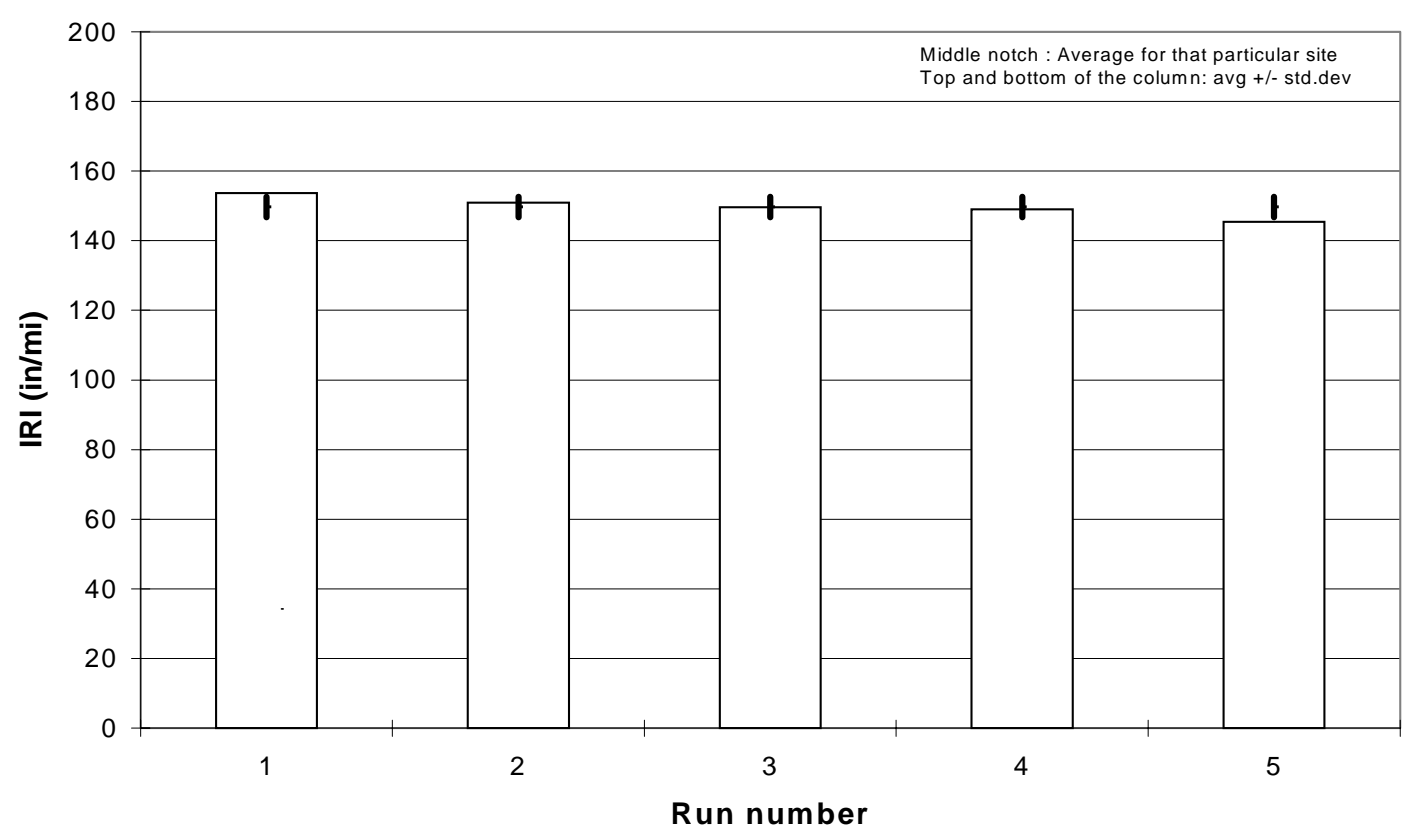

Figure A-12 IRI for replicate runs at Prairie Street (Site 3 with board) of Vendor B 


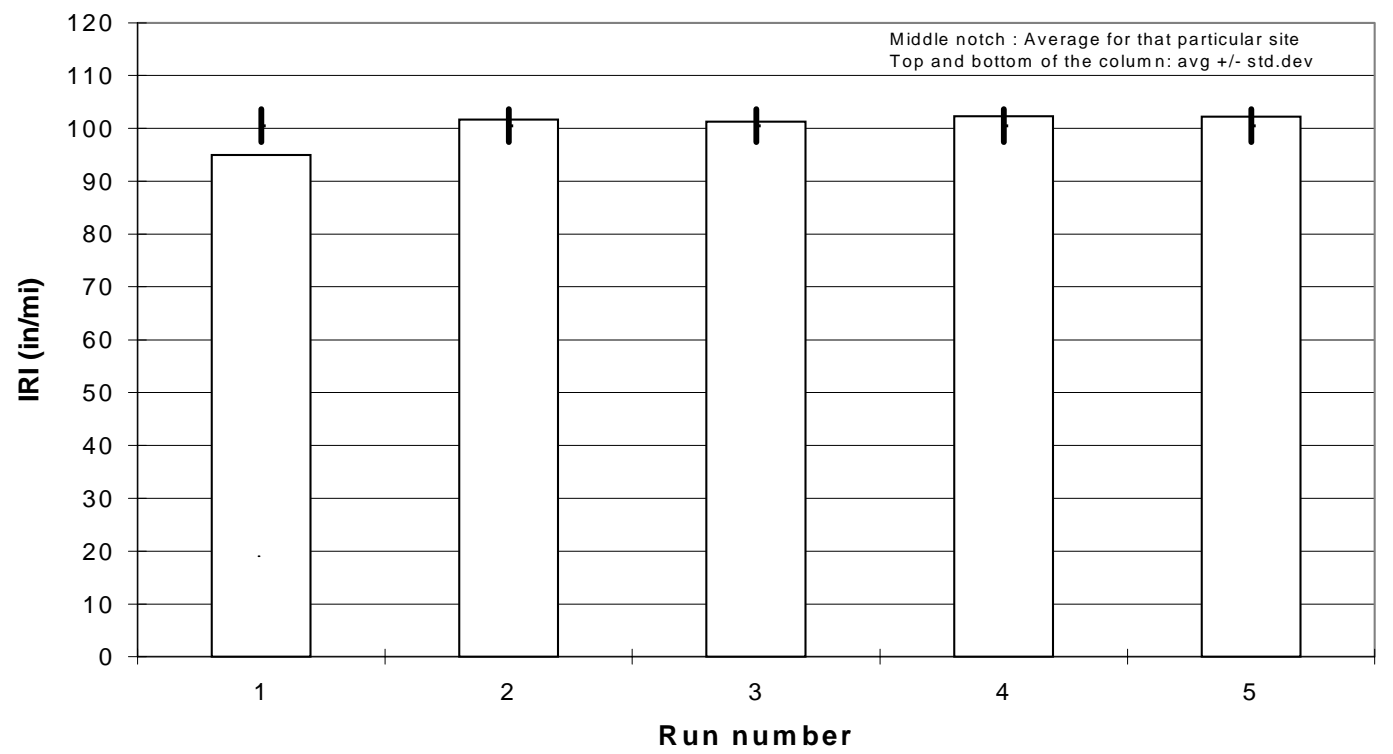

Figure A-13 IRI for replicate runs at Prairie Street (Site 3 without board) of Vendor B

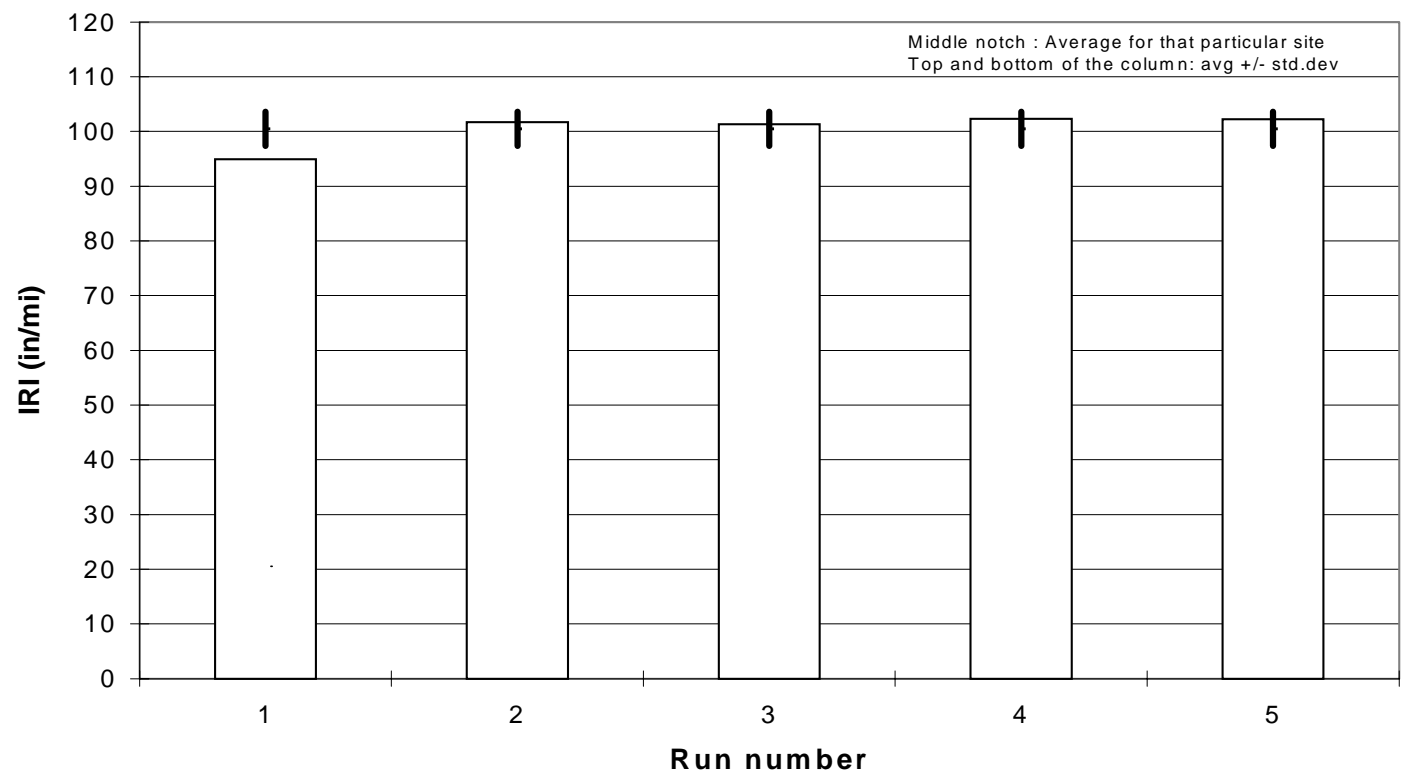

Figure A-14 IRI for replicate runs at Vinton Street (Site 4) of Vendor B 


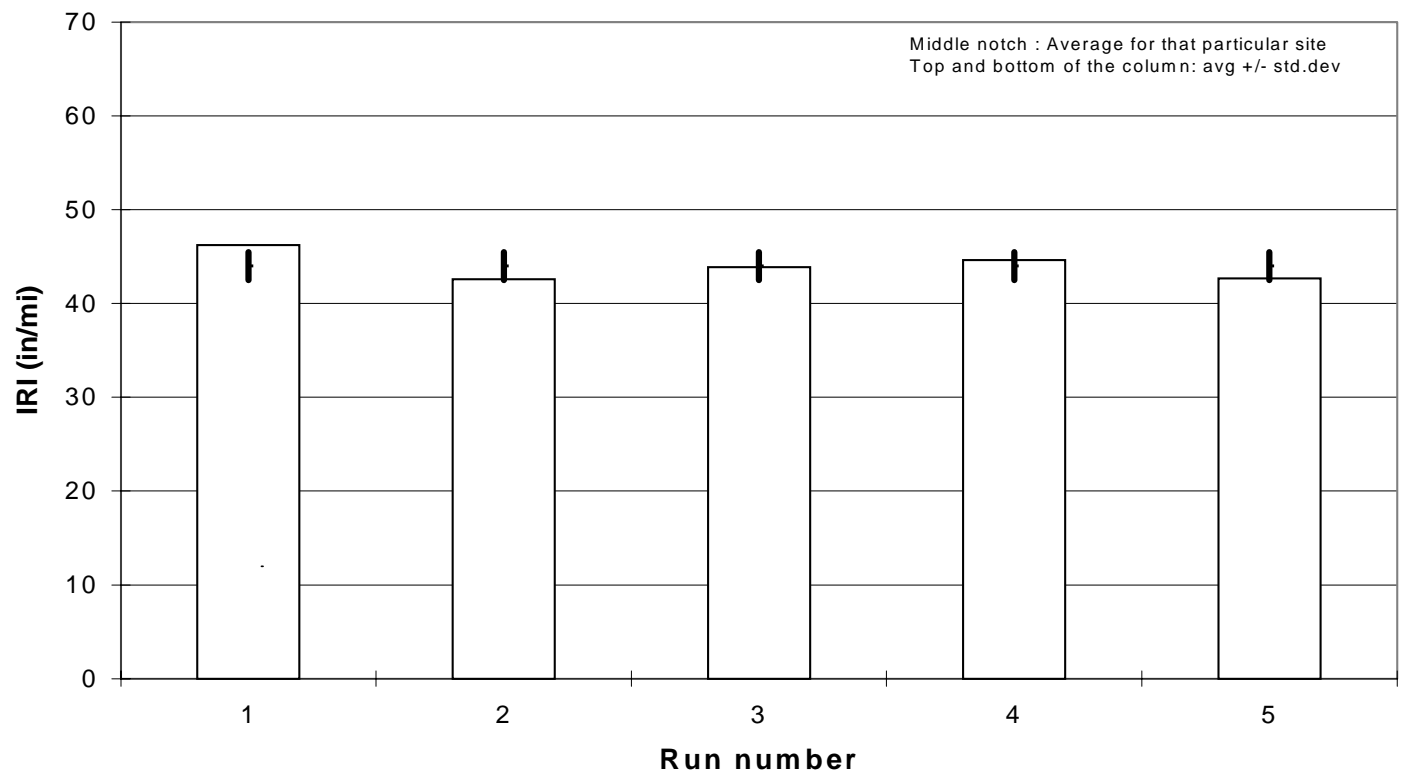

Figure A-15 IRI for replicate runs at US 231A (Site 5) of Vendor B

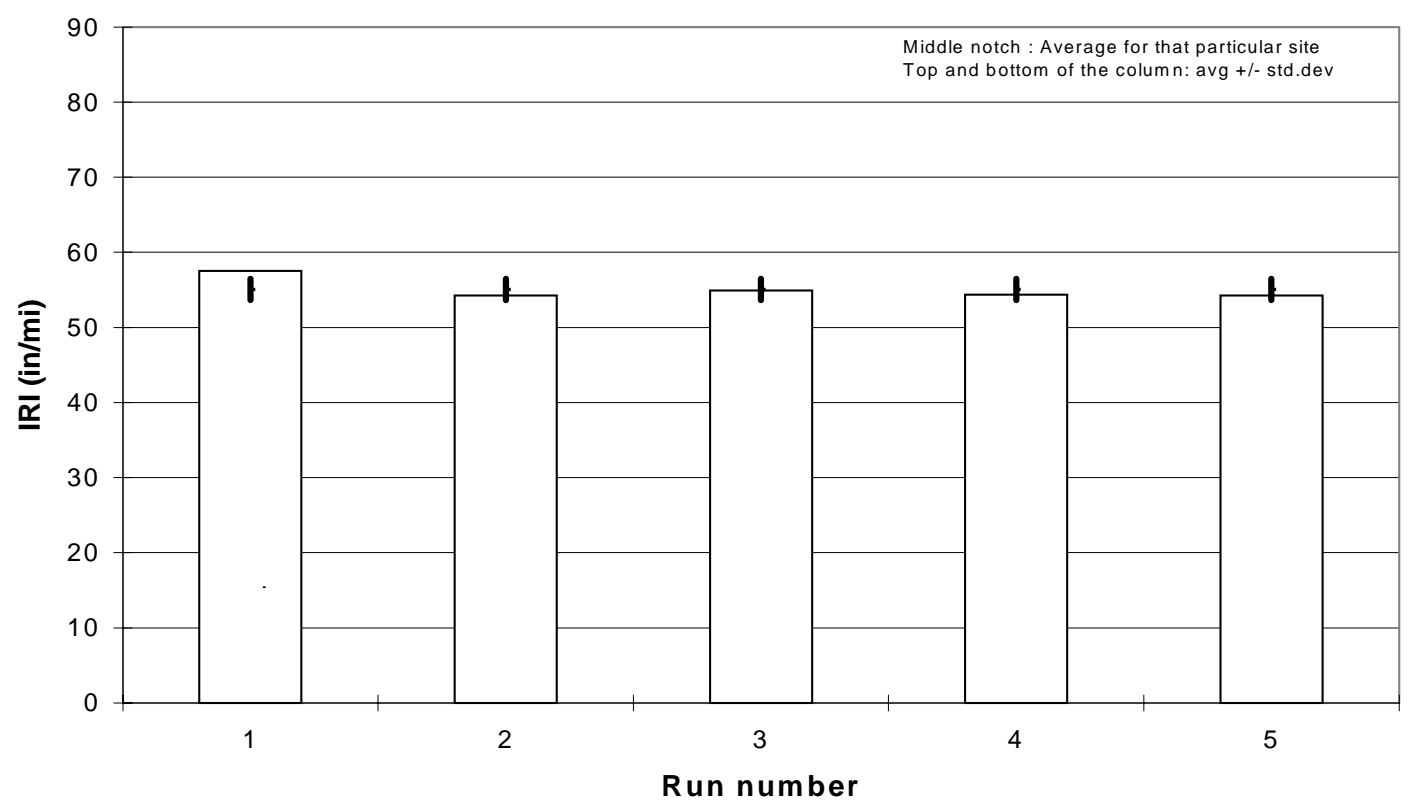

Figure A-16 IRI for replicate runs at US 231B (Site 6) of Vendor B 


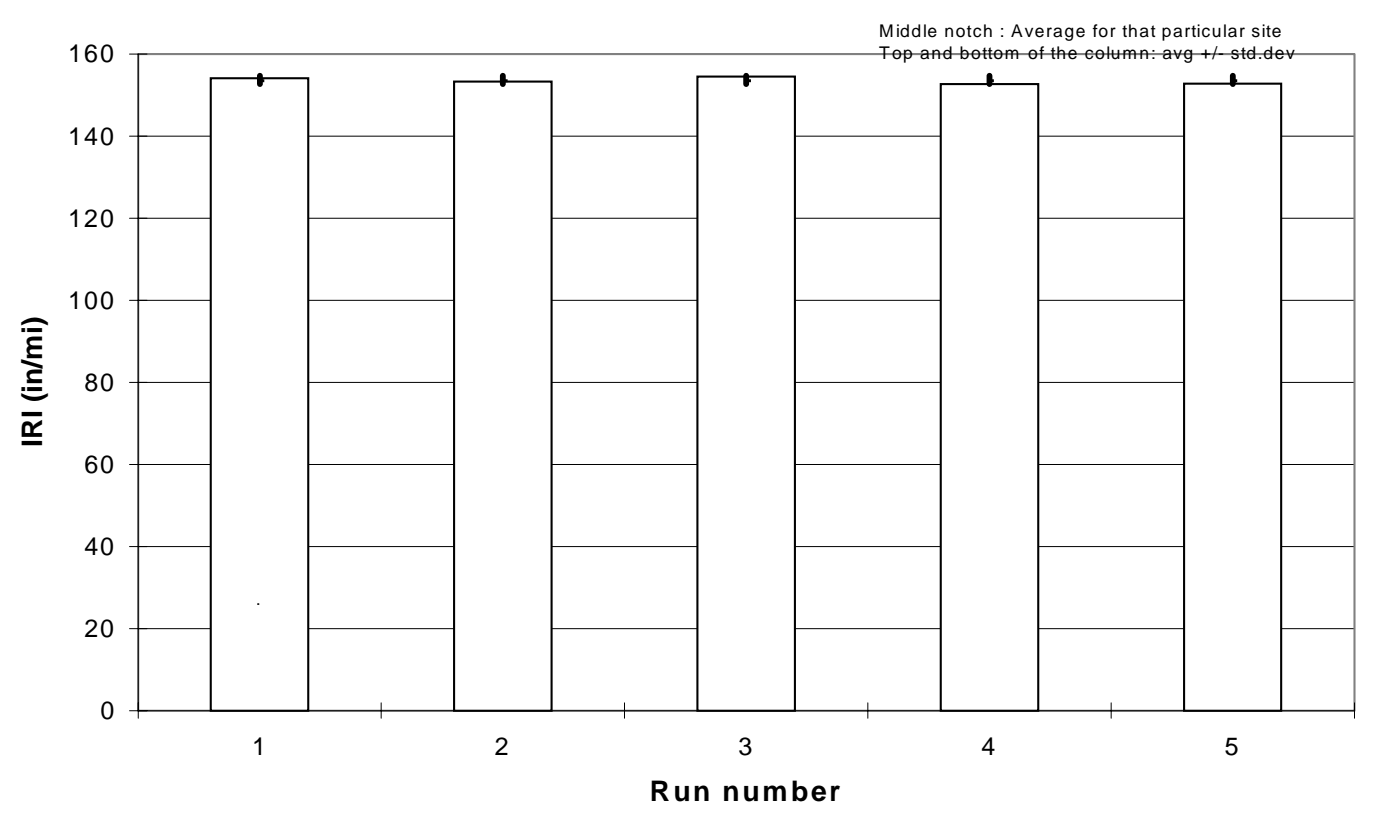

Figure A-17 IRI for replicate runs at Covington (Site 1) of Vendor C

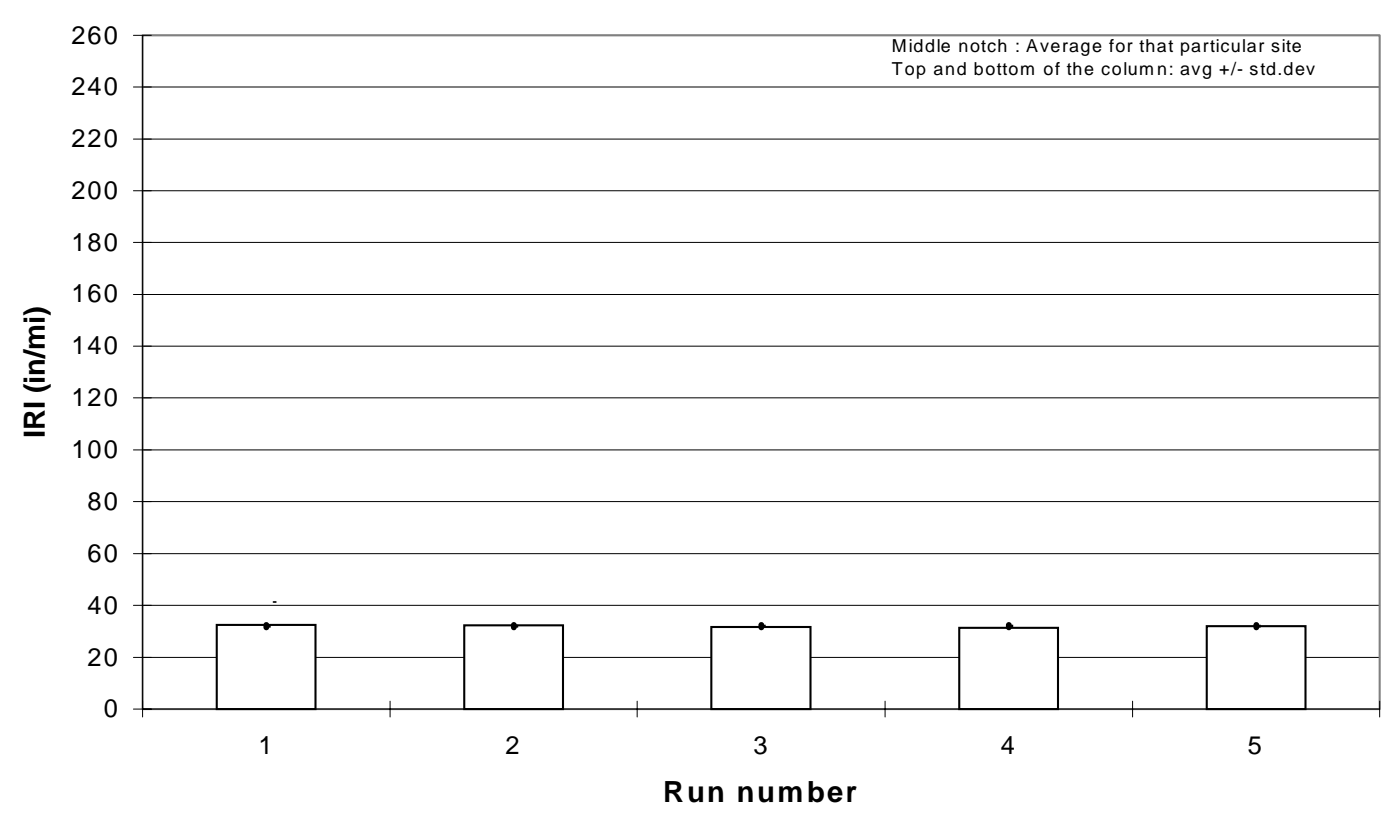

Figure A-18 IRI for replicate runs at I-65 (Site 2 with board) of Vendor C 


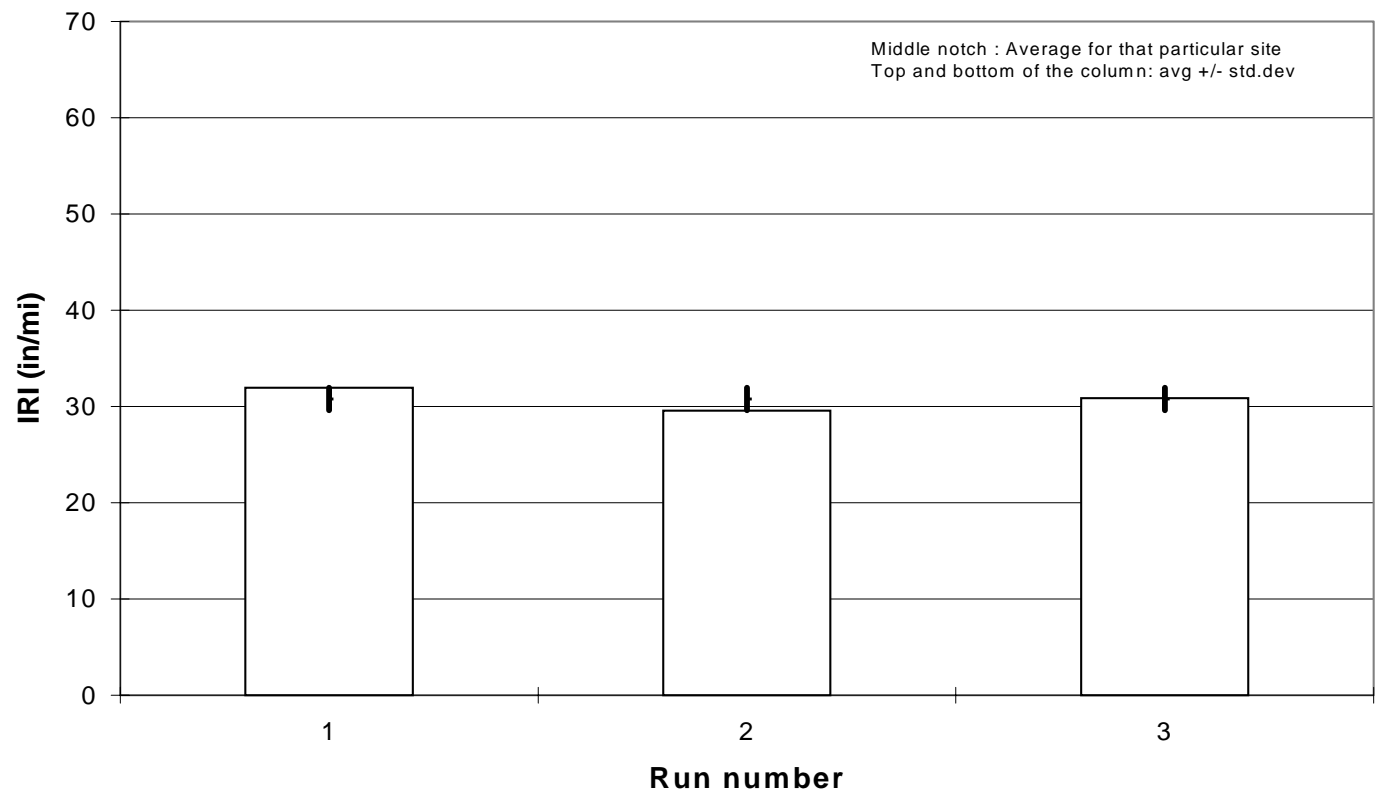

Figure A-19 IRI for replicate runs at I-65 (Site 2 without board) of Vendor C

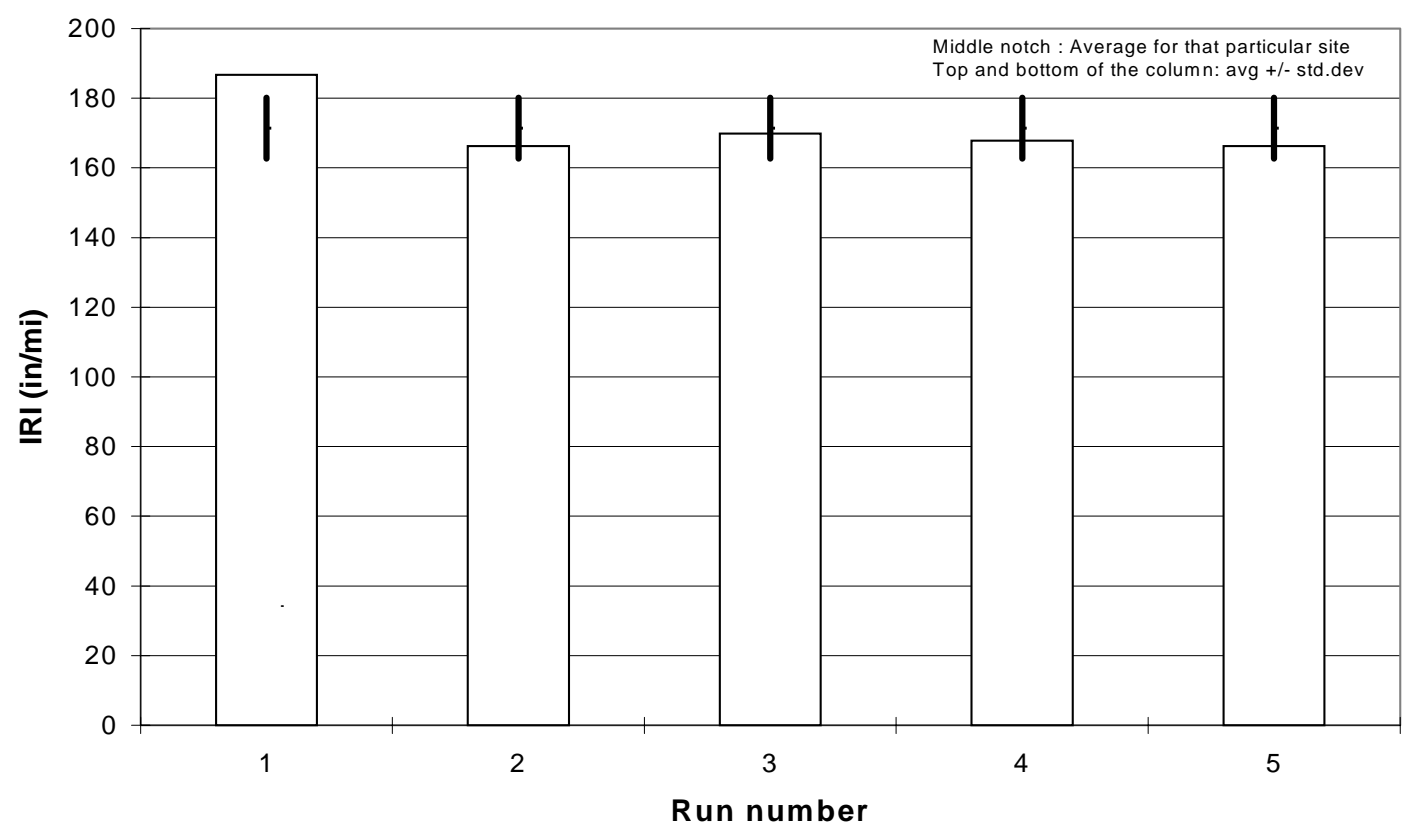

Figure A-20 IRI for replicate runs at Prairie Street (Site 3 with board) of Vendor C 


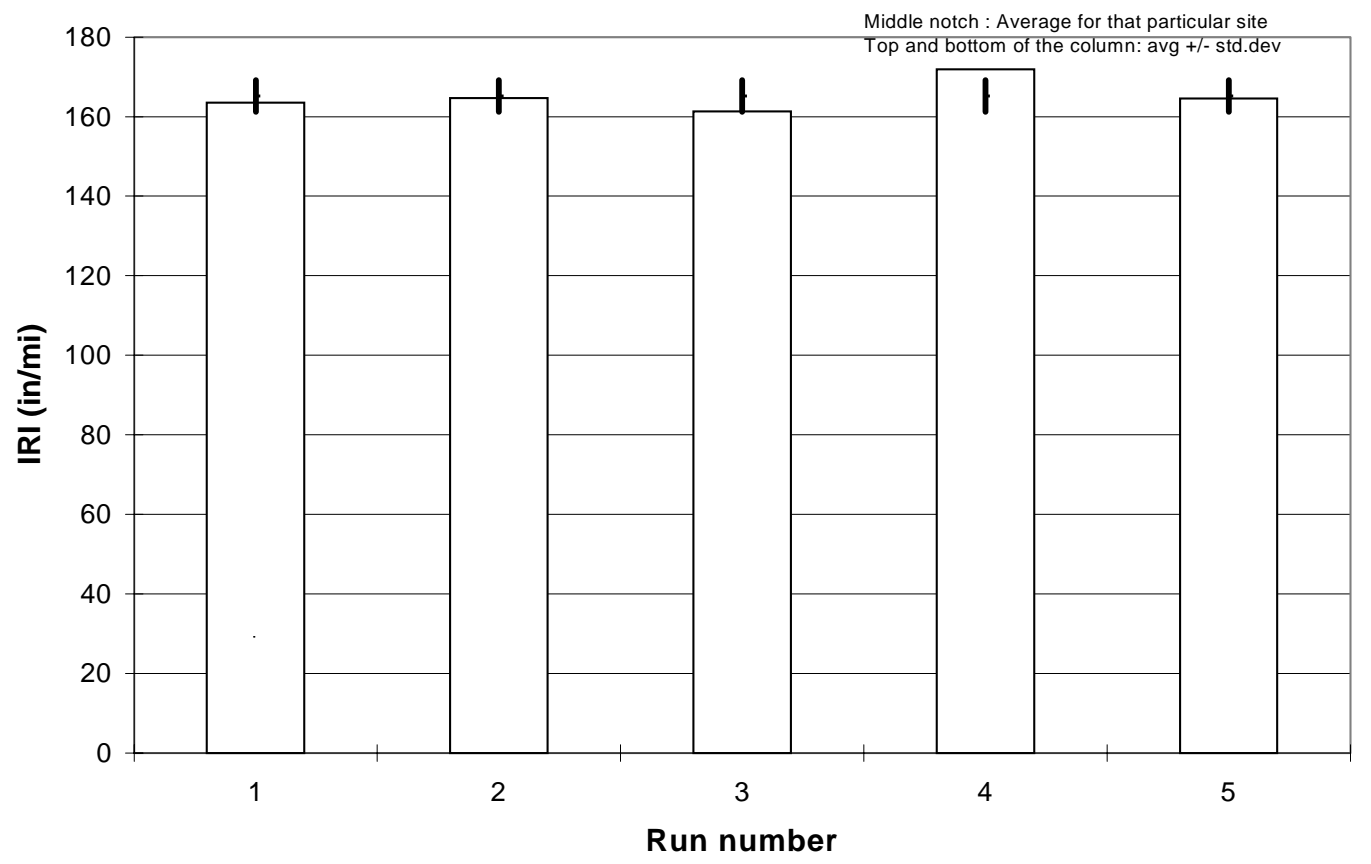

FigureA-21 IRI for replicate runs at Prairie Street (Site 3 without board) of Vendor $\mathbf{C}$

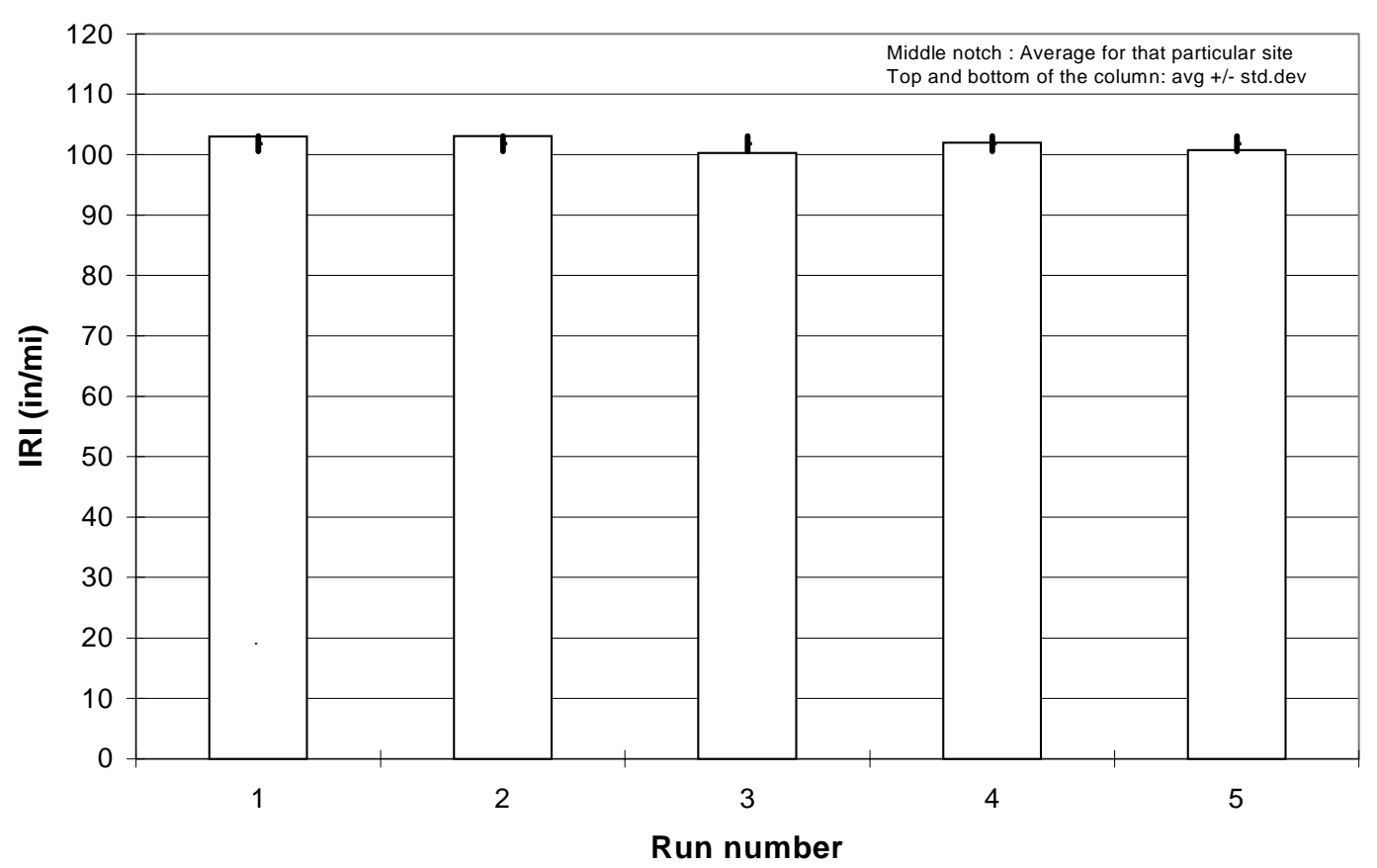

Figure A-22 IRI for replicate runs at Vinton Street (Site 4) of Vendor C 


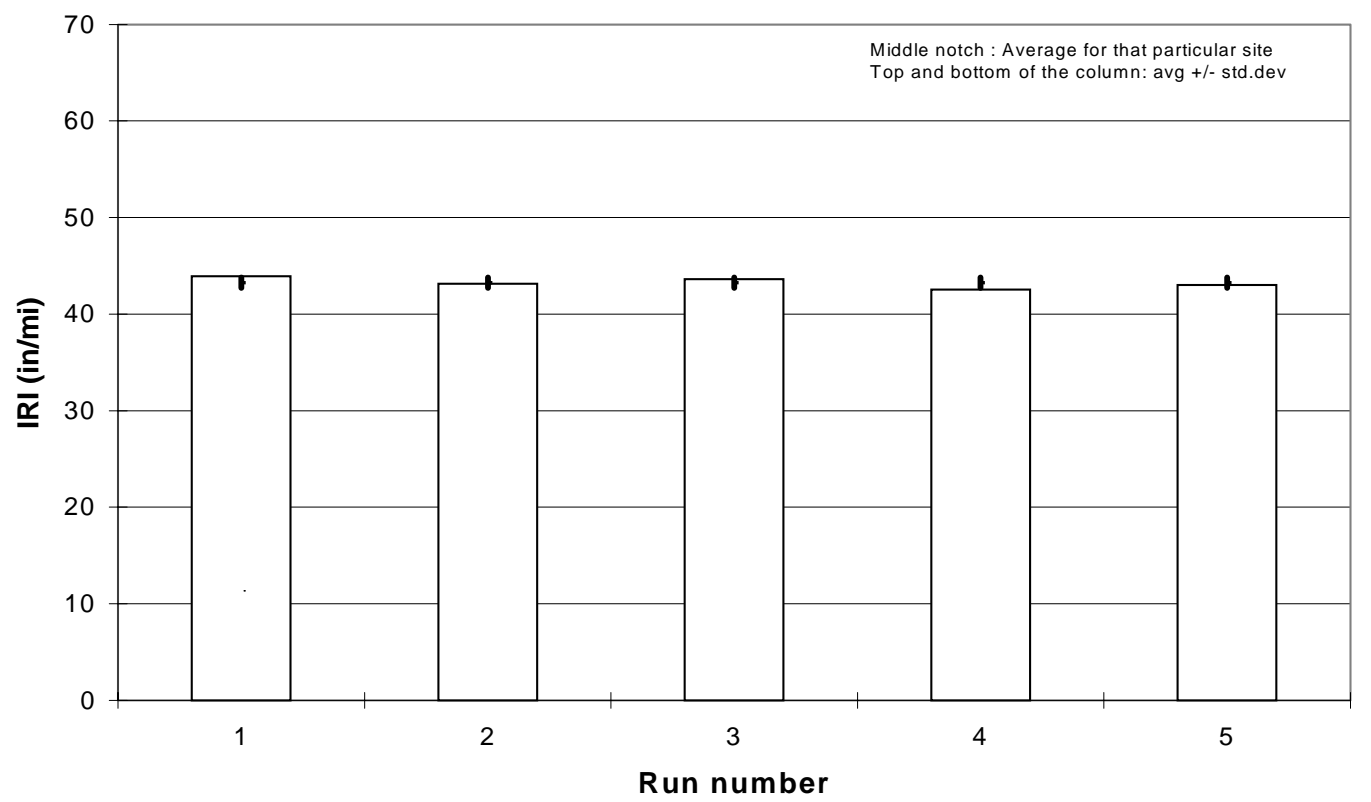

Figure A-23 IRI for replicate runs at US 231A (Site 5) of Vendor C

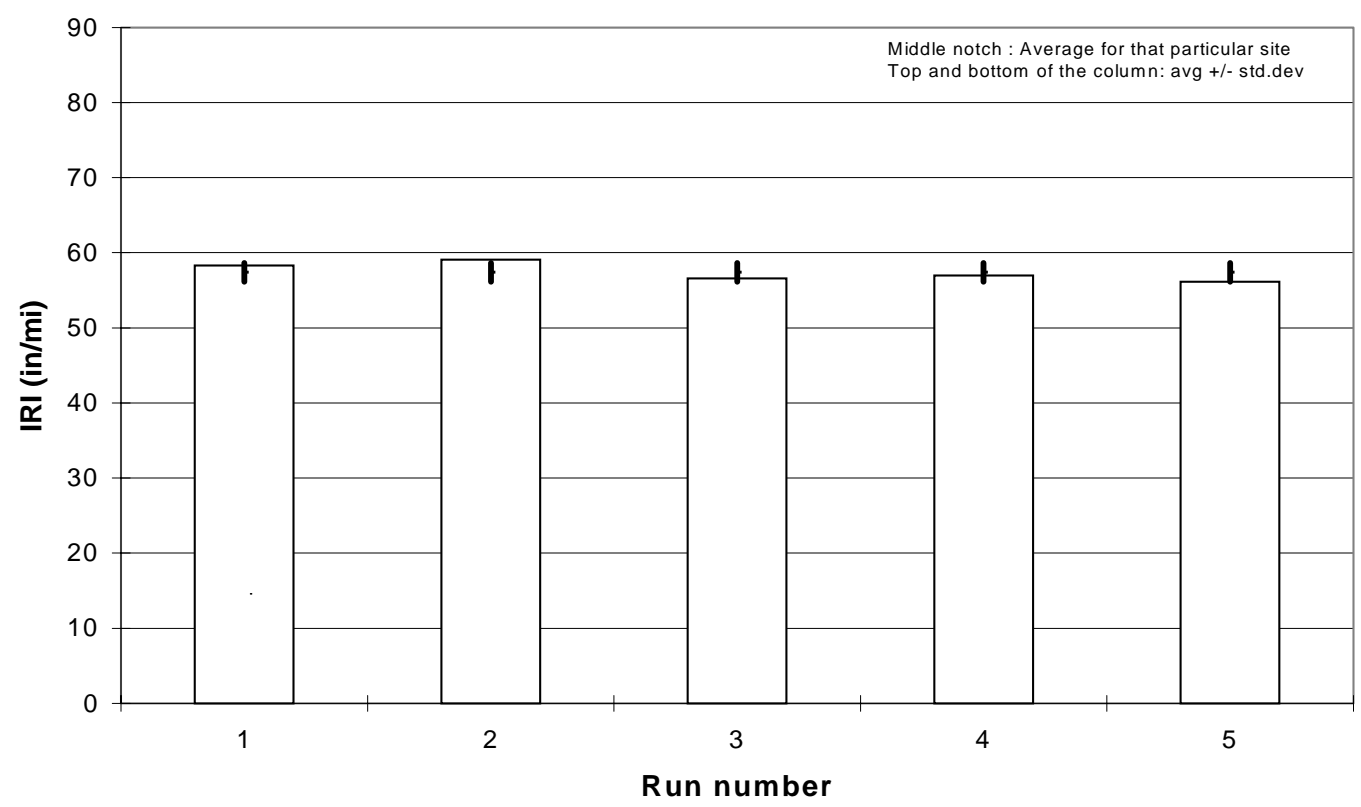

Figure A-24 IRI for replicate runs at US 231B (Site 6) of Vendor C 


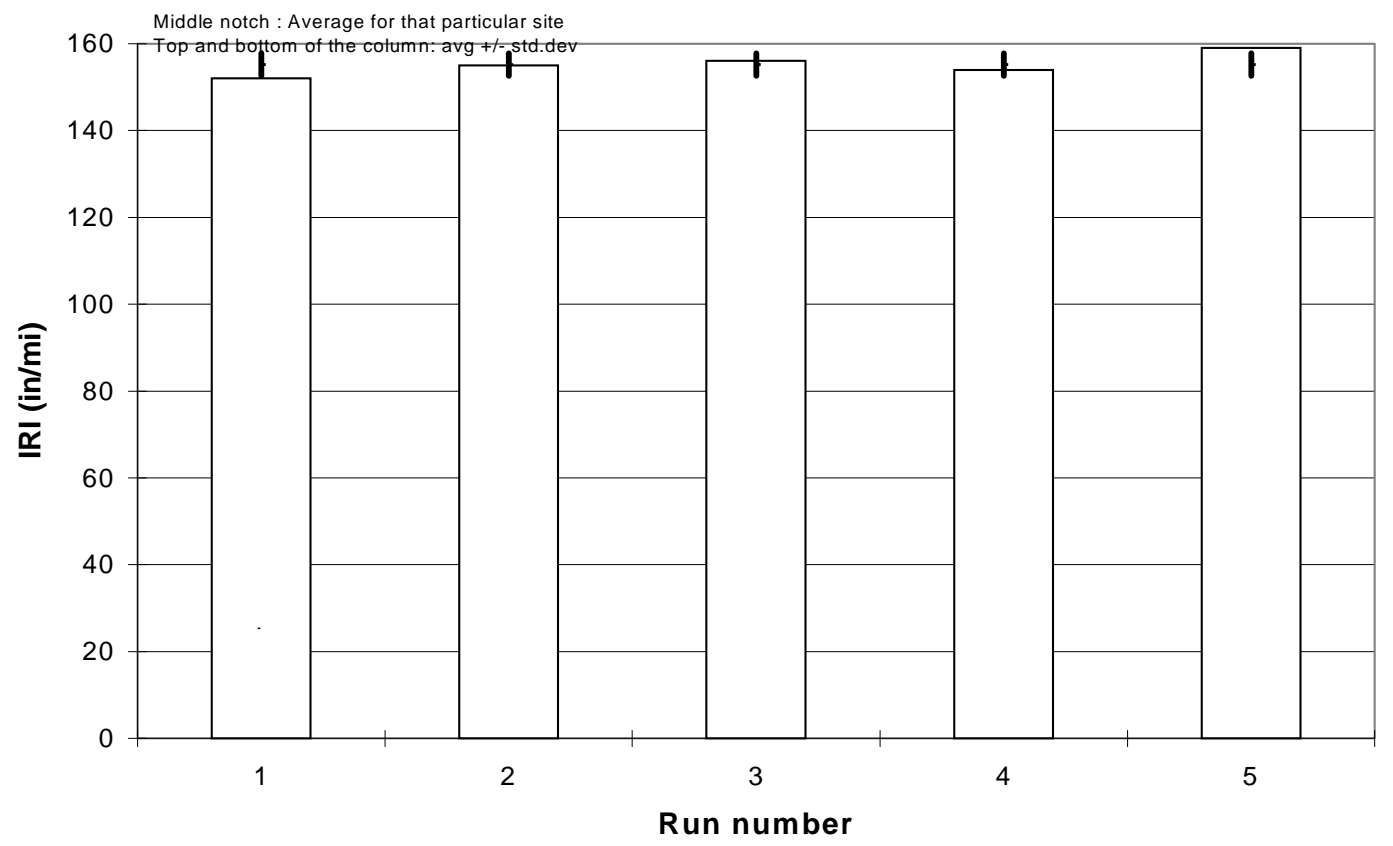

Figure A-25: IRI for replicate runs at Covington (Site 1) of Vendor D

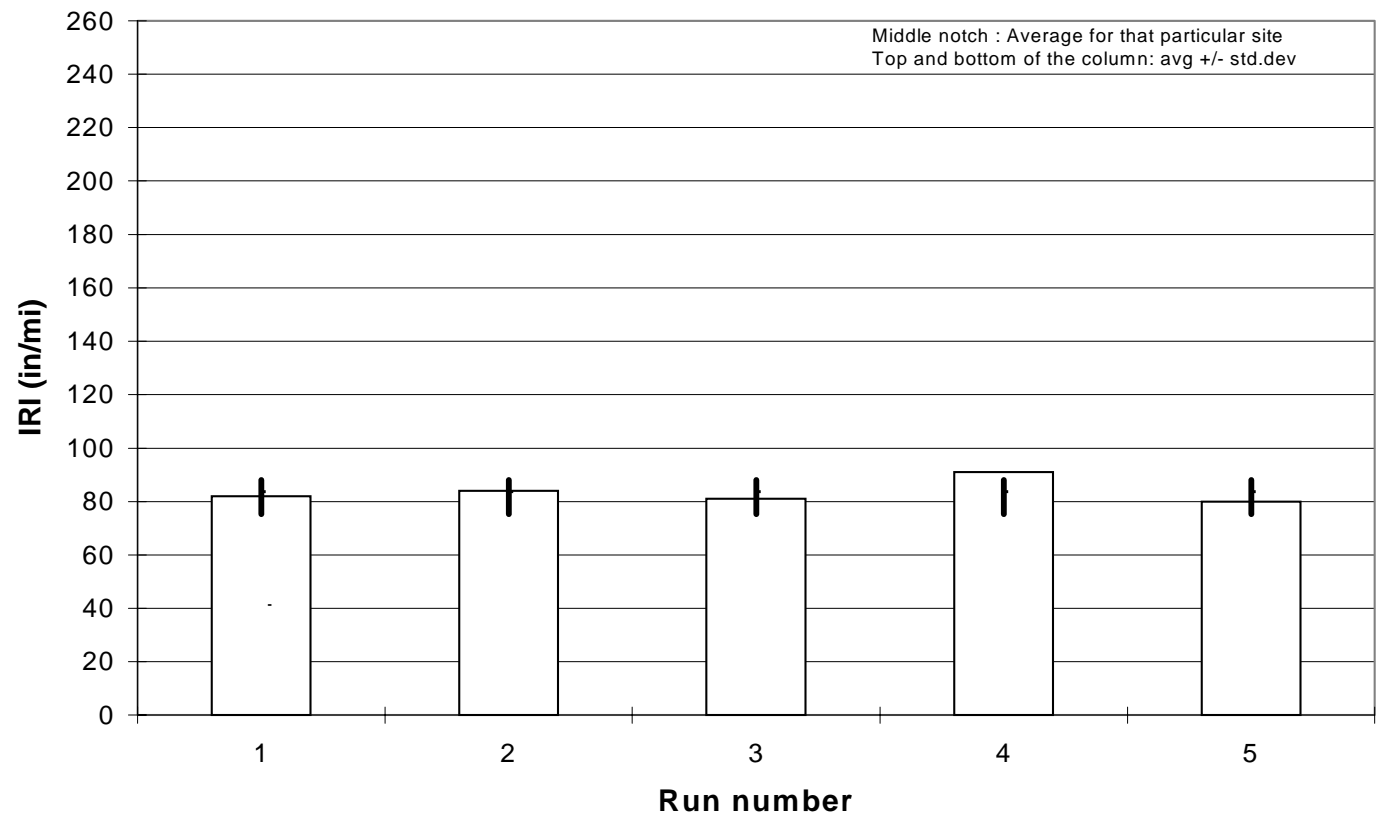

Figure A-26 IRI for replicate runs at I-65 (Site 2 with board) of Vendor D 


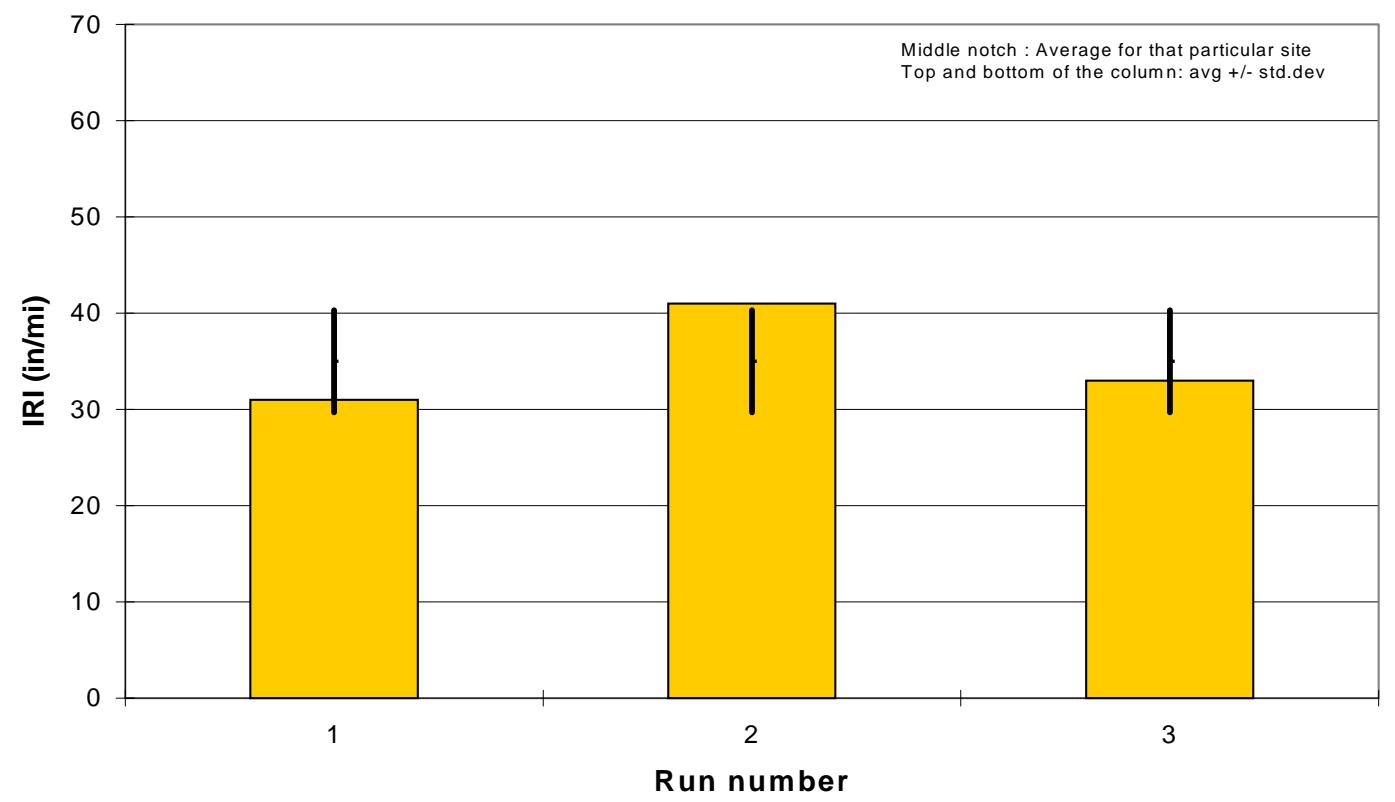

Figure A-27 IRI for replicate runs at I-65 (Site 2 without board) of Vendor D

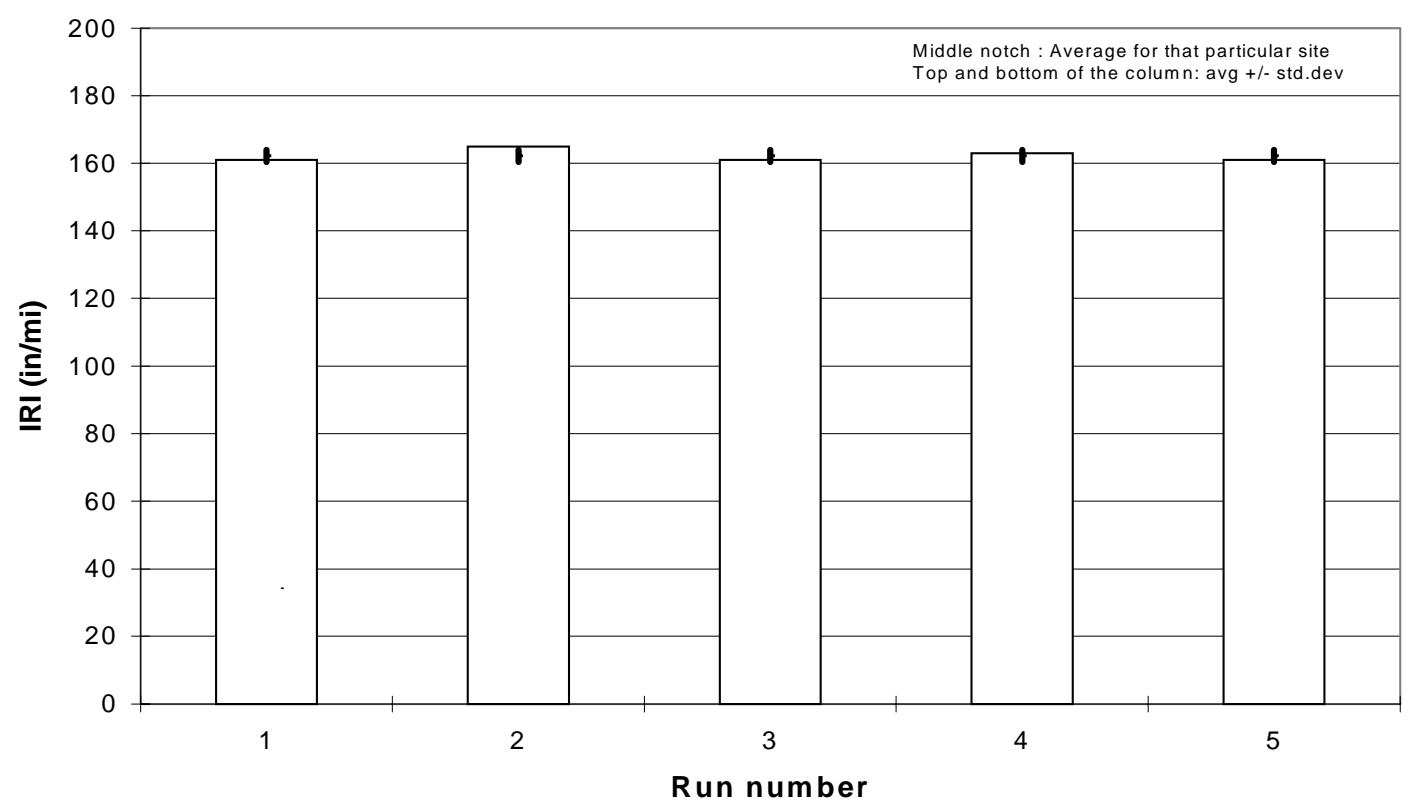

Figure A-28 IRI for replicate runs at Prairie Street (Site 3 with board) of Vendor D 


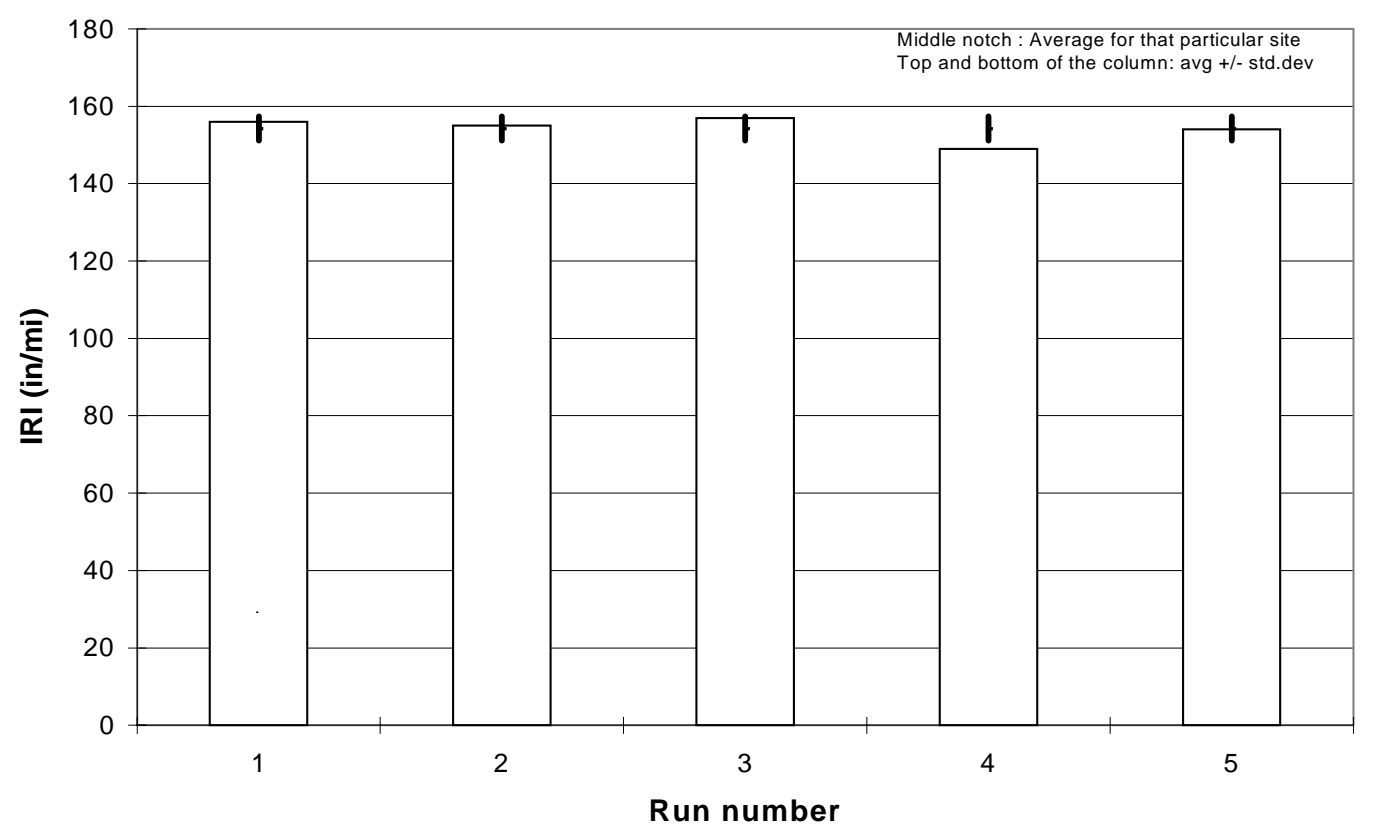

Figure A-29 IRI for replicate runs at Prairie Street (Site 3 without board) of Vendor D

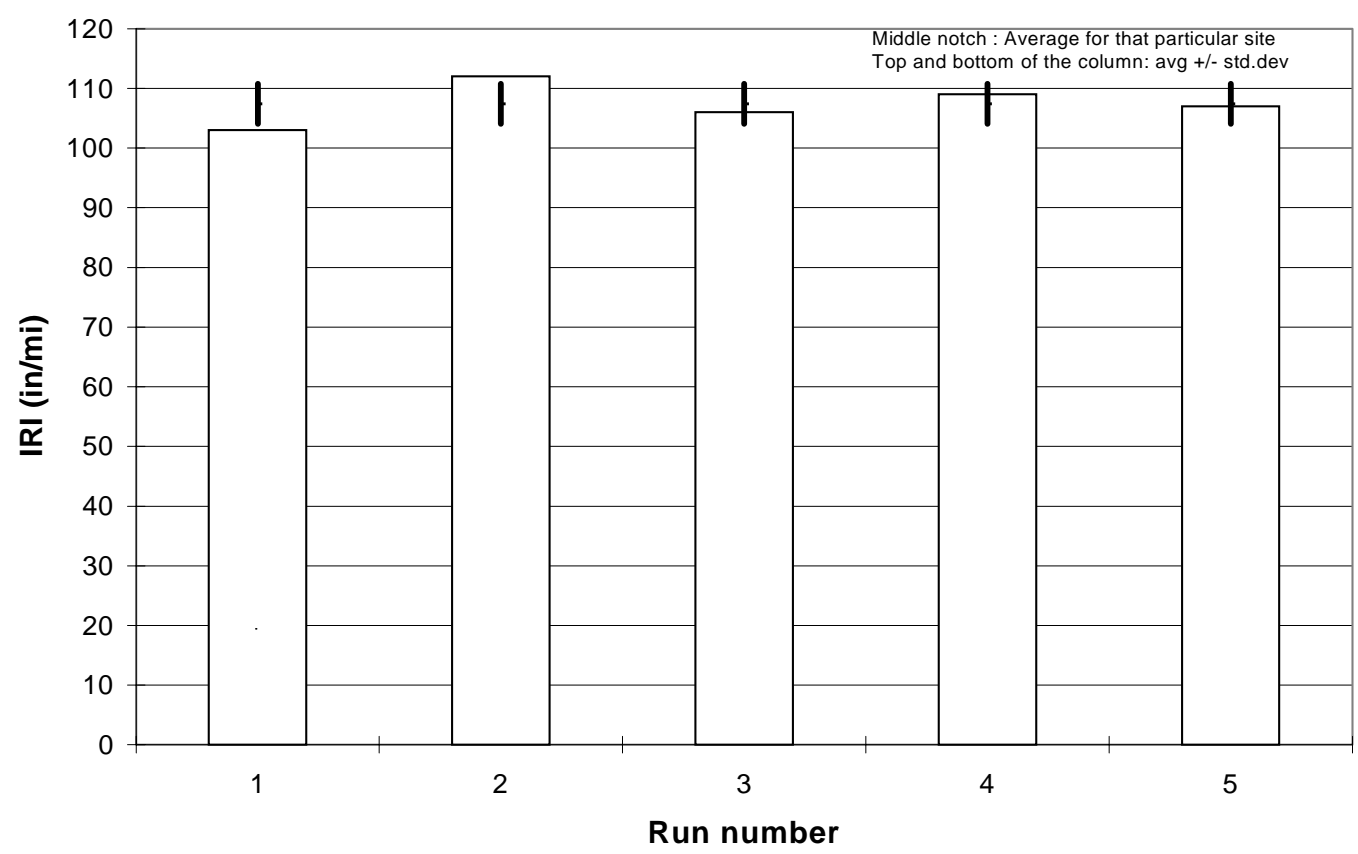

Figure A-30 IRI for replicate runs at Vinton Street (Site 4) of Vendor D 


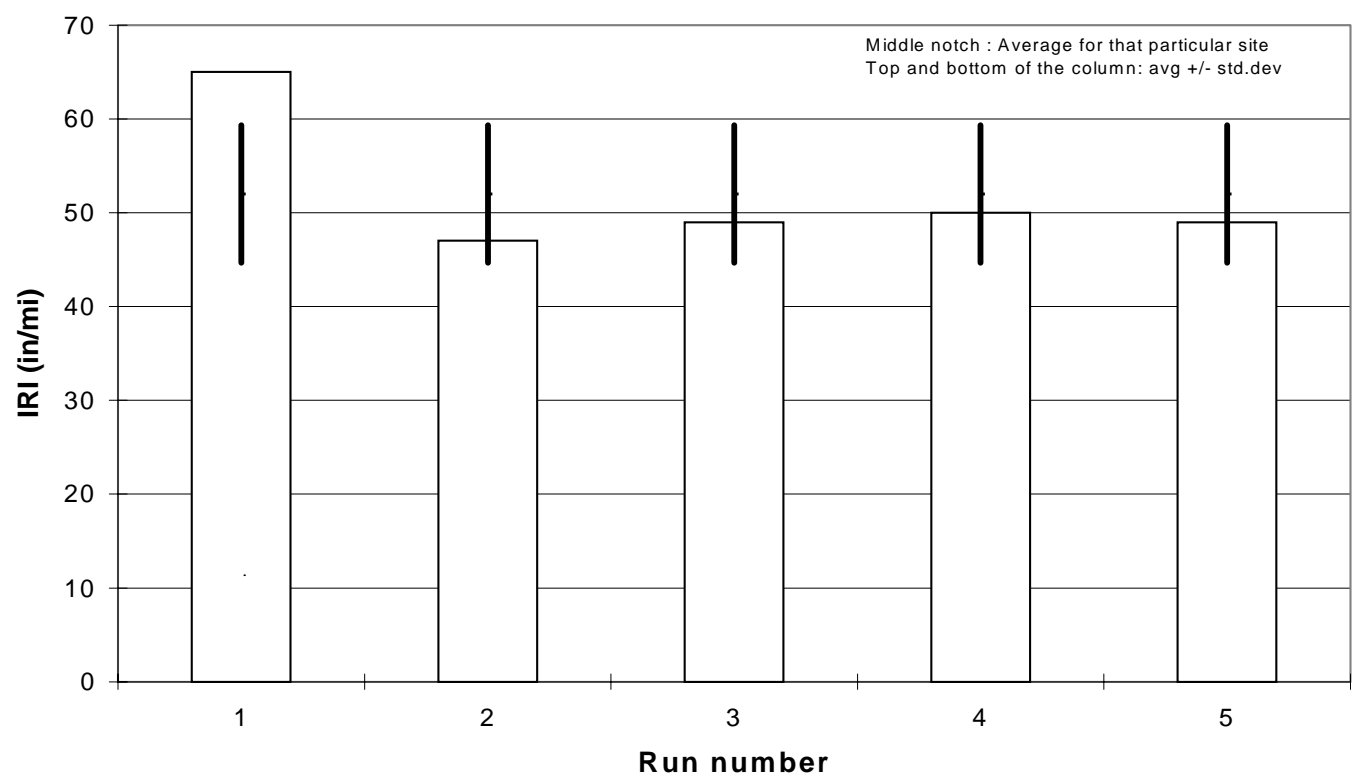

Figure A-31 IRI for replicate runs at US 231A (Site 5) of Vendor D

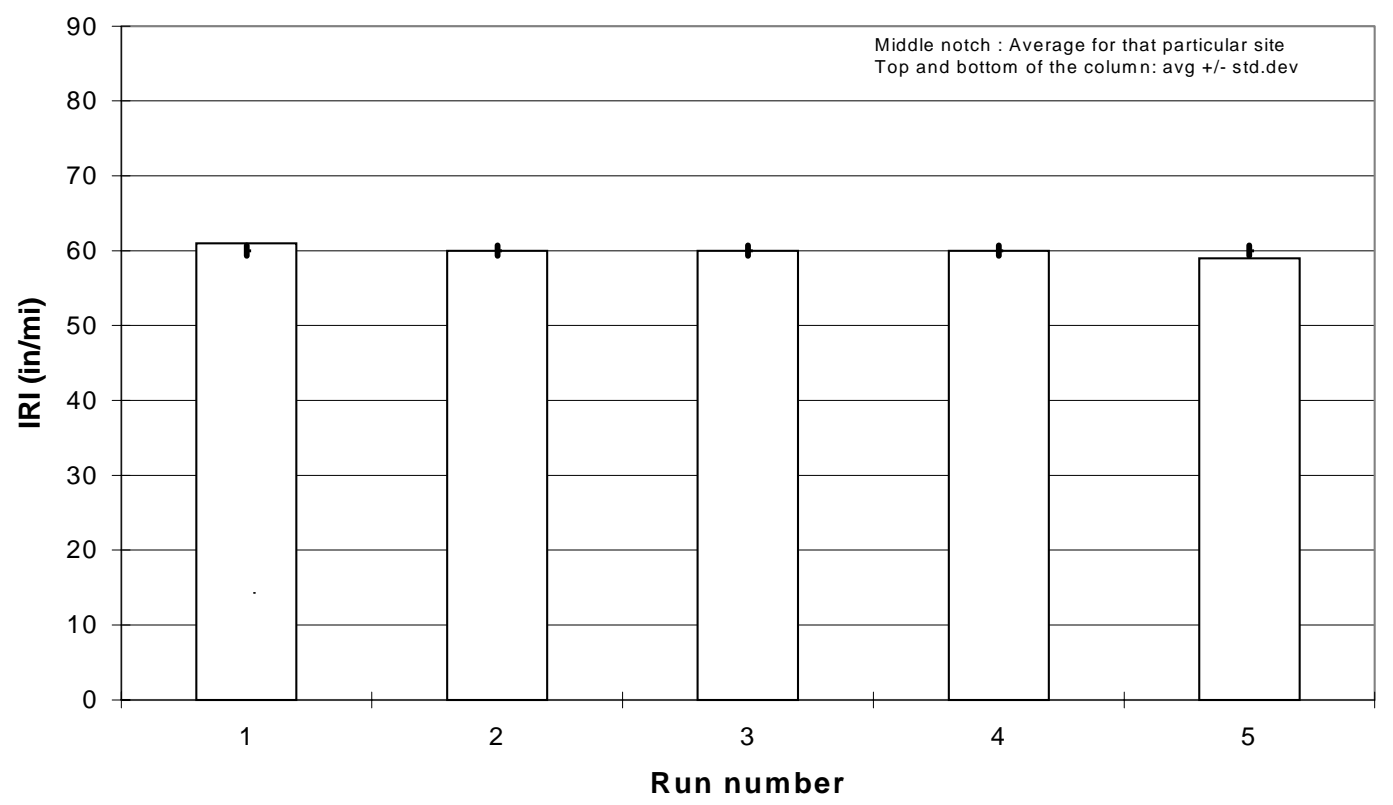

Figure A-32 IRI for replicate runs at US 231B (Site 6) of Vendor D 
APPENDIX B - PI-0.0" Comparison-Within Vendors 


\section{APPENDIX B - PI-0.0” Comparison-Within Vendors}

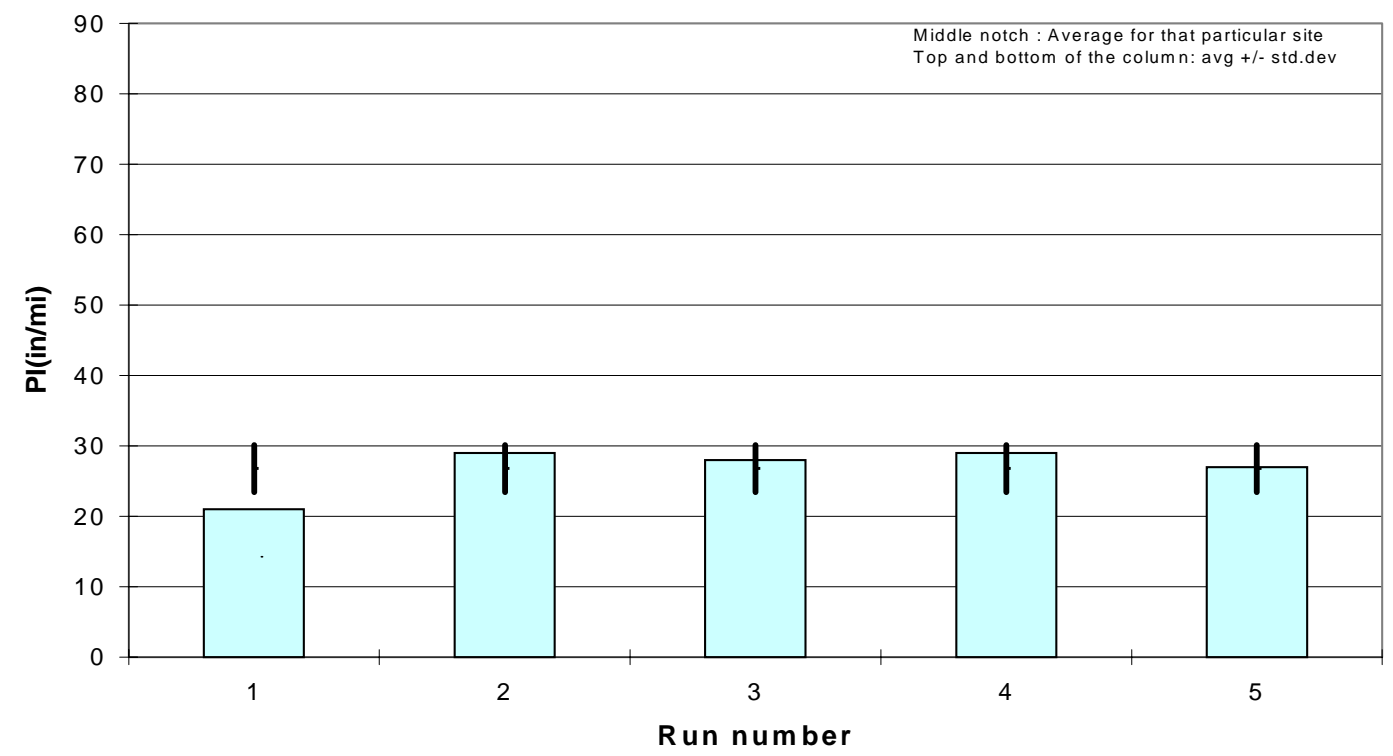

Figure B-1 PI-0.0”' blanking band for replicate runs at Covington (Site 1) of

Vendor A

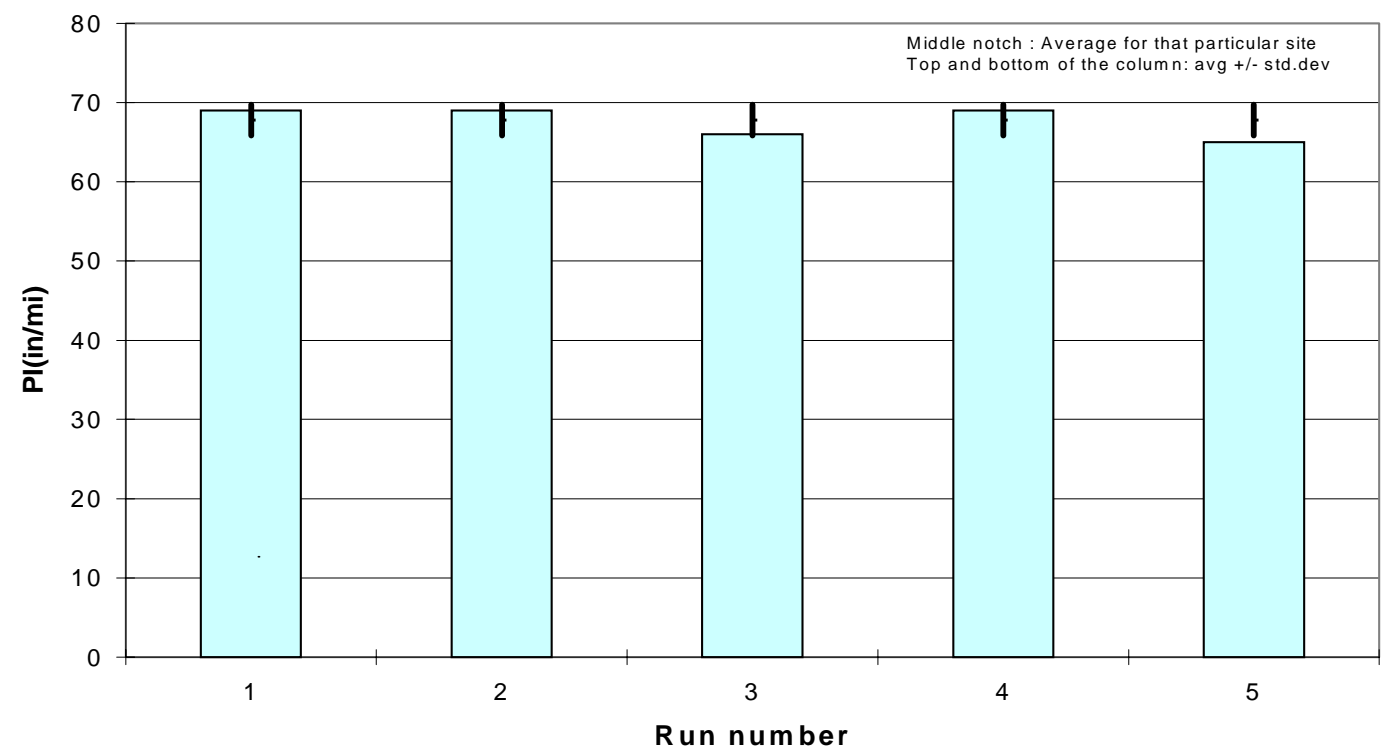

Figure B-2 PI-0.0" blanking band for replicate runs at I-65 (Site 2 with board) of

Vendor A 


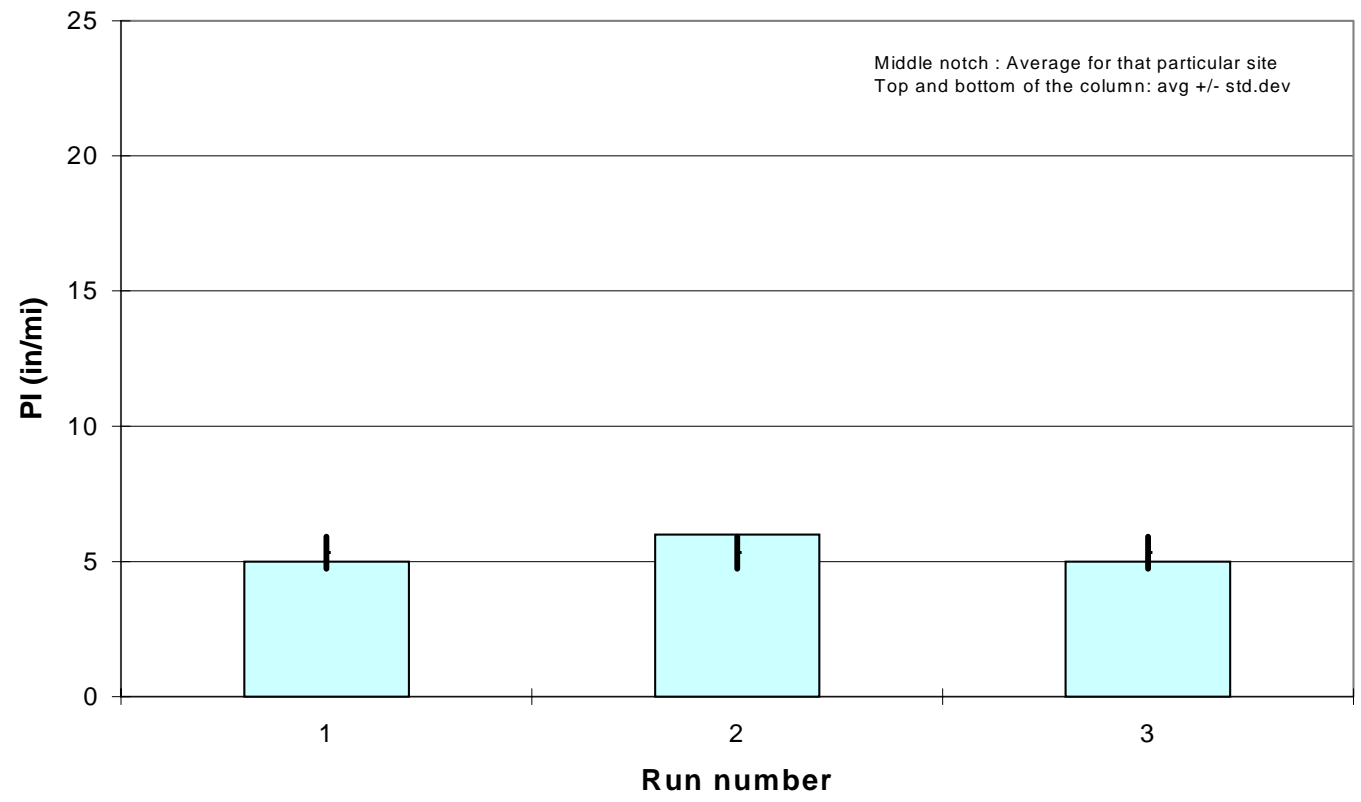

Figure B-3 PI-0.0" blanking band for replicate runs at I-65 (Site 2 without board) of Vendor A

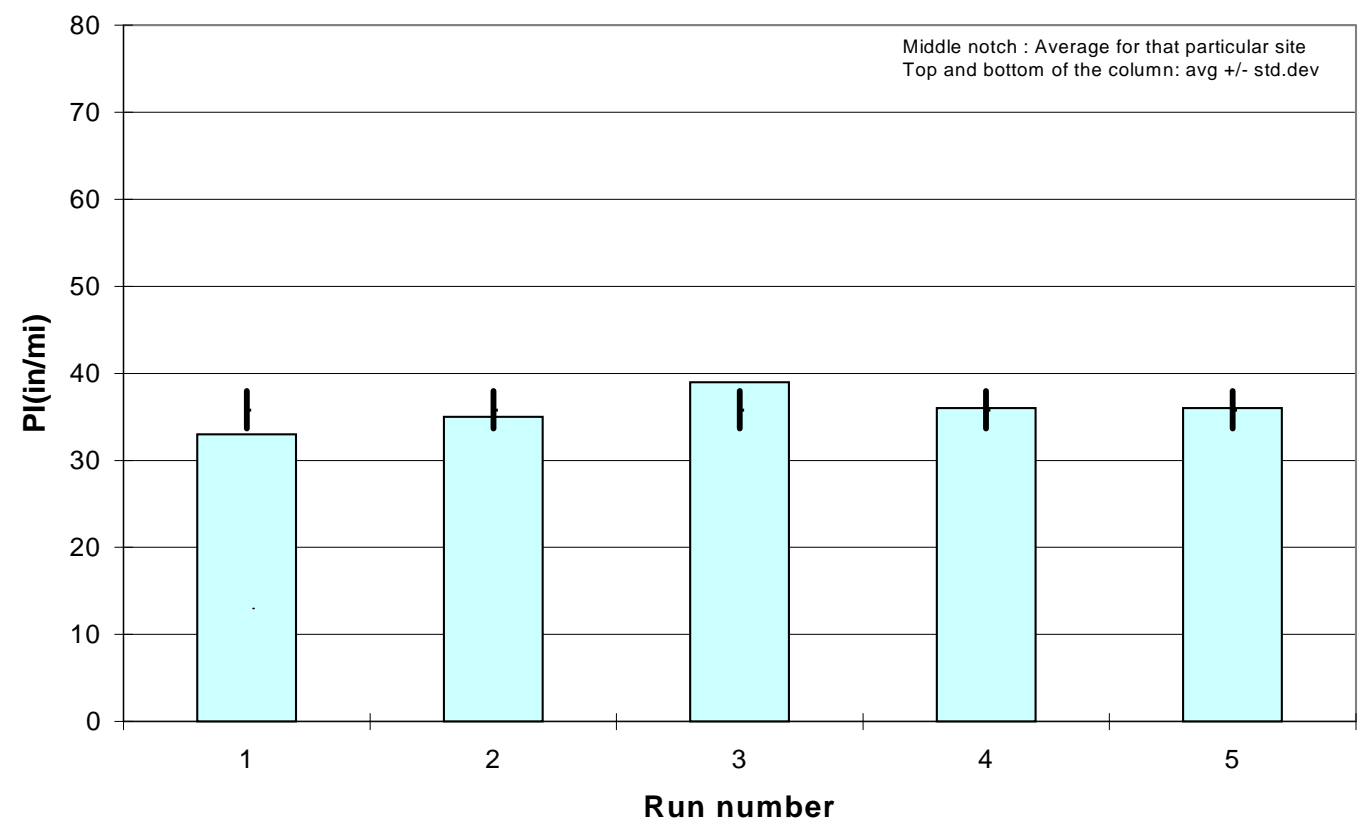

Figure B-4 PI-0.0" blanking band for replicate runs at Prairie Street (Site 3 with board) of Vendor A 


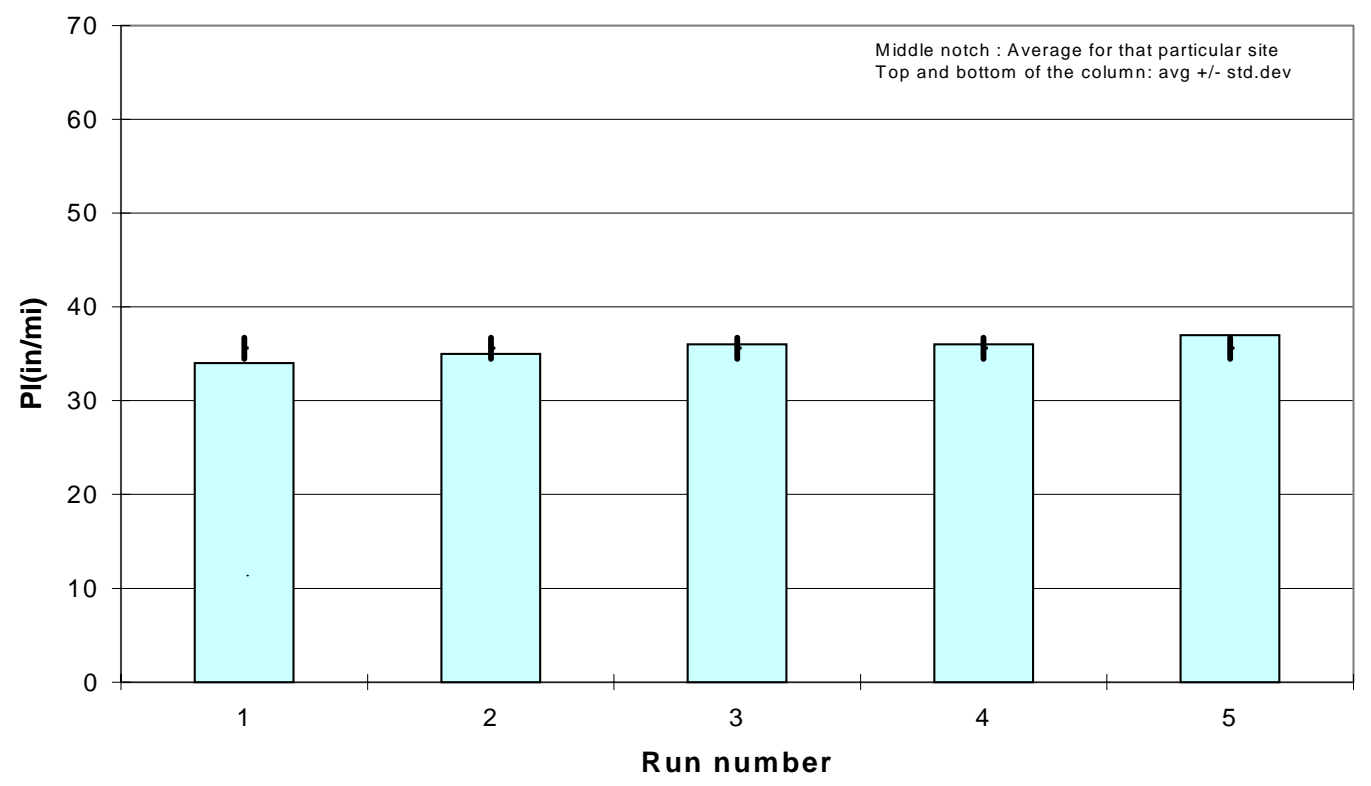

Figure B-5 PI-0.0” blanking band for replicate runs at Prairie Street

(Site 3 without board) of Vendor A

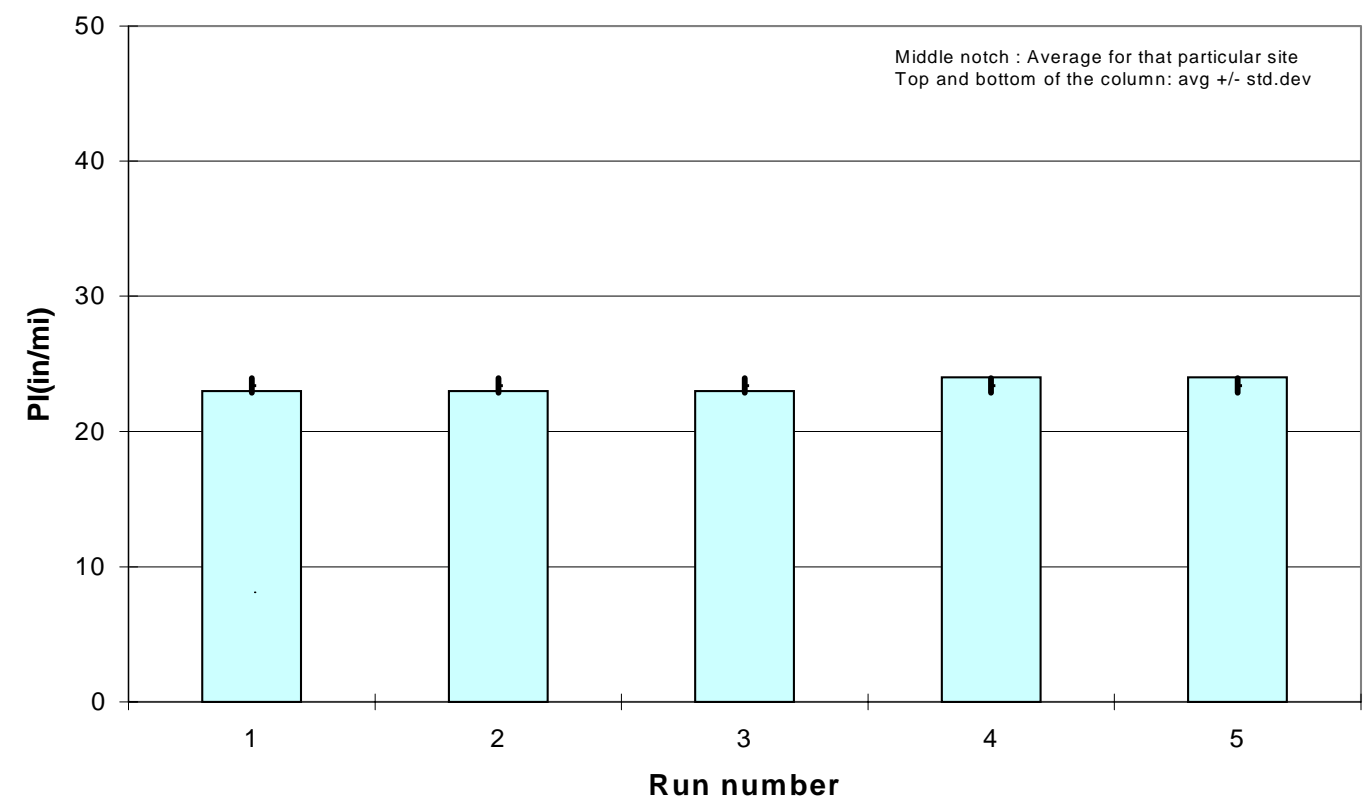

Figure B-6 PI-0.0”' blanking band for replicate runs at Vinton Street (Site 4) of Vendor A 


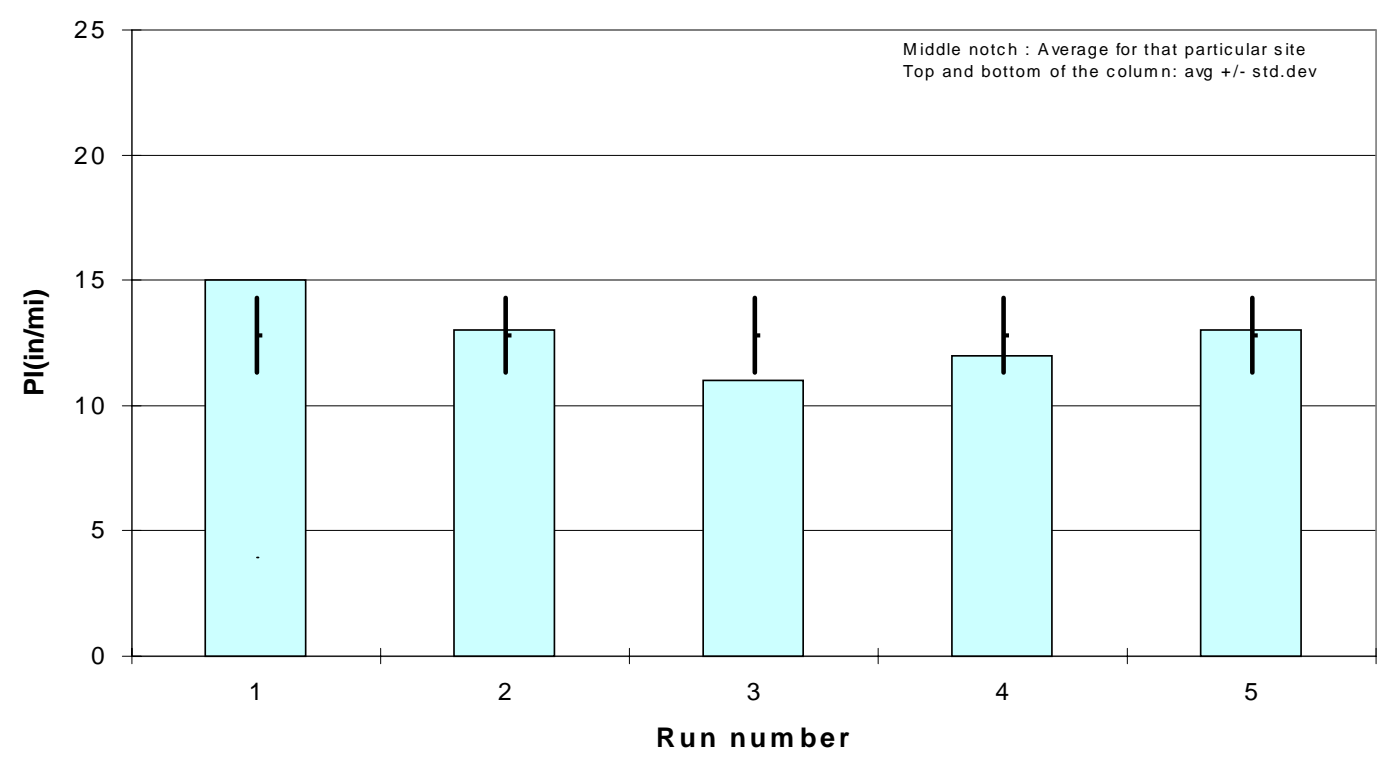

Figure B-7 PI-0.0" blanking band for replicate runs at US 231A (Site 5) of Vendor A

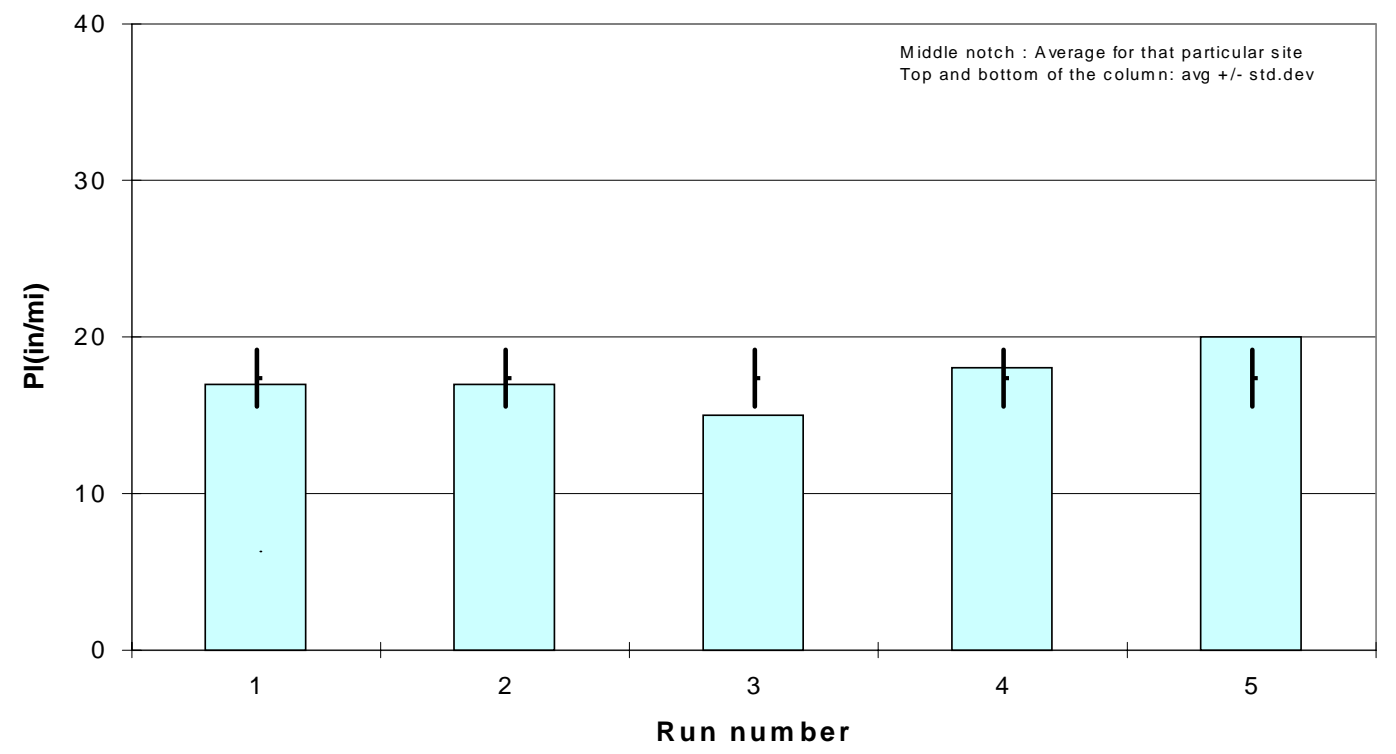

Figure B-8 PI-0.0" blanking band for replicate runs at US 231B (Site 6) of Vendor A 


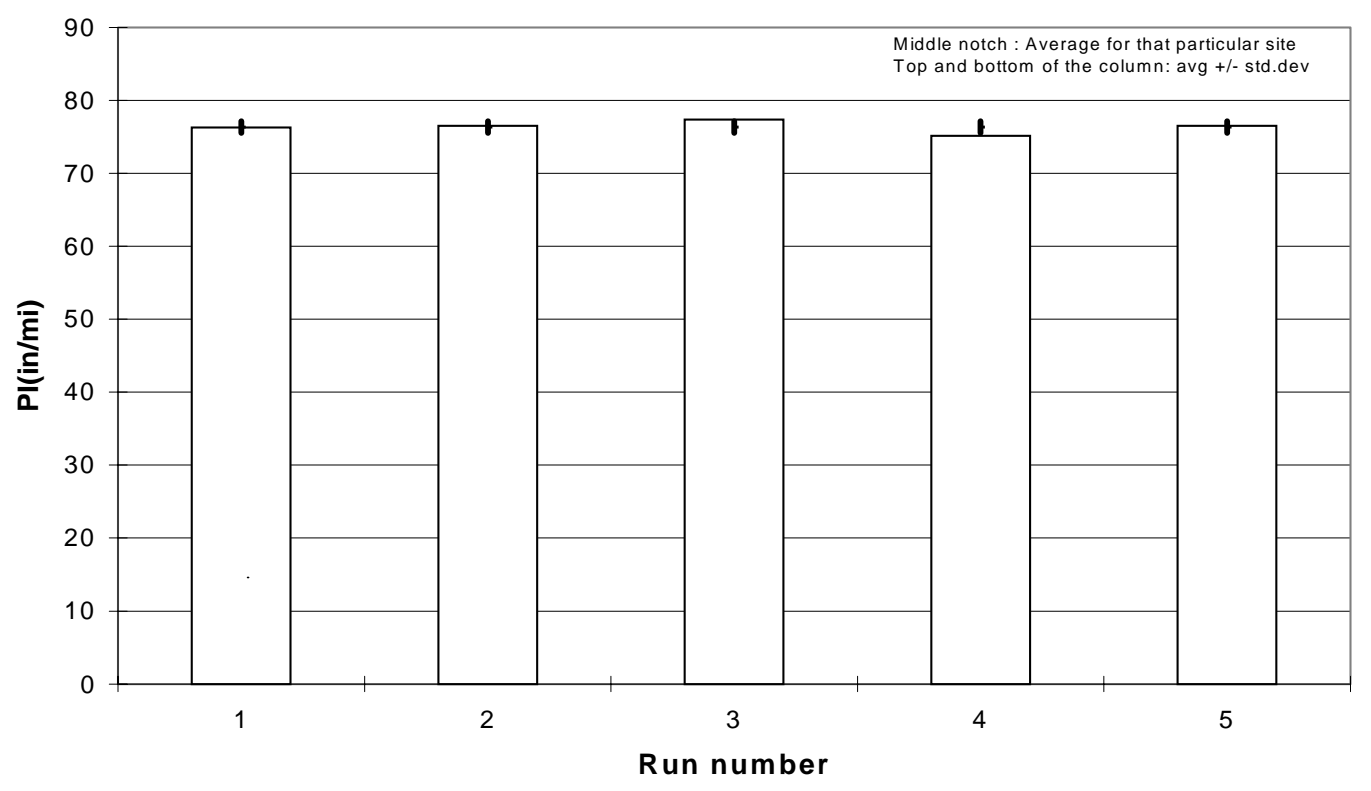

Figure B-9 PI-0.0”' blanking band for replicate runs at Covington (Site 1) of

Vendor B

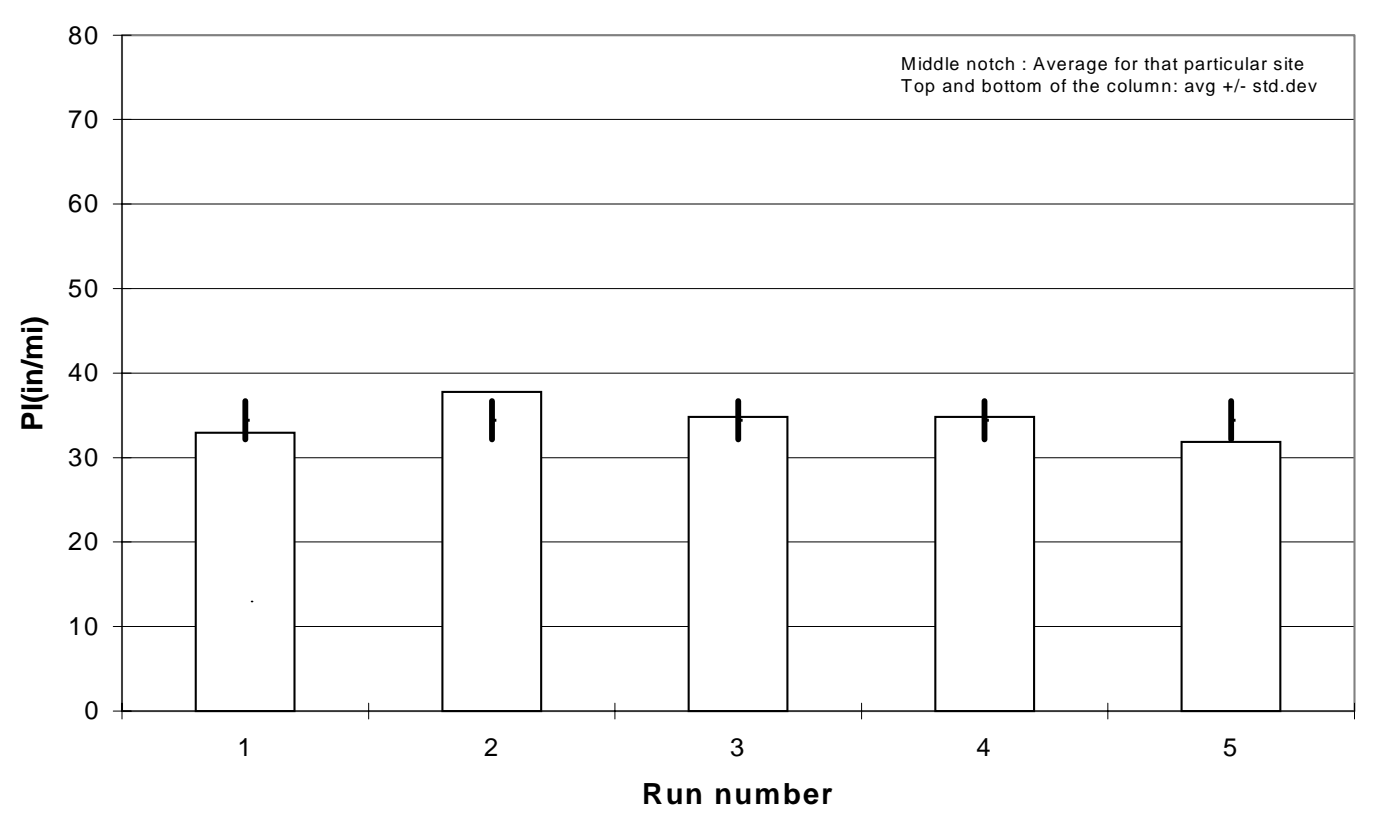

Figure B-10 PI-0.0" blanking band for replicate runs at I-65 (Site 2 with board) of Vendor B 


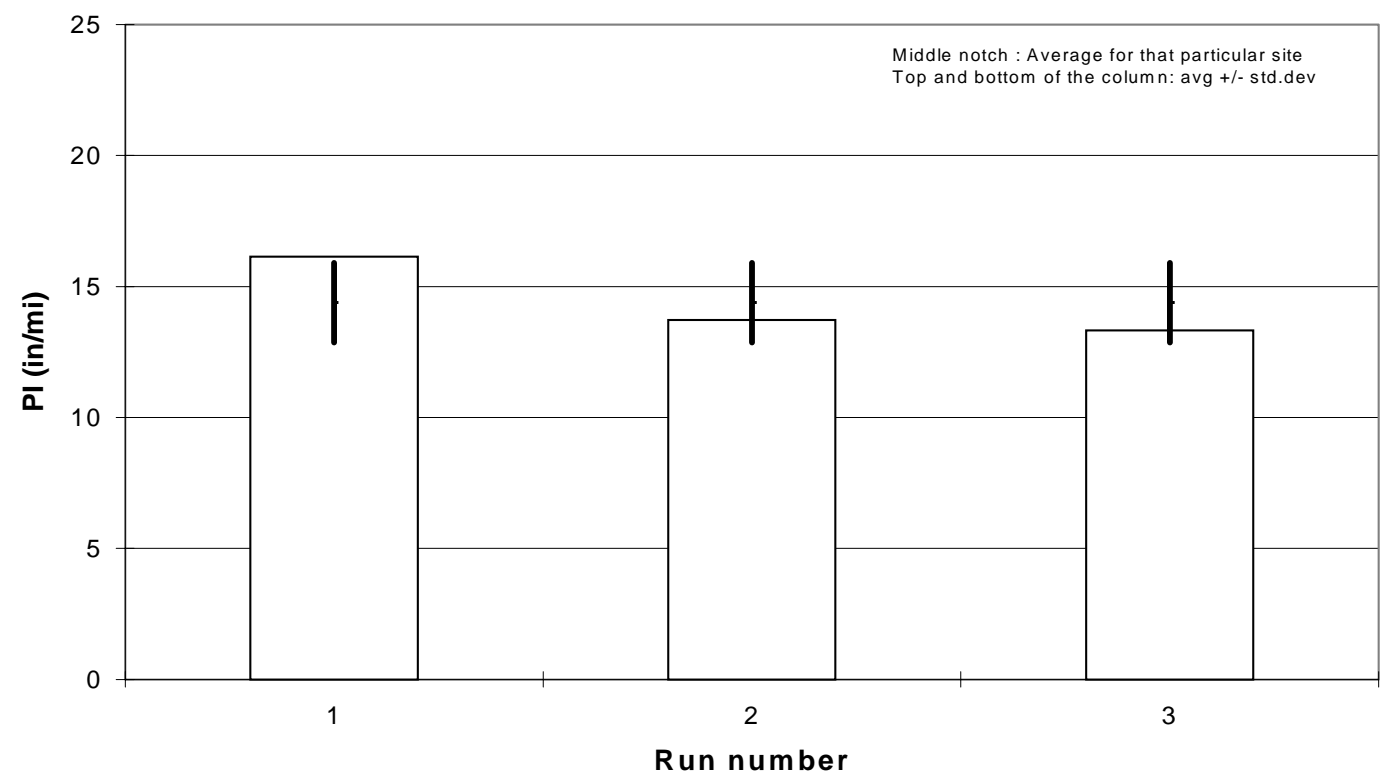

Figure B-11 PI-0.0" blanking band for replicate runs at I-65 (Site 2 without board) of Vendor B

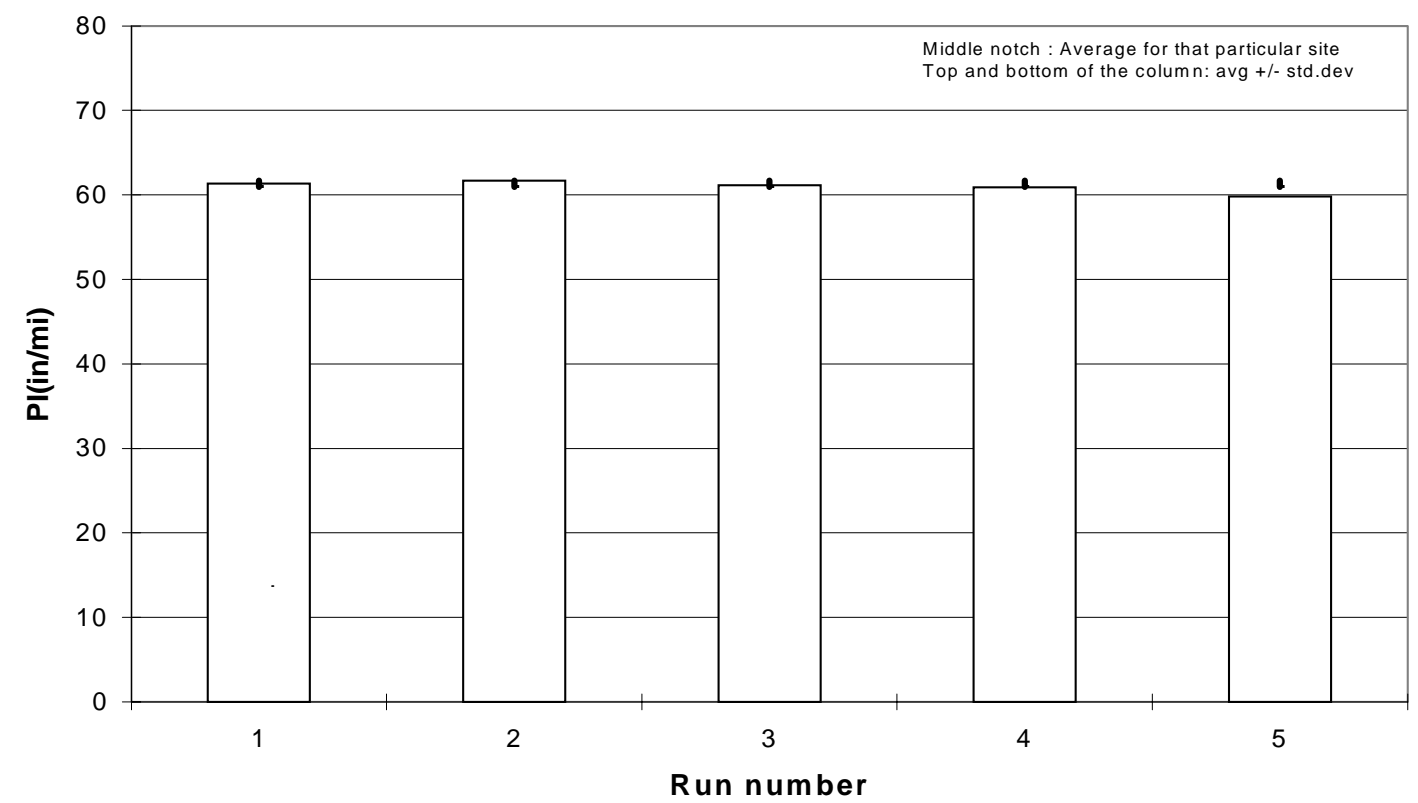

Figure B-12 PI-0.0" blanking band for replicate runs at Prairie Street

(Site 3 with board) of Vendor B 


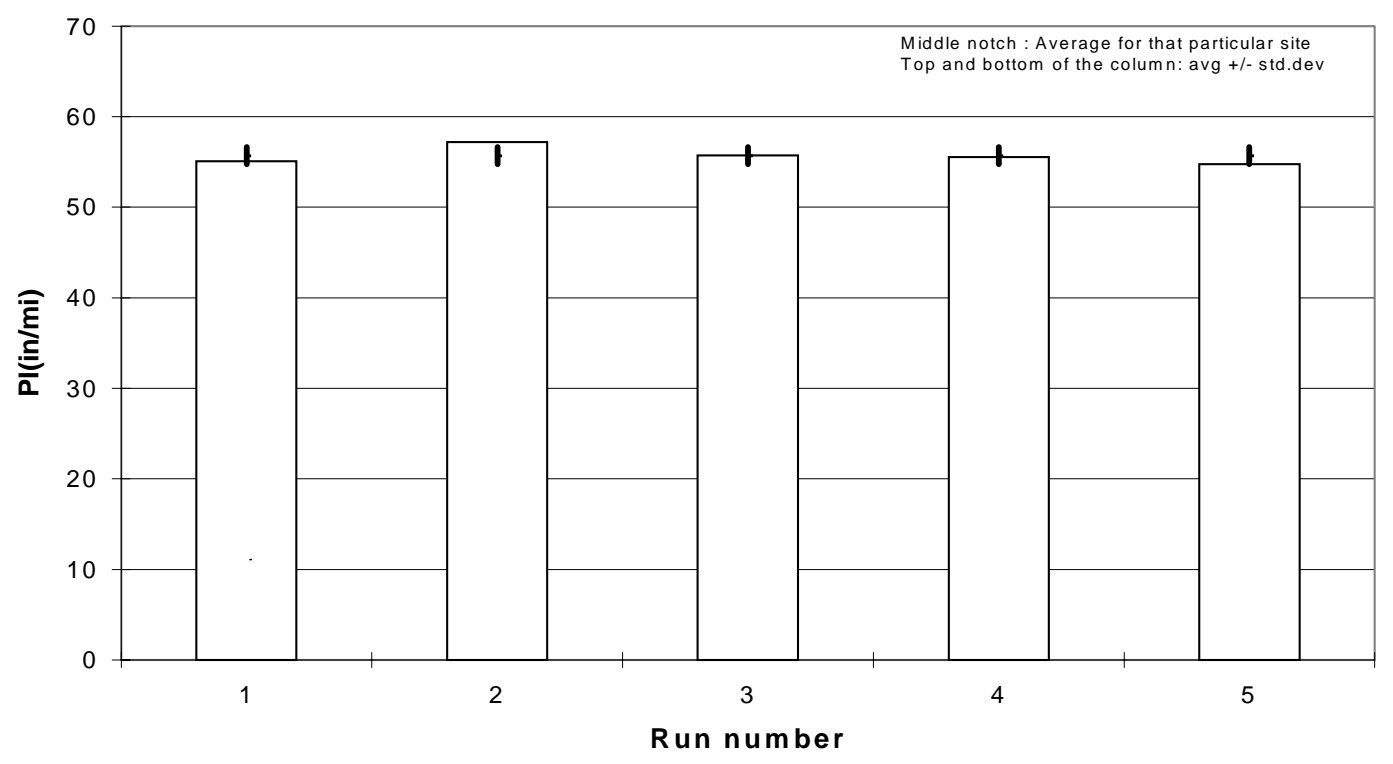

Figure B-13 PI-0.0” blanking band for replicate runs at Prairie Street

(Site 3 without board) of Vendor B

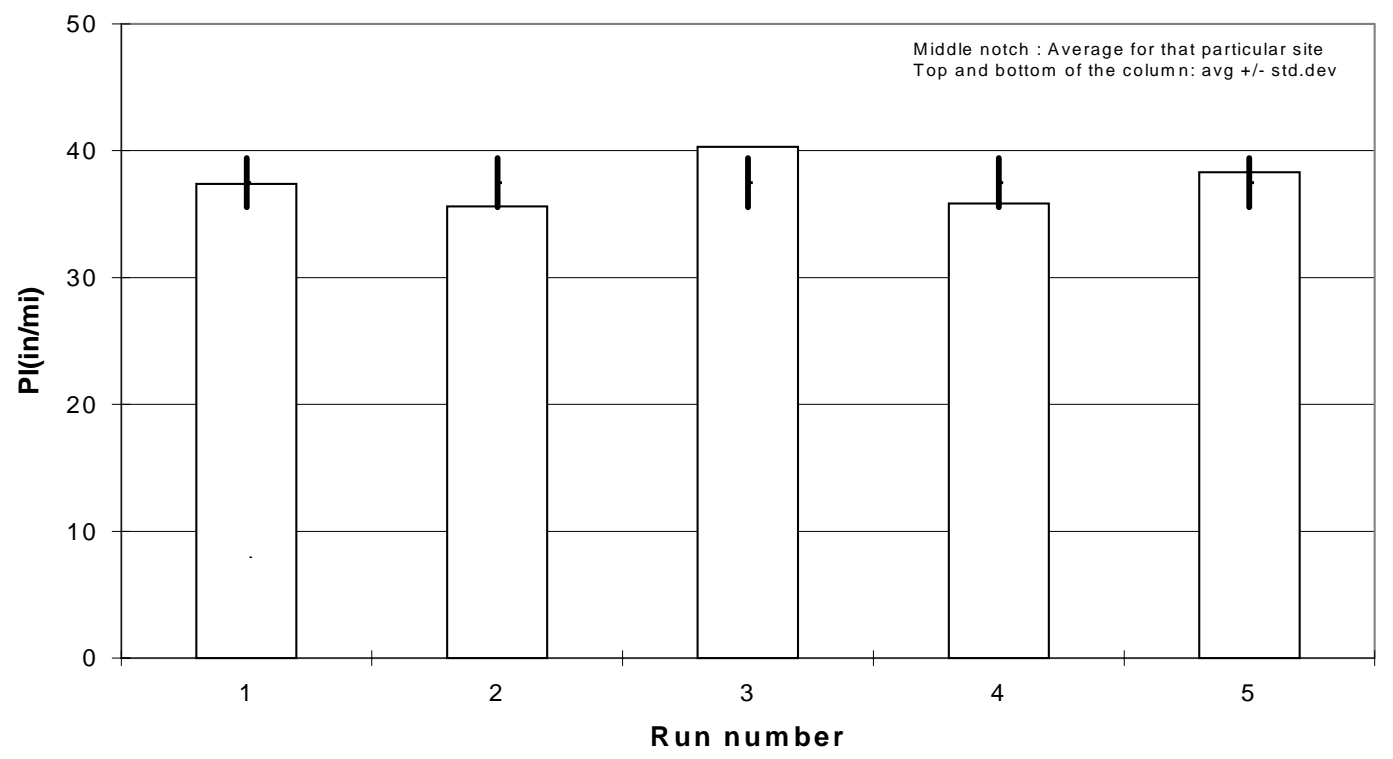

Figure B-14 PI-0.0'blanking band for replicate runs at Vinton Street (Site 4) of Vendor B 


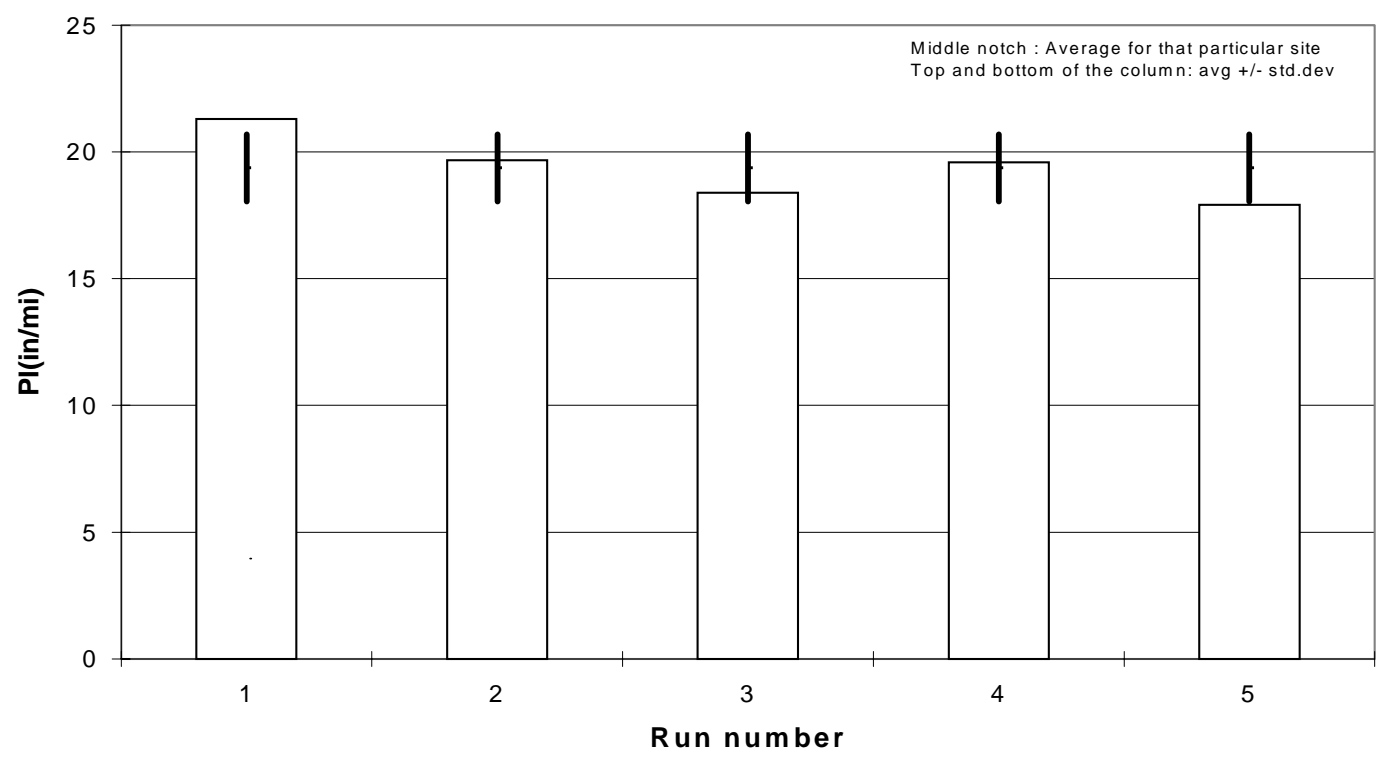

Figure B-15 PI-0.0'blanking band for replicate runs at US231A (Site 5) of Vendor B

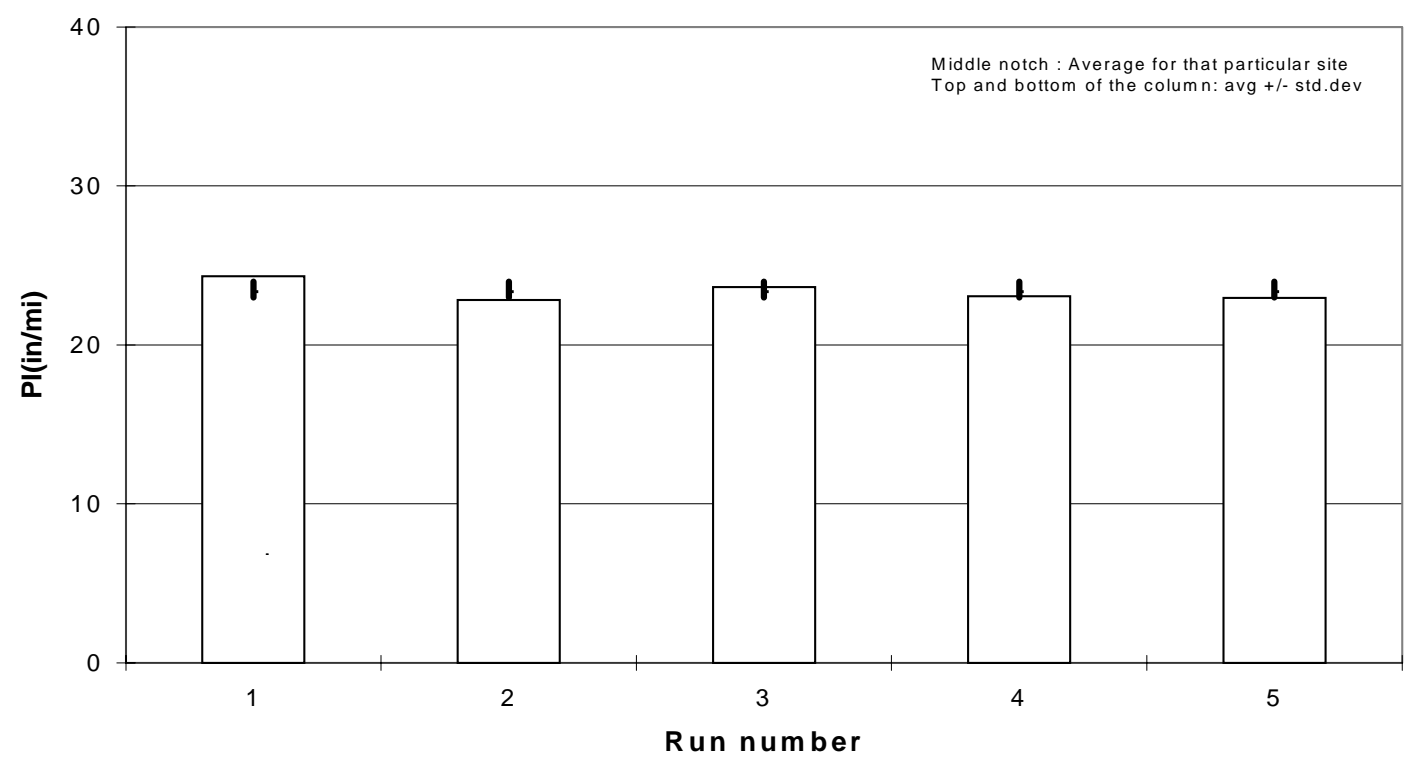

Figure B-16 PI-0.0'blanking band for replicate runs at US231B (Site 6) of Vendor B 


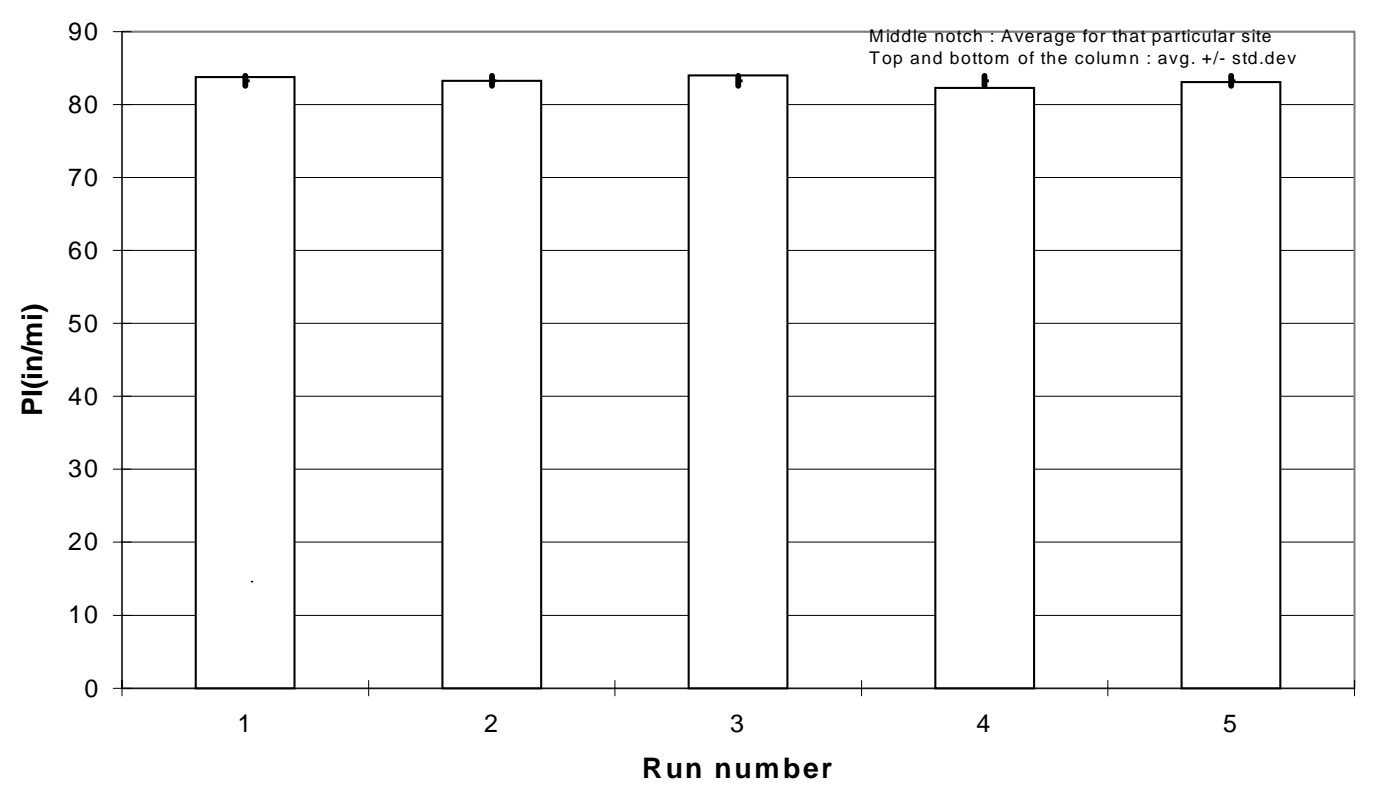

Figure B-17 PI-0.0’'blanking band for replicate runs at Covington Street (Site 1) of

Vendor C

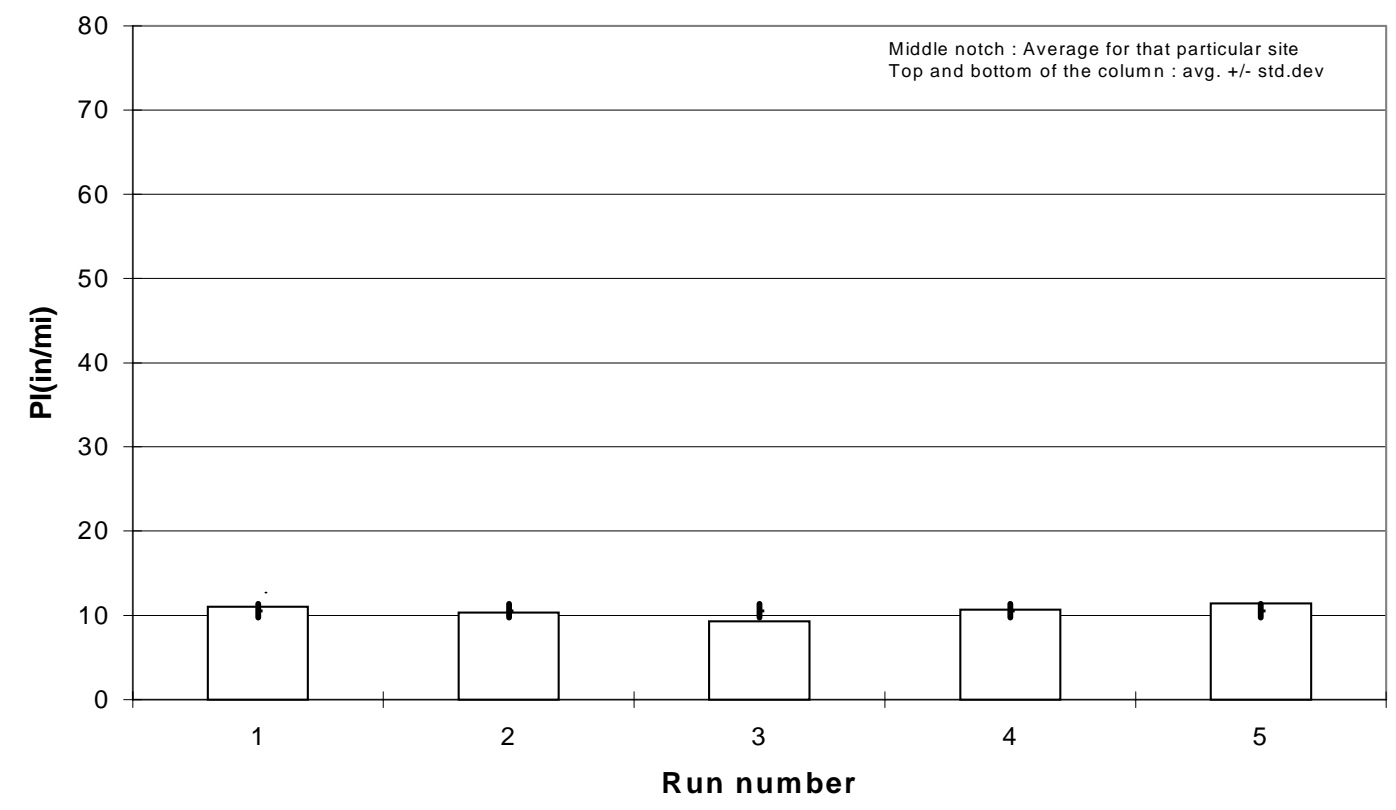

Figure B-18 PI-0.0'’blanking band for replicate runs at I-65 (Site 2 with board) of Vendor C 


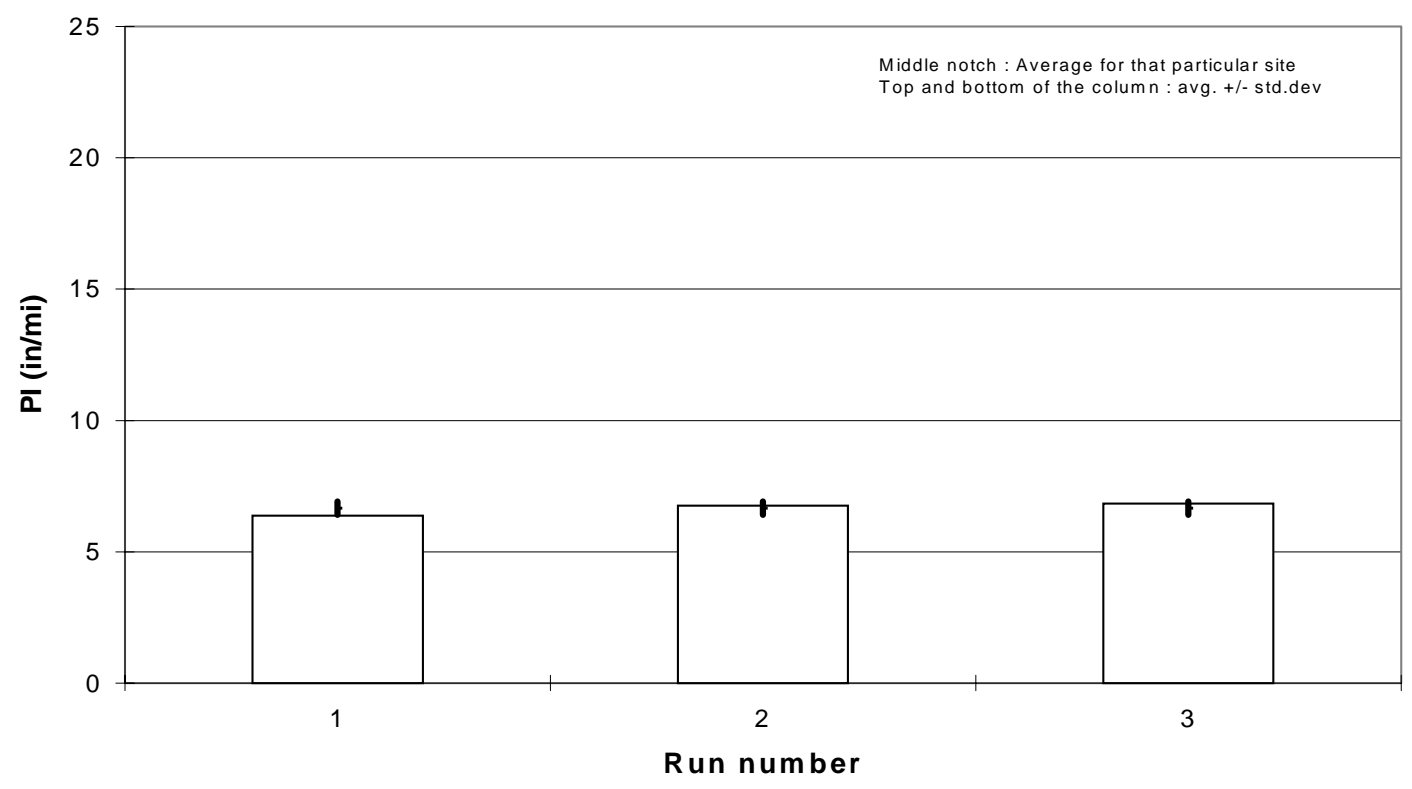

Figure B-19 PI-0.0’'blanking band for replicate runs at I-65

(Site 2 without board) of Vendor $C$

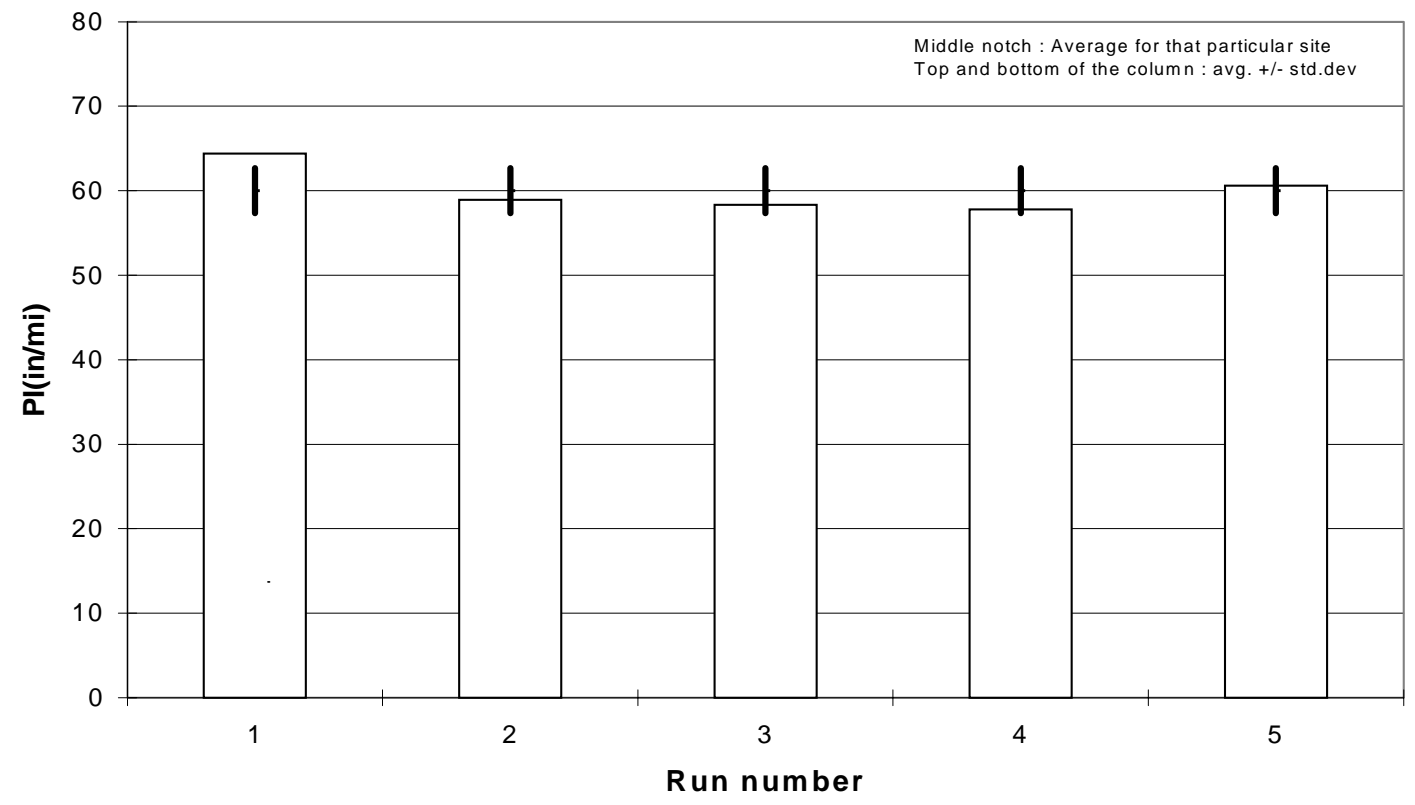

Figure B-20 PI-0.0'blanking band for replicate runs at Prairie Street

(Site 3 with board) of Vendor C 


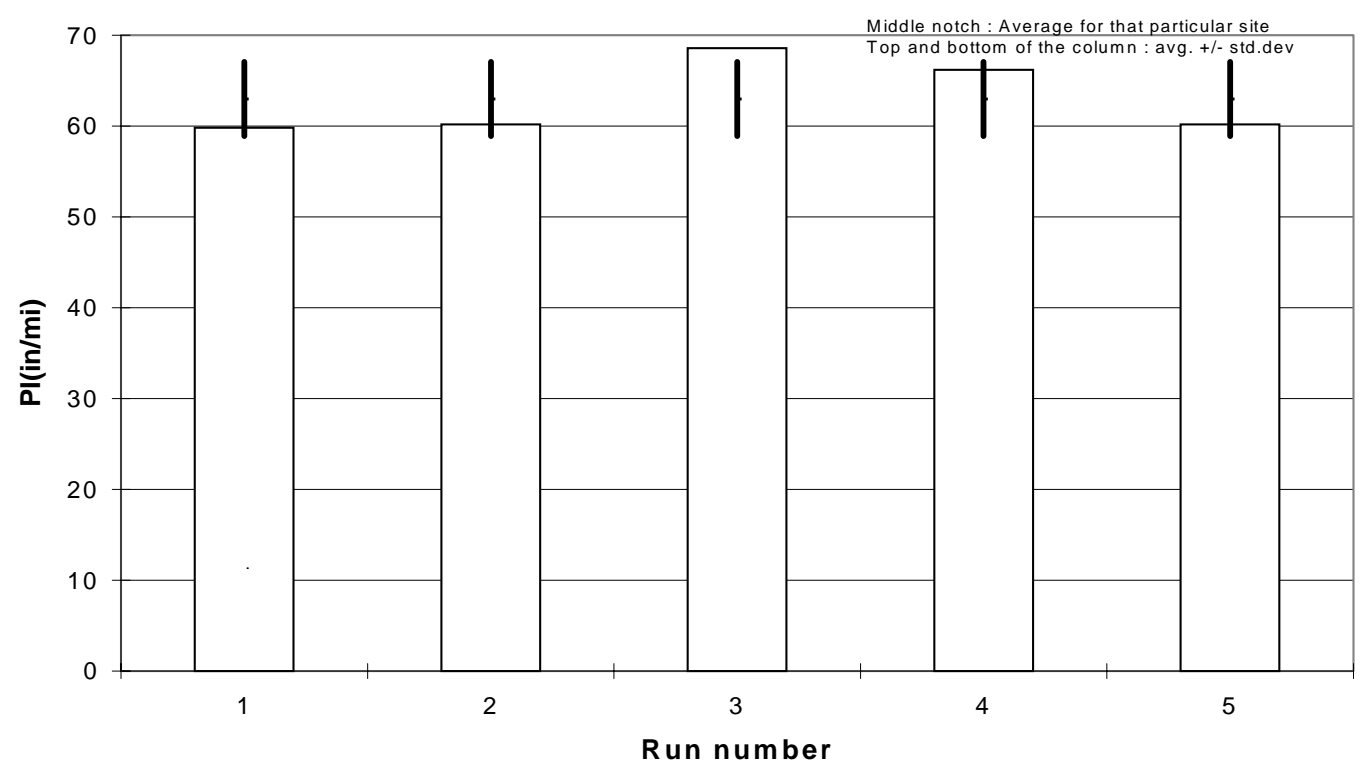

Figure B-21 PI-0.0’blanking band for replicate runs at Prairie Street

(Site 3 without board) of Vendor C

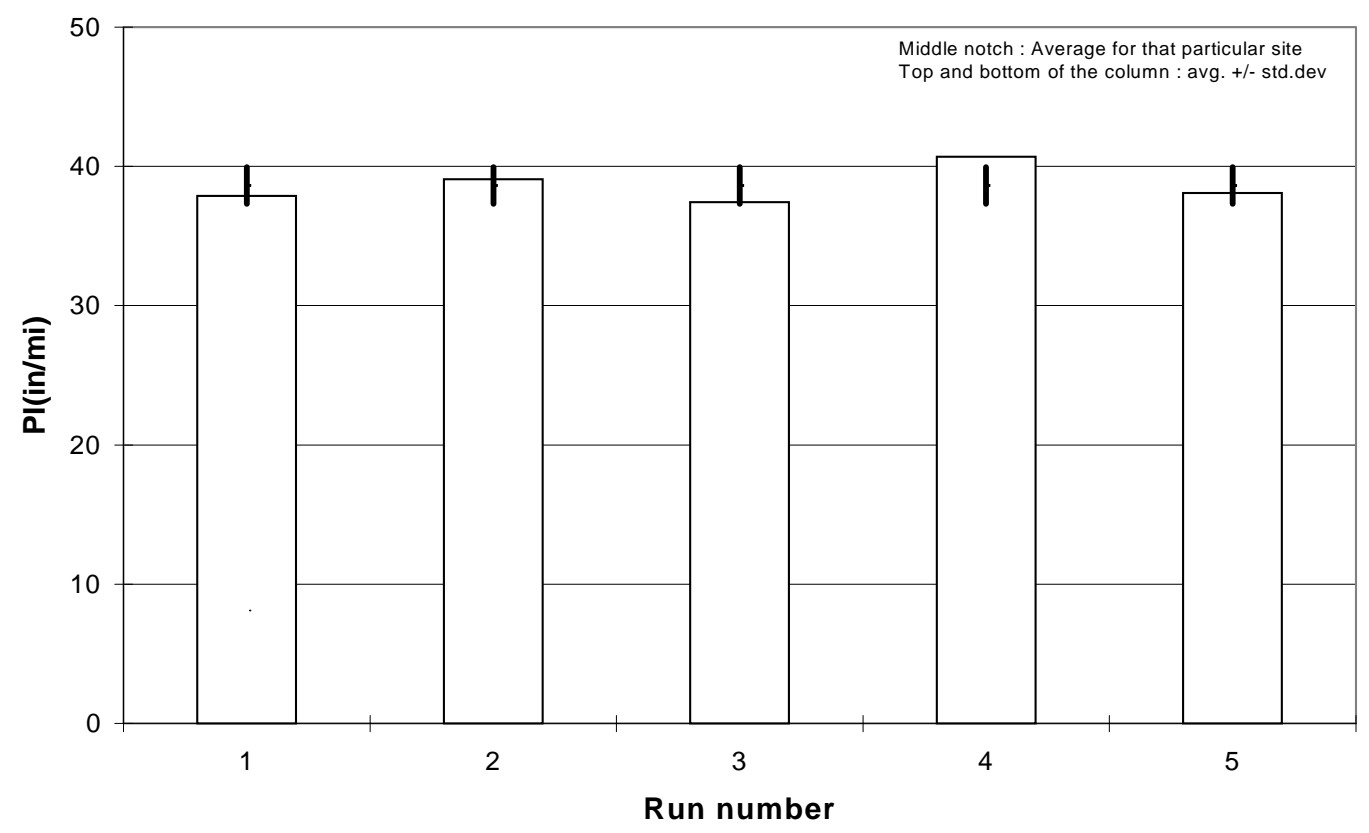

Figure B-22 PI-0.0'blanking band for replicate runs at Vinton Street (Site 4) of Vendor C 


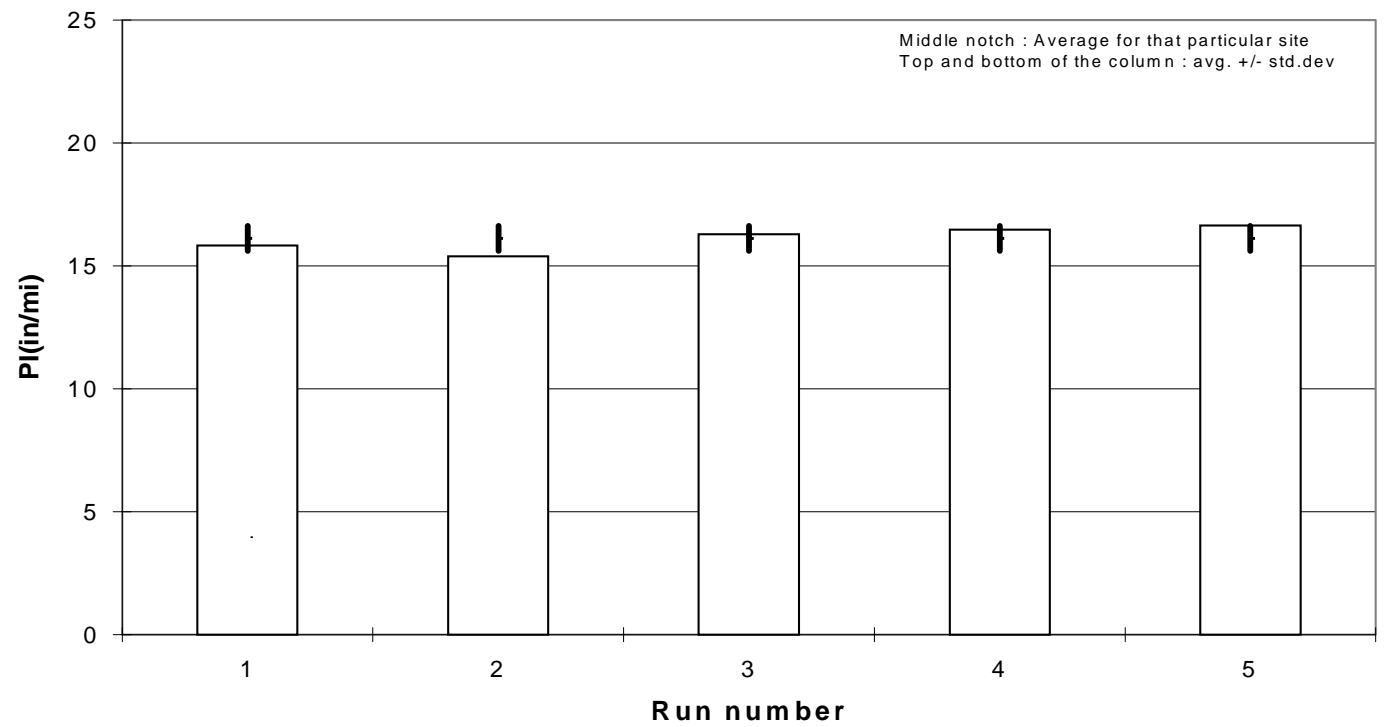

Figure B-23 PI-0.0'blanking band for replicate runs at US 231A (Site 5) of

Vendor C

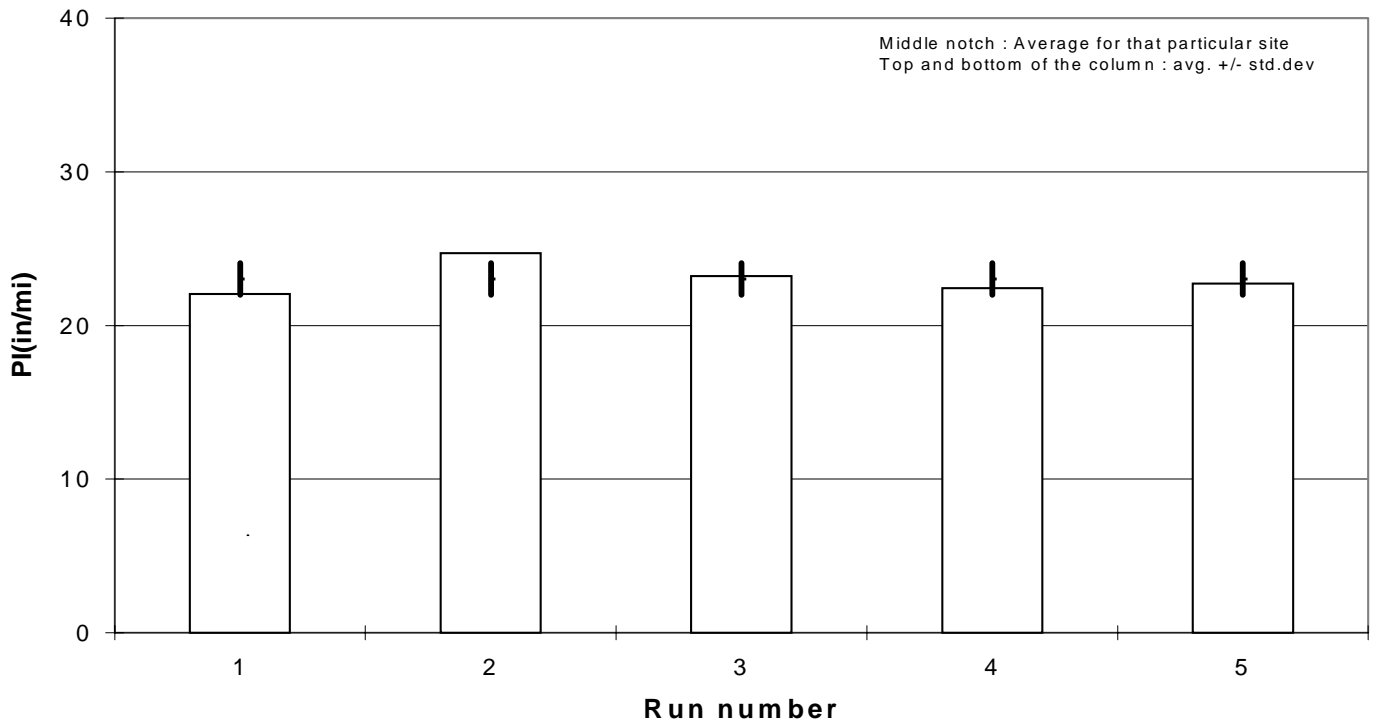

Figure B-24 PI-0.0"'blanking band for replicate runs at US 231B (Site 6) of Vendor C 


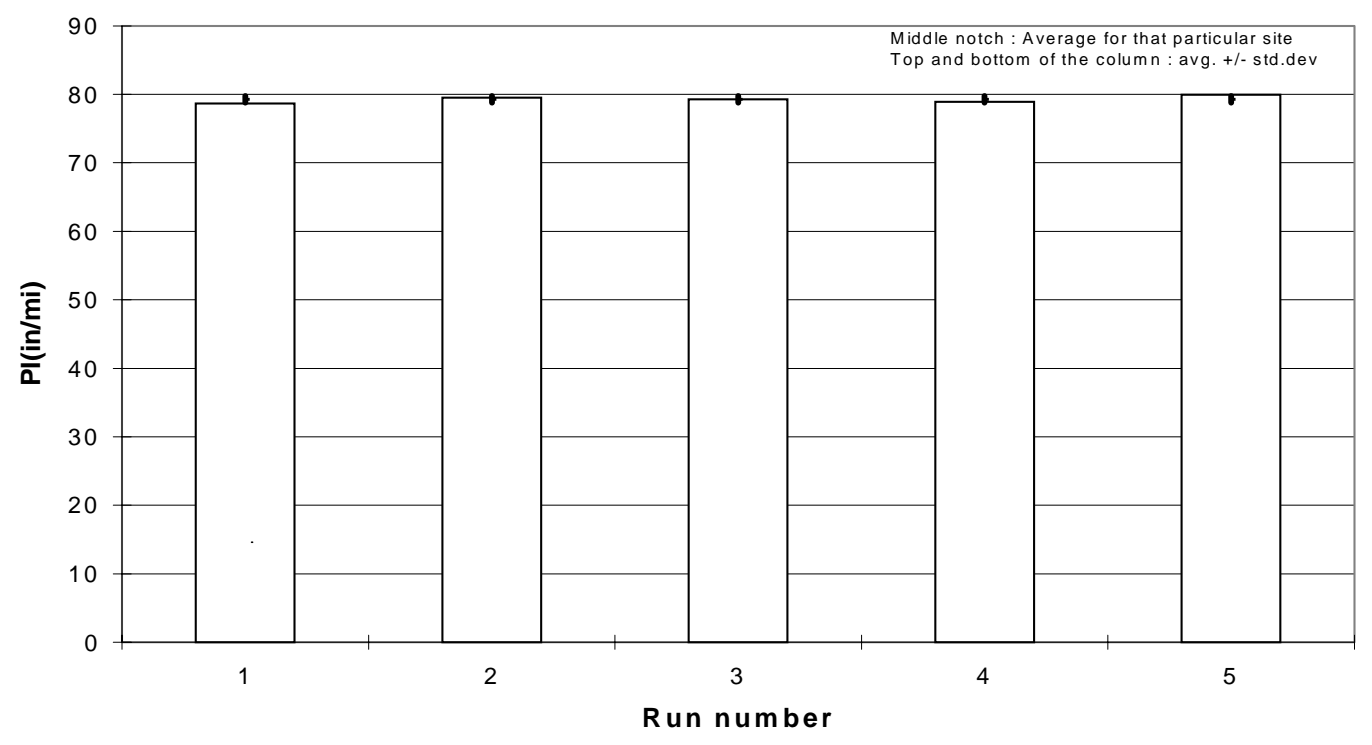

Figure B-25 PI-0.0’'blanking band for replicate runs at Covington Street (Site 4)

\section{of Vendor D}

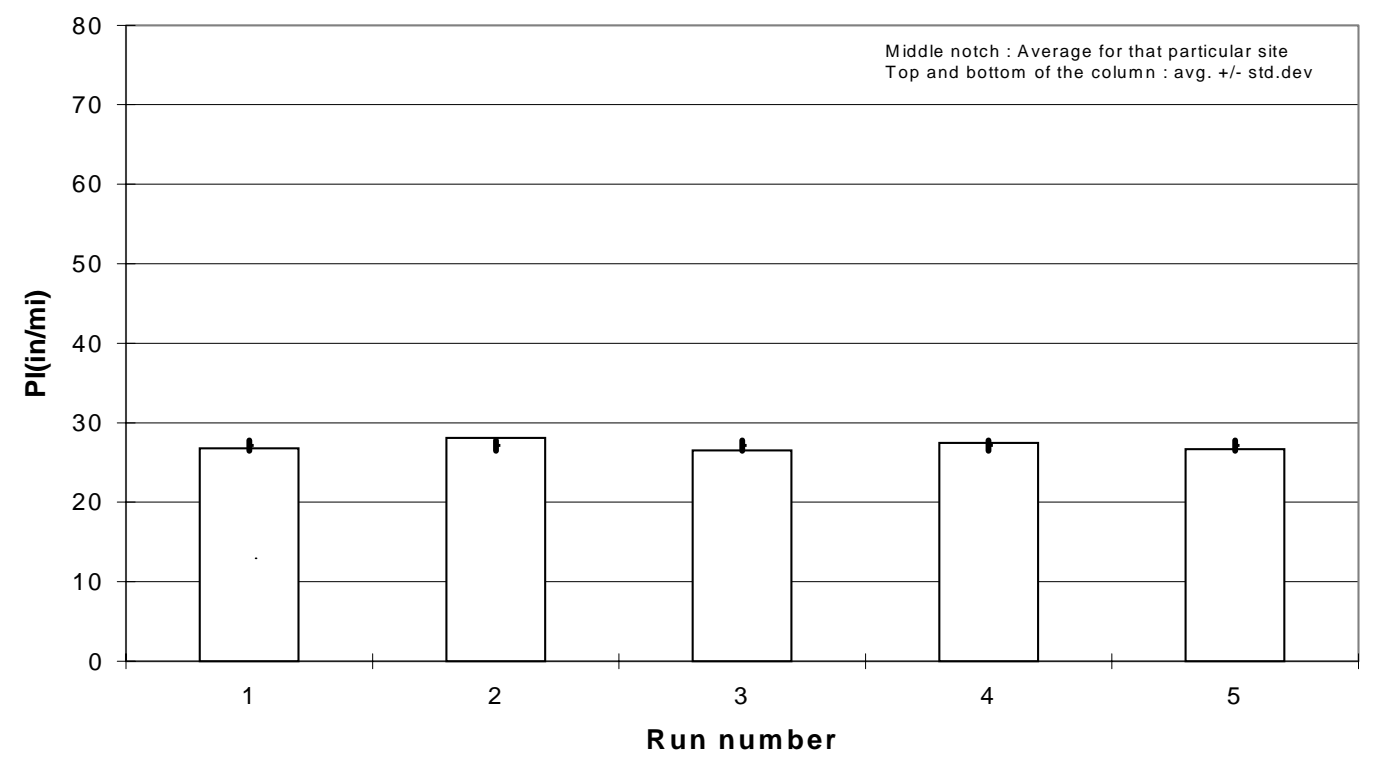

Figure B-26 PI-0.0'blanking band for replicate runs at I-65 (Site 2 with board) of Vendor D 


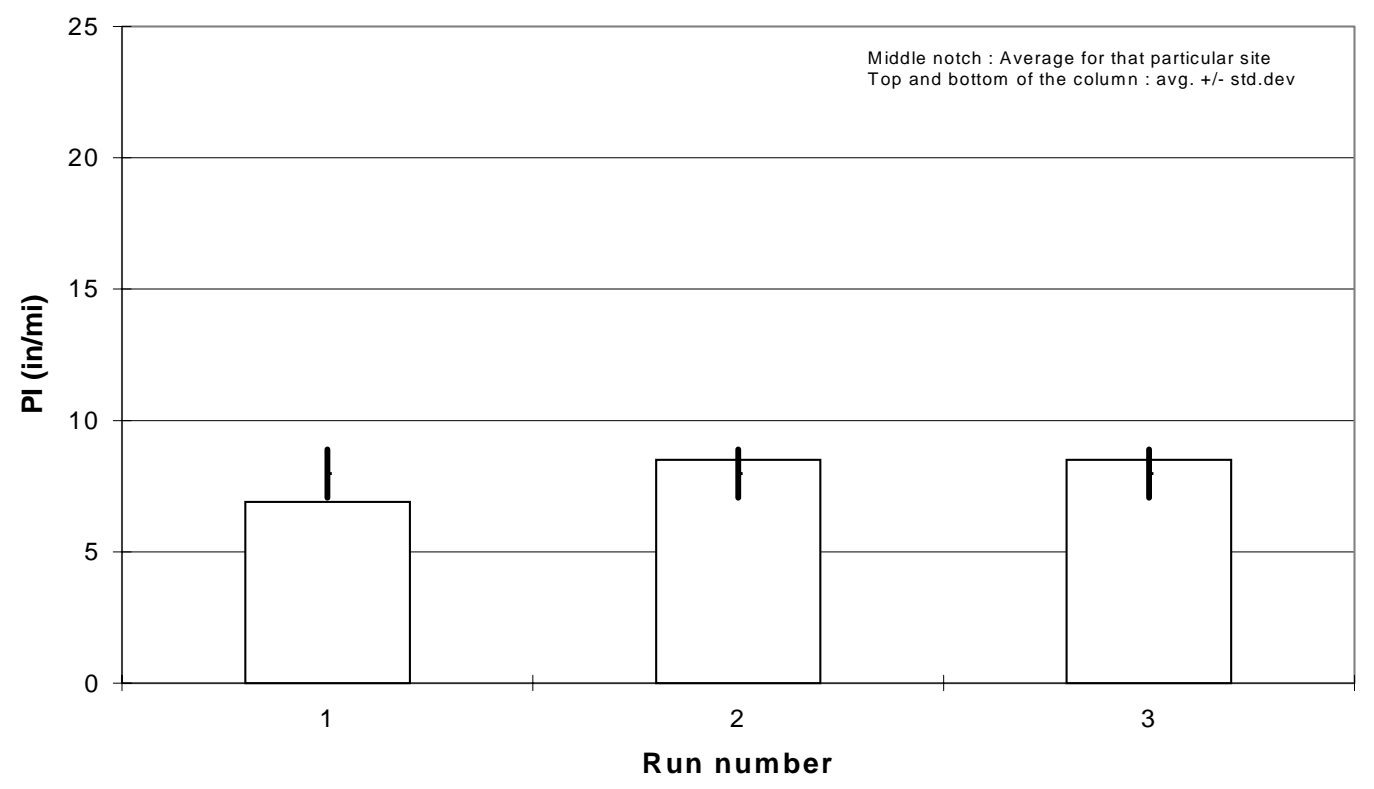

Figure B-27 PI-0.0’'blanking band for replicate runs at I-65 (Site 2 without board) of Vendor D

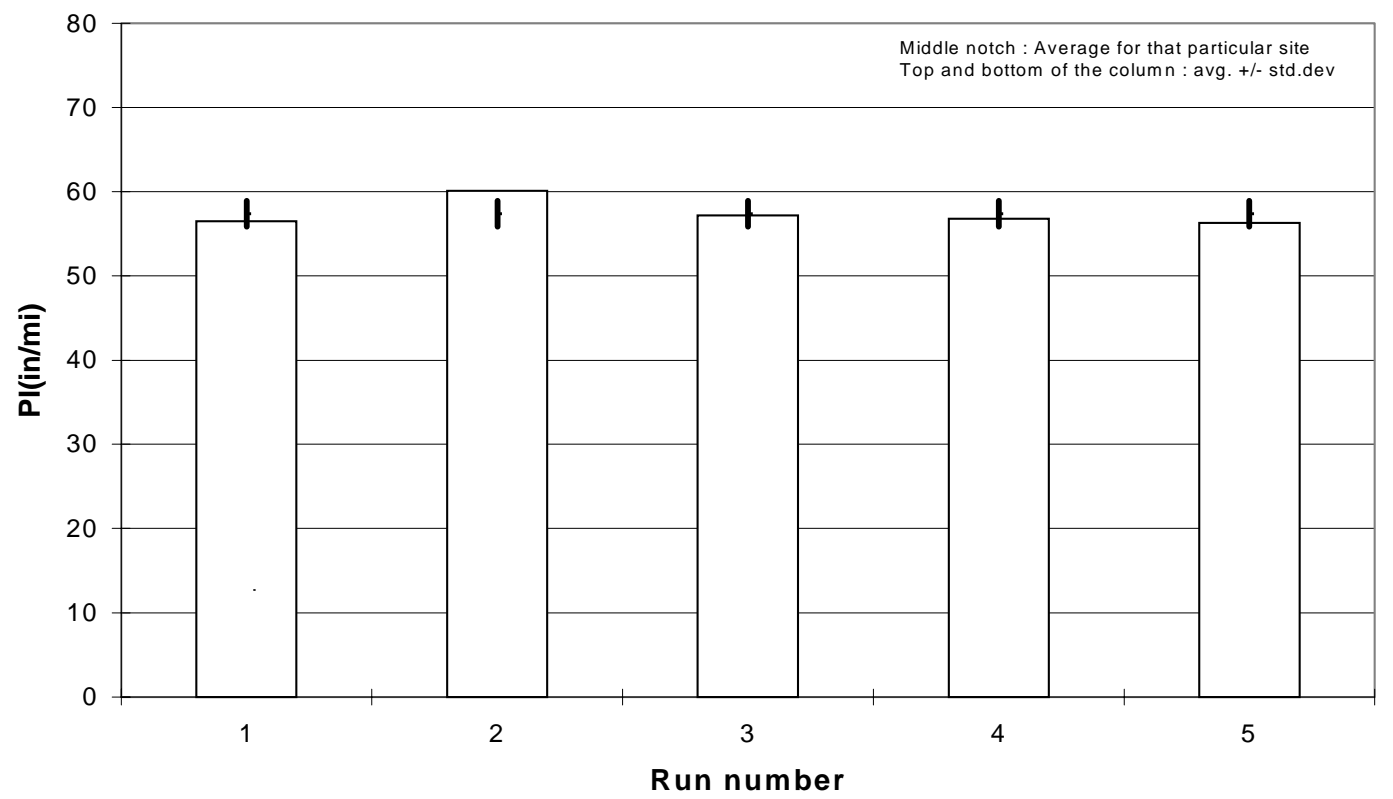

Figure B-28 PI-0.0'blanking band for replicate runs at Prairie Street (Site 3 with board) of Vendor $D$ 


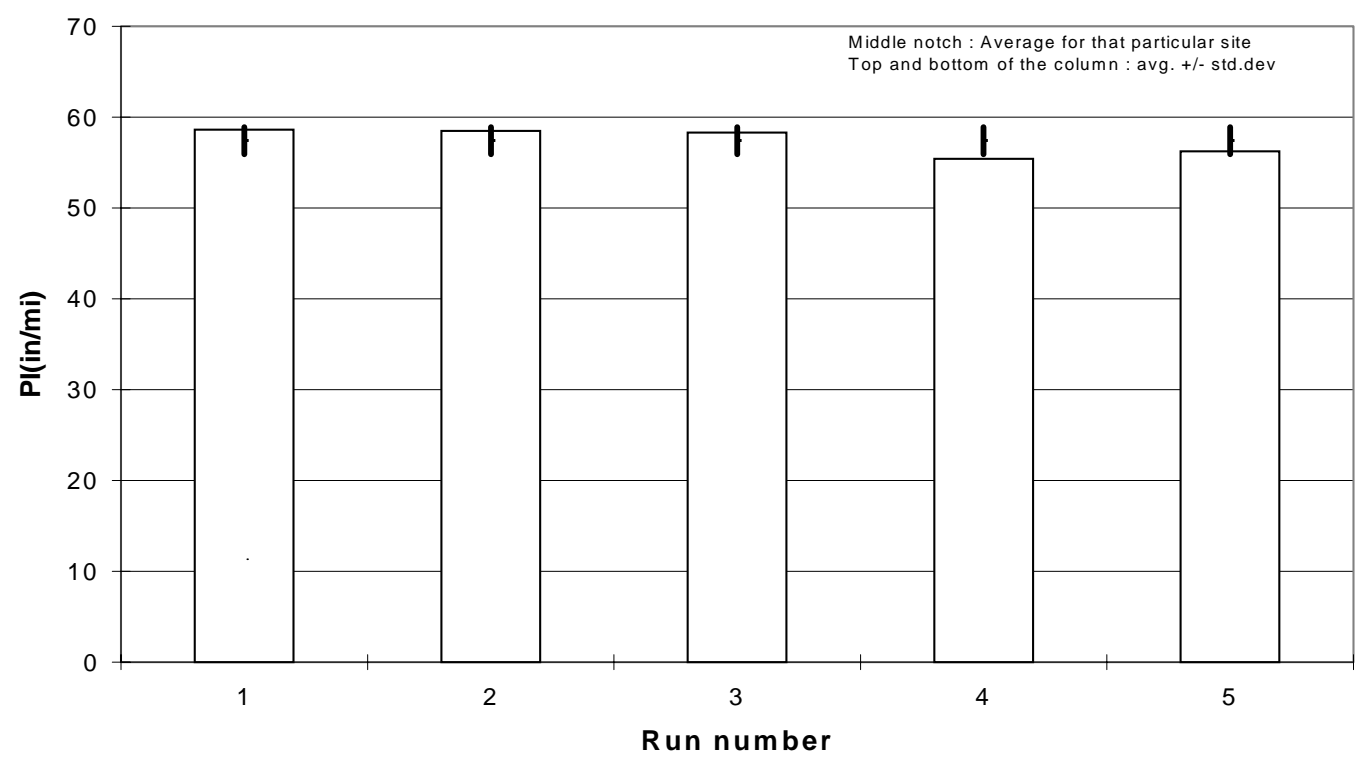

Figure B-29 PI-0.0’blanking band for replicate runs at Prairie Street

(Site 3 without board) of Vendor D

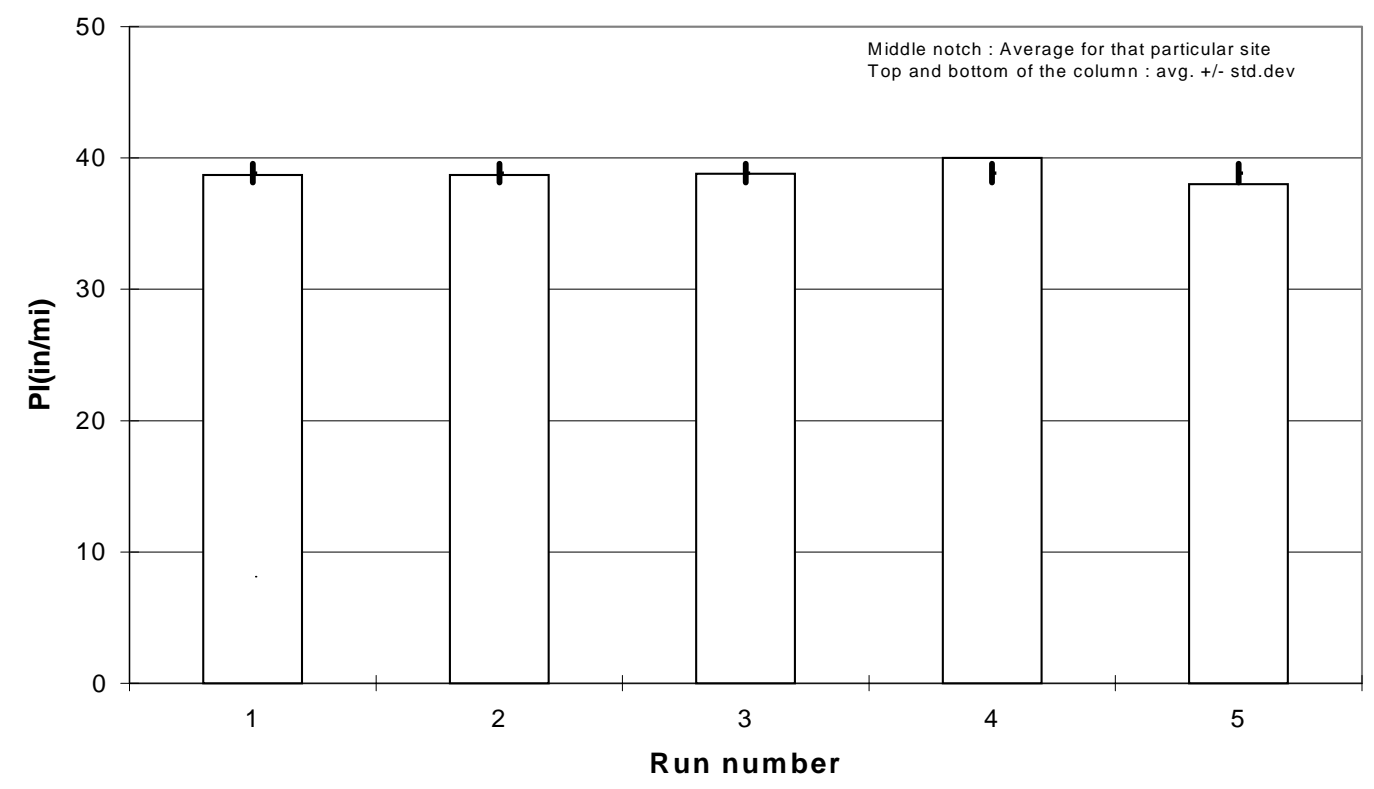

Figure B-30 PI-0.0'blanking band for replicate runs at Vinton Street (Site 4) of

Vendor D 


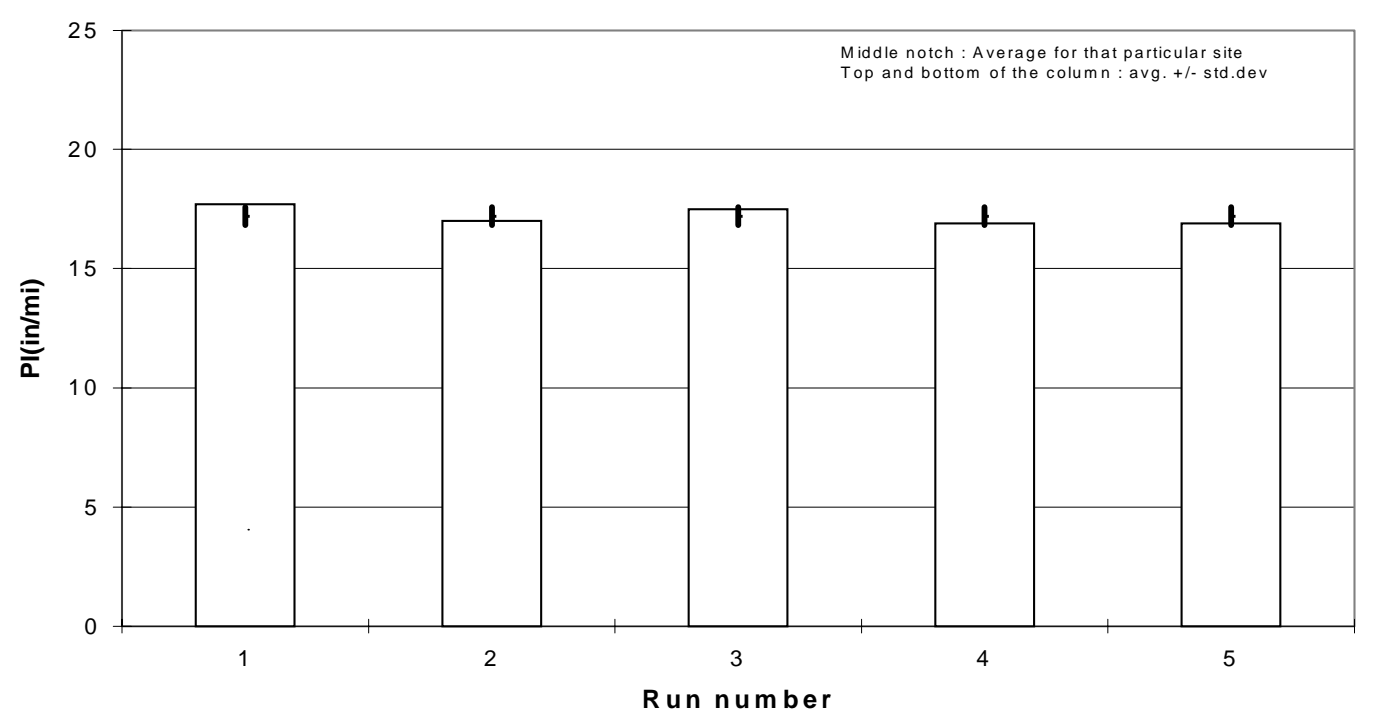

Figure B-31 PI-0.0'blanking band for replicate runs at US231A (Site 5) of Vendor D

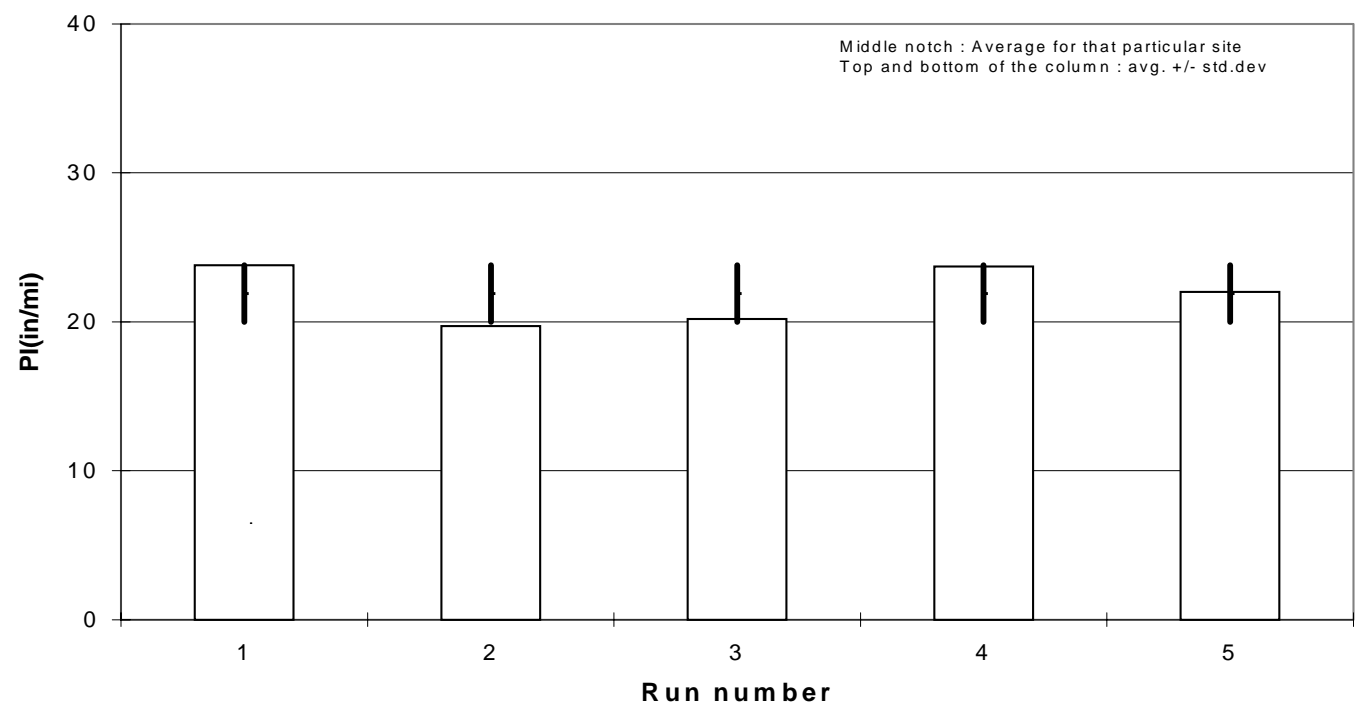

Figure B-32 PI-0.0'blanking band for replicate runs at US231B (Site 6) of Vendor D 
APPENDIX C - PI-0.2” Comparison-Within Vendors 


\section{APPENDIX C - PI-0.2” Comparison-Within Vendors}

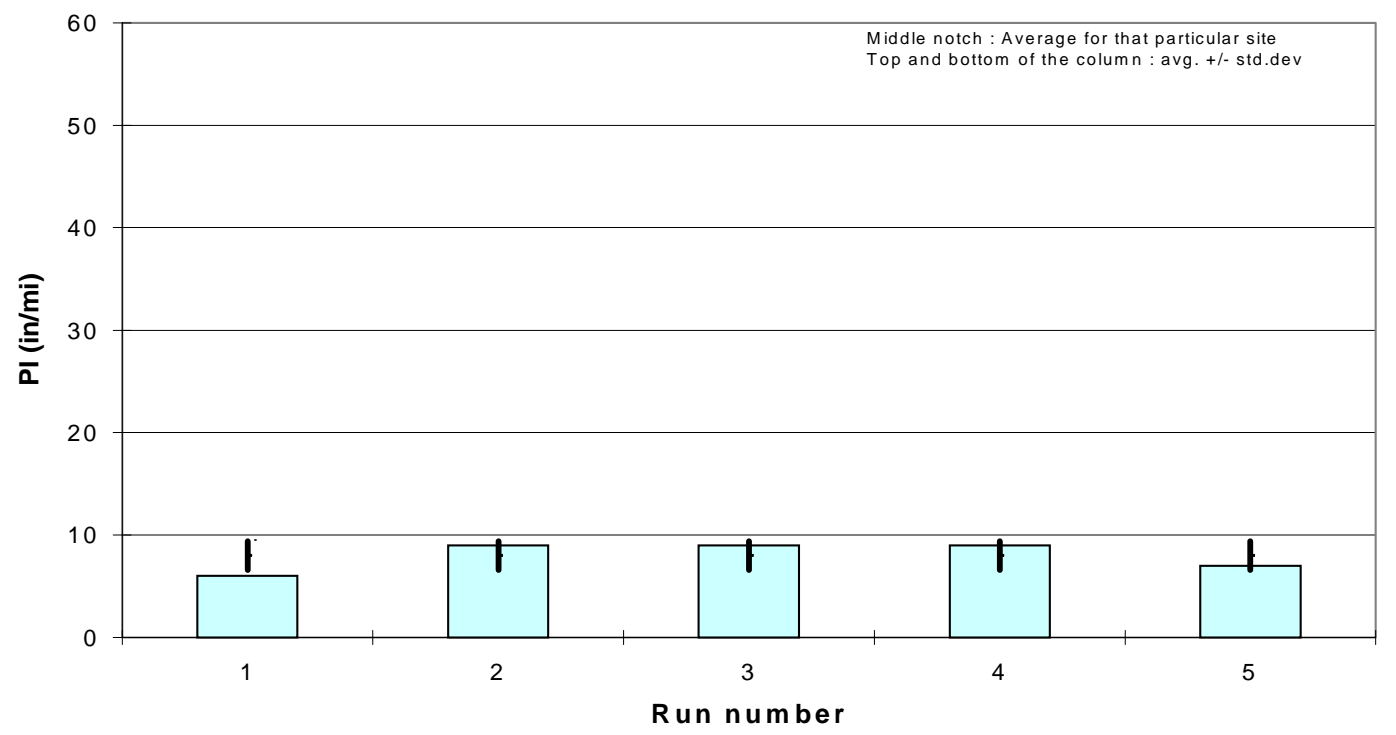

Figure C-1 PI-0.2”' blanking band for replicate runs at Covington (Site 1) of

Vendor A

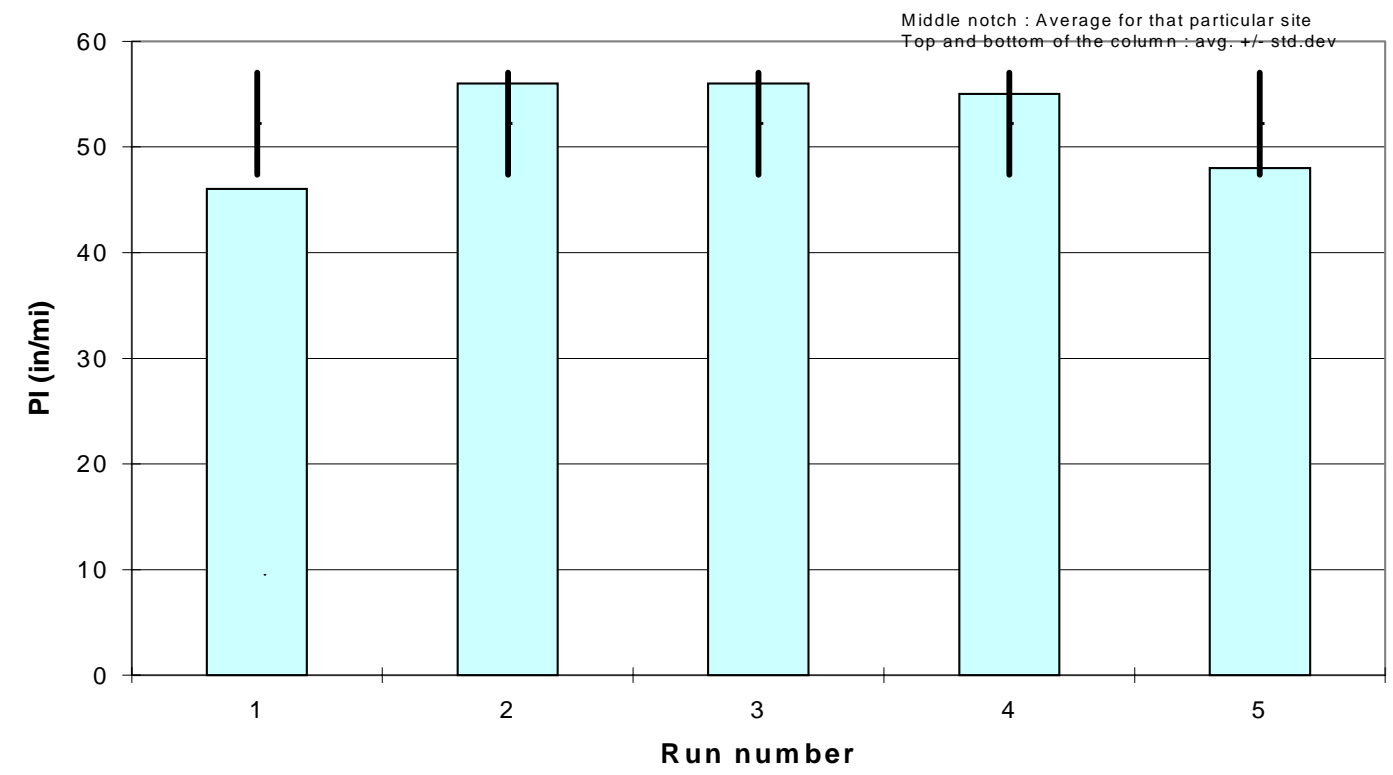

Figure C-2 PI-0.2” blanking band for replicate runs at I-65 (Site 2 with board) of Vendor A 


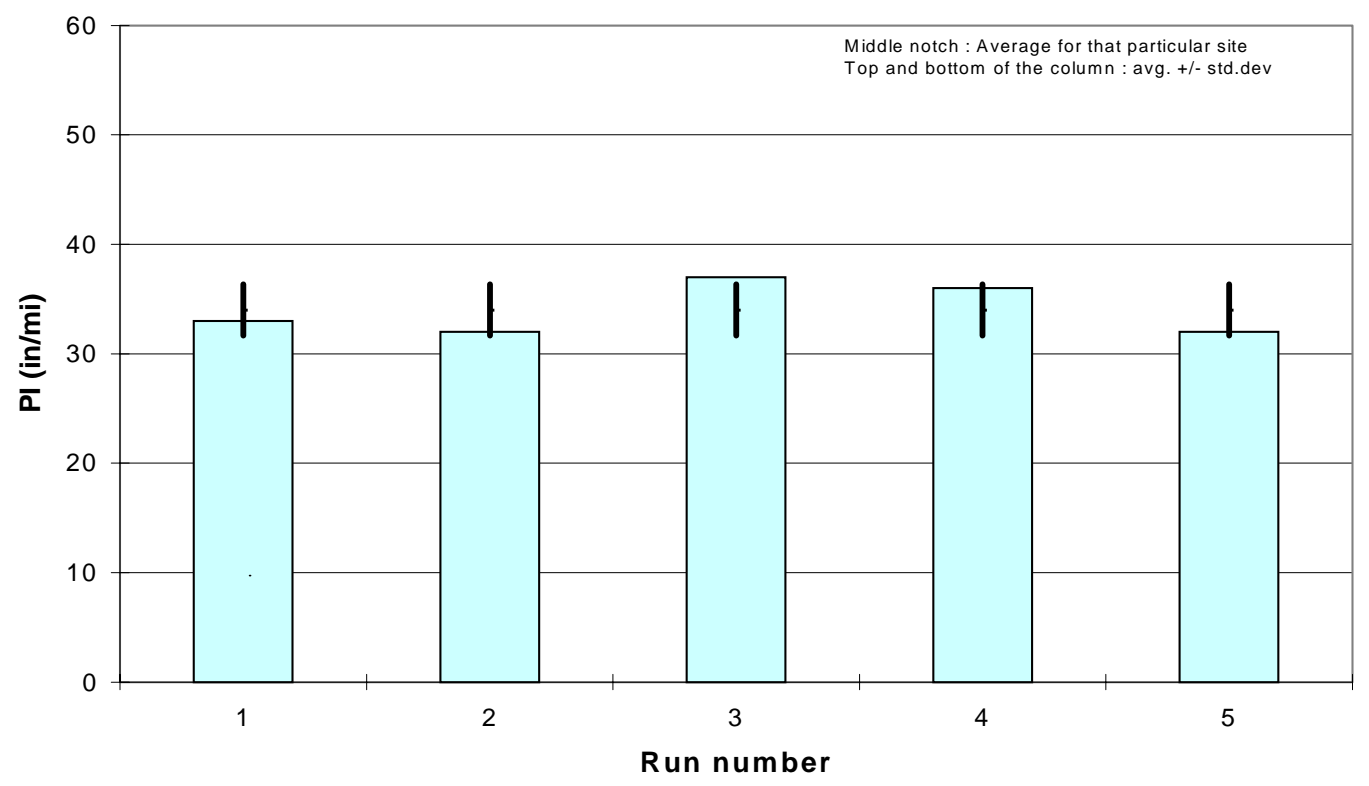

Figure C-3 PI-0.2" blanking band for replicate runs at Prairie Street

(Site 3 with board) of Vendor A

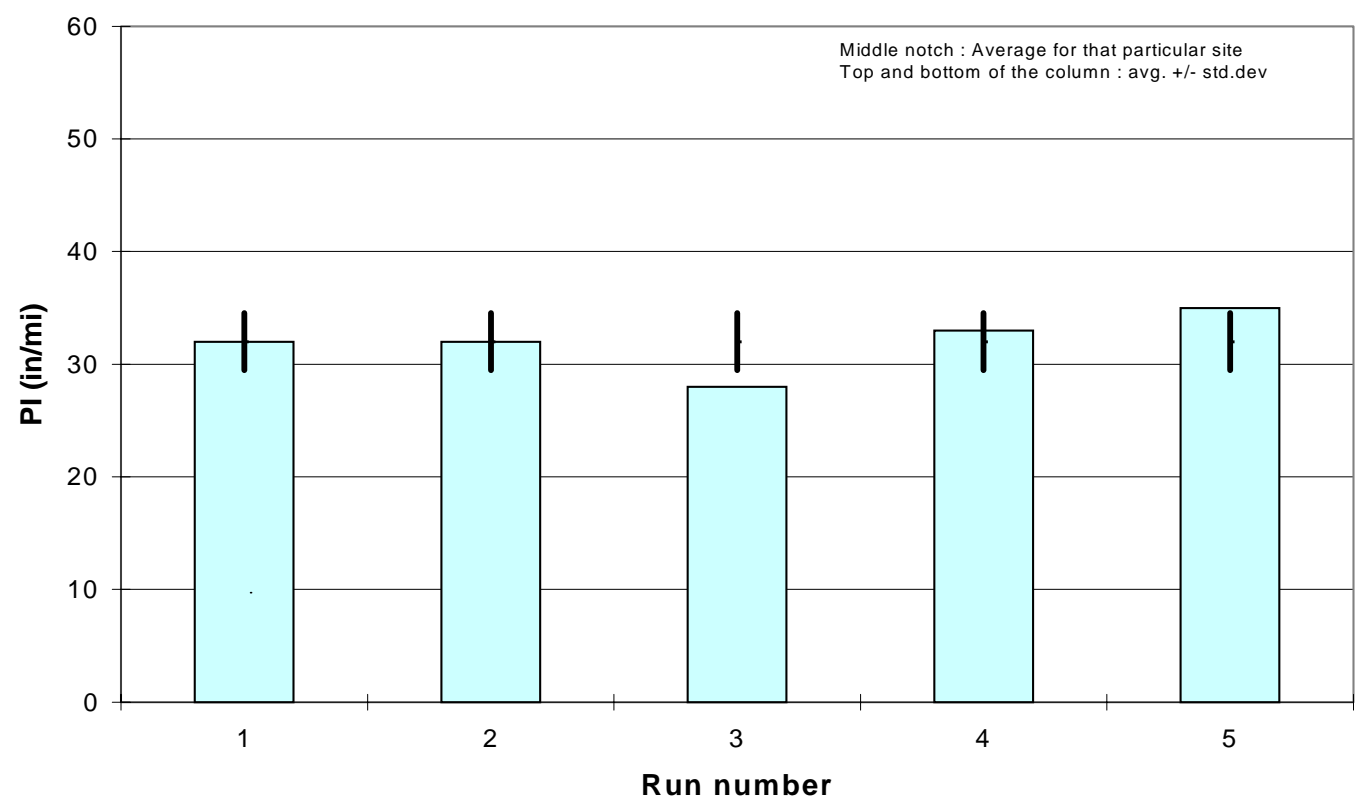

Figure C-4 PI-0.2" blanking band for replicate runs at Prairie Street

(Site 3 without board) of Vendor A 


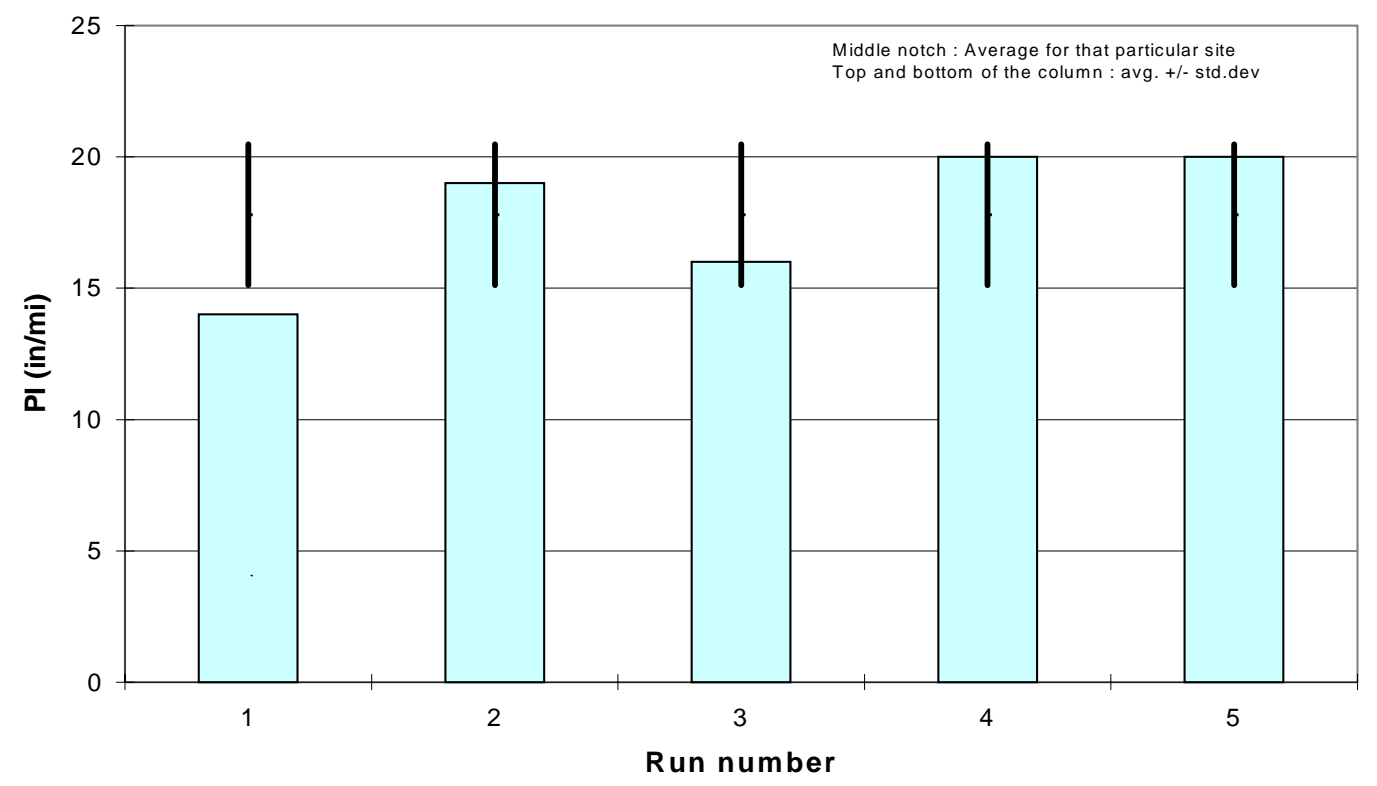

Figure C-5 PI-0.2" blanking band for replicate runs at Vinton Street (Site 4) of

Vendor A

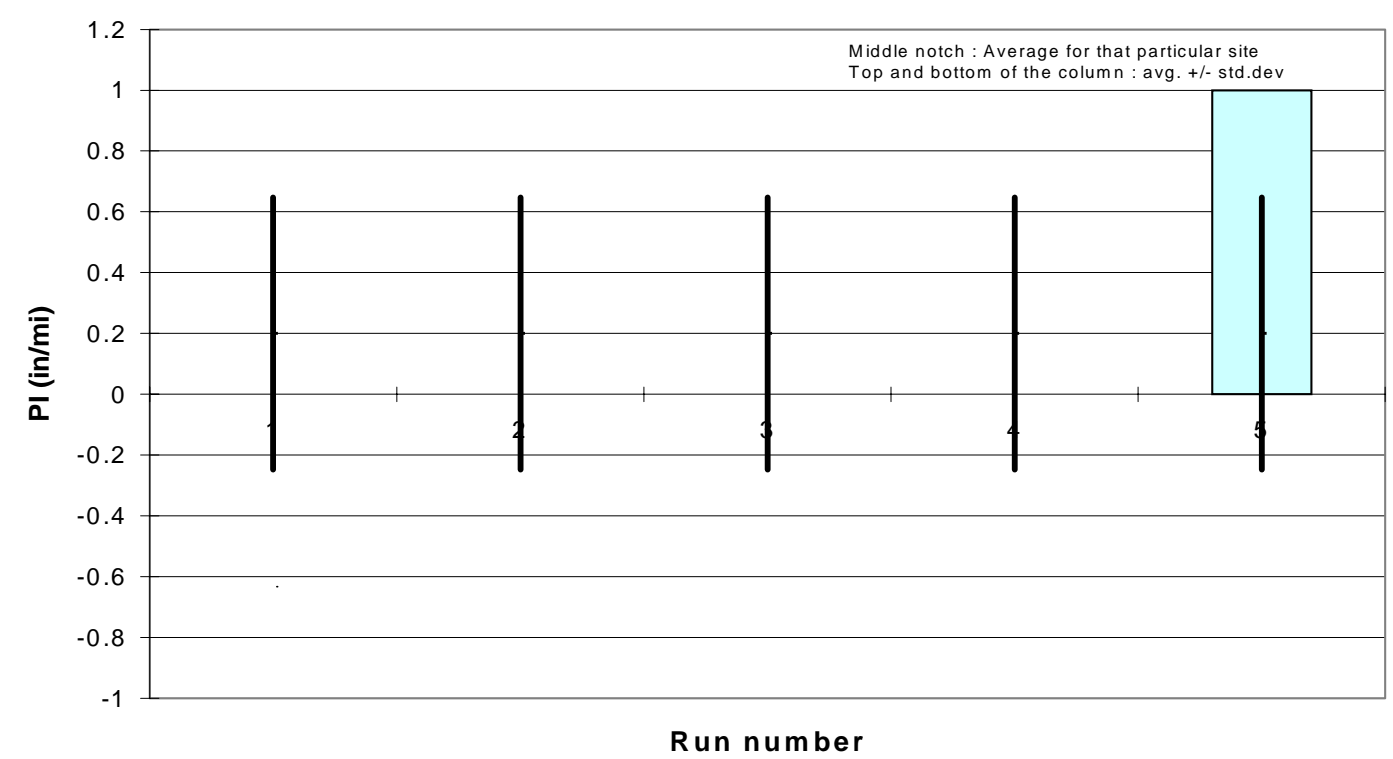

Figure C-6 PI-0.2" blanking band for replicate runs at US 231A (Site 5) of

Vendor A 


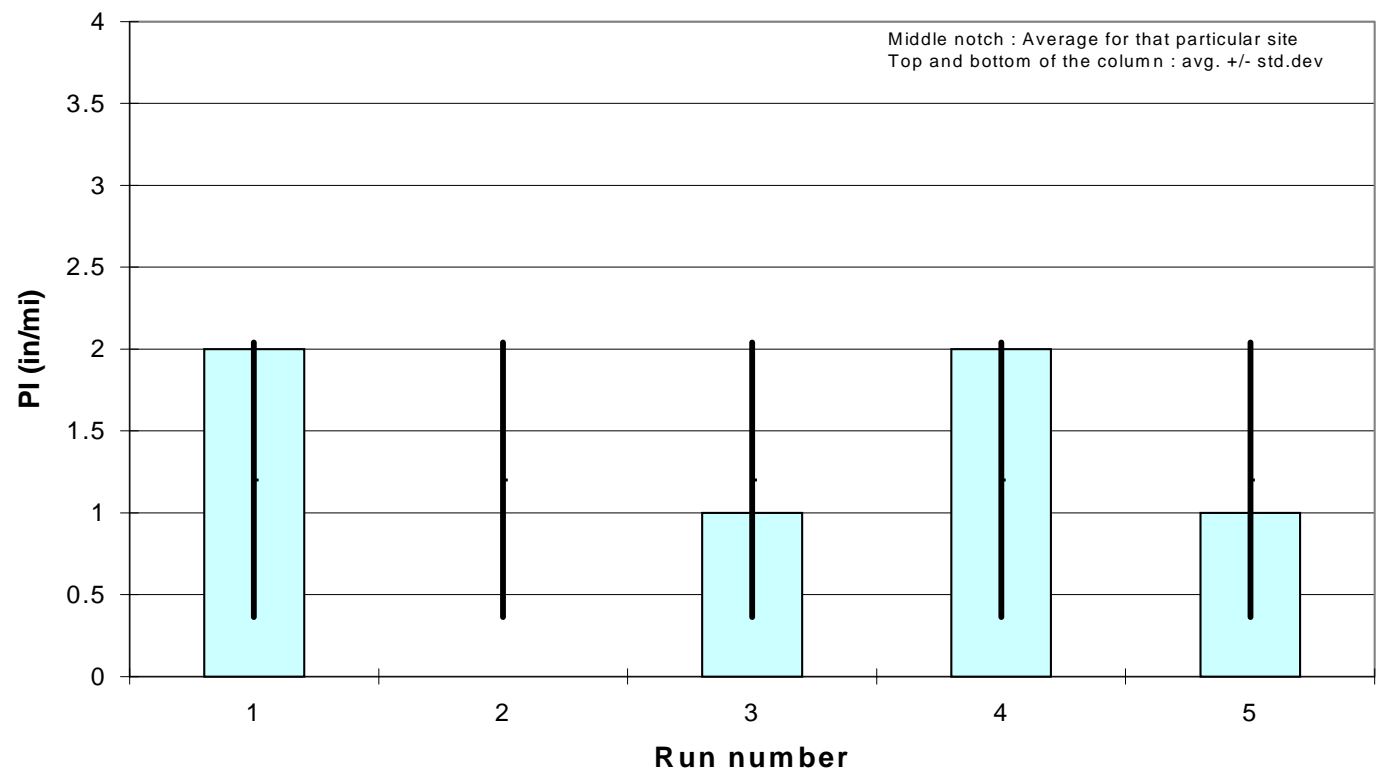

Figure C-7 PI-0.2" blanking band for replicate runs at US-231B (Site 6) of

Vendor A

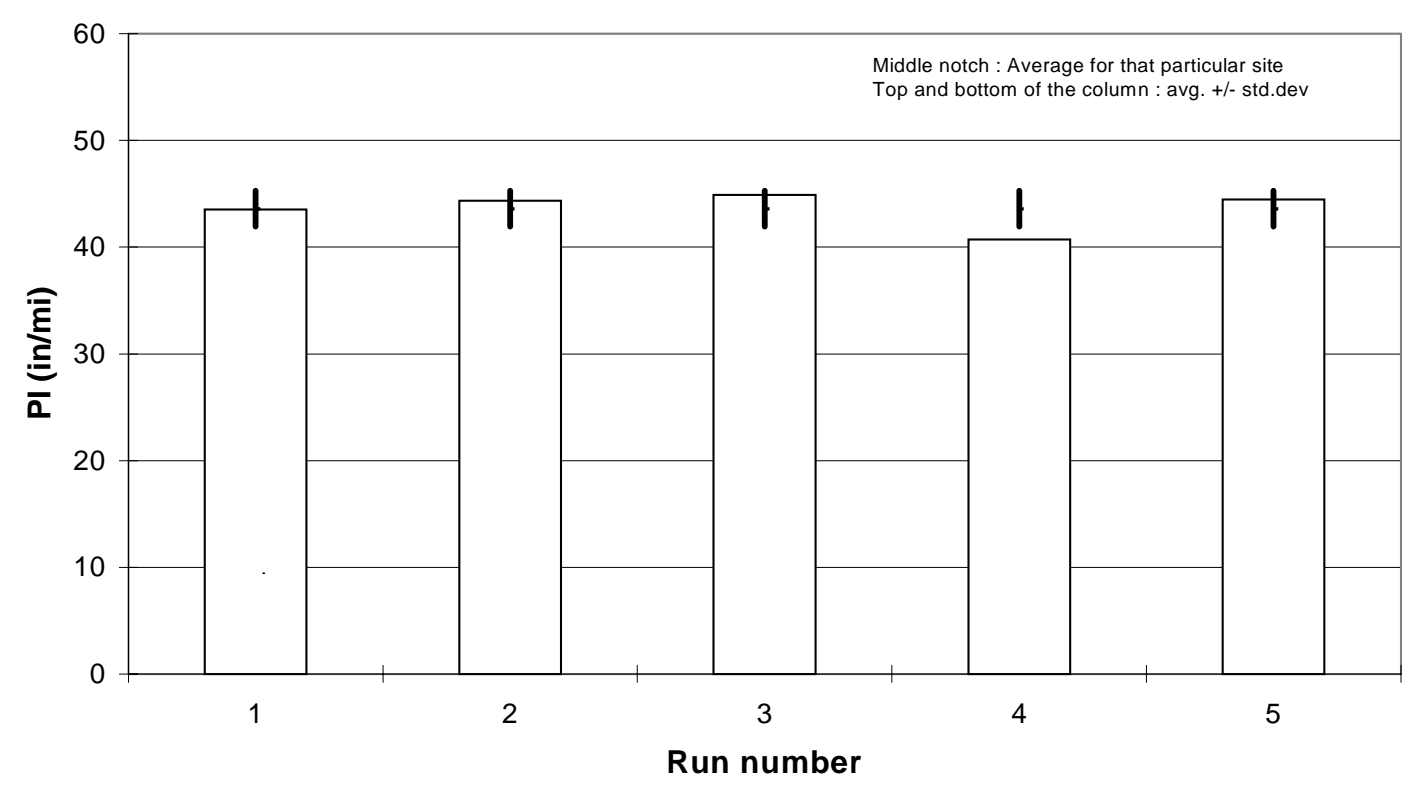

Figure C-8 PI-0.2" blanking band for replicate runs at Covington (Site 1) of Vendor B 


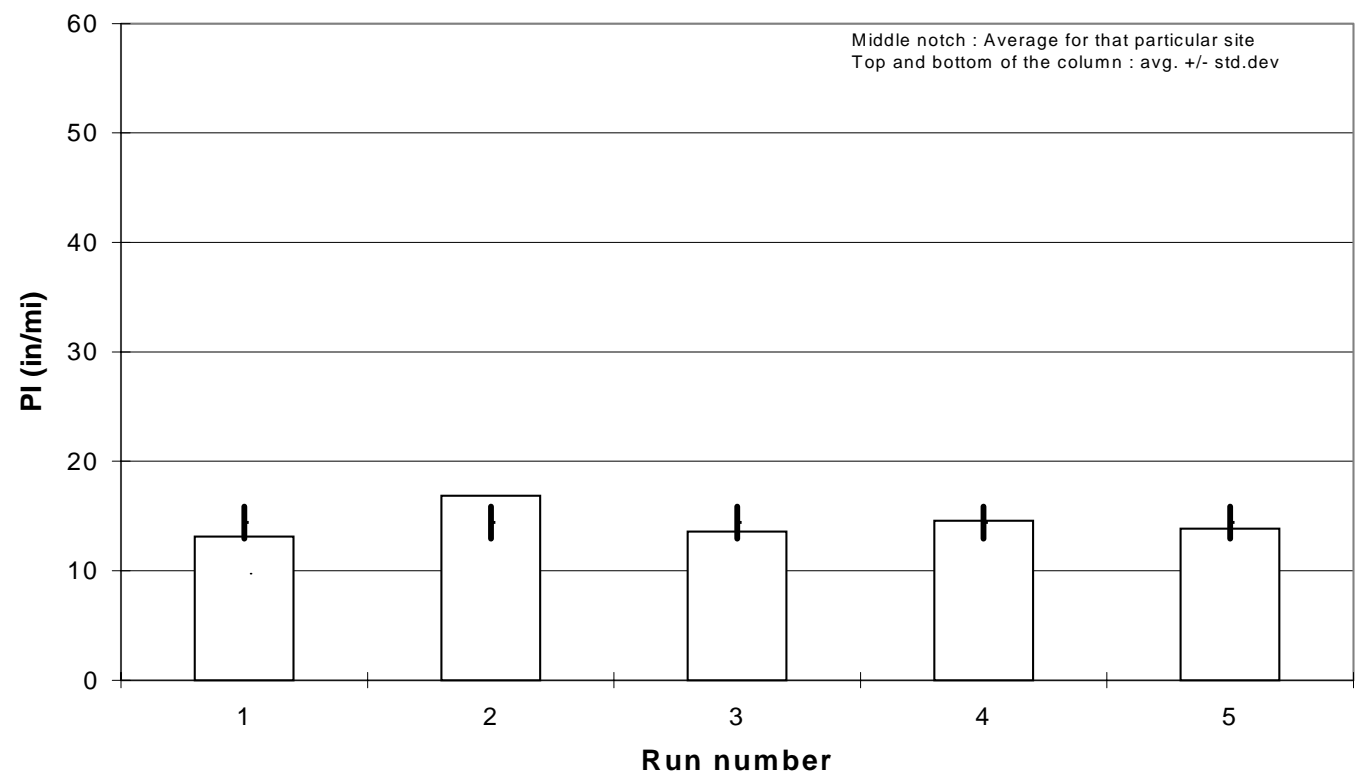

Figure C-9 PI-0.2" blanking band for replicate runs at I-65 (Site 2 with board) of Vendor B

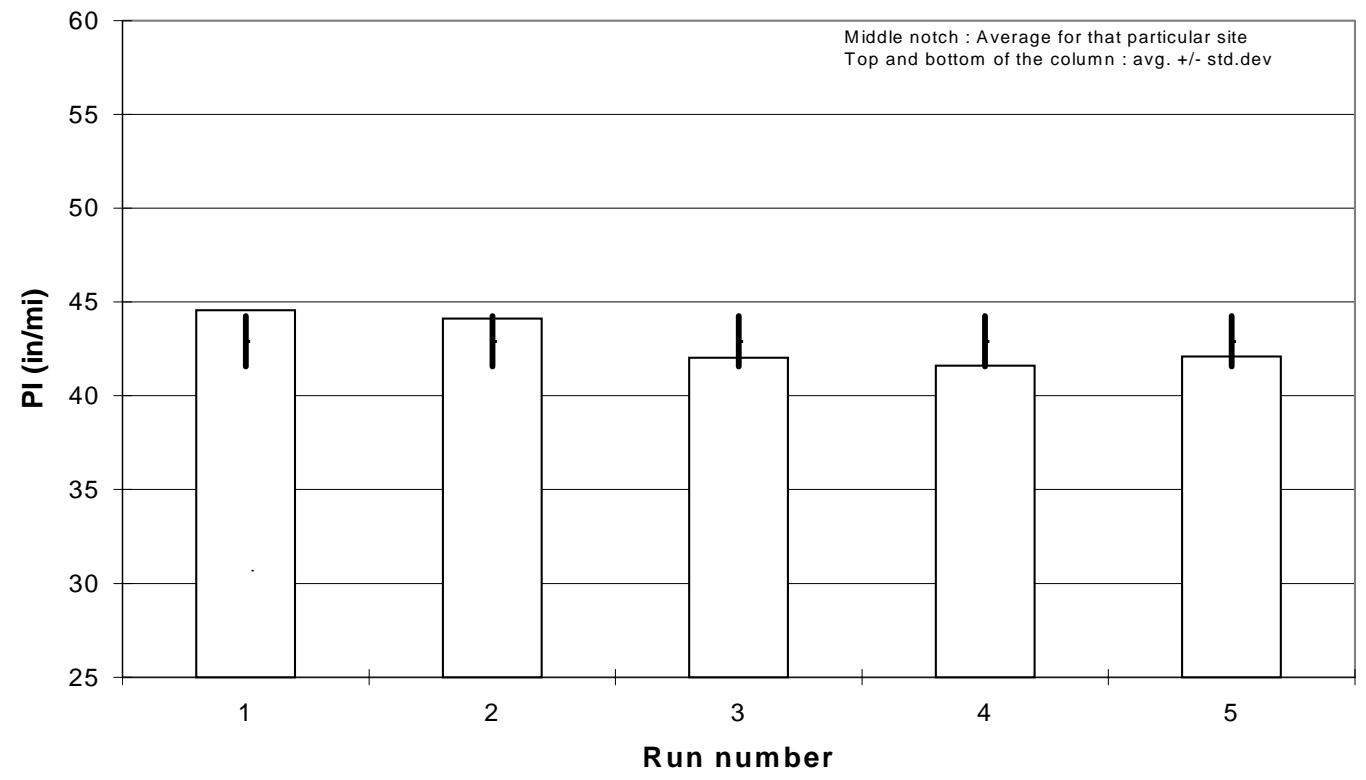

Figure C-10 PI-0.2" blanking band for replicate runs at Prairie Street (Site 3 with board) of Vendor B 


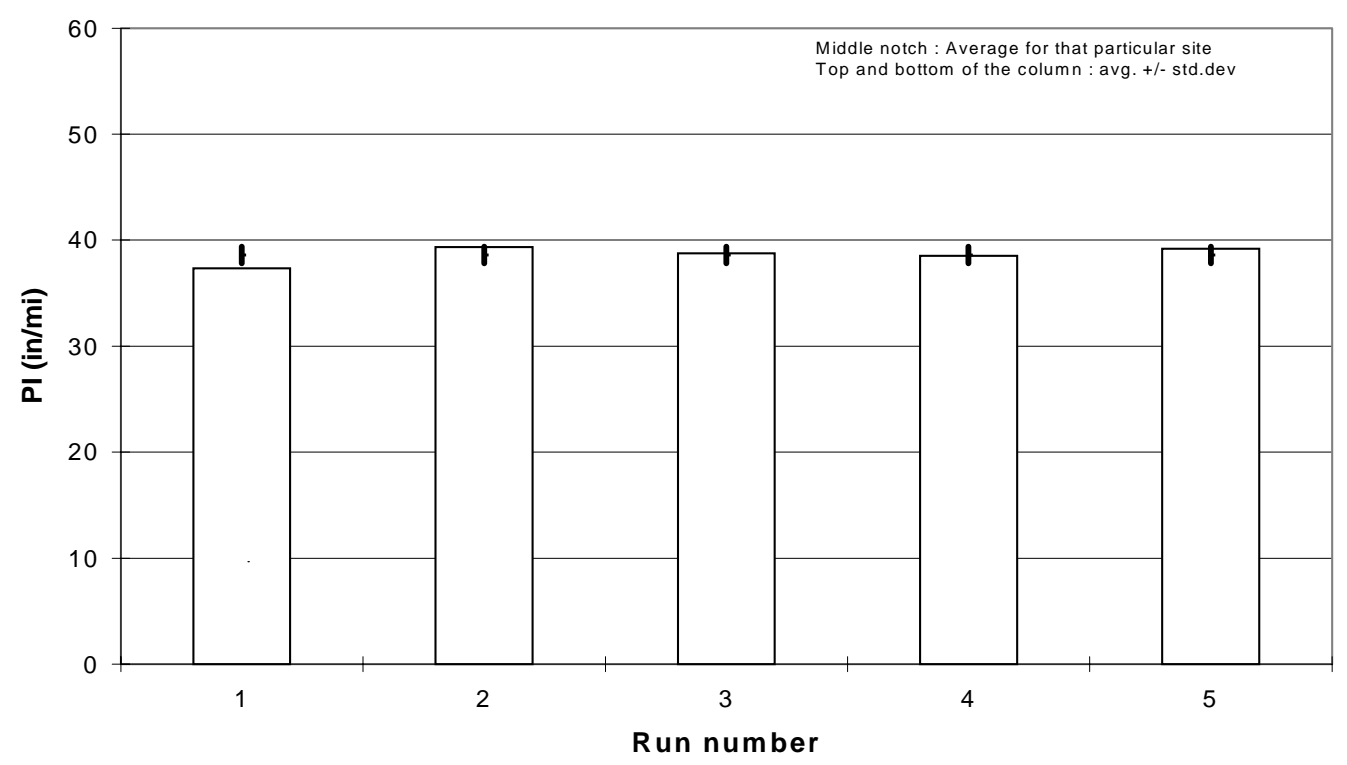

Figure C-11 PI-0.2" blanking band for replicate runs at Prairie Street

(Site 3 without board) of Vendor B

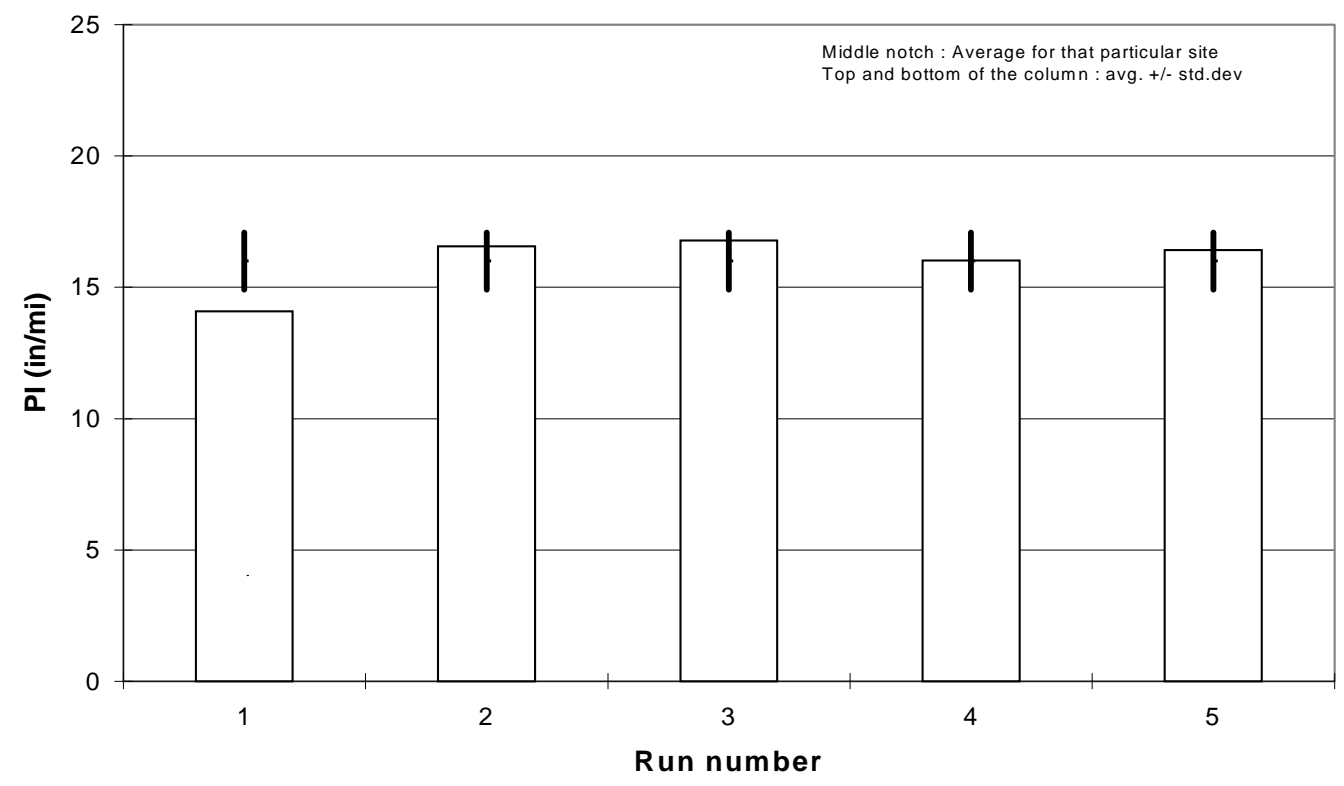

Figure C-12 PI-0.2" blanking band for replicate runs at Vinton Street (Site 4) of Vendor B 


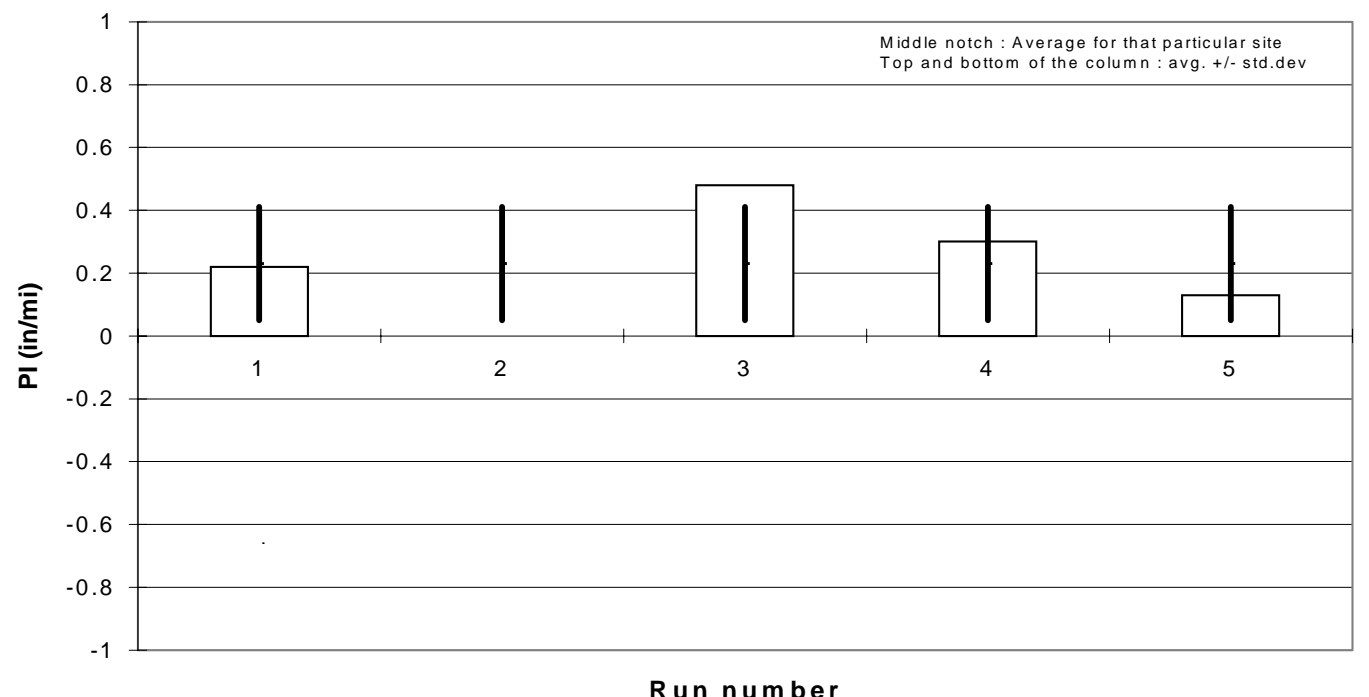

Figure C-13 PI-0.2" blanking band for replicate runs at US 231A (Site 5) of

Vendor B

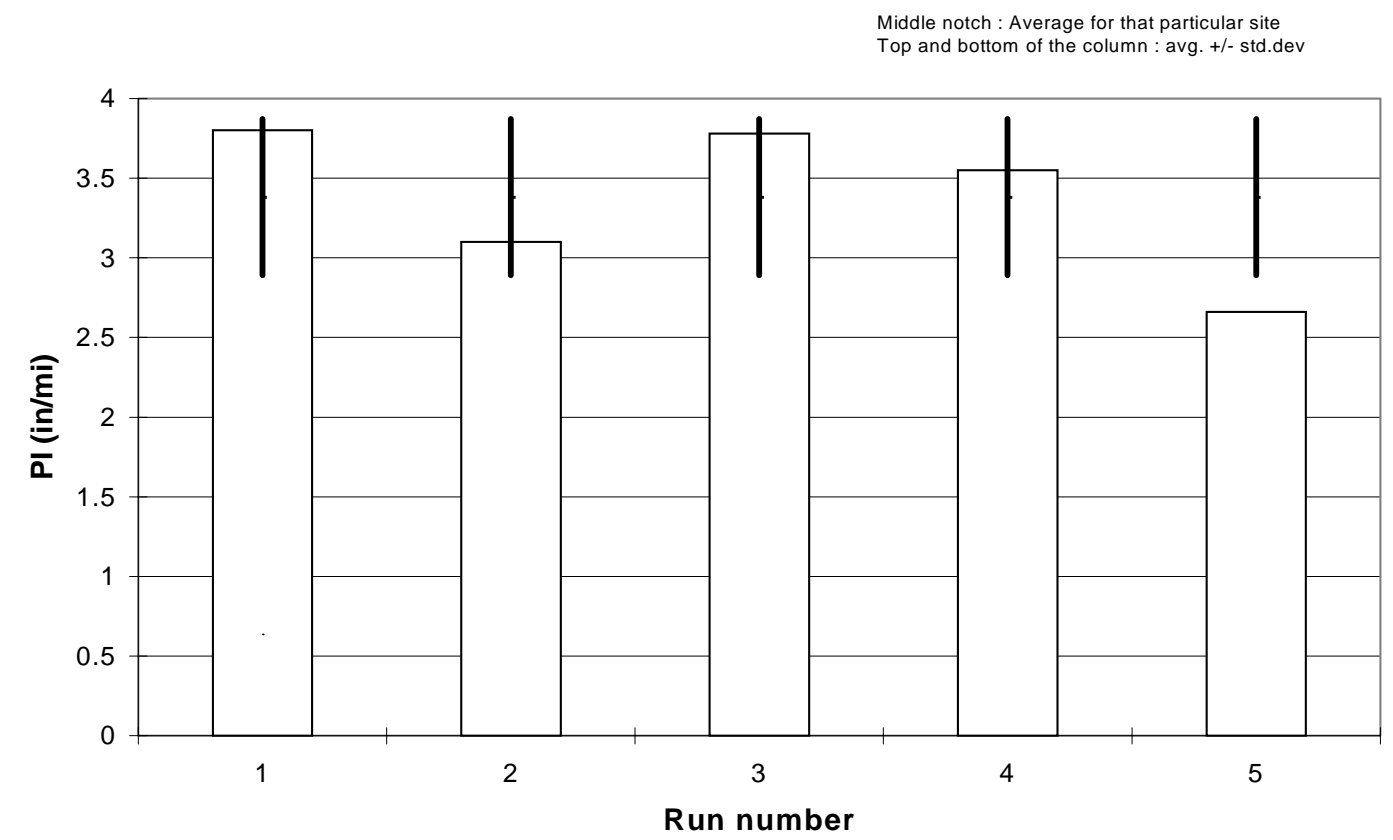

Figure C-14 PI-0.2" blanking band for replicate runs at US 231B (Site 6) of Vendor B 


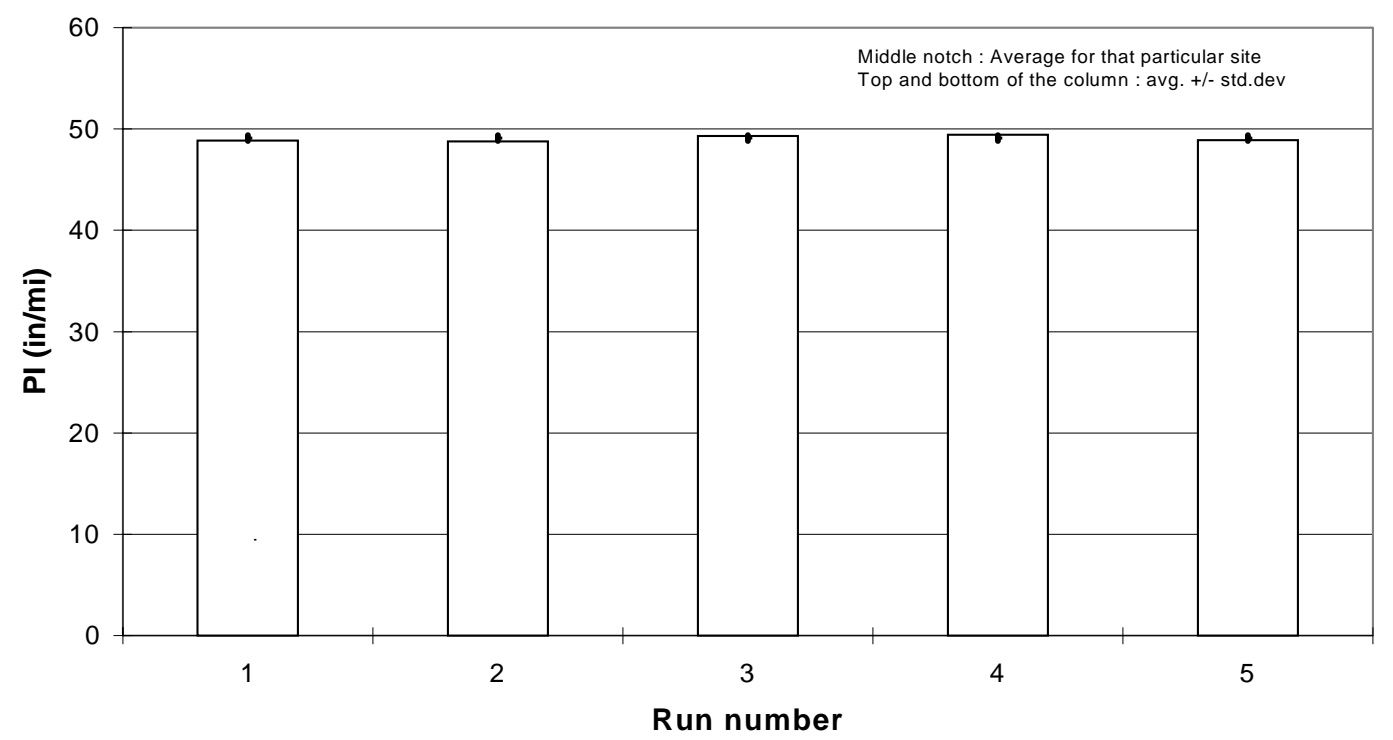

Figure C-15 PI-0.2" blanking band for replicate runs at Covington (Site 1) of

Vendor C

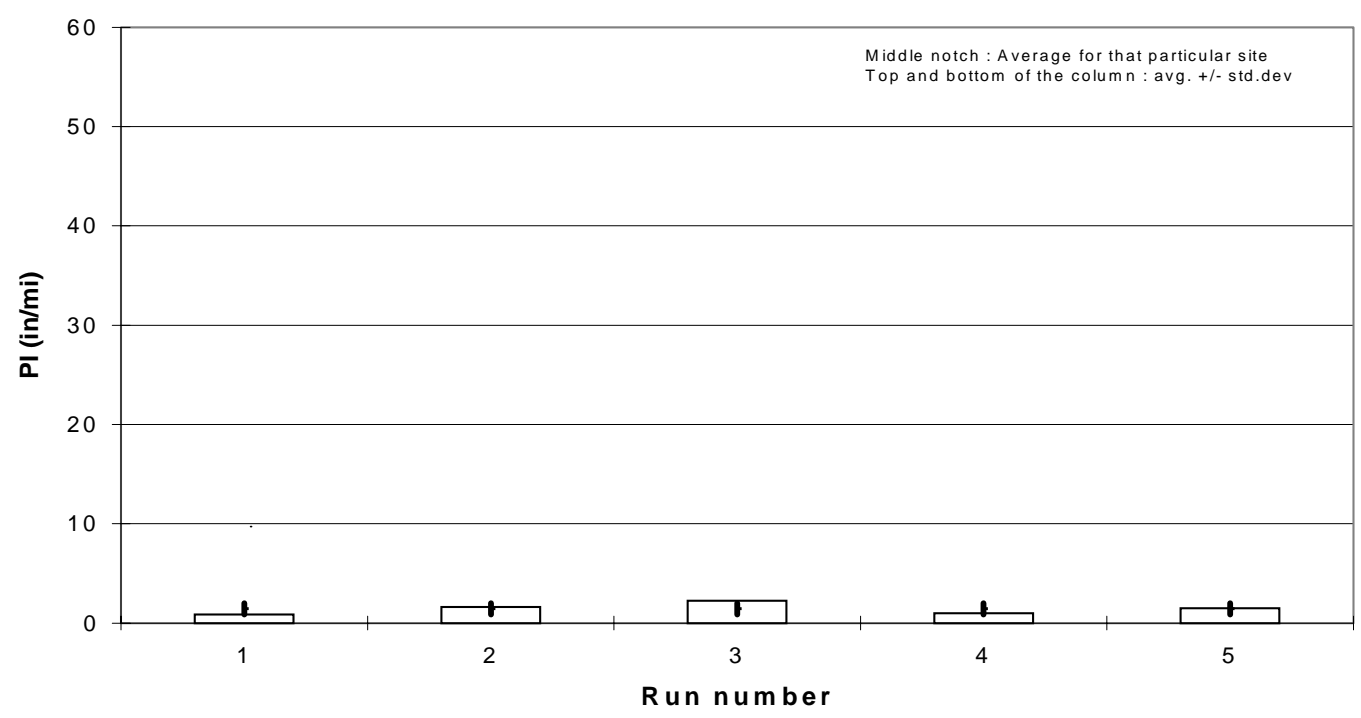

Figure C-16 PI-0.2" blanking band for replicate runs at I-65 (Site 2 with board)

of Vendor C 


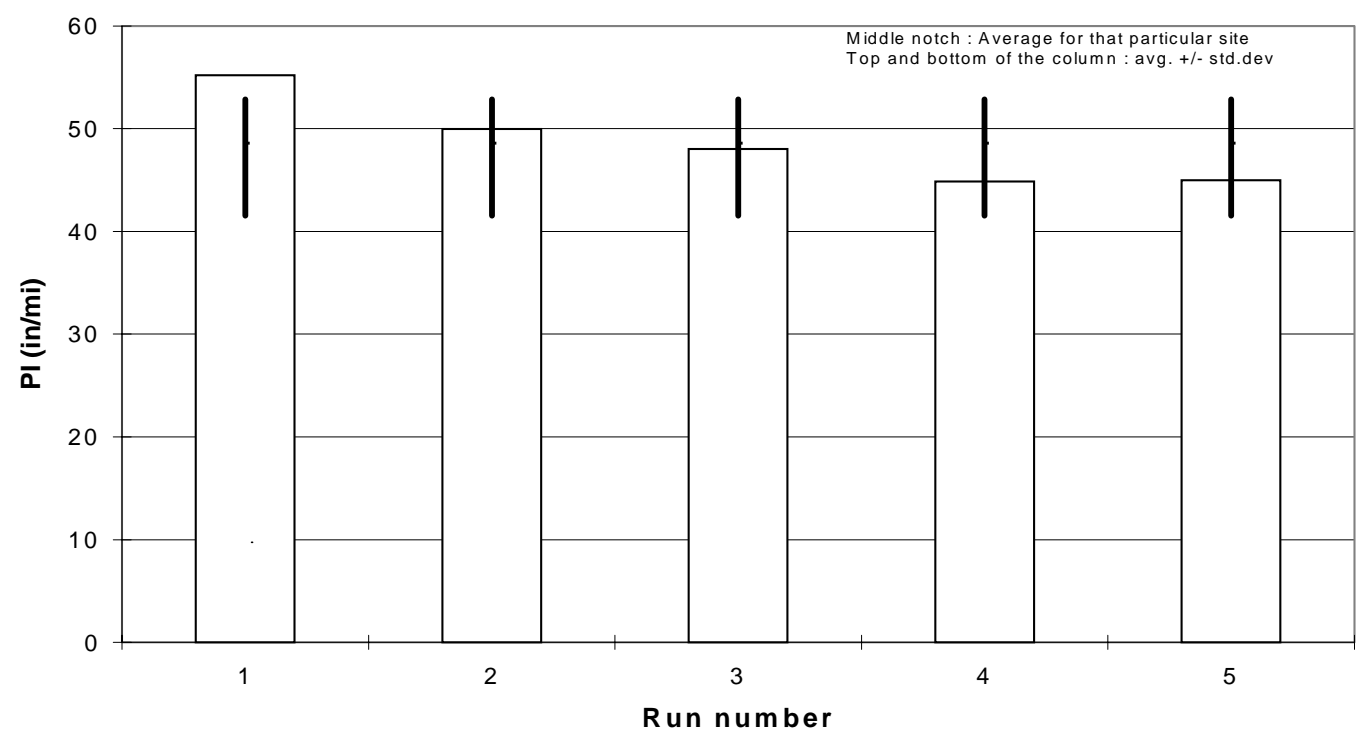

Figure C-17 PI-0.2" blanking band for replicate runs at Prairie Street

(Site 3 with board) of Vendor C

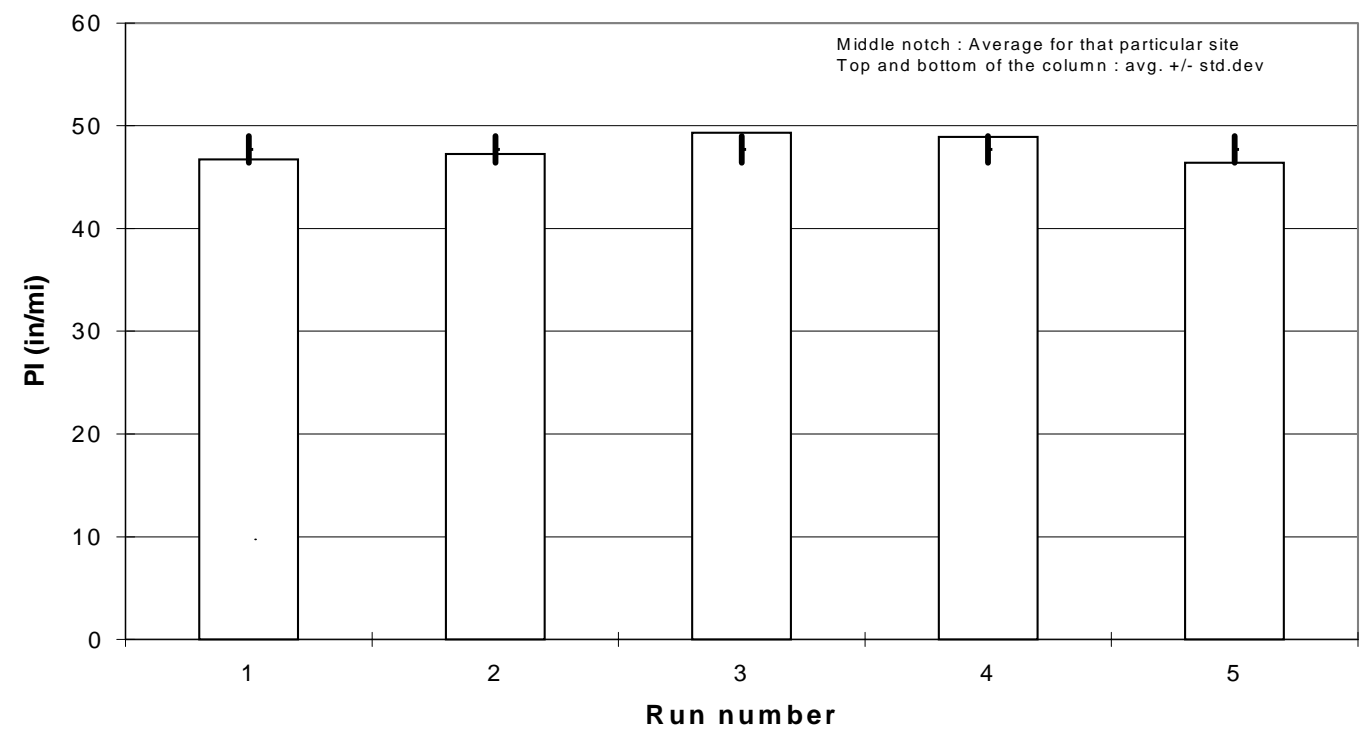

Figure C-18 PI-0.2" blanking band for replicate runs at Prairie Street

(Site 3 without board) of Vendor C 


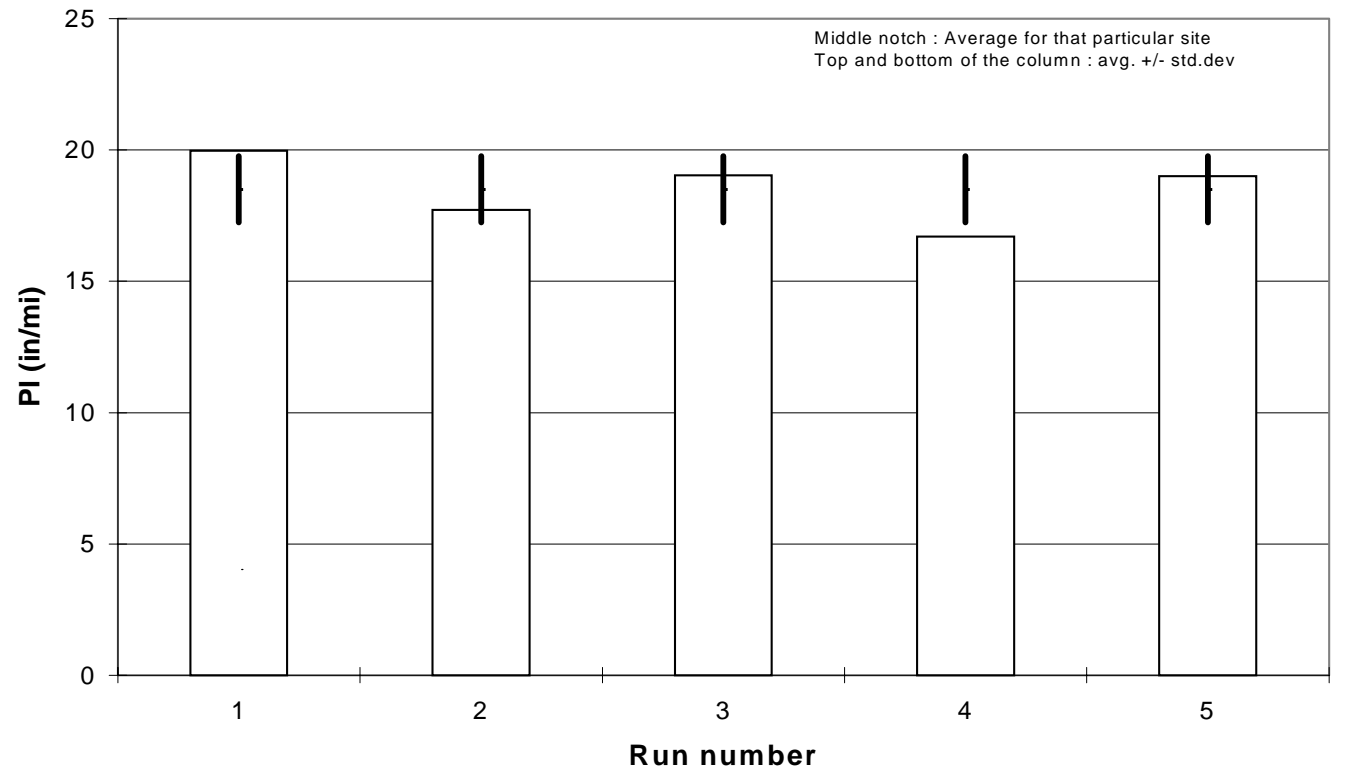

Figure C-19 PI-0.2" blanking band for replicate runs at Vinton Street (Site 4)

of Vendor C

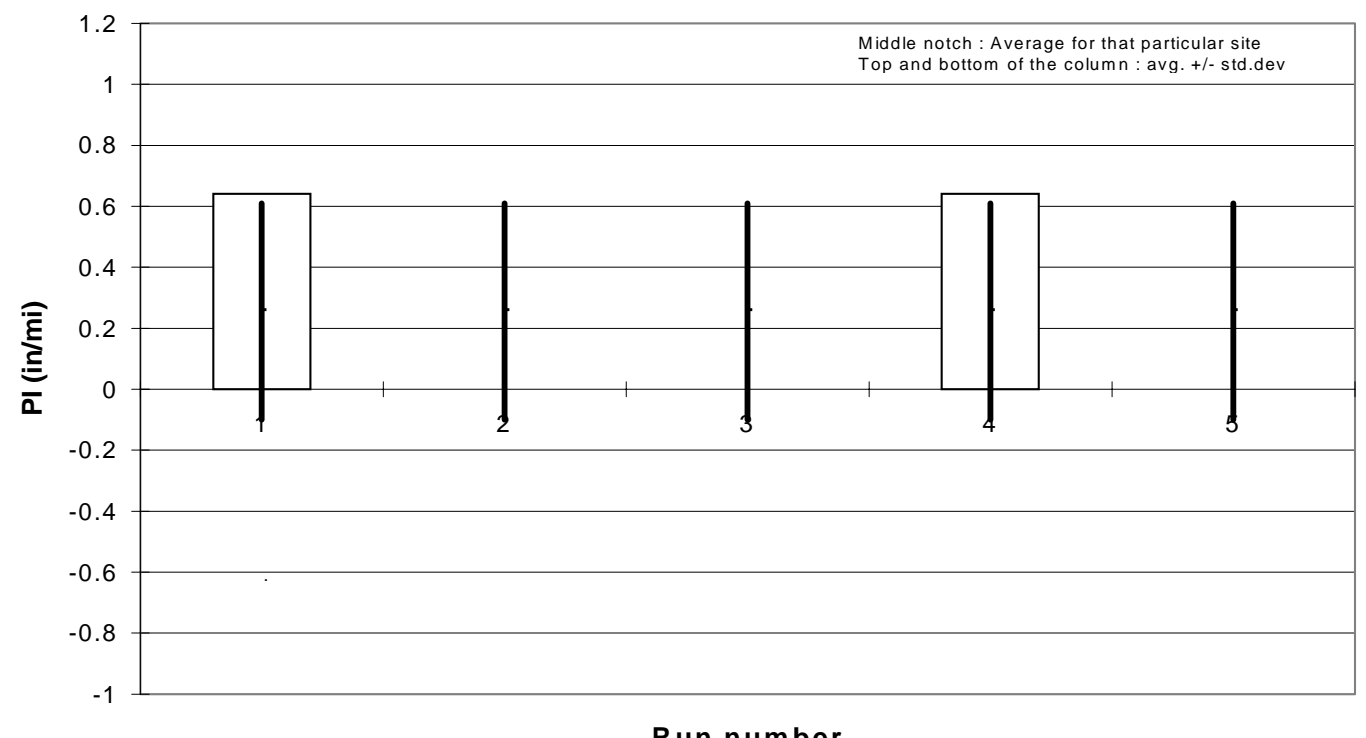

Run number

Figure C-20 PI-0.2" blanking band for replicate runs at US 231A (Site 5) of Vendor C 


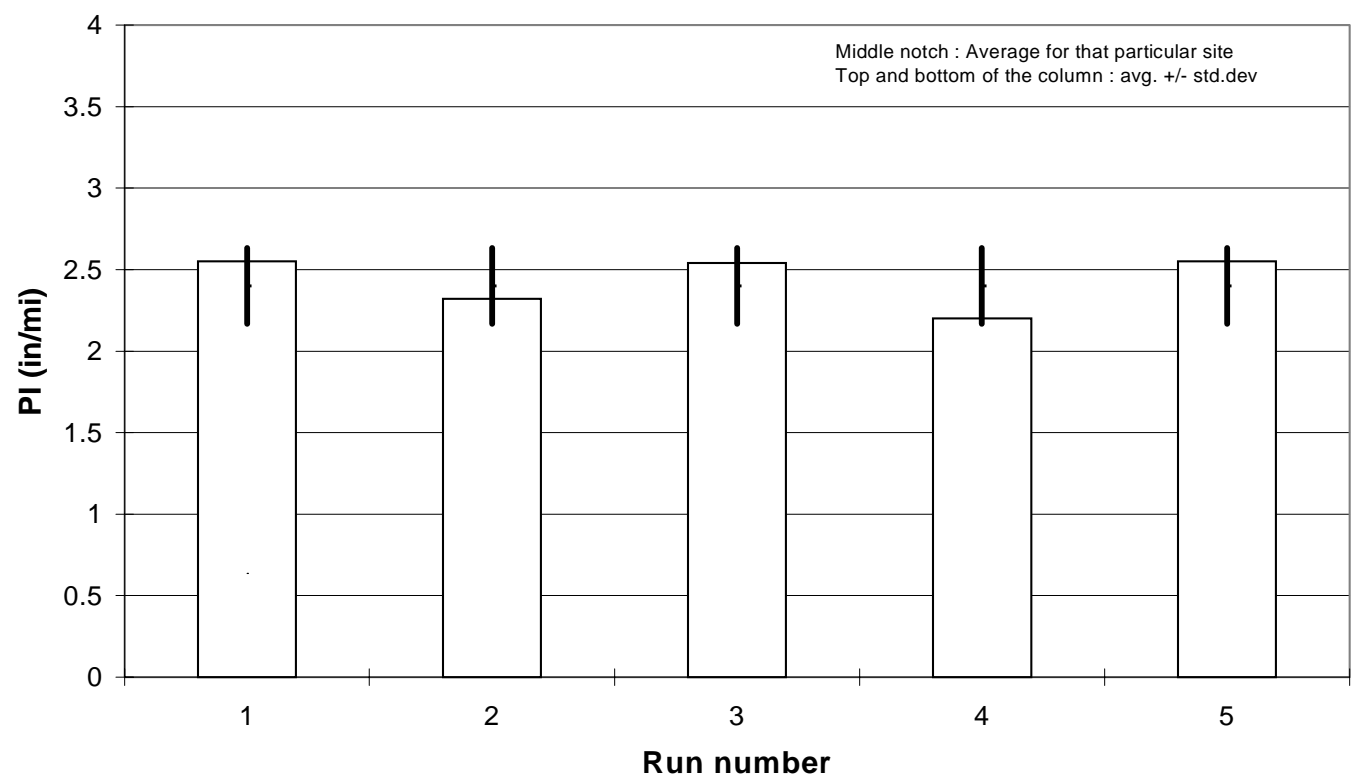

Figure C-21 PI-0.2" blanking band for replicate runs at US 231B (Site 6) of Vendor C

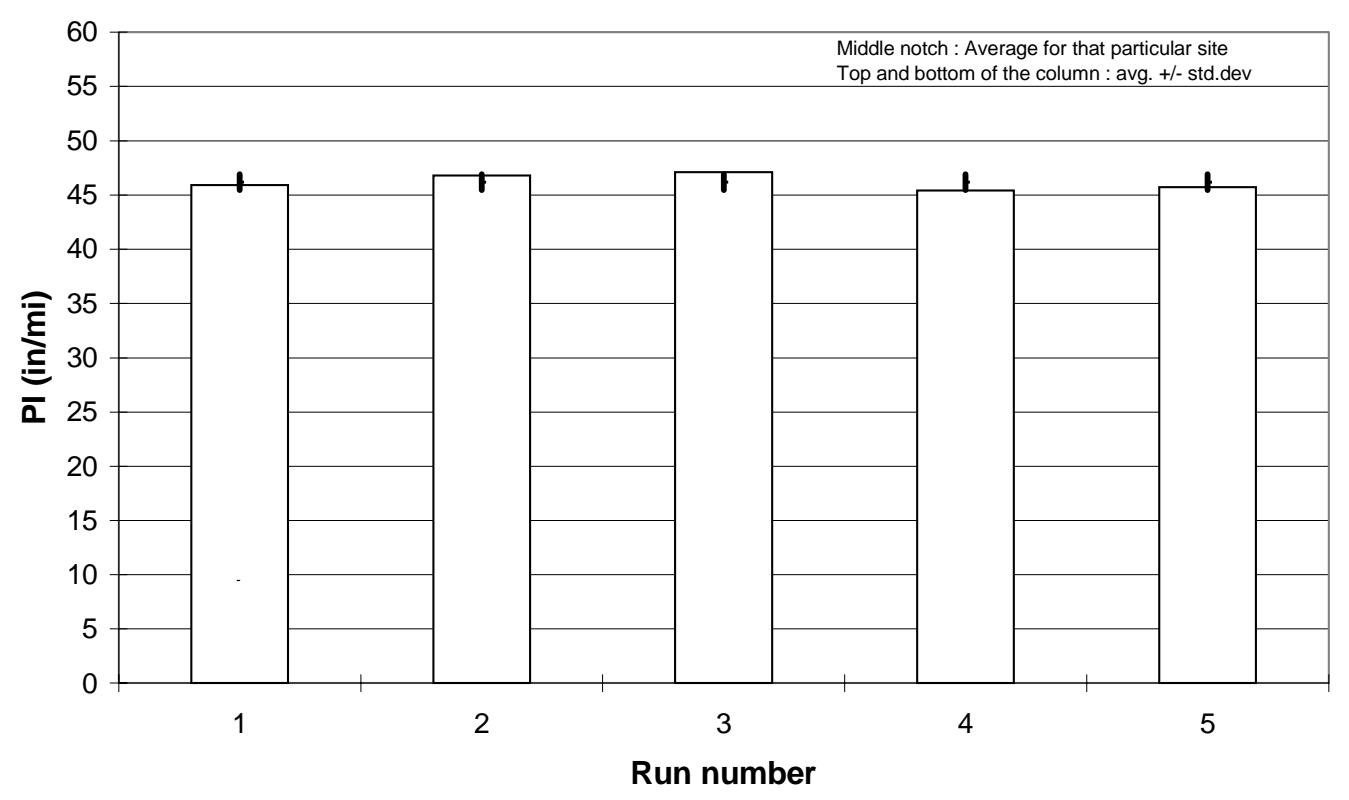

Figure C-22 PI-0.2" blanking band for replicate runs at Covington (Site 1) of Vendor D 


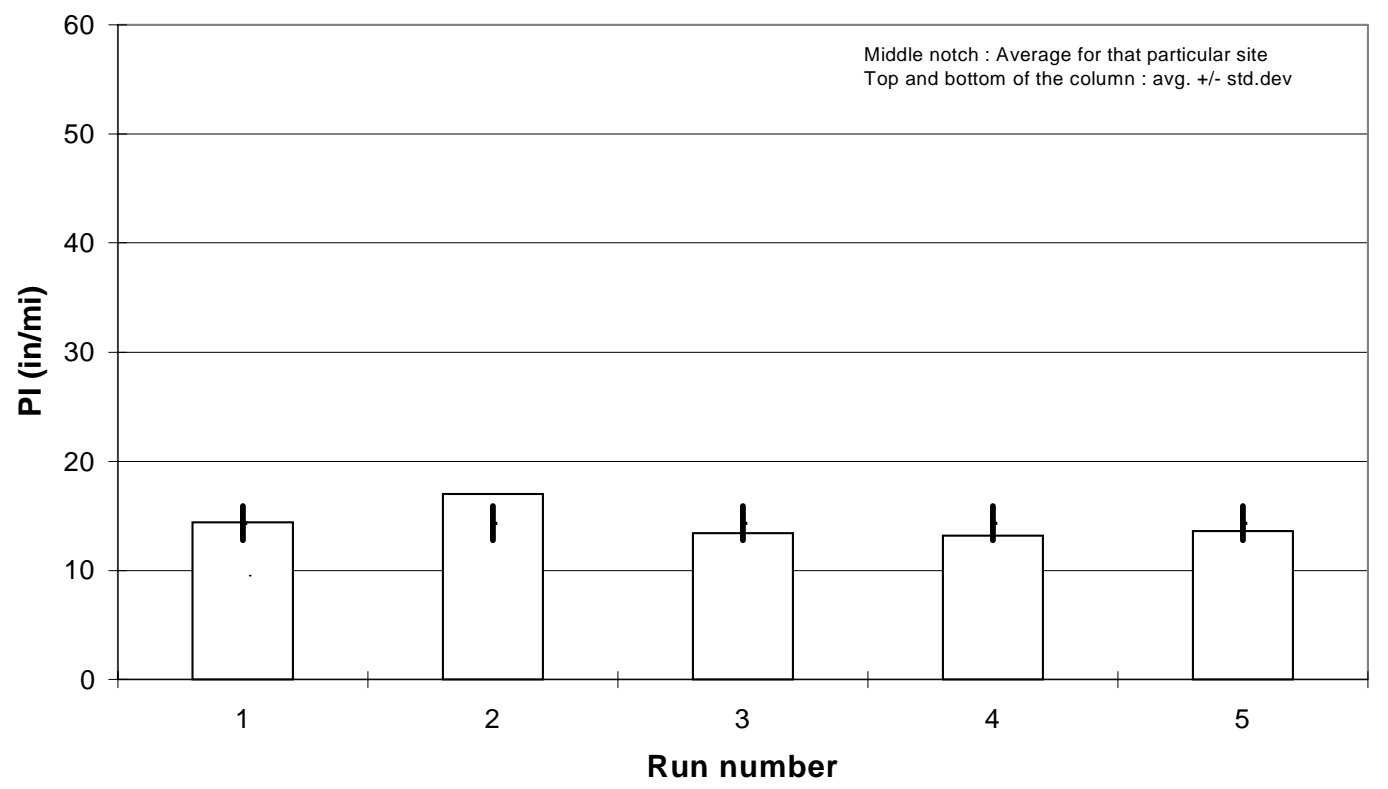

Figure C-23 PI-0.2" blanking band for replicate runs at I-65 (Site 2 with board) of Vendor D

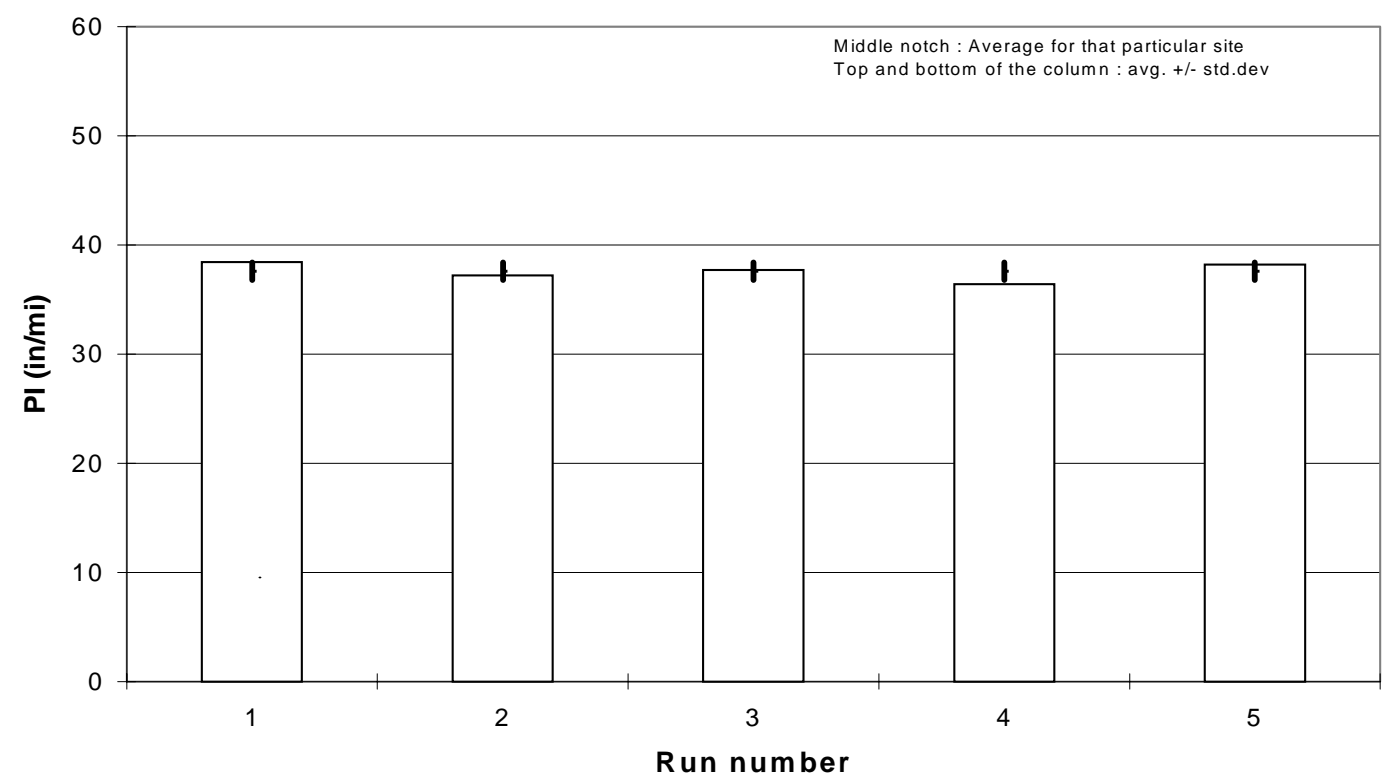

Figure C-24 PI-0.2" blanking band for replicate runs at Prairie Street (Site 3 with board) of Vendor D 


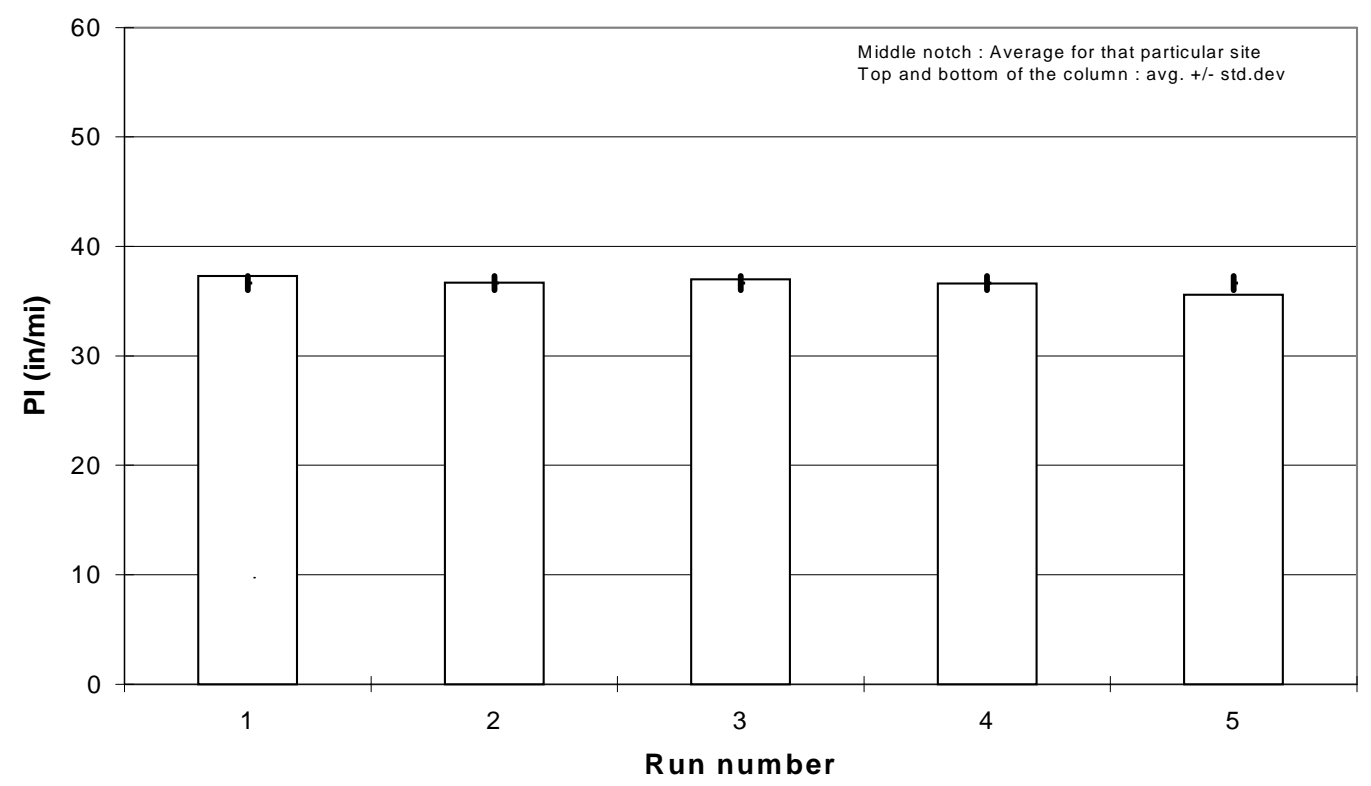

Figure C-25 PI-0.2" blanking band for replicate runs at Prairie Street

(Site 3 without board) of Vendor D

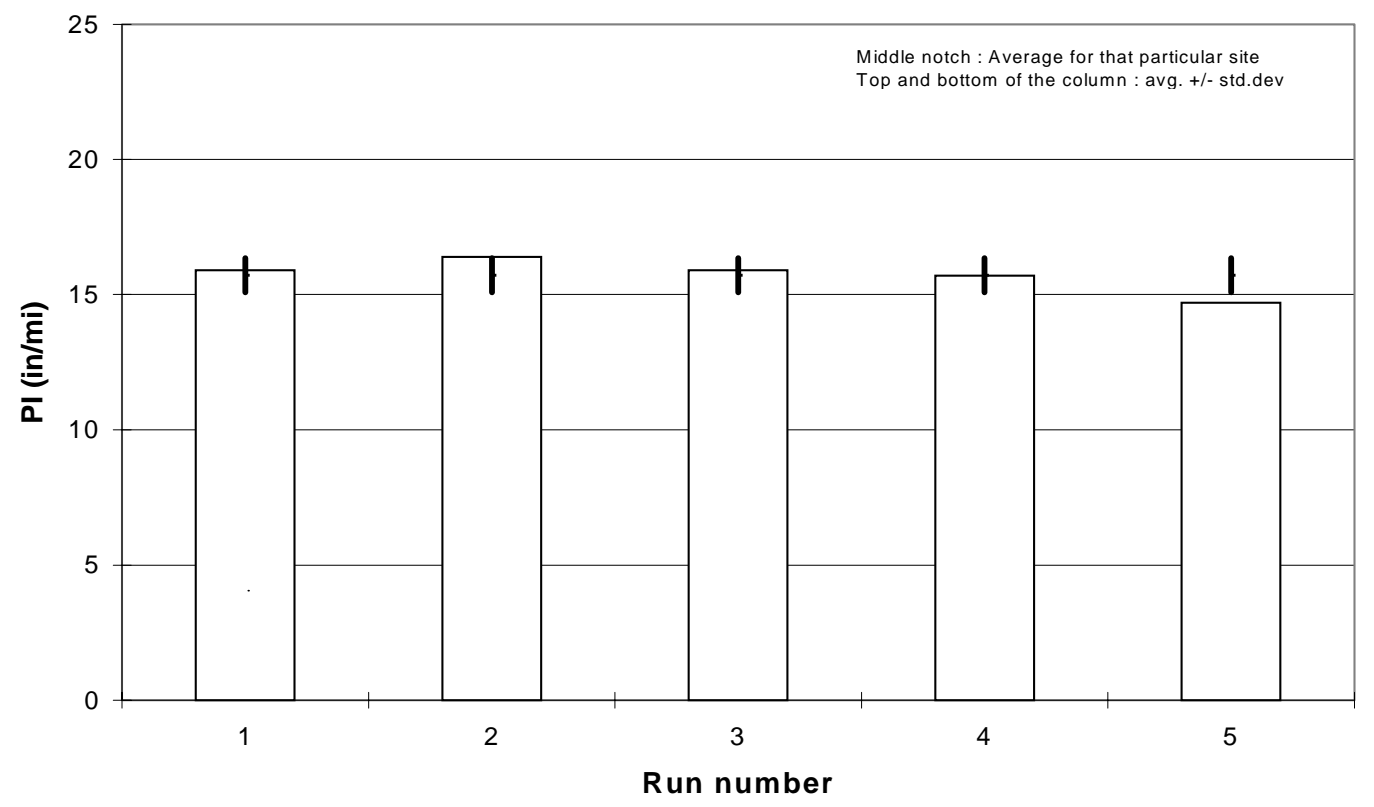

Figure C-26 PI-0.2" blanking band for replicate runs at Vinton Street (Site 4) of Vendor D 


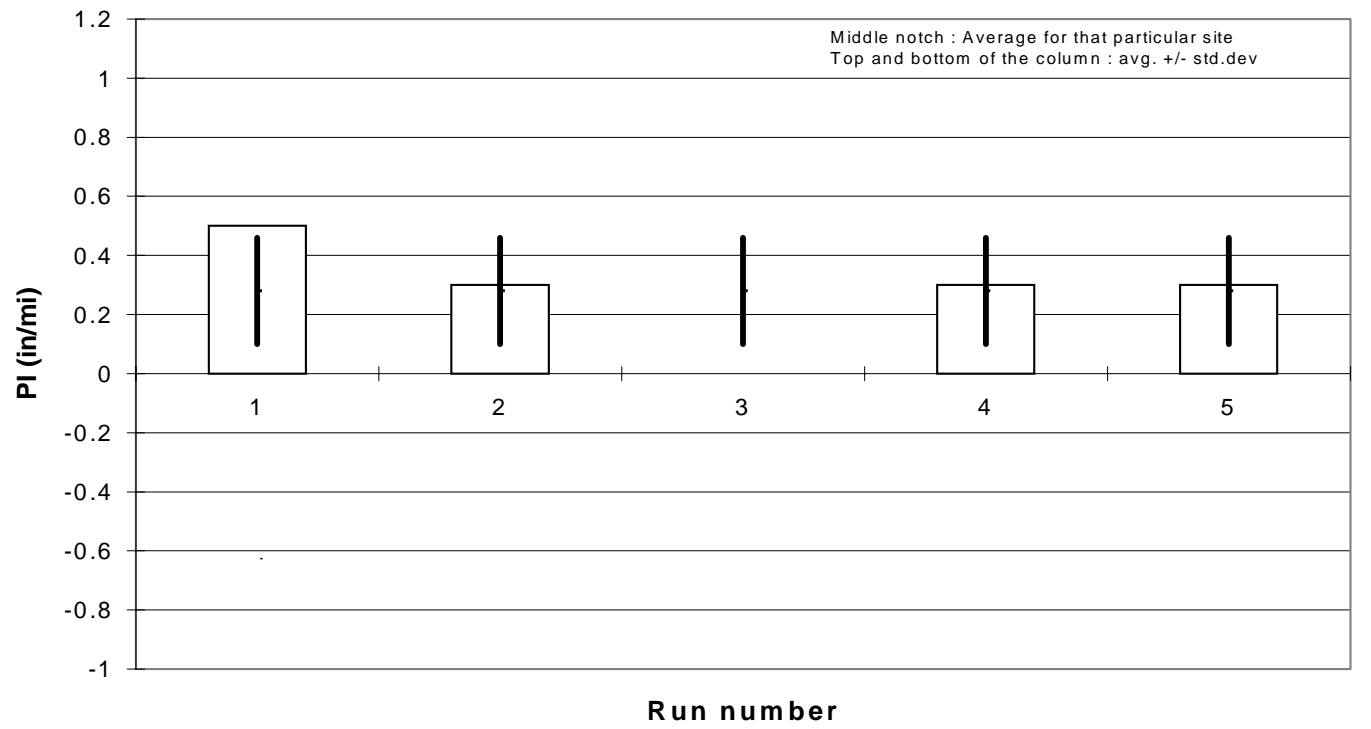

Figure C-27 PI-0.2" blanking band for replicate runs at US 231A (Site 5) of

Vendor D

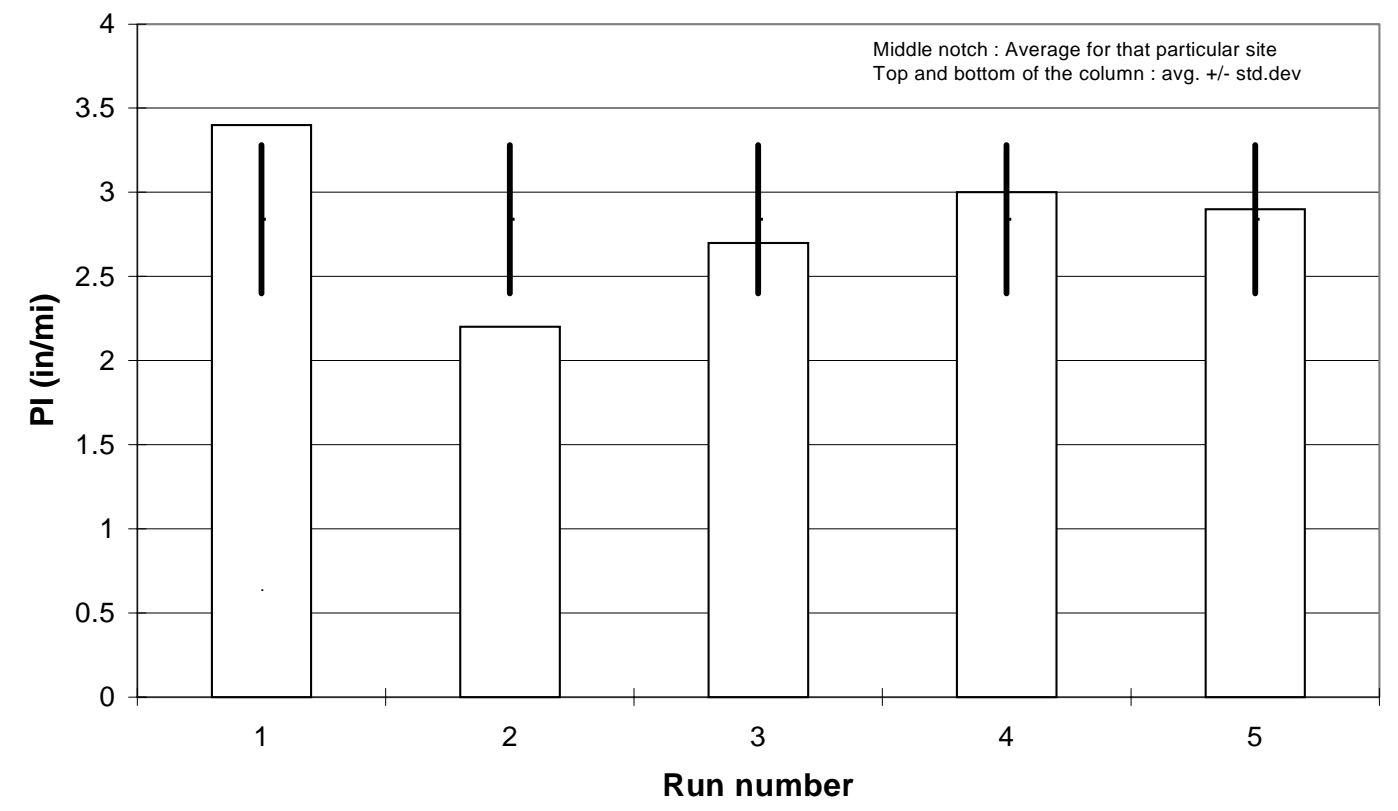

Figure C-28 PI-0.2" blanking band for replicate runs at US 231B (Site 6) of Vendor D 
APPENDIX D - Between Vendors Comparison of Smoothness Indices (IRI, PI-0.0” and PI-0.2”) 


\section{APPENDIX D - Between Vendors Comparision of Smoothness Indices (IRI, PI-0.0" and PI-0.2”)}

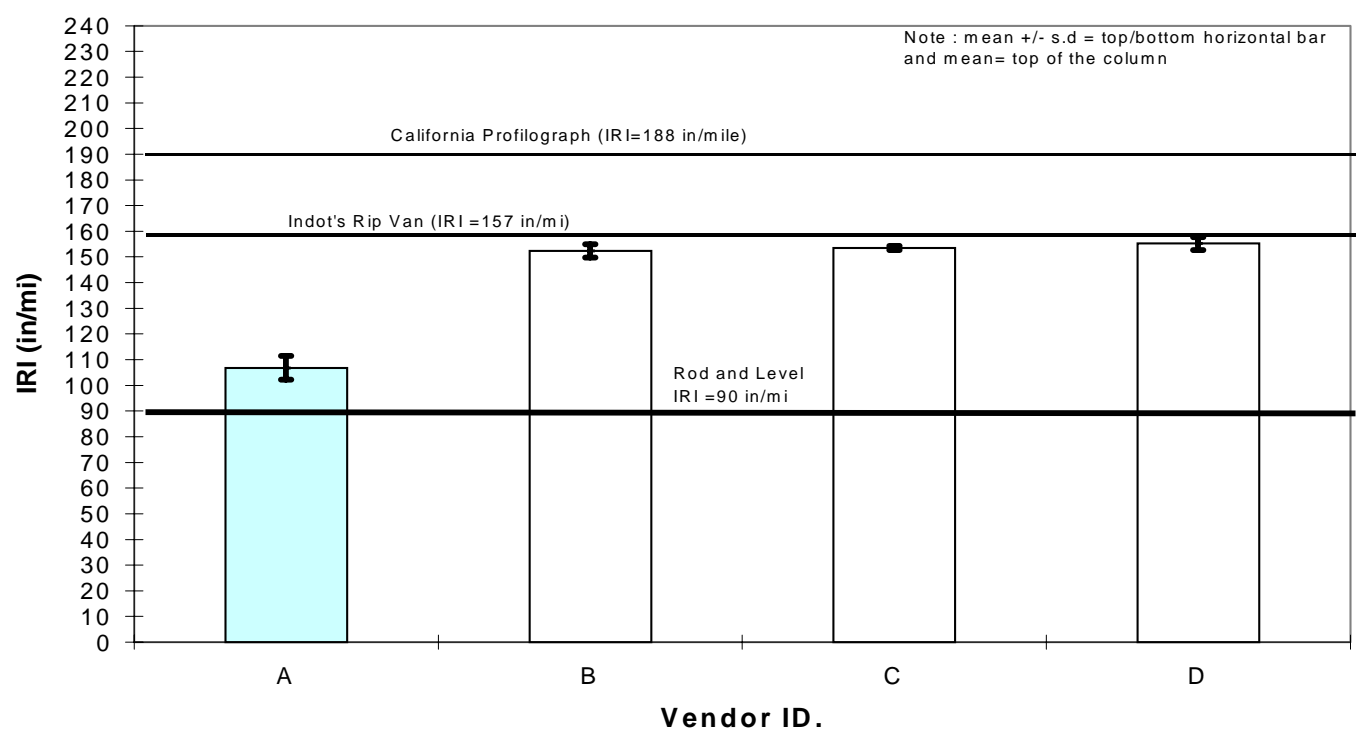

Figure D-1 IRI Between Vendor Comparison at Covington (Site 1)

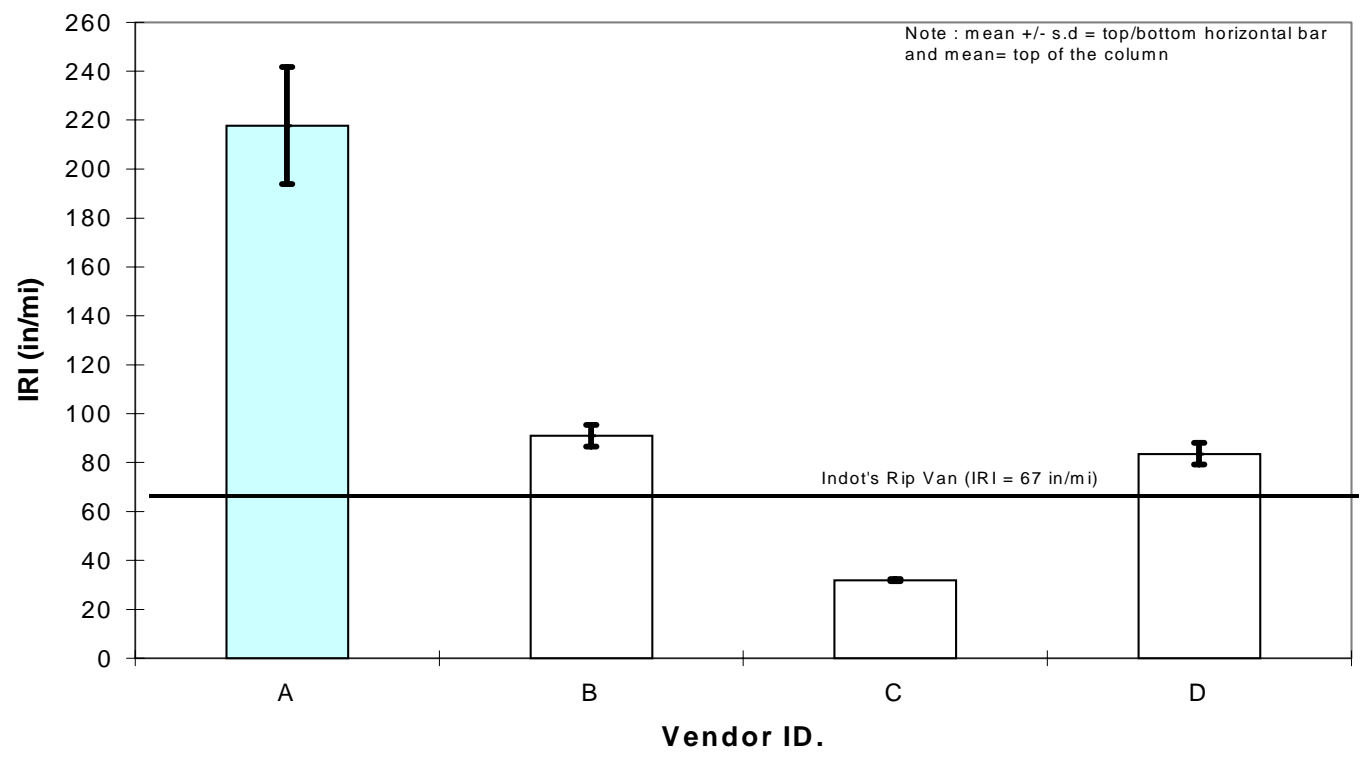

Figure D-2 IRI Between Vendor Comparison at I-65 (Site 2 with board) 


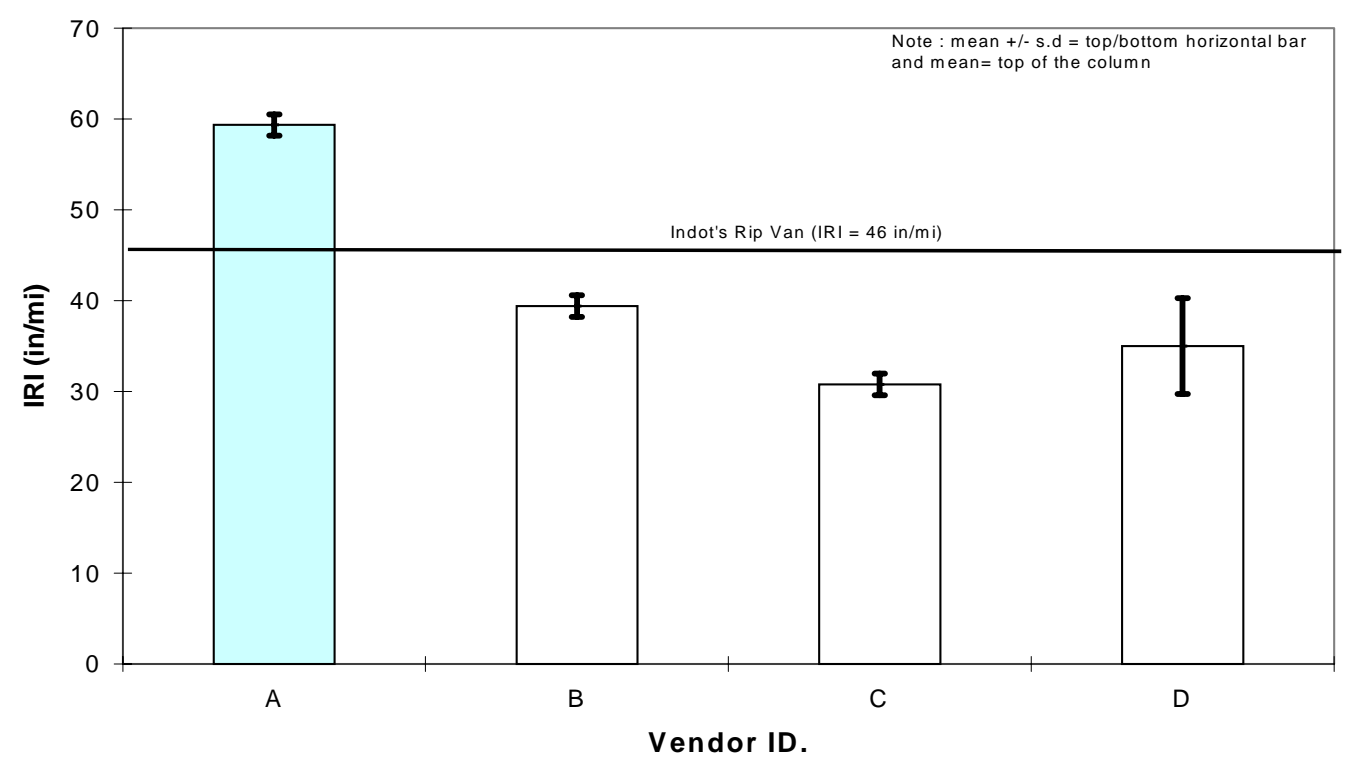

Figure D-3 IRI Between Vendor Comparison at I-65 (Site 2 without board)

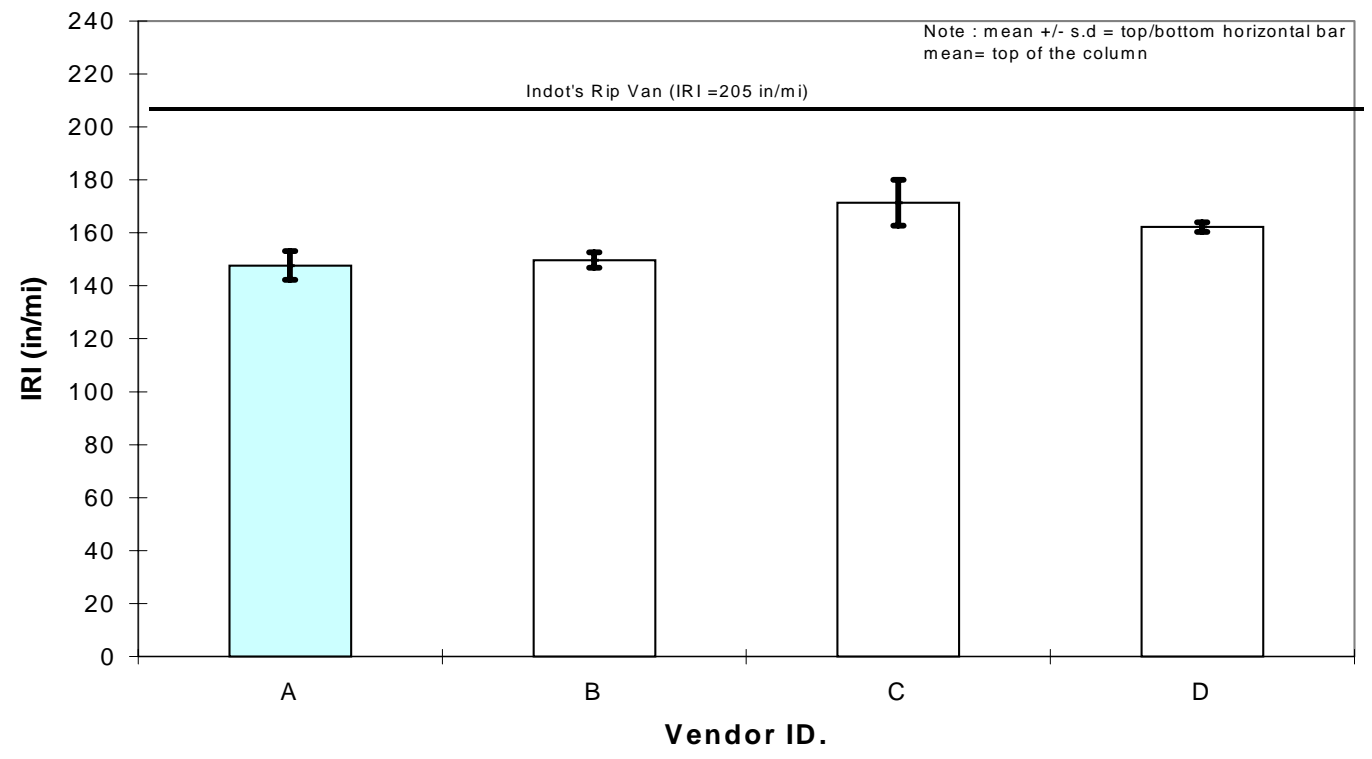

Figure D-4 IRI Between Vendor Comparison at Prairie Street (Site 3 with board) 


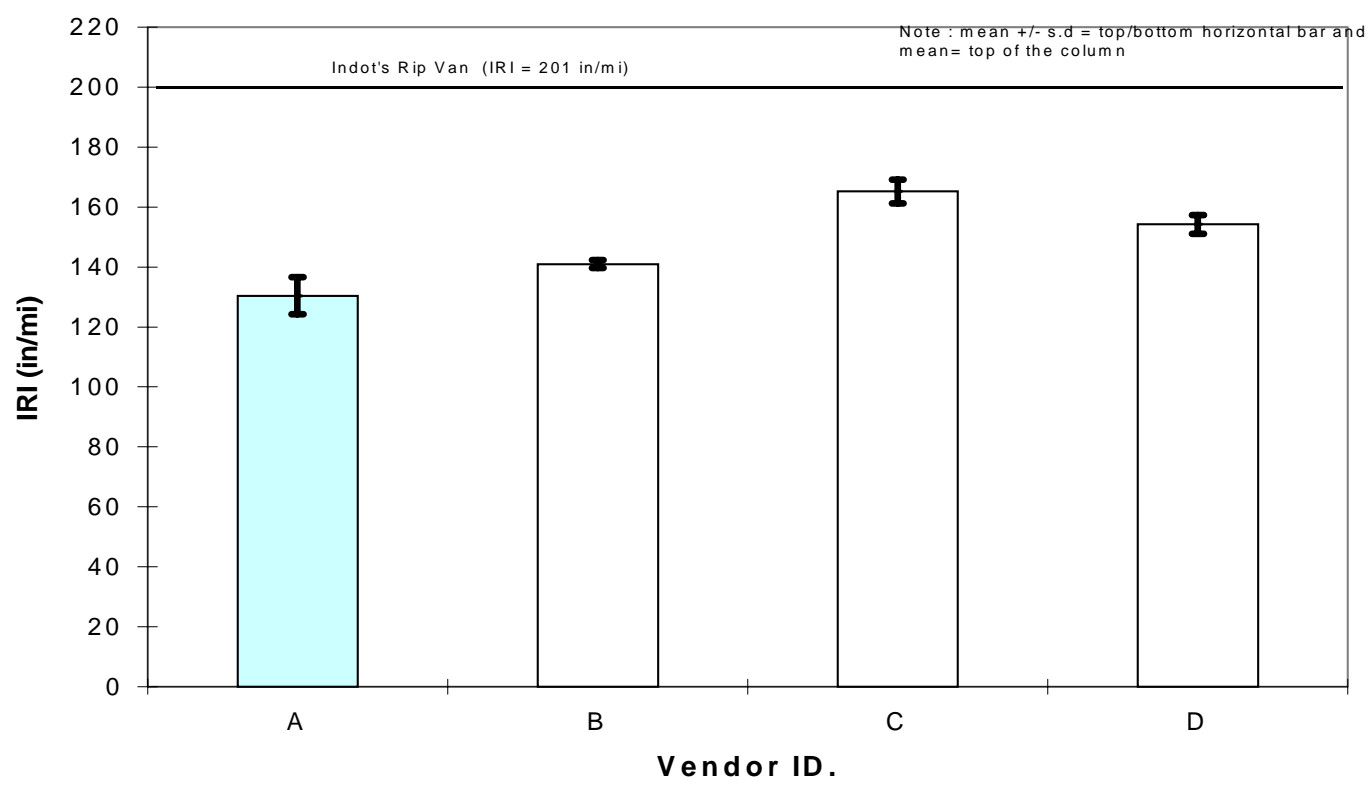

Figure D-5 IRI Between Vendor Comparison at Prairie Street

(Site 3 without board)

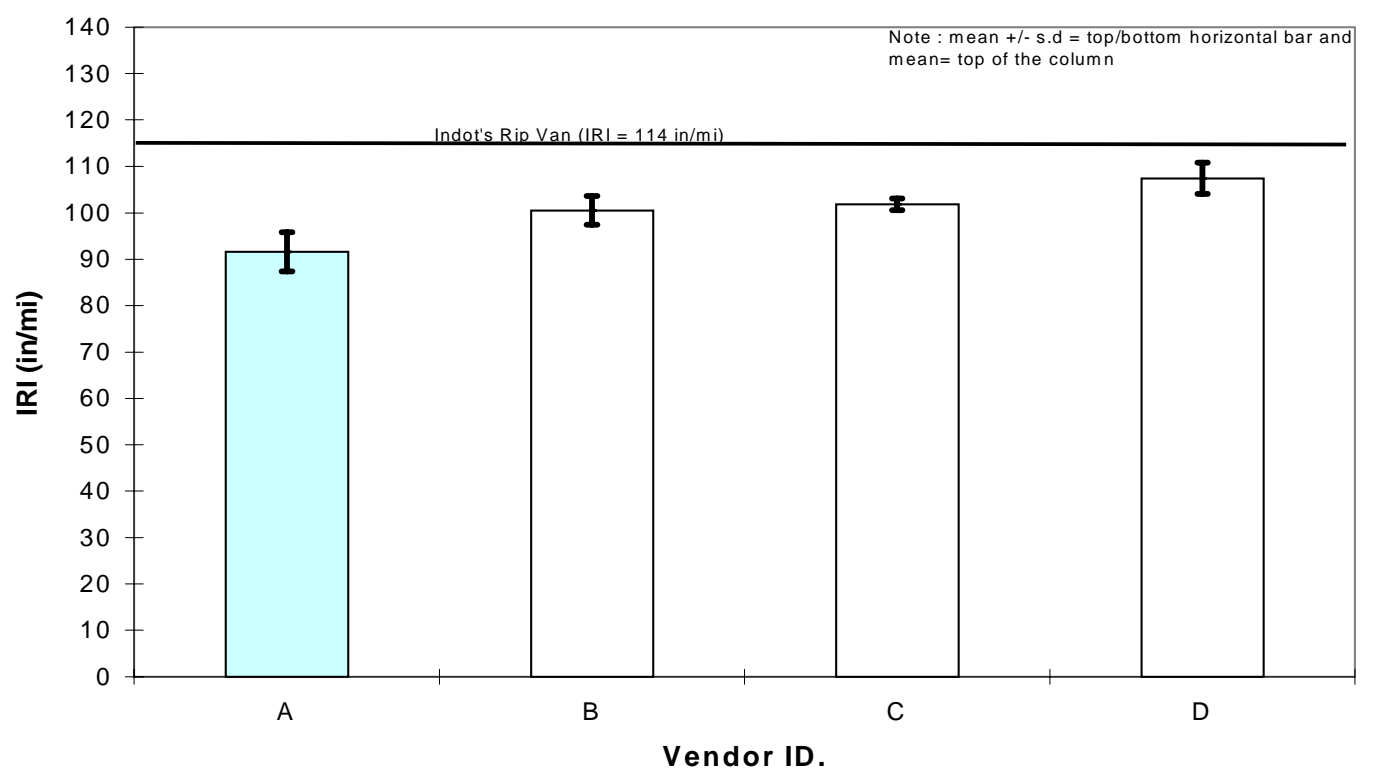

Figure D-6 IRI Between Vendor Comparison at Vinton Street (Site 4) 


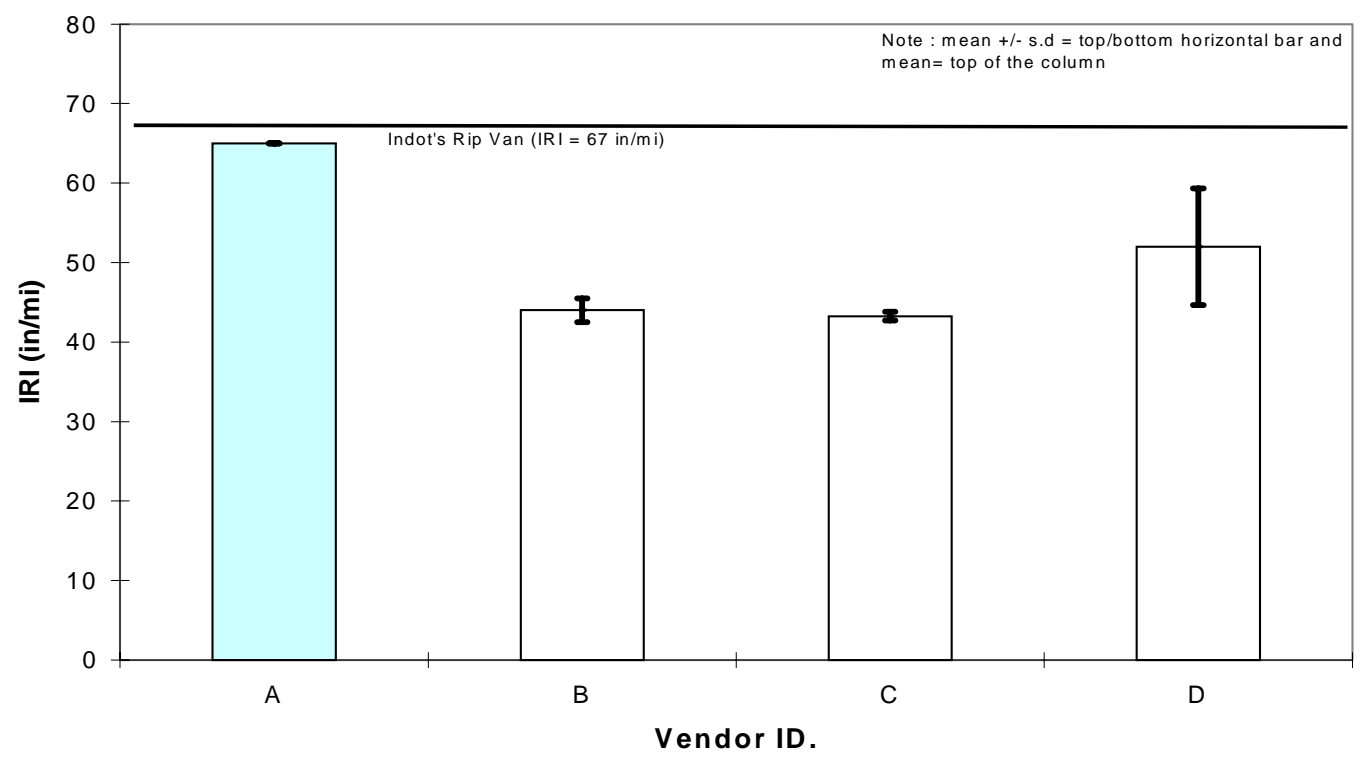

Figure D-7 IRI Between Vendor Comparison at US 231A (Site 5)

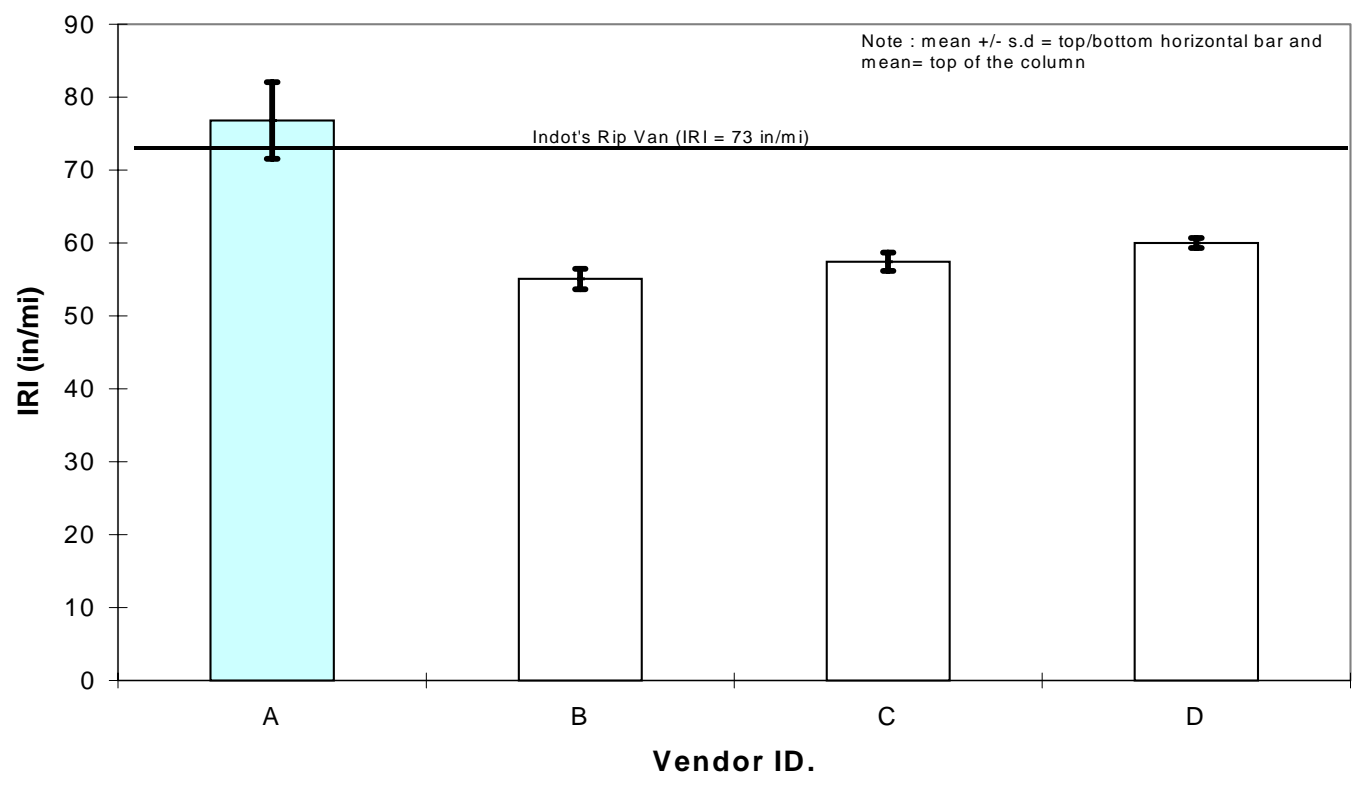

Figure D-8 IRI Between Vendor Comparison at US 231B (Site 6) 


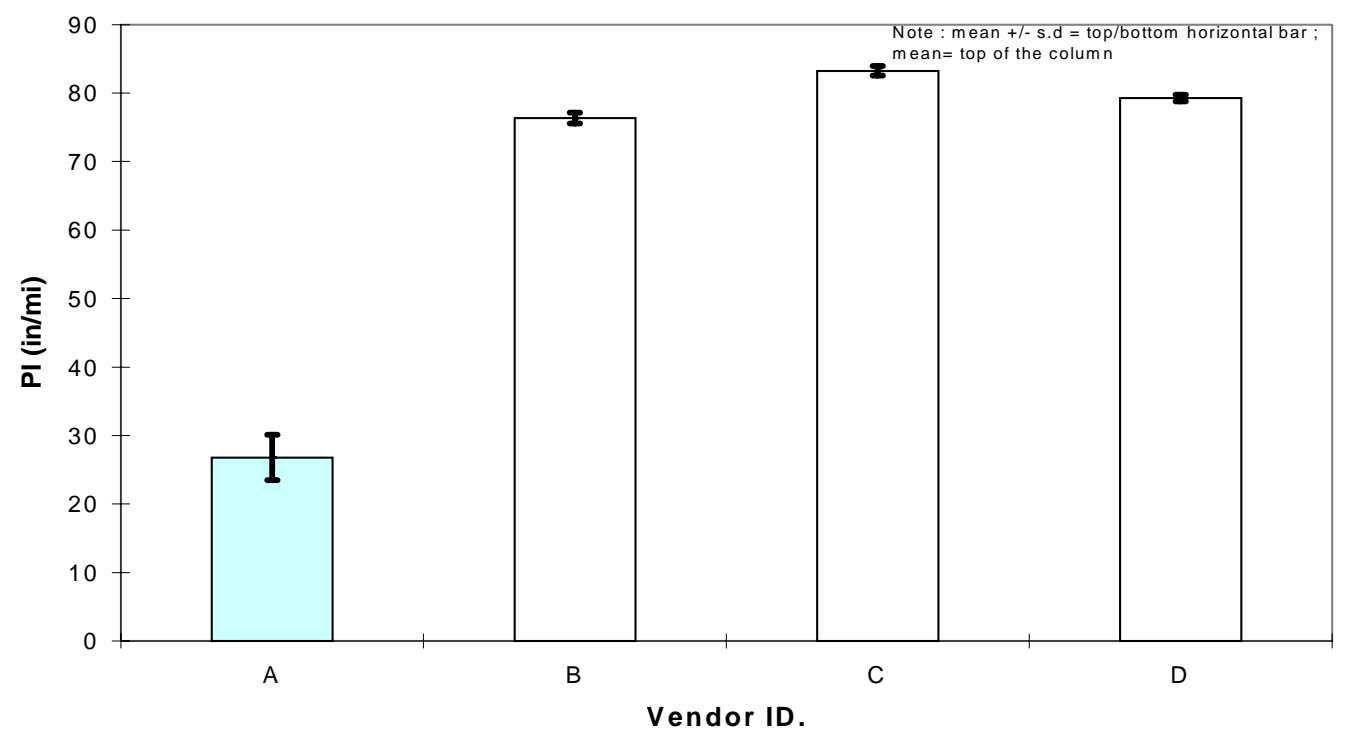

Figure D-9 PI-0.0”' Between Vendor Comparison at Covington (Site 1)

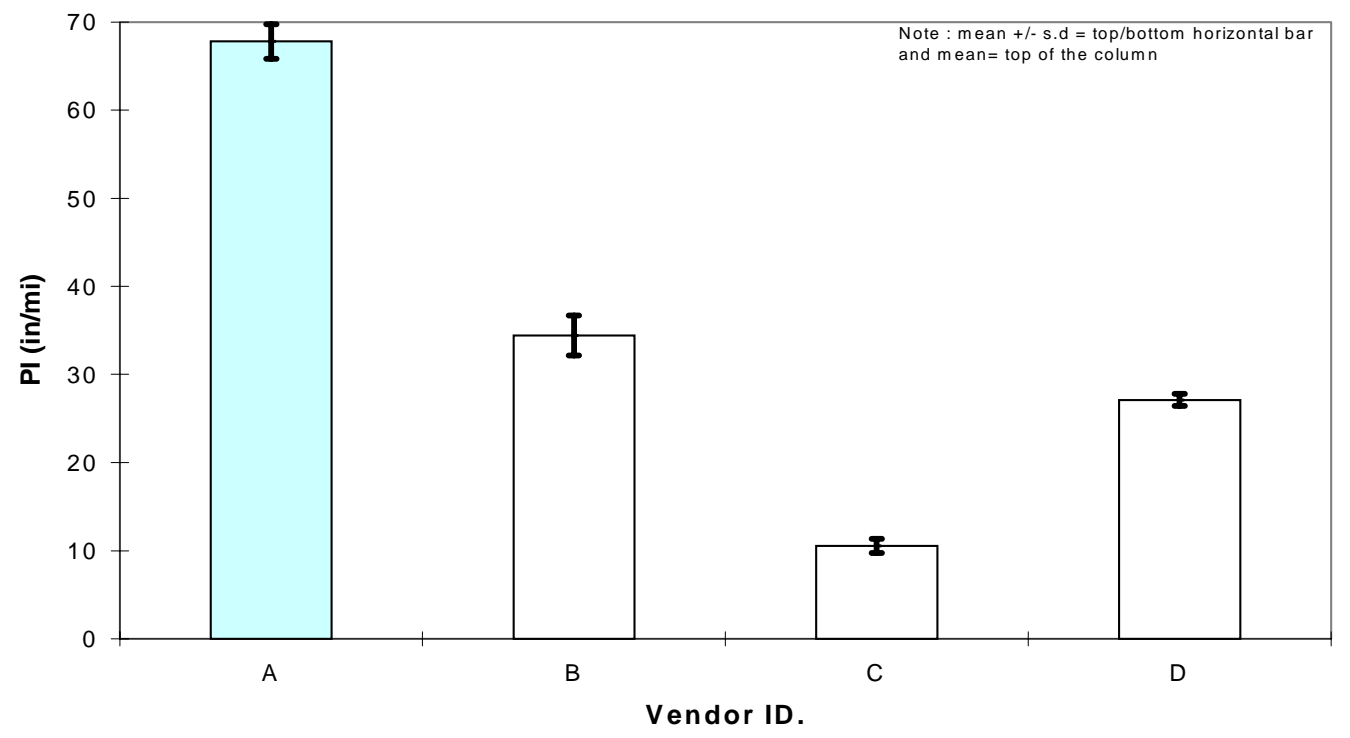

Figure D-10 PI-0.0”' Between Vendor Comparision at I-65 (Site 2 with board) 


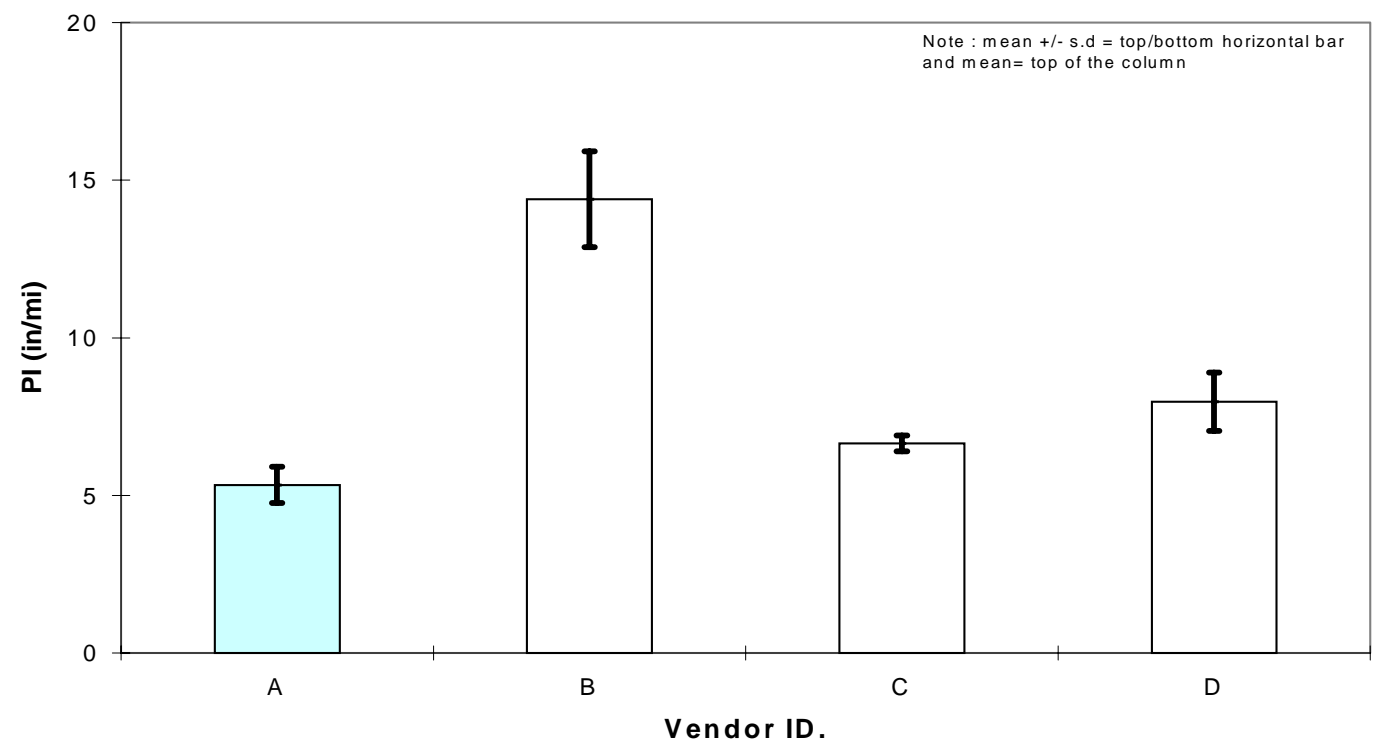

Figure D-11 PI-0.0”' Between Vendor Comparison at I-65 (Site 2 without board)

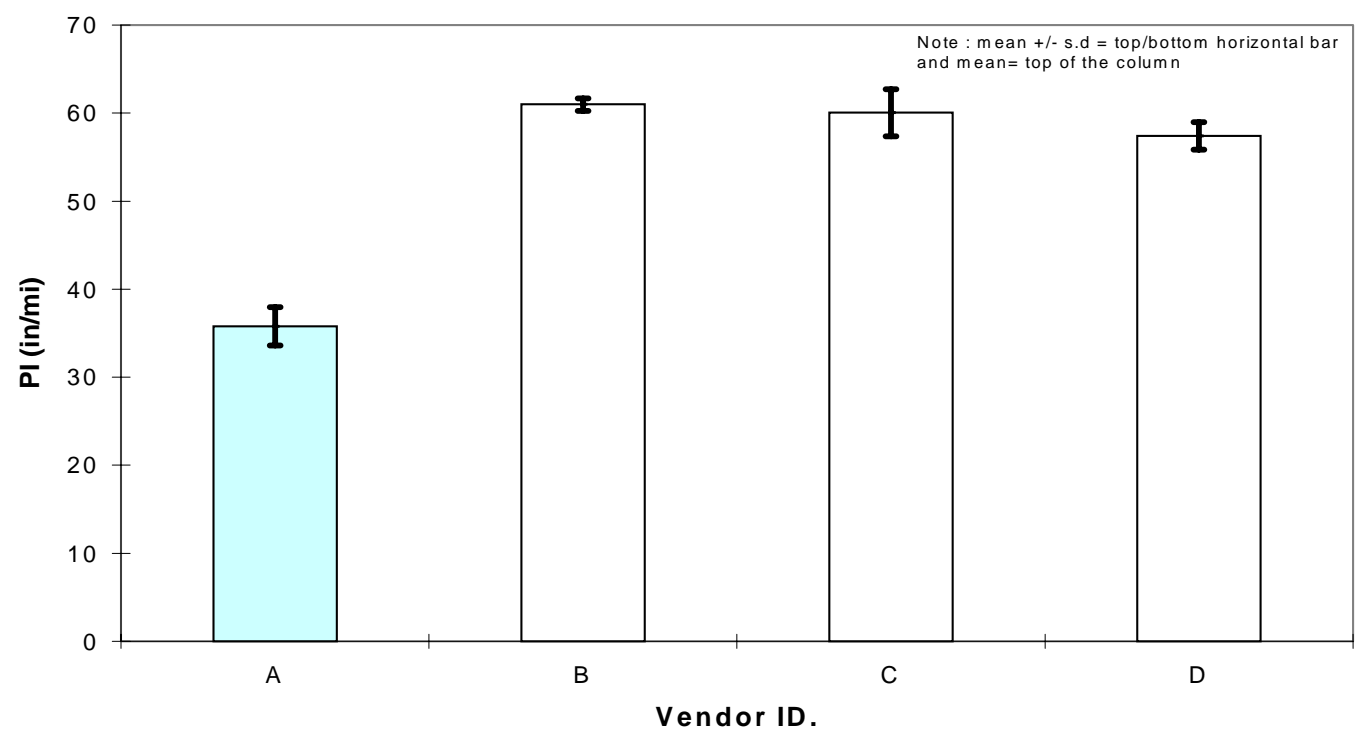

Figure D-12 PI-0.0" Between Vendor Comparison at Prairie Street (Site 3 with board) 


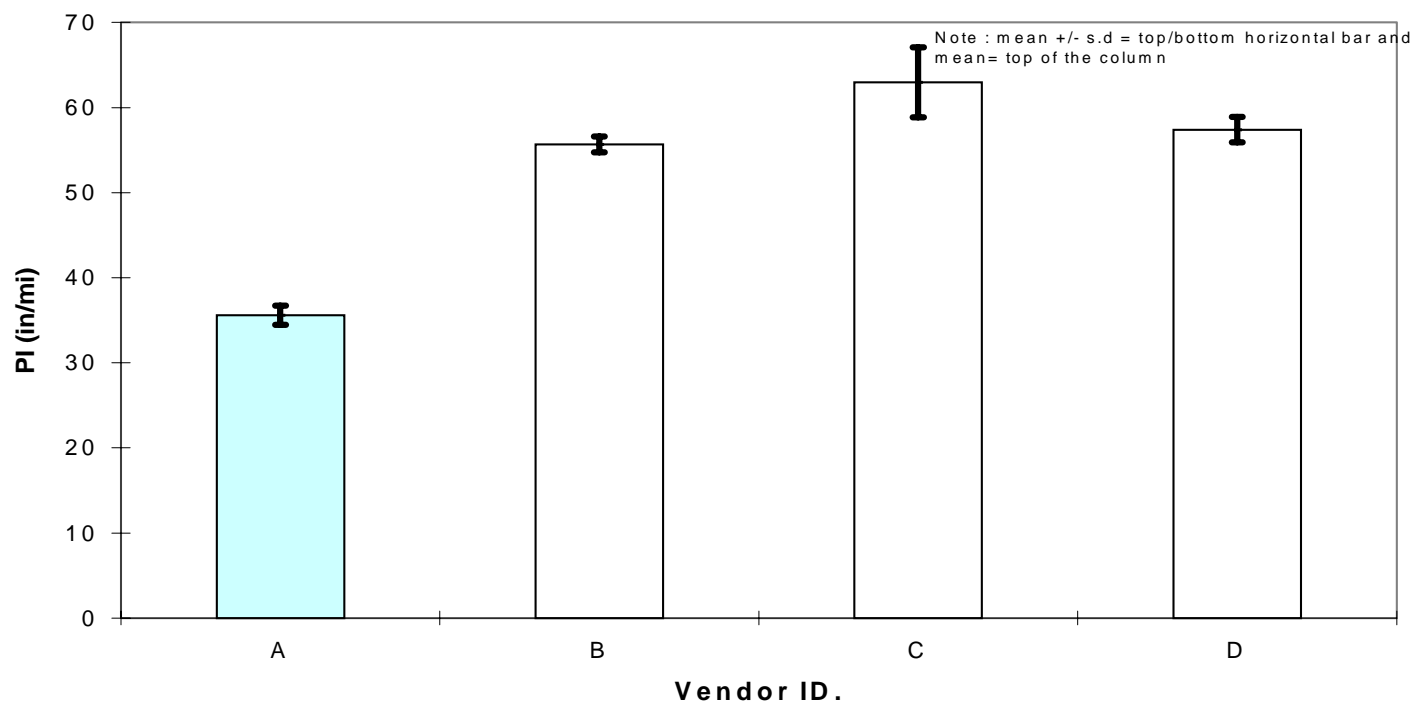

Figure D-13 PI-0.0” Between Vendor Comparison at Prairie Street

(Site 3 without board)

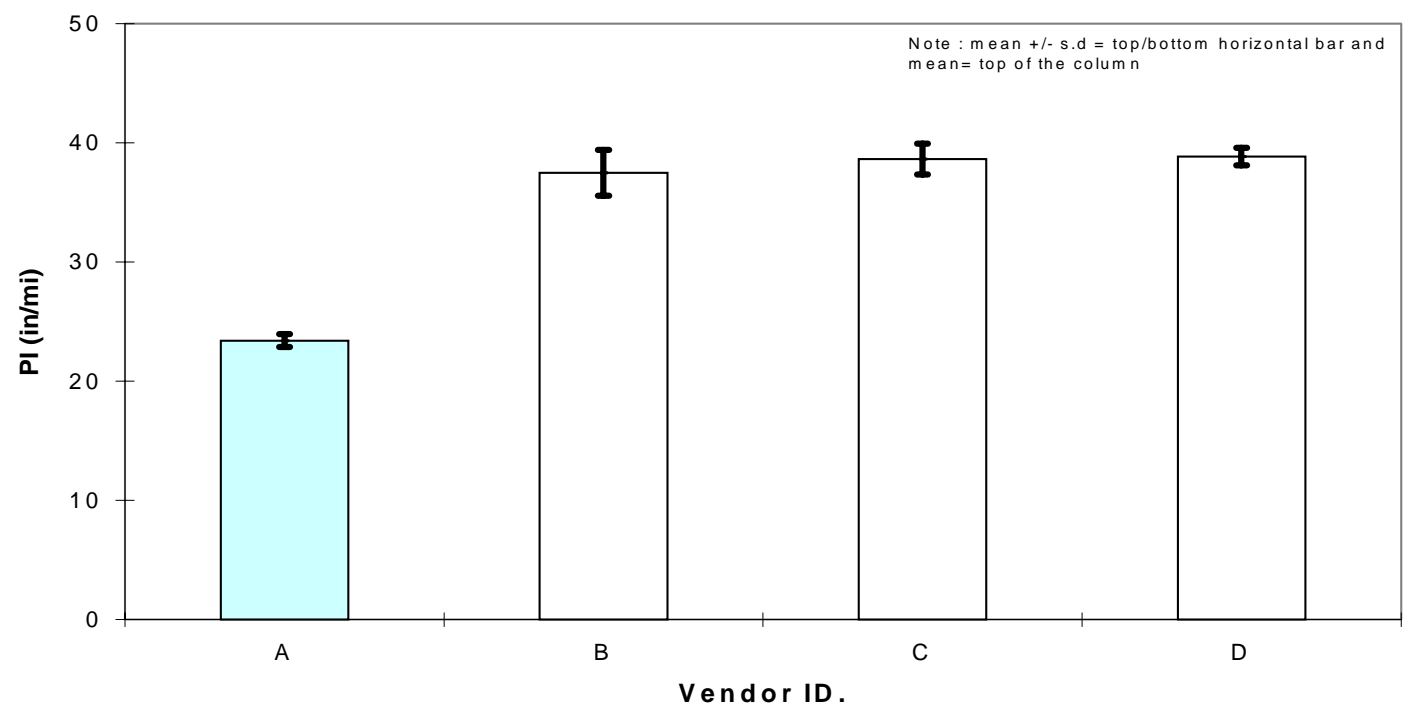

Figure D-14 PI-0.0” Between Vendor Comparison at Vinton Street (Site 4) 


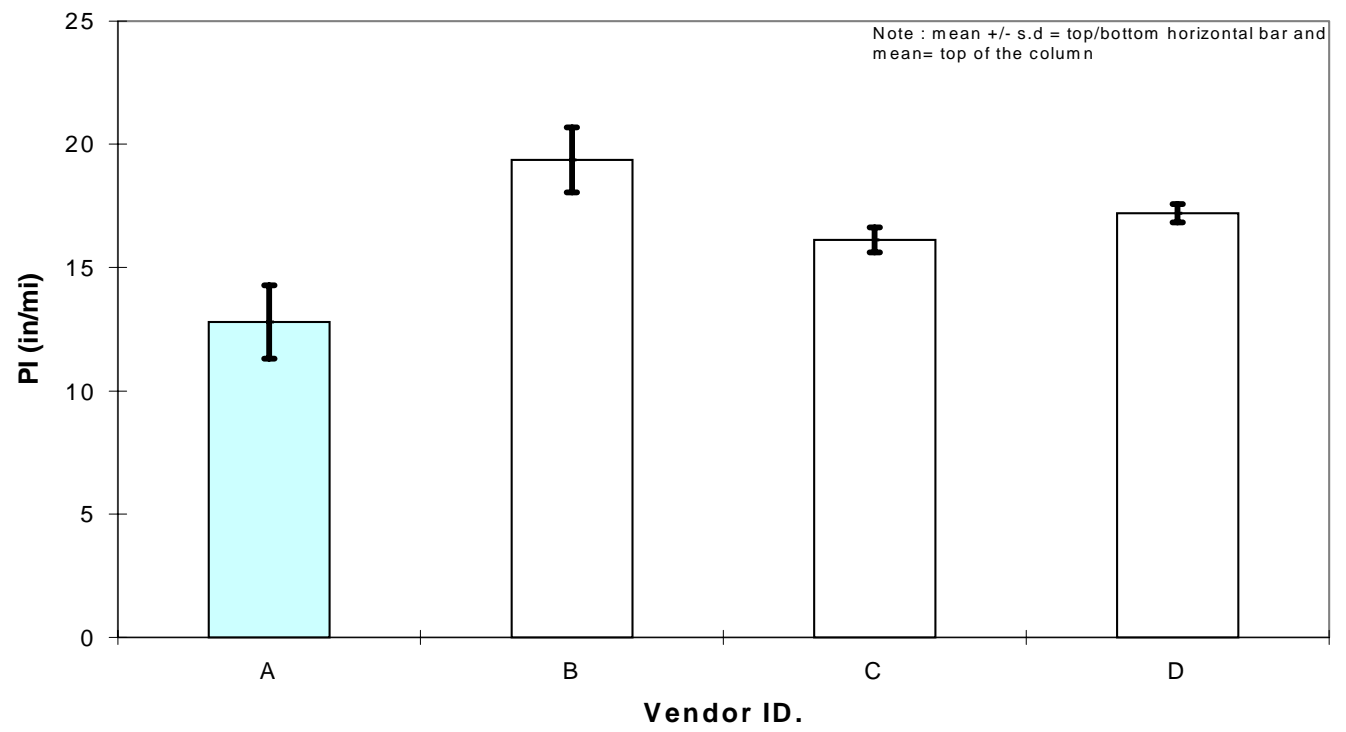

Figure D-15 PI-0.0” Between Vendor Comparison at US 231A (Site 5)

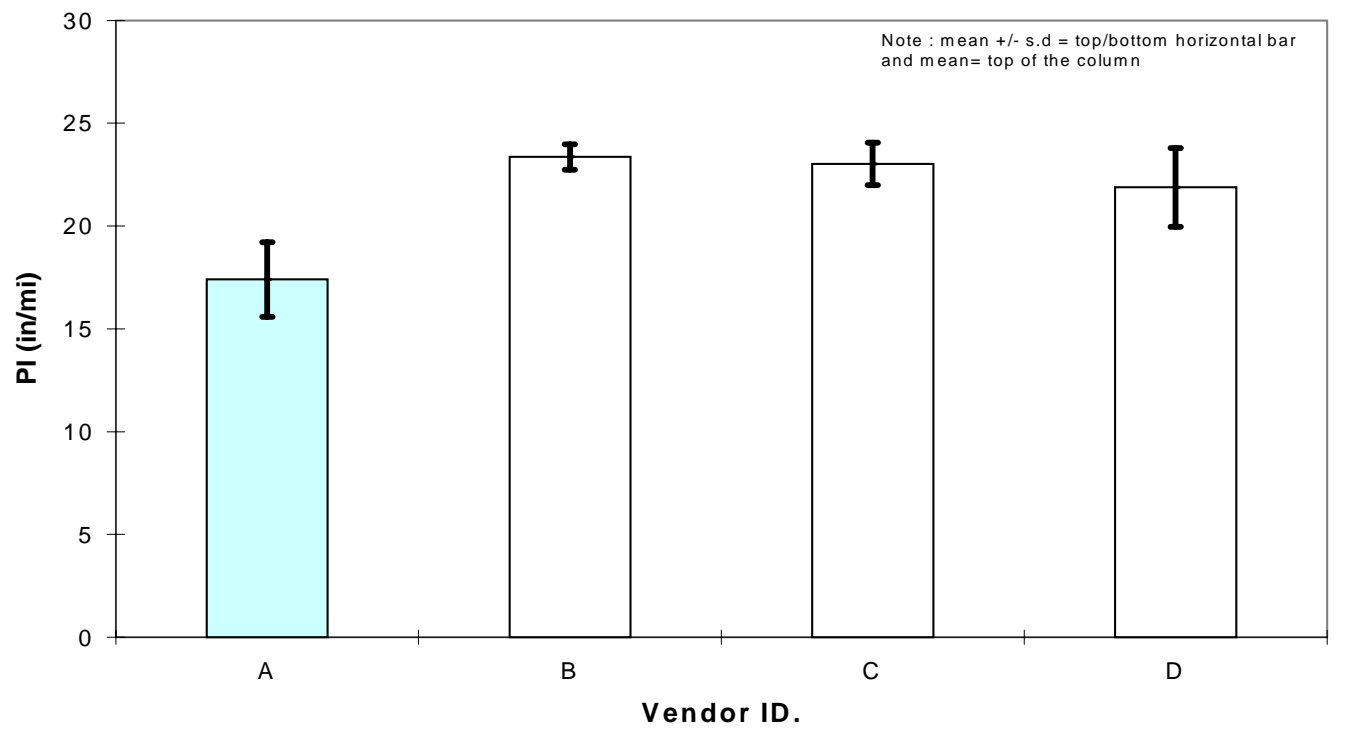

Figure D-16 PI-0.0” Between Vendor Comparison at US 231B (Site 6) 


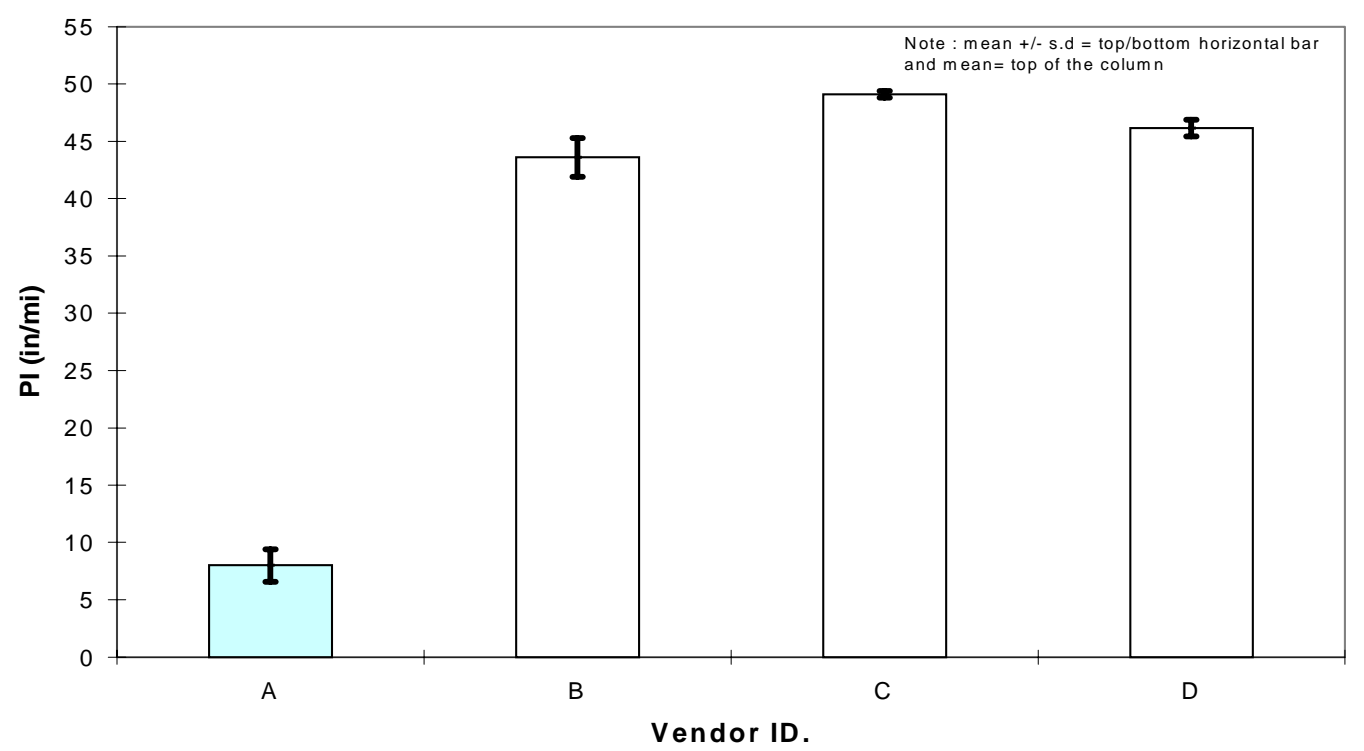

Figure D-17 PI-0.2”' Between Vendor Comparison at Covington (Site 1)

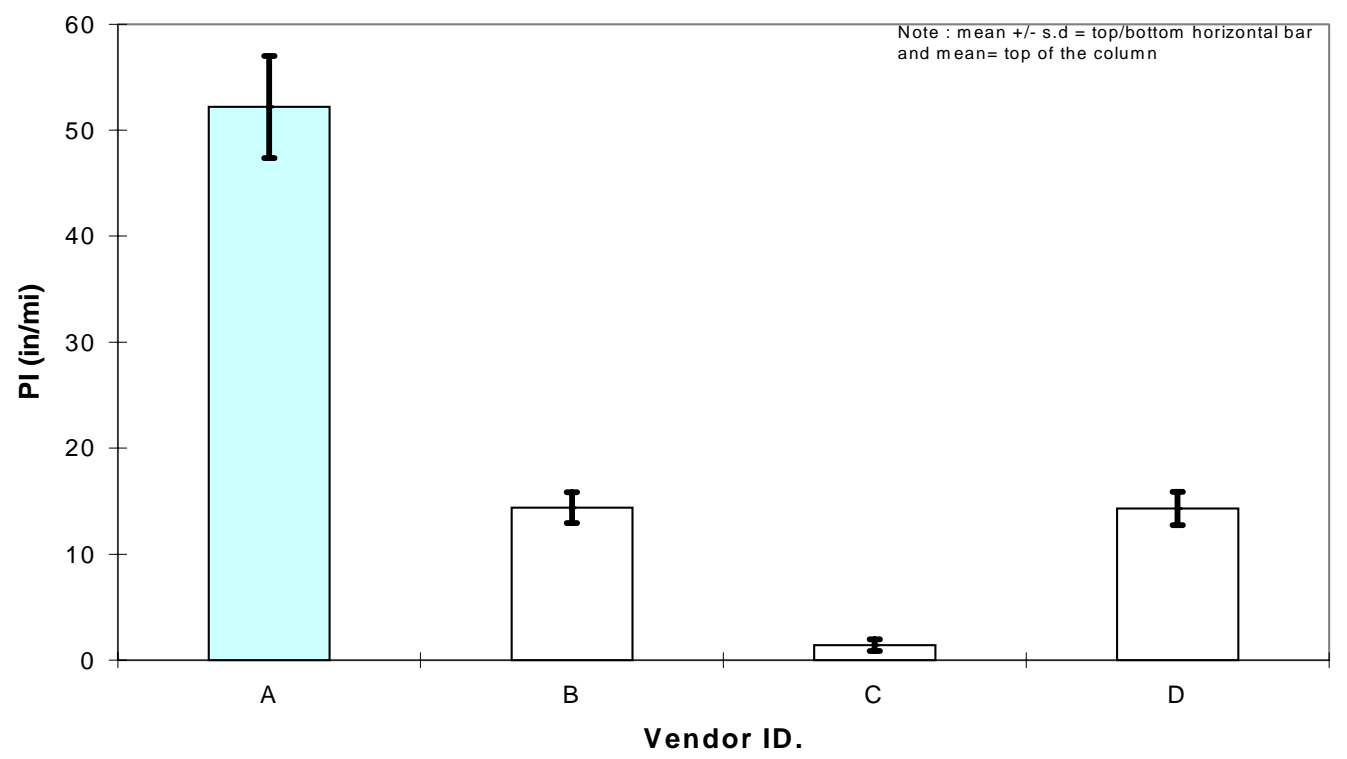

Figure D-18 PI-0.2”' Between Vendor Comparison at I-65 (Site 2 with board) 


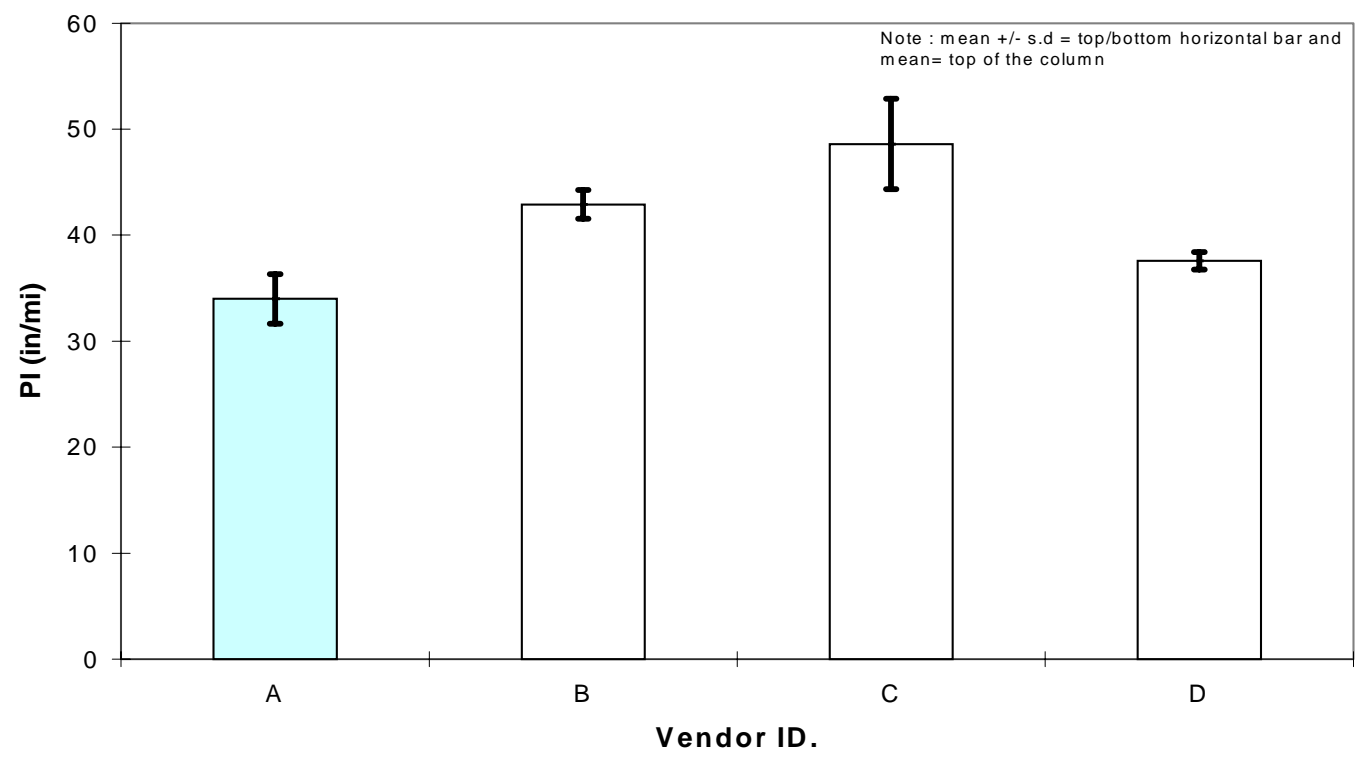

Figure D-19 PI-0.2” Between Vendor Comparison at Prairie Street

(Site 3 with board)

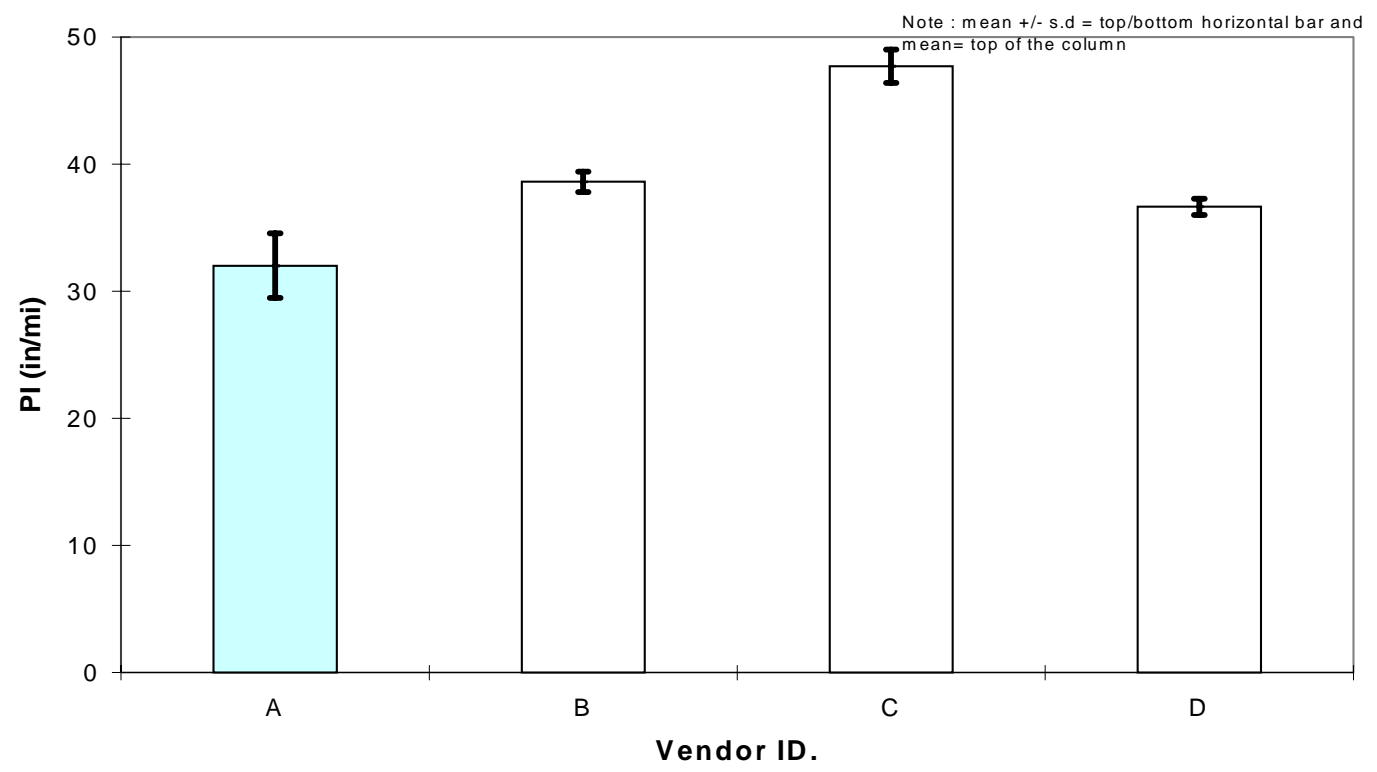

Figure D-20 PI-0.2”' Between Vendor Comparison at Prairie Street

(Site 3 without board) 


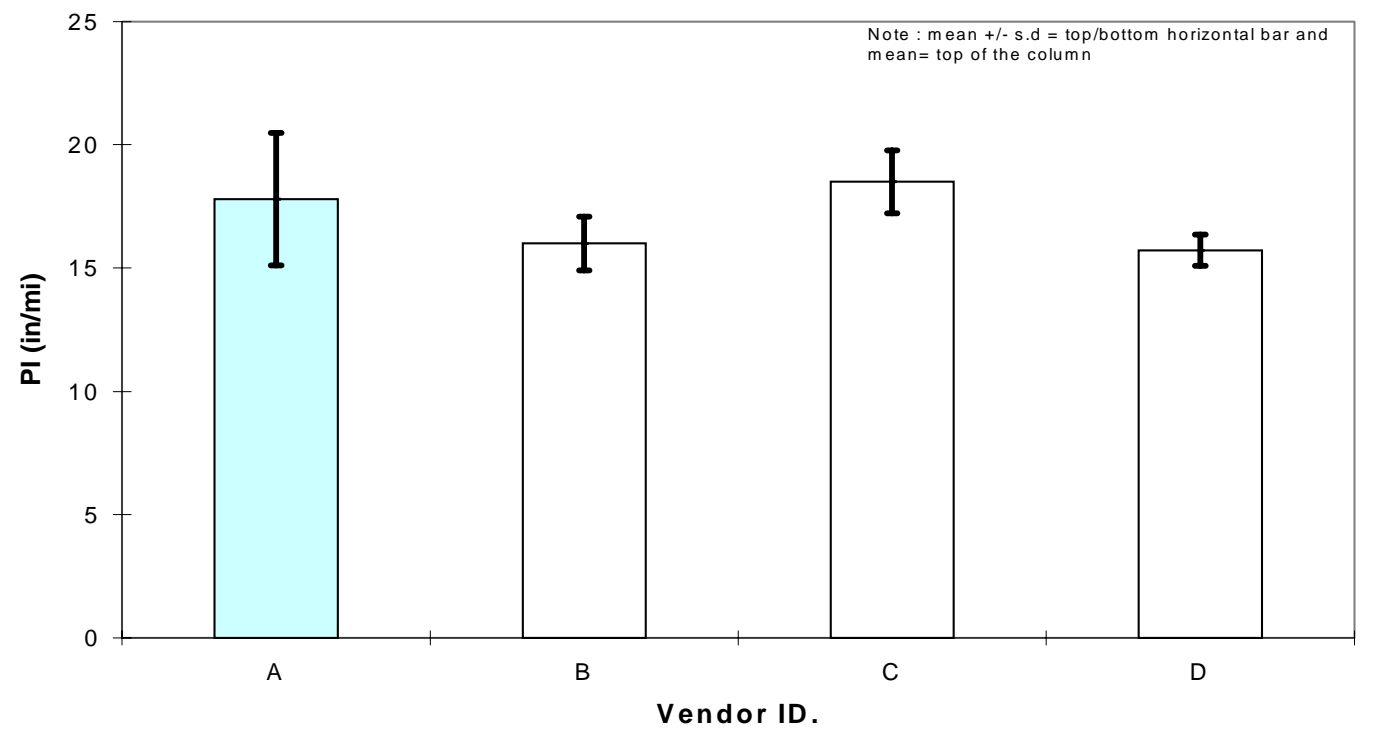

Figure D-21 PI-0.2”' Between Vendor Comparison at Vinton Street (Site 4)

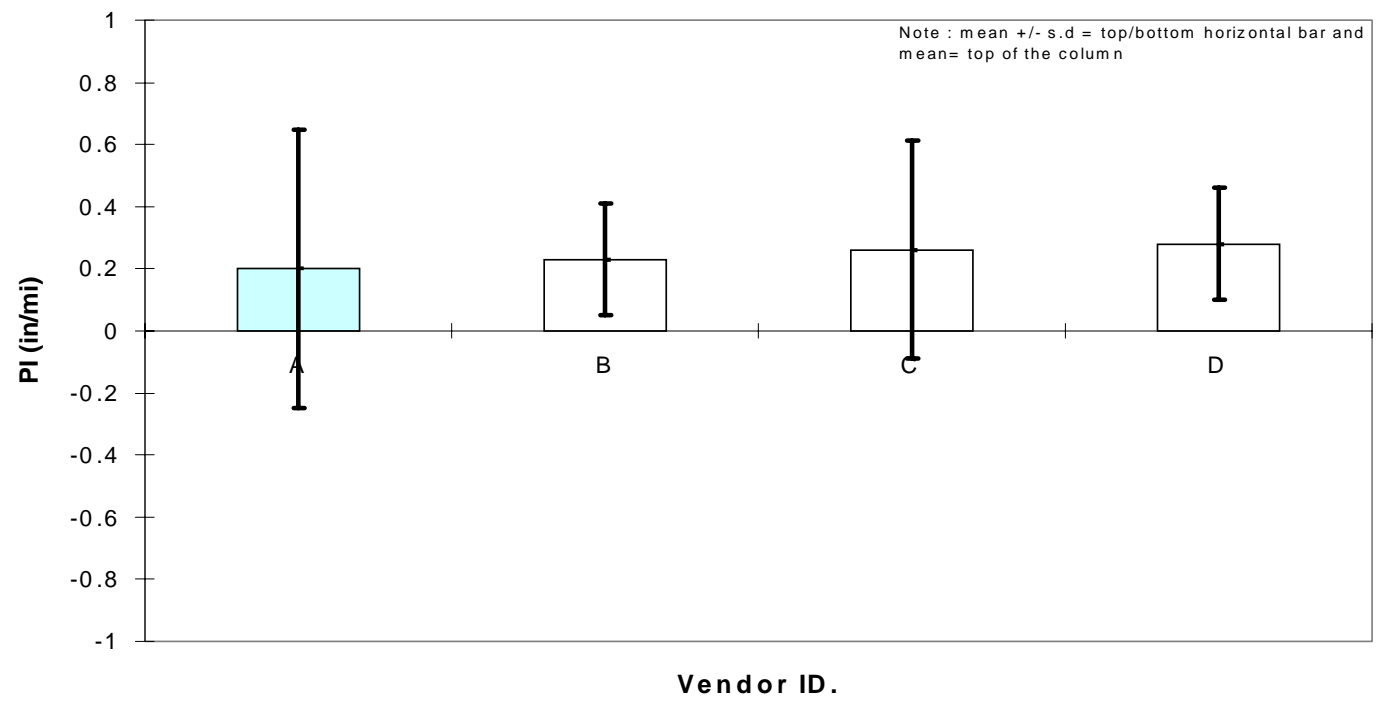

Figure D-22 PI-0.2”' Between Vendor Comparison at US 231A (Site 5) 


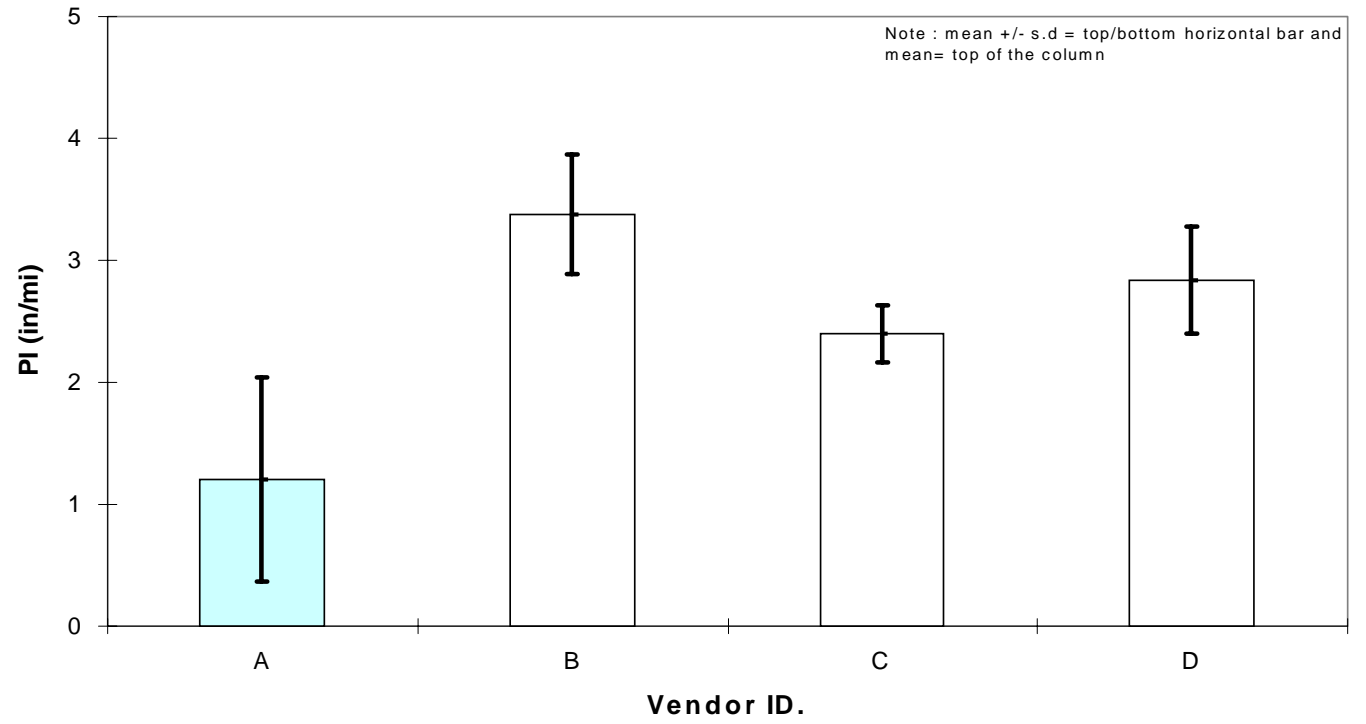

Figure D-23 PI-0.2” Between Vendor Comparison at US 231B (Site 6) 


\section{APPENDIX E - Within Vendor Profile Comparison}




\section{APPENDIX E - Within Vendor Profile Comparison}

\section{Part 1- Profiles of Vendor A}

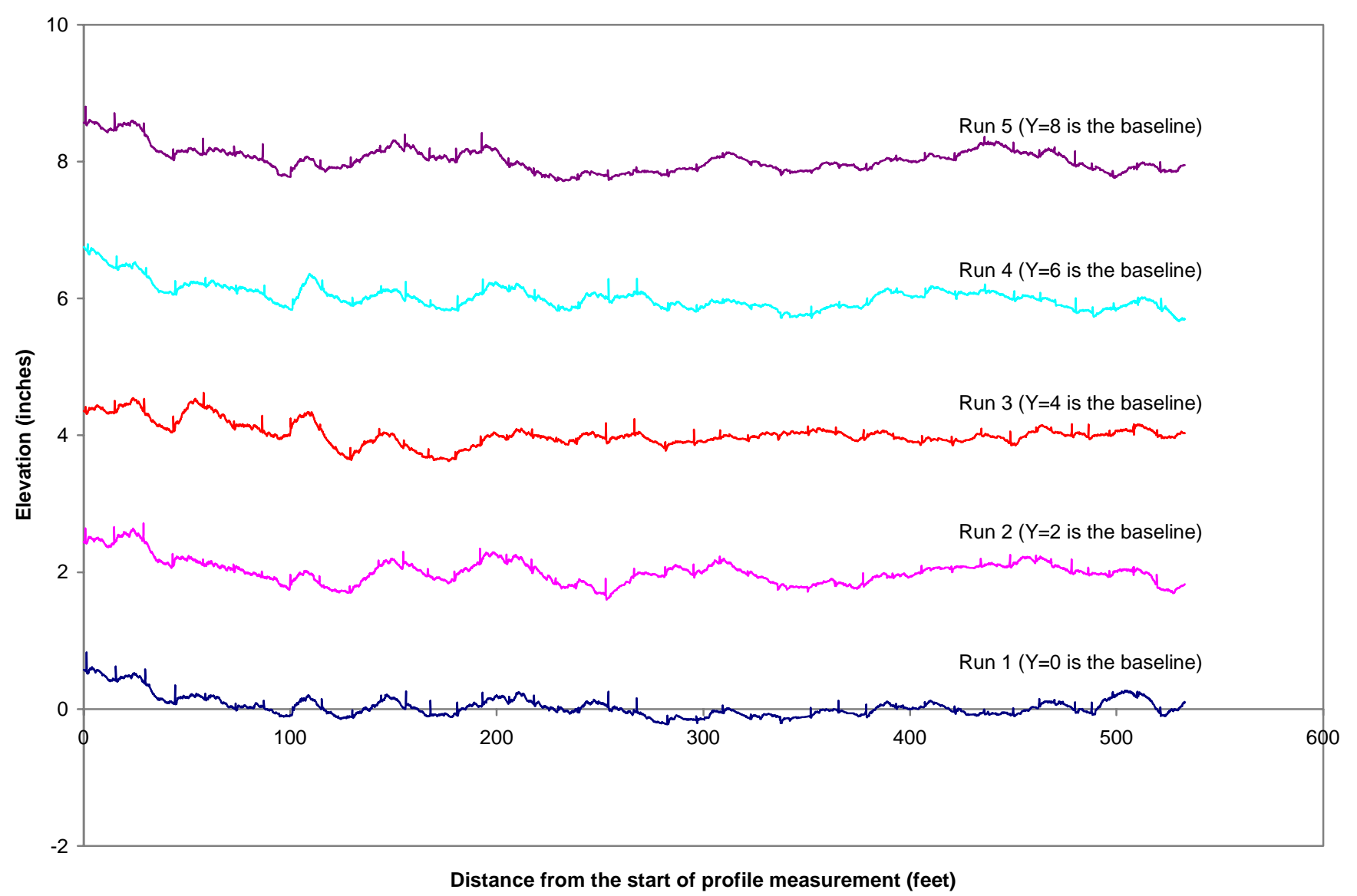

Figure E1-1 Profiles from replicate runs for Vendor A at Covington Street (Site 1) 


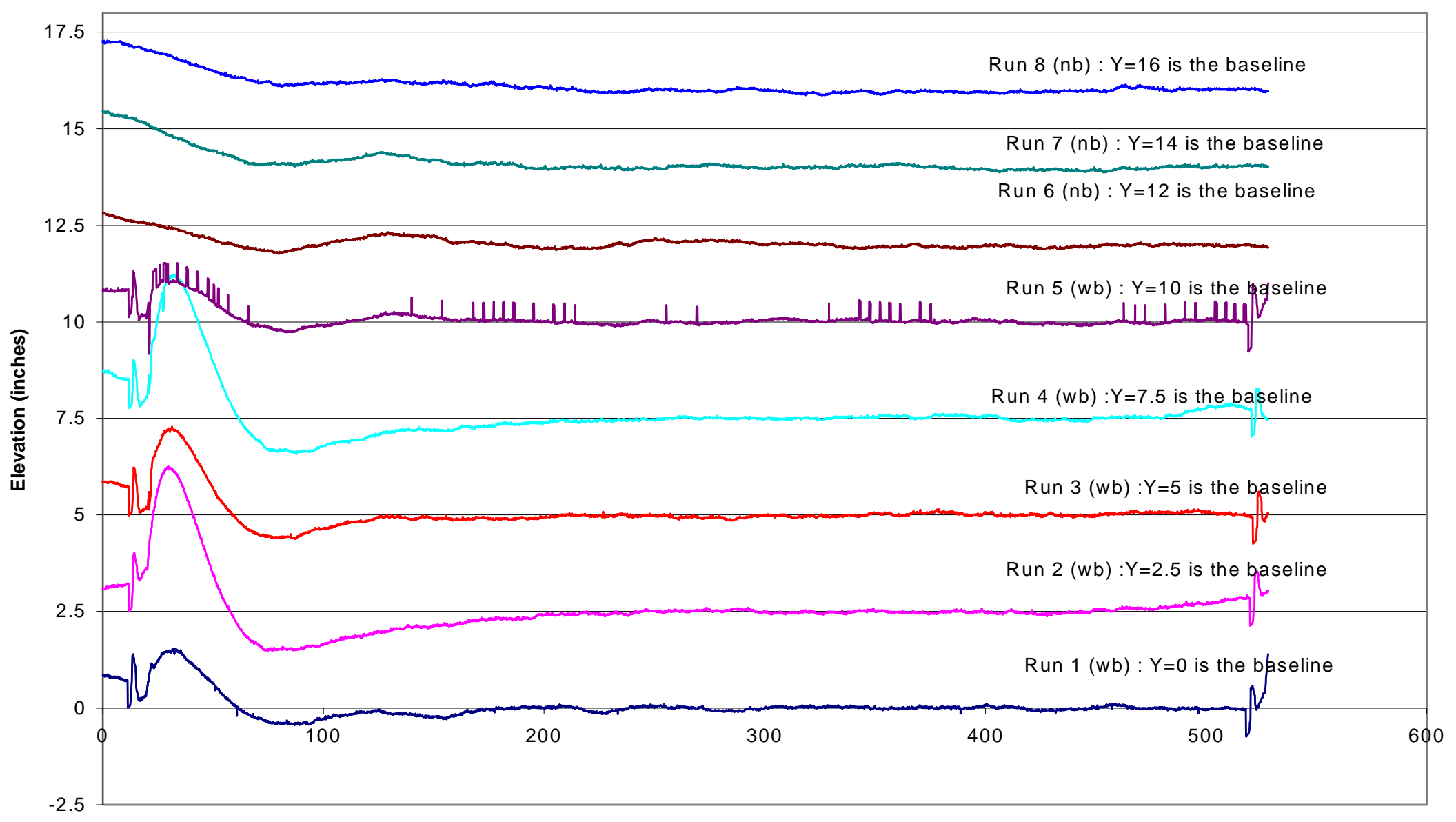

Distance from the start of profile measurement (feet)

Figure E1-2 Profiles from replicate runs for Vendor A at I-65 (Site 2 and 2b) 


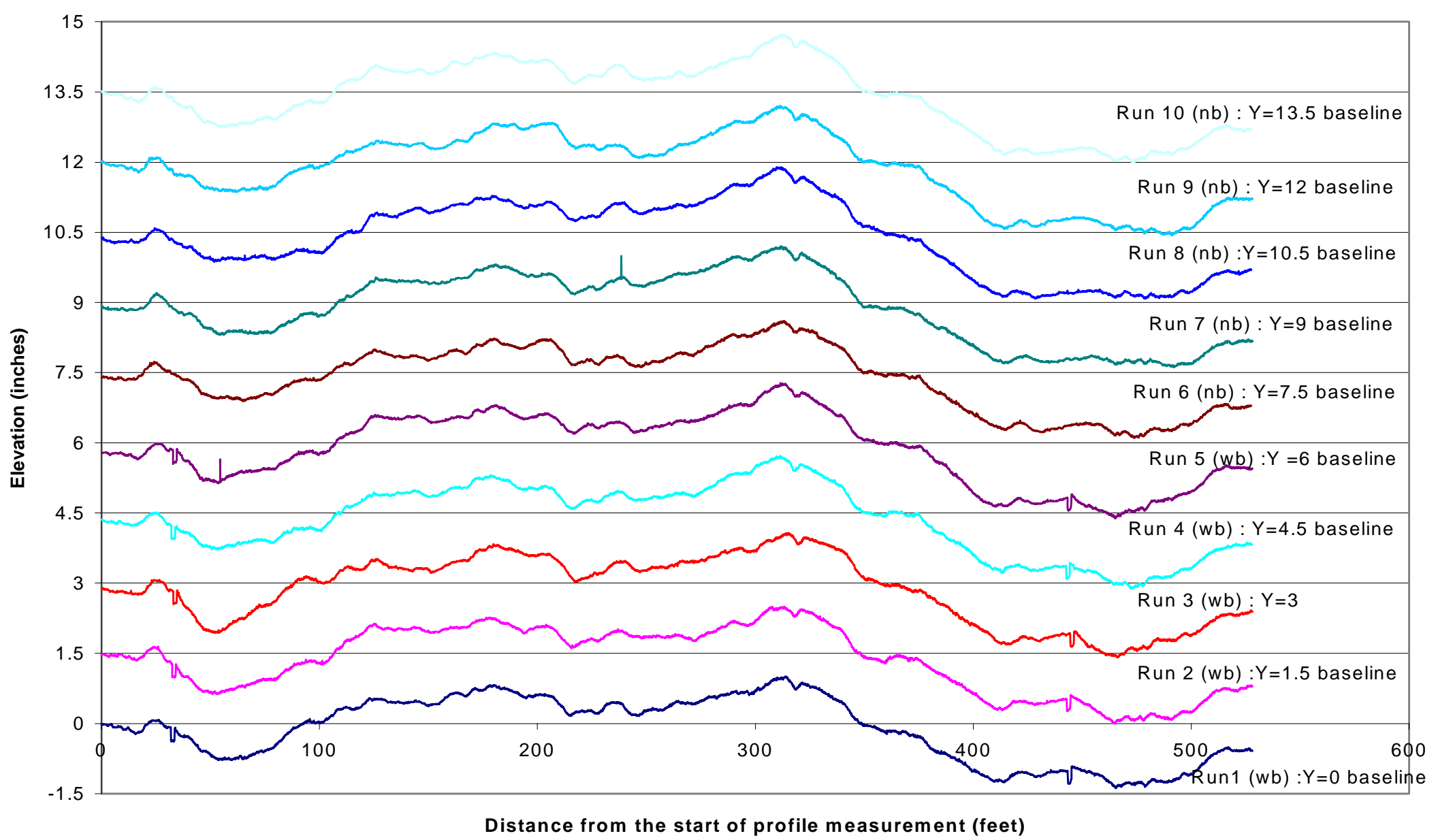

Figure E1-3 Profiles from replicate runs for Vendor A at Prairie Street (Site 3 and 3b) 


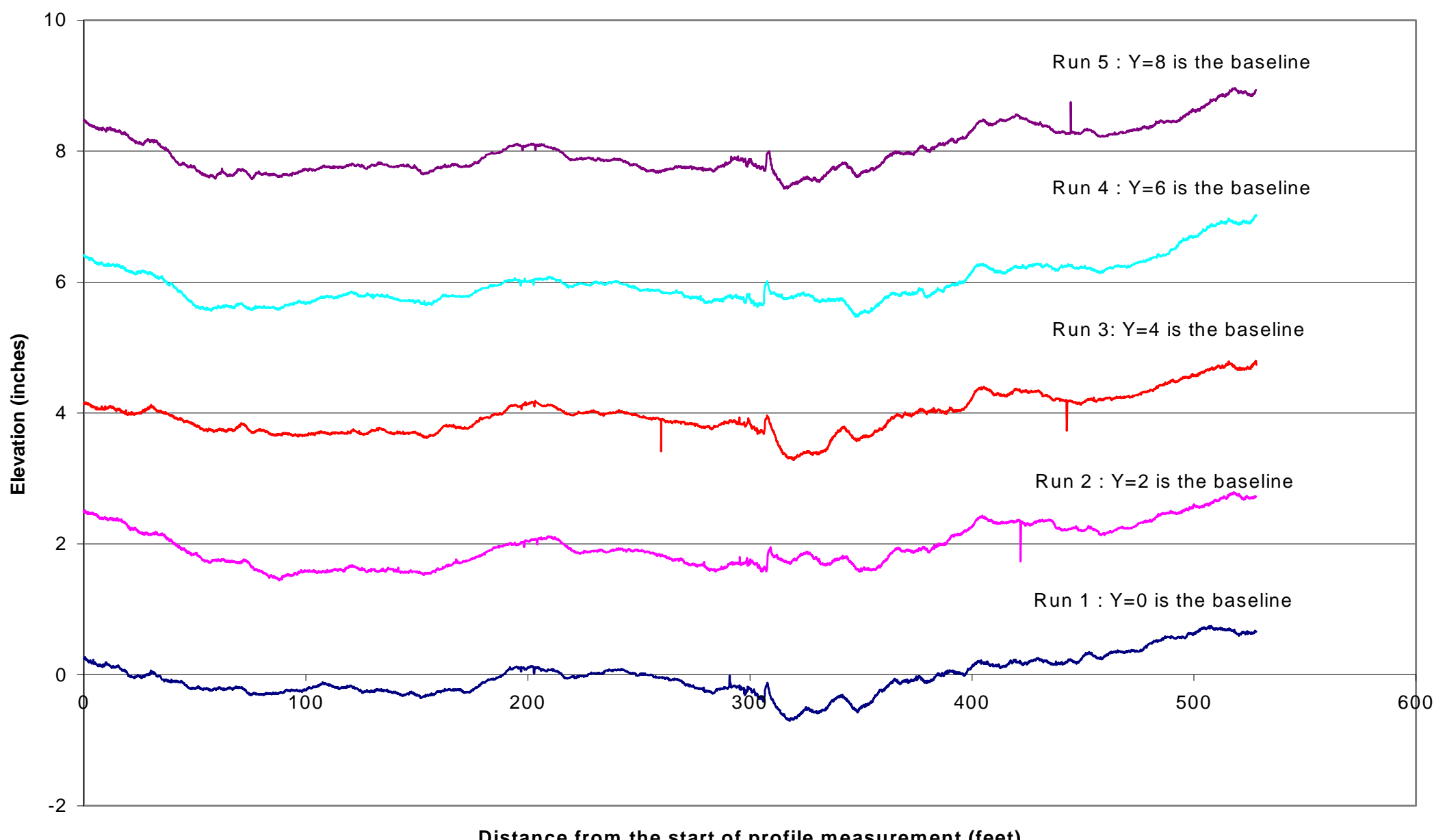

Figure E1-4 Profiles from replicate runs for Vendor A at Vinton Street (Site 4) 


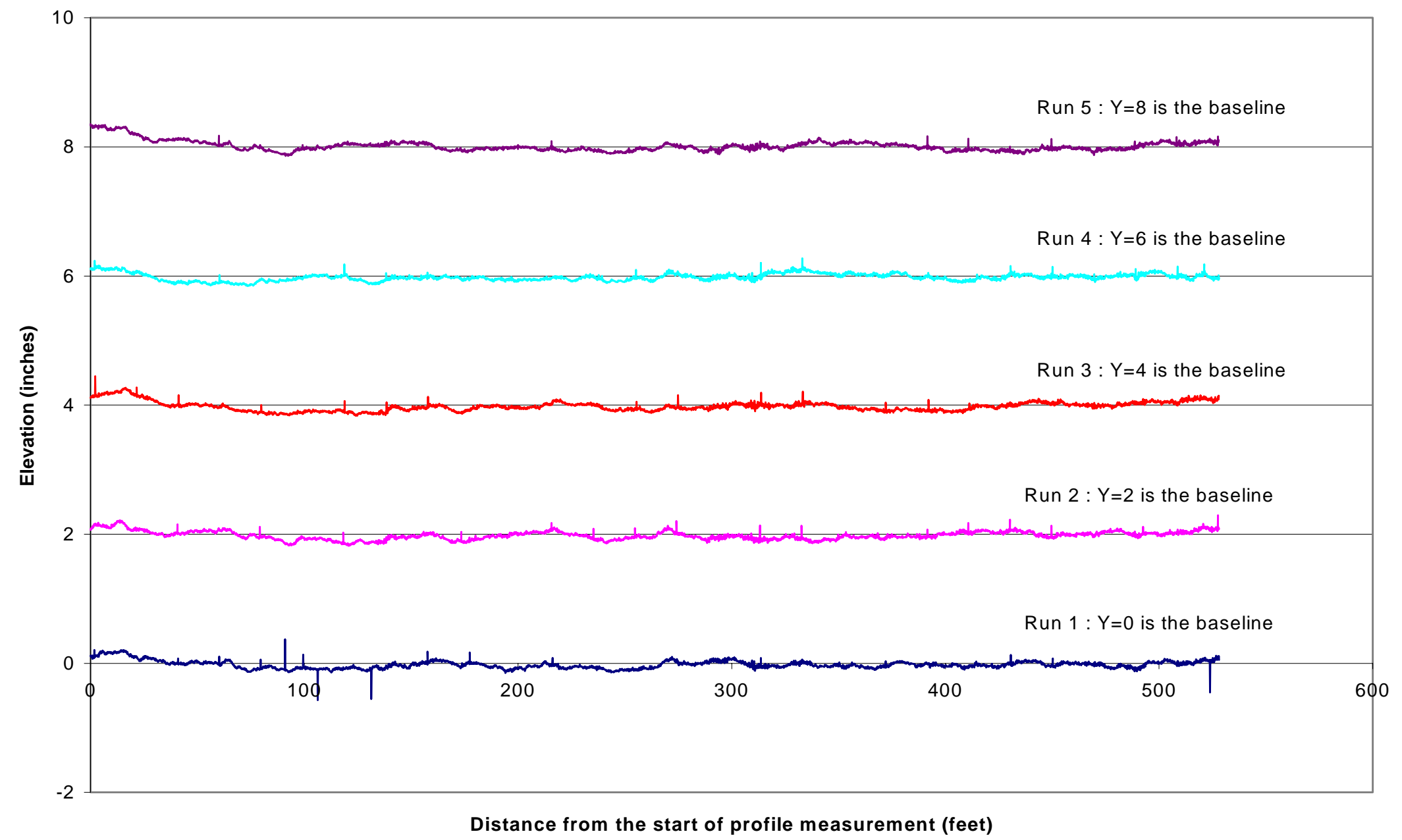

Figure E1-5 Profiles from replicate runs for Vendor A at US 231A (Site 5) 


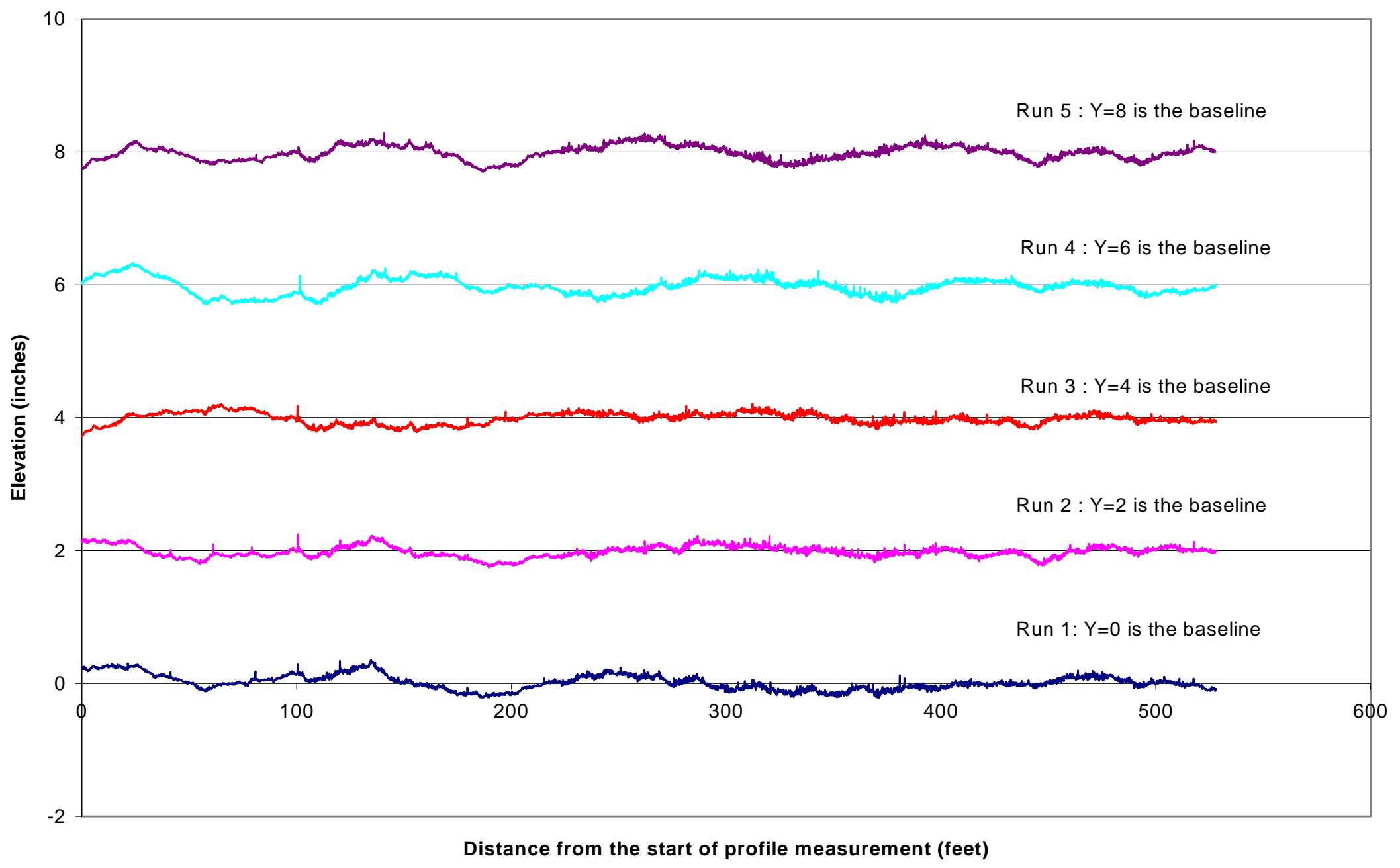

Figure E1-6 Profiles from replicate runs for Vendor A at US 231B (Site 6) 


\section{Part 2 - Profiles of Vendor B}

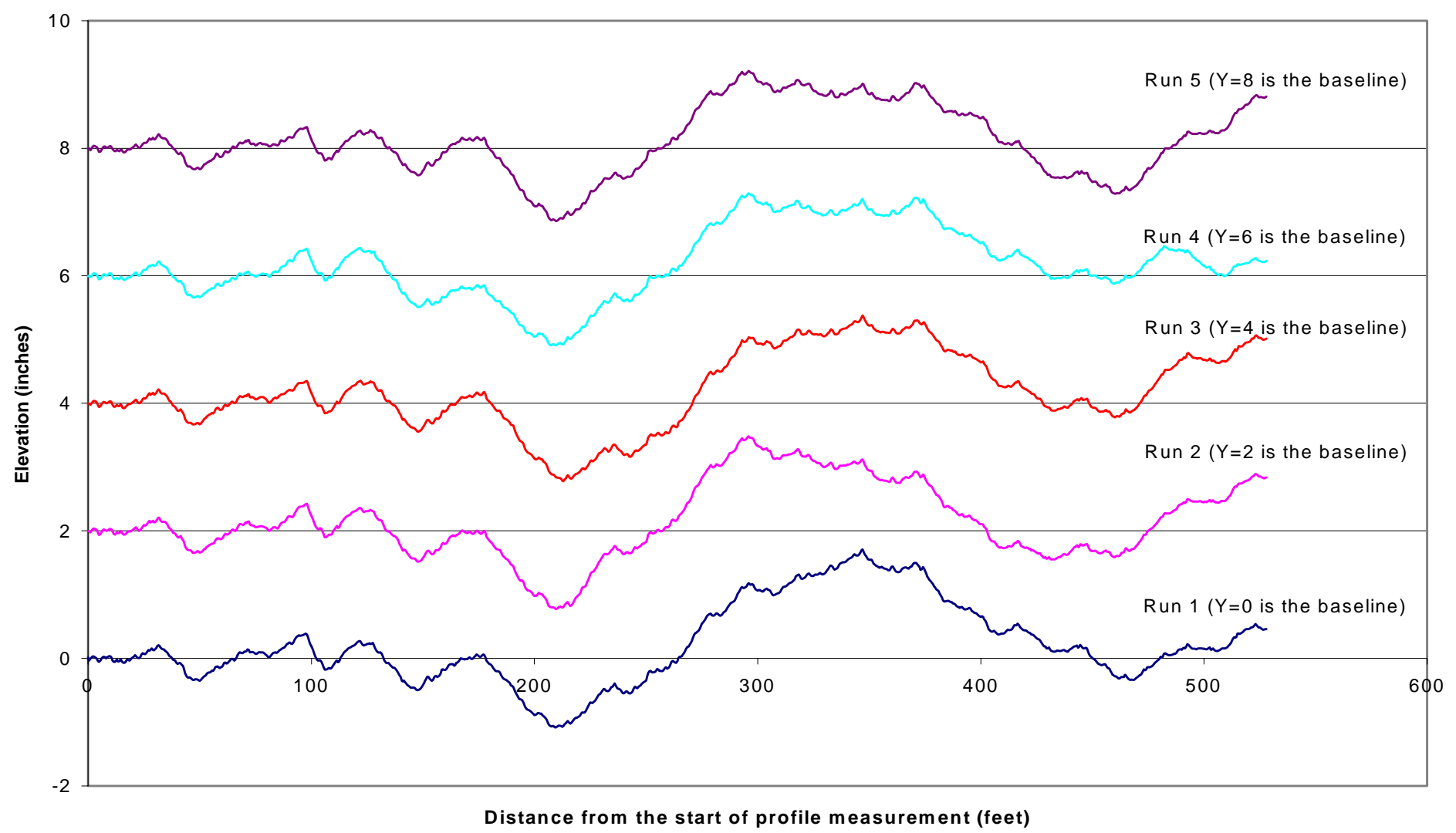

Figure E2-1 Profiles from replicate runs for Vendor B at Covington Street (Site 1) 


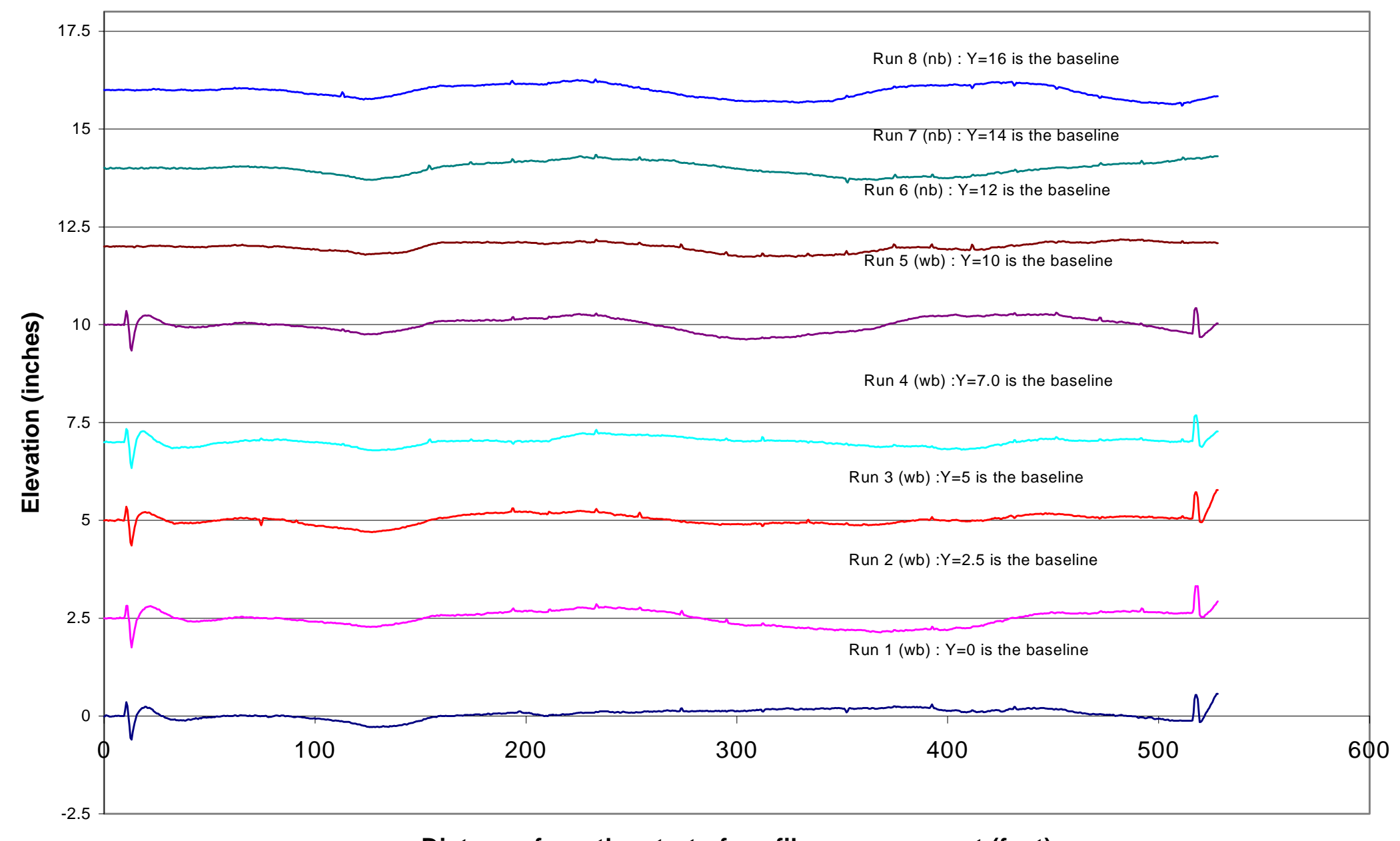

Distance from the start of profile measurement (feet)

Figure E2-2 Profiles from replicate runs for Vendor B at I-65 (Site 2 and 2b) 


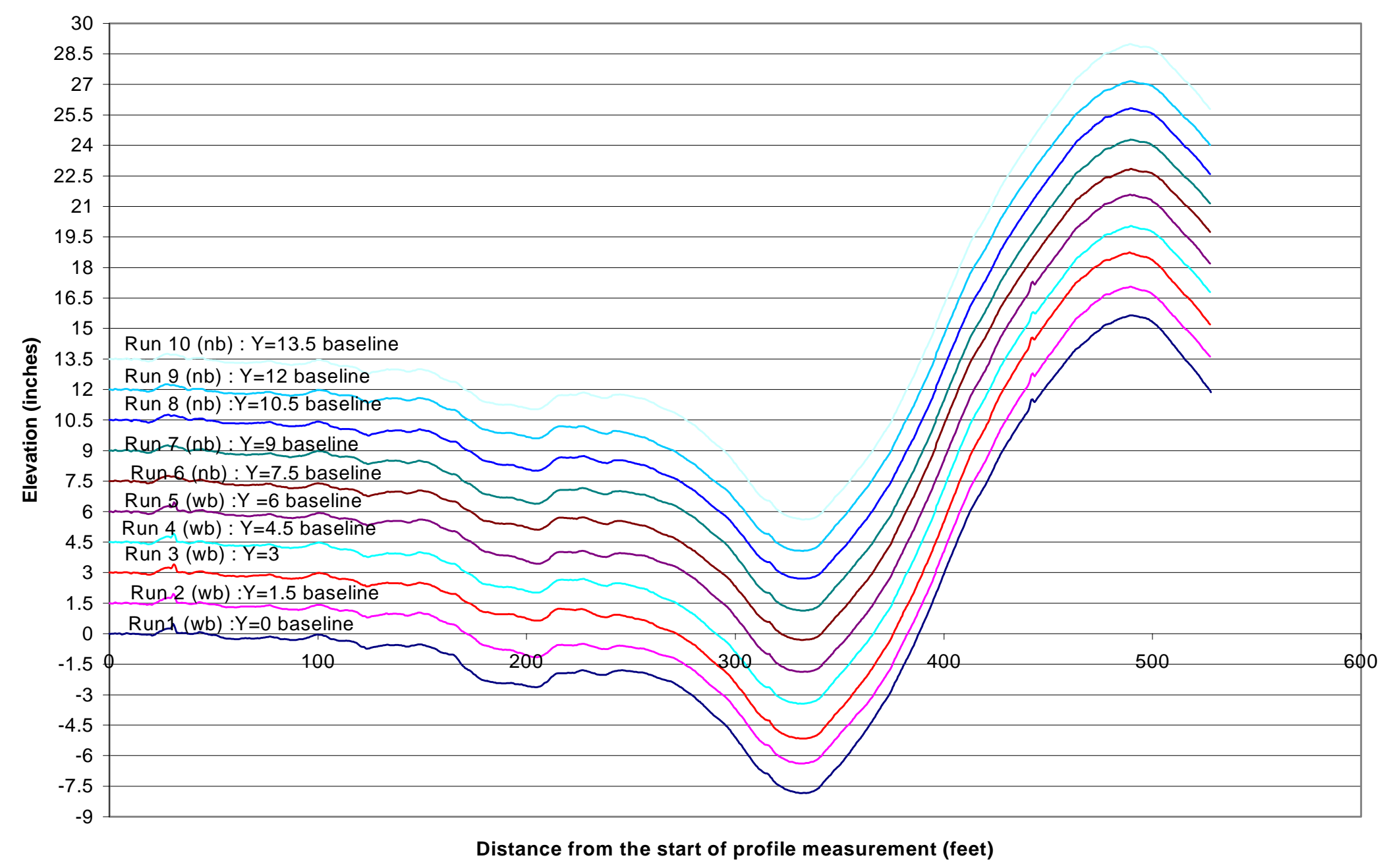

Figure E2-3 Profiles from replicate runs for Vendor B at Prairie Street (Site 3 and 3b) 


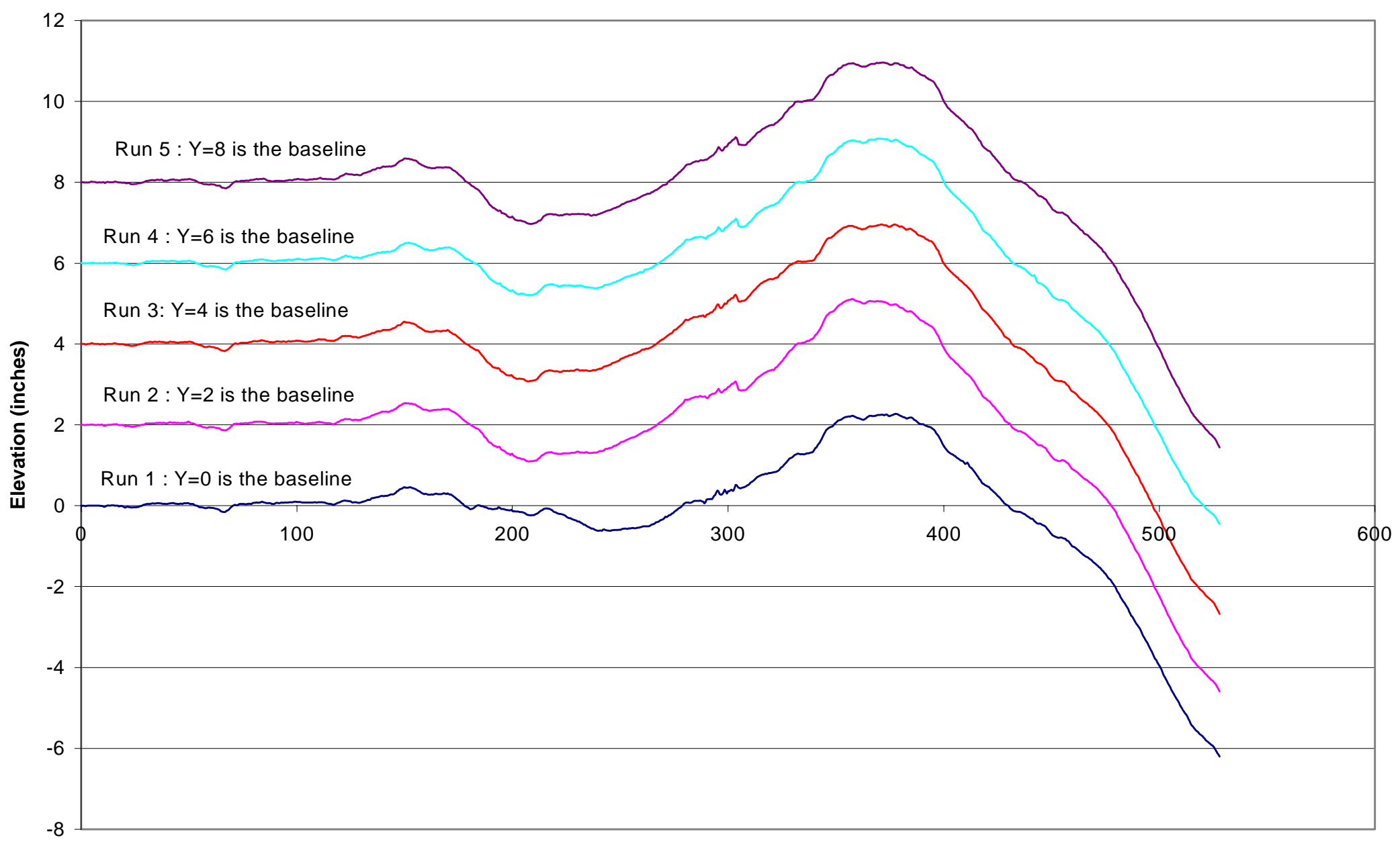

Distance from the start of profile measurement (feet)

Figure E2-4 Profiles from replicate runs for Vendor B at Vinton Street (Site 4) 


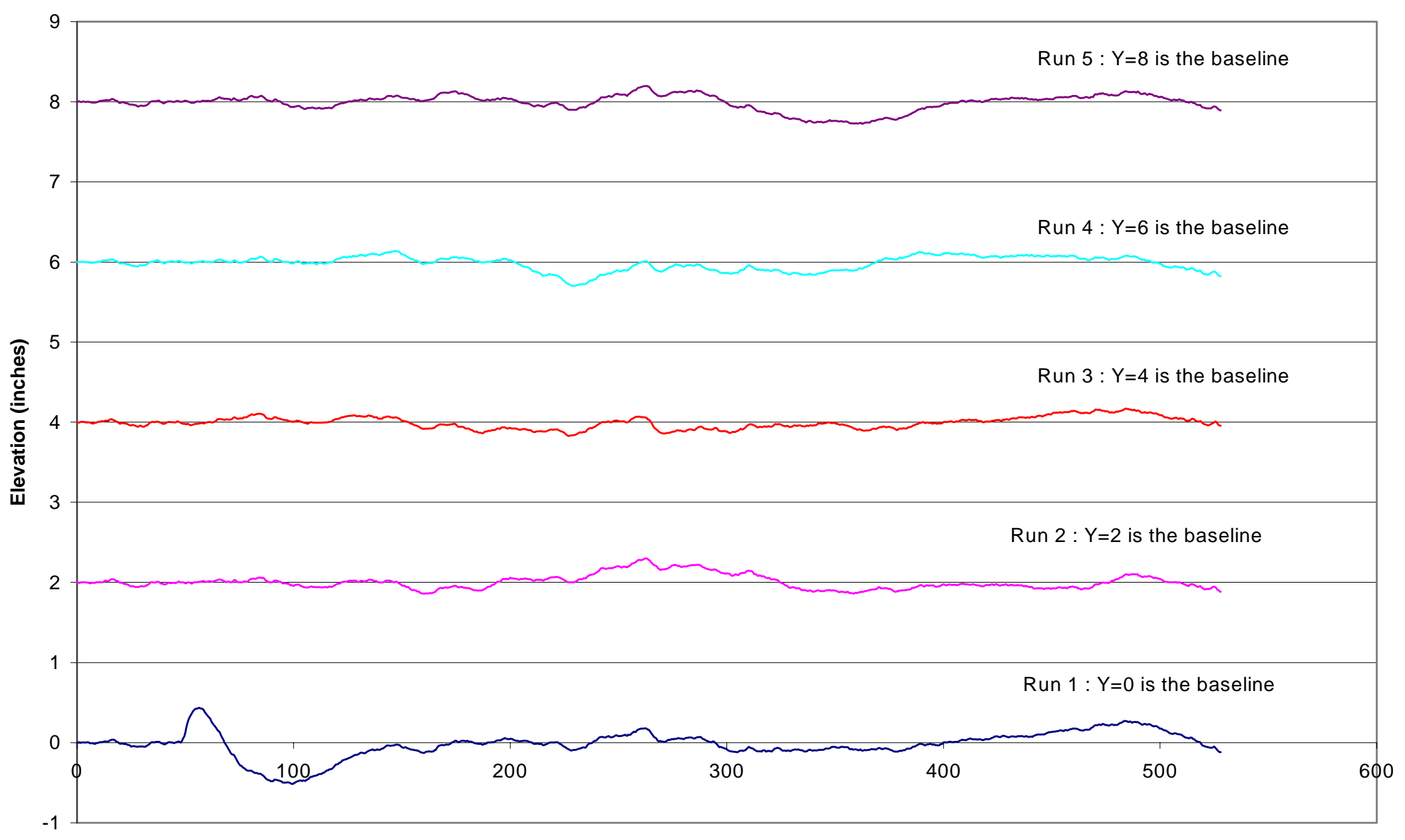

Distance from the start of profile measurement (feet)

Figure E2-5 Profiles from replicate runs for Vendor B at US 231A (Site 5) 


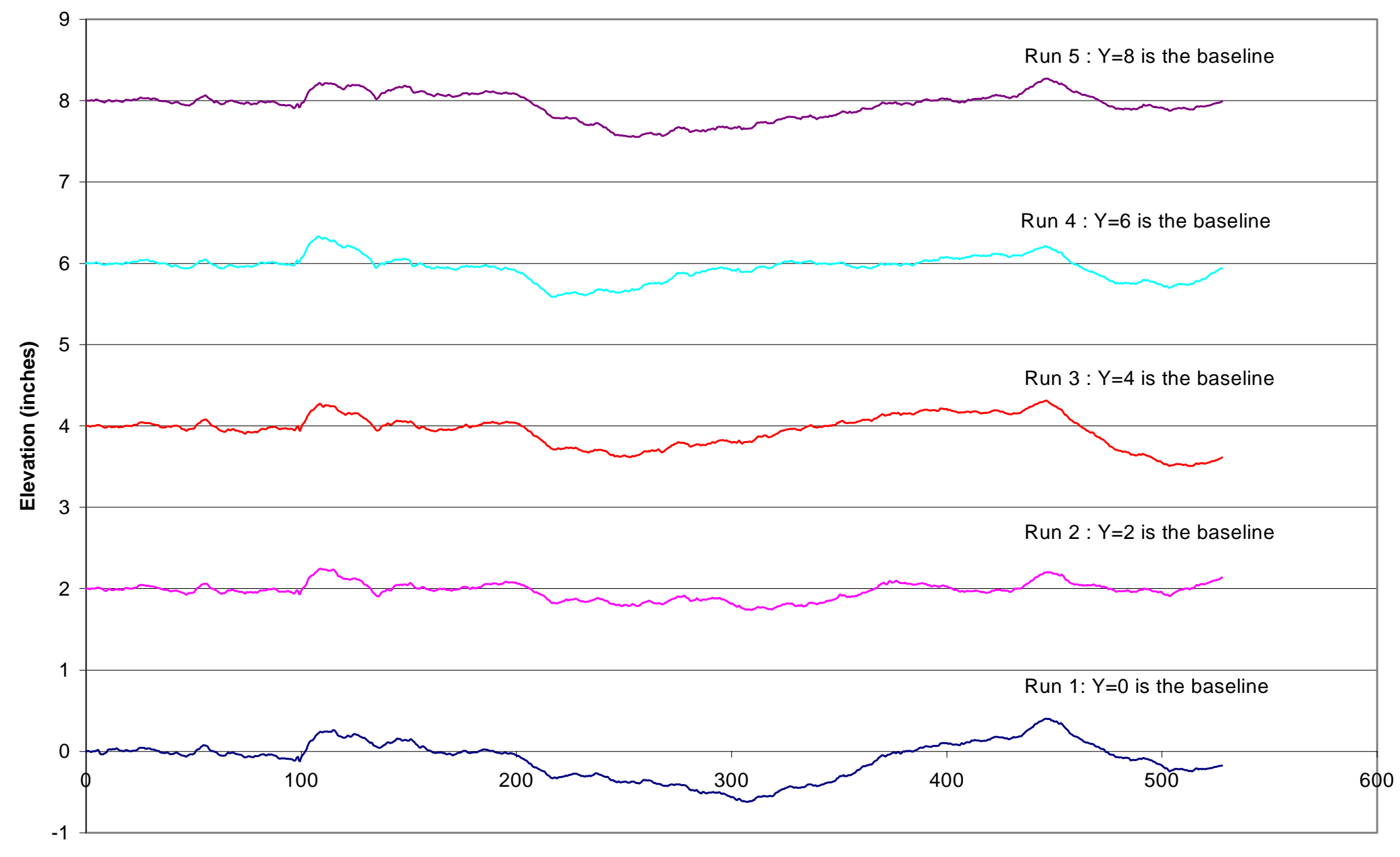

Distance from the start of profile measurement (feet)

Figure E2-6 Profiles from replicate runs for Vendor B at US 231B (Site 6) 


\section{Part 3 - Profiles of Vendor C}

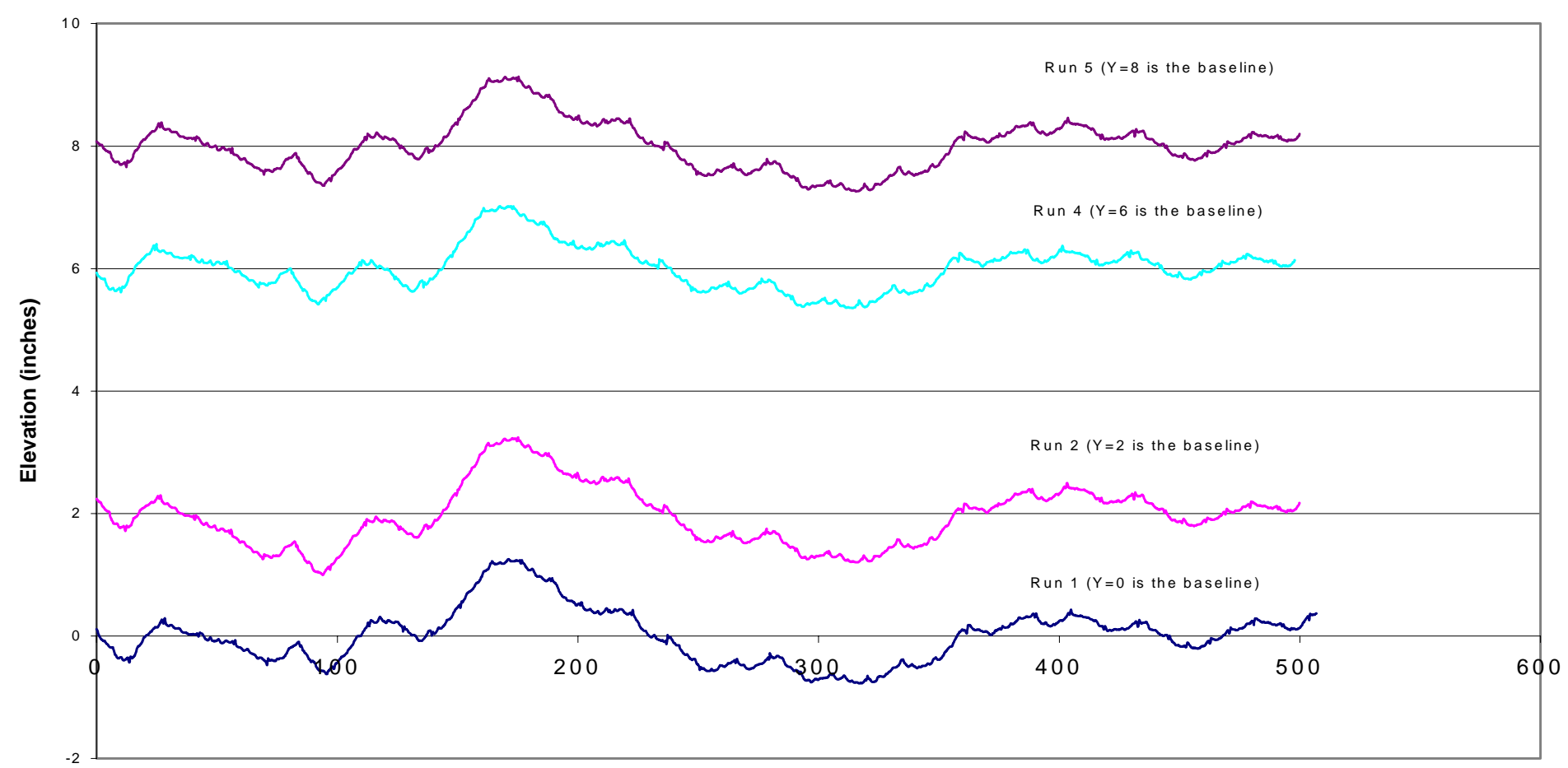

Distance from the start of profile measurement (feet)

Figure E3-1 Profiles from replicate runs for Vendor C at Covington Street (Site 1) 


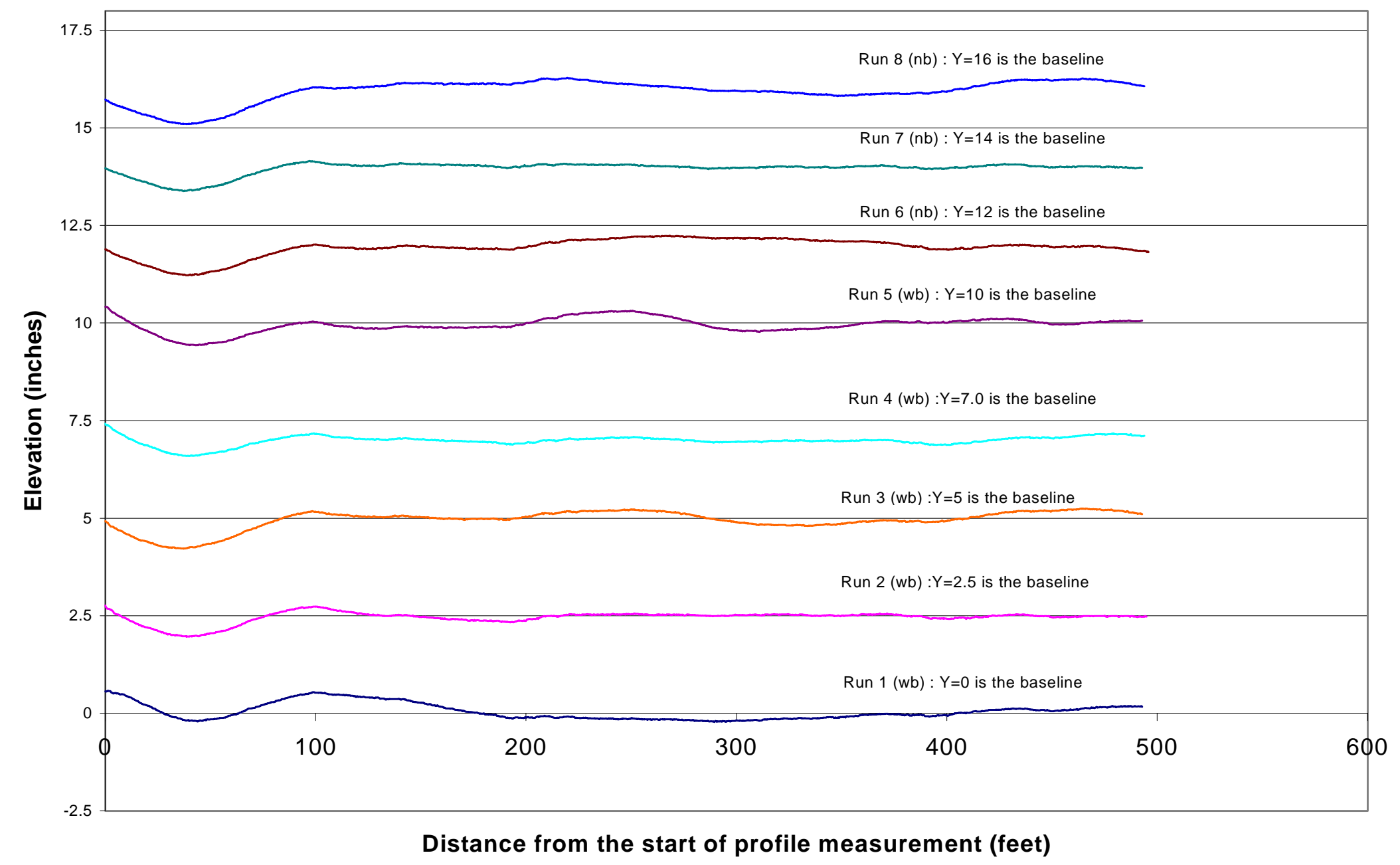

Figure E3-2 Profiles from replicate runs for Vendor C at I-65 (Site 2 and 2b) 


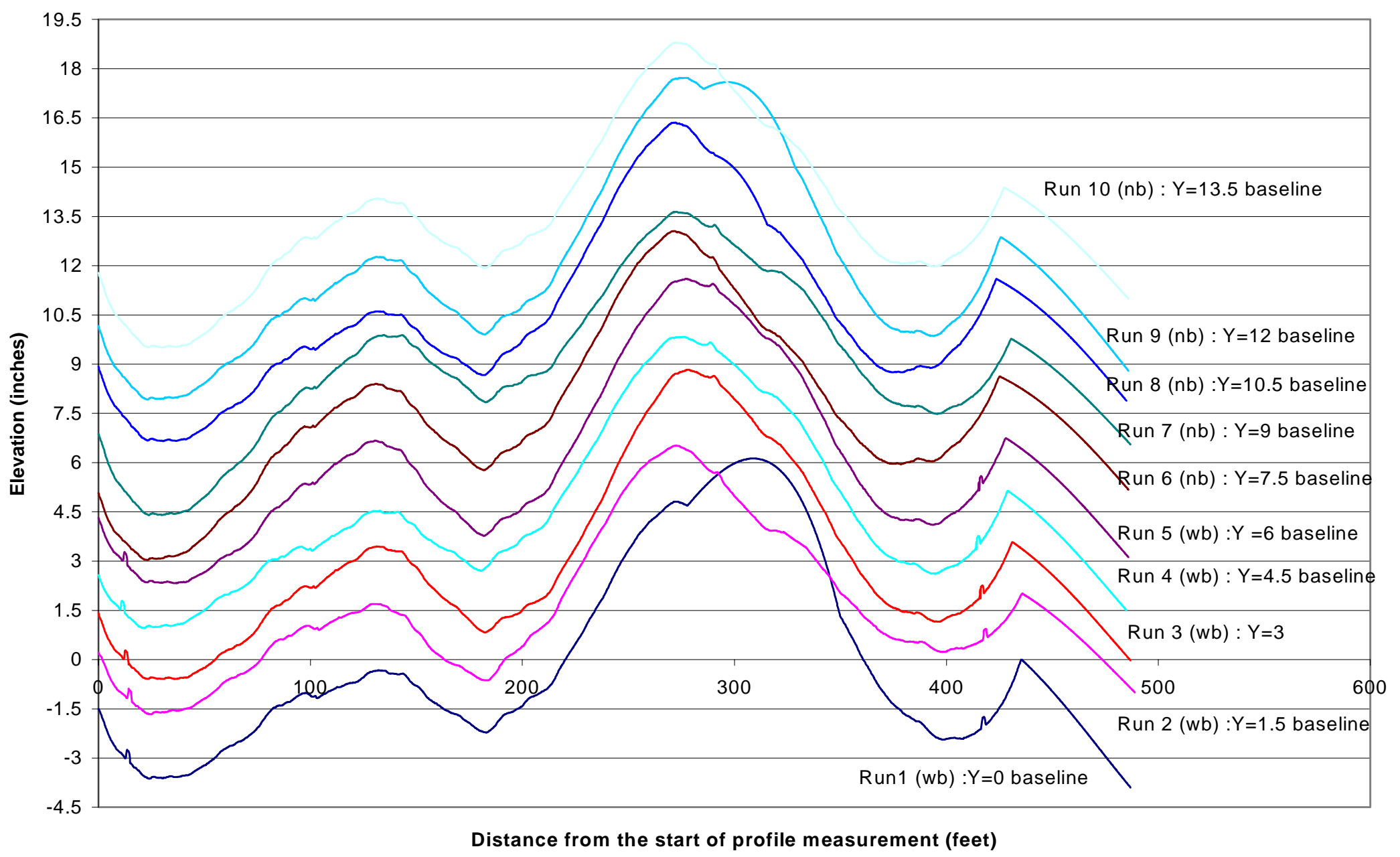

Figure E3-3 Profiles from replicate runs for Vendor C at Prairie Street (Site 3 and 3b) 


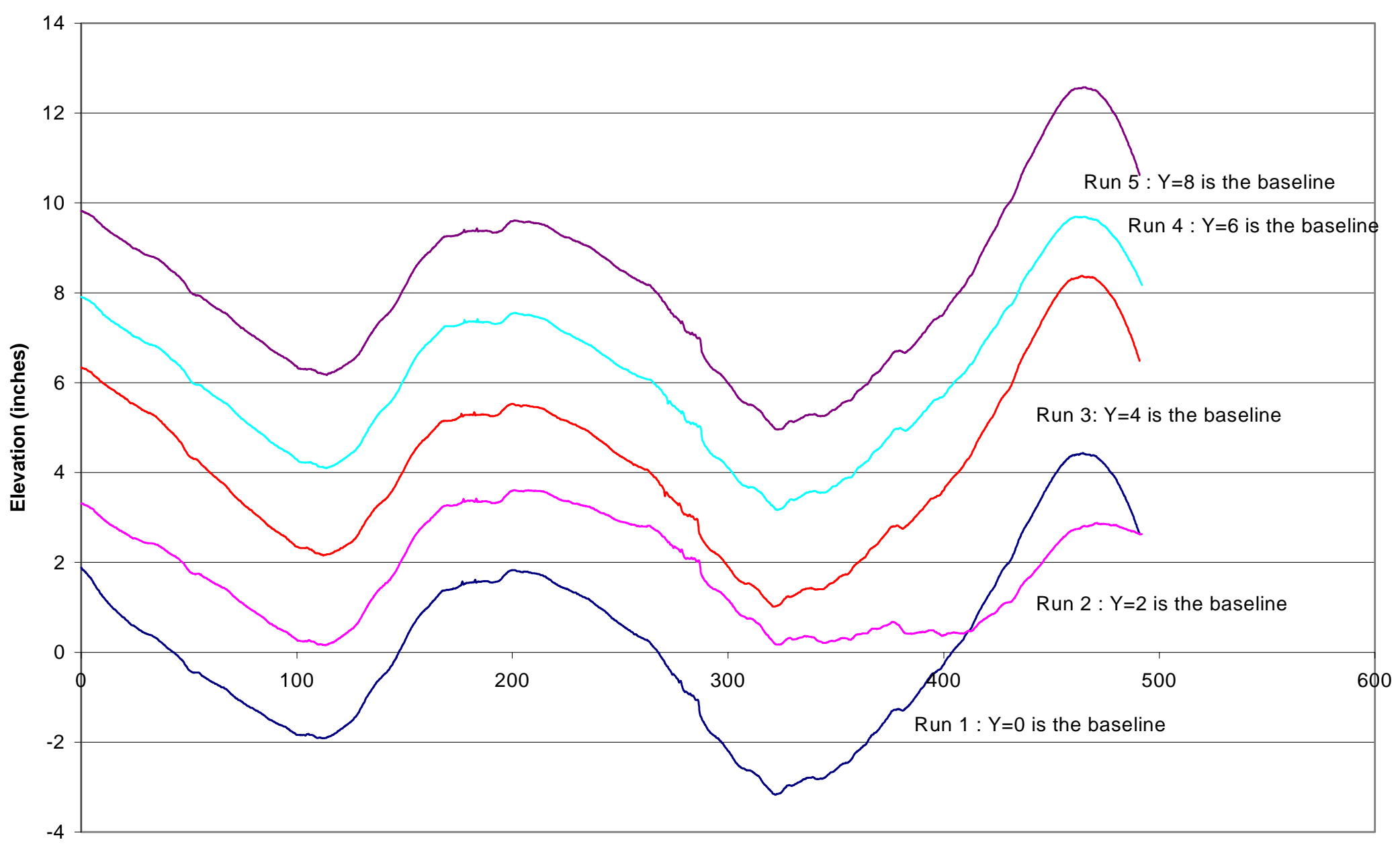

Distance from the start of profile measurement (feet)

Figure E3-4 Profiles from replicate runs for Vendor C at Vinton Street (Site 4) 


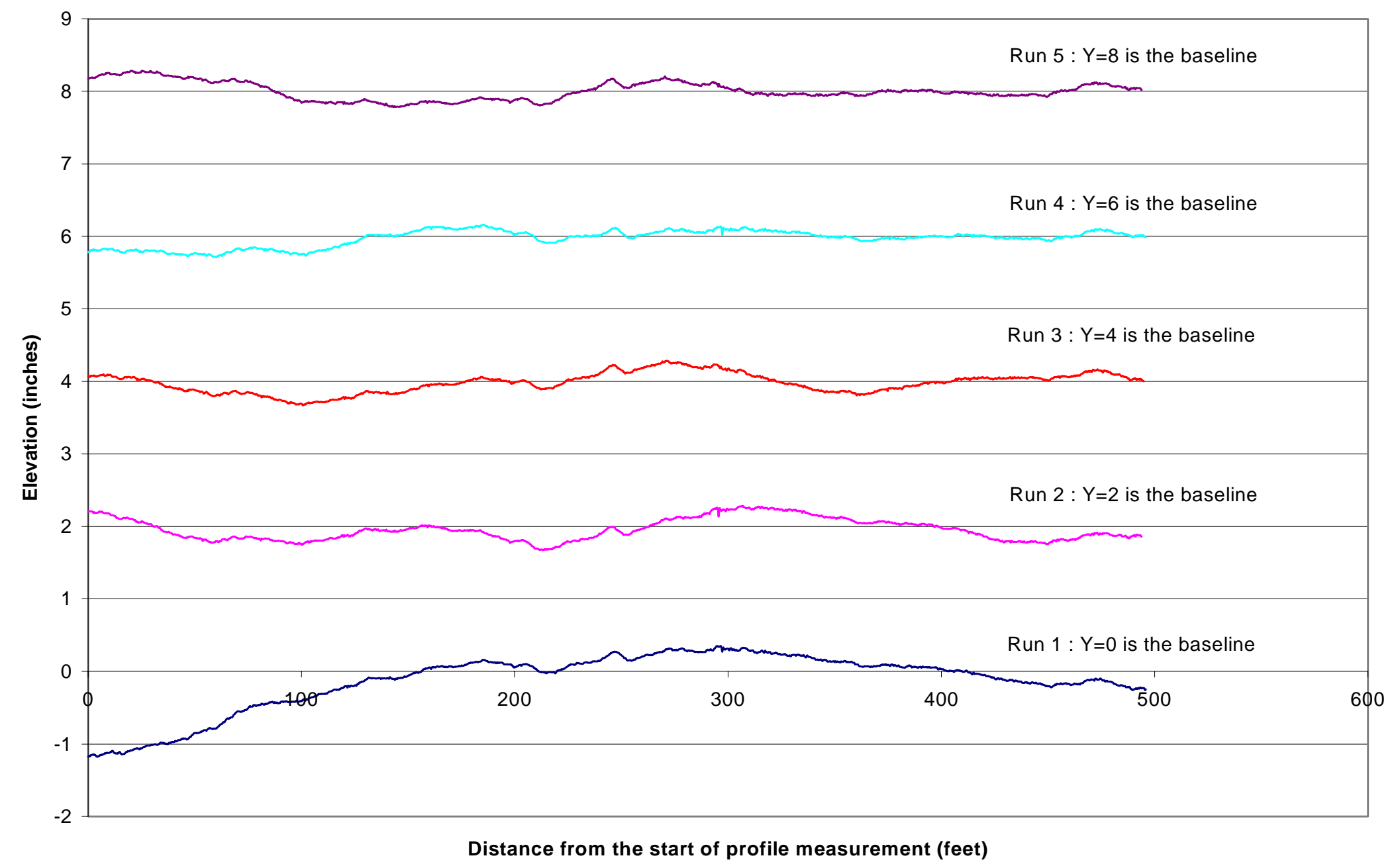

Figure E3-5 Profiles from replicate runs for Vendor C at US 231A (Site 5) 


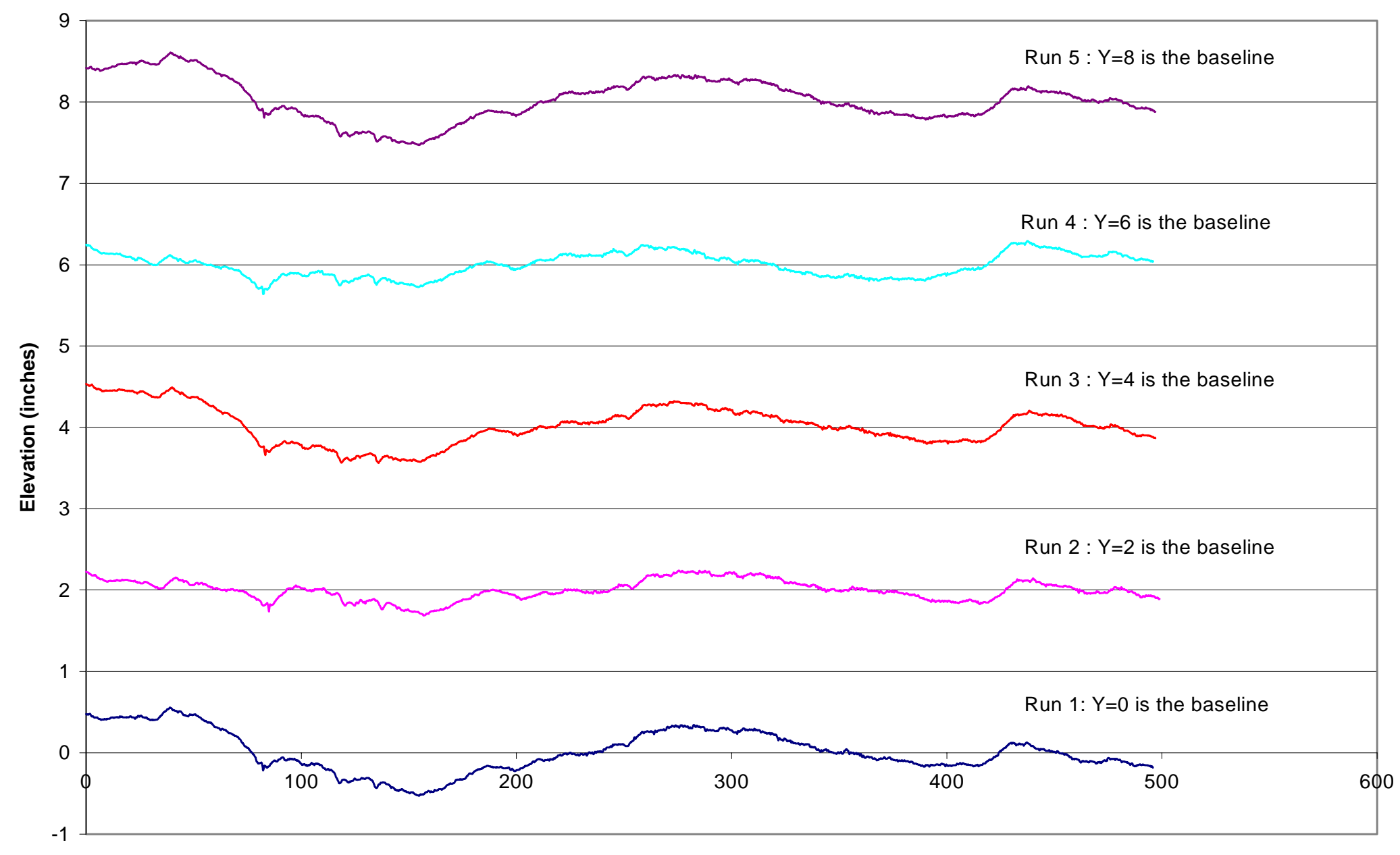

Distance from the start of profile measurement (feet)

Figure E3-6 Profiles from replicate runs for Vendor C at US 231B (Site 6) 


\section{Part 4 - Profiles of Vendor D}

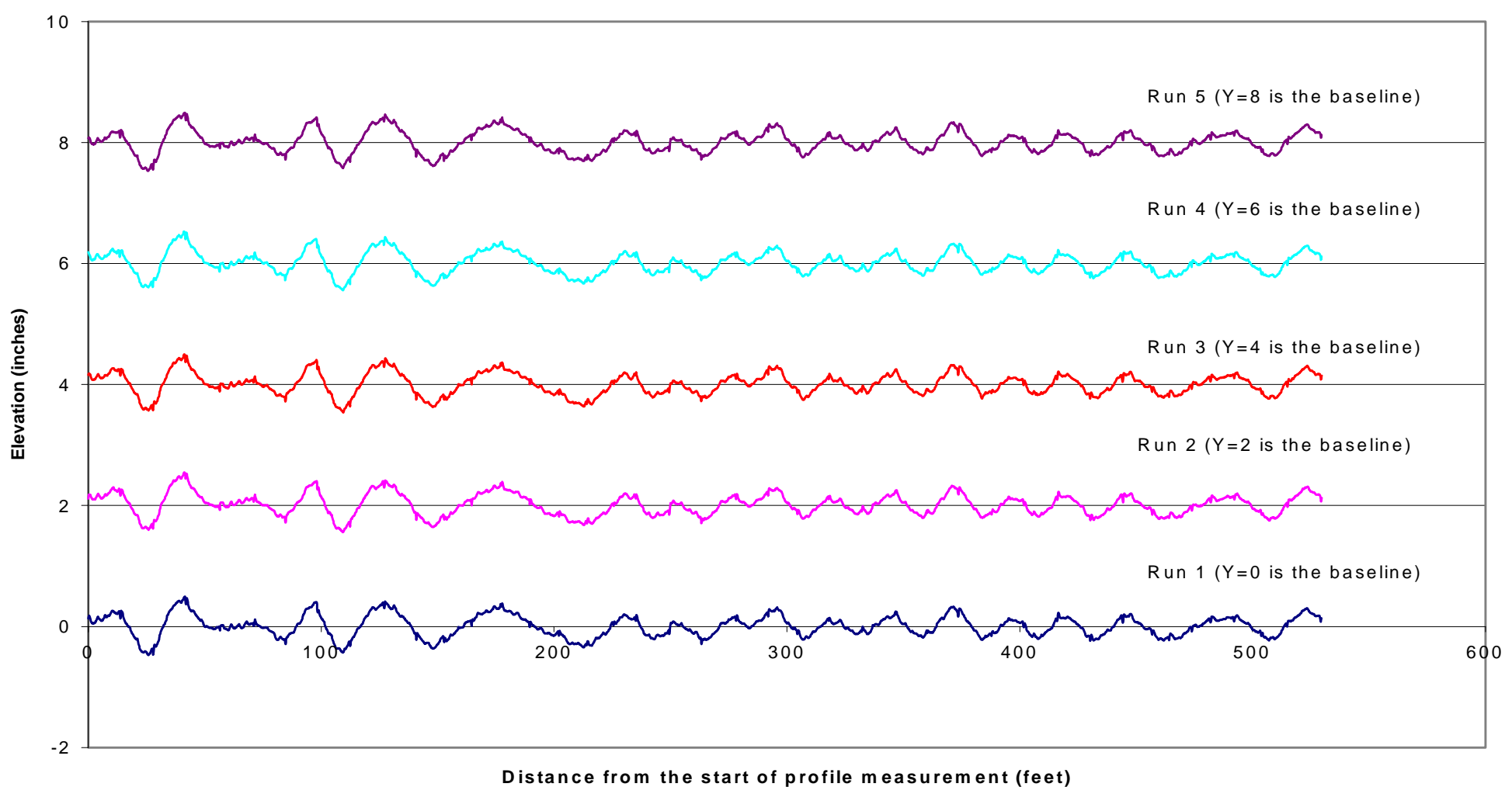

Figure E4-1 Profiles from replicate runs for Vendor D at Covington Street (Site 1) 


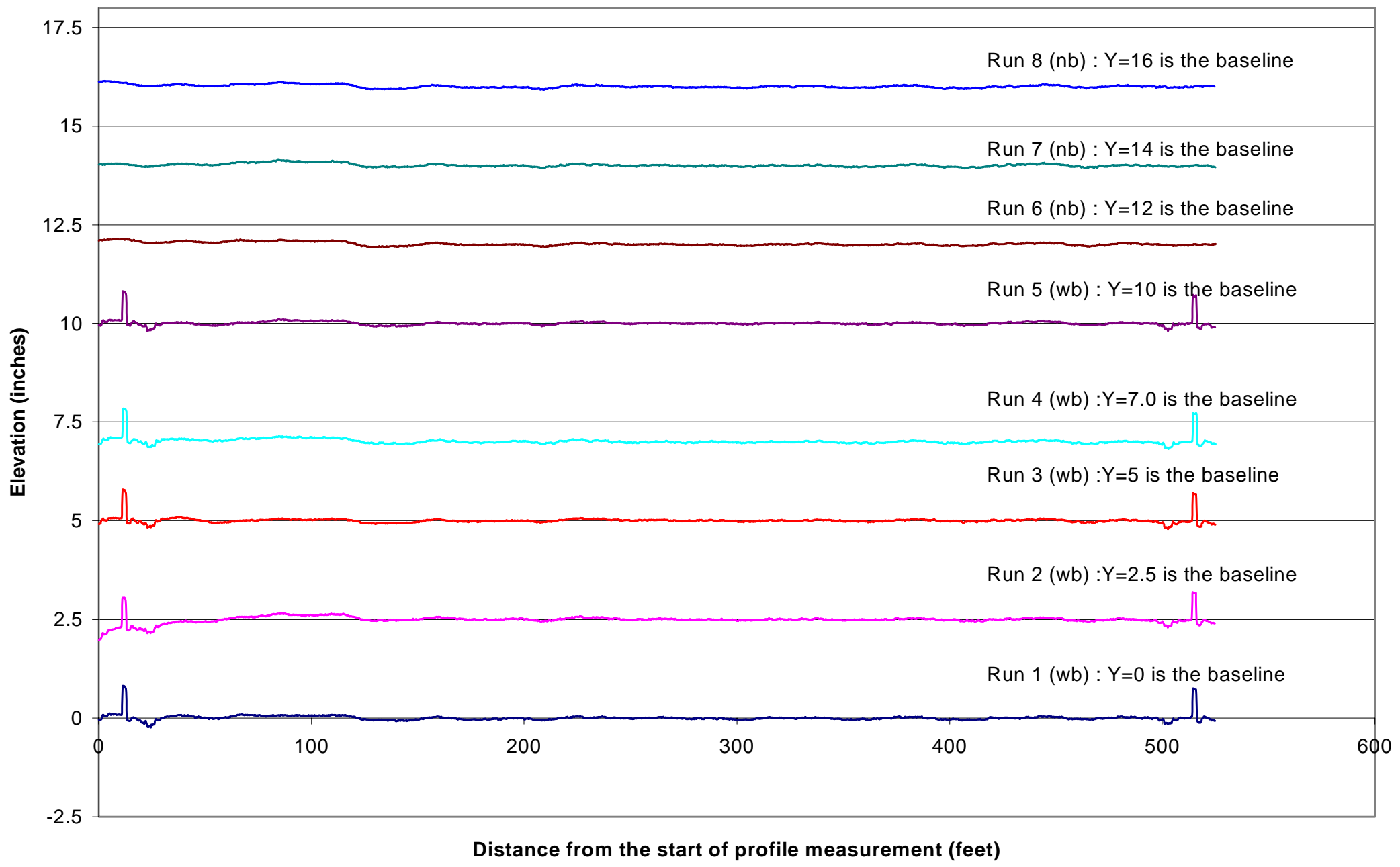

Figure E4-2 Profiles from replicate runs for Vendor D at I-65 (Site 2 and 2b) 


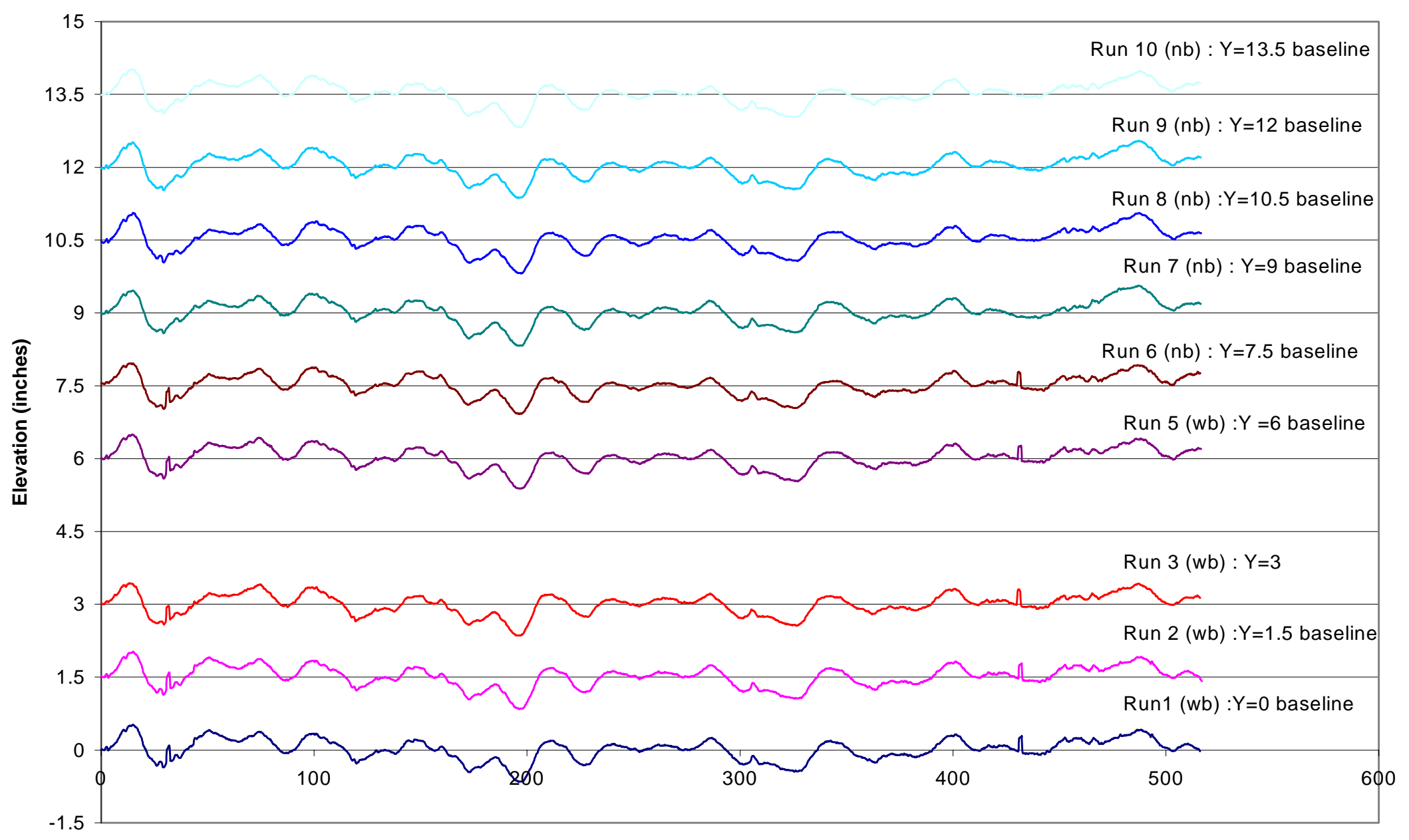

Distance from the start of profile measurement (feet)

Figure E4-3 Profiles from replicate runs for Vendor D at Prairie Street (Site 3 and 3b) 


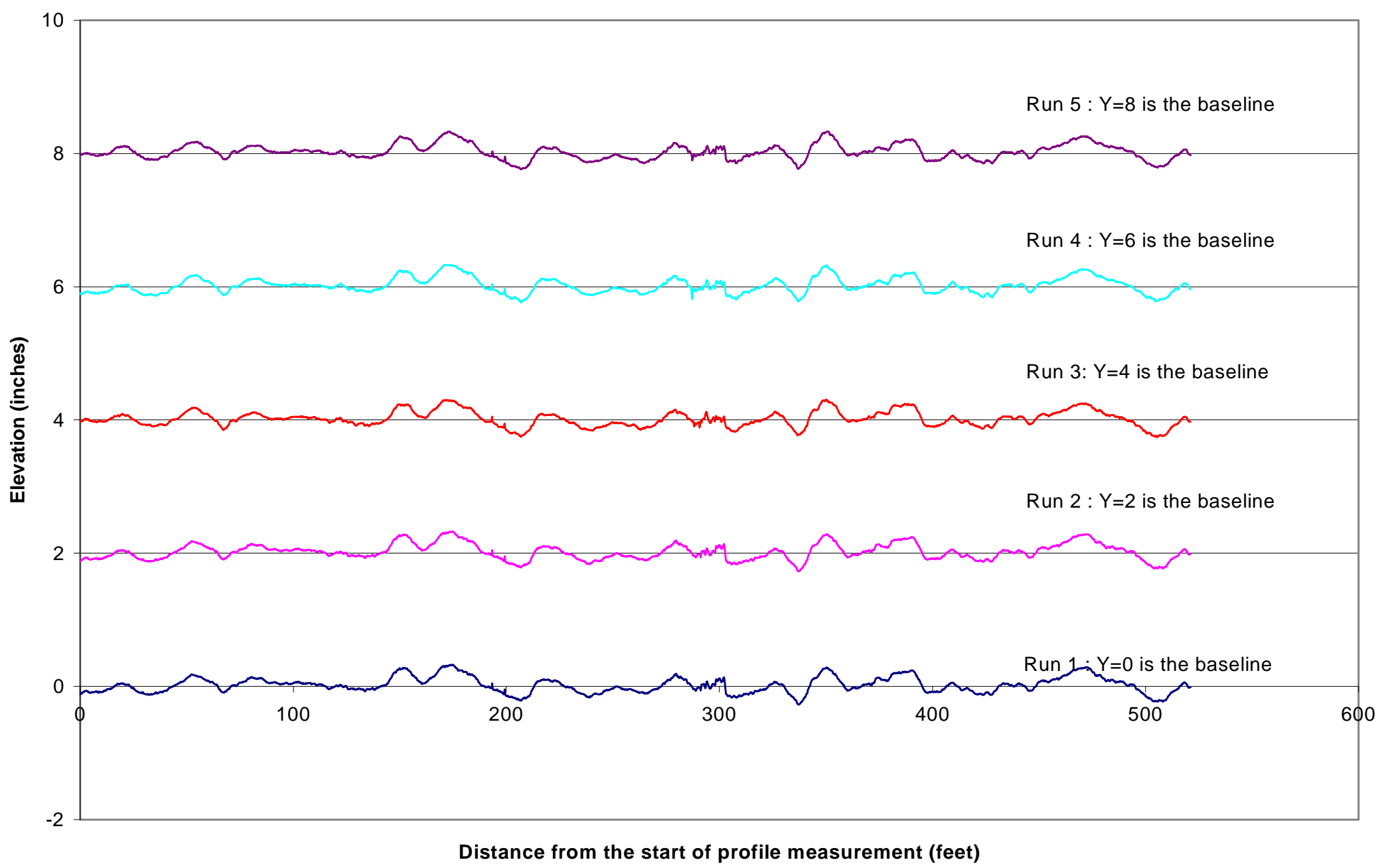

Figure E4-4 Profiles from replicate runs for Vendor D at Vinton Street (Site 4) 


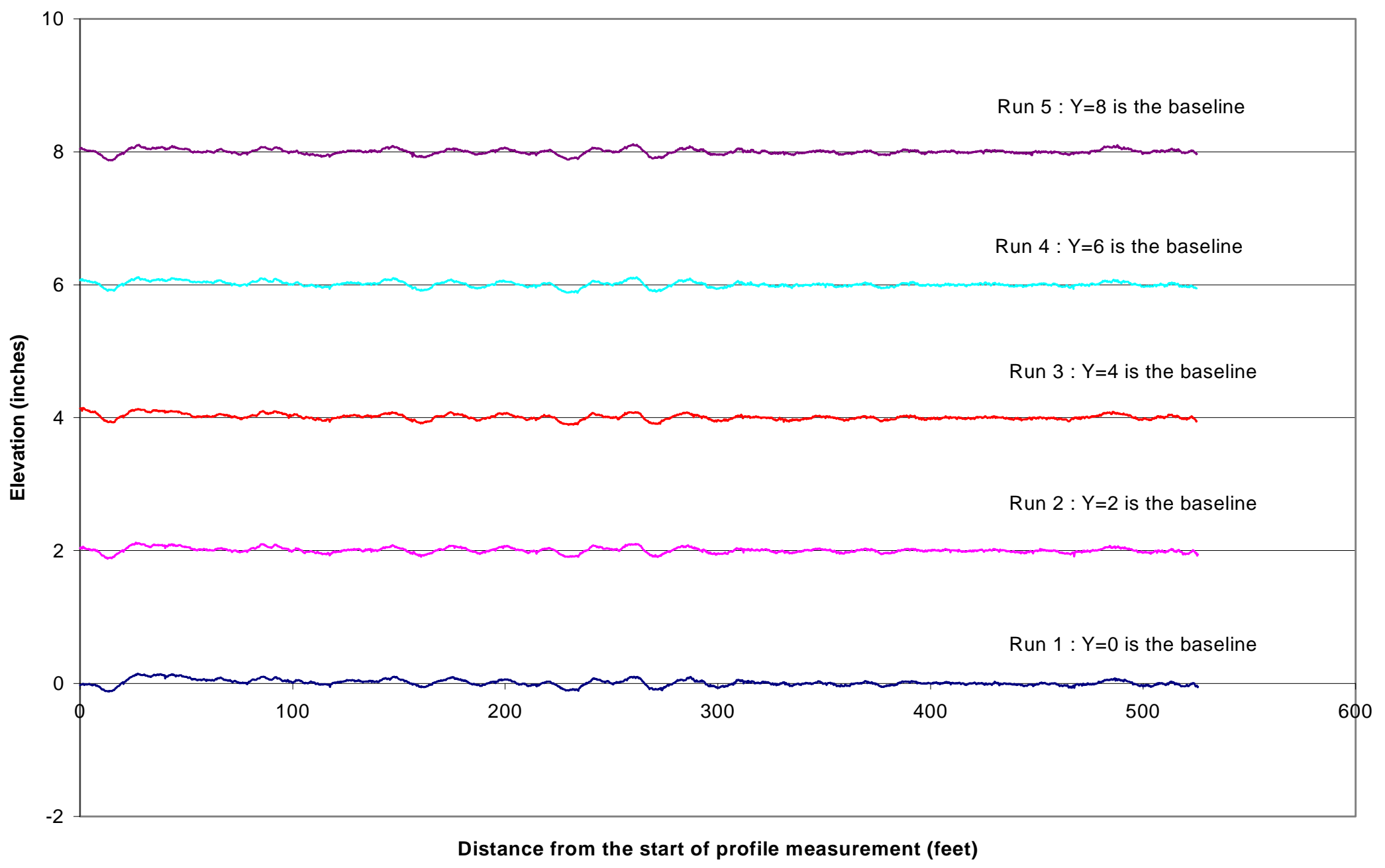

Figure E4-5 Profiles from replicate runs for Vendor D at US 231A (Site 5) 


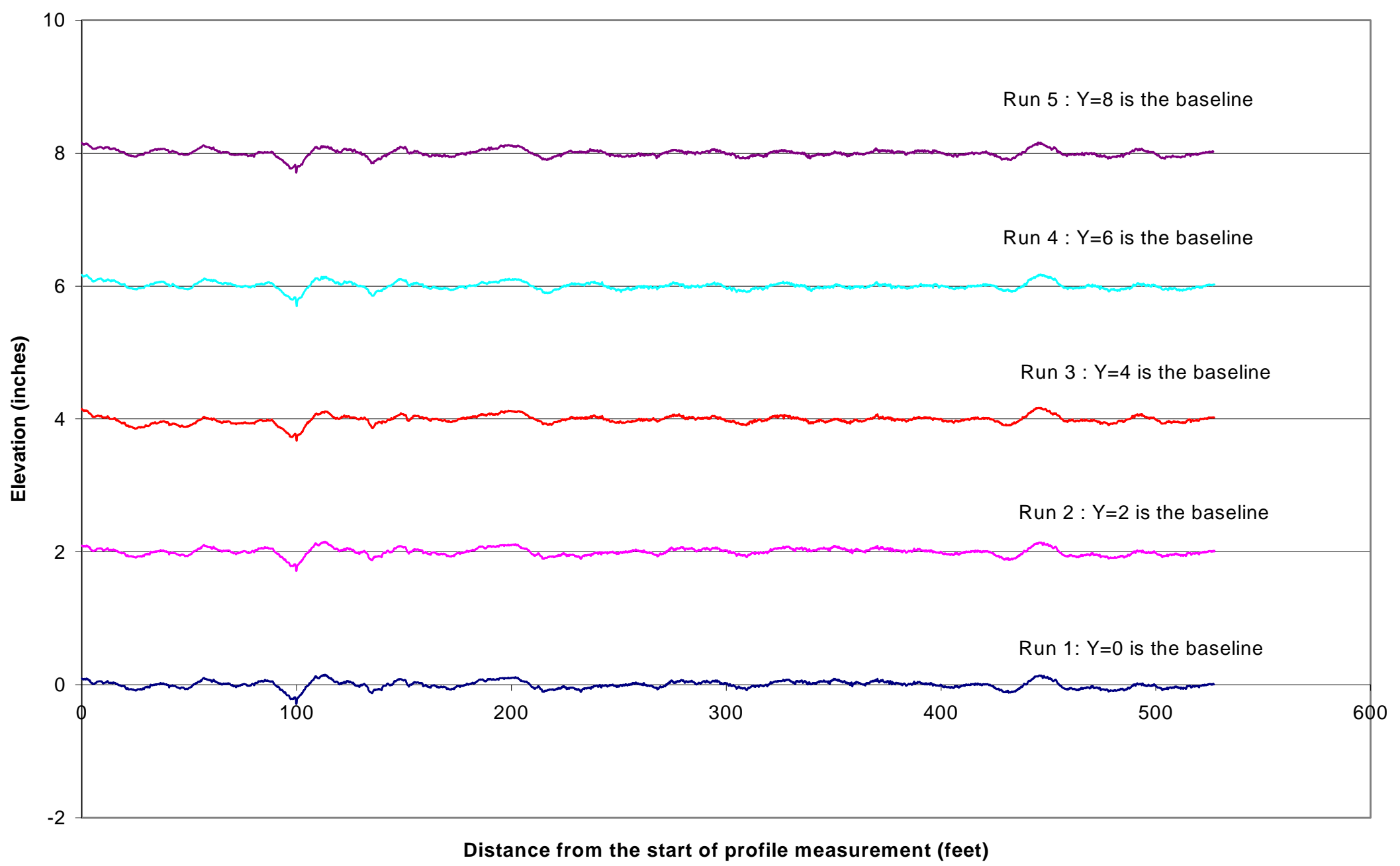

Figure E4-6 Profiles from replicate runs for Vendor D at US 231B (Site 6) 


\section{Appendix F - Between Vendor Profile Comparison}




\section{Appendix F - Between Vendor Profile Comparison}

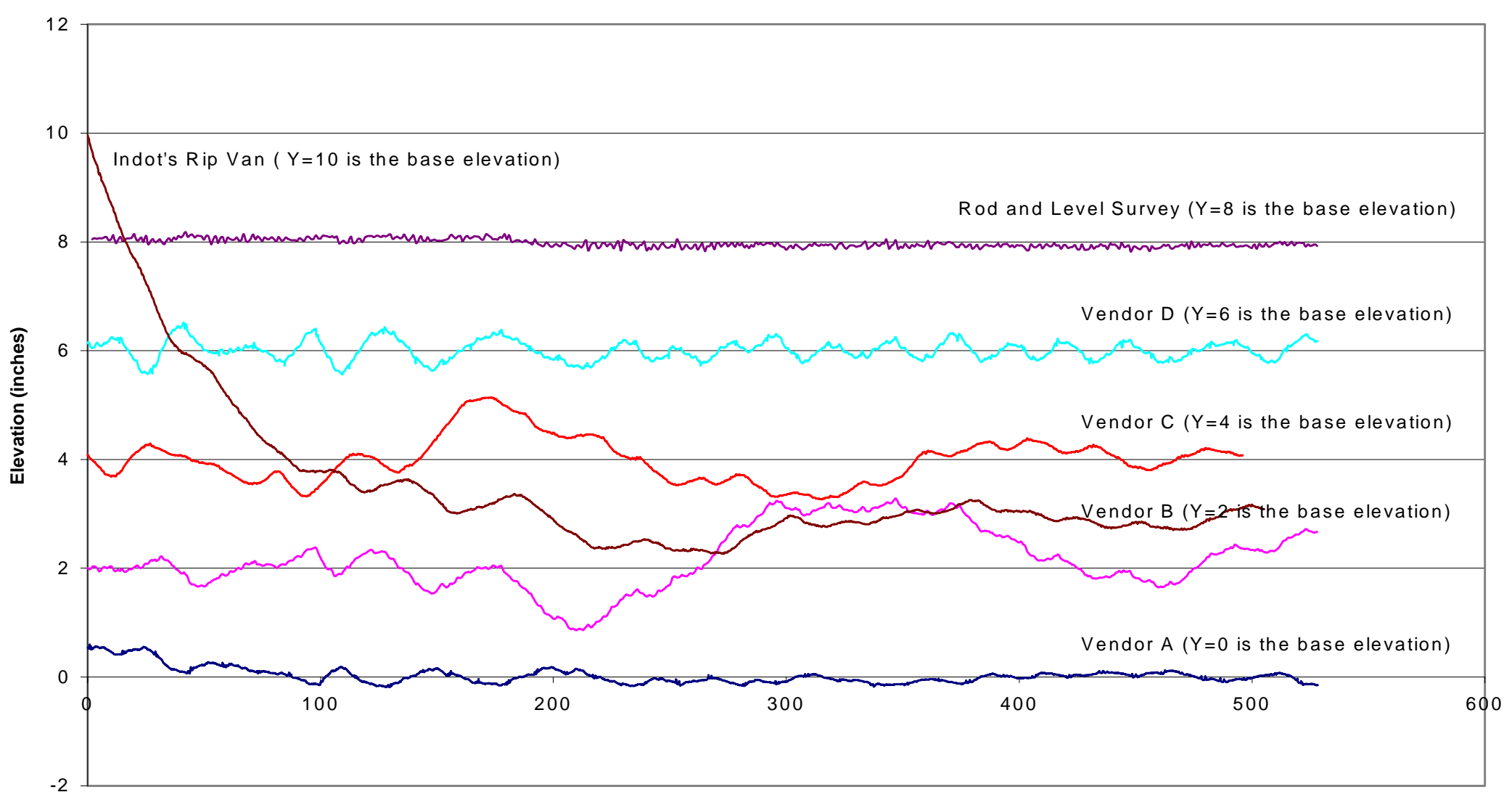

Distance from the start of profile measurement (feet)

Figure F-1 Between Vendors Profile Comparison for Covington Street (Site 1) 


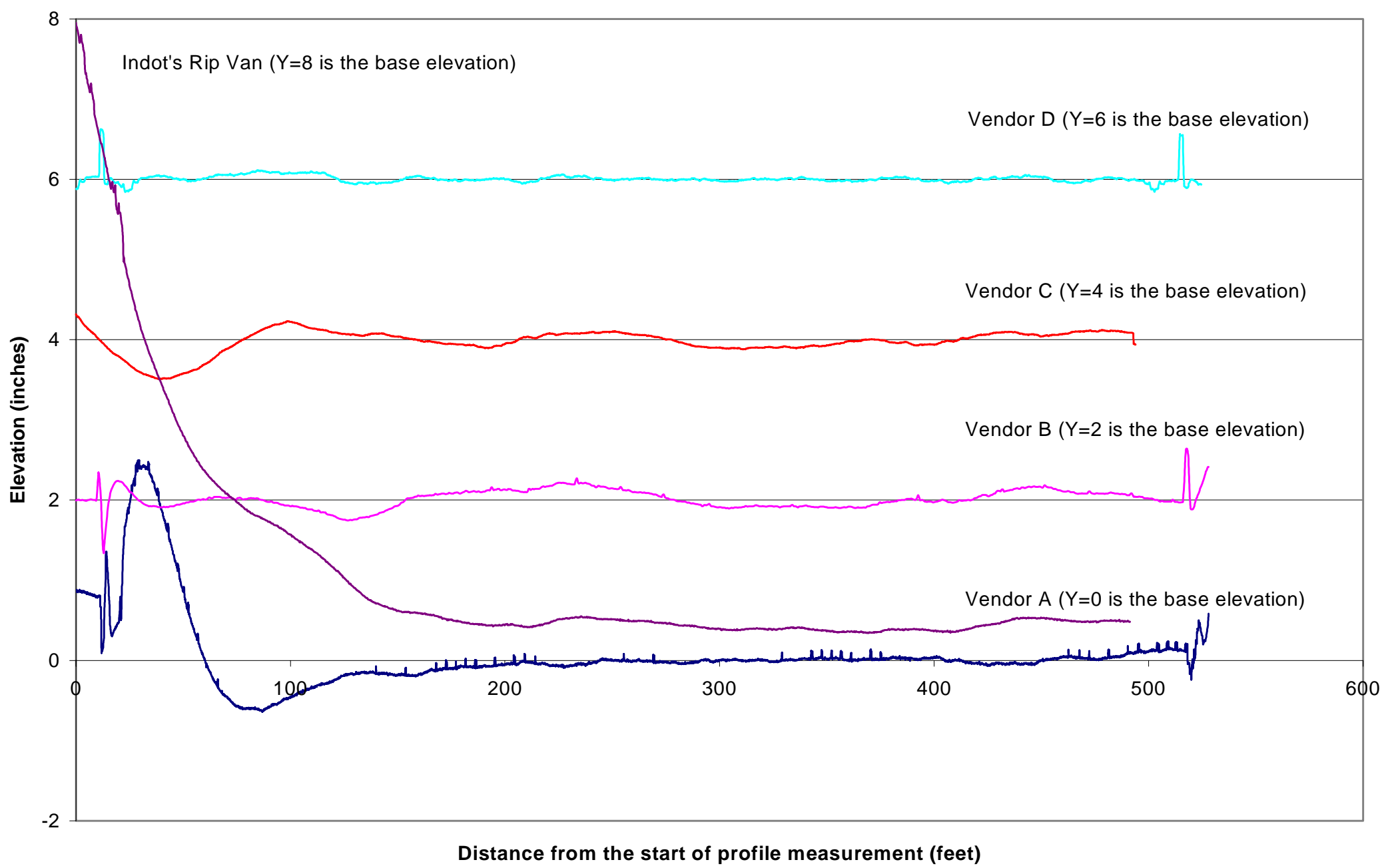

Figure F-2 Between Vendors Profile Comparison for I-65 with Plywood Strips (Site 2) 


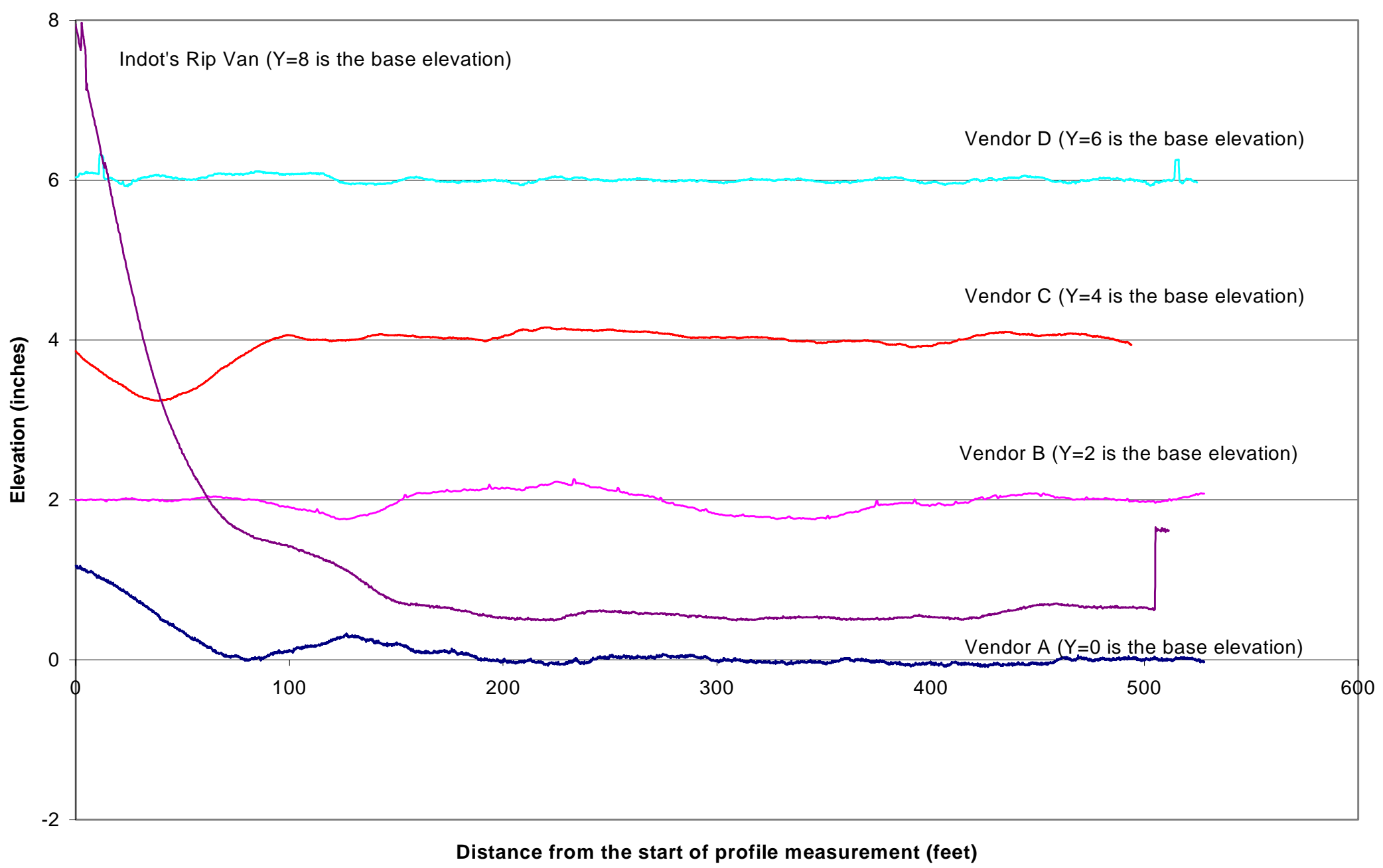

Figure F-3 Between Vendors Profile Comparison for I-65 without Plywood Strips (Site 2b) 


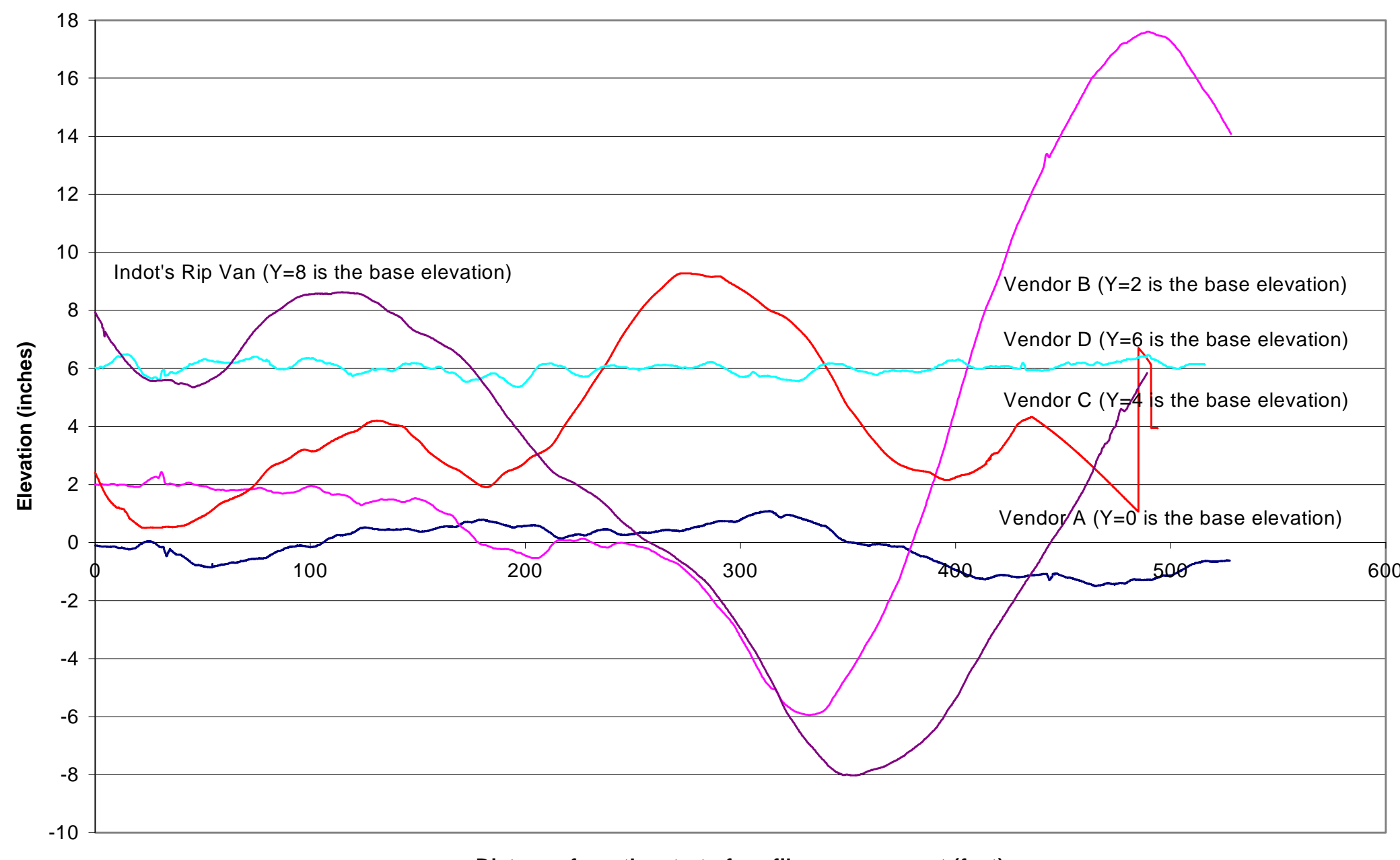

Distance from the start of profile measurement (feet)

Figure F-4 Between Vendors Profile Comparison for Prairie Street with Plywood Strips (Site 3) 


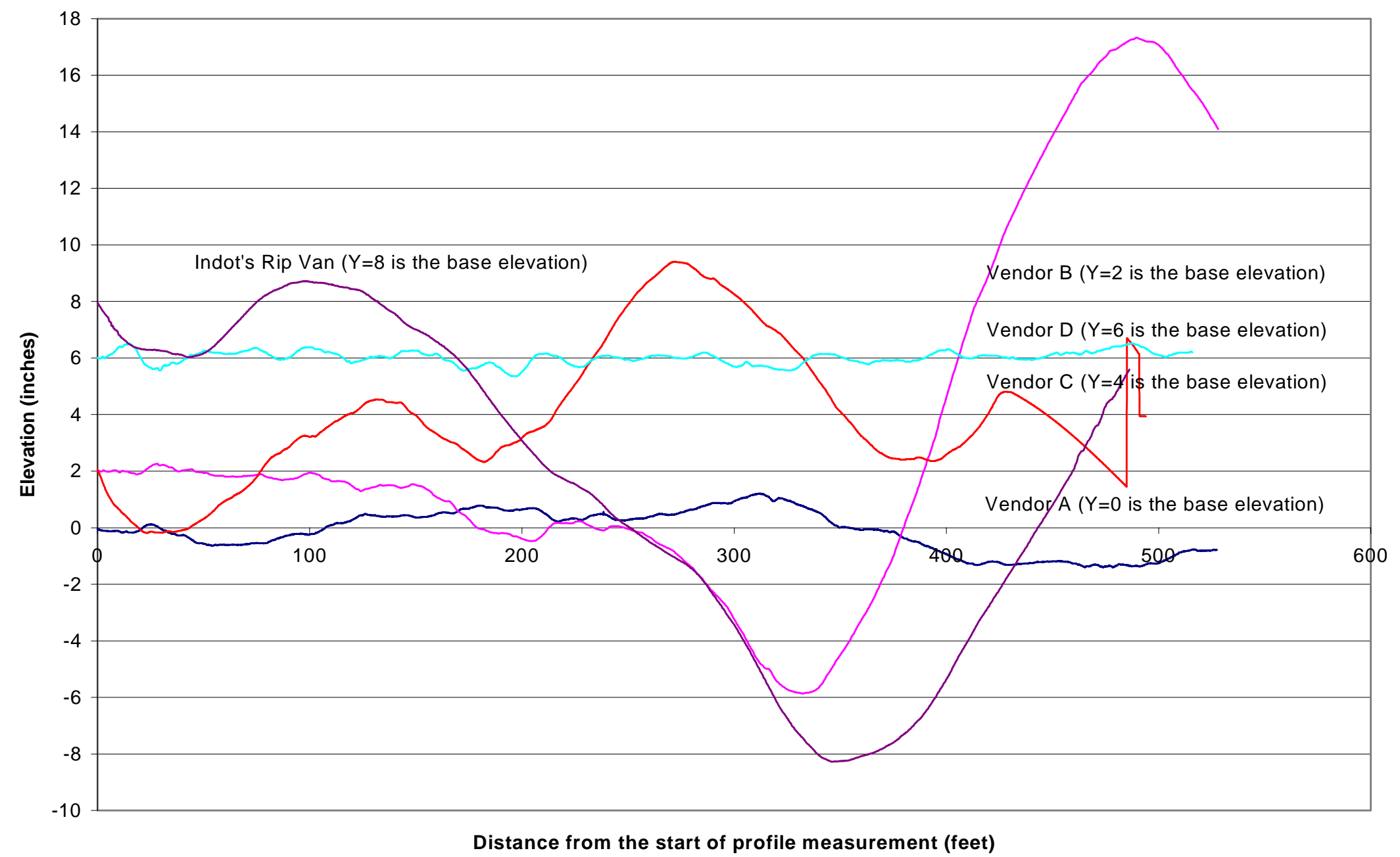

Figure F-5 Between Vendors Profile Comparison for Prairie Street without Plywood Strips (Site 3b) 


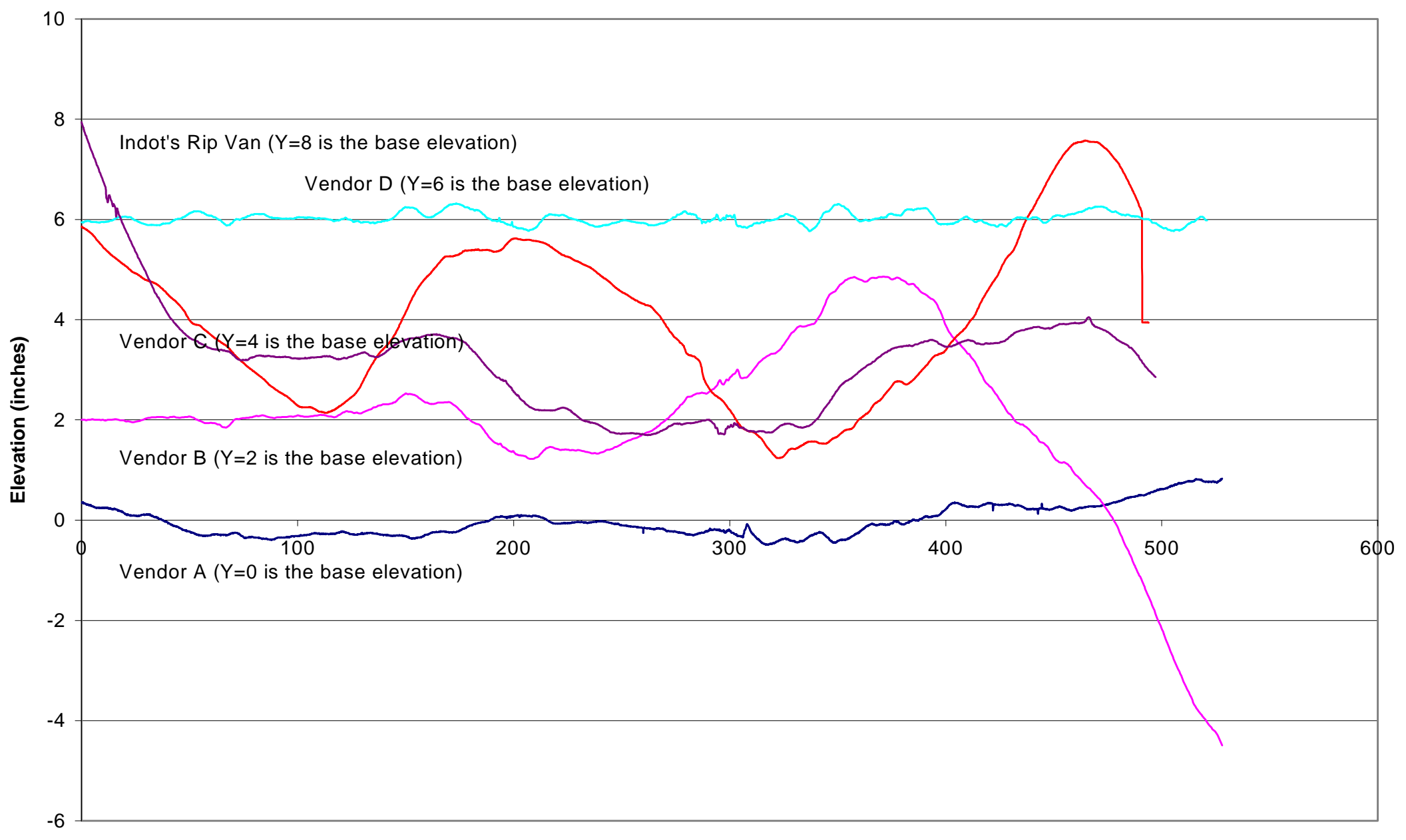

Distance from the start of profile measurement (feet)

Figure F-6 Between Vendors Profile Comparison for Vinton Street (Site 4) 


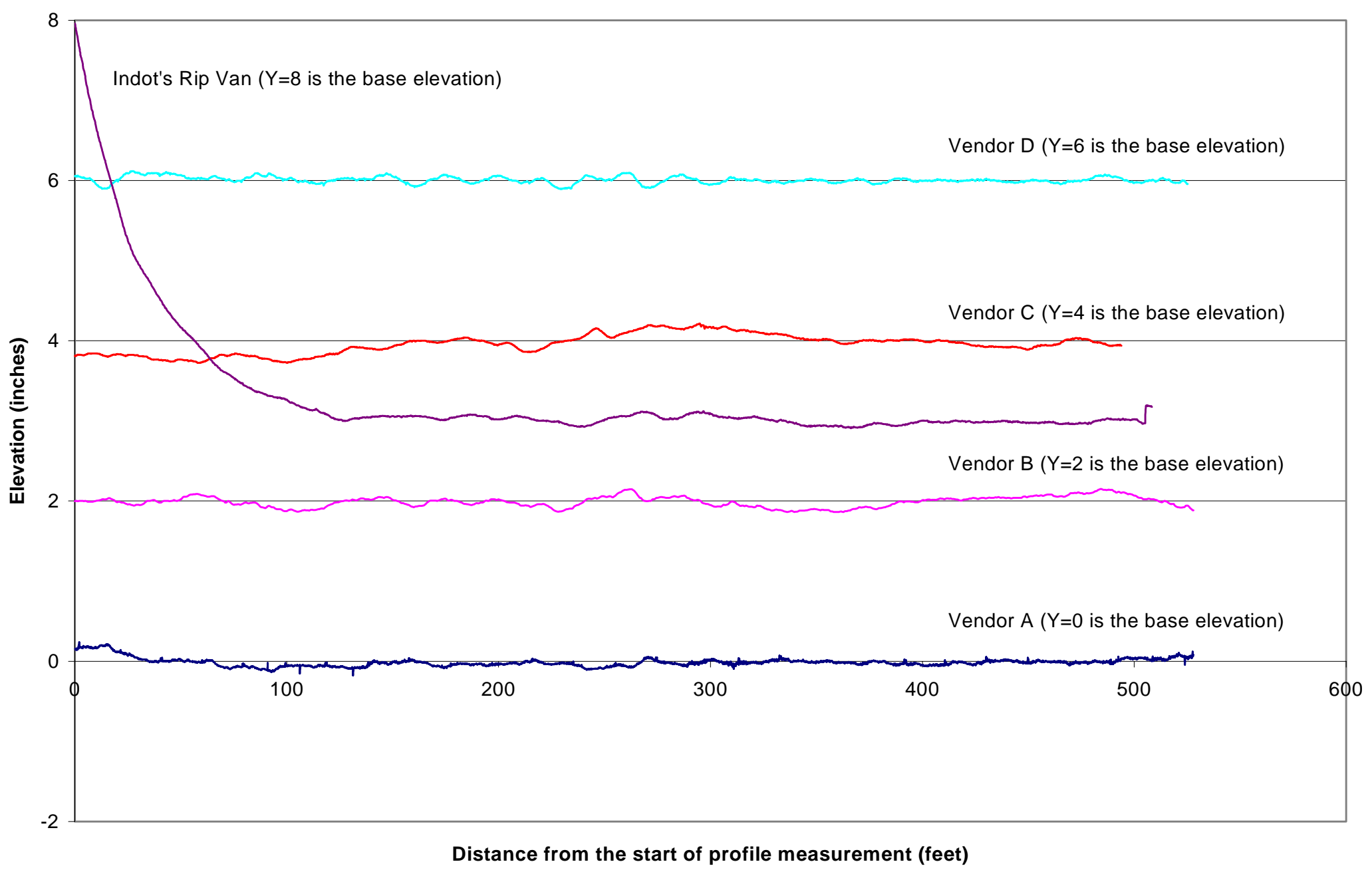

Figure F-7 Between Vendors Profile Comparison for US 231A (Site 5) 


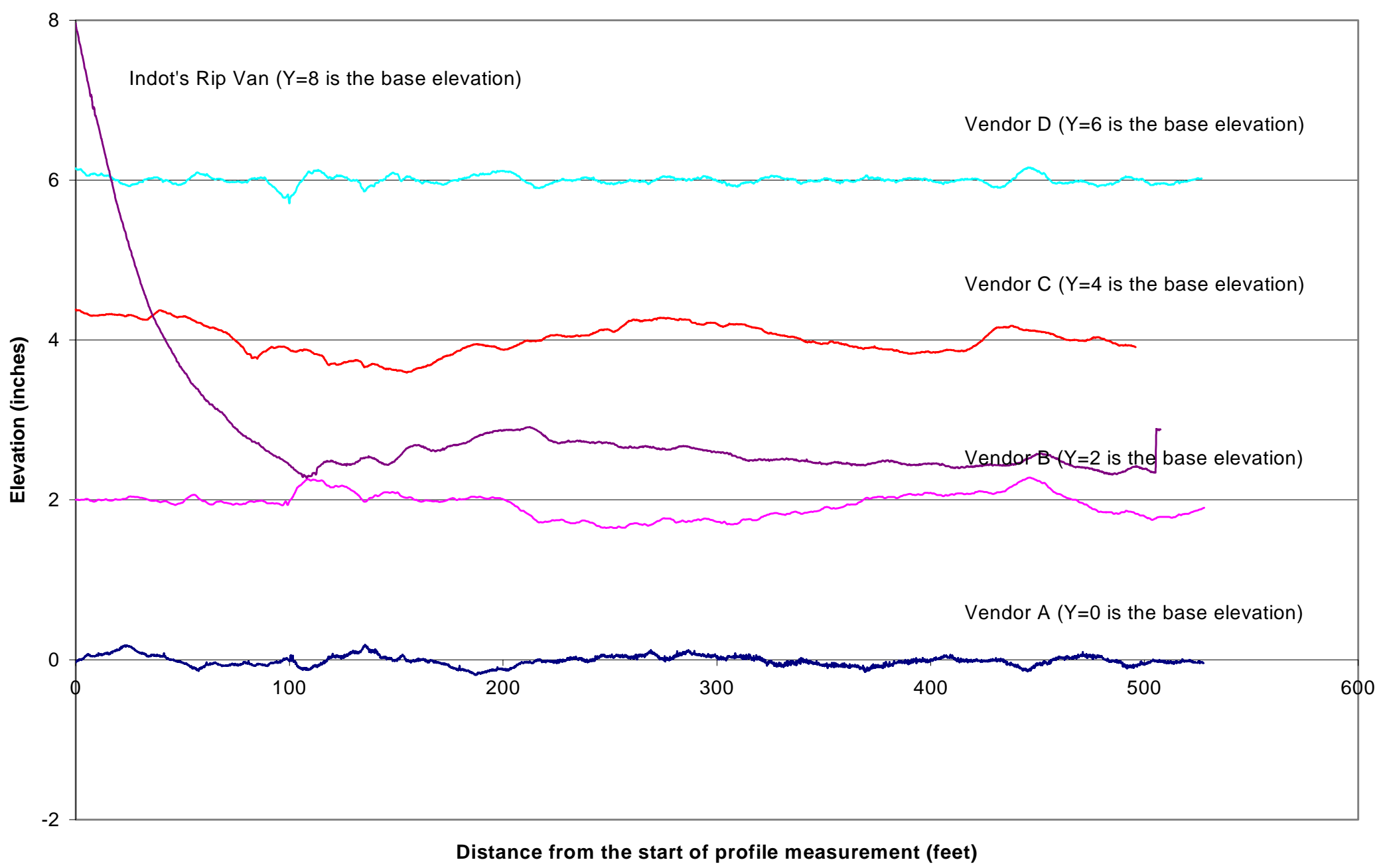

Figure F-8 Between Vendors Profile Comparison for US 231B (Site 6) 
Appendix G - Summary $h$ and $k$ analysis 


\section{Appendix G - Summary $h$ and $k$ analysis}

Case 1 - All profilers, all sites (with and without board) and all replicates for the field test data from Indiana

Table G-1-1 Case 1 IRI Consistency Statistics

\begin{tabular}{|c|c|c|c|c|c|c|c|c|c|c|c|c|c|c|c|c|}
\hline \multirow{3}{*}{ Vendor } & \multicolumn{16}{|c|}{ Site } \\
\hline & \multicolumn{2}{|c|}{1} & \multicolumn{2}{|c|}{2 with board } & \multicolumn{2}{|c|}{2 no board } & \multicolumn{2}{|c|}{3 with board } & \multicolumn{2}{|c|}{3 no board } & \multicolumn{2}{|c|}{4} & \multicolumn{2}{|c|}{5} & \multicolumn{2}{|c|}{6} \\
\hline & $\mathrm{h}$ & $\mathrm{k}$ & $\mathrm{h}$ & $\mathrm{k}$ & $\mathrm{h}$ & $\mathrm{k}$ & $\mathrm{h}$ & $\mathrm{k}$ & $\mathrm{h}$ & $\mathrm{k}$ & $\mathrm{h}$ & $\mathrm{k}$ & $\mathrm{h}$ & $\mathrm{k}$ & $\mathrm{h}$ & $\mathrm{k}$ \\
\hline $\mathrm{A}$ & -1.5 & 1.56 & 1.41 & 1.94 & 1.44 & 0.41 & -0.91 & 1 & -1.14 & 1.53 & -1.33 & 1.33 & 1.38 & 0 & 1.47 & 1.87 \\
\hline $\mathrm{B}$ & 0.44 & 0.87 & -0.19 & 0.36 & -0.14 & 0.42 & -0.72 & 0.55 & -0.44 & 0.33 & 0.03 & 0.98 & -0.7 & 0.4 & -0.74 & 0.49 \\
\hline $\mathrm{C}$ & 0.49 & 0.27 & -0.94 & 0.04 & -0.82 & 0.42 & 1.23 & 1.61 & 1.15 & 0.98 & 0.23 & 0.4 & -0.77 & 0.14 & -0.5 & 0.44 \\
\hline $\mathrm{D}$ & 0.56 & 0.86 & -0.28 & 0.35 & -0.49 & 1.87 & 0.4 & 0.33 & 0.43 & 0.77 & 1.08 & 1.06 & 0.09 & 1.95 & -0.23 & 0.25 \\
\hline
\end{tabular}

h-critical $=1.49 \quad$ k-critical $=1.66 \quad$ bolded numbers indicate values greater than h- or k-critical

Table G-1-2 Case 1 PI with 0.0 inch blanking band Consistency Statistics

\begin{tabular}{|c|c|c|c|c|c|c|c|c|c|c|c|c|c|c|c|c|}
\hline \multirow{3}{*}{ Vendor } & \multicolumn{16}{|c|}{ Site } \\
\hline & \multicolumn{2}{|c|}{1} & \multicolumn{2}{|c|}{2 with board } & \multicolumn{2}{|c|}{2 no board } & \multicolumn{2}{|c|}{3 with board } & \multicolumn{2}{|c|}{3 no board } & \multicolumn{2}{|c|}{4} & \multicolumn{2}{|c|}{5} & \multicolumn{2}{|c|}{6} \\
\hline & $\mathrm{h}$ & $\mathrm{k}$ & $\mathrm{h}$ & $\mathrm{k}$ & $\mathrm{h}$ & $\mathrm{k}$ & $\mathrm{h}$ & $\mathrm{k}$ & $\mathrm{h}$ & $\mathrm{k}$ & $\mathrm{h}$ & $\mathrm{k}$ & $\mathrm{h}$ & $\mathrm{k}$ & $\mathrm{h}$ & $\mathrm{k}$ \\
\hline $\mathrm{A}$ & -1.49 & 1.89 & 1.36 & 1.23 & -0.81 & 0.61 & -1.49 & 1.13 & -1.45 & 0.5 & -1.5 & 0.44 & -1.31 & 1.42 & -1.46 & 1.25 \\
\hline $\mathrm{B}$ & 0.37 & 0.45 & -0.02 & 1.43 & 1.45 & 1.61 & 0.62 & 0.37 & 0.23 & 0.41 & 0.39 & 1.54 & 1.09 & 1.27 & 0.71 & 0.43 \\
\hline $\mathrm{C}$ & 0.63 & 0.38 & -1.02 & 0.51 & -0.48 & 0.26 & 0.54 & 1.39 & 0.84 & 1.78 & 0.54 & 1.04 & -0.09 & 0.49 & 0.58 & 0.72 \\
\hline $\mathrm{D}$ & 0.48 & 0.27 & -0.33 & 0.42 & -0.15 & 0.98 & 0.32 & 0.81 & 0.38 & 0.65 & 0.57 & 0.58 & 0.3 & 0.36 & 0.17 & 1.32 \\
\hline
\end{tabular}

h-critical $=1.49 \quad \mathrm{k}$-critical $=1.66 \quad$ bolded numbers indicate values greater than $\mathrm{h}$ - or $\mathrm{k}$-critical

Table G-1-3 Case 1 PI with 0.2 inch blanking band Consistency Statistics

\begin{tabular}{|c|c|c|c|c|c|c|c|c|c|c|c|c|c|c|c|c|}
\hline \multirow{3}{*}{ Vendor } & \multicolumn{16}{|c|}{ Site } \\
\hline & \multicolumn{2}{|c|}{1} & \multicolumn{2}{|c|}{2 with baord } & \multicolumn{2}{|c|}{2 no board } & \multicolumn{2}{|c|}{3 with board } & \multicolumn{2}{|c|}{3 no board } & \multicolumn{2}{|c|}{4} & \multicolumn{2}{|c|}{5} & \multicolumn{2}{|c|}{6} \\
\hline & $\mathrm{h}$ & $\mathrm{k}$ & $\mathrm{h}$ & $\mathrm{k}$ & $\mathrm{h}$ & $\mathrm{k}$ & $\mathrm{h}$ & $\mathrm{k}$ & $\mathrm{h}$ & $\mathrm{k}$ & $\mathrm{h}$ & $\mathrm{k}$ & $\mathrm{h}$ & $\mathrm{k}$ & $\mathrm{h}$ & $\mathrm{k}$ \\
\hline $\mathrm{A}$ & -1.49 & 1.21 & 1.44 & 1.82 & 0 & 0 & -1.06 & 0.92 & -1.02 & 1.68 & 0.59 & 1.66 & -1.16 & 1.44 & -1.35 & 1.54 \\
\hline $\mathrm{B}$ & 0.36 & 1.44 & -0.28 & 0.55 & 0 & 0 & 0.33 & 0.53 & -0.02 & 0.52 & -0.75 & 0.68 & -0.42 & 0.58 & 1 & 0.9 \\
\hline $\mathrm{C}$ & 0.64 & 0.24 & -0.87 & 0.21 & 0 & 0 & 1.23 & 1.67 & 1.36 & 0.86 & 1.1 & 0.79 & 0.44 & 1.13 & -0.06 & 0.43 \\
\hline $\mathrm{D}$ & 0.49 & 0.63 & -0.29 & 0.59 & 0 & 0 & -0.5 & 0.32 & -0.32 & 0.42 & -0.94 & 0.39 & 1.13 & 0.57 & 0.42 & 0.81 \\
\hline
\end{tabular}

h-critical $=1.49 \quad$ k-critical $=1.66 \quad$ bolded numbers indicate values greater than h- or k-critical

Case 2 - All profilers, all sites (only without board data) and all replicates for the field test data from Indiana 
Table G-2-1 Case 2 IRI Consistency Statistics

\begin{tabular}{|c|c|c|c|c|c|c|c|c|c|c|c|c|}
\hline \multirow{3}{*}{ Vendor } & \multicolumn{12}{|c|}{ Site } \\
\hline & \multicolumn{2}{|c|}{1} & \multicolumn{2}{|c|}{2 no board } & \multicolumn{2}{|c|}{3 no board } & \multicolumn{2}{|c|}{4} & \multicolumn{2}{|c|}{5} & \multicolumn{2}{|c|}{6} \\
\hline & $\mathrm{h}$ & $\mathrm{k}$ & $\mathrm{h}$ & $\mathrm{k}$ & $\mathrm{h}$ & $\mathrm{k}$ & $\mathrm{h}$ & $\mathrm{k}$ & $\mathrm{h}$ & $\mathrm{k}$ & $\mathrm{h}$ & $\mathrm{k}$ \\
\hline $\mathrm{A}$ & -1.50 & 1.56 & 1.44 & 0.41 & -1.14 & 1.53 & -1.33 & 1.33 & 1.38 & 0.00 & 1.47 & 1.87 \\
\hline $\mathrm{B}$ & 0.44 & 0.87 & -0.14 & 0.42 & -0.44 & 0.33 & 0.03 & 0.98 & -0.70 & 0.40 & -0.74 & 0.49 \\
\hline $\mathrm{C}$ & 0.49 & 0.27 & -0.82 & 0.42 & 1.15 & 0.98 & 0.23 & 0.40 & -0.77 & 0.14 & -0.50 & 0.44 \\
\hline $\mathrm{D}$ & 0.56 & 0.86 & -0.49 & 1.87 & 0.43 & 0.77 & 1.08 & 1.06 & 0.09 & 1.95 & -0.23 & 0.25 \\
\hline
\end{tabular}

$\mathrm{h}$-critical $=1.49 \quad \mathrm{k}$-critical $=1.66 \quad$ bolded numbers indicate values greater than $\mathrm{h}$ - or $\mathrm{k}$-critical

Table G-2-2 Case 2 PI with 0.0 inch blanking band Consistency Statistics

\begin{tabular}{|c|c|c|c|c|c|c|c|c|c|c|c|c|}
\hline \multirow{3}{*}{ Vendor } & \multicolumn{12}{|c|}{ Site } \\
\hline & \multicolumn{2}{|c|}{1} & \multicolumn{2}{|c|}{2 no board } & \multicolumn{2}{|c|}{3 no board } & \multicolumn{2}{|c|}{4} & \multicolumn{2}{|c|}{5} & \multicolumn{2}{|c|}{6} \\
\hline & $\mathrm{h}$ & $\mathrm{k}$ & $\mathrm{h}$ & $\mathrm{k}$ & $\mathrm{h}$ & $\mathrm{k}$ & $\mathrm{h}$ & $\mathrm{k}$ & $\mathrm{h}$ & $\mathrm{k}$ & $\mathrm{h}$ & $\mathrm{k}$ \\
\hline $\mathrm{A}$ & -1.49 & 1.89 & -0.81 & 0.61 & -1.45 & 0.50 & -1.50 & 0.44 & -1.31 & 1.42 & -1.46 & 1.25 \\
\hline B & 0.37 & 0.45 & 1.45 & 1.61 & 0.23 & 0.41 & 0.39 & 1.54 & 1.09 & 1.27 & 0.71 & 0.43 \\
\hline $\mathrm{C}$ & 0.63 & 0.38 & -0.48 & 0.26 & 0.84 & 1.78 & 0.54 & 1.04 & -0.09 & 0.49 & 0.58 & 0.72 \\
\hline $\mathrm{D}$ & 0.48 & 0.27 & -0.15 & 0.98 & 0.38 & 0.65 & 0.57 & 0.58 & 0.30 & 0.36 & 0.17 & 1.32 \\
\hline
\end{tabular}

h-critical $=1.49 \quad \mathrm{k}$-critical $=1.66 \quad$ bolded numbers indicate values greater than $\mathrm{h}$ - or $\mathrm{k}$-critical

Table G-2-3 Case 2 PI with 0.2 inch blanking band Consistency Statistics

\begin{tabular}{|c|c|c|c|c|c|c|c|c|c|c|c|c|}
\hline \multirow{3}{*}{ Vendor } & \multicolumn{12}{|c|}{ Site } \\
\hline & \multicolumn{2}{|c|}{1} & \multicolumn{2}{|c|}{2 no board } & \multicolumn{2}{|c|}{3 no board } & \multicolumn{2}{|c|}{4} & \multicolumn{2}{|c|}{5} & \multicolumn{2}{|c|}{6} \\
\hline & $\mathrm{h}$ & $\mathrm{k}$ & $\mathrm{h}$ & $\mathrm{k}$ & $\mathrm{h}$ & $\mathrm{k}$ & $\mathrm{h}$ & $\mathrm{k}$ & $\mathrm{h}$ & $\mathrm{k}$ & $\mathrm{h}$ & $\mathrm{k}$ \\
\hline A & -1.49 & 1.21 & 0.00 & 0.00 & -1.02 & 1.68 & 0.59 & 1.66 & -1.16 & 1.44 & -1.35 & 1.54 \\
\hline B & 0.36 & 1.44 & 0.00 & 0.00 & -0.02 & 0.52 & -0.75 & 0.68 & -0.42 & 0.58 & 1.00 & 0.90 \\
\hline $\mathrm{C}$ & 0.64 & 0.24 & 0.00 & 0.00 & 1.36 & 0.86 & 1.10 & 0.79 & 0.44 & 1.13 & -0.06 & 0.43 \\
\hline $\mathrm{D}$ & 0.49 & 0.63 & 0.00 & 0.00 & -0.32 & 0.42 & -0.94 & 0.39 & 1.13 & 0.57 & 0.42 & 0.81 \\
\hline
\end{tabular}

$\mathrm{h}$-critical $=1.49 \quad \mathrm{k}$-critical $=1.66 \quad$ bolded numbers indicate values greater than $\mathrm{h}$ - or $\mathrm{k}$-critical

Case 3 - All profilers except the profiler from Vendor A, all sites (with and without board) and all replicates for the

\section{field test data from Indiana}


Table G-3-1 Case 3 IRI Consistency Statistics

\begin{tabular}{|c|c|c|c|c|c|c|c|c|c|c|c|c|c|c|c|c|}
\hline \multirow{3}{*}{ Vendor } & \multicolumn{16}{|c|}{ Site } \\
\hline & \multicolumn{2}{|c|}{1} & \multicolumn{2}{|c|}{2 with board } & \multicolumn{2}{|c|}{2 no board } & \multicolumn{2}{|c|}{3 with board } & \multicolumn{2}{|c|}{3 no board } & \multicolumn{2}{|c|}{4} & \multicolumn{2}{|c|}{5} & \multicolumn{2}{|c|}{6} \\
\hline & $\mathrm{h}$ & $\mathrm{k}$ & $\mathrm{h}$ & $\mathrm{k}$ & $\mathrm{h}$ & $\mathrm{k}$ & $\mathrm{h}$ & $\mathrm{k}$ & $\mathrm{h}$ & $\mathrm{k}$ & $\mathrm{h}$ & $\mathrm{k}$ & $\mathrm{h}$ & $\mathrm{k}$ & $\mathrm{h}$ & $\mathrm{k}$ \\
\hline B & -0.92 & 1.2 & 0.69 & 1.23 & 1.01 & 0.38 & -1.05 & 0.55 & -1.03 & 0.44 & -0.75 & 1.13 & -0.5 & 0.35 & -0.98 & 1.21 \\
\hline $\mathrm{C}$ & -0.14 & 0.37 & -1.15 & 0.13 & -0.99 & 0.37 & 0.95 & 1.61 & 0.97 & 1.32 & -0.39 & 0.47 & -0.65 & 0.12 & -0.03 & 1.08 \\
\hline $\mathrm{D}$ & 1.06 & 1.19 & 0.46 & 1.22 & -0.01 & 1.65 & 0.1 & 0.33 & 0.06 & 1.03 & 1.14 & 1.22 & 1.15 & 1.69 & -1.02 & 0.61 \\
\hline
\end{tabular}

$\mathrm{h}$-critical $=1.15 \quad \mathrm{k}$-critical $=1.56 \quad$ bolded numbers indicate values greater than $\mathrm{h}$ - or $\mathrm{k}$-critical

Table G-3-2 Case 3 PI with 0.0 inch blanking band Consistency Statistics

\begin{tabular}{|c|c|c|c|c|c|c|c|c|c|c|c|c|c|c|c|c|}
\hline \multirow{3}{*}{ Vendor } & \multicolumn{16}{|c|}{ Site } \\
\hline & \multicolumn{2}{|c|}{1} & \multicolumn{2}{|c|}{2 with board } & \multicolumn{2}{|c|}{2 no board } & \multicolumn{2}{|c|}{3 with board } & \multicolumn{2}{|c|}{3 no board } & \multicolumn{2}{|c|}{4} & \multicolumn{2}{|c|}{5} & \multicolumn{2}{|c|}{6} \\
\hline & $\mathrm{h}$ & $\mathrm{k}$ & $\mathrm{h}$ & $\mathrm{k}$ & $\mathrm{h}$ & $\mathrm{k}$ & $\mathrm{h}$ & $\mathrm{k}$ & $\mathrm{h}$ & $\mathrm{k}$ & $\mathrm{h}$ & $\mathrm{k}$ & $\mathrm{h}$ & $\mathrm{k}$ & $\mathrm{h}$ & $\mathrm{k}$ \\
\hline $\mathrm{B}$ & -0.94 & 1.2 & 0.85 & 1.57 & 1.14 & 1.47 & 0.81 & 0.39 & -0.79 & 0.37 & -1.14 & 1.37 & 1.09 & 1.56 & 0.78 & 0.47 \\
\hline $\mathrm{C}$ & 1.05 & 1.02 & -1.1 & 0.56 & -0.73 & 0.24 & 0.3 & 1.46 & 1.12 & 1.59 & 0.43 & 0.93 & -0.87 & 0.6 & 0.35 & 0.79 \\
\hline $\mathrm{D}$ & -0.11 & 0.72 & 0.25 & 0.46 & -0.41 & 0.89 & -1.12 & 0.85 & -0.34 & 0.58 & 0.71 & 0.52 & -0.22 & 0.44 & -1.13 & 1.46 \\
\hline
\end{tabular}

h-critical $=1.15 \quad$ k-critical $=1.56 \quad$ bolded numbers indicate values greater than $\mathrm{h}$ - or $\mathrm{k}$-critical

Table G-3-3 Case 3 PI with 0.2 inch blanking band Consistency Statistics

\begin{tabular}{|c|c|c|c|c|c|c|c|c|c|c|c|c|c|c|c|c|}
\hline \multirow{3}{*}{ Vendor } & \multicolumn{16}{|c|}{ Site } \\
\hline & \multicolumn{2}{|c|}{1} & \multicolumn{2}{|c|}{2 with board } & \multicolumn{2}{|c|}{2 no board } & \multicolumn{2}{|c|}{3 with board } & \multicolumn{2}{|c|}{3 no board } & \multicolumn{2}{|c|}{4} & \multicolumn{2}{|c|}{5} & \multicolumn{2}{|c|}{6} \\
\hline & $\mathrm{h}$ & $\mathrm{k}$ & $\mathrm{h}$ & $\mathrm{k}$ & $\mathrm{h}$ & $\mathrm{k}$ & $\mathrm{h}$ & $\mathrm{k}$ & $\mathrm{h}$ & $\mathrm{k}$ & $\mathrm{h}$ & $\mathrm{k}$ & $\mathrm{h}$ & $\mathrm{k}$ & $\mathrm{h}$ & $\mathrm{k}$ \\
\hline B & -0.98 & 1.57 & 0.58 & 1.14 & 0 & 0 & -0.03 & 0.31 & -0.4 & 0.82 & -0.5 & 1.06 & -1.03 & 0.72 & 1.03 & 1.22 \\
\hline $\mathrm{C}$ & 1.02 & 0.26 & -1.15 & 0.43 & 0 & 0 & 1.01 & 1.62 & 1.14 & 1.37 & 1.15 & 1.23 & 0.07 & 1.4 & -0.97 & 0.58 \\
\hline $\mathrm{D}$ & -0.03 & 0.68 & 0.57 & 1.23 & 0 & 0 & -0.99 & 0.52 & -0.74 & 0.67 & -0.66 & 0.61 & 0.96 & 0.72 & -0.06 & 1.09 \\
\hline
\end{tabular}

$\mathrm{h}$-critical $=1.15 \quad \mathrm{k}$-critical $=1.56 \quad$ bolded numbers indicate values greater than $\mathrm{h}$ - or k-critical

Case 4 - All lightweight profilers, all sites, and five randomly selected replicates for the field-test data from

\section{Connecticut}


Table G-4 Case 4 IRI Consistency Statistics

\begin{tabular}{|c|c|c|c|c|c|c|}
\hline \multirow{3}{*}{ Vendor } & \multicolumn{7}{|c|}{ Site } & \multicolumn{2}{c|}{3} \\
\cline { 2 - 7 } & \multicolumn{2}{|c|}{1} & \multicolumn{2}{c|}{2} & $\mathrm{~h}$ & $\mathrm{k}$ \\
\cline { 2 - 7 } & $\mathrm{h}$ & $\mathrm{k}$ & $\mathrm{h}$ & $\mathrm{k}$ & 1.15 & 0.53 \\
\hline $\mathrm{A}$ & -1.03 & 1.31 & 0.59 & 1.68 & 0.76 & 0.64 \\
\hline $\mathrm{B}$ & -0.2 & 1.31 & -1.39 & 1.23 & 0.09 & 2.06 \\
\hline $\mathrm{C}$ & -0.15 & 0.57 & -0.48 & 0.34 & -1.22 & 0.16 \\
\hline $\mathrm{D}$ & 1.67 & 1.03 & 0.05 & 0.6 & -0.78 & 0.21 \\
\hline $\mathrm{E}^{*}$ & -0.28 & 0.42 & 1.23 & 0.46 & \\
\hline
\end{tabular}

$\mathrm{h}$-critical $=1.75 \mathrm{k}$-critical $=1.71$ bolded numbers indicate values greater than $\mathrm{h}$ - or $\mathrm{k}$ - critical

* Profiler is from Trigg Industries

Case 5 - All the common lightweight profilers, all sites (considering only without board data from Indiana field tests), and five replicates for the combined data from the field tests conducted at Connecticut and Indiana 
Table G-5 Case 5 IRI Consistency Statistics for the combined data from Indiana and Connecticut

\begin{tabular}{|c|c|c|c|c|c|c|c|c|c|c|}
\hline \multirow{3}{*}{ Vendor } & \multicolumn{10}{|c|}{ Site } \\
\cline { 2 - 12 } & \multicolumn{1}{|c|}{1 (Conn.) } & \multicolumn{2}{|c|}{2 (Conn.) } & \multicolumn{2}{c|}{3 (Conn.) } & \multicolumn{2}{c|}{1 (Indiana) } & \multicolumn{2}{|c|}{2 (Indiana) } \\
\cline { 2 - 12 } & $\mathrm{h}$ & $\mathrm{k}$ & $\mathrm{h}$ & $\mathrm{k}$ & $\mathrm{h}$ & $\mathrm{k}$ & $\mathrm{h}$ & $\mathrm{k}$ & $\mathrm{h}$ & $\mathrm{k}$ \\
\hline $\mathrm{A}$ & -0.97 & 1.19 & 1.06 & 1.53 & 0.92 & 0.47 & $\mathbf{- 1 . 5}$ & 1.58 & 1.44 & 0.41 \\
\hline $\mathrm{B}$ & -0.24 & 1.20 & -1.28 & 1.12 & 0.55 & 0.57 & 0.44 & 0.82 & -0.14 & 0.42 \\
\hline $\mathrm{C}$ & -0.19 & 0.52 & -0.21 & 0.31 & -0.1 & $\mathbf{1 . 8 5}$ & 0.49 & 0.27 & -0.82 & 0.42 \\
\hline $\mathrm{D}$ & 1.40 & 0.94 & 0.43 & 0.55 & -1.36 & 0.14 & 0.57 & 0.88 & -0.49 & $\mathbf{1 . 8 7}$ \\
\hline
\end{tabular}

\section{(continued )}

\begin{tabular}{|c|c|c|c|c|c|c|c|c|}
\hline \multirow{3}{*}{ Vendor } & \multicolumn{9}{|c|}{ Site } \\
\cline { 2 - 9 } & \multicolumn{2}{|c|}{3 (Indiana) } & \multicolumn{2}{c|}{4 (Indiana) } & \multicolumn{2}{c|}{5 (Indiana) } & \multicolumn{2}{c|}{6 (Indiana) } \\
\cline { 2 - 9 } & $\mathrm{h}$ & $\mathrm{k}$ & $\mathrm{h}$ & $\mathrm{k}$ & $\mathrm{h}$ & $\mathrm{k}$ & $\mathrm{h}$ & $\mathrm{k}$ \\
\hline $\mathrm{A}$ & -1.14 & 1.53 & -1.33 & 1.33 & 1.38 & 0 & 1.47 & $\mathbf{1 . 8 7}$ \\
\hline $\mathrm{B}$ & -0.44 & 0.33 & 0.03 & 0.98 & -0.7 & 0.4 & -0.74 & 0.49 \\
\hline $\mathrm{C}$ & 1.15 & 0.98 & 0.23 & 0.4 & 0.09 & 0.14 & -0.5 & 0.44 \\
\hline $\mathrm{D}$ & 0.43 & 0.77 & 1.08 & 1.06 & -0.77 & $\mathbf{1 . 9 5}$ & -0.23 & 0.25 \\
\hline
\end{tabular}

h-critical $=1.49 \quad k$-critical $=1.66$ bolded numbers indicate values greater than $h$ - or $k$ - critical 